\title{
Evaluation of meal and deworming programs for primary schools in rural Senegal
}

Citation for published version (APA):

Diallo, F. L. (2014). Evaluation of meal and deworming programs for primary schools in rural Senegal. [Doctoral Thesis, Maastricht University]. Datawyse / Universitaire Pers Maastricht. https://doi.org/10.26481/dis.20140617fd

Document status and date:

Published: 01/01/2014

DOI:

10.26481/dis.20140617fd

Document Version:

Publisher's PDF, also known as Version of record

\section{Please check the document version of this publication:}

- A submitted manuscript is the version of the article upon submission and before peer-review. There can be important differences between the submitted version and the official published version of record. People interested in the research are advised to contact the author for the final version of the publication, or visit the DOI to the publisher's website.

- The final author version and the galley proof are versions of the publication after peer review.

- The final published version features the final layout of the paper including the volume, issue and page numbers.

Link to publication

\footnotetext{
General rights rights.

- You may freely distribute the URL identifying the publication in the public portal. please follow below link for the End User Agreement:

www.umlib.nl/taverne-license

Take down policy

If you believe that this document breaches copyright please contact us at:

repository@maastrichtuniversity.nl

providing details and we will investigate your claim.
}

Copyright and moral rights for the publications made accessible in the public portal are retained by the authors and/or other copyright owners and it is a condition of accessing publications that users recognise and abide by the legal requirements associated with these

- Users may download and print one copy of any publication from the public portal for the purpose of private study or research.

- You may not further distribute the material or use it for any profit-making activity or commercial gain

If the publication is distributed under the terms of Article $25 \mathrm{fa}$ of the Dutch Copyright Act, indicated by the "Taverne" license above, 


\title{
Evaluation of Meal and Deworming Programs for Primary Schools in Rural Senegal
}

\author{
Fatoumata Lamarana Diallo ${ }^{1}$
}


Universitaire Pers Maastricht ISBN 9789461593436 


\title{
Evaluation of Meal and Deworming Programs for Primary Schools in Rural Senegal
}

\author{
DISSERTATION \\ to obtain the degree of Doctor at \\ Maastricht University, \\ on the authority of the Rector Magnificus, Prof. dr. L.L.G. Soete, \\ in accordance with the decision of the Board of Deans, \\ to be defended in public on Tuesday, 17 June 2014, at 12.00 hours
}

by

Fatoumata Lamarana Diallo

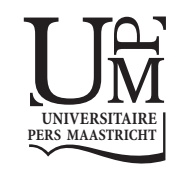




\section{Supervisor}

Prof. dr. Théophile T. Azomahou

\section{Co-supervisors}

Prof. Abdoulaye Diagne, Dakar, Senegal

Dr. Wladimir Raymond, Luxembourg

\section{Assessment Committee}

Prof. Dr. Pierre Mohnen (Chair)

Prof. dr. Mbaye Diene (University Cheikh Anta Diop. UCAD and CRES, Senegal)

Dr. Franziska Gassmann

Prof. dr. Norbert Janz (Aachen University of Applies Sciences, Germany)

Prof. dr. François Laisney (University of Strassbourg, France)

Prof. Dr. Bart Verspagen

Financial support for this dissertation has been provided by: 'Nouveau Programme de Troisième Cycle Inter-universitaire (NPTCI)', United Nations University at Maastricht (UNU-MERIT) and 'Ministère de l'Enseignement Supérieur et de la Recherche" of Senegal. 


\section{Dedication}

To my Daughter Hadja Binta and my Husband Abdourahmane Sow 


\section{Summary}

Program evaluation is an important tool to inform policy makers about the effectiveness of programs and the efficient allocation of resources. Several studies have examined the impact of school feeding and deworming programs on educational outcomes in developing countries. However, conflicting evidence still exists regarding the effectiveness of these programs on school performance. Also, there are little evidences on the cost effectiveness of these programs.

Although nutritional and health experiences have been developed in recent years in Senegal, there is no empirical study gearing towards assessing the impacts of meal and deworming programs on educational outcomes, that also addresses issues related to the complementarity or substitutability of the two programs.

This thesis aims at filling this gap. We use experimental and observational data and rely on both program evaluation and structural approaches. The thesis is organized in 5 chapters. After introduction in Chapter 1, Chapter 2 uses observational data to assess the impacts of meal and deworming programs taken on isolation. We study the impact of these programs on school performances measured by test scores, while elaborating on the determinants of test scores. This explains that the meal and the deworming programs can increase pupils' scores. The impact of each program is more important for pupils who are in school with association of mothers. Deworming in school has a positive effect on pupils' performance, while the deworming at home has a negative impact on pupils' scores. In addition, the deworming program increases significantly the scores of pupils who are in school with association of their mothers or school project. Among the determinants of test scores, education expenditure, household size, Koranic school, early childhood institution and existence of a college in the village have a positive effect on pupil's performance, while controls such as disturbed courses, temporary shelters, gender of teacher and absenteeism of teacher have negative impact on scores. In terms of policy analysis, the Local Average Treatment Effect (LATE), reveals that if the objective is to increase scores, the most cost-effective way is to combine the canteen and the association of parents compared to other options including management committee, school project, school cooperative and reduction of teacher absence.

Chapter 3 uses the observational data to assess the school feeding and deworming programs as a package on school performances (aggregate score, French score, math score, enrollment, repetition and dropout rates), while elaborating on the determinants of these performances. In this chapter we develop a model for two treatments, which makes it possible to estimate a variety of treatment effects, while also addressing issues related to complementarity or substitutability of programs. We found out that both meal and deworming programs increase scores, but the combination of the two programs is more beneficial in terms of increasing pupils' achievements. The sequence of implementation does matter. The two programs are complementary in the goal of increasing scores and promotion rates. However, they are substitutes with the aim at reducing dropouts. The cost-effectiveness analysis shows that deworming is by far cheaper than school meals. However, the implementation of meals program before deworming is more cost-effective than the reverse. In terms of the determinants of scores, the results found in this chapter are consistent with those in Chapter 2. In addition, deworming at home impact scores negatively, while existence of latrine and existence of hand washing device in school impact scores positively.

An interesting and intuitive result is that having a literate household head increases the enrollment rate. In contrast, the enrollment rate declines with school expenses. This result is important for education policy in terms of reducing the illiteracy rate in rural areas where the living standard is very low. Indeed, if policy makers seek to encourage families to send their children to school, it would be interesting to ensure that the cost of schooling is not too high. Two other results are worth noting: having a male teacher and holding arable land have negative effect on enrollment.

As a whole, our findings in terms of determinants of school performances presented in Chapters 
2 and 3 suggest that despite the fact that temporary shelters reduce dropout rates with having positive effects on enrollment and promotion rates, it also contributes to reducing pupils' achievement (scores). In addition, even if school manual can improve on the competence of pupils, when the number of pupils per manual become higher (more than 4 pupils per manual), the effect seems to be negative on scores. Implementing a policy gearing towards reducing teachers' absenteeism and disturbances will improve educational quality. It also seems important to promote more experienced teachers (from 33 years old) and female teachers. Finally, encouraging them to have professional qualification CAP and CEAP, supporting teachers with High school diploma and more as an academic qualification, while increasing the duration of continuing training for teachers are some of the measure towards improving pupil achievement. Chapter 4 uses an experimental data to assess the impact of the meal program on school performances in the presence of attrition and partial adherence. Estimation results show that the school feeding program significantly increases pupils' performances and enrollment rate, while reducing dropout rate. However, the program increases the repetition rate and decreases promotion rate, which is unexpected. This may follow from the measures taken by the Government of Senegal consisting in removing repetition during the first year of each step in the primary cycle and capped at $5 \%$ maximum rate of repetition at the end of each step.

We also found out that, the meal program has greater effect for girls compared to boys in math score, while boys outperform the girls in French score.

The cost-benefit analysis conducted in this chapter shows that the meal program is justified in terms of economic gains, while the result of the cost-effectiveness analysis is consistent with findings in Chapter 3.

Chapter 5 summarizes the key findings of all chapters, with some policy implications. It also discusses the limitations of the thesis and justifies the need for some future research projects.

Overall, this thesis provides empirical evidence on the impact of meal and deworming programs on school performances using both experimental and non-experimental studies. To our knowledge, this is the first study to show that the combination of meal and deworming programs as package would be more effective in terms of increasing school performances while giving the effect of each intervention and several other effects to inform policy makers. 


\section{Acknowledgements}

Writing this dissertation has been like going through a long journey often inhabited by strong emotions. Thank God, I have finally been able to manage all emotions in a calm, serene and wise manner.

I have to express sincere thanks and appreciation to several people and institutions that have helped me go through this journey. First of all, writing this dissertation has been made possible through a partnership between the United Nations University-Maastricht Economic and Social Research Institute on Innovation and Technology (UNU-MERIT) in Maastricht and the 'Consortium pour la Recherche Économique et Sociale (CRES)' in Dakar. I would like to take this opportunity to thank Prof. Luc Soete former Director of UNU-MERIT and current Rector Magnificus of Maastricht University, Prof. Bart Verspagen current Director of UNU-MERIT, Prof. Abdoulaye Diagne current Director of CRES and Prof. Théophile Azomahou for the scientific cooperation they have developed between the two institutions. This partnership is very instrumental towards intellectual growth in Africa, particularly for supporting young African researchers from francophone countries in Africa. Thanks to this partnership, I have benefited from two research visits to UNU-MERIT in 2010 (early June to late August 2010) and in 2011 (early February to late December 2011). During these two research visits, UNU-MERIT provided me with ideal conditions that helped me carry out my research with the opportunity to write my dissertation at Maastricht University. I would also like to express my sincere gratitude to my team of supervisors Prof.dr. Théophile Azomahou, Prof. Abdoulaye Diagne and Dr. Wladimir Raymond. Despite their numerous engagements, they were able to allocate time to supervise my thesis with rigor and keen attention. They shared their knowledge without limitations, and their guidance and supports have helped immensely to complete the thesis. I would particularly like to thank Prof. Azomahou for agreeing to promote my thesis, for believing in me and for his utmost patience. I am grateful to the members of the reading committee of this thesis, Prof.dr. Pierre Mohnen, Prof.dr. Mbaye Diene, Dr. Franziska Gassmann, Prof.dr. Norbert Janz, Prof.dr. François Laisney and Prof.dr. Bart Verspagen, for their helpful comments and suggestions which helped to improve the final draft of the thesis. Furthermore, I am deeply indebted to Prof. Pierre Mohnen for his advice, comments, suggestions and numerous discussions we had during my research visits at UNU-MERIT. I would also like to thank Wilma Coenegrachts, Marc Vleugels, Eveline in de Braek, Eric Engelen, Ad Notten and many others for their hospitality and support during my research visits at UNU-MERIT.

Secondly, I am also grateful to the 'Nouveau Programme de Troisième Cycle Inter-Universitaires (NPTCI)' that provided me a 3 years scholarship by selecting me for the NPTCI PhD program. I would also like to express my gratitude to the 'Ministère de l'Enseignement Supérieur et de la Recherche' of Senegal that provided me with a grant at the end of my thesis by selecting me for the 'Projet d'Appui à la Promotion des Enseignantes-chercheures du Sénégal (PAPES)'.

Thirdly, this thesis uses data collected in a project carried out with financial and scientific support from the Partnership for Economic Policy (PEP), with funding from the Department for International Development (DFID) of the United Kingdom (or UK Aid), and the Government of Canada through the International Development Research Center (IDRC). The CRES coordinated the project and conducted surveys in partnership with the Senegalese Ministry of Education and the World Food Programme (WFP). I would like to thank the PEP who provided financial and technical support to the project. I would also like to express my gratitude to the CRES, particularly the Director Prof. Abdoulaye Diagne for all supports, especially for providing me a suitable environment to carry out my research during recent years. I am indebted to Prof. Mbaye Diene, Prof. François J. Cabral, and all the staff of CRES and PEP Africa for their help and supports, particularly Fatou Cissé, Gaye Daffé, Mamadou Dansokho, Doudou Ndiaye, Boubacar Diao, Michel Faye, Jean J. Badji, Yaya Ky, Harouna Wassogo, Mbaye Ndoumbé Gueye, Mrs Thiam, Mame Sokhna Thiaw, Aissatou Diop, Awa Diop and Marietou Mbaye to name a few. In addition, the support provided by the Ministry of Education of Senegal 
and the World Food Programme (WFP) have been very instrumental. I would like to thank Mr. Abdoulaye Touré chief of the Division of School Canteens (DCaS) at the Ministry of Education of Senegal, as well as Mr. Moth Gueye, Mr. Elhadj Seck and all their colleagues for their help during the collection of additional information. In addition, I would also like to thank Mr. Allouseyni Sy from the 'Institut National d'Etudes d'Action pour le Développement de l'Education (INEADE)', Mr Elhadj Malick Dia from the 'Direction de la Planification et de la Réforme de l'Education (DPRE)', Mrs Khady Diallo from the 'Division du Contrôle Médical Scolaire (DCMS)', Mr Abdoulaye Faye from the World Food Program (WFP) and Mr Diouf and Mr Dieng from the 'Direction des Examens et Concours (DEXCO)' for their help, advice and guidance. I would also like to thank conference participants of the Africa Econometric Society (AES, 2011) in Nairobi, the participants of the first year PhD fellows presentation June, 2011 at UNU-MERIT, the Africa Research Workshop in October, 2011 at UNU-MERIT, 'les Jeudis de BAME/IFPRI' in May, 2013 at the Internationale Food Policy Research Institute (IFPRI) Dakar, the 'Rencontres de la Recherche Economique du CRES' in April, 2013 at CRES and participants from the Doctoriales organized at the Cheikh Anta Diop University in March, 2013 for their useful comments and discussions.

Fourthly, I am deeply indebted to my parents and my friends who have helped me during difficult moment and with whom I often shared good times: You lend me a hand and gave me a taste of life whenever I was on the floor during the drafting of my thesis. To my husband: Many thanks for his moral and financial supports. I am aware of his patience, his understanding and sacrifices he had made and continues making towards my success. Many thanks: To my mother and my father for their supports, encouragement and for taking care of my little daughter during my research visit to UNU-MERIT; To my brothers Mr. Thierno M. Dian 'Koto', Elhadj Ibrahim and Moustapha for their support and encouragement; To my sister-in-low Dalanda Bah for facilitating the stay of my daughter at Labé; To my mother-in-law Nénan Mariama for taking care of my little daughter during my multiple trips and especially during my first research visit to UNU-MERIT; To my best friend Dr. Facinet Conté for his help, support and encouragement. I would also like to make a special mention to Amadou Dicko, Yaya Ky, Ousmane Birba, Kalie Sillah and Bakary Manga who helped me immensely. I am grateful to Marone's family for their support and advice. I am also grateful to the family of Wladimir Raymond for their great hospitality.

Finally, I would like to express my sincere thanks to all the people or institutions who have directly or indirectly contributed towards the realization of this thesis: The research center 'Consortium pour la Recherche Économique en Afrique (CREA)' in Dakar, the NGO 'Action et Developpement (ACDEV)', Ibrahim Sadou, Anne-Sophie Robillard, Mar Cathy Dieng, Saly Faye, Abdoul Salam Wane, Chimère Somparé and all my teachers since the primary school. 
Dedication $\quad$ i

Summary $\quad$ iii

Acknowledgements $\quad$ v

1 General Introduction 1

1.1 Motivation and objectives of the thesis . . . . . . . . . . . . 2

1.2 History of meal and deworming programs in Senegal . . . . . . . . . . . . 4

1.3 Program evaluation v.s. structural approaches . . . . . . . . . . . . 6

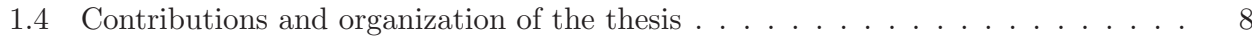

2 Non-experimental Assessment of Meal and Deworming Programs 11

2.1 Introduction . . . . . . . . . . . . . . . . . . . . . . . 11

2.2 Taking stock of the literature . . . . . . . . . . . . . . . . 13

2.2.1 Determinants of school achievement . . . . . . . . . . . . . 13

2.2.2 Deworming and school feeding programs: some facts . . . . . . . . . . 15

2.3 Description of the data . . . . . . . . . . . . . . . . . . . 17

2.3 .1 Variables . . . . . . . . . . . . . . . . . . . 18

2.3 .2 Descriptive statistics . . . . . . . . . . . . . . . . . . 22

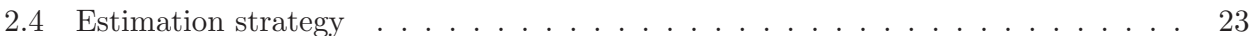

2.4.1 The Heckman selection framework . . . . . . . . . . . . . . . . . 23

2.4.2 The Roy model . . . . . . . . . . . . . . . . . . . . . . 26

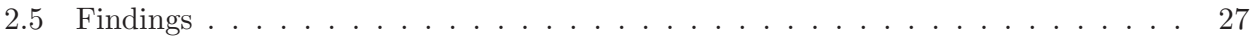

2.5.1 Determinants of school achievement: a summary of findings . . . . . . . 28

2.5.2 Effects of meal and deworming programs on scores . . . . . . . . . . . 38

2.6 Policy analysis . . . . . . . . . . . . . . . . . . . . . . . 43

2.6.1 Impact of programs on some subgroups . . . . . . . . . . . . . . . . 43

2.6 .2 LATE as policy instrument . . . . . . . . . . . . . . . . . . . 44

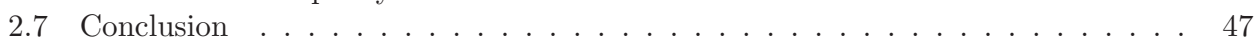

3 Packaging Meal and Deworming Programs ${ }^{1} \quad 48$

3.1 Introduction . . . . . . . . . . . . . . . . . . . . . . 48

3.2 Insights from the literature . . . . . . . . . . . . . . . . . . 50

3.2 .1 School meal programs . . . . . . . . . . . . . . . . . 50

3.2 .2 Deworming programs . . . . . . . . . . . . . . . 52

3.3 Programs package: variables and descriptive statistics . . . . . . . . . . 53 
3.3.1 Variables ............................. 54

3.3.2 Descriptive statistics . . . . . . . . . . . . . . . . . 57

3.4 Econometric specifications . . . . . . . . . . . . . . . . . . . . . 60

3.4.1 The double-index selection model . . . . . . . . . . . . . . . . . . . . 60

3.4.2 The generalized Roy's model . . . . . . . . . . . . . . . . . . . . . . 65

3.5 Findings ............................. . . 73

3.5.1 Determinants of school performances: Selections and outcomes . . . . . . 73

3.5.2 Effects of meal and deworming on school performances . . . . . . . . . . . 78

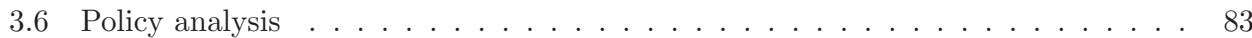

3.6.1 Treatment effect heterogeneity . . . . . . . . . . . . . . . . . . . 83

3.6.2 Cost-effectiveness analysis . . . . . . . . . . . . . . . . . . . . . 84

3.7 Conclusion ............................. . . . 89

4 Impact of Meal Program on School Performances: A Randomized Experiment 91

4.1 Introduction . . . . . . . . . . . . . . . . . . . . . . . . . . . . . 91

4.2 Experimental design, data and variables . . . . . . . . . . . . . . . . . . . . . . . . . . . . . . . . . . . . .

4.2.1 Sampling strategy ...................... . . . . 93

4.2.2 Data and variables ......................... 96

4.3 Descriptive statistics . . . . . . . . . . . . . . . . . . . . . . . . . . . . 98

4.3.1 Descriptive statistics before program . . . . . . . . . . . . . . . . . 98

4.3.2 Descriptive statistics after program . . . . . . . . . . . . . . 108

4.4 Econometrics specification . . . . . . . . . . . . . . . . . . . . . . 111

4.4.1 Partial adhesion and attrition: framework . . . . . . . . . . . . . 111

4.4.2 Partial adhesion and attrition: the model . . . . . . . . . . . . . . . . 113

4.5 Findings . . . . . . . . . . . . . . . . . . . . . . . 114

4.5.1 Impact of school feeding on pupils' performances . . . . . . . . . . . . . 115

4.5.2 Impact of canteens on internal efficiency of schools . . . . . . . . . . . . 121

4.6 Policy analysis . . . . . . . . . . . . . . . . . . . . . . . . 127

4.6.1 Cost-effectiveness analysis . . . . . . . . . . . . . . . . . . 127

4.6 .2 Cost-benefit analysis . . . . . . . . . . . . . . . . . . . 128

4.7 Conclusion . . . . . . . . . . . . . . . . . . . . . . . . 129

5 General Conclusion $r$

5.1 Summary of findings . . . . . . . . . . . . . . . . . . . . . . . . . . . 132

5.2 Policy implications . . . . . . . . . . . . . . . . . . . . . . . . . . 135

5.3 Limitations of the thesis . . . . . . . . . . . . . . . . . . . 137

5.4 Future research .......................... . . 137

$\begin{array}{lr}\text { Bibliography } & 139\end{array}$

$\begin{array}{lr}\text { Appendix } & 146\end{array}$

$\begin{array}{lr}\text { A Appendix Chapter 2 } & 147\end{array}$

$\begin{array}{lrr}\text { B Appendix Chapter } 3 & 173\end{array}$

C Appendix Chapter $4 \quad 202$

$\begin{array}{lr}\text { Summary in Dutch } & 209\end{array}$

Biography $r 212$ 
2.1 Treatment parameters . . . . . . . . . . . . . . . . . . 27

2.2 Determinants of scores: Meal, HSF (FIML) . . . . . . . . . . . . . . 28

2.3 Determinants of scores: Deworming, HSF (FIML) _ . . . . . . . . . . . . 30

2.4 Treatment effects: Meal . . . . . . . . . . . . . . . . . . . . . . . . 39

2.5 Treatment effects: Meal, Propensity score matching . . . . . . . . . . . . . 40

2.7 Treatment effects: Deworming programs . . . . . . . . . . . . . . . . . . 42

2.8 Treatment effects: Deworming, Propensity score matching . . . . . . . . . . . . 43

2.10 Impacts heterogeneity: Meal . . . . . . . . . . . . . . . . . . . . . . . . . . 44

2.11 Impacts heterogeneity: Deworming . . . . . . . . . . . . . . . . . . . 44

2.12 Local Average Treatment Effect (LATE): Meal . . . . . . . . . . . . . . . . . 45

2.13 Comparing some options using LATE: Policy analysis . . . . . . . . . . . . . 46

3.1 Average treatment effects in the double-index selection model . . . . . . . . . . . 68

3.2 Treatment effect parameters in the generalized Roy model . . . . . . . . . . . . 70

3.3 Average Treatment Effects for scores: DISM . . . . . . . . . . . . . . . . . . . . 79

3.4 Treatment Effects for scores: Generalized Roy's model . . . . . . . . . . . . . . . 80

3.5 Substitution and Sequential Effects for scores: Generalized Roy's model . . . . . 81

3.6 Average Treatment Effects for rates . . . . . . . . . . . . . . . . . . 82

3.7 Treatment Effects for rates: Generalized Roy's . . . . . . . . . . . . . . . . . 83

3.8 Program cost per year: Meal . . . . . . . . . . . . . . . . . 85

3.9 Program cost per year: Deworming . . . . . . . . . . . . . . . . 85

3.10 Cost effectiveness analysis for scores . . . . . . . . . . . . . . 86

3.11 Cost effectiveness analysis for rates . . . . . . . . . . . . . . 88

4.1 Schools eligible to the experiment . . . . . . . . . . . . . . . . . . . 94

4.2 Kolmogorov-Smirnov test: School level . . . . . . . . . . . . . . . . . 100

4.3 Mann-Whitney test: School level . . . . . . . . . . . . . . . . . . 100

4.4 Hotelling test: School level . . . . . . . . . . . . . . . . . . . . . 101

4.5 MANOVA on quantitative variables: School level . . . . . . . . . . . . . . . . . 101

4.6 Kolmogorov-Smirnov test: pupil level . . . . . . . . . . . . . . . . . . 103

4.7 Mann-Whitney test: pupil level . . . . . . . . . . . . . . . . . . . . . 103

4.8 Hotelling test: Pupil level . . . . . . . . . . . . . . . . . . . . . . . . . . 104

4.9 MANOVA on quantitative variables: Pupil level . . . . . . . . . . . . . . . 105

4.10 Hotelling test: attritors versus non-attritors . . . . . . . . . . . . 106

4.11 Hotelling test for treatment group: attritors vs. non-attritors . . . . . . . . . . 107

4.12 Hotelling test for control group: attritors vs. non-attritors . . . . . . . . . . . 107 
4.13 Hotelling test on attritors: treatment vs. control . . . . . . . . . . . . . . 108

4.14 Treatment planned versus treatment received . . . . . . . . . . . . . . . . . . 109

4.15 Comparison of adhesion status . . . . . . . . . . . . . . . . . 110

4.16 Complier versus Noncomplier . . . . . . . . . . . . . . . . . . . . . 112

4.17 Effect of school feeding on pupils' performance . . . . . . . . . . . . 115

4.18 Effect of school feeding on pupils' performance by gender . . . . . . . . . . . . 118

4.19 Effect of school feeding on pupils' performance by grade . . . . . . . . . . . . 120

4.20 Effect of school feeding on internal efficiency . . . . . . . . . . . . . . . . . . . . . . . . . . . . . . .

4.21 Effect of school feeding on internal efficiency by gender . . . . . . . . . . . . . . 124

4.22 Effect of school feeding on internal efficiency by grade . . . . . . . . . . . . . 126

4.23 Cost effectiveness . . . . . . . . . . . . . . . . . . . . . . . 128

A.1 List and definition of variables . . . . . . . . . . . . . . . 147

A.2 Summary statistics: Meal . . . . . . . . . . . . . . . . . 150

A.3 Summary statistics: Deworming . . . . . . . . . . . . . . . . . . 151

A.4 Propensity score estimation: Probit regression . . . . . . . . . . . . . . . 152

A.5 Determinants of scores: Meal, HSF (2-step) . . . . . . . . . . . . . . . . 153

A.6 Determinants of scores: Deworming, HSM (2-step) . . . . . . . . . . . . . 154

A.7 Determinants of scores: Meal, Roy regression (ML) . . . . . . . . . . . . . . 156

A.8 Determinants of scores: Deworming, Roy regression (ML) . . . . . . . . . . 158

A.9 Determinants of scores: Meal, Roy regression(2-step) . . . . . . . . . . . . . 160

A.10 Determinants of scores: Deworming, Roy regression (2-step) . . . . . . . . . . 163

A.11 Matching test: Meal . . . . . . . . . . . . . . . . . 166

A.13 Matching test: Deworming . . . . . . . . . . . . . . . . . 166

A.15 Deworming in school: Heckman selection framework (FIML) . . . . . . . . 166

A.16 Deworming in school: Heckman selection framework (2-step) . . . . . . . . . 168

A.17 Deworming at home: Heckman selection framework (FIML) . . . . . . . . . . . 169

A.18 Deworming at home: Heckman selection framework (2-step) . . . . . . . . . 171

B.1 Programs package: Summary statistics . . . . . . . . . . . . . . . . . 180

B.2 Determinants of scores: DISM (FIML) . . . . . . . . . . . . . . 182

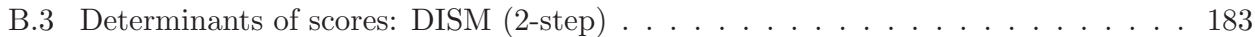

B.4 Determinants of scores: Generalized Roy's model . . . . . . . . . . . . . . . . . . 185

B.5 Determinants of rates: Generalized Roy's model . . . . . . . . . . . . . . . . . 187

B.6 Determinants of rates: DISM, (2-step) . . . . . . . . . . . . . . . 190

C.1 Levene and Student tests: School level . . . . . . . . . . . . . . . . . . . 203

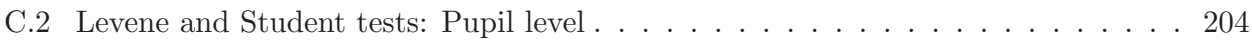

C.3 Descriptive statistics on the attrition status . . . . . . . . . . . . . 205

C.4 Descriptive statistics of attritors by group . . . . . . . . . . . . . . . 206

C.5 Descriptive statistics after program . . . . . . . . . . . . . . . . 207 
2.1 Relation between aggregate score and school manual. . . . . . . . . . . . . . . . . . 32

2.2 Relation between French score and school manual. . . . . . . . . . . . . . . . . 32

2.3 Relation between math score and class size. . . . . . . . . . . . . . . . . 33

2.4 Relation between aggregate score and teacher age. . . . . . . . . . . . . . 33

2.5 Relation between math score and teacher age. . . . . . . . . . . . . . . . 34

2.6 Relation between aggregate score and pupil age. . . . . . . . . . . . . 35

2.7 Relation between French score and pupil age. . . . . . . . . . . . . . . . 36

2.8 Relation between math score and pupil age. . . . . . . . . . . . . . . 36

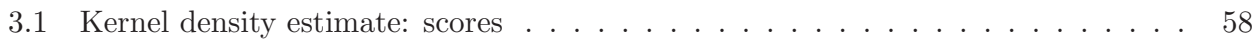

3.2 Kernel density estimate: rates . . . . . . . . . . . . . . . . . . . . . . . 59

4.1 Histogram of posterior distribution for aggregate score . . . . . . . . . . 116

4.2 Histogram of posterior distribution for French and math scores . . . . . . . . . 117

4.3 Histogram of posterior distribution for dropout and enrollment rates . . . . . . . 122

4.4 Histogram of posterior distribution for repetition and promotion rates . . . . . . 123

A.1 Geographical areas covered by the meal and deworming programs . . . . . . . . 149

B.1 Distribution of treatment effects for aggregate score . . . . . . . . . . . . . 192

B.2 Distribution of treatment effects for French score . . . . . . . . . . . . . . . . 193

B.3 Distribution of treatment effects for math score. . . . . . . . . . . . . . . . . . 194

B.4 Distribution of sequential and substitution effects on the treated: scores . . . . 195

B.5 Distribution of sequential and substitution effects on the nontreated: scores . . . 196

B.6 Distribution of treatment effects for enrollment rate . . . . . . . . . . . . . 197

B.7 Distribution of treatment effects for promotion rate . . . . . . . . . . . . . . 198

B.8 Distribution of treatment effects for dropout rate . . . . . . . . . . . . . . 199

B.9 Distribution of sequential and substitution effects on the treated: rates . . . . . 200

B.10 Distribution of sequential and substitution effects on the nontreated: rates . . . . 201 


\section{General Introduction}

In developing countries, hundreds of millions of children suffers from poverty, health problems, morbidity and malnutrition. This situation results to fewer years of schooling for these children as compared to those in developed countries. Recent studies have shown that impairment of health and child malnutrition reduced learning time, attendance, concentration in class and therefore academic performances.

The United Nations estimate shows that more than one third of children aged under 5 have growth problems whereas hundreds of millions more suffer from tropical diseases, malaria and intestinal parasites (OMS, 2000). Intestinal worms are endemic in tropical and subtropical regions and mostly resulting to symptoms such as abdominal pain, diarrhea and exhaustion. The consequences are particularly devastating for children. This is because it leads to malnutrition, increased susceptibility to other infections and slower growth during a critical period of childhood. Infected and non-dewormed children generally become ill, which results in slower cognitive development, increased absenteeism at school and adversely affects school performance.

Moreover, the reduction or elimination of malnutrition is often sought for its intrinsic benefits. However, there is evidence showing that better nutrition of children has significant physical and mental benefits. Severe malnutrition can cause delays or even deficiencies in cognitive development. Malnourished children enter school late, mostly exposed to repetition and dropout rates, and they are also likely to have weak scores on assessments in learning. Owing to its negative impacts on health and education, malnutrition weakens human capital formation. Thus, malnutrition issues and lack of child health are considered as important issues in developing countries.

As the lack of health and malnutrition of children have a negative impact on education, policies that improve health of children and their nutrition will also improve their academic performance (Glewwe and Miguel, 2008). Given the importance of keeping children in school and enhancing their performance, any policy designed to improve health and nutrition is an important mechanism in achieving the Millennium Development Goals (MDGs).

Since the implementation of school feeding and deworming aims at eradicating malnutrition and improving child health, the assumption is that these programs can improve school performance. However, until recently empirical evidence of policies implemented in that respect in Sub-Saharan Africa are scarce and have not been conclusive. 


\subsection{Motivation and objectives of the thesis}

Education is seen as a driving factor of development. The MDGs call for reducing hunger and improvement of universal primary education by 2015. However, until recently, there has not been any rigorous assessment on how best to achieve these goals. As it is difficult to attract and keep children in school in developing countries because of poverty, improvements in school quality and modest incentives, like providing meals at school or rations to take home, could be effective means to attract children to school, maintain them, and improve pupils' performance.

School feeding has been of great public interest in recent years. It is considered as a strategy to achieve the objectives of Education for All; goals set by the international community in 2000 in Dakar. To meet these objectives, the 'Global Initiative Food for Education' was launched by the USA in 2001 and was replaced in 2002 by the 'Food for Education' (FFE) program. These programs are often accompanied by medical treatment aiming at eliminating intestinal helminths. The interventions usually target specific beneficiaries, i.e. geographical areas, schools, and in some cases students or families. However, the implementation of these programs is more expensive than some other programs (rations for home, health or school inputs for example), which also aim to improve school performance. Therefore, school feeding is not considered to be the only lever to improving school performance. Among others, deworming, directly linked to health is also another important tool. The impacts of these programs on school performance and their comparison in terms of cost effectiveness are considered important issues for NGOs, governments and donors. But until recently, related empirical work has been inconclusive. Participation in school (enrollment and attendance), academic performance (test score), cognitive development, dropout and repetition rates are the main outcomes most often used by economists. There is a lack of evidence on the impact of FFE programs on repetition and dropout rates.

Depending on the type of meals provided, the size of food rations and the duration of the program, there is conflicting evidence on how school meals improve cognitive development and test performance in mathematics and in literature (Adelman et al. (2008)). There is also conflicting evidence regarding the effect of these programs on school performance measured through scores, repetition and dropout rates. While some authors find positive effects (Vermeerrch and Kremer, 2004; Cueto and Chinen, 2007; Ahmed, 2004; Simeon and Grantham-McGregor, 1989, etc.), others find negative effects (Ahmed and del Ninno, 2002; Ahmed, 2004), while others did not find any effects (Ahmed, 2004, Kazianga et al., 2009, Tan et al., 1999, Simeon and Grantham-McGregor, 1989).

Generally, it has been shown that lunch programs have more impact in areas of low schooling and on children suffering from severe malnutrition. Program impacts may be higher when combined with complementary programs which improve the characteristics of schools and children's health. Lugaz (2006) pointed out that issues related to the implementation of school meals including targeting errors, poor quality food or poor hygiene can affect the impact of programs. Therefore, more information is needed on how to improve the impact of these programs on school performance through more effective targeting, changing the size and food composition, or the provision of complementary inputs (such as health-related or school inputs).

Unlike empirical studies on the effects of the meal program, evidence on the impact of intestinal deworming on school performance (test score) is spotty in Africa and empirical evaluations suffer from several methodological drawbacks. To our knowledge, the main study was conducted by Miguel and Kremer (2004) who assess the impact of randomized intestinal deworming on school participation, health, nutrition and the scores of students in schools in Kenyan schools. The results show that despite reduction in absenteeism, there is no evidence that deworming increases the scores of students. The authors also concluded that the deworming program is less expensive than other alternatives in increasing school participation. Nokes et al. (1992) evaluated a treatment of whipworm in Jamaica and concluded that the treatment improves some cognitive functions. However, other outcomes, including those related to academic performance, do not seem to have changed significantly. A study conducted in South Africa, Kvalsig et al. 
(1991) did not find any relationship between drug treatment, cognitive development and school performance. Miguel and Kremer (2004) emphasize that deworming could improve test scores by increasing the time spent at school or through the improvement of learning, but it could also potentially reduce the test results because of congestion or negative side effects. In the literature, we find no empirical studies that estimate the impact of a joint program of school meals and deworming programs on educational outcomes (test scores, repetition, promotion, enrollment and dropout rates).

In Senegal, under the leadership of 'Programme Décennal de l'Education et de la Formation (PDEF)', significant progress has been made in providing access to primary education during the 2000s. The gross primary school enrollments increased from $71.6 \%$ in 2002 to $92.5 \%$ in 2009. However, the education system of the country is still marked by a weak ability to advance a significant proportion of pupils from the beginning to the end of an education cycle, with a high dropouts and repeaters. Efforts made in favor of primary education since 2000 have led to a rapid increase in the gross enrollment rate and significantly reduced the repetition rate. Indeed, to fight repetition, the government has taken a number of administrative actions such as removing repetition during the first year of each step in the primary cycle and capped at 5 $\%$ the maximum rate of repetition in the late stage of each cycle. However, decisions on the administrative nature will not succeed by themselves in solving the problem of keeping children at school. This is much more an issue of demand for education. Primary education in the country is still characterized by high dropout rates. Therefore, keeping children in school and improving their academic performances are among the main challenges facing the education system towards achieving universal education in 2015. With poverty and vulnerability in rural areas, there is evidence of the most pronounced delay in enrollment, school dropout and repetition rates. The food insecure households have low incomes and a high proportion of their expenditure concerns food. However, their diets are poor, and the weakness in the nutritional quality is compounded by the presence of children having intestinal worms. PAM (2006) observed $31 \%$ of the cases related to nutritional deficiency in school age and adolescence, and a predominance of nutritional anemia among children 4 -14 years old, with $62 \%$ for boys against $38 \%$ for girls. In addition, for some children, the distances to schools are long, and they are not always able to bring food to school. Thus, fatigue, hunger and poor health can reduce pupils' performance and increase repetition and dropout. That is why, fighting malnutrition has emerged as an important dimension of educational policy in Senegal, especially in areas experiencing poverty and vulnerability. For over 10 years, the government of Senegal with the support from international organizations introduced nutritional and health programs in some rural areas. The National Agency for Medical School Control ('Division Nationale du Contrôle Médical Scolaire, DCMS') of the Ministry of Education leads a program focused exclusively on deworming and medical monitoring in rural public schools.

Currently, a policy of generalization of meal and deworming programs is in sight. That is why, it is important to conduct rigorous analysis to assess the impacts of these programs on school performances and also to compare them in terms of cost and efficiency. The reports of the Ministry of Education, the World Food Programme (WFP) and NGOs only provide a statement of positive impacts of school canteens (meal) on educational outcomes. However, these assessments suffer not only from several methodological drawbacks, but also, none of them has investigated whether combined meal and deworming programs has positive impact on educational outcomes, and whether the two programs are complementary or substitutable. Even less, none of these studies endeavour to find out whether the impact of programs on school performances varies according to the sequence of implementation of interventions. Our thesis seeks to fill these gaps by evaluating the effects of meal and deworming programs on academic performances of pupils and internal efficiency of schools in rural Senegal.

The main objective of this thesis is to provide empirical evidence on the impact of meal and deworming programs on school performances in rural Senegal, while elaborating on the 
determinants of these performances. The specific objectives are as follows: i) analyze the impact of the meal and deworming programs on school performances separately while elaborating on the determinants of scores using observational data; ii) develop a model for multiple treatments, which makes possible to estimate a range of treatment effects, while addressing issues related to the complementarity or substitutability of the meal and deworming programs using observational data; iii) use experimental data to assess the impact of meal program on school performance.

\subsection{History of meal and deworming programs in Senegal}

School feeding is not a recent practice. Historically, countries implement school feeding programs in order to increase enrollment and attendance, as well as provide food to the poorest pupils. In developed countries, given the high level of enrollment rates, the main objective of school feeding is to adapt to the pace of work of parents by giving pupils the opportunity to enjoy a healthy meal at school. In developing countries, school feeding programs are implemented to improve educational (enrollment, attendance, dropout, repetition, academic performance) and nutritional (Height for age, weight for height, dietary intake) outcomes. Most of them are initiated by the World Food Program (WFP).

In Senegal, the government has always emphasized school canteens in the development of basic education. Indeed, school meals or school canteens have existed since 1963 and represent the activity through which WFP and other NGO like Countepart International or Caritas support the education sector in Senegal. ${ }^{1}$ School canteens are designed to provide students with a regular food supply in vulnerable areas and to promote children's access to quality basic education, especially for girls. These interventions support the government in achieving universal education for all children by the year 2015 which is one of the goals of the 'Programme Décennal de l'Education et de la Formation (PDEF)' and the Millennium Development Goals (MDGs). Since the 1970s, WFP is supporting the Government in achieving universal enrollment of children by the year 2015 which is one of the objectives of the PDEF and the MDGs. In addition, the school canteens are also specific interventions of WFP to strengthen social cohesion. The programs help displaced children or those returning in conflict areas to feed normally. The WFP also implements canteens to respond to the rising food prices. The action of WFP exclusively targets pupils attending public elementary schools, children supported in pre-schools and kindergartens which are supervised by the 'Agence Nationale de la Case des Tout-Petits (ANCTP)'. To be assisted by WFP, the school must meet the following conditions: i) be located in an area of insecure food supply or particularly affected by higher prices, ii) be located in rural or peri-urban areas, iii) preferably have a minimum size of 50 pupils and maximum of 600 (management concerns requirement), iv) have an operational management committee including active women's among members, v) show a commitment of the local community to develop infrastructure such as storage, even cooking based on local materials, vi) have an acceptable standard of hygiene.

Initially, WFP's school feeding covered the following regions: Fatick, Kaolack, Tambacounda and Matam (under the 'Programme Pays' 2007-2011) and the regions Ziguinchor, Kolda and Sédhiou (under the 'Intervention Prolongée de Secours et de Redressement (IPSR)' carried out since 2003 in Casamance Natural). Under the action plan, WFP has strengthened the school feeding program in its initial intervention areas: Fatick, Kaolack, Kaffrine, Tambacounda, Kedougou, Matam, Ziguinchor, Kolda and Sédhiou were considered as priority areas. Later on, the action plan has been extended to other regions: Diourbel, Louga and Thies. The program involves a total of 12 regions out of 14 in Senegal.

WFP activities are implemented within the framework of two programs, namely the 'Programme Pays (PP)' which extends from 2007 to 2011 and the IPSR program for the 2008-2010 and following. Both programs are developed in accordance with United Nations Development Assistance (Plan-cadre des Nations Unies pour l'Aide au Développement, UNDAF), based on

\footnotetext{
${ }^{1}$ See DCaS (2011) for details on the history of school meals in Senegal.
} 
the new draft Poverty Reduction Strategy (2006-2010) of the Government of Senegal and the MDGs. Specific interventions (IPSR) consisting of two components operating for the 'postconflict rehabilitation in the 'Casamance Naturelle' to assist vulnerable populations affected by poor harvests and fighting against the impacts of price increases in Senegal. Although the action plan of WFP covers the entire national territory, the school feeding program gives priority to areas located in regions already identified as most vulnerable to food insecurity, ${ }^{2}$ meaning regions whose gross enrollment ratio (GER) is below the national average $(86.0 \%)$ and regions with proven rates of global acute malnutrition ('Malnutrition Aigue Globale, MAG') above 10 $\%^{3}$

The setting-up of the activity is carried out in coordination with the Ministry of National Éducation through its national agency of school feeding (Division des Cantines Scolaires, DCaS) established in 2009, with the financial and technical support from WFP and other partners such as Caritas and Kinkeliba NGOs.

The supplies consist of cereals, pulses, oil fortified with vitamin A and iodized salt. Pupils receive a hot meal at midday Monday to Friday throughout the school year. This meal should include help to improve concentration and learning. The delivery of food in schools is provided on a quarterly basis. ${ }^{4}$

The management committee of each school is responsible for managing school canteens (food preparation, food storage etc.). Parents are also involved in management through the composition of these committees, participation in financial contributions for the provision of ingredients not provided by WFP such as vegetables, fish etc. The follow-up of schools which have benefitted from feeding is done by a person designated within each academic inspection in collaboration with DCaS and WFP.

The few empirical studies undertaken and the assessments of teachers report positive effects of school feeding on schooling. The qualitative observations made are also positive but no quantitative study was conducted to evaluate the effect of food assistance to schools in Senegal until 2004. In a WFP/UNESCO report, Akakpo (2004) examined the impact of WFP's school feeding on schooling and pupils' achievement in the certificate of elementary end studies (Certificat de Fin d'Études Élémentaire, CFEE) in rural primary schools in three regions of Senegal (Fatick, Kaolack and Tambacounda). The author concludes that schooling is much better in beneficiaries schools and that the presence of feeding increases the chances of success in the CFEE. In addition to some methodological limitations, the author did not use test scores, repetition rate, and dropout rate as outcome.

In schools receiving meals, usually deworming is conducted in collaboration with the 'Division du Contrôle Médical Scolaire (DCMS)' of the Ministry of education and the United Nations Children's Fund (UNICEF). Deworming aims at improving the health and nutritional status of pupils and their academic performance. Deworming includes providing each pupil with a $500 \mathrm{mg}$ tablet of mebendazole or albendazole $400 \mathrm{mg}$ every 6 months. A pupil absent the day of deworming will receive his/her tablet once return to school. A report by the Ministry of education through the DCMS concluded that regular deworming of children improves their health and nutritional status. However, it has no effect on their scores in French and in mathematics (DCMS, 2003). In this report, the methodological approach consisted of simple comparison of the outcome variables before and after deworming without further considerations of sampling issues. This reveals some methodological weaknesses in the estimation of treatments effects.

The methods used for program evaluations are subject to a lively debate in the econometric literature. Over the past two decades, several methods were developed for assessing the impact

\footnotetext{
${ }^{2}$ The survey of 'Sécurité Alimentaire en Situation d'Urgence (ESASU)' conducted by WFP has identified areas considered as vulnerable to food insecurity.

${ }^{3}$ These rates were collected in September 2008 in the survey 'Standardized Monitoring and Assessment of Relief and Transitions (SMART)'. The United Nations Children's Fund (UNICEF) supported the Ministry of Health in collaboration with WFP and the World Health Organization (WHO) for this survey.

${ }^{4}$ See DCaS (2011) for further details.
} 
of development programs. This theoretical and empirical literature is built upon a combination of statistical and econometric approaches.

Traditional (structural) economic models were used in assessments of policy or program. However, after 60 years of their application, economists have realized the difficulties in identifying and estimating the parameters of interest that could allow them to answer issues that were first mentioned by the Cowles Commission. The apparent limitations of these methods in the 1970s and 1980s led to the development of two other methodological approaches. The first is what is termed by Heckman (2010) as approach to program evaluation, referring to RCTs (Randomized Controlled Trial) and quasi-experimental methods (matching, propensity score matching, LATE, regression discontinuity, etc.). The second represents the nonparametric version of the structural approach. ${ }^{5}$

The literature on methods for evaluating program has reached a level of maturity that earned it to be an important tool in many areas of empirical research in economics. However, considerable controversy exists about the approaches used for the identification and estimation of program effects. Recently, a lively debate between the program evaluation approach and structural approaches was raised by Deaton (2009, 2010), Heckman and Urzua (2009), Heckman (2010) and Imbens (2010). ${ }^{6}$ Relying on Marschak (1953), Heckman (2010) built a bridge between the two approaches by leveraging the strengths of each. In what follows we summarized the essence of the debate before dealing briefly with the conciliation offered by Heckman (2010).

\subsection{Program evaluation v.s. structural approaches}

An experimental evaluation consists of randomly sampling of an eligible population into two groups before the introduction of the program. Randomization is a procedure that assigns individuals to either a treatment group or a control group. In the literature on program evaluation, randomization is the term often used for randomized controlled trials (experiments) because it is the procedure commonly used to construct homogeneous groups of treatment and control, thereby eliminating the selection bias. The quasi-experimental approach (or quasirandomization) consists of sampling randomly ex-post the population into two groups and conditionally on their characteristics. As it is possible to consider that the quasi-randomization is a conditional randomization, in what follows, analyzing program evaluation versus structural approaches comes sometimes to contrast randomization (quasi randomization and randomization) versus non-randomization (structural approaches).

The recent literature on program evaluation in economics is based on the counterfactual model of causality of Rubin. This model defines causality using an experimental framework. As a matter of fact, it provides practitioners false sense that random assignment or randomization is the most convincing way to identify causal effects (Heckman, 2010). Proponents of this approach considered randomization as an ideal and considered instrumental variables or matching or regression discontinuity as a substitute for randomization while noting that these alternatives are all available (Imbens, 2010).

However, Heckman and Urzua (2009) and Deaton (2009) argue that, even perfectly executed, randomization does not meet all the important economic issues. There are examples of issues for which structural models perform better than controlled experiments (Heckman, 1992, Heckman and Vytlacil 2007a,b). Randomized experiments have become very popular in the evaluation of development programs. Duflo (2004) claims that the randomized controlled trials of projects should generate knowledge that could be used everywhere as an 'international public good'.

\footnotetext{
${ }^{5}$ The program evaluation approach draws some of its terminology from medical science where the 'intervention' is often synonymous with the adoption of a treatment. In this thesis, the use of the terms such as treatment, interventions, program or policy means the same thing. They call for public intervention, a subsidy policy, program development or alternative actions that could be undertaken.

${ }^{6}$ The LATE in particular and the structural approaches are subject to intense debates. The controversy focuses on the identification and estimation of causal effects (Deaton, 2010; Heckman, 2010; Imbens, 2010).
} 
Freedman (2006) says that the experiments provide more reliable data than the causal studies using observational data. Imbens (2010) note that if the experiments are feasible, they are clearly preferable in the identification of causal effects because no approach has as much credibility as a randomized experiment. However, this does not prevent from implementing observational studies even in the case where randomized experiments are feasible.

Despite the popularity of randomized controlled trials, some authors argue that randomization has no special ability to produce more credible knowledge than other methods. Actual experiences (randomization), and quasi-experiments (quasi-randomization) are often subjected to practical problems that question any statistical or epistemic superiority of these methods. Deaton (2009) and Heckman and Urzua (2009) criticized the program evaluation approach with a particular emphasis on the increased use of instrumental variables and natural experiments in labor economics and randomized controlled trials in development economics. They direct their harshest critics to the Local Average Treatment Effect (LATE) parameter. ${ }^{7}$ The technique of instrumental variables has evolved as a mechanism to disentangle the causal influences. When the instrument is the result of 'natural experiments', true exogeneity is claimed. The purity of result has fueled the enthusiasm of 'experimentalists'. While Heckman and Urzua (2009) argue that it is difficult to use instrumental variables estimator to meet an interesting economic question, Deaton (2010) writes that he is struggling to make sense of the LATE. Imbens (2010) argues that the methodological approach in the case of randomized experiments and quasi-randomized is unassailable. For Imbens, none of the arguments put forward by Deaton (2010) or Heckman and Urzua (2009) could weaken the fact that randomization is widely recognized. However, Imbens stated that in reality, randomized experiments are generally not perfect and that their implementation can be improved. In addition, Imbens (2010) considers that the quality of experimental data and the design of the studies must be distinguished from choice of models and estimation methods. Based on such distinction, several empirical studies have attempted to reproduce the results from randomized evaluations using both experimental and non experimental methods. The results are mixed because some conclude that non-experimental methods cannot fully replicate the experimental results, while others find that they can reproduce experimental results. ${ }^{8}$

According to Imbens, the main concern with the current trend on the credibility of randomized experiments is that researchers may try to avoid major issues for which randomization is difficult or conceptually impossible and for which natural experiments are not available. However, in response to issues for which randomized experiments are possible, randomization is superior to any other approach in terms of credibility. Moreover, much of the debate between structural and program evaluation approaches focuses on issues of internal and external validity of a study. The problem is that in general the randomized experiments may be successful in terms of internal validity but less in terms of external validity compared to structural models. However Duflo et al. (2008) emphasize that problems of selection, imperfect compliance, and attrition to externalities are potential drawbacks to the internal validity of randomized evaluations. They discuss ways to eliminate these problems either in ex-ante or ex-post analysis.

In many disciplines, internal validity is emphasized. However, when the objective is to generalize a program on a given population, external validity is as important as the internal validity. In biomedical science, if one can easily extend a vaccine to prevent infection to other populations, in economics, the question of external validity is more difficult to solve. Imbens (2010) proposed to use structural models in order to solve this problem.

Despite the differences between program evaluation and structural approaches, conciliation

\footnotetext{
${ }^{7}$ The parameter LATE is introduced by Imdens and Angrist (1994). It represents the average impact of treatment on individuals who move from the status of a non participant to that of participants when we move from an instrument to another.

${ }^{8}$ See Lalonde (1986) in the case of the labor market, Todd and Wolpin (2006) and Attanasio, et al. (2009) for other experiments in an attempt to replicate experimental results using non-experimental approaches including structural models.
} 
is possible by assuming that for many economic policy issues, it is not necessary to achieve a complete identification of the models. In this line, Heckman (2010) proposes to make the balance thanks to Marschak's principle. Indeed, during the first generations of structural models in econometrics, Marschak (1953) stated that many problems of policy analysis do not require to identify or to fully estimate the structural parameters in contrast to what the pioneers of the structural analysis (Cowles Commission) and generations of structural economists who succeeded them did. Marshak suggests the principle of making combinations of invariant structural parameters that are often easier to identify than the individual parameters themselves. The combination of invariant structural parameters does not require knowledge of individual parameters. This approach has the advantage of drawing attention to how to respond clearly economic policy issues. The proposed approach often requires less complex calculation because it focuses on a more limited range of policy issues compared to issues targeted by the pioneers of structural analysis. Heckman (2010) built upon this idea and suggested an approach termed Marschak's principle as a middle way between the structural and the program evaluation (especially the LATE) approaches. Marschak's principle makes it easier to determine the structural parameters and improves upon program evaluation by producing estimates which are relevant to economic policy. ${ }^{9}$ Heckman (2010) links Roy (1951)'s model to that of Imbens and Angrist (1994). The approach he proposes makes the interpretation of the LATE easier and broadens the number of questions that the LATE could answer. It addresses the interpretation of estimates and their political relevance. It explores how to make the most of the data using the economic content to define issues of concern and the statistics that are useful to answer these questions.

The summary made above of the debate published in Journal of Economic Literature highlights that unanimity on the superiority of the randomization or quasi-randomization compared with the structural approach is far from being reached. This can be understood easily if we notice that some authors even offer to take the middle ground of the two. Both program evaluation and structural approaches have important features. Program evaluation models are generally simpler than structural models. Identifying the effects of a program is often more transparent than the identification of structural parameters. At the same time, economic issues and the relevance of treatment effects in program evaluation are often not very clear. Structural approaches produce more interpretable parameters which are best suited to conduct counterfactual policy analysis (Heckman, 2010). Currently, the distinction between these two literatures is strong enough. However, Heckman and Vytlacil (2007a, p. 4851) outline that it could disappear in the future, "As the treatment effect literature is extended to address the more general set of policy forecasting problems entertained in the explicitly economic literature, the distinction between the two approaches will vanish although it is currently very sharp".

In this thesis, we use two approaches emphasizing the structural approach because it better suited to meet the economic policy issues raised in the work. We seek to simultaneously estimate the determinants of school performance indicators in the presence of endogenous treatment indicators that represent selection processes. We are interested in calculating the average treatment effect (ATE), the average treatment effect on the treated (ATET), the average treatment effect on the untreated (ATNT), the local average treatment effect (LATE) and several relative effects. $^{10}$

\subsection{Contributions and organization of the thesis}

In terms of contribution, this thesis contributes to the literature in several aspects. From a methodological point of view, the thesis proposes models of selection for the case of two treatments when data are non experimental. Empirically, the application of this framework allows us to draw important conclusions for policy makers and NGOs. A cost-effectiveness analysis of

\footnotetext{
${ }^{9}$ See Heckman (2010) for a further details.

${ }^{10}$ See Heckman and Vytlacil (2007a) for details on these effects; also see Chapters 2 and 3.
} 
a school feeding program compared to deworming is implemented. In addition, the variability of the impact of two programs on improving school performances is studied. Thus this thesis contributes to the growing literature on the impact of school feeding programs and deworming in developing countries. In terms of policy analysis, this thesis provides new perspectives to the empirical literature on the evaluation of development programs in Africa, in particular, those relating to meal and deworming programs. The thesis can also inform policy makers (government and NGOs) on the effectiveness of programs implemented these years and guide them in choosing between several options depending on the objectives.

The thesis uses unpublished data (observational and experimental) from deworming and school meals programs conducted in rural Senegal. The observational data used in Chapter 2 and Chapter 3 are novel in the sense that both programs have been implemented at the same time since 2007-2008 by the World Food Programme (WFP) and supported by the Senegalese government to contribute to the objectives of the MDGs. Therefore, it is not experimental data and the beneficiary population (about 5000 pupils) is significantly higher than in the experimental data. In addition, the design of the sampling in the observational data is unique and rich in terms of program implementation as it involves mutually non-exclusive treatments. To date, these observational data have never been used and no evaluation of these programs had been undertaken.

In 2009, an experimental school lunch program supported by the Policy Impact Evaluation Research Initiative (PIERI) was launched with the coordination of the Consortium pour la Recherche Économique et Sociale (CRES) in collaboration with the Senegalese Ministry of Education and the World food programme (WFP). The experimental data used in Chapter 4 consists on two primary data sets collected before (baseline data) and after (follow-up data) the implementation of the school feeding program. During the collection of baseline data for the experiment (RCT), the evaluators discovered the existence of programs package that had been implemented by the WFP and the Senegalese Ministry of Education. The baseline data for the RCT were contaminated with pupils treated with deworming, school meal program and both (package programs). Thereafter, the RCT has been corrected taking account of the contamination. Fortunately, data opportunity arose for us to take advantage of the existence of such unique package program and the richness of information to conduct a study on impact assessment.

The outcome variables are aggregate score, French score, mathematics score, enrollment, repetition, promotion and dropout rates. Pupils' characteristics (age, gender, etc.), the characteristics of their families as well as those of schools and teachers are documented. The description of the observational data and variables used is discussed in Chapters 2 and 3, while the description of the experimental data is presented in Chapter 4. The thesis is organized as follows.

Chapter 2 uses observational data to measure the effect of deworming and school meal programs on pupils' performance, while elaborating on the determinants of these performances. In this chapter, we used both program evaluation (propensity score matching) and structural models. The structural framework has the advantage of allowing for the joint estimation of a performance equation and a selection mechanism, while estimating the effect of the program. To our knowledge, there is no study that sought to measure both the impact of a meal and a deworming program, while estimating the determinants of school performance in a joint framework.

Chapter 3 seeks to assess the impact of school feeding and deworming programs as a package on academic performance (test scores), retention (drop-out rates) and repetition rates while elaborating on the determinants of school performance. Governments or NGOs are generally implementing packages of programs for their effectiveness and because the implementation of a unique program is more expensive as compared to a package. However, researchers usually analyze package program separately (Chapter 2), or present a combined effect of the package because of the unavailability of appropriate tools that enable them to estimate the effects of 
exclusive programs, while providing the effect of the package in a unified framework. In Chapter 3, we propose to fill this gap by developing a model for multiple treatments, which allows estimating a variety of treatment effects while also addressing issues related to the complementarity or substitutability of programs. Evaluating multiple programs requires the identification and estimation of many different treatment effects which makes the analysis complex but more interesting.

Chapter 4 uses experimental data to estimate the effect of a meal program on school performances. In recent years, experimental studies are increasingly used to assess the impact of development projects because randomized controlled trials should generate knowledge that could be used everywhere as an 'international public good', see Duflo (2004). However, despite their advantages over observational studies, randomized experiments are often faced with difficulties. In fact, in controlled social science experiments, researchers usually often do not have perfect control of their target population because participants may choose not to follow instructions on the random assignment to the treatment (partial adhesion problem) or not to respond (attrition problem), Horiuchi et al. (2007) and Esterling et al. (2011). In this regard, some authors including Horiuchi et al. (2007) and Esterling et al. (2011) argued that a good analysis must take problems related to attrition and partial adhesion into account simultaneously. Unfortunately, in the literature, the majority of existing studies does not address one of the two problems, although this invalidates causal inference.

In this chapter, we measures the impact of an experimental school feeding program on school performance in the presence of attrition and partial adherence in rural Senegal. We use a statistical method that can overcome the problems of partial adherence and attrition simultaneously.

Chapter 5 summarizes the key findings from all chapters and their policy implications, discusses the limitations of the thesis and presents some future research projects. 
CHAPTER 2

Non-experimental Assessment of Meal and Deworming Programs

\subsection{Introduction}

In developing countries, several empirical studies have attempted to assess the impact of meal and deworming programs on school performance. However, scholarly contributions on Africa are spotty, despite the fact that program evaluation is an important tool to inform policy makers about the impact of programs and the efficient allocation of resources for new or existing policies. ${ }^{1}$

From a methodological point of view, several academic contributions have developed statistical and econometric tools to assess the effects of programs or policies. Two main families of methods are widely used in impact evaluation: program evaluation approaches (in particular the so-called Local Average Treatment Effect, hereafter LATE) and structural approaches based on the Roy (1951) or Tobit 5 type model. ${ }^{2}$ Approaches for evaluating programs are based on randomization and quasi-randomization. These approaches consider among others instrumental variables methods, Propensity Score Matching or regression discontinuity as substitutes for randomization (Imbens, 2010). However, Heckman and Urzua (2009) and Deaton (2010) pointed out that even perfectly executed, randomization does not answer all the relevant economic issues raised by policy programs. Structural models generally produce more information about preferences than programs evaluation approaches (Heckman and Vytlacil, 2007a, 2007b). However, approaches for evaluating programs are successful in making within sample inference while structural approaches prove robust for both within sample and out of sample inferences. As previously discussed in Chapter 1 (Section 1.3), both inferences are important because it is only when both are used that assessment of a policy becomes more informative for decision makers.

From an empirical point of view, Adelman et al. (2008) provided a review of assessment of the effect of Food for Education (FFE) programs on education outcomes. The authors showed conflicting evidence on the ability of school meals to improve pupils' cognitive development test performance in mathematics and language. Some studies found that school meal programs lead to a significant increase in academic performance as measured by test scores, while other studies found a negative effect or no impact. Vermeersch and Kremer (2004) used data from a randomized breakfast program in Kenyan kindergartens. The authors showed that the program improved student learning, but only for those whose teachers were more experienced at the beginning of the program. Cueto and Chinen (2007) examined the impact of an experimental program of school breakfasts in primary schools in Peru. The results showed that children in the treatment group, who are in 'multigrade' schools and 'simple flow' performed better in coding

\footnotetext{
${ }^{1}$ Policy makers are seen here as governments, aid donors and the development community at large.

${ }^{2}$ See Heckman and Taber (2008) for a presentation of the Roy model; Maddala, 1983 for Tobit models.
} 
tests, arithmetic and reading.

Studies of the impact of deworming on school achievement are even rarer. Kvalsvig et al. (1991) examined the impact of deworming in South Africa and concluded that the drug treatments had some effects on school performance, but some effects were not significant. Miguel and Kremer (2004) studied the impact of an experimental deworming program (supported by the World Bank) on (among other outcomes) scores of pupils in Kenyan primary schools. The authors showed that there is no evidence that deworming increases the scores of students. The study also indicates that not taking into account deworming externalities may underestimate the effects of deworming programs.

To the best of our knowledge, there is no study that sought to measure both the impact of a lunch program and a deworming program, while estimating the determinants of school performance in a joint framework. This study uses a unique and recent data set from a school lunch and deworming program in rural Senegal to assess their effects on pupils' achievement measured by test scores while elaborating on the determinants of test scores. To this end, we use observational data on 159 schools from an experimental program called PIERI. ${ }^{3}$ This program was lead by the 'Consortium pour la Recherche Economique et Sociale' (CRES) and the Senegalese Ministry of Education. The sample contains data on school and teacher characteristics, pupils' achievements (score in French, Maths, aggregate score), pupils' and household characteristics, community characteristics, shocks variables, geographical location and treatment indicators.

In this chapter we use both approaches (program evaluation vs. structural models) for two reasons. Firstly, for comparison purpose, we wanted to have the most robust results. The propensity score used here allows us to estimate the Average Treatment effect on the Treated (ATET), while the structural specification informs about the average treatment effect on the entire sample. Secondly, we are not only interested in assessing the effectiveness of canteen and deworming on pupils' school performance — both approaches can do this job but we are also interested in the joint estimation of the determinants of school performance and the participation model (selection equation or the probability to benefit from the programs). In that context, the structural model better suits our purpose. With such a framework in mind, we can specify a two system equations: one equation for the outcome (performance equation) and another for the treatment (selection equation). It is clear that the program evaluation approach (in particular the propensity score matching) cannot do that as it is only designed for the purpose of evaluation.

In addition, this chapter distinguishes between deworming at home and deworming in school. We have one indicator for meal program (33.73\% of pupils benefiting from the meal program) and three indicators for deworming. For the school or home deworming (25.88\% of pupils dewormed in school or at home) we refer to as deworming program throughout the text. We refer to the school deworming (11.80\% of pupils dewormed in school) as deworming in school, and the house deworming (16.08\% of pupils dewormed at home) as deworming at home. It is important to emphasize that the deworming program is not the sum of the deworming in school and at home. Furthermore, we do not take the combination of deworming in school and deworming at home into account. Also, in terms of policy analysis, this study uses the local average treatment effect (LATE) as an instrument to analyze economic policy.

The results show that deworming in school has a positive effect on pupils' performance while deworming at home has a negative impact on pupils' scores. In addition, the deworming program increases the scores of pupils who are in school with association of pupils' mothers or school project.

In terms of policy analysis, results using the LATE show that in increasing scores the most cost-effective way is to combine the canteen and the association of parents.

The rest of the chapter is structured as follows. In section 2.2 we provide a summary of the state-of-the-art literature on school feeding and deworming programs. Section 2.3 describes the data used. In Section 2.4 we present the econometric specifications. Section 2.5 summarizes

\footnotetext{
${ }^{3}$ PIERI (Policy Impact Evaluation Research Initiative).
} 
estimation results. Section 2.6 presents a policy analysis before the conclusion in the Section 2.7 .

\subsection{Taking stock of the literature}

In this section, we first provide a summary of literature review on the determinants of school performance before presenting an empirical review on the impact of deworming and school feeding programs on educational outcomes.

\subsubsection{Determinants of school achievement}

Among the determinants of school performance, school factors and those related to the family environment were the most commonly used by researchers in recent years. In developed countries, some studies have concluded that family environment is the most important factor in explaining school performance. In contrast, other studies have found significant effects of school factors and weak effects of family environment on educational outcomes for students in the case of developing countries (Coleman et al. 1966; Fuller and Clarke 1994). It is important to note that outside of school, individual and family factors, other elements such as socioeconomic and health shocks can have consequences on student outcomes.

School and teacher characteristics. There is a common perception that school inputs have a positive impact on school performance. Indicators such as class size, expenditure per pupil, pupil/teacher ratio, salary, age, experience and academic level of the teacher, have been used in some studies as school inputs. In addition to these factors, teaching practices and school composition may help to explain why some schools are more effective than others with identical levels of resources.

In fact, research on the effects of reducing class size on student performance has not produced consistent findings. Glass and Smith (1979) showed, firstly, that the relationship between class size and student achievement exists. Secondly, they found out that the effect of reducing classes is not linear. Hoxby (2000), in the case of the United States fails to find the effect of class size on student performance. It shows that smaller class sizes may not be cost-effective. While some studies show that students in small classes have higher student achievement (Piketty 2004), others show that the reduction of the class size still leave uncertainties (Davies, 2003).

On the other hand, while some research in developed countries tends to conclude that expenditure per pupil and the quality of school resources have little impact on pupil learning, studies on developing countries show that physical inputs such as textbooks, libraries and good training of teachers determine student's performance (Lockheed et al. 1986). The most expensive inputs such as science laboratories, higher teacher salaries and reducing class sizes appear to have little effect (Cohen and Rossmiller, 1987). In summary, the physical inputs are more important in a context where school resources are inadequate and unevenly distributed, notably in developing countries. The impact is however less in a context where a minimum level of core resources has been reached (developed countries).

For teacher characteristics, Bressoux (1996) analyzed the impact of the experience and training of teachers on students' achievement. He found out that experience and training have positive effects on pupil achievement, especially in mathematics. In contrast, the quadratic effect of experience of teachers is not significant. However, neither training nor experience can reduce the initial gap between the best and worst students. More recent studies have found a weak positive impact of teacher experience on student achievement (Clotfelter et al. 2006). Krueger (1999) obtained a low explanatory power of teacher characteristics. Qualifications of the teachers have no significant effect, while the experience has a small positive effect. The latter effect is quadratic: it is maximum at about 20 years of experience. Regarding the age of the teacher, if one assumes 
that the operation of classroom instruction is positively affected by maturity of teacher, one can expect a positive correlation between age of teacher and performance. In a study conducted in the "Republique Centreafricaine", Diambomba (1997) observed a positive relationship between teacher's age and performance in French. It is important to note that in the literature some authors have found no correlation between the age of the teacher and pupils' scores.

The impact of the professional training of teachers has also been less studied. Angrist and Lavy (2001) showed that the improvement of educational performance resulting from professional training seems important in religious schools. In addition, a cost-effectiveness analysis shows that teacher training is a less costly strategy to increase academic achievement compared to decreasing of class size or increasing the number of course hours. More recent studies (Kane et al. 2008) have found a small effect of professional training on performance. The research leads to mixed results regarding the impact of the academic level of teachers (academic qualification) on student achievement (Clotfelter et al. 2006).

Pupils characteristics. Among individual factors considered as having the most influence on student achievement, the literature mention the gender of pupil, the ability of pupils, their perception of themselves, their physical and mental health. Our data refer only to gender and physical health status of children (whether the child was ill in the last 3 months or no). In addition to these two variables, we included other variables: pupils' age, attendance early childhood institution and attendance in Koranic school. The literature shows that school performance varies by gender. Although some studies indicate that boys are better than girls in maths and science, others show that gender differences are not statistically significant and others show mixed results. The health status of children may affect their academic success by addressing cognitive abilities and their academic learning skills. Regarding health indicators, nutritional indicators and indicators directly related to health such as experience of a period of prolonged illness, the presence of parasites in the body or physical infirmity have been used.

Many studies linking nutritional deficiencies with educational outcomes have shown the importance of nutrition as a determinant of academic progress. The evidence of the impact of severe malnutrition on cognitive abilities is particularly convincing for children under 5 years (Grantham-McGregor et al., 2000). Malnutrition during this period of life can lead to cognitive disabilities which remain throughout school age. Even short-term nutritional deficiencies such as not taking breakfast, can cause cognitive dysfunction at school age (Simeon and Grantham McGregor, 1989). Many researchers argue that there is a link between the eating habits of pupils such as taking lunch, and school performance, Taras (2005). The effect of parasitic infections was also studied by Grigorenko et al. (2002). These authors found that these infections are common in children between 5 and 15 years. They cause anemia and have some effects on cognitive ability and school participation. However, mixed results are noted concerning the direction of the relationship between the health of the child and his school performance (El Hioui et al. 2008). This view is consistent with results found by other authors such as Fu et al. (2007) who noted that there is no significant relationship between the academic achievement of children and their nutritional status.

Household characteristics. To explain the unequal achievement of pupils, studies have focused on the role of socioeconomic status, family structure (gender of household head), family size and parenting practices. Numerous studies indicate that marked disparities in schooling and the acquisition of knowledge are associated with the socioeconomic status of parents (Ryan and Adams 1998). Lockheed et al. (1989), found significant effects of social class on student performance in mathematics and languages. In Africa, studies have found the parents' socioeconomic status as a determinant of academic failure (Lawson-Body, 1993). In contrast, Curtis and Phipps (2000), found that there is a weak link that is statistically insignificant between the situation of poverty or the household income and educational outcomes for students. Family structure is often controlled by the variable gender of household head in the literature. In fact, in some African contexts, "female head of household" seems to be associated with significant educational 
opportunities for children (Fuller and Liang 1999, Lloyd and Blanc 1996).

In addition, studies on the impact of family size on student achievement have resulted in very controversial results. In the United States an inverse relationship between number of siblings and school performance was found (Blake, 1989). However, Anh et al. (1998) point out that the negative relationship between number of siblings and schooling disappears when socioeconomic status is added as control. Chernichovsky (1985) in Botswana showed that the number of children aged 7-14 years in the household is positively correlated with the performance of pupils. In Kenya, Buchmann (2000) found no relationship.

Community characteristics. Studies that examined the effects of community characteristics on school's performance remain limited compared to those that examine the effects of individual variables and school.

Shocks variables. There are some shocks related to schooling that can lower student achievement. These shocks are for example the delay in starting the courses or the temporary closure of classes. They reduce the time of student learning and, therefore, the results. In primary education, teachers' strikes and high absenteeism rates constitute to the main source of temporary closure of classes.

\subsubsection{Deworming and school feeding programs: some facts}

Several studies have evaluated school feeding and deworming programs in underdeveloped countries. However contributions on Africa are very spotty. Some studies conclude that school meal programs have a positive effect on scores while others conclude on a negative effect or no effect. So we can say that empirical studies do not draw the same conclusions (Ahmed (2004), Vermeersch and Kremer (2004), Kazianga et al. (2009), Powell et al. (1998), Jacoby et al. (1996), Akakpo (2004), Ahmed and del Ninno (2002)). Studies do not always seek to measure the same relation between programs and the associated outcomes. Moreover, the nature of interventions may differ depending on the goal of the study. Studies can be differentiated from a number of criteria. We focus here on outcome variables and the nature of the intervention as criterion of differentiation. For the nature of intervention, we limit ourselves only to deworming and school feeding (including take-home rations) programs and for outcome variables we will focus on academic achievement and cognitive development.

The impact of deworming programs on educational outcomes is less documented than the impact of school feeding programs. To our knowledge, there are few studies on the impact of deworming on test scores. One significant study was conducted by Miguel and Kremer (2004) in the case of schools in Kenya. They studied the impact of a randomized deworming program on test scores among others. The results show no evidence that deworming increases test scores of students. In using the Kenya Life Panel Survey (KLPS) to document the long-term impact of deworming program on school achievement, the authors found that the program has no effect on tests score even in the long-term. Miguel and Kremer (2004) outlined that deworming could improve test scores both by increasing time spent in school and by improving learning, but it could also potentially reduce test scores through congestion or negative peer effects. In the case of Jamaica, Nokes et al. (1992) evaluated a treatment of whipworms and found that the academic performance did not change significantly. For South Africa, Kvalsvig et al. (1991) examined the impact of a deworming program and concluded that the treatment had some effect on school performance (tests of sustained attention), while for memory function tests the effects were not significant.

Soewondo et al. (1989) and Seshadri and Gopaldas (1989) have worked on Indian and Indonesian data. They found a significant impact of iron supplementation on cognitive development and school performance of anemic children. Pollitt et al. (1989) didn't find such an impact in Thailand. For an extensive literature on iron supplementation, see Nokes et al. (1998).

Several studies have examined the impact of school feeding programs on the performance measured as test scores. Despite the popularity of such programs, evidence on their impact on 
educational outcomes is mixed. While some authors found a positive impact (Vermeersch and Kremer 2004, Cueto and Chinen 2007, Ahmed 2004, Simeon and Grantham-McGregor 1989), others found a negative impact (Ahmed and del Ninno 2002, Ahmed 2004, Kazianga et al. 2009) or simply found no evidence (McEwan 2013, Kazianga et al. 2009, Ahmed 2004, Vermeersch and Kremer 2004, Simeon and Grantham-McGregor 1989, Tan et al. 1999). Ahmed (2004) in the case of Bangladesh studied the impact of a school lunch program on student outcomes (aggregate, math and language scores). Using data on test scores for 1648 students in fifth grade elementary school, he found that the aggregate scores of students who are in schools that received the program are $15.7 \%$ higher than students in schools that were in the control group. However, from a breakdown of scores, the author found that the improvement is mainly due to an increase in math score. Also, by controlling characteristics (such as children, households and schools) in particular the number of pupils in a class, the author found that the program has a significant and negative effect on scores in English. However, the size of the impact is still quite low. Also for Bangladesh, Ahmed and del Ninno (2002) concluded that school meal programs had a negative and significant impact on test scores of pupils who benefit from the program in fourth-year primary schools. Investigating whether this result was due to the increase in class size in treated schools, the authors found that students in the control group performed the same way as students in schools receiving the program. The authors also indicated that the differences between the two groups follow a decrease in scores of pupils who benefit from the program. They concluded that this result stems from the low level of socio-economic status of beneficiaries. Vermeersch and Kremer (2004) in western Kenya, quantified the effects of subsidized school meals on student outcomes using randomized data obtained from a breakfast program in kindergarten. The authors found an improvement in student learning, but only for children in schools where teachers were more experienced at the beginning of the program. Kazianga et al. (2009) in northern rural Burkina Faso used a randomized trial to assess the impact of school meal and take-home ration on health and education outcomes for poor children. The experience lasted one school year and the authors concluded that there was no significant impact on raw scores in mathematics. McEwan (2013) in the case of Chile evaluates the impact of higher-calorie meals on the education outcomes of public, rural schools and their students. Using a regression-discontinuity design and administrative data, the author found no evidence that additional calorie affect the outcome variables including school enrollment and attendance, first-grade enrollment age and grade repetition, and fourth grade test scores. Cueto and Chinen (2007) in three departments of Peru studied the impact of an experimental program of school breakfasts in primary schools. The outcome variables were, among others, test scores on standardized coding, arithmetic and reading. The results showed that children in the treatment group who are enrolled in multigrade schools and uni-grade performed better in coding test, arithmetic and reading. Tan et al., (1999) in the case of Philippines evaluated the impact of a school feeding program on the academic performance of students in the first year of primary school. They found that school feeding either alone or with parent-teacher partnerships, had a positive and significant effect on tests scores in English. In addition, school feeding where parents and teachers are involved had a significant positive impact on math scores. One can see then that some studies conclude that the lunch program at school has a positive impact on student achievement, others find the opposite, or simply that these programs have no significant effect on student scores.

On the aspect of cognitive development, several studies have sought to measure the impact of feeding programs on children. Among them, we can cite the effect of breakfast programs on cognitive performance of children (Pollitt, 1995). In general, the impacts are higher for children with low nutritional status (Adelman et al., 2008). Simeon and Grantham-McGregor (1989) in the case of Jamaica concluded that breakfast had no effect on the cognitive performance of children with a normal weight and height for their age, while breakfast increases the performance 
of children at risk. Vermeersch and Kremer (2004) in Kenya also concluded that the breakfast program for children has no impact on their cognitive abilities. The intervention has benefited the boys in terms of weight, but there was no impact on girls (weight or height) and on the height of boys. Whaley et al. (2003) in rural Kenya using a randomized program of school feeding studied the impact of animal foods, meat and milk on the cognitive development of children at primary school. Their result shows that food of animal origin as well as energy has a positive effect on the results of children in cognitive tests such as arithmetic and perceptions. One limitation in their study was that there were only three schools in each group. So, it is possible that the results are due to differences in quality between the groups. We can note that school feeding programs may have an impact on cognitive development, although the size and nature of the effect varies considerably depending on the program. The effect varies also depending on the micronutrient contained in the food served, or the initial nutritional status of children or the used measure of cognitive development.

To summarize, from this literature review, we can see that empirical studies on the impact of deworming and school feeding programs on school performance in Senegal are almost nonexistent. ${ }^{4}$ It is important to also note that there are few studies that identify the different impacts of these programs on children by gender. The impact of school feeding programs on student achievement has not been carefully analyzed in relation to the inputs of schooling and class size. Finally, in the literature we found no work that tries to measure in the same study the impact of deworming and meal programs on student achievement (test scores) while evaluating the determinants of school performance.

\subsection{Description of the data}

The school feeding or Food For Education (FFE) is the activity through which the World Food Programme (WFP) has supported the education sector in Senegal since the 1960s. This intervention aims at providing pupils with a regular diet and to promote children's access to basic education quality, especially girls. This intervention supports the government in achieving universal education for all children by the year 2015 which is one of the goals of the Ten-Year Education and Training (PDEF) and Millennium Development Goals (MDGs). The Ministry of Education, through the 'Direction de l'administration générale et de l'équipement (DAGE)' also funds a school feeding program. Its 'Division du Contrôle Médical Scolaire (DCMS)' also leads another program which focuses exclusively on deworming and medical monitoring in rural public schools.

In this study, we use primary data collected by the 'Consortium pour la Recherche Economique et Sociale (CRES)' and the Ministry of Education as part of an experimental program on school canteens and deworming in four rural areas in Senegal namely: Diourbel, Fatick, Kolda and Sédhiou. ${ }^{5}$ The sampling strategy involved first selecting a number of areas for experimentation based on the following criteria: i) poor areas, ii) existence of a standardized assessment, iii) location of intervention must be priority areas for partners (such as WFP) that support the experiment. ${ }^{6}$ Then, schools eligible for the program were randomly selected based on the following criteria: i) never had a school canteen, ii) never had access to a package of health services (deworming and supplementation iron), iii) have an enrollment of at least 50 pupils and at most 600 , iv) have at least second and fourth year classes of primary school, v) have a functioning management committee, vi) be located in rural areas. Finally, a random selection of 20 pupils in each class was conducted in each selected school. Because of imperfect information on schools

\footnotetext{
${ }^{4}$ There is one study of Akakpo, 2004 on effect of school feeding program, however the author did not use as outcome variables, the scores of students.

${ }^{5}$ See the map of Senegal in the appendix A.2 for geographical areas covered by meal and deworming programs in this thesis.

${ }^{6}$ The Senegalese education system implements, for nearly a decade, a major innovation in assessing the performance of elementary school pupils. This approach is called 'standardized assessment'.
} 
meeting the selection criteria, it was found that some schools in the sample have already had a deworming and/or canteens program for years.

Therefore, we have observational data of 159 randomly selected schools from which we were able to form the following groups: a group of pupils that benefit from a meal program, a group of pupils that are dewormed (at school or at home), a group of pupils that are dewormed at home, a group of pupils that are dewormed at school and the control group that receives nothing.

\subsubsection{Variables}

The data available concern schools and teachers' characteristics; pupils and households' characteristics; communities' characteristics and geographical location. The variables used in the estimation are: i) the outcome or school performance variables (aggregate score, French and math scores), ii) the determinants of performance or control variables that can be grouped as follows: those related to schools and teacher characteristics (temporary shelters, school manual, class size, distance to school, water point, playground, meals near school, gender of teacher, age of teacher, continuing training, professional and academic diploma of teacher), pupils characteristics (gender, age, number of hours, grade, Koranic school, early childhood institution, snack and pupil eat fill), household characteristics (food and education expenditures, household size, livestock, gender of household head, literacy of household head, marital status and land), community characteristics (college and parents school) and shocks variables (disturbed courses, absenteeism, number of pregnancies, number of marriages, ill last three months preceding the survey, and health expenditures of household). We also have the treatment dummies and region dummies. The definition of variables is documented in Table A.1 in the appendix. The control variables in the performance equation were chosen based on both their availability in the database and their relevance in terms of the literature on the determinants of academic performance. Some control variables are specific to the context of the study and are therefore particularly interesting. We will mention then during the description of variables.

Schools' characteristics.

Generally, it is assumed that schools with better infrastructure will perform better. School and teacher characteristics taken into account are: the number of classes in temporary shelters, the number of students per manual 'school manual', the class size, the distance between the school and the pupil's home, existence of a water point in school 'water point', existence of a playground 'playground', the possibility for students to eat near their school 'meal near school', gender of teacher, teacher's age, continuing training received by the teacher, professional and academic diploma of teacher. Among these variables, those that contextualize our study are temporary shelters and the opportunity for students to eat near their school. For variable temporary shelters, we expect a negative effect on scores. In Senegal, to overcome the lack of classrooms in some areas, temporary shelters were set up to receive pupils. These are usually precarious straw constructions which become unusable during the rainy season. For 'meal near school', we expect a positive effect on pupil achievement. For the variable school manual which represents the number of pupils per textbook, we expect a negative effect on the scores. It is generally accepted that schools having sufficient school manual often perform better than those not having. However, if the number of pupils sharing a manual is high, this will impact negatively on performances. About the variable class size, research and experiments on the effects of a reduction classes on pupils' achievement have not led to consistent conclusions. While some studies show that pupils in small classes have better academic results (Piketty, 2004), others show that the reduction of class size still leaves uncertainty (Davies, 2003). Glass and Smith (1979) showed that the relation between class size and pupils' performance is not linear. We expect a positive relationship between distance to school and pupils' achievement. Distance to school is equal to one if the pupil's house is located less than $1 \mathrm{~km}$ from the school and zero if the distance is higher than one $\mathrm{km}$. We expect a positive effect of variables 'water point' and 'playground' on pupil achievement. In addition, to take into account a possible non-linearity 
that may exist between some variables (school manual and class size) and pupils' performance, we introduced their square.

For the teacher characteristics, in the Senegalese context, we expect that a female teacher will have a positive influence on students particularly girls who identify themselves more easily to a female teacher.

Regarding the age of the teacher, we assume a positive effect. We introduced the square of the teacher's age to take into account possible non-linearity that may exist between this variable and scores. Indeed, if we assume that the operation of classroom instruction is positively affected by the maturity of the teachers, we can expect a positive correlation between teacher's age and school performance, Schwille et al. (1991). It is important to remember that in the literature, there is no consensus on the relationship between the age of the teacher and school performance. Undoubtedly, the teacher's age can interact with both experience and education level of the teacher. This means that some teachers having the same age but with different levels of education and different experience do not necessarily have the same effects on student achievement.

Concerning academic qualification of teachers, we made a distinction between those who have the National certificate (Brevet) and those who have the High school diploma and more. Regarding the impact of academic diploma of teacher on student performance, studies led to mixed results (Clotfelter et al. 2006). For continuing training of teacher, we expect a positive effect on scores because training improves teaching skills of teachers.

Also, in the literature, there is no consensus on the effect of teacher's professional diploma on scores. In fact, according to CONFEMEN (1999), the teachers trained for a year in Burkina Faso and Cameroon are less satisfactory results in 5th year than those who had no professional training. In Senegal, the same phenomenon is reflected for teachers who received two years of training as compared to those who received only one year. It seems that the initial training is the basis of this result (CONFEMEN, 1999).

Regarding the variable continuing training, we expect a positive effect on pupils' performance because continuing training should improve teaching methods of teachers. However, it is important to note that the impact of continuing training on the performance of pupils brings a positive effect if the training covers several periods, otherwise you can even record a negative effect (UNESCO, 2000).

Pupils' characteristics. Pupils' characteristics taken into account are gender, age, number of hours that the child spends on household work per day, attending at the koranic school, attending a early childhood institution, bring a snack to school and eat his/her fill.

For the gender of pupils, some studies indicate that boys are better than girls in mathematics and science (Felouzis, 1997). Others studies show that gender differences are not statistically significant and sometimes mixed (Ma, 2007). We anticipate that boys perform better than girls because girls in rural areas are more prone to domestic work. In addition, some families always doubt the interest of the education of girls.

We also introduced the pupil's age and its square to highlight the non-linearity that may exist between the age of the pupils and their performance. The literature mentions a positive effect of pupils's age on scores (Schwille et al. 1991) or a negative effect, depending on whether pupils enroll late to school or have had a lot of repetitions.

Regarding the variable 'number of hour', we expect a negative effect on school performance. In fact, children who are forced to spend a high number of hours on domestic activities are exposed to poor school performance because it reduces their learning time.

Attending an early childhood institution and or a koranic school are specific to the context of our study. The Koranic schools, usually found in Muslim countries, is an informal private educational structure that provides religious education based on memorization. In Senegal, 95.9\% of the population are muslim. We now understand that this structure, albeit informal, is highly developed in the Senegalese society. This school is known for developing the capacity 
of learning and memory of students because children learn to memorize at an early age. It is expected that these two variables positively affect student achievement.

Two other control variables are the fact to eat fill at home and the fact to bring a snack to school. These two variables are not frequently used in the literature. Recall that the study focuses on a population in rural areas and these indicators tell us about the nutritional well-being of students. We anticipate that these two variables have a positive effect on pupils' performance.

Households' characteristics. Among the households' characteristics of pupils, we have included: food and education expenditures, household size per adult equivalent, literacy of the household head, gender of household head, marital status, possession of an arable land and livestock owned by the household.

The variable food expenditure is an indicator of standard of living of the household. We expect a positive correlation with performance. The variable education expenditure, we also expect a positive relationship with performance because more parents invest in the education of children, more they will follow the children at home.

Household size per adult equivalent has also been used as a control variable. Studies on the impact of family size on schooling in developing countries have led to highly controversial results that do not allow generalizable conclusions. We expect a positive relationship between family size and pupils' performance. We believe that in large size households, it is likely to have seniors persons who are already in college or high school and who should be willing to guide the youngest.

Literacy of household head is another important variable in the explanation of the performance of the student. In fact, a literate environment has a positive influence on the child's ability to learn read, write and do arithmetic if however, the child is well supported at home.

Household characteristics also consist of their marital status. Since more than $95 \%$ of household heads are married, we have grouped the terms of this variable to married and unmarried (unmarried included singles, divorced, concubine and widowed). We expect that children living with parents married will have better performance, although individual experiences suggest that children living with a single mother divorced or widowed are generally more successful compared to children living with both parents in a large polygamous household.

Two others important variables not mentioned in the literature are the household ownership of arable land and the number of livestock owned by the household. Despite the fact that these two variables are indicators of richness, we expect that they will have a negative effect on children's performance. In terms of arable land, especially in the context of Senegal, rural households still continue to use their children for domestic and farm work. It is therefore likely that children living in households with farmland are asked to work in the fields, which will result in reducing the learning time of pupil. The same reasoning applies to the variable livestock owned by the household. The probability is that the higher the size of livestock, the more children in the household are exposed to work.

Community characteristic. Studies examining the effects of community characteristics on pupils' performance are still limited compared to those examining the effects of individual, family and school variables. However, factors associated with neighborhood can have both, positive and negative influences on school performance. In this research, the characteristics of the environment taken into account are: the existence of a college in the village of the student, the fact for students to live in a community where some children do not go to school because parents are not interested in school.

Regarding the existence of a college, we expect a positive effect on performance. Indeed, parents who have not the possibility to help their children to pursue studies in a remote village after obtaining the primary certificate will invest little in their child's education, because they are convinced that he will leave school prematurely. In addition, living in a village where there is a college implies that there are seniors who are in college and can help the younger ones with their studies. Regarding variable 'Parents school', we expect a negative effect on performance. Living 
in a community where some children do not go to school because parents are not interested could negatively impact on the scores. In such communities, a lot of children are generally used to work to help their families. We believe that living in this context is likely to have an adverse effect on the performance of children who go to school because there possibility of ripple effect.

Shocks variables. Among shocks, we have those directly affecting the child, those affecting his school, his household and those acting on the environment in which he lives. Shock variable directly affecting pupils which is available is: being sick during the last three months preceding the survey ('sick last 3 month'). The health of students is a major element for the explanation of the performance. Being sick the last three months preceding the survey can reduce the time of school attendance and learning ability therefore the performance of pupil. We expect a negative effect of this variable on student performance.

Shocks variable affecting school of pupils are: the number of pregnancies 'Pregnancies' and marriages registered during the school year, teacher absenteeism and the existence of disturbances which entrained delays in starting courses.

For variable number of pregnancy and number of marriage, we expect negative effect on performance. Girls in rural areas are generally exposed to early marriage and pregnancy. If marriage or pregnancy does not result automatically by a pure and simple dropout of the pupil, it becomes extremely difficult to focus on her studies due to family responsibilities.

Regarding the variable absenteeism, we expect a negative effect of this variable on performance, because if the teacher is often absent, the learning time will reduced, as well as the performance. The existence of disturbances having driven delays in the start of the courses also reduces the learning time of pupils. We hypothesized a negative effect of this variable on pupils' performance.

Shock variable affecting the household of the pupil is: health expenditure. We expect a negative effect of this variable on performance of pupil. In fact, a high health expenditure shows that there have been cases of illness in the household. The illness of the pupil has a negative effect on the learning time. In addition, illness of a family member (parent, etc.) can affect the pupil psychologically. It is important to note that low health expenditure does not necessarily signify that there are no illness cases in the household concerned. It may be that the household is unable to provide medical needs for their members.

In addition, having regarded some specific characteristic area of intervention for which information is not available, we introduced the indicator regions among control variables. It is dummies variables for Diourbel Fatick, Kolda and Sedhiou. The reference region is Fatick which has the largest number of pupils.

Having also consider the choice of control variables in the selection equation of the meal program at school, we used the criteria set by WFP and the division of school canteens. Among the criteria, there is a lack of information in the database on some variables such as being located in an area food insecure or particularly affected by rising prices. The variables are the total number of students in the school, the distance between the school and pupils' homes, the existence of a management committee in the school, an association of parents, a cooperative school, a grant from the Rural Council, a water point, a disturbances that delays starting courses, a storage, and the gender of pupil. We assume that the distance to school has a negative influence on the probability to benefit from the program, while the forms of associations and infrastructure that exist within the school will act positively on the probability of having the program. Similarly, we anticipate disturbances in courses and having more boys in the school act negatively on the probability to benefit from the meal program. For the choice of control variables in the selection equation of deworming program, we based this on the criteria listed by the Ministry in the meal program and our intuition. The variables are: The total number of students, the existence of a management committee of the school, an parents' association, a cooperative school, a water point, a medicine box, gender of the pupil and literacy of the household head. 


\subsubsection{Descriptive statistics}

We summarize the statistics of variables in Tables A.2 and A.3 in the appendix, respectively for meal and deworming programs. The statistics are provided by group (treatment and control group). Additionally, the last column of each block reports the test whether variables are statistically different across treatment and control groups.

For the meal program (Table A.2 in the appendix), the summary statistics show that on average the treatment group perform better than the treatment group in terms of the scores (aggregate, French and math). The mean of aggregate, French and math scores for the treatment group are $42.445,41.877$ and 42.890 respectively, whereas they are $37.587,38.228$ and 36.949 for the control group. There is a significant difference between the two groups as showed by the t-test. We also note that there is no significant difference between the two groups for the following variables: total pupils, number of hours, food expenditure, household size, cooperative school, meal near school, professional qualification CAP, other professional qualification, academic qualification (high school diploma), academic qualification (national certificate), gender of pupil, grade, Koranic school, sick last 3 months, pupils eat fill, literacy of household head, marital status, land and parents school. On average, there are about 187 pupils per school in each group. Pupils spends on average 3 hours per day to do household work, also, The household size is about 11 persons on average and we note the same food expenditure in each group. In each group, about $56 \%$ of teachers have 'brevet' as academic qualification, while only $44 \%$ have a 'high school diploma' as academic qualification. In the treatment group, about $48 \%$ of the pupils are boys, while in the control group, there are about $50 \%$ of boys. But there is no significant difference between the two averages. In the treatment group, approximately $27 \%$ of pupils became sick during the last three months preceding the survey while it is $24.4 \%$ in the control group, but there is no significant difference between the two averages. Also, on average, in each group, $16 \%$ of household heads are literate, $95 \%$ are married and $95 \%$ have their own arable land. In each group, $49 \%$ of children live in a community where some children do not go to school because their parents are not interested in sending them to school.

For some variables such as temporary shelters, school manual, pregnancies, teacher's age, education expenditure, health expenditure, distance to school, playground, management committee, association of parents, association of mothers, rural council grant, water point, disturbed courses, storage, professional qualification CEAP, gender of household head, Fatick and Sédhiou, the treatment group shows the highest average with a significant difference. However, if we take the variables, class size, marriages, pupil's age, livestock, school project, medicine box, gender of teacher, no professional diploma, continuing training, absenteeism, early childhood institution, snack, college, Diourbel and Kolda, the control group displays the highest average with a significant difference. So far, around $92 \%$ of schools in the treatment group have a playground while it is around $75 \%$ for schools in the control group. Also, about $90 \%$ of schools in the treatment group have had a delay in starting the courses because of disturbances. This figure is about $74.5 \%$ for schools in the control group. Also, one can note that $20 \%$ of teachers in the treatment group have no professional diploma compared to about $32 \%$ in the control group. We noted that $35 \%$ of teachers in the treatment group had a CEAP as professional qualification, while there were $19 \%$ in the control group. For continuing training, about $65 \%$ of teachers had benefited in the control group compared to $45 \%$ in the treatment group. It is important to note that $8 \%$ of the teacher in the control group are often absent, while only around $4 \%$ in the treatment group is not often present. Likewise, about $4 \%$ of pupils in the treatment group have attended an early childhood institution. This figure is about $7 \%$ for pupils in the control group. Also, about $5 \%$ of pupils in the treatment group bring snack at school. This figure is about $10 \%$ for pupils in the control group. About $24 \%$ of schools in the control group are located in a village having a college, while this figure is only about $13 \%$ for schools in the treatment group. Majority of treated schools are located in Fatick while the majority of schools in the control group are in Kolda. To summarize, the treatment group performs better in terms of scores. 
Regarding the deworming program, the statistical summary (Table A.3 in the appendix) shows that on average, for scores, there are no significant differences between the treatment and control groups as documented by the t-test. In fact, in the treatment group one note 37.495, 37.680 and 37.161 for aggregate, French and Math scores. In the control group, one note 37.587, 38.228 and 36.949 for aggregate, French and Math scores respectively. Also, for a number of control variables such as health class size, marriages, food expenditure, household size, meal near school, medicine box, gender of teacher, professional qualification CEAP, other professional qualification, gender of pupil, grade, koranic school, gender of household head, marital status, land and the area of Sédhiou, there is no significant difference between the two groups. In each group, the average class size is about $36 \%$ pupils, the household size is about 11 persons and $12 \%$ of schools have medicine box. Also, around $49 \%$ of pupils are boys in the treatment group whereas $51 \%$ in the control group, $27.6 \%$ of pupils did a Koranic school in the treated group and $29.6 \%$ in the control group. We observe that around $95 \%$ of households are married. Likewise in each group, about $94 \%$ of household have an arable land. However, for temporary shelters, total pupils, pregnancies, teacher's age, education expenditure, distance to school, playground, management committee, cooperative school, association of parents, association of mothers, rural council grant, water point, storage, professional qualification CAP, High school diploma, absenteeism, early childhood institution, snack, sick last 3 months, pupils eat fill, literacy of household head parents school and Fatick, the control group displays the lowest average with a significant difference, whereas the treatment group displays the lowest average with a significant difference for all remaining variables. For example, about $85.6 \%$ of schools are in less than $1 \mathrm{~km}$ from pupil's home for the treatment group, while only $78 \%$ of schools in the control report the same. Also about $52.6 \%$ of schools in the treatment group have a water point, while only about $39.5 \%$ of schools in the control group have.

\subsection{Estimation strategy}

Observational studies typically use non random samples. In these situations, where the sample is either fully or partly based on values taken by the outcome response variable, parameter estimates are likely to be inconsistent unless corrective measures are applied. Such samples are broadly defined as selected samples. Two types of specifications are suited for this purpose: i) the dummy endogenous selection model originally developed by Heckman (1979), which is fully integrated into the evaluation literature, ii) the Roy model which dates from Roy (1951). In what follows, we apply these two specifications to our study.

As previously outlined, we are interested not only in assessing the effectiveness of meal program, but also in the study of determinants of outcomes. In this regard, we found the structural model most appropriate for our purpose. With such a framework we can specify a two equations system: one equation for the outcome (performance equation) and another for the treatment (selection equation). The system can be estimated using Full Information Maximum Likelihood (FIML) or the Control Function (CF) approach. Moreover, as the structural specification imbeds a treatment (selection) equation which is the basic material of the Propensity Score Matching (PSM), we only present the structural model (see Maddala, 1983 and Greene, 2011). ${ }^{7}$ The generic model combines a Probit selection equation (treatment equation) which is assumed to stem from an unobserved latent variable, and a regression equation (performance equation).

\subsubsection{The Heckman selection framework}

This specification relies on an exclusion restriction, meaning that a variable determines participation in the program but not the outcome of the program itself. Unlike to 'matching' which

\footnotetext{
${ }^{7}$ For a presentation of the Propensity Score Matching (PSM) method see Imbens and Wooldridge, 2009; Caliendo and Kopeinig, 2008.
} 
is viewed as 'selection on observable', the Heckman approach enables 'selection on [observables and] unobservables'. As outlines by Blundell and Costa Dias (2008), a comparison of both approaches turns to be very informative in understanding the advantages and limitations of these methods.

i) Firstly, we assume a latent Probit selection mechanism (selection equation):

$$
\begin{aligned}
z_{i}^{*} & =\mathbf{w}_{i}^{\prime} \boldsymbol{\alpha}+\mu_{i} \text { with } \mu_{i} \sim \mathrm{N}[0,1] \\
z_{i} & =\mathbf{1}_{\left[z_{i}^{*}>0\right]}
\end{aligned}
$$

where $\mathbf{w}$ stands for the selection controls, and $z_{i}=1$ if treated, $\mu_{i}$ is the disturbance.

ii) Secondly, we assume a linear regression with sample selection (performance equation):

$$
y_{i}=\mathbf{x}_{i}^{\prime} \boldsymbol{\beta}+\delta z_{i}+\varepsilon_{i}
$$

We assume that equations (1-1) and (1-2) are linked by a bivariate normal distribution conditionally on $\left(\mathbf{w}_{i}, \mathbf{x}_{i}\right)$ :

$$
\left(\begin{array}{c}
\varepsilon_{i} \\
\mu_{i}
\end{array}\right) \sim \mathrm{N}\left[\left(\begin{array}{l}
0 \\
0
\end{array}\right),\left(\begin{array}{cc}
\sigma & \rho \\
\rho & 1
\end{array}\right)\right]
$$

Next, we will implement two estimation strategies to estimate the effect of interventions on the entire population, that is to say the Average Treatment Effect (ATE): the Full Information Maximum Likelihood (FIML) and the Control Function (CF) which relies on the Heckman $(1976,1979)$ two-step procedure.

\section{Full Information Maximum Likelihood (FIML)}

We assume that $\mu_{i} \mid \varepsilon_{i} \sim \mathrm{N}\left(\frac{\rho}{\sigma} \varepsilon_{i}, 1-\rho^{2}\right)$. The probabilities for $z_{i}=1$ (treated) and $z_{i}=0$ (untreated) are then defined as:

- Treated:

$$
\mathbb{P}\left(z_{i}=1 \mid, \mathbf{w}_{i}, \varepsilon_{i}\right)=\Phi\left(\frac{\mathbf{w}_{i}^{\prime} \boldsymbol{\alpha}+\rho \varepsilon_{i} / \sigma}{\sqrt{1-\rho^{2}}}\right)
$$

where $\Phi(\cdot)$ denotes the standard normal cumulative distribution, and $\rho$ is the correlation coefficient between $\varepsilon_{i}$ and $u_{i}$.

- Untreated:

$$
\mathbb{P}\left(z_{i}=0 \mid \mathbf{w}_{i}, \varepsilon_{i}\right)=\Phi\left(\frac{-\left(\mathbf{w}_{i}^{\prime} \boldsymbol{\alpha}+\rho \varepsilon_{i}\right)}{\sqrt{1-\rho^{2}}}\right)
$$

Let $\theta=(\boldsymbol{\beta}, \boldsymbol{\alpha}, \sigma, \rho)$ denotes the set of parameters and the functions $f_{2}(\cdot), f_{1}(\cdot)$ respectively bivariate and univariate normal density functions. The log-likelihood function for the full model is the joint density for the observed data. The FIML estimator $\hat{\theta}_{\text {FIML }}$ is obtained as:

$$
\hat{\theta}_{\mathrm{FIML}}=\underset{\theta \in \Theta}{\arg \max } \ln L
$$

where

$$
\begin{aligned}
L & =\prod_{i=1}^{N}\left[\int_{-\infty}^{-\mathbf{w}^{\prime} \alpha} f_{2}\left(\mu_{i}, y_{i}\right) d \mu_{i}\right]^{1-z_{i}}\left[\int_{-\mathbf{w}^{\prime} \alpha}^{\infty} f_{2}\left(\mu_{i}, y_{i}\right) d \mu_{i}\right]^{z_{i}} \\
& =\prod_{i=1}^{N}\left[f_{1}\left(y_{i}\right) \int_{-\infty}^{-\mathbf{w}^{\prime} \alpha} f_{1}\left(\mu_{i} \mid y_{i}\right) d \mu_{i}\right]^{1-z_{i}}\left[f_{1}\left(y_{i}\right) \int_{-\mathbf{w}^{\prime} \alpha}^{\infty} f_{1}\left(\mu_{i} \mid y_{i}\right) d \mu_{i}\right]^{z_{i}}
\end{aligned}
$$


and

$$
\begin{array}{rlrl}
\ln L & =\sum_{i=1}^{n} \ln \Phi\left[\left(2 z_{i}-1\right)\left(\frac{\mathbf{w}_{i}^{\prime} \boldsymbol{\alpha}+\left(y_{i}-\mathbf{x}_{i}^{\prime} \boldsymbol{\beta}-\delta z_{i}\right)(\rho / \sigma)}{\sqrt{1-\rho^{2}}}\right)\right] \\
& -\frac{1}{2} \ln \left[\frac{\exp \left(y_{i}-\mathbf{x}_{i}^{\prime} \boldsymbol{\beta}-\delta z_{i}\right)^{2}}{\sigma^{2} \sqrt{2 \pi}}\right] & \text { for } z_{i}=(0,1)
\end{array}
$$

\section{Two-step or Control Function}

The rationale of the Control Function approach is in controlling for the part of the disturbance in the outcome relation that is correlated with the treatment dummy indicator. This method involves a two-step procedure. The first step relies on a Probit estimation of the selection equation to obtain $\mathbb{P}\left(z_{i}=1 \mid \mathbf{w}_{i}\right)=\Phi\left(\mathbf{w}_{i}^{\prime} \boldsymbol{\alpha}\right)$. Then, the conditional outcome expectation in (1-2) is modified as

$$
\mathbb{E}\left(y_{i} \mid z_{i}\right)=\mathbf{x}_{i}^{\prime} \boldsymbol{\beta}+\delta z_{i}+\lambda h_{i}
$$

where $\lambda=\rho \sigma$ and $h_{i}$ is the Control Function:

$$
h_{i}= \begin{cases}\frac{\phi\left(\mathbf{w}_{i}^{\prime} \hat{\boldsymbol{\alpha}}\right)}{\Phi\left(\mathbf{w}_{i}^{\prime} \hat{\boldsymbol{\alpha}}\right)} & \text { if } z_{i}=1 \\ \frac{-\phi\left(\mathbf{w}_{i}^{\prime} \hat{\boldsymbol{\alpha}}\right)}{1-\Phi\left(\mathbf{w}_{i}^{\prime} \hat{\boldsymbol{\alpha}}\right)} & \text { if } z_{i}=0\end{cases}
$$

where $\phi$ denotes the standard normal density function. The second step consists in an OLS estimation of $\boldsymbol{\beta}, \delta$ and $\lambda$ in the regression (1-8) augmented with the Control Function $h_{i}$. This modified regression allows us to handle the part of the disturbance that is correlated with the selection mechanism. By including $h_{i}$ in the outcome relation, we are able to disentangle the true impact of treatment from the selection mechanism. accounts for the difference between treated and untreated.

The estimation of the parameters $\sigma^{2}$ and $\rho$ are obtained as $\hat{\sigma}^{2}=\left(\varepsilon^{\prime} \varepsilon+\hat{\lambda}^{2} \sum_{i=1}^{N} \varphi_{i}\right) / N$ and $\hat{\rho}=\hat{\lambda} / \hat{\sigma}$ with $\varphi_{i}=h_{i}\left(h_{i}+\mathbf{w}_{i}^{\prime} \hat{\boldsymbol{\alpha}}\right)$.

\section{Treatment effects}

The estimated effect of a program is obtained by computing the difference in expected school performance between participants and nonparticipants: $\mathbb{E}\left(y_{i} \mid z_{i}=1\right)-\mathbb{E}\left(y_{i} \mid z_{i}=0\right)$. We have

$$
\mathbb{E}\left(y_{i} \mid z_{i}=1\right)=\mathbf{x}_{i}^{\prime} \boldsymbol{\beta}+\delta+\mathbb{E}\left(\varepsilon_{i} \mid z_{i}=1\right)
$$

and

$$
\mathbb{E}\left(y_{i} \mid z_{i}=0\right)=\mathbf{x}_{i}^{\prime} \boldsymbol{\beta}+\mathbb{E}\left(\varepsilon_{i} \mid z_{i}=0\right)
$$

Taking the difference of the above terms leads to

$$
\begin{aligned}
\mathbb{E}\left(y_{i} \mid z_{i}=1\right)-\mathbb{E}\left(y_{i} \mid z_{i}=0\right) & =\delta+\mathbb{E}\left(\varepsilon_{i} \mid z_{i}=1\right)-\mathbb{E}\left(\varepsilon_{i} \mid z_{i}=0\right) \\
& =\delta+\mathbb{E}\left(\varepsilon_{i} \mid u_{i}>-\mathbf{w}_{i}^{\prime} \boldsymbol{\alpha}\right)-\mathbb{E}\left(\varepsilon_{i} \mid u_{i}<-\mathbf{w}_{i}^{\prime} \boldsymbol{\alpha}\right) \\
& =\delta+\lambda\left[\frac{\phi\left(-\mathbf{w}_{i}^{\prime} \boldsymbol{\alpha}\right)}{1-\Phi\left(-\mathbf{w}_{i}^{\prime} \boldsymbol{\alpha}\right)}\right]+\lambda\left[\frac{\phi\left(-\mathbf{w}_{i}^{\prime} \boldsymbol{\alpha}\right)}{\Phi\left(-\mathbf{w}_{i}^{\prime} \boldsymbol{\alpha}\right)}\right] \\
& =\delta+\lambda\left[\frac{\phi\left(\mathbf{w}_{i}^{\prime} \boldsymbol{\alpha}\right)}{\Phi\left(\mathbf{w}_{i}^{\prime} \boldsymbol{\alpha}\right)\left\{1-\Phi\left(\mathbf{w}_{i}^{\prime} \boldsymbol{\alpha}\right)\right\}}\right]
\end{aligned}
$$

where $\lambda=\rho \sigma$, the standard error of which is approximated by $\operatorname{Var}(\lambda) \approx \mathbf{D}\{\operatorname{atanh}(\rho) \ln \sigma\} \mathbf{D}^{\prime}$, where $\mathbf{D}$ is the Jacobian of $\lambda$ with respect to $\operatorname{atanh}(\rho)$ and $\ln \sigma$. If the correlation coefficient $\rho$ is zero then the estimation procedure is reduced to an OLS. As a result, the difference in expected outcome between treated and untreated is simply $\delta$. 


\subsubsection{The Roy model}

The Roy model has the advantage of considering selection on both observables and unobservables while relaxing the assumption of homogeneity of treatment by introducing heterogenous treatment effects. Thereby, we are able to identify and estimate not only the average treatment effect, but also treatment effect on the treated and on the untreated. The model with endogenous treatment and different outcome equations is

$$
\begin{aligned}
T_{i}^{*} & =\gamma^{\prime} \mathbf{w}_{i}+\mu_{i}, \quad T_{i}=\mathbf{1}\left[T_{i}^{*}>0\right], \quad i=1, \cdots, N \\
y_{i 1} & =\boldsymbol{\beta}_{1}^{\prime} \mathbf{x}_{i}+\varepsilon_{i 1}, \\
y_{i 0} & =\boldsymbol{\beta}_{0}^{\prime} \mathbf{x}_{i}+\varepsilon_{i 0}
\end{aligned}
$$

where $T_{i}^{*}$ in equation (1-11) is a latent variable, the observed counterpart of which is $T_{i}$, and $\mathbf{1}$ [ ] denotes the indicator function which takes on the value 1 if the corresponding latent variable is positive, and 0 otherwise. Relations (1-12) and (1-13) are respectively the outcome equations for participants and non-participants, $\boldsymbol{\gamma}$ and $\boldsymbol{\beta}$ 's denote vectors of parameters to be estimated, and $\mu_{i}, \varepsilon_{1}$ and $\varepsilon_{0}$ the denote error terms in the corresponding equations. Our estimation strategies are the maximum likelihood estimation and the two-step control function approaches.

\section{Maximum Likelihood (ML)}

We assume joint normality for the three disturbances conditionally on $\left(\mathbf{w}_{i}, \mathbf{x}_{i}\right)$;

$$
\left(\begin{array}{l}
\mu_{i} \\
\varepsilon_{i 1} \\
\varepsilon_{i 0}
\end{array}\right) \sim N\left[\left(\begin{array}{l}
0 \\
0 \\
0
\end{array}\right),\left(\begin{array}{ccc}
1 & & \\
\rho_{\mu_{\varepsilon_{1}}} \sigma_{\varepsilon_{1}} & \sigma_{\varepsilon_{1}}^{2} & \\
\rho_{\mu_{\varepsilon_{0}}} \sigma_{\varepsilon_{0}} & 0 & \sigma_{\varepsilon_{0}}^{2}
\end{array}\right)\right]
$$

Observe here that the null correlation term corresponds to $\operatorname{Cov}\left(\varepsilon_{0}, \varepsilon_{1}\right)=\rho_{\varepsilon_{0} \varepsilon_{1}} \sigma_{\varepsilon_{0}} \sigma_{\varepsilon_{1}}$ as the outcome equations are assumed not to be related. The likelihood function is then given by

$$
\begin{aligned}
L & =\prod_{i=1}^{N}\left[\int_{-\infty}^{-\gamma^{\prime} \mathbf{w}_{i}} f_{2}\left(\mu_{i}, y_{i 0}\right) d \mu_{i}\right]^{1-T_{i}}\left[\int_{-\gamma^{\prime} \mathbf{w}_{i}}^{\infty} f_{2}\left(\mu_{i}, y_{i 1}\right) d \mu_{i}\right]^{T_{i}} \\
& =\prod_{i=1}^{N}\left[f_{1}\left(y_{i 0}\right) \int_{-\infty}^{-\gamma^{\prime} \mathbf{w}_{i}} f_{1}\left(\mu_{i} \mid y_{i 0}\right) d \mu_{i}\right]^{1-T_{i}}\left[f_{1}\left(y_{i 1}\right) \int_{-\gamma^{\prime} \mathbf{w}_{i}}^{\infty} f_{1}\left(\mu_{i} \mid y_{i 1}\right) d \mu_{i}\right]^{T_{i}}
\end{aligned}
$$

where the functions $f_{2}(\cdot)$ and $f_{1}(\cdot)$ stand respectively for bivariate and univariate normal density functions. By replacing these functions by the population analogues, the likelihood function becomes

$$
L=\prod_{i=1}^{N}\left[\frac{1}{\sigma_{\varepsilon_{0}}} \phi\left(\zeta_{0}\right) \Phi\left(\frac{-\gamma^{\prime} \mathbf{w}_{i}-\rho_{\mu_{i} \varepsilon_{i 0}} \zeta_{0}}{\sqrt{1-\rho_{\mu_{i} \varepsilon_{i 0}}^{2}}}\right)\right]^{1-T_{i}}\left[\frac{1}{\sigma_{\varepsilon_{1}}} \phi\left(\zeta_{1}\right) \Phi\left(\frac{\gamma^{\prime} \mathbf{w}_{i}+\rho_{\mu_{i} \varepsilon_{i 1}} \zeta_{1}}{\sqrt{1-\rho_{\mu_{i} \varepsilon_{i 1}}^{2}}}\right)\right]^{T_{i}}
$$

where $\zeta_{k}=\frac{y_{i k-\boldsymbol{\beta}_{k}^{\prime} \mathbf{x}_{i}}}{\sigma_{\varepsilon_{k}}}$, with $k=(0,1)$, and $\phi(\cdot)$ and $\Phi(\cdot)$ denote respectively the standard normal probability density and the normal cumulative distribution functions. The parameters vector estimates are obtained by maximizing the $\log$-likelihood $\ln L$ using standard numerical procedures (e.g. Newton-Raphson). Standard error estimates are obtained using the inverse Hessian or outer product of gradient. 


\section{Two-step (2-step)}

In order to estimate the Roy model using the Heckman's two-step method applied to the truncated means, we write the regression function for each subpopulation as

$$
\begin{aligned}
& E\left(y_{i 1} \mid T=1, \mathbf{x}, \mathbf{w}\right)=\boldsymbol{\beta}_{1}^{\prime} \mathbf{x}_{i}+\operatorname{Cov}\left(\varepsilon_{i 1}, \mu_{i}\right) \lambda_{1}\left(\boldsymbol{\gamma}^{\prime} \mathbf{w}_{i}\right) \\
& E\left(y_{i 0} \mid T=0, \mathbf{x}, \mathbf{w}\right)=\boldsymbol{\beta}_{0}^{\prime} \mathbf{x}_{i}+\operatorname{Cov}\left(\varepsilon_{i 0}, \mu_{i}\right) \lambda_{0}\left(\boldsymbol{\gamma}^{\prime} \mathbf{w}_{i}\right),
\end{aligned}
$$

where $\lambda_{1}\left(\gamma^{\prime} \mathbf{w}_{i}\right)=\frac{\phi\left(\gamma^{\prime} \mathbf{w}_{i}\right)}{\Phi\left(\gamma^{\prime} \mathbf{w}_{i}\right)}$ and $\lambda_{0}\left(\gamma^{\prime} \mathbf{w}_{i}\right)=-\frac{\phi\left(\gamma^{\prime} \mathbf{w}_{i}\right)}{1-\Phi\left(\gamma^{\prime} \mathbf{w}_{i}\right)}$ are the inverse Mills ratios. In terms of parameters to be estimated, these regressions can be rewritten as:

$$
\begin{aligned}
& y_{i 1}=\boldsymbol{\beta}_{1}^{\prime} \mathbf{x}_{i}+\rho_{\mu_{\varepsilon_{1}}} \sigma_{\varepsilon_{1}} \lambda_{1}\left(\boldsymbol{\gamma}^{\prime} \mathbf{w}_{i}\right)+\eta_{i 1} \\
& y_{i 0}=\boldsymbol{\beta}_{0}^{\prime} \mathbf{x}_{i}+\rho_{\mu_{\varepsilon_{0}}} \sigma_{\varepsilon_{0}} \lambda_{0}\left(\boldsymbol{\gamma}^{\prime} \mathbf{w}_{i}\right)+\eta_{i 0},
\end{aligned}
$$

where $E\left(\eta_{i 1} \mid \mathbf{x}_{i}, \lambda_{1}\right)=E\left(\eta_{i 0} \mid \mathbf{x}_{i}, \lambda_{0}\right)=0$. In relations (1-18) and (1-19), $\lambda_{1}\left(\gamma^{\prime} \mathbf{w}_{i}\right)$ and $\lambda_{0}\left(\gamma^{\prime} \mathbf{w}_{i}\right)$ do enter as additional controls, the parameters of which $\rho_{\mu_{\varepsilon_{1}}} \sigma_{\varepsilon_{1}}$ and $\rho_{\mu_{\varepsilon_{0}}} \sigma_{\varepsilon_{0}}$ have to be estimated in addition to parameters vector $\boldsymbol{\beta}_{1}$ and $\boldsymbol{\beta}_{0}$. Thereby, the estimation proceeds as follows:

1. Obtain consistent and efficient (under normality) estimates for $\gamma$ by estimating a probit using maximum likelihood. Compute $\lambda_{1}\left(\gamma^{\prime} \mathbf{w}_{i}\right)$ and $\lambda_{0}\left(\gamma^{\prime} \mathbf{w}_{i}\right)$ given the predictions.

2. Use $\lambda_{1}\left(\gamma^{\prime} \mathbf{w}_{i}\right)$ and $\lambda_{0}\left(\gamma^{\prime} \mathbf{w}_{i}\right)$ as additional controls along side $\mathbf{x}_{i}$ and apply OLS to equations (1-18) and (1-19). Since we use the estimates of the $\lambda$ 's, the conventional standard errors are not valid and need to be corrected by using simulation techniques or bootstrap.

\section{Treatment effects}

The computations of the treatment effects are summarized in Table 2.1, where the covariance term is given by $\operatorname{Cov}\left(\varepsilon_{i 1}-\varepsilon_{i 0}, \mu_{i}\right)=\rho_{\mu_{i} \varepsilon_{i 1}} \sigma_{\varepsilon_{i 1}}-\rho_{\mu_{i} \varepsilon_{i 0}} \sigma_{\varepsilon_{i 0}}$. For the $\operatorname{LATE}(\mathbf{x})$, we apply the formulæ for double truncation to obtain the term $\lambda_{01}=\frac{\phi\left(\boldsymbol{\gamma}^{\prime} \mathbf{w}_{i}\right)-\phi\left(\gamma^{\prime} \tilde{\mathbf{w}}_{i}\right)}{\Phi\left(\gamma^{\prime} \tilde{\mathbf{w}}_{i}\right)-\Phi\left(\boldsymbol{\gamma}^{\prime} \tilde{\mathbf{w}}_{i}\right)}$, where $\tilde{\mathbf{w}}_{i}$ stands for a 'policy instrument'. For these effects, the associated parameters ATE, ATET, ATENT and LATE can be retrieved by simply integrating out or averaging over the sample.

Table 2.1: Treatment parameters (single treatment Roy model)

\begin{tabular}{lll}
\hline \hline & & \\
Parameter & Definition & Assumptions (model) \\
\hline & & \\
$\operatorname{ATE}(\mathbf{x})^{a}$ & $E\left(y_{i 1}-y_{i 0} \mid \mathbf{x}\right)$ & $\mathbf{x}_{i}\left(\boldsymbol{\beta}_{1}^{\prime}-\boldsymbol{\beta}_{0}^{\prime}\right)=: \vartheta(\mathbf{x})$ \\
$\operatorname{ATET}(\mathbf{x}, \mathbf{w})^{b}$ & $E\left(y_{i 1}-y_{i 0} \mid T=1, \mathbf{x}\right)$ & $\vartheta(\mathbf{x})+\operatorname{Cov}\left(\varepsilon_{i 1}-\varepsilon_{i 0}, \mu_{i}\right) \lambda_{1}$ \\
$\operatorname{ATENT}(\mathbf{x}, \mathbf{w})^{c}$ & $E\left(y_{i 1}-y_{i 0} \mid T=0, \mathbf{x}\right)$ & $\vartheta(\mathbf{x})-\operatorname{Cov}\left(\varepsilon_{i 1}-\varepsilon_{i 0}, \mu_{i}\right) \lambda_{0}$ \\
$\operatorname{LATE}(\mathbf{x}, \tilde{\mathbf{w}}, \mathbf{w})^{d}$ & $E\left(y_{i 1}-y_{i 0} \mid T(\mathbf{w})=0, T(\tilde{\mathbf{w}})=1, \mathbf{x}\right)$ & $\vartheta(\mathbf{x})+\operatorname{Cov}\left(\varepsilon_{i 1}-\varepsilon_{i 0}, \mu_{i}\right) \lambda_{01}$ \\
& $E\left(y_{i 1}-y_{i 0} \mid-\gamma^{\prime} \tilde{\mathbf{w}}_{i} \leq \mu_{i}<-\gamma^{\prime} \mathbf{w}_{i}, \mathbf{x}\right)$ & \\
\hline \hline
\end{tabular}

\footnotetext{
(a) Average treatment effect

(b) Average treatment effect on the treated

(c) Average treatment effect on the untreated

(d) Local average treatment effect
}

\section{$2.5 \quad$ Findings}

As we have previously indicated, we used both the propensity score matching framework (hereafter PSM) which can be viewed as given by relation (1-1), and the structural model presented 
in the previous section to assess the impact of meal and deworming programs. As we have also mentioned, the structural model has the advantage of allowing to jointly estimate a performance equation (1-2) and a selection equation (1-1) while estimating the effect of the program. One important aspect of the structural model is whether both equations are related via the correlation term $\rho$ between errors. If the correlation is zero, both equations are unrelated and the problem reduced to one estimable by OLS and the effect of the program is $\delta$.

In our framework, the PSM yields the ATET and the structural approach allows to have a greater number of effects (ATE, ATET, ATENT and LATE).

\subsubsection{Determinants of school achievement: a summary of findings}

For the determinants of performance, we conducted estimations. For example, taking the meal program, we have made estimates with the Heckman selection framework (FIML and two step) and estimates with the Roy model (FIML and two step). With the deworming program, we did also estimations with the Heckman selection framework (FIML and two step) and the Roy model (FIML and two step). For reasons of space, we present only estimates on meal program (FIML) and deworming program (FIML). The other estimates are reported in the appendix. If there is no difference between the results presented and those in the appendix, we will not mention the later. Otherwise, we will highlight the differences.

The results for the determinants of school performance are given in Tables 2.2 and 2.3 . The LR test in the two tables shows that $\rho$ is significant for the aggregate and math scores, and not significant for the score in French in Table 2.2 and significant for all scores in Table 2.3 meaning that we cannot reject the null hypothesis that the two error terms are unrelated.

Table 2.2: Determinants of scores: Meal, $\mathrm{HSF}^{a}$ regression (FIML)

\begin{tabular}{|c|c|c|c|c|c|c|}
\hline \multirow[b]{2}{*}{ Variable } & \multicolumn{2}{|c|}{ Aggregate score } & \multicolumn{2}{|c|}{ French score } & \multicolumn{2}{|c|}{ Math score } \\
\hline & Coef. & Std. Err. & Coef. & Std. Err. & Coef. & Std. Err. \\
\hline Performance Eq. & & & & & & \\
\hline Temporary shelters & $-0.766^{* *}$ & 0.345 & -0.434 & 0.369 & $-1.166^{* * *}$ & 0.386 \\
\hline School manual & $6.731^{* * *}$ & 1.107 & $6.784^{* * *}$ & 1.185 & $6.668^{* * *}$ & 1.240 \\
\hline School manual squared & $-0.619^{* * *}$ & 0.195 & $-0.940^{* * *}$ & 0.209 & -0.295 & 0.219 \\
\hline Class size & $-0.241^{* *}$ & 0.114 & -0.077 & 0.122 & $-0.3948^{* * *}$ & 0.128 \\
\hline Class size squared & 0.002 & 0.001 & -0.0002 & 0.001 & $0.004^{* *}$ & 0.001 \\
\hline Number of pregnancies & $-3.544^{* * *}$ & 1.035 & $-3.749^{* * *}$ & 1.108 & $-3.259^{* * *}$ & 1.158 \\
\hline Number of marriages & -0.544 & 0.734 & -1.251 & 0.786 & 0.068 & 0.822 \\
\hline Teacher's age & $-1.385^{* *}$ & 0.656 & -1.127 & 0.703 & $-1.547^{* *}$ & 0.734 \\
\hline Teacher's age square & $0.023^{* *}$ & 0.009 & 0.016 & 0.010 & $0.028^{* *}$ & 0.011 \\
\hline Pupil's age & $7.088^{* * *}$ & 2.108 & $8.436^{* * *}$ & 2.257 & $5.845^{* *}$ & 2.361 \\
\hline Pupil's age square & $-0.283^{* * *}$ & 0.097 & $-0.347^{* * *}$ & 0.104 & $-0.225^{* *}$ & 0.109 \\
\hline Number of hours & -0.019 & 0.534 & -0.113 & 0.572 & 0.073 & 0.596 \\
\hline Food expenditure $^{b}$ & -0.782 & 0.768 & -1.090 & 0.822 & -0.584 & 0.859 \\
\hline Education expenditure ${ }^{b}$ & $0.937^{* * *}$ & 0.296 & $1.149^{* * *}$ & 0.317 & $0.754^{* *}$ & 0.332 \\
\hline Health expenditure ${ }^{b}$ & -0.268 & 0.186 & -0.188 & 0.199 & -0.362 & 0.208 \\
\hline Household size ${ }^{c}$ & $0.210^{* * *}$ & 0.065 & $0.215^{* * *}$ & 0.070 & $0.205^{* * *}$ & 0.073 \\
\hline Livestock & 0.007 & 0.019 & -0.004 & 0.020 & 0.020 & 0.021 \\
\hline Distance to school & 1.377 & 0.940 & 1.424 & 0.997 & 1.320 & 1.061 \\
\hline Playground & 0.373 & 1.056 & 0.090 & 1.132 & 0.696 & 1.179 \\
\hline Water point & $2.447^{* * *}$ & 0.885 & $1.583^{*}$ & 0.939 & $3.267^{* * *}$ & 0.997 \\
\hline Disturbed courses & $-6.318^{* * *}$ & 1.161 & $-5.087^{* * *}$ & 1.239 & $-7.663^{* * *}$ & 1.301 \\
\hline Meals near school & -0.118 & 0.911 & -1.276 & 0.975 & 1.071 & 1.021 \\
\hline Gender of teacher & $-4.731^{* * *}$ & 1.145 & $-3.913^{* * *}$ & 1.225 & $-5.608^{* * *}$ & 1.282 \\
\hline
\end{tabular}


Table 2.2 - continued

\begin{tabular}{|c|c|c|c|c|c|c|}
\hline \multirow[b]{2}{*}{ Variable } & \multicolumn{2}{|c|}{ Aggregate score } & \multicolumn{2}{|c|}{ French score } & \multicolumn{2}{|c|}{ Math score } \\
\hline & Coef. & Std. Err. & Coef. & d. Err. & Coef. & td. Err. \\
\hline No professional qualif. ${ }^{d}$ & $6.267^{* * *}$ & 1.041 & $5.063^{* * *}$ & 1.116 & $7.469^{* * *}$ & 1.165 \\
\hline Professional qualif. CAP & $2.801^{* *}$ & 1.412 & $3.081^{* *}$ & 1.511 & $2.707^{*}$ & 1.583 \\
\hline Professional qualif. CEAP & $4.474^{* * *}$ & 1.097 & $2.021^{*}$ & 1.175 & $6.796^{* * *}$ & 1.228 \\
\hline High school diploma \& more & 0.222 & 0.888 & -1.233 & 0.952 & $1.658^{*}$ & 0.994 \\
\hline Continuing training & -0.191 & 0.789 & -0.371 & 0.845 & -0.174 & 0.883 \\
\hline Absenteeism & -1.131 & 1.583 & -0.423 & 1.706 & -2.030 & 1.762 \\
\hline Gender of pupil & $1.409^{*}$ & 0.723 & 0.704 & 0.766 & $1.922^{* *}$ & 0.817 \\
\hline Garde & $-12.622^{* * *}$ & 1.032 & $-19.553^{* * *}$ & 1.105 & $-5.646^{* * *}$ & 1.155 \\
\hline Koranic school & $2.663^{* * *}$ & 0.813 & $2.625^{* * *}$ & 0.870 & $2.781^{* * *}$ & 0.910 \\
\hline Early childhood inst. & $4.930^{* * *}$ & 1.563 & $5.810^{* * *}$ & 1.673 & $3.967^{* *}$ & 1.751 \\
\hline Snack & 1.499 & 1.315 & 1.477 & 1.408 & 1.736 & 1.472 \\
\hline Sick last 3 months & 0.075 & 0.824 & 0.723 & 0.883 & -0.490 & 0.922 \\
\hline Pupils eat fill & $2.929^{* *}$ & 1.165 & $2.618^{* *}$ & 1.248 & $3.198^{* *}$ & 1.303 \\
\hline Gender of household head & -1.142 & 1.365 & -2.288 & 1.462 & 0.067 & 1.528 \\
\hline Lit. of household head & $-1.659^{*}$ & 1.006 & -1.175 & 1.077 & $-2.377^{* *}$ & 1.127 \\
\hline Marital status & 1.130 & 1.872 & 2.368 & 2.006 & -0.079 & 2.095 \\
\hline Land & -2.325 & 1.670 & -1.011 & 1.787 & $-3.384^{*}$ & 1.870 \\
\hline Parents school & $-2.997^{* * *}$ & 0.827 & $-3.938^{* * *}$ & 0.882 & $-1.962^{* *}$ & 0.926 \\
\hline College & $7.284^{* * *}$ & 1.109 & $6.675^{* * *}$ & 1.186 & $7.942^{* * *}$ & 1.244 \\
\hline Diourbel & $6.877^{* * *}$ & 1.811 & $4.950^{* *}$ & 1.932 & $8.788^{* * *}$ & 2.028 \\
\hline Kolda & -0.656 & 1.298 & -0.087 & 1.388 & -1.096 & 1.454 \\
\hline Sedhiou & -0.826 & 1.904 & -3.694 & 2.042 & 1.805 & 2.130 \\
\hline Meal & 2.814 & 2.010 & $4.932^{* *}$ & 2.205 & 1.182 & 2.164 \\
\hline Intercept & 27.078 & 17.813 & 21.637 & 19.071 & 31.385 & 19.948 \\
\hline \multicolumn{7}{|l|}{ Selection Eq. } \\
\hline Total pupils & 0.0005 & 0.0003 & $0.0005^{*}$ & 0.0003 & $0.0004^{*}$ & 0.0003 \\
\hline Distance to school & $0.132^{*}$ & 0.075 & $0.129^{*}$ & 0.075 & 0.136 & 0.075 \\
\hline Management committee & 0.095 & 0.069 & 0.092 & 0.070 & 0.096 & 0.068 \\
\hline Association of mothers & $0.686^{* * *}$ & 0.072 & $0.696^{* * *}$ & 0.073 & $0.700^{* * *}$ & 0.071 \\
\hline Cooperative school & -0.006 & 0.067 & -0.013 & 0.068 & 0.008 & 0.066 \\
\hline Rural council grant & $0.859^{* * *}$ & 0.088 & $0.816^{* * *}$ & 0.088 & $0.879^{* * *}$ & 0.086 \\
\hline Water point & -0.030 & 0.075 & -0.035 & 0.076 & -0.027 & 0.075 \\
\hline Disturbed courses & $0.967^{* * *}$ & 0.095 & $0.983^{* * *}$ & 0.096 & $0.955^{* * *}$ & 0.095 \\
\hline Storage & $0.821^{* * *}$ & 0.074 & $0.842^{* * *}$ & 0.074 & $0.795^{* * *}$ & 0.074 \\
\hline Gender of pupil & -0.087 & 0.062 & -0.087 & 0.062 & -0.087 & 0.062 \\
\hline Intercept & $-2.709^{* * *}$ & 0.160 & $-2.702^{* * *}$ & 0.161 & $-2.717^{* * *}$ & 0.159 \\
\hline $\operatorname{atanh} \rho$ & $0.246^{* * *}$ & 0.075 & 0.088 & 0.076 & $0.334^{* * *}$ & 0.072 \\
\hline $\ln \sigma$ & $2.850^{* * *}$ & 0.016 & $2.907^{* * *}$ & 0.014 & $2.973^{* * *}$ & 0.017 \\
\hline$\rho$ & 0.241 & 0.071 & 0.088 & 0.076 & 0.322 & 0.065 \\
\hline$\sigma$ & 17.289 & 0.280 & 18.312 & 0.270 & 19.552 & 0.335 \\
\hline$\lambda$ & 4.172 & 1.266 & 1.612 & 1.401 & 6.307 & 1.336 \\
\hline \multicolumn{7}{|l|}{ LR test $\left(H_{0}: \rho=0\right)$} \\
\hline$\chi_{2}(1)$ & \multicolumn{2}{|c|}{9.16} & \multicolumn{2}{|c|}{1.28} & \multicolumn{2}{|c|}{18.95} \\
\hline$P>\chi_{2}$ & \multicolumn{2}{|c|}{0.0020} & \multicolumn{2}{|c|}{$\begin{array}{l}1.20 \\
0.257\end{array}$} & \multicolumn{2}{|c|}{0.000} \\
\hline Log likelihood & \multicolumn{2}{|c|}{-11140.128} & \multicolumn{2}{|c|}{-11302.72} & \multicolumn{2}{|c|}{-11406.014} \\
\hline \# Observations & \multicolumn{2}{|c|}{2369} & \multicolumn{2}{|c|}{2369} & \multicolumn{2}{|c|}{2369} \\
\hline Wald $\chi_{2}(46)$ & \multicolumn{2}{|c|}{604.12} & \multicolumn{2}{|c|}{775.16} & \multicolumn{2}{|c|}{505.38} \\
\hline$P>\chi_{2}$ & 0.0 & & 0.0 & & 0.0 & \\
\hline
\end{tabular}

Notes $^{a}$ : Heckman selection framework; ${ }^{b}:$ In log; ${ }^{c}$ : Household size per adult equivalent; ${ }^{d}$ : qualification.

Significance levels (bootstrap): $\quad *: 10 \% \quad * *: 5 \% \quad * * *: 1 \%$

This supports our modeling strategy that both equations should be estimated jointly. The 
sign of the coefficients in the selection equation are the same for the three scores. The same holds for significance apart from the variables 'total pupils' which is not significant for the aggregate score and 'distance to school' which is not significant for the math score. For example, total pupils, association of mothers, rural council grant, disturbed courses, storage, distance to school impact positively the probability for a pupil to benefit from a meal program. Similar results are found when using the two step estimation. These results are expected, as these controls are specific indicators used by the World Food Program (WFP) and the Senegalese Ministry of education to select the schools that will participate in the program. However, in the same line, one would expect that having an operational management committee and a water point will be among the determinants of selection, which surprisingly is not the case. When we compare these findings to those obtained from the PSM estimation (see Table A.4 in the appendix), we have quite similar results except for the variable water point which is positive and significant now.

Table 2.3: Determinants of scores: Deworming, $\mathrm{HSF}^{a}$ regression (FIML)

\begin{tabular}{|c|c|c|c|c|c|c|}
\hline \multirow[b]{2}{*}{ Variable } & \multicolumn{2}{|c|}{ Aggregate score } & \multicolumn{2}{|c|}{ French score } & \multicolumn{2}{|c|}{ Math score } \\
\hline & Coef. & Std. Err. & Coef. & Std. Err. & Coef. & Std. Err. \\
\hline Performance Eq. & & & & & & \\
\hline Temporary shelters & 0.537 & 0.393 & 0.572 & 0.419 & $0.793^{*}$ & 0.442 \\
\hline School manual & 1.096 & 1.087 & $2.560^{* *}$ & 1.150 & 0.307 & 1.224 \\
\hline School manual squared & 0.110 & 0.190 & $-0.331^{*}$ & 0.200 & $0.460^{* *}$ & 0.214 \\
\hline Class size & -0.162 & 0.119 & 0.056 & 0.126 & $-0.488^{* * *}$ & 0.134 \\
\hline Class size squared & 0.0008 & 0.001 & -0.002 & 0.001 & $0.005^{* * *}$ & 0.001 \\
\hline Number of pregnancies & $-6.453^{* * *}$ & 1.116 & $-8.082^{* * *}$ & 1.150 & $-4.428^{* * *}$ & 1.256 \\
\hline Number of marriages & -0.200 & 0.680 & 0.203 & 0.709 & -0.399 & 0.764 \\
\hline Teacher's age & $-2.527^{* * *}$ & 0.624 & $-3.540^{* * *}$ & 0.656 & $-2.201^{* * *}$ & 0.702 \\
\hline Teacher's age square & $0.035^{* * *}$ & 0.009 & $0.047^{* * *}$ & 0.009 & $0.033^{* * *}$ & 0.010 \\
\hline Pupil's age & $6.974^{* * *}$ & 1.950 & $7.698^{* * *}$ & 2.040 & $6.366^{* * *}$ & 2.193 \\
\hline Pupil's age square & $-0.277^{* * *}$ & 0.091 & $-0.315^{* * *}$ & 0.096 & $-0.247^{* *}$ & 0.103 \\
\hline Number of hours & -0.180 & 0.626 & -0.432 & 0.667 & -0.174 & 0.703 \\
\hline Food expenditure ${ }^{b}$ & 0.706 & 0.738 & -0.045 & 0.773 & $1.367^{*}$ & 0.830 \\
\hline Education expenditure ${ }^{b}$ & 0.252 & 0.275 & 0.264 & 0.283 & 0.223 & 0.309 \\
\hline Health expenditure $^{b}$ & 0.083 & 0.166 & 0.129 & 0.173 & 0.038 & 0.187 \\
\hline Household size $^{c}$ & $0.265^{* * *}$ & 0.063 & $0.234^{* * *}$ & 0.066 & $0.283^{* * *}$ & 0.071 \\
\hline Livestock & -0.001 & 0.020 & -0.023 & 0.021 & 0.019 & 0.023 \\
\hline Distance to school & 1.001 & 0.908 & 1.456 & 0.950 & 0.598 & 1.022 \\
\hline Playground & $1.573^{*}$ & 0.954 & $2.671^{* * *}$ & 1.008 & $1.995^{*}$ & 1.073 \\
\hline Water point & -0.009 & 0.965 & $-3.954^{* * *}$ & 1.063 & 1.200 & 1.084 \\
\hline Disturbed courses & $-4.220^{* * *}$ & 0.990 & $-2.132^{* *}$ & 1.028 & $-5.899^{* * *}$ & 1.112 \\
\hline Meals near school & -0.537 & 0.908 & $-2.157^{* *}$ & 0.955 & 0.486 & 1.024 \\
\hline Gender of teacher & $-3.913^{* * *}$ & 1.127 & -1.416 & 1.182 & $-5.151^{* * *}$ & 1.267 \\
\hline No professional qualif. ${ }^{d}$ & 0.115 & 1.034 & -0.889 & 1.085 & 1.778 & 1.163 \\
\hline Professional qualif. CAP & -0.670 & 1.246 & -1.593 & 1.301 & -0.233 & 1.401 \\
\hline Professional qualif. CEAP & $4.483^{* * *}$ & 1.169 & $3.107^{* *}$ & 1.244 & $5.546^{* * *}$ & 1.318 \\
\hline High school diploma \& more & $1.811^{* *}$ & 0.882 & 0.848 & 0.922 & $2.368^{* *}$ & 0.993 \\
\hline Continuing training & -0.518 & 0.797 & -0.570 & 0.833 & -0.596 & 0.899 \\
\hline Absenteeism & $-4.528^{* * *}$ & 1.285 & $-4.322^{* * *}$ & 1.352 & $-6.771^{* * *}$ & 1.449 \\
\hline Gender of pupil & 0.579 & 0.725 & 0.646 & 0.847 & 1.266 & 0.831 \\
\hline Garde & $-14.211^{* * *}$ & 0.983 & $-20.854^{* * *}$ & 1.028 & $-7.389^{* * *}$ & 1.106 \\
\hline Koranic school & $3.499^{* * *}$ & 0.798 & $4.028^{* * *}$ & 0.832 & $3.136^{* * *}$ & 0.898 \\
\hline Early childhood inst. & 2.027 & 1.313 & $2.744^{* *}$ & 1.370 & 1.213 & 1.473 \\
\hline Snack & -0.290 & 1.148 & -0.483 & 1.197 & -0.661 & 1.289 \\
\hline
\end{tabular}


Table 2.3 - continued

\begin{tabular}{|c|c|c|c|c|c|c|}
\hline \multirow[b]{2}{*}{ Variable } & \multicolumn{2}{|c|}{ Aggregate score } & \multicolumn{2}{|c|}{ French score } & \multicolumn{2}{|c|}{ Math score } \\
\hline & Coef. & Std. Err. & Coef. & Std. Err. & Coef. & td. Err. \\
\hline Sick last 3 months & -0.263 & 0.770 & 0.273 & 0.800 & -0.792 & 0.865 \\
\hline Pupils eat fill & 0.256 & 1.195 & 1.332 & 1.259 & -0.396 & 1.349 \\
\hline Gender of household head & -0.649 & 1.263 & -1.319 & 1.317 & -0.184 & 1.421 \\
\hline Lit. of household head & 0.466 & 1.015 & -0.918 & 1.159 & -0.079 & 1.155 \\
\hline Marital status & 2.847 & 1.789 & 2.121 & 1.870 & 3.161 & 2.011 \\
\hline Land & -2.404 & 1.590 & -2.348 & 1.643 & -2.385 & 1.789 \\
\hline Parents school & $-3.143^{* * *}$ & 0.798 & $-2.219^{* * *}$ & 0.848 & $-3.463^{* * *}$ & 0.899 \\
\hline College & $1.801^{*}$ & 1.029 & 1.269 & 1.085 & $2.994^{* *}$ & 1.161 \\
\hline Diourbel & 0.865 & 1.703 & 0.289 & 1.786 & 1.283 & 1.913 \\
\hline Kolda & $-9.844^{* * *}$ & 1.241 & $-9.737^{* * *}$ & 1.304 & $-9.794^{* * *}$ & 1.399 \\
\hline Sedhiou & $-9.116^{* * *}$ & 2.276 & $-9.503^{* * *}$ & 2.407 & $-10.967^{* * *}$ & 2.554 \\
\hline Dworming & $-13.046^{* * *}$ & 3.529 & $22.635^{* * *}$ & 2.390 & $-16.443^{* * *}$ & 3.431 \\
\hline Intercept & $47.868^{* * *}$ & 16.778 & $60.688^{* * *}$ & 17.505 & $37.559^{* *}$ & 18.884 \\
\hline \multicolumn{7}{|l|}{ Selection Eq. } \\
\hline Total pupils & $0.0004^{*}$ & 0.0002 & $0.0003^{*}$ & 0.0002 & $0.0005^{* *}$ & 0.0002 \\
\hline Management committee & $0.175^{* * *}$ & 0.063 & $0.325^{* * *}$ & 0.051 & $0.170^{* * *}$ & 0.059 \\
\hline Association of mothers & $0.222^{* * *}$ & 0.067 & 0.095 & 0.064 & $0.240^{* * *}$ & 0.066 \\
\hline Cooperative school & $0.1717^{* * *}$ & 0.060 & -0.040 & 0.052 & $0.187^{* * *}$ & 0.058 \\
\hline Water point & $0.179^{* * *}$ & 0.064 & $0.169^{* * *}$ & 0.062 & $0.172^{* * *}$ & 0.064 \\
\hline Medicine box & $-0.328^{* * *}$ & 0.088 & $0.126^{*}$ & 0.073 & $-0.333^{* * *}$ & 0.082 \\
\hline Gender of pupil & -0.050 & 0.053 & $-0.089^{*}$ & 0.052 & -0.050 & 0.053 \\
\hline Lit. of household head & $0.157^{* *}$ & 0.069 & $0.164^{* *}$ & 0.068 & $0.149^{* *}$ & 0.069 \\
\hline Intercept & $-0.944^{* * *}$ & 0.071 & $-0.869^{* * *}$ & 0.066 & $-0.962^{* * *}$ & 0.070 \\
\hline $\operatorname{atanh} \rho$ & $0.454^{* * *}$ & 0.128 & $-0.882^{* * *}$ & 0.093 & $0.541^{* * *}$ & 0.112 \\
\hline $\ln \sigma$ & $2.877^{* * *}$ & 0.031 & $3.039^{* * *}$ & 0.030 & $3.015^{* * *}$ & 0.030 \\
\hline$\rho$ & 0.425 & 0.425 & -0.707 & 0.046 & 0.494 & 0.084 \\
\hline$\sigma$ & 17.771 & 0.452 & 20.885 & 0.643 & 20.398 & 0.631 \\
\hline$\lambda$ & 7.557 & 2.082 & -14.776 & 1.395 & 10.086 & 2.011 \\
\hline \multicolumn{7}{|l|}{ LR test $\left(H_{0}: \rho=0\right)$} \\
\hline$\chi_{2}(1)$ & \multicolumn{2}{|c|}{4.80} & \multicolumn{2}{|c|}{22.46} & \multicolumn{2}{|c|}{11.04} \\
\hline$P>\chi_{2}$ & \multicolumn{2}{|c|}{0.028} & \multicolumn{2}{|c|}{0.000} & \multicolumn{2}{|c|}{0.000} \\
\hline Log likelihood & \multicolumn{2}{|c|}{-12011.633} & \multicolumn{2}{|c|}{-12134.14} & \multicolumn{2}{|c|}{-12302.472} \\
\hline \# Observations & \multicolumn{2}{|c|}{2487} & \multicolumn{2}{|c|}{2487} & \multicolumn{2}{|c|}{2487} \\
\hline Wald $\chi_{2}(46)$ & \multicolumn{2}{|c|}{730.52} & \multicolumn{2}{|c|}{1119.30} & \multicolumn{2}{|c|}{546.79} \\
\hline$P>\chi_{2}$ & \multicolumn{2}{|c|}{0.000} & \multicolumn{2}{|c|}{0.000} & \multicolumn{2}{|c|}{0.000} \\
\hline
\end{tabular}

Notes $^{a}$ : Heckman selection framework; ${ }^{b}:$ In log; ${ }^{c}$ : Household size per adult equivalent; ${ }^{d}$ : qualification.

Significance levels (bootstrap): $\quad *: 10 \% \quad * *: 5 \% \quad * * *: 1 \%$

Regarding the determinants of performance as measured by test scores, as mentioned in the description of variables, we distinguished four categories of controls: those related to schools (temporary shelters, school manual, class size, teacher's age, pupil's age, distance to school, playground, water point, meals near school, gender of teacher, professional and academic qualification of teacher, continuing training), pupils (gender, grade, age, Koranic school, early childhood institution, snack, pupils eat at fill) household characteristics (food and education expenditures, household size, livestock, literacy of household head, gender of household head, marital status and land), community (parents school and existence of college in the village) and shocks variables (number of pregnancies, number of marriages, absenteeism, disturbed courses, sick last 3 months and health expenditures). Most of the control variables are of the expected sign when significant. Some others are not conformed on intuition. However, put in the specific context of rural Africa experience, they become rather highly interesting. This is the case of the relation 
between class size and math score as we will see later. For controls related to school characteristics, as expected, increasing temporary shelters has a negative effect on pupils' performance (aggregate, French and math scores). However, the result is not significant for French score. In temporary shelters, there are no adapted benches tables, blackboard that meet the requirement and sometimes no roof. That is why the quality of learning in temporary shelters are very bad.

For variables number of pupils per manual 'School manual', class size and teacher's age as mentioned above, we included the linear and the square terms to account for possible nonlinearities. In fact, for school manual which varies between 0.2 to 7 pupils per manual, Figures 2.1 and 2.2 show inverted U-shaped relations with scores, meaning that the number of pupils per manual increase the scores until a point from which the variable has a negative effect on performance. The turning point is 5 pupils for the aggregate score and 4 pupils for the French score. It is important to note that the nonlinear part of school manual is not significant for the math score. Our reading is that even if school manual has a capacity to ameliorate the competence of pupils, when the number of pupils per manual become higher this has a negative impact on scores because the learning time decreases with increasing of the number of pupils per manual. CONFEMEN (1999) shows that French and mathematics manuals have a positive effect on learning with a larger impact for the French manuals.

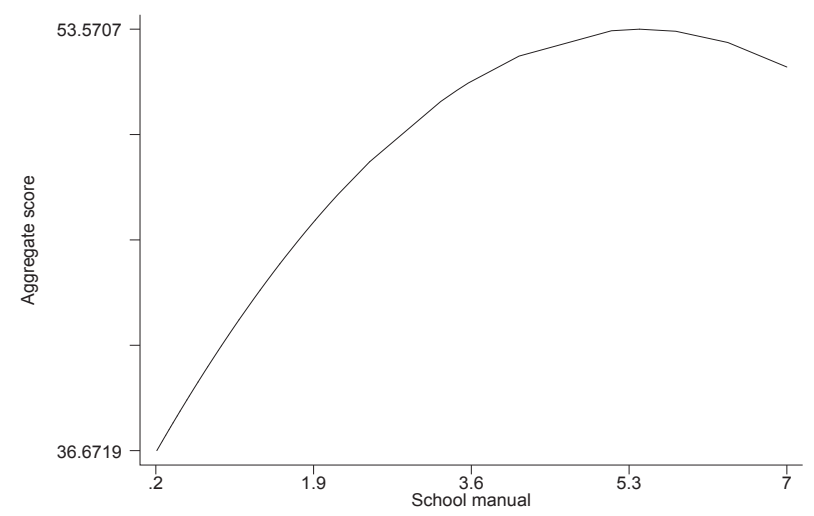

Figure 2.1: Meal: Relation between aggregate score and school manual.

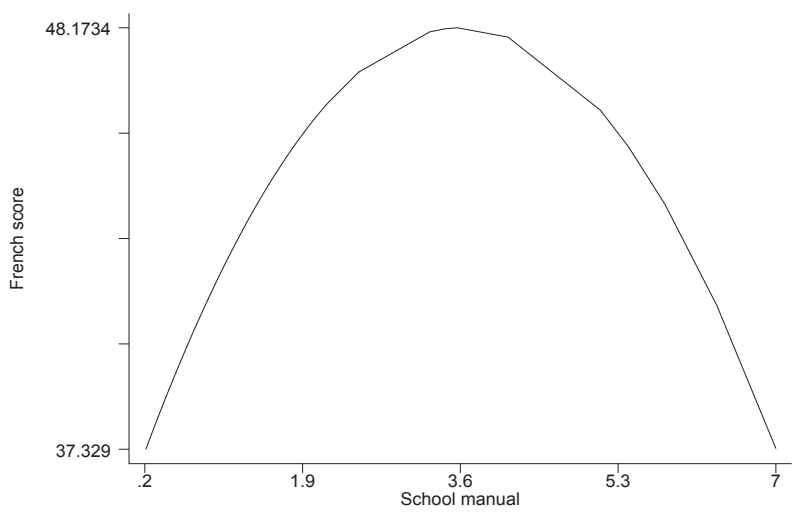

Figure 2.2: Meal: Relation between French score and school manual. 
Class size displays a U-shaped relation with math score. Note that this variable ranges from 4 to 82 pupils. Firstly, the performance decreases with increasing class size which is consistent with some findings in the literature on performance. However, starting from 47 pupils, the performance increases which is also consistent with other findings (see Figure 2.3). It is important to note that neither class size nor its square are significant for the French score.

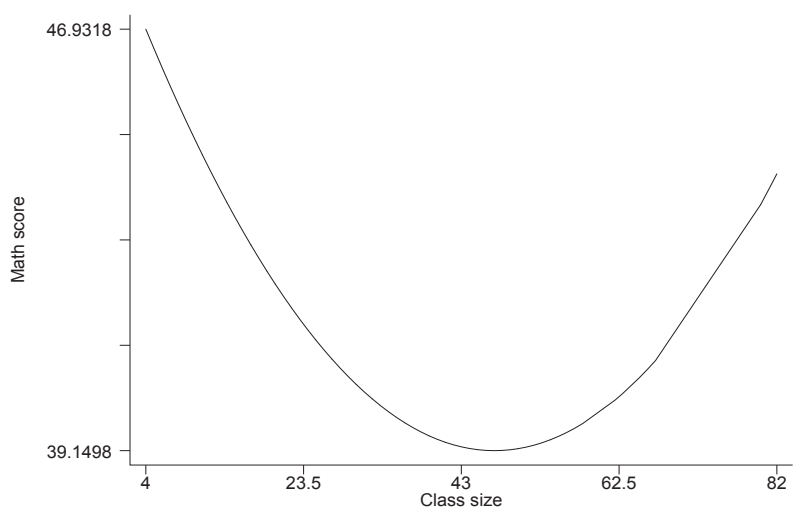

Figure 2.3: Meal: Relation between math score and class size.

As mentioned above, the effects of a reduction of class size on pupil achievement have not led to consistent conclusions in the literature (Davies, 2003). How to explain that large classes can perform? In these specific rural areas, large classes may impact positively math score. This result could be linked to the strategy of multigrade classes which is practiced in some rural schools in Senegal. The strategy based on multigrade classes is to put together in a classroom, pupils from several different grades under the supervision of only one teacher. Generally, if the practice of multigrade classes solves the problems of enrollment, it greatly reduces the learning time of pupils and consequently their academic performance. We believe that some small class sizes could be in multigrade classes, which would explain the negative correlation between small class size and the math score that we found. With big class size, it is difficult or not possible to practice the multigrade system. We think that, in this rural area, the small class sizes could be in multigrade class whereas the big class sizes could not.

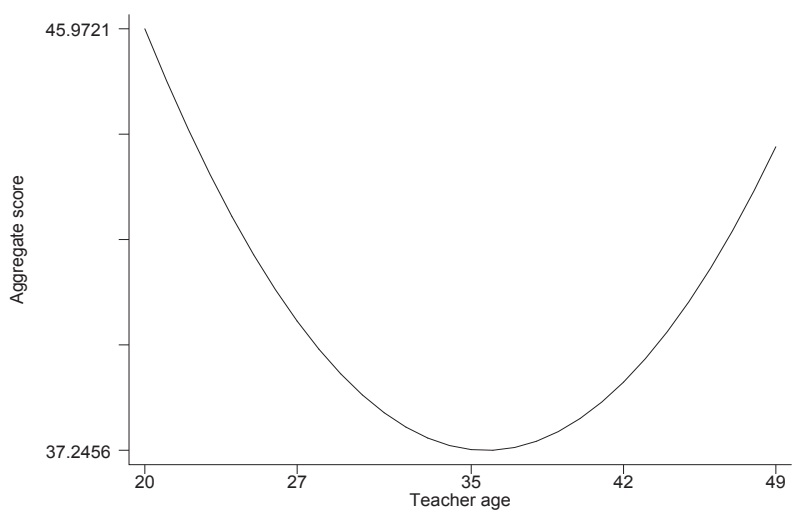

Figure 2.4: Meal: Relation between aggregate score and teacher age. 


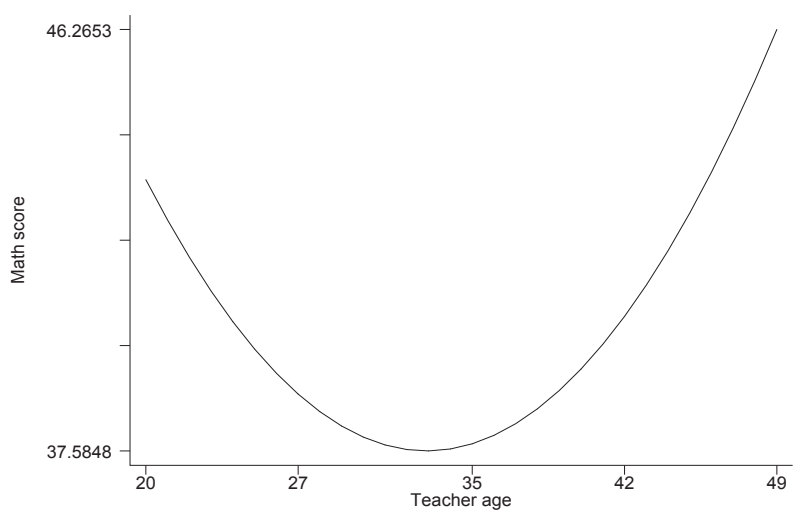

Figure 2.5: Meal: Relation between math score and teacher age.

The teacher's age also shows a similar U-shaped picture. This variable ranges from 20 to 53. The linear term is negative while the square term is positive for scores. This means that the teacher's age affect negatively the scores until a certain threshold at which additional age affect positively on the performances. We observe that the turning point is 36 and 33 respectively for aggregate and math scores (See Figures 2.4 and 2.5).

Always for controls related to school characteristics, one can note that variables gender of teacher $($ male $=1)$ and teacher absenteeism have a negative effect on scores, as expected, while variables water point, professional qualification and academic qualification (High School Diploma) have a positive effect on pupils' performance. In fact, for variable: gender of teacher, the result shows that pupils with female teachers perform better than those with male teachers. This result is consistent with the finding of Jarousse and Mingat (1989) who found similar results relying on data from Togo. For control absenteeism, the results are not surprising because when teachers are often absent, it reduces the learning time of pupils. For variable water point, the result is intuitive because schools with better infrastructure will record the best performance. Regarding professional qualification, the results show that pupils supervised by a teacher with the professional qualification CAP and CEAP perform better than those taught by a teacher with another professional qualification which is the reference. This result is intuitive. In fact, in Senegal, the two most important professional qualifications are the CAP (Certificat d'Aptitude Pédagogique, meaning 'Pedagogical Aptitude Certificate') and CEAP (Certificat Elémentaire d'Aptitude Pédagogique, which is 'Basic Pedagogical Aptitude Certificate'). The surprising result is that pupils taught by teacher with no professional diploma perform better than those supervised by a teacher with other professional degree. This result is consistent with a finding of CONFEMEN (2007) in Senegal. In rural areas, the result can be explained in two ways. Firstly, it may be that those teachers without professional diploma are awaiting to be officially graduated because most often they have already passed the written examination. Secondly, their level of motivation is much more important than teachers with professional qualification. In fact, teachers without professional qualification can be controlled at any time and this control is crucial for their career. So the performance of pupils under their care is reflected in the state of their job. As a result, it is likely that the motivation of those teachers is a push factor which leads them to teach pupils very well. This may also explain why pupils supervised by teachers without professional qualification are more effective.

For the academic qualification of teachers, the results from the DESM model show that pupils supervised by a teacher with the High School Diploma perform better in mathematics compared to those supervised by a teacher with the national certificate. As pointed out by Rivers and 
Sanders (2002), this result suggests that teachers with High School Diploma and more have a higher level of knowledge in mathematics than those with national certificate.

When one takes the deworming estimations, we always have the same result as in the meal regressions. If one takes the Roy's model into account, one has the same results for the untreated. However, if one takes the treated, one observes a negative relation between the scores and the proportion of pupils who are supervised by a teachers with High School Diploma. This result is consistent with the findings of CONFEMEN(1999).

Regarding controls related to pupils' characteristics, variable pupil's age shows an inverted U-shaped relation. Broadly, getting older has a negative effect on performance. In the sample, the variable varies between 6 to 15 years old. In fact, the results show that the age of pupil has a positive effect on scores until a certain threshold at which the age has a negative impact on the scores. The thresholds are 13, 12 and 13 years old respectively for aggregate, French and math scores (see figures 2.6, 2.7 and 2.8). This finding is consistent with observations and can be related to several factors which can explain the presence of pupils having age that is higher than the normal in primary school. These factors are for example temporary dropout of the pupil, repetition of the pupil or adverse family conditions for the pupil, etc. This result is observed for all estimations methods.

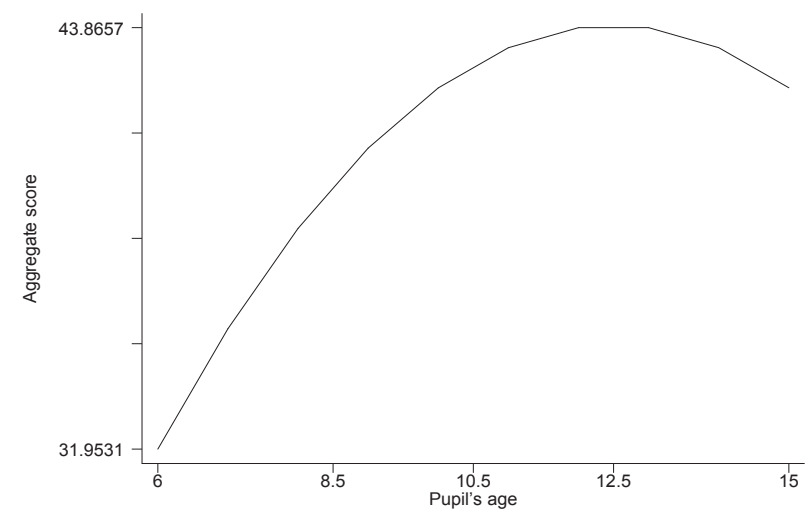

Figure 2.6: Meal: Relation between aggregate score and pupil age. 


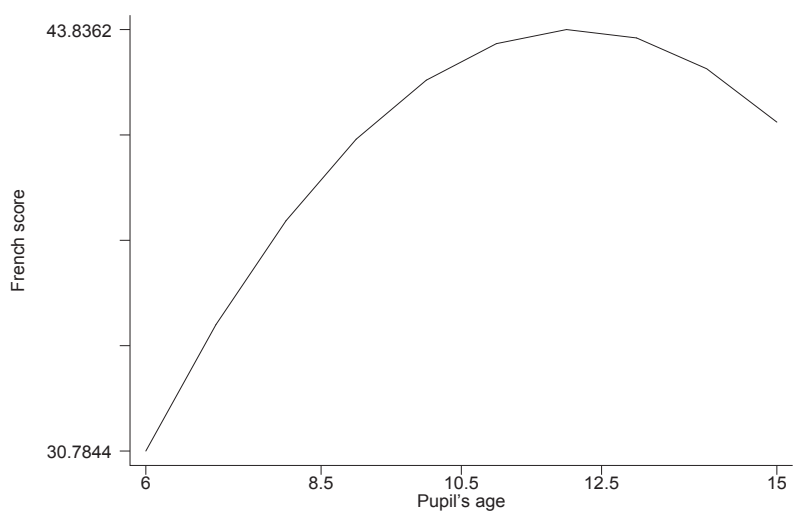

Figure 2.7: Meal: Relation between French score and pupil age.

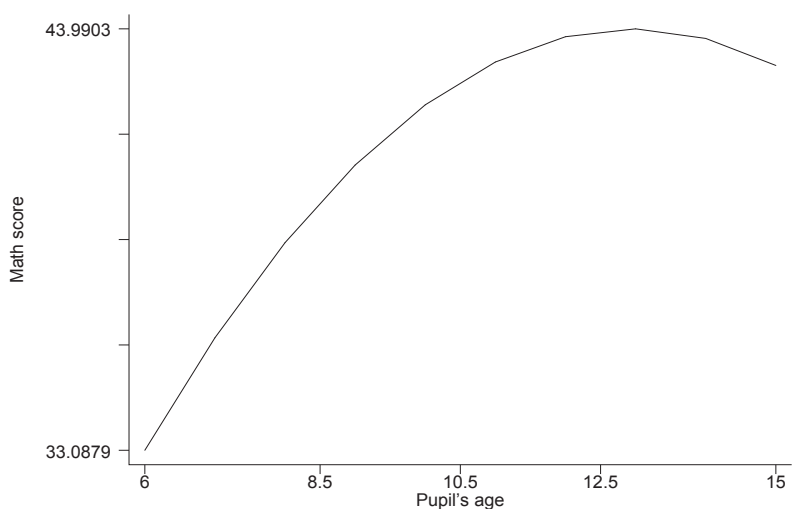

Figure 2.8: Meal: Relation between math score and pupil age. 
Other control variables related to pupils' characteristic, gender, Koranic school, early childhood institution, eat at fill have a positive effect on performance. One observes that boys perform better than girls in scores and the results are significant for math and aggregate scores. This results is consistent with the findings of Felouzis (1997). In the Roy estimations, one observes that girls perform better in French scores in the group of pupils who benefit from the meal or the deworming program. The presence of meal programs or deworming improves the nutritional status of pupils. In addition, the presence of canteen allows girls to stay in school all day. So they do not have domestic chores before going to school in the afternoon. Therefore they can concentrate better in the afternoon in the presence of a school meal.

For control variables Koranic school which is an informal school and early childhood institution which is a modern pre-school, the results show a positive impact on scores. This result is intuitive and well known in the literature. Both types of schools are known to develop children's ability to learn at an early age. Particularly, Koranic school is known for developing learning ability and memory because children learn by heart very early. For control pupils eat at fill, as anticipated, one observe a positive effect on scores. In fact the study focused on a population in rural areas and eating at fill at home is an indicator of nutritional well-being. So one can understand that this pupil performs better than pupils who do not eat at fill.

Regarding household characteristics, the results show that education expenditure and household size have a positive effect on performances. For education expenditure, one knows that the more parents invest in the education of their children the more they will supervise kids at home. For household size per adult equivalent as expected, one observes a positive effect on scores. As mentioned in the description of variables, we believe that large households are likely to have seniors students who are already in college or high school and are willing to coach the youngest. It is important to also remember that studies on the effect of family size on education outcomes in developing countries have led to highly controversial results.

Another important variable is the literacy of household head. Contrary to our expectation, results show a negative impact of this variable on aggregate and math scores. In fact, as mentioned in the description of the variables, the only fact of having a literate parent is not enough to obtain good results. This requires monitoring at home. Therefore, one can think that children with literate parents are possibly not well monitored by their parents at home. For variable land, as expected, the result shows a negative impact on scores. The result is only significant for math score. In Senegal, rural households still continue to use their children for domestic and farm work. So children living in households with farmland probably have to work in the fields, which will result in reducing their learning time and therefore their performances.

Regarding community characteristics, control "parents school" shows a negative effect on scores while 'college' shows a positive one. These two results are expected. For the control "parents school", living in a community where some children do not go to school because their parents are not interested is likely to have adverse effects on the performance of children who go to school because there is a big chance that it has a spill-over effect.

For the control college, the result can be viewed as an indirect effect. As mentioned in the description of variables, the result can be explained from two angles. Firstly, living in a community where there is a college implies that parents are encouraged to invest in their children's education because they know that children can continue studying in the village after obtaining the primary certificate. Secondly, living in a village having a college implies that there are more advanced seniors (brother or sister) who are in college and who can guide the youngest with their studies. However, we have no information on the fact that a pupil would have a brother or sister in a college.

Regarding shocks variables, controls number of pregnancies registered in school, absenteeism of teacher, disturbed courses and health expenditure have negative effects on scores. These results are expected, for example the presence of absenteeism and disturbed courses will reduce the learning time of pupils as well as their performances. 
Regarding regions, the results show that pupils living in Diourbel perform better than those living in Fatick which is the reference.

Finally the coefficient of the meal program is positive and significant for French outcomes meaning that having a meal program affects positively on pupils' performance. Also, coefficient of the deworming program is negative for aggregate and math scores, but positive for French score. However, it is worth stressing that these estimations do not reveal causalities but rather correlations. We now turn to the causal relation as given by the treatment effects from the PSM and the structural models.

\subsubsection{Effects of meal and deworming programs on scores}

As outlined earlier, our framework allows identifying and estimating the average treatment effects on the population (ATE); the average treatment effects on the treated (ATET); and the average treatment effects on the non-treated (ATENT) depending on the model used. In the endogenous selection model (Heckman selection framework), we can identify only the ATE. In the PSM we can identify the ATET and in the Roy model in addition to identifying the ATE and ATET, we can also calculate the ATENT. Regarding the ATENT, i.e, the average effect on the group of pupils who do not receive the meal program, the purpose is to inform policy makers on the rationale for extending the programs to this subpopulation of pupils.

The combination of the average treatment effect on the treated (ATET) and the average treatment effect on the non-treated (ATENT) provides a more comprehensive view of the effectiveness of a given program and allows to choose a result from a wide alternatives of options.

\section{School feeding program}

From the foregoing, we computed the ATE of the meal program using the relation (1-10) for the Heckman selection framework. For the Roy model, we used relations in Table 2.1 to compute ATE, ATET and ATENT. See Table 2.4 for results on various effects computed from the Heckman selection framework and the Roy model. For the PSM specification, the average treatment effects on the treated (see Tables 2.5, 2.8) is based on various matching methods or algorithms in order to check the robustness of the estimates. ${ }^{8}$ Results based on structural approaches (see Table 2.4) show a positive impact of meal program on scores. Likewise, from the DESM (both FIML and two step), one notes a positive average treatment's effect (ATE) of the meal program on all scores. One observes that the ATE on math score is higher than the ATE on aggregate and French scores.

\footnotetext{
${ }^{8}$ In order to check the balancing between groups (treated vs. control), we also report the matching tests in Tables A.11 to A.13 in the appendix.
} 


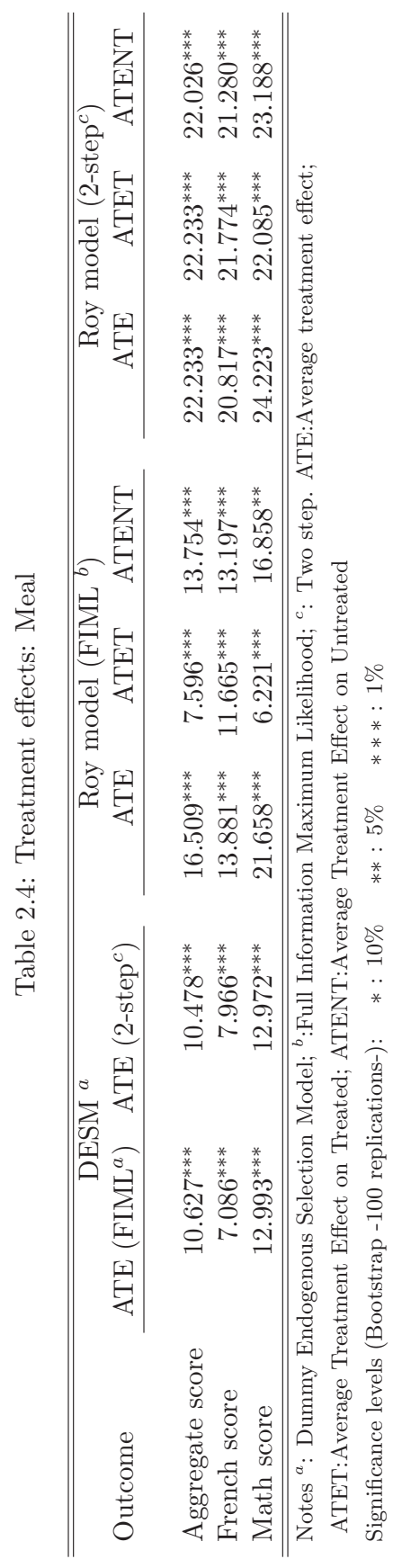


From the Roy model, the ATE computed goes in the same direction as the ATE obtained from the DESM. However, it is important to note that the ATE from the Roy model is higher than those from de DESM. As mentioned above, in addition to the ATE the Roy model allow us to compute treatment effect on treated and on non-treated. The results on average treatment's effect on treated (ATET), show that the meal program has a positive impact on the pupils who benefit from the program. One can note that the ATE is higher than the ATET. This can be explained by the fact that the ATENT is higher than the ATET. For the ATENT, results show a positive effect of the meal program on all scores. The ATENT on math score is higher than the ATENT on aggregate and French score.

Results from the PSM specification (see table 2.5) show a positive and significant ATET for aggregate and French scores using only the kernel matching and a positive and significant ATET using the Nearest Neighbors matching (K-NN), the Nearest Neighbors matching with 5 neighbors $(\mathrm{K}-\mathrm{NN}(5))$ and the kernel procedure for the math score.

Table 2.5: Treatment effects: Meal, Propensity score matching (ATET)

\begin{tabular}{|c|c|c|c|}
\hline \multirow[b]{2}{*}{ Score } & \multicolumn{3}{|c|}{ Meal } \\
\hline & Coef. & Std. Err. & Boot. $^{a}$ \\
\hline \multicolumn{4}{|l|}{ Aggregate } \\
\hline $\mathrm{K}-\mathrm{NN}^{b}$ & 2.879 & 2.804 & 2.153 \\
\hline $\mathrm{K}-\mathrm{NN}(5)^{b}$ & 2.928 & 1.425 & 1.832 \\
\hline Kernel & 3.757 & $0.774^{* * *}$ & $0.805^{* * *}$ \\
\hline \multicolumn{4}{|l|}{ French } \\
\hline $\mathrm{K}-\mathrm{NN}^{b}$ & -1.241 & 2.884 & 2.954 \\
\hline $\mathrm{K}-\mathrm{NN}(5)^{b}$ & 0.548 & 1.514 & 1.791 \\
\hline Kernel & 2.683 & $0.859^{* * *}$ & $0.888^{* * *}$ \\
\hline \multicolumn{4}{|l|}{ Math } \\
\hline $\mathrm{K}-\mathrm{NN}^{b}$ & 6.965 & $3.232^{* *}$ & $2.313^{* * *}$ \\
\hline $\mathrm{K}-\mathrm{NN}(5)^{b}$ & 5.266 & $1.584^{* * *}$ & $1.872^{* * *}$ \\
\hline Kernel & 5.062 & $0.841^{* * *}$ & $0.895^{* * *}$ \\
\hline
\end{tabular}

\section{Deworming programs: school vs. home}

For the deworming program, as mentioned earlier, we distinguished between deworming program, school deworming program and home deworming program. It is important to remember that the deworming program is not the sum total of the two programs. Furthermore, we do not take into account the intersection of school deworming and home deworming. The effects of deworming on scores are reported in 2.7 , for structural models and in table 2.8 , for the PSM method.

From the structural models, Table 2.7 given the effect of the deworming in school, the deworming at home and the deworming program (in school or at home) in the first, second and last column of the table respectively.

Due to a small number of observations regarding pupils dewormed at school or at home, we have not been able to estimate the Roy model for these subgroups. That is why we do not have the ATET and ATENT for school and home deworming. Regarding the school deworming, Table 2.7 shows a positive impact on all scores using either FIML or two step. This result means that the deworming in school program has a positive effect on the entire population (ATE). The impact is more important for the math score. If we take the deworming at home, the table 
shows a negative impact on all scores (both with FIML and two step). This result could be explained by the nature of the drug used by rural households to deworm children. They use either traditional or modern deworming drugs, and one knows that several cases of side effects in terms of fatigue or diarrhea have been reported. In addition, the use of traditional drugs is not without consequences because this could lead to the reduction of pupils' performance. Regarding the deworming program, Table 2.7 shows a negative impact on all scores for the entire population, for the treated and for the non-treated. 


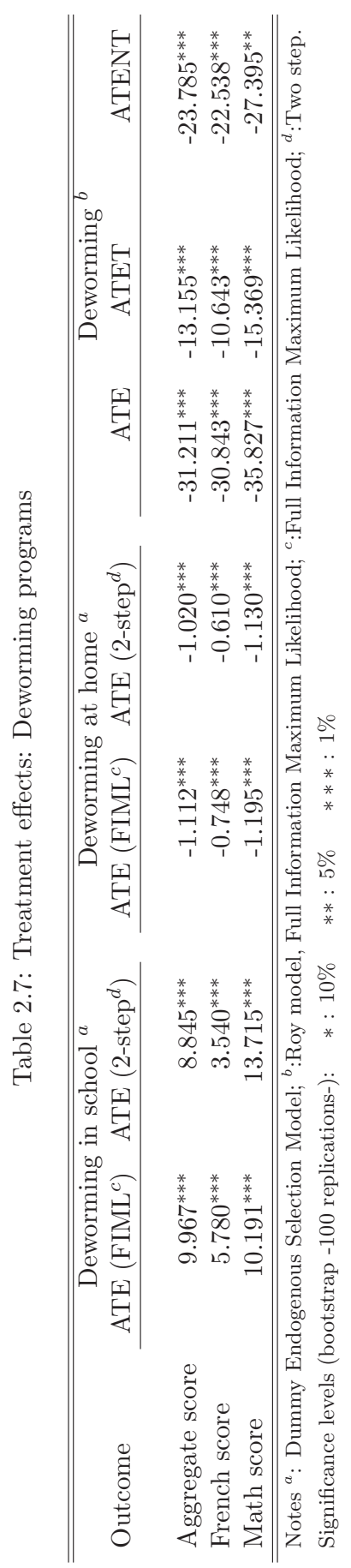


The ATET from the PSM (see Table 2.8) shows a negative and significant effect on both the aggregate and math scores in the case of the Kernel matching methods. For the score in French, we have a negative impact for all algorithms. ${ }^{9}$

Table 2.8: Treatment effects: Deworming, Propensity score matching (ATET)

\begin{tabular}{|c|c|c|c|}
\hline \multirow[b]{2}{*}{ Score } & \multicolumn{3}{|c|}{ Deworming } \\
\hline & Coef. & Std. Err. & Boot. $^{a}$ \\
\hline \multicolumn{4}{|l|}{ Aggregate } \\
\hline $\mathrm{K}-\mathrm{NN}^{b}$ & -3.404 & 2.206 & 2.113 \\
\hline $\mathrm{K}-\mathrm{NN}(5)^{b}$ & -1.228 & 0.934 & 1.635 \\
\hline Kernel & -1.927 & $0.766^{* *}$ & $0.802^{* *}$ \\
\hline \multicolumn{4}{|l|}{ French } \\
\hline $\mathrm{K}-\mathrm{NN}^{b}$ & -5.874 & $2.390^{* *}$ & $2.183^{* * *}$ \\
\hline $\mathrm{K}-\mathrm{NN}(5)^{b}$ & -2.762 & $1.224^{* *}$ & $1.277^{* *}$ \\
\hline Kernel & -1.680 & $0.851^{* *}$ & $0.854^{* *}$ \\
\hline \multicolumn{4}{|l|}{ Math } \\
\hline $\mathrm{K}-\mathrm{NN}^{b}$ & 0.358 & 2.437 & 2.081 \\
\hline $\mathrm{K}-\mathrm{NN}(5)^{b}$ & 1.092 & 1.256 & 1.445 \\
\hline Kernel & -1.713 & $0.835^{* *}$ & $0.789^{* *}$ \\
\hline \multicolumn{4}{|c|}{ Note: ${ }^{a}$ Bootstraping standard errors; } \\
\hline
\end{tabular}

\subsection{Policy analysis}

\subsubsection{Impact of programs on some subgroups}

We have estimated the effects of the meal program, but it is also interesting to study these effects on some subgroups. This exercise allows us to emphasize the variability of the effects for different recipients. It is thought for example that the program's impact is different according to gender or according to whether there is an association around the school as we know that the operation of a school canteen requires a minimum of organization. In this subsection, using the structural approach only, we present the impact heterogeneity of the meal and deworming programs by gender, according to whether there is a school project in the school, an association of pupil mothers, a school cooperative, and by grade or education level of the pupils followed.

In Table 2.10, one can see that the impact of the meal program on the scores is positive for boys and girls. However, the impact on the math score of boys is higher than for girls, while the the reverse applies for the French score. Thus one can say that the effect of the meal program is not uniform by gender.

In addition, an analysis according to the existence of an association of mothers of pupils is conducted. We found that the meal program has a positive effect on all scores for pupils who are in schools with and without association of mothers. However, the impact of the meal program is higher in schools with an association of mothers.

For the grade, Table 2.10 shows that the effect of the meal program on the math score is higher for pupils in CP, while the effect on the French score is higher for pupils in CE2.

\footnotetext{
${ }^{9}$ The matching test reported in Table A.13 in the appendix indicates that on average, the treated and control group show similar characteristics after matching in view of the variables used.
} 
Table 2.10: Impacts heterogeneity: Meal

\begin{tabular}{lccc}
\hline \hline & Aggregate & French & Math \\
Gender of pupil & & & \\
Male & $11.018^{* * *}$ & $7.570^{* * *}$ & $14.107^{* * *}$ \\
Female & $10.465^{* * *}$ & $8.905^{* * *}$ & $12.315^{* * *}$ \\
\hline Association of mother & & & \\
With & $14.596^{* * *}$ & $17.640^{* * *}$ & $12.372^{* * *}$ \\
Without & $8.904^{* * *}$ & $6.994^{* * *}$ & $10.780^{* * *}$ \\
\hline Grade & & & \\
CE2 & $10.333^{* * *}$ & $9.823^{* * *}$ & $10.701^{* * *}$ \\
CP & $11.828^{* * *}$ & $8.104^{* * *}$ & $15.593^{* * *}$ \\
\hline \hline
\end{tabular}

Significance levels (bootstrap): $\quad *: 10 \% \quad * *: 5 \% \quad * * *: 1 \%$

Regarding the deworming program, Table 2.11 shows that the impact on the scores of girls is positive, while it is negative for the scores of boys. This means that the program has a positive effect on girls' performance contrary to boys' scores. In addition, the table shows that the deworming program has a positive impact on the pupils' performance in school with association of mothers or school project.

Table 2.11: Impacts heterogeneity: Deworming

\begin{tabular}{lrrr}
\hline \hline & Aggregate & French & Math \\
Gender of pupil & $-2.094^{* * *}$ & $-3.693^{* * *}$ & $-0.456^{* * *}$ \\
Male & $0.734^{* * *}$ & $0.123^{* * *}$ & $1.143^{* * *}$ \\
Female & & & \\
\hline School project & $0.668^{* * *}$ & $1.091^{* * *}$ & $0.249^{* * *}$ \\
With & $-0.386^{* * *}$ & $-1.508^{* * *}$ & $0.679^{* * *}$ \\
Without & & & \\
\hline Association of mother & $1.659^{* * *}$ & $0.205^{* *}$ & $5.096^{* * *}$ \\
With & $-0.175^{* * *}$ & $-0.222^{* * *}$ & $-0.203^{* * *}$ \\
Without & & & \\
\hline Grade & $-0.253^{* * *}$ & $-0.704^{* * *}$ & $0.109^{* * *}$ \\
CE2 & $-0.593^{* * *}$ & $-1.769^{* * *}$ & $0.582^{* * *}$ \\
CP
\end{tabular}

Significance levels (bootstrap): $\quad *: 10 \% \quad * *: 5 \% \quad * * *: 1 \%$

We can thus summarize by saying that having an association of mothers and a school project would lead to positive effect of both meal and deworming programs on pupils' scores. This implies that even if a meal or deworming program is implemented at school, if a number of conditions are not met, the program cannot improve pupils' achievement.

\subsubsection{LATE as policy instrument}

The local average treatment effect (LATE), introduced by Imdens and Angrist (1994), is an interesting parameter in terms of economic policies, Blundell and Costa Dias (2008). It represents the average gain to participate in a program for individuals called to receive the program due to a change in one variable that Imbens and Angrist (1994) called instrument. In the context of our study, the LATE is the average impact to benefit from the school canteen for a group of pupils that is passed from the status of non-beneficiaries to the status of beneficiaries. At the same time one provides to the group of pupils a school input called instrument. The instruments used are: 
management committee, school project, cooperative school, association of parents, association of mothers, eliminating absenteeism of teachers and providing lot of textbook to pupils.

The management committee has three specialized committees responsible for food management, community involvement and cooking in the school. So, one can easily understand that the presence of a functional management committee in a school is important for the proper operation of the school canteen. The school project is a management system tool that can improve the quality of learning. The management of a school project is in the responsibility of a management committee.

The cooperative school is also another association around the school. Ideally, it is a group of adults and pupils who implement an educational project. Parents of pupils are grouped around associations such as the pupils' parents or mother. These associations ensure the smooth operation of school canteens and respect for the financial contribution of up to 200 FCFA per pupil per month to purchase ingredients not provided by WFP. The parents also help to supply firewood for cooking meals and support the construction of kitchens and warehouse for food storage. Reduction of teacher absenteeism is important. For school manual, it is generally accepted that pupils in schools with lots of textbooks perform better than pupils in schools with few textbooks.

From the relationship contained in Table 2.1, the LATE was calculated for each instrument.

Table 2.12: Local Average Treatment Effect (LATE) ${ }^{a}$ : Meal

\begin{tabular}{|c|c|c|c|c|c|c|}
\hline \multirow[b]{2}{*}{ Policy instruments } & \multicolumn{2}{|c|}{ Aggregate score } & \multicolumn{2}{|c|}{ French score } & \multicolumn{2}{|c|}{ Math score } \\
\hline & FIML & 2-step & FIML & 2-step & FIML & 2-step \\
\hline Management committee & $33.371^{* * *}$ & $30.671^{* * *}$ & $24.146^{* * *}$ & 30.135 & $44.668^{* * *}$ & $33.568^{* * *}$ \\
\hline School project & $30.311^{* * *}$ & $27.281^{* * *}$ & $21.502^{* * *}$ & $26.179^{* * *}$ & $42.585^{* * *}$ & $32.306^{* * *}$ \\
\hline Cooperative School & $40.349^{* * *}$ & $36.630^{* * *}$ & $34.825^{* * *}$ & $37.062^{* * *}$ & $53.155^{* * *}$ & $44.035^{* * *}$ \\
\hline Association of mothers & $23.382^{* * *}$ & $20.177^{* * *}$ & $14.542^{* * *}$ & $19.587^{* * *}$ & $34.359^{* * *}$ & $23.194^{* * *}$ \\
\hline Association of parents & $45.292^{* * *}$ & $43.828^{* * *}$ & $37.095^{* * *}$ & $41.081^{* * *}$ & $57.166^{* * *}$ & $50.639^{* * *}$ \\
\hline Absence & $22.515^{* * *}$ & $23.290^{* * *}$ & $13.083^{* * *}$ & $13.916^{* * *}$ & $37.830^{* * *}$ & $38.798^{* * *}$ \\
\hline Textbooks & $24.951^{* * *}$ & $22.451^{* * *}$ & $15.160^{* * *}$ & $21.061^{* * *}$ & $35.932^{* * *}$ & $25.349^{* * *}$ \\
\hline
\end{tabular}

Note $^{a}$ : Estimate from the Roy model.

Significance levels (bootstrap -100 replications-): $\quad *: 10 \% \quad * *: 5 \% \quad * * *: 1 \%$

Table 2.12 shows results on various LATE depending on the instrument used. The association of parents gives the greatest LATE for all scores. After the association of parents, we have the cooperative school, management committee, then the school project, eliminating absenteeism of teachers and finally the provision of textbooks in sufficient quantity. However, in order to identify the best option it is important to link the cost of each input to the corresponding average effect, i.e the corresponding LATE. This analysis can be made through a cost-effectiveness analysis which is a form of economic analysis that compares the relative costs and outcomes (effects) of two or more courses of action. It is important to notice that the cost effectiveness analysis made here is not about comparing the canteen program with another option in the aim to increase pupils' performance. It is a question of comparing several options in the presence of the canteen program in order to obtain the best result at a lower cost.

To conduct the analysis, we need the cost of the meal program and the cost related to each input. For the cost of the canteen, based on information from the WFP, the annual cost of the canteen per student is approximately 13100 CFA. For the school project, based on information on the region of Matam from the Ministry of education, we estimated an approximate cost per 
pupil for the year 2010. The total cost for the school project for the year is $78,978,651 \mathrm{CFA}$ and the total number of pupils in the region was 87,971 in 2010 . So the cost of a school project per year is around 900 CFA. Although Matam is not part of regions covered in our study, we still felt that the cost of a school project in Matam can be a rough average cost for other regions. Then, the annual cost of a canteen combined with a school project per student is 14000 CFA. From Diagne (2012, page 220), we have the cost of increasing the effective learning time of pupils by the establishment of an administrative system to monitor the attendance of teachers. This cost is 111 CFA per year and per pupil. So the annual cost of a canteen combined with monitoring the attendance of teachers is about 13211 CFA. Diagne (2012, page 220) also gives us the cost of improving the staffing of pupils' textbooks. He estimated that the cost of a textbook that can be used for two years is 2305 CFA. In this study we divided this cost by 2 to get the annual cost of a textbook. So, the cost of a book per year is 1151 CFA and the cost of a canteen combined with improving the staffing of pupils' textbooks is 14252. For management committee, school cooperative, association of parents and mothers, we will only use the cost of the canteen (13100 CFA). For these forms of association, a local organization can allow to make them operating without a budget from the government.

To assess the cost-effectiveness, in Table 2.13 we used first the cost of each option (cost of the canteen plus the cost of the instrument) divided by the percentage of additional score which is the corresponding LATE.

Table 2.13: Comparing options using LATE: Policy analysis

\begin{tabular}{lrrr}
\hline \hline & Aggregate & French & Math \\
\hline Cost $^{a}$ & & & \\
Management committee & 13100 & 13100 & 13100 \\
School project & 14000 & 14000 & 14000 \\
Cooperative school & 13100 & 13100 & 13100 \\
Association of mothers & 13100 & 13100 & 13100 \\
Association of parents & 13100 & 13100 & 13100 \\
Absence & 13200 & 13200 & 13200 \\
Textbook & 14300 & 14300 & 14300 \\
\hline Percentage of additional score ${ }^{b}$ & & & \\
Management committee & 33.4 & 24.1 & 44.7 \\
School project & 30.3 & 21.5 & 42.6 \\
Cooperative school & 40.3 & 34.8 & 53.2 \\
Association of mothers & 23.4 & 14.5 & 34.3 \\
Association of parents & 45.3 & 37.1 & 57.2 \\
Absence & 22.5 & 13.1 & 37.8 \\
Textbook & 25 & 15.2 & 36 \\
\hline Cost per percentage of additional score & & & \\
Management committee & 393 & 543 & 293 \\
School project & 462 & 651 & 329 \\
Cooperative school & 325 & 376 & 246 \\
Association of mothers & 560 & 901 & 381 \\
Association of parents & 289 & 353 & 229 \\
Absence & 587 & 1010 & 349 \\
Textbook & 571 & 940 & 397 \\
\hline \hline
\end{tabular}

Table 2.13 shows that the most cost effective option is the combination of the canteen and the association of parents. Having taken the aggregate and French scores, the results of the analysis show that after association of parents, one observes cooperative school, the management committee, the school project, the association of mothers, the textbook and the eliminating of absenteeism of teachers. Meaning that, eliminating absenteeism of teacher is the least profitable option when one wants to increase the aggregate and French scores of pupils by 1 point. If one takes the math score, the least profitable option is improving the staffing of pupils' textbooks. 


\subsection{Conclusion}

In this study, we have assessed the effects of school feeding and deworming programs on school performance measured through aggregate score, French score and mathematic score. We use a observational database of 159 schools in four rural areas in Senegal: Diourbel, Fatick, Kolda and Sedhiou. We use both program evaluation (Propensity Score Matching) and structural approaches. The structural framework has the advantage of allowing for the joint estimation of a performance equation and a selection mechanism while estimating the effect of the program. Our study distinguishes between deworming at home and deworming in school. It is important to emphasize that the deworming program is not the sum total of the deworming in school and at home. Furthermore, we do not take into account the intersection of deworming in school and at home. Also, in terms of policy analysis, this study uses the local average treatment effect (LATE) as an instrument to analyze economic policy.

The main results that emerge from this study are the following: i) The results show that the meal program can significantly increase pupils performances. The impact is more important for pupils who are in a school with an association of mothers. ii) The deworming in school has a positive effect on pupils' performance while the deworming at home has a negative impact on pupils' scores. Our reading is that it is essential to assist rural households to deworm children in households. This will avoid the use of traditional medicines (that have adverse consequences on pupils' performance). In addition, the deworming program significantly increases the scores of pupils who are in a school with an association of mothers or a school project. iii) Among the determinants of test scores (schools and teacher, pupils', household and community characteristics, and shocks variables), school manual, class size, age of teacher and pupil's age display a non-linear relations with the scores. Particularly, class size displays a U-shaped relation meaning that the performance (aggregate and math scores) decreases with increasing class size which is consistent with the findings in the literature on performance. However, starting from a certain threshold, the performance increases with respect to class size. In the context of rural Senegal, large classes may have a positive impact on test scores. Among other controls, education, expenditure, household size per equivalent adult, water point, Koranic school, early childhood institution, existence of a college in the village have a positive effect on pupils' performance, while controls such as disturbed courses, gender of teacher (male), absenteeism of teacher and parents school have negative impact on scores. In terms of policy analysis, results show that the impact of programs varies depending on the gender of the pupil, the existence of an association around the school or the educational level of the pupil.

An extension of this study would be to introduce unobserved heterogeneity in an econometric specification designed for such purpose. Another extension would be to pay attention to the variability (complementarity vs. substitutability) of the impact of the two programs on educational outcomes. Finally, our data also raise a methodological challenge that would be to elaborate on a structural model to estimate the impact of double or multi-program. However, accounting for this is by no means trivial. This is the objective of the next chapter. 
CHAPTER 3

\section{Packaging Meal and Deworming Programs ${ }^{1}$}

\subsection{Introduction}

Policymakers are prone to comparing the relative benefits of different programs rather than the impact of a single program. This leads to many relevant policy questions when multiple programs are implemented. Some of such policy questions includes whether joint implementation of a package be more effective than each programs if performed separately? whether programs are complementary or suplementary? What is the impact of a given treatment compared to the impact of another treatment? Whether the cumulative impact of two programs is greater than the sum of the impacts of each separately? This chapter provides some answers to these policy questions.

Deworming and school meals programs have been widely implemented in developing countries namely: Latin America, South America, Asia and Africa. They are considered as important driving forces to improve enrollment, attendance and pupils' academic performance in developing countries. However, no consensus has emerged on the role and the efficiency of such interventions. In this study, we focus on neglected aspects of these interventions by taking into account the interdependence between both programs. To this end, we propose an econometric framework that extends the basic endogenous selection methods à la Heckman and the Roy model.

This chapter seeks to assess the impact of school feeding and deworming programs as a package on school performance (test scores), inscription (enrollment rate), progression (promotion rate) and retention (dropout rate), while elaborating on the determinants of these performances. To the best of our knowledge, previous contributions have been considered either as a single program or a package of programs as a purpose of study. Package of programs are usually implemented by policy makers because of their efficiency and also because implementing package programs is less expensive than single programs. Banerjee and Duflo (2009) argued that the cost of organizing an experiment may be high. Then it is worth implementing multiple experiments or multiple treatments at the same time on the same population in order to assess alternative variants of the program. However, due to the unavailability of appropriate tools that may enable researchers to jointly estimate the effect of package, and at the same time to disentangle the effects within a unified setting, most studies analyze these programs either separately or consider the package as unique programs; or do not consider on the interactions between programs in the package. Evaluating multiple programs requires the identification and estimation of many different treatment effects which makes the analysis more complex.

\footnotetext{
${ }^{1}$ Co-authors: Théophile T. Azomahou (United Nations University, UNU-MERIT and Maastricht University) and Wladimir Raymond (STATEC, Luxembourg)
} 
Some 'multiple treatment experiments' albeit in a very different context and using different methodology include among others Banerjee et al. (2007), Duflo et al. (2006) and Olken (2007). However, it is worthwhile to note that multiple treatments in these empirical papers differ from what we study here.A apart from the fact that these papers were not interested in canteen or deworming programs, the authors did not jointly study their treatments and its interactions.

From a methodological perspective, Lechner (2001), Frolich (2004), Heckman and Vytlacil (1998) and Wooldridge (2003) studied identification and estimation strategies for the evaluation of policies with multiple programs. Lechner (2001) extended the conventional two state framework of the Rubin model to the case of multiple mutually exclusive treatment and discusses various measures of the causal effects. The author discussed the identification of these effects under the Conditional Independence Assumption (CIA). The results explain that low dimensional balancing scores, similar to the ones valid in the case of only two treatments exist and can be used for identification of various causal effects. Lechner (2001) outlined a matching estimator and showed that for specific parameters, like the treatment effect on the treated, the multiple program nature of the policy can be ignored, because individuals who are not in programs of interest, are not needed for identification. In Frolich (2004), different nonparametric strategies to solve the selection bias problem and to identify average treatment effects have been inspected. The study outlines that the difference-in-difference and the instrumental variable approach often identify only the treatment effect using participation versus non-participation, and do not allow a comparison between different treatments. Lechner (2001) and Frolich (2004) are based on propensity score matching.

Heckman and Vytlacil (1998) and Wooldridge (2003) used instrumental variables (IV). Heckman and Vytlacil (1998) developed models for counterfactuals and causality that build on Cowles Commission econometrics. The authors generalized the IV approach to consider models with multiple outcomes. They proposed both ordered and unordered choice models and defined treatment effects for a general multiple treatment problem and presented conditions for the application of IV for identifying a variety of new treatment parameters. Rather than relying on IV to estimate the local average treatment effect, Wooldridge (2003) imposed assumptions that identify the average effect for general kinds of treatment based on the correction function approach. The author developed a correlated random coefficient model with multiple treatments which is more robust than the plug-in estimators of Heckman and Vytlacil (1998).

From the methodological viewpoint, the approach used in this chapter departs from the previous studies on several aspects. First of all, the foregoing contribution is aiming at providing framework for analyzing experimental data such as randomization. One need to recall that we are dealing with observational data. More specifically, we do not require instrumental variables like Wooldridge (2003). It is well known that Instrumental variables do correct the endogeneity issue alongside ruling out the simultaneity aspect of the selection decision which is crucial to us. Moreover, we are not in a pure propensity score matching paradigm as in the case of Lechner (2001) and Frolich (2004) as we require the joint estimation of selection and outcome equations. Propensity score matching for multiple treatments can easily be retrieved from the first step estimation of our procedure by elaborating on multiple selection. Lastly, our sampling process generates mutually non-exclusive data as it will become clear in Section 3.3.

In this chapter, we propose a structural framework for double-index selection where treatment variables are endogenous. Firstly, we use a double endogenous selection model, and secondly we use a generalized Roy (1951) framework. For each specification, we propose two estimation procedures: The Full Information Maximum Likelihood (FIML) and the Control Function (twostep estimation) à la Heckman $(1978,1979)$. One difficulty in the two-step procedure is that the conditional expectations in the second step regression function involve the truncated trivariate normal distribution. We compute these expectations relying on the moment-generating function formula along the lines of Muthén (1990). As for the inference, it is well known that the conventional standard errors of the parameters estimated in the second step are not valid and 
need to be corrected by generalizing the results of Heckman $(1978,1979)$, or by using simulation or bootstrapping methods. Our framework has the advantage of allowing the identification and the estimation of a wide and rich range of treatment effects: global effects (effects of programs taken together), exclusive effects (disentangled effects), relative effects (effect of a program versus the effect of another program), additional effects (effect following from having another program in addition), sequential and substitution effects. We also tackle issues related to complementarity versus substitutability of programs.

The implementation of the assessment uses a very rich and recent observational data for approximately 5,000 pupils in rural Senegal. The data relates to canteen and deworming programs, pupils' achievements (score in French, Maths, aggregate score, enrollment rates, promotion and dropout rates), their characteristics (age, sex, etc.), their households' and schools' characteristics. The use of these data is interesting not only for the underlying above mentioned development issues, but also because it raises methodological challenges.

The results show that the package of meal and deworming is more beneficial in terms of increasing pupils' achievements. The sequence of implementation does matter. The two programs are complementary in increasing scores and promotion rates. Instead, they are substitutes with the aim of reducing dropouts. The cost-effectiveness analysis shows that deworming is by far cheaper than the school meals. However, the implementation of meals program before deworming is more cost-effective than the reverse.

The chapter proceeds as follows. Section 3.2 takes stock of the literature on canteen and deworming program and their effectiveness on school achievement in developing countries. Section 3.3 describes the data and variables. We propose two econometrics frameworks: the doubleindex selection model and the generalized Roy's model in section 3.4. Estimation results are discussed in Section 3.5. Section 3.6 presents a treatment effect heterogeneity and performs a cost-effectiveness analysis. The final section summarizes and discusses implications of the results, and elaborates its limitations.

\subsection{Insights from the literature}

In this section, we discuss salient features and evaluation studies from the literature, focusing on issues related to the nature of interventions: school meals vs. deworming. Do these programs achieve their ultimate objectives? We shall also highlight the main conclusions which they have led. The list of references cited below is by no means exhaustive. School feeding (or other nutrition) and deworming programs are the subject of great interest in recent years in many developing countries. Some empirical studies found out that school meal and take-home ration programs induce a significant increase in pupil achievement as measured by test scores. However, some other studies found no evidence or a negative effect of the programs on test scores (Ahmed and del Ninno (2002) and Kazianga et al. (2009)). Thereafter our literature survey is targeted at the nature of the interventions.

\subsubsection{School meal programs}

Most empirical studies that evaluate school meals or feeding programs used participation in school (which includes enrollment and attendance) as outcome. Some conclude that school meal programs have a significant positive impact on attendance and enrollment. On the other hand, some other studies outlined that school feeding programs have a positive impact on learning outcomes measured by test scores. As pointed out by Ahmed (2004), Vermeersch and Kremer (2004), Powell et al. (1998), Jacoby et al. (1996) and Akakpo (2004) school feeding does not have the same impact on all recipients. This raises the issue of causal heterogeneity we will highlight later in this study.

Ahmed (2004) evaluated the impact of the school lunch program in Bangladesh on student 
outcomes using test scores for 1648 pupils in fifth grade elementary school. The author revealed that the aggregate scores of pupils who received the program are $15.7 \%$, higher than pupils in the control group. Relying on the disaggregated scores, the also author found out that the improvement is mainly due to an increase in math score. By controlling the characteristics of children, households and schools the number of pupils in particular, he observed that the program has a significant and negative effect on scores in English. However, the impact is not sizeable. Ahmed and del Ninno (2002) found that the FFE program in Bangladesh had a negative and significant impact on test scores for pupils who benefited from the program in fourth-year primary schools. The authors also indicated that the difference between the two groups came from a decrease in scores of pupils who enjoyed the program. This finding stems from the low socio-economic status of beneficiaries. Vermeersch and Kremer (2004) quantified the effects of subsidized school meals on student outcomes using randomized data from a breakfast program in kindergarten in western Kenya. Results show that children in the treatment group attended school $35.9 \%$ of the time, compared to $27.4 \%$ in the control group. The difference in attendance is one third in the comparison group. The authors also showed that there is an improvement in student learning, but only for children in schools where teachers were more experienced at the beginning of the program. Kazianga et al. (2009) used a randomized trial to assess the impact of school meal and take-home ration on health and education outcomes for poor children in northern rural Burkina Faso. After one year academic experiment, the authors found that both programs increased girls' enrollment but there was no significant impact on raw scores in mathematics. Also, the interventions had caused an increase in absenteeism in households, with low use of child labor while it decreased for household that had a relatively large use of child labor. Powell et al. (1998) used data on 814 children in the fifth year in primary schools in rural Jamaica. The children were randomly divided into two groups. The treated group received a breakfast containing 576 to $703 \mathrm{kcal}$ and $27 \mathrm{~g}$ of protein. The control group received a placebo consisting of a slice of orange with $18 \mathrm{kcal}$ every day during eight months of school year. School attendance rates records showed a slight increase for children in the treatment group. This impact was greater for malnourished children than for well fed children. However, these impacts are relatively small compared to the extent of the real impact because participation rates were about $70 \%$ in both groups. Jacoby et al. (1996) found that a breakfast program in Peru increases attendance rates of pupils in fourth and fifth year of primary school. The authors found that there was no significant difference in the rates of school attendance between the two groups before the implementation of the program. During the implementation of the program, they found out that attendance increased by $0.58 \%$ in schools for the treatment group and decreased by $2.98 \%$ in the control group.

Akakpo (2004) examined the impact of school canteens on enrollment and attendance and on student achievement with certificates of completion of elementary study (FEAC) in rural public schools in three regions (Fatick, Kaolack and Tambacounda) of WFP intervention in Senegal. The analysis includes the schools belonging to the WFP program for at least two years, those who recently joined the program and those who participated in any of the WFP programs. The results showed that school attendance is far better in schools where WFP operates, as enrollment grew by $12 \%$ per year while the increase is only $8 \%$ in the group of schools where WFP does not operate. The schooling of girls is particularly high in schools where WFP has been operating and the number pupils grew by $15 \%$ per year against $10 \%$ in schools without canteens. Cueto and Chinen (2007) examined the impact of an experimental program of school breakfasts in primary schools in three departments in Peru. The outcome variables were test scores on standardized coding, arithmetic and reading as well as attendance, enrollment and dropout rates. The results showed that children in the program have high rates of attendance and low dropout rate, compared to children not receiving the program. In addition, children in the treatment group who are enrolled in multigrade schools and uni-grade have perform better in coding test, arithmetic and reading. 
Several studies have sought to measure the impact of feeding programs at schools on the cognitive development of children. Simeon and Grantham-McGregor (1989) used data on rural Jamaica and found that breakfast had no effect on the cognitive performance of children with normal weight and height for their age while breakfast increases the performance of children at risk. Whaley et al. (2003) studied the impact of animal foods (meat and milk) on the cognitive development of children at primary school in rural Kenya using a randomized program. The authors concluded that the quality and quantity of food can predict the performance in arithmetic. The study shows that food of animal origin as well as energy have a positive effect on the results of children in cognitive tests such as arithmetic and perceptions.

\subsubsection{Deworming programs}

Compared to school feeding studies, evaluation research on the effect of deworming programs on educational outcome is less documented. Miguel and Kremer (2004) assessed the impact of a randomized deworming program in Kenyan schools. The results showed that absenteeism in the treatment group was 25\% lower than in the control group. Moreover, deworming increases school attendance by 0.14 percent per treated pupil on average. Miguel and Kremer (2004) also studied the impact of deworming on test scores. The results showed that despite the reduction in absenteeism, there is no evidence that deworming increased students scores. Additionally, the authors use the Kenya Life Panel Survey (KLPS) to document the long-term impact of deworming program on school achievement, cognitive skills, labor market, fertility, marital choice, health, physical strength and personal happiness. The authors found that children's health and participation in school are increasing not only for students in the treatment group but also for students whose primary schools are located within $6 \mathrm{~km}$ from treated schools. In particular the impact was significant on schools located within $3 \mathrm{~km}$. The impact on nearby schools seem to be due to the reduction of transmission of the disease (positive externalities) thank to the intervention. A key finding of the study is that the failure to take these externalities into account leads to a significant underestimation of the benefits of the intervention and the actual cost of deworming. However, increased participation in schools is not reflected in the results of tests score. In addition, the authors presented cost-effectiveness analysis showing that the intervention is cost effective, but this intervention not does improve basic skills.

Bobonis et al. (2006) conducted a randomized trial in India as part of a health program that provides iron supplementation and deworming to children aged from 2 to 6 years in 200 kindergartens in poor urban areas of Delhi. After five months of treatment, the authors found significant weight gains and a reduction of one fifth of absenteeism. This finding is consistent with the results of Miguel and Kremer (2004). Subsequently, Bobonis et al. (2006) have tried to obtain estimates after 2 years of program implementation. However, the attrition of the sample and the apparently non-random entry of new children in kindergartens make it difficult to obtain unbiased estimates of impacts in the long run. An important channel through which the gains of preschool attendance in Bobonis et al. (2006) could affect the long run entry of new children in kindergartens is an improvement over time in academic performance in primary school. In fact, $71 \%$ of parents in the field study of India argued that the improved outcomes in primary school has been a motivation for sending their children to preschool. In this study, children received both iron supplementation and deworming. However, the study does not distinguish between the effects of both treatments meaning that iron supplementation and deworming were considered together as one program.

Other studies focus on the impact of parasitic infection on cognitive development. Kvalsig et al. (1991) examined the impact of whipworms and other parasites in South Africa and found no association between drug treatment and educational attainment or memory function. Nokes et al. (1992) evaluated a treatment of whipworms in Jamaica and concluded that cognitive functions improved after undergoing the treatment, but other outcomes, particularly those related to academic performance, does not seem to have changed significantly. 
The main lesson from this brief literature review is that school meal and deworming interventions as well as the potential policy implications have received substantial attention by scholars. The debate has reached a state of maturity thanks to all the impact evaluation studies that helped accumulate a rich and substantial knowledge of the success and failure of these programs. However, no consensus has emerged yet as the conclusions are highly mixed and controversy. This paper aims at contributing to this heated debate by exploring in depth an important aspect of the problem which has so far been neglected: the package aspect of nutrition and health interventions.

\subsection{Programs package: variables and descriptive statistics}

As we have outlined earlier, the design of the sampling of the two programs is unique and rich. This richness follows from the fact that the two treatments in our analysis are mutually nonexclusive. This means that having deworming does not prevent pupils from benefiting from meals. As a result, some of pupils get only deworming, some only meals, some get both and other received nothing. This section presents the variables and descriptive statistics. Before that it is important to understand the data structure and the motivation of the econometric specification.

Data generation process and programs package

We have the case of two non-exclusive treatments $T_{1}$ and $T_{2}$. In this case, some individuals receive only $T_{1}$ whereas others receive only $T_{2}$. Some others receive both $T_{1}$ and $T_{2}$. As a result, the treatments here are typically mutually non-exclusive events. As we are in the case of a package consisting of two treatments, we have four regimes, that are, $T_{1} T_{2}, T_{1}\left(1-T_{2}\right)$, $\left(1-T_{1}\right) T_{2}$ and $\left(1-T_{1}\right)\left(1-T_{2}\right)$.

In terms of impact analysis, this type of data sampling makes the study particularly rich as compared to the case where all individuals in the treated group receive both treatments or when the interventions are mutually exclusive, which means that deworming and meals cannot be implemented at the same time. ${ }^{2}$ In other words, the occurrence of deworming does not automatically rule out the occurrence of meals and vice versa. This kind of data arrangement offers the possibility to study not only the impact of each intervention as single, but also the impact of the combination of the two programs and several relative effects. Moreover, it helps to design a specification that aims at testing the complementarity vs. substitutability of the two programs.

Programs package: Area covered and data sampling

In this study, we used a primary data (non-experimental) collected by the 'Consortium for Social and Economic Research (CRES)' and the Ministry of Education as part of an interventions of school canteens (meal) and deworming. For intervention areas, as mentioned earlier, four regions (Fatick, Kolda, and Diourbel Sédhiou) of Senegal were chosen. Central regions (Diourbel and Fatick) are mainly composed of farmers and are closer to the capital (Dakar), while those in the South (Kolda and Sédhiou) are very isolated and are mainly ranchers as main economic activity. The southern regions also suffer from poor living conditions and lack of access to basic infrastructure, which prevent people getting out of poverty. In 2003, the regions of Kolda and Sédhiou are part of southern regions classified as a very low risk management capacity whereas Fatick is part of regions with average management capacity of risk and Diourbel is considered as a region with good capacity to manage risk (PAM, 2003). ${ }^{3}$ These four regions are

\footnotetext{
${ }^{2}$ See Brodaty et al. (2001) and Lechner (2001) for multiple mutually exclusive treatments analysis with nonexperimental data.

${ }^{3}$ In Senegal, rural ares, most risks that people are facing are natural hazards such as drought, land degradation, animal diseases, pests and flooding. The consequences of these risks are felt on agricultural production (production loss, lower yields), livestock (cattle decreased, decreased production of animal products) and household incomes because they will lose a portion of income from the sale of agricultural and animal products. In addition there are other risks such as economic risks associated with rising prices of basic necessities, lower prices for agricultural
} 
characterized by weak school enrollment and high prevalence of poverty and vulnerability. As a result of low harvests, food insecurity in these regions reduced not only household income, but also impoverished their diet. Therefore, kids are exposed to acute malnutrition. It has been recorded $31 \%$ of nutritional deficiency cases at school age and adolescence and a prevalence of nutritional anemia for children at 4 to 14 years, with $62 \%$ of boys and $38 \%$ of girls (PAM, 2006).

The sample study is made up of pupils in the second and fourth year of primary school (hereafter CP and CE2) in these four regions. Several reasons have guided the selection of these two classes or grade. For reasons of costs of investigations, it was difficult to include into the interventions all pupils in each school. It should also be noted that all pupils within a school benefit from school lunch. On the other hand, if at the end of the second year the pupil cannot read and write, the probability to fail before the end of the education cycle is very strong. In addition, if after four years of primary education, skills in reading and writing are not acquired, it is unlikely that the pupils cannot learn the basic skills that education is supposed to provide. The interventions take about two years. We have a observational database of 159 schools with about 5500 pupils of which 2800 are at second year (CP) and 2850 at fourth year (CE2) randomly selected from a more large population. The pupils were offered the two programs: deworming $\left(T_{1}\right)$ and or meal $\left(T_{2}\right)$ as described above. Relying on that we formed the four groups: a group of pupils that benefit from the meal program only, a group of pupils that are dewormed only, a group of pupils that received the two programs and a control group that receives nothing.

Since this data set has not been used previously, we conducted an important work of data cleaning and cleansing in order to detect and correct corrupt or inaccurate records (typographical errors, etc.), and to also make data valid, consistent, accurate and complete. Moreover, the merging of the four survey questionnaires was challenging. Part of the merging identifier for households' questionnaires was not accurate and has been restored, which took enormous time. Score variables were imputed using the databases on scores from the 'Institut National d'Étude et d'Action pour le Développement de l'Éducation (INEADE)' who is responsible for administering tests to pupils and to enter the data.

\subsubsection{Variables}

The variables used in this study are of two types: the outcome or performance variables (aggregate score, French score, math score, enrollment rate, promotion rate and dropout rate) and the determinants of performance or control variables. We have gathered the controls into four categories: pupils' characteristics, households' characteristics, schools and teachers's characteristics, the characteristics of the community where pupils live and the geographic location or regions (Diourbel Fatick, Kolda and Sédhiou). The treatment indicators are the response variables in the selection mechanism. The definitions of all variables are summarized in Table A.1 in the appendix. The control variables were chosen based on data availability from the survey sample and their relevance for the analysis. Some controls are specific to the context of the study and therefore are of particular interest. We mention them as such and also elaborate a bit on the rationale of their use.

The characteristics of pupils. Pupils' characteristics are: gender, age, class, having attended a Koranic school, early childhood institution, being sick in the last three months preceding the survey, eat his/her fill, bring a snack to school and be dewormed at home. It is worth noticing that deworming at home is not part of the deworming program studied. The latter consists only of being dewormed at school. ${ }^{4}$

The gender of pupils can influence their academic performance. Although some studies indicate that boys perform better than girls in mathematics and in science Felouzis (1997), and

products and livestock and cattle theft. Any region that doesn't have ability to manage risk is highly vulnerable to food insecurity. This has a negative impact on children's education, nutrition and school performance.

${ }^{4}$ Remark: Deworming at home usually involves the use of traditional medecines or drugs by families without any control of a practitioner. 
others have explained that gender difference is not statistically significant, and that sometimes results are mixed (Ma, 2007). In the context of rural areas in Senegal, we anticipate that boys perform better than girls because girls are more prone to domestic work. In addition, some families still question the value of education of girls and are very reluctant to promote the schooling of girls. The age of pupils may have a negative effect on their achievement in case where there is a delay in school progress due to the grade repetition. However, if one considers that the delay follows from late entry to school, the literature mentions a positive relation between age and academic achievement (Schwille et al., 1991). Health status is a major determinant of performance. In the survey sample, it was asked whether a pupil has being sick the last three months preceding the survey. Intuitively, being sick will reduce learning ability and the number of school attendance.

Attending an early childhood institution and a Koranic school are specific to the context of the study. Indeed, the development of structures like nursery that aims at supporting children of young age are still underdeveloped. ${ }^{5}$ The Koranic school, usually found in Muslim countries, is an informal private structure that provides religious education to kids. In Senegal, $95.9 \%$ of the population practice Islam. Albeit informal, Koranic schools are very common in the society. Children usually go to Koranic school between the ages of 5 and 6 years. These schools are very popular and contribute to a tremendous development of kids capacity to learn and memorize at young age. We can say that the learning mechanism in these schools is a memory based learning. We hypothesis that these two variables (early childhood institution and a Koranic school) will positively influence the academic performance of pupils. Two other variables that are not frequently used in the literature are to eat at fill at home and bringing a snack to school. Remember that the study focused on rural population. These food indicators provide information on the nutritional well-being of children. We also anticipate that these two variables act positively on pupils' performance.

The characteristics of schools and teachers. Schools' characteristics taken into account are: the number of classes in provisional shelters, the distance between school and the home of pupils, the class size, the number of pupils per textbook, the existence of latrines, hand washing device, association of pupils' parents, water point, opportunity for pupils to eat near school, disruptions that delay the kick-off of classes, absenteeism of teachers and schools stating that the tuition fee or school cost is high. Among these variables, those that allow us to contextualize the study are classes in provisional shelters, hand washing device, association of pupils' parents, the opportunity for pupils to eat near their school and disruptions that delay the start of classes.

For the variable temporary shelters, we expect a negative effect on the scores but a positive effect on enrollment, dropout and repetition rate. In Senegal, temporary shelters have been set up to overcome the lack of classrooms in some rural areas. They are usually precarious straw constructions which become unusable during the rainy season. Through the office of the association of parents, the community can control over the school, which could have a positive effect on the effectiveness of schools. We give serious consideration to the quality of sanitation in schools through the variables latrines and hand washing device. We expect a positive effect of these variables on pupils' performance. The same goes to pupils having a meal near their school. There are disturbances resulting to delays in the start of the course reduces the learning time. We hypothesize a negative effect of this variable on pupils' achievement. In the Senegalese context, there are often disruptions or strikes by teachers as well as flood cases that often cause delay in opening of classes. This situation reduces the learning time of pupils. The school cost variable may impact negatively on the enrollment rate because if costs are too high households may not be able to enroll their children.

Teachers' characteristics are: gender, age, training received by teachers, professional and academic qualifications and absenteeism. In the Senegalese context, we also expect that a

\footnotetext{
${ }^{5}$ In Senegal, enrollment in kindergarten is very low. In relation to the provision of education, institutions to support early childhood are: community houses, houses for toddlers, nursery schools and kindergartens.
} 
female teacher has a positive influence on pupils' achievement particularly girls who identify themselves more easily to a teacher female. Regarding the age of teacher, we assume a positive effect. We introduced the square of the age of the teacher to take into account a possible nonlinearity that may exist between the age and pupils' performance. It is important to note that in the literature, some authors have found no evidence between the age of the teacher and pupils' scores. Concerning the academic degree of teachers, we made the distinction between the national certificate, which is a diploma certifying the acquisition of general knowledge at the end of the secondary education and the 'High School Diploma' which is the national graduate certifying the acquisition of high school. ${ }^{6}$ The literature reports mixed results regarding the effect of the academic level of teachers on pupils' achievement (Clotfelter et al. 2006). Regarding the variable continuing training, we expect a positive effect on pupils' achievement. Indeed, training improves the teaching skills of teachers. There is no consensus in the literature as to the effect of the continuing training of the teacher. According to CONFEMEN (1999), the teacher's trained for a year in Burkina Faso and Cameroon have less satisfactory results than those trained for five (5) years. In Senegal, the same phenomenon is reflected for teachers who received two years of training compared to those who only received one year.

The characteristics of households. Here we have included some control variables usually found in the literature such as education spending, health care spending, literacy of the household head, gender of household head and marital status. Two other control variables which are important in our context but not often mentioned in the literature are whether the household owns arable land and cattle. Despite the fact that these two variables could be regarded as indicators of wealth, we expect that they will have a negative effect on pupils' academic performance and also on enrollment, promotion and dropout rates. As we previously outlined, in rural areas, it is likely that pupils living in households with farmland are required by the families to work in the fields or for domestic work. ${ }^{7}$ This will result in keeping them away from school. The same rationale applies to the variable number of head of cattle owned by the household. The assumption is that the higher the head of cattle the higher the probability that pupils are exposed to labor. This variable is relevant in explaining the dropout because the communities who practice livestock are generally nomadic and move permanently with their families.

With regard to marital status, more than $95 \%$ of household heads are married. We then create two marital statuses consisting of married and unmarried people where unmarried includes singles, unmarried, divorced and widowed. We expect that pupils living in married couple will have a much better performance. Some facts suggest that children living with a single divorced mother or widowed are generally more successful than children living in a large polygamous household.

The community characteristics. Studies that have examined the effects of neighborhood or community factors on pupils' performance are still scarce. These factors could have both positive and negative effect on pupils' performance. In our study, we use the following indicators: the existence of a college in the village, living in a community in which some children do not go to school because parents are not interested in school, or some children do not attend school because they only go to Koranic school and the number of primary schools in the village. These variables are context-specific and can enrich our understanding of the determinants of pupils' achievement.

Regarding the existence of a college, we expect a positive effect on performance. Parents who have no means to support their children to further their studies in a remote village after their primary certificate will invest little in the education of their children. This could lead to premature termination of schooling. In addition, living in a village where there is a college

\footnotetext{
${ }^{6}$ In the French system, the national certificate is denoted 'Le diplôme national du brevet (DNB)' and the High School Diploma is called 'Baccalauréat'. The latter doesn't mean bachelor's degree. It is equivalent to the 'General Education Diploma'.

${ }^{7}$ Remind that agriculture and livestock are the main economic activities in the target rural regions of the programs under study.
} 
implies that there are facilities for further study and possibly also that there are senior pupils in these colleges who can help the younger ones in primary schools. Regarding the variable parents who are not interested in school, we expect a negative effect. The number of primary schools in a community inform about educational opportunities. So it is expected that this variable will have a positive effect on enrollment. Lastly, to take into account certain unobserved characteristics of the areas where the programs were implemented, we introduced region dummies. The region of reference is Fatick because it has the largest number of schools and students.

\subsubsection{Descriptive statistics}

The distribution of the sample pupils according to the programs received is shown in the bottom of Table B.1. About $4 \%$ of pupils receive both programs, $8 \%$ receive deworming but not meal, $23 \%$ receive meal but not deworming and $65 \%$ have not received any of the two programs. Table B.1 also summarizes descriptive by treatment status. To check whether there is any difference between groups, we provide mean difference tests. In what follows, we provide salient features on outcome indicators: scores (aggregate, French and Math) and enrollment rate, promotion and dropout rates).

We observe that, on average, pupils who received the package of two programs have the highest academic results: 47.66, 45.24 and 50.08 respectively for the aggregate scores and the scores in math and French. Those who have only deworming have the lowest aggregated average score and score in French (36.69 and 35.36 respectively). The lowest average score in math is 36.96 and this occurs in the untreated group. As indicated in the test of mean difference, the differences between groups are significant except between untreated and deworming groups for the aggregate score and score in math.

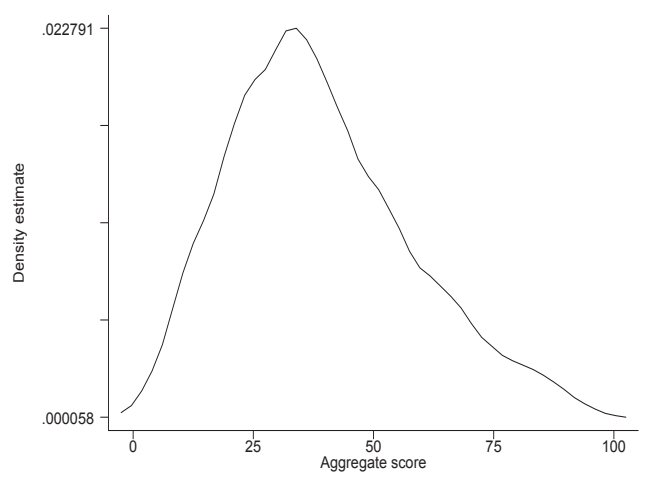

The figure 3.1 displays the distribution of the three scores. These distributions are basically uni-modal. Moreover, these distributions are fairly clustered around the sample averages. The major information we draw from this distribution is that the academic performance of pupils is quite low. It shows that most pupils have scores below the central value (50). It is therefore clear that improving the academic performance of pupils became an objective of policy makers because this improvement will contribute to human capital formation.

For the outcomes enrollment, promotion and dropout rates, statistics are reported in the same Table. For a given outcome variable, the rate is calculated as given in the appendix B. We observe negative average rate of enrollment for the deworming group, package and untreated. This rate is positive for the meal group (7.6\%). Negative values mean that the number of students enrolled in school at date $t-1$ is greater than the number of entries in $t$. In our data collected, we observe that some schools that reported no registration at $t$, had registered in $t-1$, thereby resulting in significant negative rates as shown on the the distribution (see Figure 3.2). 

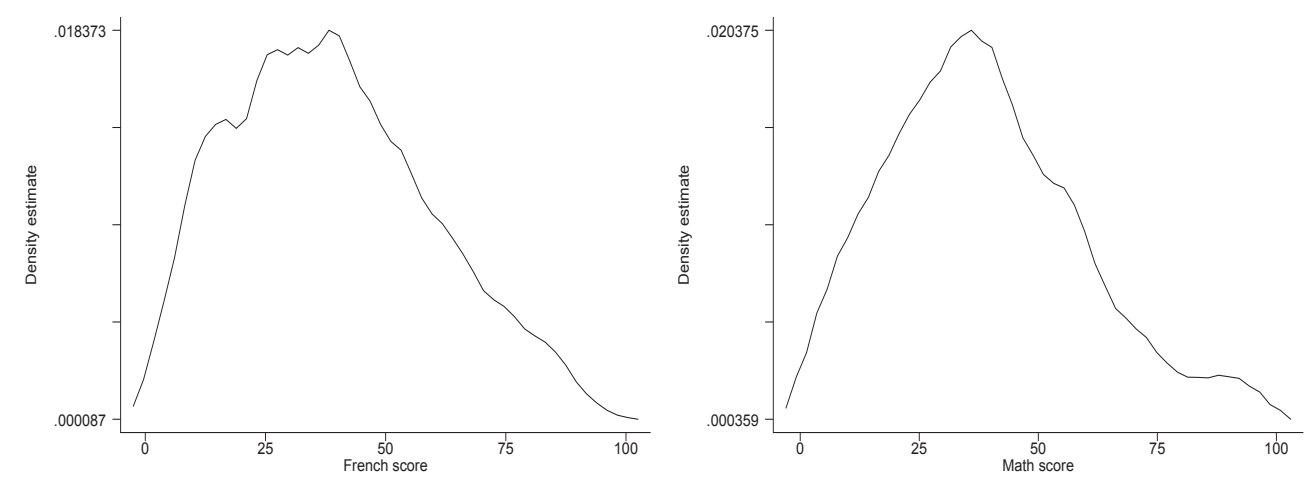

Figure 3.1: Kernel density estimate. [Top]: Aggregate score [Bottom-left]: French score [Bottomright]: Math score

These enrollment statistics are particularly instructive in several respects. Several criticisms and doubts persist as for the objectives assigned to the school feeding and deworming programs. This is due to the fact that these programs can be diverted from their original objectives, which would explain the high attractiveness of schools in areas where these programs are implemented. This is also because they could allow families to meet the food needs of their children. With a lot of caution, we may tentatively say that the observed positive average of enrollment rate applies only for the meals group, while the average rates of other groups are negative.

The average promotion rate is highest in the group of pupils who received the package $(81.89 \%)$, followed by the untreated group (79.15\%), meal group (78.76\%) and deworming group $(73.34 \%)$. Regarding the dropout rate, it is lowest in the package group $(10.2 \%)$ and highest in the untreated group $(16.6 \%)$. Note that the test of mean difference indicates that the average enrollment rate between the deworming group $(-32.28 \%)$ and the untreated group $(-31.40 \%)$ is not significantly different. The same goes for the average promotion rate between the untreated group $(79.15 \%)$ and the meal group $(78.76 \%)$, and the average dropout rate between deworming group $(15.18 \%)$ and meal group (15.07\%). 
Figure 3.2 provides additional picture about the distribution of the three rates. The enrollment rate has a bi-modal dominant distribution: with a mode around negative values and another around zero. It is clear that the distribution of enrollment rate is dominated by negative values. Promotion and dropout rates have a dominant uni-modal distribution. A significant proportion of schools are located around $85 \%$ with regard to the promotion rate and another smaller mode is shown around $65 \%$. Overall, we observe that a significant proportion of the population experienced relatively high rates of promotion. The dropout rate meanwhile shows a dominant mode around a value slightly lower at $15 \%$ and another smaller mode around $33 \%$. This indicates that most schools experienced a relatively low dropout rates.
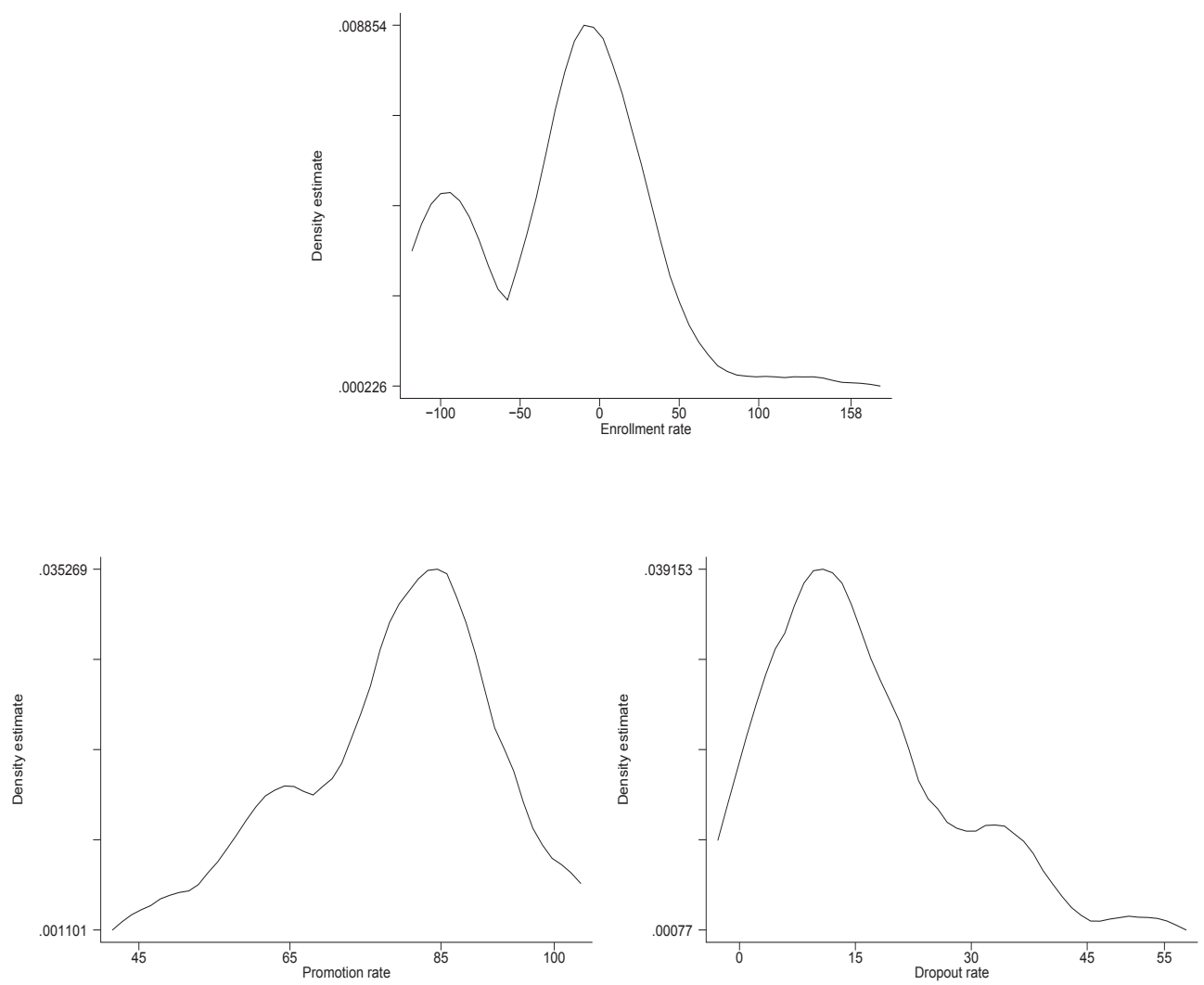

Figure 3.2: Kernel density estimate [Top]: Enrollment rate [Bottom-left]: Promotion rate [Bottom-right]: Dropout rate

In conclusion, as indicated by the test of mean differences in Table B.1 in the appendix, we observe significant differences between the averages of several variables including outcomes and controls. We can conclude that experimental methods based on randomization are not suited to assess the impact of the package of programs that we study. In the following sections, we develop and apply appropriate non-experimental methods to our data. 


\subsection{Econometric specifications}

\subsubsection{The double-index selection model}

Let $T_{1 i}^{*}$ and $T_{2 i}^{*}$ denote two latent (unobserved) variables denoting the reasons for pupil $i$ ( $i=$ $1, \cdots, N)$ to receive treatment 1 (deworming) and treatment 2 (school meal) respectively. These variables are assumed to be functions of observed characteristics of the pupil or the household he/she belongs to, which we denote by $\mathbf{w}_{j i}(j=1$ or 2$)$. Formally,

$$
\begin{aligned}
& T_{1 i}^{*}=\gamma_{1}^{\prime} \mathbf{w}_{1 i}+\mu_{1 i}, \\
& T_{2 i}^{*}=\gamma_{2}^{\prime} \mathbf{w}_{2 i}+\mu_{2 i},
\end{aligned}
$$

where $\gamma_{j}$ denotes the vectors of parameters to be estimated, and $\mu_{j i}$ denotes the error terms. We assume that $\mu_{j i} \perp \mathbf{w}_{j i}$. The observed counterparts to $T_{1 i}^{*}$ and $T_{2 i}^{*}$, denoted by $T_{1 i}$ and $T_{2 i}$, are defined as

$$
\begin{aligned}
& T_{1 i}=\mathbf{1}\left[T_{1 i}^{*}>0\right], \\
& T_{2 i}=\mathbf{1}\left[T_{2 i}^{*}>0\right],
\end{aligned}
$$

where $\mathbf{1}[$.$] denotes the indicator function which takes on the value 1$ if the corresponding latent variable is positive, and 0 otherwise. In other words, if the unobserved reasons for pupil $i$ to receive treatment $j$ are sufficiently valid, i.e. $T_{j i}^{*}>0$, the pupil does receive the treatment, in which case $T_{j i}=1$. If not, the pupil does not receive the treatment, i.e. $T_{j i}=0$. The outcome for pupil $i, y_{i}$, in terms of achievement (e.g. scores, repetition and dropout rates) is given by

$$
y_{i}=\boldsymbol{\beta}^{\prime} \mathbf{x}_{i}+\delta_{1} T_{1 i}+\delta_{2} T_{2 i}+\theta T_{1 i} T_{2 i}+\varepsilon_{i},
$$

where $\mathbf{x}_{i}$ denotes the control variables, $\boldsymbol{\beta}, \delta_{j}$ and $\theta$ are parameter vectors to be estimated; ${ }^{8}$. $\mathbf{x}_{i}$ also contains an intercept whose coefficient will be the effect of the absence of treatment on the outcome; $\varepsilon_{i}$ denotes the error term capturing among other things the effect of unobserved factors on the outcome. Since $T_{1 i}$ and $T_{2 i}$ are endogenous, $\mathbb{E}\left(\varepsilon_{i} \mid T_{1 i}, T_{2 i}, \mathbf{x}_{i}\right) \neq 0$. By including the interaction term $T_{1 i} T_{2 i}$, as additional regressor in equation (1-5), we can isolate the exclusive effect of either treatment and their joint effect. Moreover, depending on the sign, $\theta$ reflects the complementarity (positive) or the substitutability between $T_{1}$ and $T_{2}$.

The model consisting of equations (1-1)-(1-5) is a generalization of the dummy endogenous variable model of Heckman (1978) in that there are two endogenous dummy variables. To estimate the model, we consider a full information maximum likelihood (FIML) approach and a generalized two-step Heckman method as described in what follow.

\section{FIML Estimation}

We make the following distributional assumption: conditionally on $\left(\mathbf{w}_{i}, \mathbf{x}_{i}\right),\left(\mu_{1 i}, \mu_{2 i}, \varepsilon_{i}\right)^{\prime}$ is normally distributed with vector mean $\mathbf{0}$ and covariance matrix $\boldsymbol{\Sigma}=\left(\begin{array}{ccc}1 & & \\ \rho_{\mu_{1} \mu_{2}} & 1 & \\ \rho_{\mu_{1} \varepsilon} \sigma_{\varepsilon} & \rho_{\mu_{2} \varepsilon} \sigma_{\varepsilon} & \sigma_{\varepsilon}^{2}\end{array}\right) .9$

The likelihood function of the model consists of four parts due to the combination of the two treatments. The contributions to the likelihood are as follows: those of pupils who benefit from deworming and canteen meals $\left(T_{1 i}=1, T_{2 i}=1\right)$, from deworming only $\left(T_{1 i}=1, T_{2 i}=0\right)$, from canteen meals only $\left(T_{1 i}=0, T_{2 i}=1\right)$, and from neither one $\left(T_{1 i}=0, T_{2 i}=0\right)$. For all four

\footnotetext{
${ }^{8}$ The vectors and matrices are bold faced while scalars are typeset normally

${ }^{9}$ With $\rho_{12} \neq 0$, equations (1-1)-(1-4) form a bivariate probit where $\sigma_{\mu_{1}}$ and $\sigma_{\mu_{2}}$ are not identified. Thus, $\sigma_{\mu_{1}}=\sigma_{\mu_{2}}=1$.
} 
categories of pupils, the outcome is observed. Formally, the likelihood is written as

$$
\begin{aligned}
& L=\prod_{i=1}^{N}\left[\int_{-\gamma_{1}^{\prime} \mathbf{w}_{1 i}}^{\infty} \int_{-\gamma_{2}^{\prime} \mathbf{w}_{2 i}}^{\infty} f_{3}\left(\mu_{1 i}, \mu_{2 i}, y_{i}\right) d \mu_{1 i} d \mu_{2 i}\right]^{T_{1 i} T_{2 i}} \\
& {\left[\int_{-\gamma_{1}^{\prime} \mathbf{w}_{1 i}}^{\infty} \int_{-\infty}^{-\gamma_{2}^{\prime} \mathbf{w}_{2 i}} f_{3}\left(\mu_{1 i}, \mu_{2 i}, y_{i}\right) d \mu_{1 i} d \mu_{2 i}\right]^{T_{1 i}\left(1-T_{2 i}\right)} } \\
& {\left[\int_{-\infty}^{-\gamma_{1}^{\prime} \mathbf{w}_{1 i}} \int_{-\gamma_{2}^{\prime} \mathbf{w}_{2 i}}^{\infty} f_{3}\left(\mu_{1 i}, \mu_{2 i}, y_{i}\right) d \mu_{1 i} d \mu_{2 i}\right]^{\left(1-T_{1 i}\right) T_{2 i}} } \\
& {\left[\int_{-\infty}^{-\gamma_{1}^{\prime} \mathbf{w}_{1 i}} \int_{-\infty}^{-\gamma_{2}^{\prime} \mathbf{w}_{2 i}} f_{3}\left(\mu_{1 i}, \mu_{2 i}, y_{i}\right) d \mu_{1 i} d \mu_{2 i}\right]^{\left(1-T_{1 i}\right)\left(1-T_{2 i}\right)} }
\end{aligned}
$$

where $f_{3}=f_{1}\left(y_{i} \mid \mathbf{x}_{i}, \mathbf{w}_{i}\right) f_{2}\left(\mu_{1 i}, \mu_{2 i} \mid y_{i}\right)$ denotes the trivariate normal density function and where $f_{2}$ and $f_{1}$ denote respectively the bivariate and the univariate normal density function. Substituting $f_{1}(\cdots) f_{2}(\cdots)$ for $f_{3}$ into equation (1-6) yields

$$
\begin{aligned}
L=\prod_{i=1}^{N} & {\left[\int_{-\gamma_{1}^{\prime} \mathbf{w}_{1 i}}^{\infty} \int_{-\gamma_{2}^{\prime} \mathbf{w}_{2 i}}^{\infty} f_{1}\left(y_{i} \mid \mathbf{x}_{i}, \mathbf{w}_{i}\right) f_{2}\left(\mu_{1 i}, \mu_{2 i} \mid y_{i}\right) d \mu_{1 i} d \mu_{2 i}\right]^{T_{1 i} T_{2 i}} } \\
& {\left[\int_{-\gamma_{1}^{\prime} \mathbf{w}_{1 i}}^{\infty} \int_{-\infty}^{-\gamma_{2}^{\prime} \mathbf{w}_{2 i}} f_{1}\left(y_{i} \mid \mathbf{x}_{i}, \mathbf{w}_{i}\right) f_{2}\left(\mu_{1 i}, \mu_{2 i} \mid y_{i}\right) d \mu_{1 i} d \mu_{2 i}\right]^{T_{1 i}\left(1-T_{2 i}\right)} } \\
& {\left[\int_{-\infty}^{-\gamma_{1}^{\prime} \mathbf{w}_{1 i}} \int_{-\gamma_{2}^{\prime} \mathbf{w}_{2 i}}^{\infty} f_{1}\left(y_{i} \mid \mathbf{x}_{i}, \mathbf{w}_{i}\right) f_{2}\left(\mu_{1 i}, \mu_{2 i} \mid y_{i}\right) d \mu_{1 i} d \mu_{2 i}\right]^{\left(1-T_{1 i}\right) T_{2 i}} } \\
& {\left[\int_{-\infty}^{-\gamma_{1}^{\prime} \mathbf{w}_{1 i}} \int_{-\infty}^{-\gamma_{2}^{\prime} \mathbf{w}_{2 i}} f_{1}\left(y_{i} \mid \mathbf{x}_{i}, \mathbf{w}_{i}\right) f_{2}\left(\mu_{1 i}, \mu_{2 i} \mid y_{i}\right) d \mu_{1 i} d \mu_{2 i}\right]^{\left(1-T_{1 i}\right)\left(1-T_{2 i}\right)} }
\end{aligned}
$$

where $f_{1}\left(y_{i} \mid \mathbf{x}_{i}, \mathbf{w}_{i}\right)=\frac{1}{\sigma_{\varepsilon}} \phi_{1}\left(\frac{y_{i}-\boldsymbol{\beta}^{\prime} \mathbf{x}_{i}-A_{i}\left(T_{1 i}, T_{2 i}\right)}{\sigma_{\varepsilon}}\right), \phi_{1}$ denotes the univariate standard normal density function and $A_{i}\left(T_{1 i}, T_{2 i}\right)$ is given by

$$
A_{i}\left(T_{1 i}, T_{2 i}\right) \equiv \delta_{1} T_{1 i}+\delta_{2} T_{2 i}+\theta T_{1 i} T_{2 i}
$$

The difficulty consists in evaluating the double integrals of equation (1-7) which result in bivariate (standard) normal cumulative distribution functions (cdfs) conditional on a third random variable, $y_{i}$. It is known that

$$
\left(\mu_{1 i}, \mu_{2 i}\right)^{\prime} \mid y_{i} \sim N\left[\left(\begin{array}{c}
\frac{\rho_{\mu_{1} \varepsilon}}{\sigma_{\varepsilon}}\left[y_{i}-\mathbb{E}\left(y_{i} \mid \mathbf{x}_{i}, T_{j}\right)\right] \\
\frac{\rho_{\mu_{2} \varepsilon}}{\sigma_{\varepsilon}}\left[y_{i}-\mathbb{E}\left(y_{i} \mid \mathbf{x}_{i}, T_{j}\right)\right]
\end{array}\right) ;\left(\begin{array}{cc}
1-\rho_{\mu_{1} \varepsilon}^{2} & \\
\rho_{\mu_{1} \mu_{2} \varepsilon} & 1-\rho_{\mu_{2} \varepsilon}^{2}
\end{array}\right)\right],
$$

with $\mathbb{E}\left(y_{i} \mid T_{1 i}, T_{2 i}, \mathbf{x i}\right)=\boldsymbol{\beta}^{\prime} \mathbf{x}_{i}+A_{i}\left(T_{1 i}, T_{2 i}\right)+\mathbb{E}\left(\varepsilon_{i} \mid T_{1 i}, T_{2 i}, \mathbf{x}_{i}\right)$, where $\mathbb{E}\left(\varepsilon_{i} \mid T_{1 i}, T_{2 i}, \mathbf{x}_{i}\right) \neq 0$ and $\rho_{\mu_{1} \mu_{2}, \varepsilon}$ denotes the partial correlation between $\mu_{1 i}$ and $\mu_{2 i}$ conditional on $y_{i}$ and is given by:

$$
\rho_{\mu_{1} \mu_{2} \varepsilon}=\frac{\rho_{\mu_{1} \mu_{2}}-\rho_{\mu_{1} \varepsilon} \rho_{\mu_{2} \varepsilon}}{\sqrt{\left(1-\rho_{\mu_{1} \varepsilon}^{2}\right)\left(1-\rho_{\mu_{2} \varepsilon}^{2}\right)}} \cdot{ }^{10}
$$

Using the symmetry property of the normal distribution, we derive the final expression of the

\footnotetext{
${ }^{10}$ See for instance Kotz et al. (2000).
} 
likelihood as

$$
\begin{aligned}
L=\prod_{i=1}^{N}\left[f_{1}\left(y_{i} \mid \mathbf{x}_{i}, \mathbf{w}_{i}\right) \Phi_{2}\left(\zeta_{1}, \zeta_{2} ; \rho_{\mu_{1} \mu_{2} \varepsilon \varepsilon}\right)\right]^{T_{1 i} T_{2 i}}\left[f_{1}\left(y_{i} \mid \mathbf{x}_{i}, \mathbf{w}_{i}\right) \Phi_{2}\left(\zeta_{1},-\zeta_{2} ;-\rho_{\mu_{1} \mu_{2}, \varepsilon}\right)\right]^{T_{1 i}\left(1-T_{2 i}\right)} \\
\\
\quad\left[f_{1}\left(y_{i} \mid \mathbf{x}_{i}, \mathbf{w}_{i}\right) \Phi_{2}\left(-\zeta_{1}, \zeta_{2} ;-\rho_{\mu_{1} \mu_{2}, \varepsilon}\right)\right]^{\left(1-T_{1 i}\right) T_{2 i}}\left[f_{1}\left(y_{i} \mid \mathbf{x}_{i}, \mathbf{w}_{i}\right) \Phi_{2}\left(-\zeta_{1},-\zeta_{2} ; \rho_{\mu_{1} \mu_{2}, \varepsilon}\right)\right]^{\left(1-T_{1 i}\right)\left(1-T_{2 i}\right)}
\end{aligned}
$$

where $\Phi_{2}$ denotes the bivariate standard normal cdf, and $\zeta_{k}$ is defined as

$$
\zeta_{k} \equiv \frac{\gamma_{k}^{\prime} \mathbf{w}_{k i}+\frac{\rho_{\mu_{k} \varepsilon}}{\sigma_{\varepsilon}}\left(y_{i}-\boldsymbol{\beta}^{\prime} \mathbf{x}_{i}-A_{i}\left(T_{1 i}, T_{2 i}\right)\right)}{\sqrt{1-\rho_{\mu_{k} \varepsilon}^{2}}}, \quad k=1,2
$$

To obtain FIML estimates of the model, we can maximize the $\log$-likelihood $\ln L$ using standard numerical techniques (e.g. Newton-Raphson). Standard errors of the estimates are obtained using the inverse Hessian or the outer product of gradient.

\section{Two-step estimation}

The regression equation (eq.(1-5)) is the equation of interest. The population regression can be written in the form of a conditional expectation, i.e.

$$
\mathbb{E}\left(y_{i} \mid T_{1 i}, T_{2 i}, \mathbf{x}_{i}\right)=\boldsymbol{\beta}^{\prime} \mathbf{x}_{i}+\delta_{1} T_{1 i}+\delta_{2} T_{2 i}+\theta T_{1 i} T_{2 i}+E\left(\varepsilon_{i} \mid T_{1 i}, T_{2 i}, \mathbf{x}_{i}\right) .
$$

Since $T_{1 i}$ and $T_{2 i}$ are endogenous, $\mathbb{E}\left(\varepsilon_{i} \mid T_{1 i}, T_{2 i}, \mathbf{x}_{i}\right) \neq 0$ and the ordinary least squares (OLS) estimator of $\boldsymbol{\beta}, \delta_{1}, \delta_{2}$ and $\theta$ is inconsistent. The endogeneity of $T_{j i}(j=1$ or 2$)$ comes from the fact that $T_{j i}$ depends on $\mu_{j i}$ and the latter is correlated with $\varepsilon_{i}$. Hence, the endogeneity is accounted for by taking the correlations $\rho_{\mu_{1} \varepsilon}$ and $\rho_{\mu_{2} \varepsilon}$ into account. The conditional expectation $\mathbb{E}\left(\varepsilon_{i} \mid T_{1 i}, T_{2 i}, \mathbf{x}_{i}\right)$ can be written as

$$
\begin{aligned}
\mathbb{E}\left(\varepsilon_{i} \mid T_{1 i}, T_{2 i}, \mathbf{x}_{i}\right)= & T_{1 i} T_{2 i} \mathbb{E}\left(\varepsilon_{i} \mid T_{1 i}=1, T_{2 i}=1, \mathbf{x}_{i}\right) \\
& +T_{1 i}\left(1-T_{2 i}\right) \mathbb{E}\left(\varepsilon_{i} \mid T_{1 i}=1, T_{2 i}=0, \mathbf{x}_{i}\right) \\
& +\left(1-T_{1 i}\right) T_{2 i} \mathbb{E}\left(\varepsilon_{i} \mid T_{1 i}=0, T_{2 i}=1\right) \\
& +\left(1-T_{1 i}\right)\left(1-T_{2 i}\right) \mathbb{E}\left(\varepsilon_{i} \mid T_{1 i}=0, T_{2 i}=0, \mathbf{x}_{i}\right) .
\end{aligned}
$$

Note the similarity between the four types of likelihood contributions. Using the definition of $T_{1 i}$ and $T_{2 i}$ (eqs. (1-3) and (1-4)) and the latent equations (eqs.(1-2), (1-14) can be written as

$$
\begin{aligned}
\mathbb{E}\left(\varepsilon_{i} \mid T_{1 i}, T_{2 i}, \mathbf{x}_{i}\right)= & T_{1 i} T_{2 i} \underbrace{E\left(\varepsilon_{i} \mid \mu_{1 i}>-\gamma_{1}^{\prime} \mathbf{w}_{1 i}, \mu_{2 i}>-\gamma_{2}^{\prime} \mathbf{w}_{2 i}, \mathbf{x}_{i}\right)}_{\mathbb{E}\left(\varepsilon_{i} \mid>,>\right)} \\
& +T_{1 i}\left(1-T_{2 i}\right) \underbrace{\mathbb{E}\left(\varepsilon_{i} \mid \mu_{1 i}>-\gamma_{1}^{\prime} \mathbf{w}_{1 i}, \mu_{2 i} \leq-\gamma_{2}^{\prime} \mathbf{w}_{2 i}, \mathbf{x}_{i}\right)}_{\mathbb{E}\left(\varepsilon_{i} \mid>, \leq\right)} \\
& +\left(1-T_{1 i}\right) T_{2 i} \underbrace{\mathbb{E}\left(\varepsilon_{i} \mid \mu_{1 i} \leq-\gamma_{1}^{\prime} \mathbf{w}_{1 i}, \mu_{2 i}>-\gamma_{2}^{\prime} \mathbf{w}_{2 i}, \mathbf{x}_{i}\right)}_{E\left(\varepsilon_{i} \mid \leq,>\right)} \\
& +\left(1-T_{1 i}\right)\left(1-T_{2 i}\right) \underbrace{\mathbb{E}\left(\varepsilon_{i} \mid \mu_{1 i} \leq-\gamma_{1}^{\prime} \mathbf{w}_{1 i}, \mu_{2 i} \leq-\gamma_{2}^{\prime} \mathbf{w}_{2 i}, \mathbf{x}_{i}\right)}_{\mathbb{E}\left(\varepsilon_{i} \mid \leq, \leq\right)} .
\end{aligned}
$$

The conditional expectations in equation (1-15) involve the truncated trivariate normal distribution. Using the moment-generating function formula along the lines of Muthén (1990), these 
expectations are shown to be (see Appendix B.2 for derivations):

$$
\begin{aligned}
& \mathbb{E}\left(\varepsilon_{i} \mid>,>\right)= \frac{\sigma_{\varepsilon} \rho_{\mu_{1} \varepsilon} \phi_{1}\left(\gamma_{1}^{\prime} \mathbf{w}_{1 i}\right)}{\Phi_{2}\left(\gamma_{1}^{\prime} \mathbf{w}_{1 i}, \gamma_{2}^{\prime} \mathbf{w}_{2 i}, \rho_{\mu_{1} \mu_{2}}\right)} \Phi_{1}\left(\frac{\gamma_{2}^{\prime} \mathbf{w}_{2 i}-\rho_{\mu_{1} \mu_{2}} \gamma_{1}^{\prime} \mathbf{w}_{1 i}}{\sqrt{1-\rho_{\mu_{1} \mu_{2}}^{2}}}\right) \\
&+\frac{\sigma_{\varepsilon} \rho_{\mu_{2} \varepsilon} \phi_{1}\left(\gamma_{2}^{\prime} \mathbf{w}_{2 i}\right)}{\Phi_{2}\left(\gamma_{1}^{\prime} \mathbf{w}_{1 i}, \gamma_{2}^{\prime} \mathbf{w}_{2 i}, \rho_{\left.\mu_{1} \mu_{2}\right)}\right)} \Phi_{1}\left(\frac{\gamma_{1}^{\prime} \mathbf{w}_{1 i}-\rho_{\mu_{1} \mu_{2}} \gamma_{2}^{\prime} \mathbf{w}_{2 i}}{\sqrt{1-\rho_{\mu_{1} \mu_{2}}^{2}}}\right) \\
& \mathbb{E}\left(\varepsilon_{i} \mid>, \leq\right)= \frac{\sigma_{\varepsilon} \rho_{\mu_{1} \varepsilon} \phi_{1}\left(\gamma_{1}^{\prime} \mathbf{w}_{1 i}\right)}{\Phi_{2}\left(\gamma_{1}^{\prime} \mathbf{w}_{1 i},-\gamma_{2}^{\prime} \mathbf{w}_{2 i},-\rho_{\left.\mu_{1} \mu_{2}\right)}\right)} \Phi_{1}\left(\frac{\rho_{\mu_{1} \mu_{2}} \gamma_{1}^{\prime} \mathbf{w}_{1 i}-\gamma_{2}^{\prime} \mathbf{w}_{2 i}}{\sqrt{1-\rho_{\mu_{1} \mu_{2}}^{2}}}\right) \\
& \mathbb{\Phi _ { 2 } ( \gamma _ { 1 } ^ { \prime } \mathbf { w } _ { 1 i } , - \gamma _ { 2 } ^ { \prime } \mathbf { w } _ { 2 i } , - \rho _ { \mu _ { 1 } \mu _ { 2 } ) }} \Phi_{1}\left(\frac{\sigma_{1}^{\prime} \mathbf{w}_{1 i}-\rho_{\mu_{1} \mu_{2}} \gamma_{2}^{\prime} \mathbf{w}_{2 i}}{\sqrt{1-\rho_{\mu_{1} \mu_{2}}^{2}}}\right) \\
& \mathbb{E}\left(\varepsilon_{i} \mid \leq,>\right)= \\
& \quad-\frac{\sigma_{\varepsilon} \rho_{\mu_{1} \varepsilon} \phi_{1}\left(\gamma_{1}^{\prime} \mathbf{w}_{1 i}\right)}{\Phi_{2}\left(-\gamma_{1}^{\prime} \mathbf{w}_{1 i}, \gamma_{2}^{\prime} \mathbf{w}_{2 i},-\rho_{\mu_{1} \mu_{2}}\right)} \Phi_{1}\left(\frac{\gamma_{2}^{\prime} \mathbf{w}_{2 i}-\rho_{\mu_{1} \mu_{2}} \gamma_{1}^{\prime} \mathbf{w}_{1 i}}{\sqrt{1-\rho_{\mu_{1} \mu_{2}}^{2}}}\right) \\
& \\
&+\frac{\sigma_{\varepsilon} \rho_{\mu_{2} \varepsilon} \phi_{1}\left(\gamma_{2}^{\prime} \mathbf{w}_{2 i}\right)}{\Phi_{2}\left(-\gamma_{1}^{\prime} \mathbf{w}_{1 i}, \gamma_{2}^{\prime} \mathbf{w}_{2 i},-\rho_{\left.\mu_{1} \mu_{2}\right)}\right)} \Phi_{1}\left(\frac{\rho_{\mu_{1} \mu_{2}} \gamma_{2}^{\prime} \mathbf{w}_{2 i}-\gamma_{1}^{\prime} \mathbf{w}_{1 i}}{\sqrt{1-\rho_{\mu_{1} \mu_{2}}^{2}}}\right)
\end{aligned}
$$

and

$$
\begin{aligned}
\mathbb{E}\left(\varepsilon_{i} \mid \leq, \leq\right)= & -\frac{\sigma_{\varepsilon} \rho_{\mu_{1} \varepsilon} \phi_{1}\left(\gamma_{1}^{\prime} \mathbf{w}_{1 i}\right)}{\Phi_{2}\left(-\gamma_{1}^{\prime} \mathbf{w}_{1 i},-\gamma_{2}^{\prime} \mathbf{w}_{2 i}, \rho_{\mu_{1} \mu_{2}}\right)} \Phi_{1}\left(\frac{\rho_{\mu_{1} \mu_{2}} \gamma_{1}^{\prime} \mathbf{w}_{1 i}-\gamma_{2}^{\prime} \mathbf{w}_{2 i}}{\sqrt{1-\rho_{\mu_{1} \mu_{2}}^{2}}}\right) \\
& -\frac{\sigma_{\varepsilon} \rho_{\mu_{2} \varepsilon} \phi_{1}\left(\gamma_{2}^{\prime} \mathbf{w}_{2 i}\right)}{\Phi_{2}\left(-\gamma_{1}^{\prime} \mathbf{w}_{1 i},-\gamma_{2}^{\prime} \mathbf{w}_{2 i}, \rho_{\mu_{1} \mu_{2}}\right)} \Phi_{1}\left(\frac{\rho_{\mu_{1} \mu_{2}} \gamma_{2}^{\prime} \mathbf{w}_{2 i}-\gamma_{1}^{\prime} \mathbf{w}_{1 i}}{\sqrt{1-\rho_{\mu_{1} \mu_{2}}^{2}}}\right)
\end{aligned}
$$

where we use the symmetry property of the normal distribution (i.e., $\forall \xi, \phi_{1}(-\xi)=\phi_{1}(\xi)$ ) and $\Phi_{1}$ and $\Phi_{2}$ denote respectively the univariate and bivariate standard normal cdf. For notational convenience, let

$$
\begin{aligned}
& \lambda_{1}^{++} \equiv \frac{\phi_{1}\left(\gamma_{1}^{\prime} \mathbf{w}_{1 i}\right)}{\Phi_{2}\left(\gamma_{1}^{\prime} \mathbf{w}_{1 i}, \gamma_{2}^{\prime} \mathbf{w}_{2 i}, \rho_{\mu_{1} \mu_{2}}\right)} \Phi_{1}\left(\frac{\gamma_{2}^{\prime} \mathbf{w}_{2 i}-\rho_{\mu_{1} \mu_{2}} \gamma_{1}^{\prime} \mathbf{w}_{1 i}}{\sqrt{1-\rho_{\mu_{1} \mu_{2}}^{2}}}\right), \\
& \lambda_{2}^{++} \equiv \frac{\phi_{1}\left(\gamma_{2}^{\prime} \mathbf{w}_{2 i}\right)}{\Phi_{2}\left(\gamma_{1}^{\prime} \mathbf{w}_{1 i}, \gamma_{2}^{\prime} \mathbf{w}_{2 i}, \rho_{\mu_{1} \mu_{2}}\right)} \Phi_{1}\left(\frac{\gamma_{1}^{\prime} \mathbf{w}_{1 i}-\rho_{\mu_{1} \mu_{2}} \gamma_{2}^{\prime} \mathbf{w}_{2 i}}{\sqrt{1-\rho_{\mu_{1} \mu_{2}}^{2}}}\right),
\end{aligned}
$$

for pupils who benefit from both deworming and canteen meals,

$$
\begin{aligned}
& \lambda_{1}^{+-} \equiv \frac{\phi_{1}\left(\gamma_{1}^{\prime} \mathbf{w}_{1 i}\right)}{\Phi_{2}\left(\gamma_{1}^{\prime} \mathbf{w}_{1 i},-\gamma_{2}^{\prime} \mathbf{w}_{2 i},-\rho_{\mu_{1} \mu_{2}}\right)} \Phi_{1}\left(\frac{\rho_{\mu_{1} \mu_{2}} \gamma_{1}^{\prime} \mathbf{w}_{1 i}-\gamma_{2}^{\prime} \mathbf{w}_{2 i}}{\sqrt{1-\rho_{\mu_{1} \mu_{2}}^{2}}}\right), \\
& \lambda_{2}^{+-} \equiv \frac{\phi_{1}\left(\gamma_{2}^{\prime} \mathbf{w}_{2 i}\right)}{\Phi_{2}\left(\gamma_{1}^{\prime} \mathbf{w}_{1 i},-\gamma_{2}^{\prime} \mathbf{w}_{2 i},-\rho_{\mu_{1} \mu_{2}}\right)} \Phi_{1}\left(\frac{\gamma_{1}^{\prime} \mathbf{w}_{1 i}-\rho_{\mu_{1} \mu_{2}} \gamma_{2}^{\prime} \mathbf{w}_{2 i}}{\sqrt{1-\rho_{\mu_{1} \mu_{2}}^{2}}}\right),
\end{aligned}
$$


for those who benefit from deworming but not from canteen meals,

$$
\begin{aligned}
& \lambda_{1}^{-+} \equiv \frac{\phi_{1}\left(\gamma_{1}^{\prime} \mathbf{w}_{1 i}\right)}{\Phi_{2}\left(-\gamma_{1}^{\prime} \mathbf{w}_{1 i}, \gamma_{2}^{\prime} \mathbf{w}_{2 i},-\rho_{\mu_{1} \mu_{2}}\right)} \Phi_{1}\left(\frac{\gamma_{2}^{\prime} \mathbf{w}_{2 i}-\rho_{\mu_{1} \mu_{2}} \gamma_{1}^{\prime} \mathbf{w}_{1 i}}{\sqrt{1-\rho_{\mu_{1} \mu_{2}}^{2}}}\right), \\
& \lambda_{2}^{-+} \equiv \frac{\phi_{1}\left(\gamma_{2}^{\prime} \mathbf{w}_{2 i}\right)}{\Phi_{2}\left(-\gamma_{1}^{\prime} \mathbf{w}_{1 i}, \gamma_{2}^{\prime} \mathbf{w}_{2 i},-\rho_{\mu_{1} \mu_{2}}\right)} \Phi_{1}\left(\frac{\rho_{\mu_{1} \mu_{2}} \gamma_{2}^{\prime} \mathbf{w}_{2 i}-\gamma_{1}^{\prime} \mathbf{w}_{1 i}}{\sqrt{1-\rho_{\mu_{1} \mu_{2}}^{2}}}\right),
\end{aligned}
$$

for those who benefit from canteen meals but not from deworming, and

$$
\begin{gathered}
\lambda_{1}^{--} \equiv \frac{\phi_{1}\left(\gamma_{1}^{\prime} \mathbf{w}_{1 i}\right)}{\Phi_{2}\left(-\gamma_{1}^{\prime} \mathbf{w}_{1 i},-\gamma_{2}^{\prime} \mathbf{w}_{2 i}, \rho_{\mu_{1} \mu_{2}}\right)} \Phi_{1}\left(\frac{\rho_{\mu_{1} \mu_{2}} \gamma_{1}^{\prime} \mathbf{w}_{1 i}-\gamma_{2}^{\prime} \mathbf{w}_{2 i}}{\sqrt{1-\rho_{\mu_{1} \mu_{2}}^{2}}}\right), \\
\lambda_{2}^{--} \equiv \frac{\phi_{1}\left(\gamma_{2}^{\prime} \mathbf{w}_{2 i}\right)}{\Phi_{2}\left(-\gamma_{1}^{\prime} \mathbf{w}_{1 i},-\gamma_{2}^{\prime} \mathbf{w}_{2 i}, \rho_{\mu_{1} \mu_{2}}\right)} \Phi_{1}\left(\frac{\rho_{\mu_{1} \mu_{2}} \gamma_{2}^{\prime} \mathbf{w}_{2 i}-\gamma_{1}^{\prime} \mathbf{w}_{1 i}}{\sqrt{1-\rho_{\mu_{1} \mu_{2}}^{2}}}\right),
\end{gathered}
$$

for those who did not benefit from deworming or meal program: untreated. The $\lambda$ 's are generalizations of the inverse Mill's ratio. Replacing the expressions of (eqs.(1-20a)-1-20h) into the conditional expectations of equation (1-15) yields

$$
\begin{aligned}
\mathbb{E}\left(\varepsilon_{i} \mid T_{1 i}, T_{2 i}, \mathbf{x}_{i}\right)=T_{1 i} T_{2 i}\left(\sigma_{\varepsilon} \rho_{\mu_{1} \varepsilon} \lambda_{1}^{++}+\sigma_{\varepsilon} \rho_{\mu_{2} \varepsilon} \lambda_{2}^{++}\right)+T_{1 i}\left(1-T_{2 i}\right)\left(\sigma_{\varepsilon} \rho_{\mu_{1} \varepsilon} \lambda_{1}^{+-}-\sigma_{\varepsilon} \rho_{\mu_{2} \varepsilon} \lambda_{2}^{+-}\right) \\
\quad+\left(1-T_{1 i}\right) T_{2 i}\left(-\sigma_{\varepsilon} \rho_{\mu_{1} \varepsilon} \lambda_{1}^{-+}+\sigma_{\varepsilon} \rho_{\mu_{2} \varepsilon} \lambda_{2}^{-+}\right)+\left(1-T_{1 i}\right)\left(1-T_{2 i}\right)\left(-\sigma_{\varepsilon} \rho_{\mu_{1} \varepsilon} \lambda_{1}^{--}-\sigma_{\varepsilon} \rho_{\mu_{2} \varepsilon} \lambda_{2}^{--}\right),
\end{aligned}
$$

which after factorization yields

$$
\begin{gathered}
\mathbb{E}\left(\varepsilon_{i} \mid T_{1 i}, T_{2 i}, \mathbf{x}_{i}\right)=\sigma_{\varepsilon} \rho_{\mu_{1} \varepsilon} \underbrace{\left[\lambda_{1}^{++} T_{1 i} T_{2 i}+\lambda_{1}^{+-} T_{1 i}\left(1-T_{2 i}\right)-\lambda_{1}^{-+}\left(1-T_{1 i}\right) T_{2 i}-\lambda_{1}^{--}\left(1-T_{1 i}\right)\left(1-T_{2 i}\right)\right]}_{h_{1}\left(T_{1 i}, T_{2 i}\right)} \\
+\sigma_{\varepsilon} \rho_{\mu_{2} \varepsilon} \underbrace{\left[\lambda_{2}^{++} T_{1 i} T_{2 i}-\lambda_{2}^{+-} T_{1 i}\left(1-T_{2 i}\right)+\lambda_{2}^{-+}\left(1-T_{1 i}\right) T_{2 i}-\lambda_{2}^{--}\left(1-T_{1 i}\right)\left(1-T_{2 i}\right)\right]}_{h_{2}\left(T_{1 i}, T_{2 i}\right)} .
\end{gathered}
$$

Since $\mathbb{E}\left(\varepsilon_{i} \mid T_{1 i}, T_{2 i}, \mathbf{x}_{i}\right) \neq 0$, one approach to estimate consistently the population regression (eq. (1-13)) consists in using the control function approach (Heckman 1978, 1979). In other words, we rewrite the regression equation as

$$
y_{i}=\boldsymbol{\beta}^{\prime} \mathbf{x}_{i}+\delta_{1} T_{1 i}+\delta_{2} T_{2 i}+\theta T_{1 i} T_{2 i}+\underbrace{\sigma_{\varepsilon} \rho_{\mu_{1} \varepsilon}}_{\eta_{1}} h_{1}\left(T_{1 i}, T_{2 i}\right)+\underbrace{\sigma_{\varepsilon} \rho_{\mu_{2} \varepsilon}}_{\eta_{2}} h_{2}\left(T_{1 i}, T_{2 i}\right)+\nu_{i},
$$

where $\mathbb{E}\left[\nu_{i} \mid \mathbf{x}_{i}, h_{1}\left(T_{1 i}, T_{2 i}\right), h_{2}\left(T_{1 i}, T_{2 i}\right)\right]=0$ and $\eta_{1}$ and $\eta_{2}$ are additional parameters to be estimated. In theory, the coefficients of equation (1-23) can be consistently estimated using OLS. In practice, one problem occurs in that $h_{1}\left(T_{1 i}, T_{2 i}\right)$ and $h_{2}\left(T_{1 i}, T_{2 i}\right)$ are unobserved as they are functions of the unobserved parameters $\gamma_{1}, \gamma_{2}$ and $\rho_{\mu_{1} \mu_{2}}$, hence the two-step approach:

1. Obtain consistent and efficient (under normality) estimates for $\gamma_{1}, \gamma_{2}$ and $\rho_{\mu_{1} \mu_{2}}$ by estimating a bivariate probit using maximum likelihood. Compute $\widehat{h}_{1}\left(T_{1 i}, T_{2 i}\right)$ and $\widehat{h}_{2}\left(T_{1 i}, T_{2 i}\right)$ by estimating the different $\lambda$ 's given in equations (1-20a)-(1-20h) using $\widehat{\gamma}_{1}, \widehat{\gamma}_{2}$ and $\widehat{\rho}_{\mu_{1} \mu_{2}}$.

2. Use $\widehat{h}_{1}\left(T_{1 i}, T_{2 i}\right)$ and $\widehat{h}_{2}\left(T_{1 i}, T_{2 i}\right)$ as regressors in equation (1-23) alongside $\mathbf{x}_{i}, T_{1 i}$ and $T_{2 i}$ and apply OLS to equation (1-23). Since we use their estimates in lieu of $h_{1}\left(T_{1 i}, T_{2 i}\right)$ and $h_{2}\left(T_{1 i}, T_{2 i}\right)$, the conventional standard errors are not valid and need to be corrected by generalizing the results of Heckman $(1976,1979)$, or by using techniques of simulation or bootstrap. 


\subsubsection{The generalized Roy's model}

Roy (1951) introduced a framework of self-selection that aims to analyze occupational choice with heterogeneous skill. This framework is one of the most important in economics and has been widely applied in many other contexts, including evaluation. The Roy (1951) model has been extended in several directions and many generalizations have been proposed (see e.g. Heckman and Taber, 2008 and Heckman and Vytlacil, 2007a, Chap.70). By generalized Roy's model, we mean here multiple selections in the Roy's framework also known as endogenous switching regression.

Based on our four regimes: $(1,1)$, the pupil receives both treatments; $(1,0)$, the pupil receives only treatment $T_{1} ;(0,1)$, the pupil only receives $T_{2}$; and $(0,0)$, the pupil receives neither treatment. The four corresponding outcomes are defined as: $y_{i 11}, y_{i 10}, y_{i 01}$ and $y_{i 00}$ respectively. The generalized model of Roy consists of equations (1-1)-(1-4) and the following equations:

$$
\begin{aligned}
& y_{i 11}=\boldsymbol{\beta}_{11}^{\prime} \mathbf{x}_{i}+\varepsilon_{i 11}, \\
& y_{i 10}=\boldsymbol{\beta}_{10}^{\prime} \mathbf{x}_{i}+\varepsilon_{i 10}, \\
& y_{i 01}=\boldsymbol{\beta}_{01}^{\prime} \mathbf{x}_{i}+\varepsilon_{i 01}, \\
& y_{i 00}=\boldsymbol{\beta}_{i 00}^{\prime} \mathbf{x}_{i}+\varepsilon_{i 00} .
\end{aligned}
$$

An individual unit cannot be present in two different regimes at the same so that the correlations between the error terms of equations (1-24a)-(1-24d) are not identified and hence set to zero.

\section{Maximum likelihood estimation}

In order to estimate the generalized Roy's model, we maintain the normality assumption. In this case, $\left(\mu_{1 i}, \mu_{2 i}, \varepsilon_{i 11}, \varepsilon_{i 10}, \varepsilon_{i 01}, \varepsilon_{00}\right)^{\prime}$ is normally distributed with vector mean $\mathbf{0}$ and covariance matrix $\boldsymbol{\Omega}$ defined as

$$
\boldsymbol{\Omega}=\left(\begin{array}{ccccccc}
1 & & & & & \\
\rho_{\mu_{1} \mu_{2}} & 1 & & & & \\
\rho_{\mu_{1} \varepsilon_{11}} \sigma_{\varepsilon_{11}} & \rho_{\mu_{2} \varepsilon_{11}} \sigma_{\varepsilon_{11}} & \sigma_{\varepsilon_{11}}^{2} & & & \\
\rho_{\mu_{1} \varepsilon_{10}} \sigma_{\varepsilon_{10}} & \rho_{\mu_{2} \varepsilon_{10}} \sigma_{\varepsilon_{10}} & 0 & \sigma_{\varepsilon_{10}}^{2} & & \\
\rho_{\mu_{1} \varepsilon_{01}} \sigma_{\varepsilon_{01}} & \rho_{\mu_{2} \varepsilon_{01}} \sigma_{\varepsilon_{01}} & 0 & 0 & \sigma_{\varepsilon_{01}}^{2} & \\
\rho_{\mu_{1} \varepsilon_{00}} \sigma_{\varepsilon_{00}} & \rho_{\mu_{2} \varepsilon_{00}} \sigma_{\varepsilon_{00}} & 0 & 0 & 0 & \sigma_{\varepsilon_{00}}^{2}
\end{array}\right)
$$

The likelihood function of the model is given by

$$
\begin{aligned}
& L=\prod_{i=1}^{N}\left[\int_{-\gamma_{1}^{\prime} \mathbf{w}_{1 i}}^{\infty} \int_{-\gamma_{2}^{\prime} \mathbf{w}_{2 i}}^{\infty} f_{3}\left(\mu_{1 i}, \mu_{2 i}, y_{i 11}\right) d \mu_{1 i} d \mu_{2 i}\right]^{T_{1 i} T_{2 i}} \\
& {\left[\int_{-\gamma_{1}^{\prime} \mathbf{w}_{1 i}}^{\infty} \int_{-\infty}^{-\gamma_{2}^{\prime} \mathbf{w}_{2 i}} f_{3}\left(\mu_{1 i}, \mu_{2 i}, y_{i 10}\right) d \mu_{1 i} d \mu_{2 i}\right]^{T_{1 i}\left(1-T_{2 i}\right)} } \\
& {\left[\int_{-\infty}^{-\gamma_{1}^{\prime} \mathbf{w}_{1 i}} \int_{-\gamma_{2}^{\prime} \mathbf{w}_{2 i}}^{\infty} f_{3}\left(\mu_{1 i}, \mu_{2 i}, y_{i 01}\right) d \mu_{1 i} d \mu_{2 i}\right]^{\left(1-T_{1 i}\right) T_{2 i}} } \\
& {\left[\int_{-\infty}^{-\gamma_{1}^{\prime} \mathbf{w}_{1 i}} \int_{-\infty}^{-\gamma_{2}^{\prime} \mathbf{w}_{2 i}} f_{3}\left(\mu_{1 i}, \mu_{2 i}, y_{i 00}\right) d \mu_{1 i} d \mu_{2 i}\right]^{\left(1-T_{1 i}\right)\left(1-T_{2 i}\right)} }
\end{aligned}
$$

where $f_{3}$ still denotes the trivariate normal density function. Using the results in subsection 
3.4.1, the likelihood function can be written as

$$
\begin{aligned}
L=\prod_{i=1}^{N}\left[\Phi_{2}\left(\omega_{11}, \xi_{11} ; \rho_{\mu_{1} \mu_{2} . \varepsilon_{11}}\right) \varphi_{11 i}\right]^{T_{1 i} T_{2 i}}\left[\Phi_{2}\left(\omega_{10},-\xi_{10} ;-\rho_{\mu_{1} \mu_{2} . \varepsilon_{10}}\right) \varphi_{10 i}\right]^{T_{1 i}\left(1-T_{2 i}\right)} \\
\quad\left[\Phi_{2}\left(-\omega_{01}, \xi_{01} ;-\rho_{\mu_{1} \mu_{2} . \varepsilon_{01}}\right) \varphi_{01 i}\right]^{\left(1-T_{1 i}\right) T_{2 i}}\left[\Phi_{2}\left(-\omega_{00},-\xi_{00} ; \rho_{\mu_{1} \mu_{2}, \varepsilon_{00}}\right) \varphi_{00 i}\right]^{\left(1-T_{1 i}\right)\left(1-T_{2 i}\right)}
\end{aligned}
$$

where $\omega_{j k}, \xi_{j k}$ and $\varphi_{j k}(j, k \in\{0,1\})$ are defined as follows

$$
\begin{aligned}
& \omega_{j k} \equiv \frac{\gamma_{1}^{\prime} \mathbf{w}_{1 i}+\frac{\rho_{\mu_{1} \varepsilon_{j k}}}{\sigma_{\varepsilon_{j k}}}\left(y_{i j k}-\boldsymbol{\beta}_{j k}^{\prime} \mathbf{x}_{i}\right)}{\sqrt{1-\rho_{\mu_{1} \varepsilon_{j k}}^{2}}} \\
& \xi_{j k} \equiv \frac{\boldsymbol{\gamma}_{2}^{\prime} \mathbf{w}_{2 i}+\frac{\rho_{\mu_{2} \varepsilon_{j k}}}{\sigma_{\varepsilon_{j k}}}\left(y_{i j k}-\boldsymbol{\beta}_{j k}^{\prime} \mathbf{x}_{i}\right)}{\sqrt{1-\rho_{\mu_{2} \varepsilon_{j k}}^{2}}} \\
& \varphi_{j k} \equiv \frac{1}{\sigma_{\varepsilon_{j k}}} \phi_{1}\left(\frac{y_{i j k}-\boldsymbol{\beta}_{j k}^{\prime} \mathbf{x}_{i}}{\sigma_{\varepsilon_{j k}}}\right)
\end{aligned}
$$

In order to obtain ML estimates of the generalized Roy's model, $\ln L$ can be maximized using standard numerical methods.

\section{Two-step estimation}

In order to estimate the model using the two-step approach, we write the regression of the subpopulation as

$$
\begin{aligned}
& \mathbb{E}\left(y_{i 11} \mid T_{1 i}=1, T_{2 i}=1, \mathbf{x}_{i}\right)=\boldsymbol{\beta}_{11}^{\prime} \mathbf{x}_{i}+E\left(\varepsilon_{i 11} \mid T_{1 i}=1, T_{2 i}=1, \mathbf{x}_{i}\right), \\
& \mathbb{E}\left(y_{i 10} \mid T_{1 i}=1, T_{2 i}=0, \mathbf{x}_{i}\right)=\boldsymbol{\beta}_{10}^{\prime} \mathbf{x}_{i}+E\left(\varepsilon_{i 10} \mid T_{1 i}=1, T_{2 i}=0, \mathbf{x}_{i}\right), \\
& \mathbb{E}\left(y_{i 01} \mid T_{1 i}=0, T_{2 i}=1, \mathbf{x}_{i}\right)=\boldsymbol{\beta}_{01}^{\prime} \mathbf{x}_{i}+E\left(\varepsilon_{i 01} \mid T_{1 i}=0, T_{2 i}=1, \mathbf{x}_{i}\right), \\
& \mathbb{E}\left(y_{i 00} \mid T_{1 i}=0, T_{2 i}=0, \mathbf{x}_{i}\right)=\boldsymbol{\beta}_{00}^{\prime} \mathbf{x}_{i}+E\left(\varepsilon_{i 00} \mid T_{1 i}=0, T_{2 i}=0, \mathbf{x}_{i}\right),
\end{aligned}
$$

where each regression is estimated using data for the corresponding subsamples. Using the results of the conditional expectations calculated in Subsection 3.4.1 in the double-index selection model at the two-step estimation, we can write the regression equations (eqs. (1-24a)-(1-24d)) as

$$
\begin{aligned}
& y_{i 11}=\boldsymbol{\beta}_{11}^{\prime} \mathbf{x}_{i}+\sigma_{\varepsilon_{11}} \rho_{\mu_{1} \varepsilon_{11}} \lambda_{1}^{++}+\sigma_{\varepsilon_{11}} \rho_{\mu_{2} \varepsilon_{11}} \lambda_{2}^{++}+\nu_{i 11}, \\
& y_{i 10}=\boldsymbol{\beta}_{10}^{\prime} \mathbf{x}_{i}+\sigma_{\varepsilon_{10}} \rho_{\mu_{1} \varepsilon_{10}} \lambda_{1}^{+-}-\sigma_{\varepsilon_{10}} \rho_{\mu_{2} \varepsilon_{10}} \lambda_{2}^{+-}+\nu_{i 10}, \\
& y_{i 01}=\boldsymbol{\beta}_{01}^{\prime} \mathbf{x}_{i}-\sigma_{\varepsilon_{01}} \rho_{\mu_{1} \varepsilon_{01} 1} \lambda_{1}^{-+}+\sigma_{\varepsilon_{01}} \rho_{\mu_{2} \varepsilon_{01}} \lambda_{2}^{-+}+\nu_{i 01}, \\
& y_{i 00}=\boldsymbol{\beta}_{00}^{\prime} \mathbf{x}_{i}-\sigma_{\varepsilon_{00}} \rho_{\mu_{1} \varepsilon_{00}} \lambda_{1}^{--}-\sigma_{\varepsilon_{00}} \rho_{\mu_{2} \varepsilon_{00}} \lambda_{2}^{--}+\nu_{i 00},
\end{aligned}
$$

with $\mathbb{E}\left(\nu_{i 11} \mid \mathbf{x}_{i}, \lambda_{1}^{++}, \lambda_{2}^{++}\right)=\cdots=\mathbb{E}\left(\nu_{i 00} \mid \mathbf{x}_{i}, \lambda_{1}^{--}, \lambda_{2}^{--}\right)=0$, and where the expressions of the $\lambda$ 's are given in equations (1-20a)-(1-20h). Equations (1-32a)-(1-32d) can be estimated separately by OLS using the two-step approach described in Subsection 3.4.1 at the two-step estimation, i.e.

1. Obtain consistent and efficient (under normality) estimates for $\gamma_{1}, \gamma_{2}$ and $\rho_{\mu_{1} \mu_{2}}$ by estimating a bivariate probit using maximum likelihood. Compute the $\widehat{\lambda}$ 's, given in equations (1-20a)-(1-20h), by using $\widehat{\gamma}_{1}, \widehat{\gamma}_{2}$ and $\widehat{\rho}_{\mu_{1} \mu_{2}}$.

2. Use the $\widehat{\lambda}$ 's as regressors in equations (1-32a)-(1-32d) alongside $\mathbf{x}_{i}$, and apply OLS to these equations. Since we use the estimates of the $\lambda$ 's, the standard errors of the estimates must be corrected once again by generalizing the results of Heckman $(1976,1979)$, or by using techniques of simulation or bootstrap. 
Several comments are in order about the rationale of the use of DISM versus Roy as well as their economic performance. Both models complement each other. They are different and deliver different estimations and treatment effects. The DISM model uses the selection processes alongside one outcome equation for the full sample. It allows to estimate the Average Treatment Effects (ATE). In addition, with the interaction term $T_{1} T_{2}$, the DISM model enables us to test for complementarity versus substitutability within the programs. The generalized Roy model is a switching regression framework with four regimes. It also uses the two selection equations but allows for different outcome equations (one for each regime). As a result, in addition to the ATE, we can estimate the Average Treatment Effects on the Treated (ATET), the Average Treatment Effects on the Non-Treated (ATET).

\section{Treatment effects}

As outlined earlier, our framework allows us to identify and estimate a wide range of treatment effects depending on the model used. In the double-index selection model we identify a class of average treatment effects. For the generalized Roy model, in addition to the average treatment effects, we provide several heterogenous treatment effects as well.

Treatment effects in the double-index selection model

Relying on the first model, we can identify several average treatment effects as summarized in Table (3.1). Let us consider them in turn.

i) Exclusive effect. Assume the two programmes $T_{1}$ and $T_{2}$. The exclusive effect of $T_{1}$ respectively $T_{2}$ is the marginal effect of $T_{1}$ resp. $T_{2}$ conditional on the fact that agents are not in the alternative programme. Such effects allow to measure the impact $T_{1}$ or $T_{2}$ only on the outcome $y$, given controls $\mathbf{x}$. This leads to the exclusive effects of $T_{1}$ and $T_{2}$.

ii) Global effect. The global effect is the effect of both programmes taken together.

iii) Additional effect. The additional effect is the effect following from having additionally another program. It is given by the difference between the global effect and the exclusive effects. This effect should be distinguished by the global and the sequential effects even if they are closely related. It is different from the global effect because the implementation starts only with one program, either meal or deworming, contrary to the global effect for which both programs are administered together starting from the beginning of the implementation. It is also different from the sequential effect because the order doesn't matter.

iv) Relative effect. Within our framework, we are able to assess the effectiveness of implementing $\left(T_{1}, T_{2}\right)$ vs. $T_{1}$ or $T_{2}$. Thus, we have the effect of package $\left(T_{1}, T_{2}\right)$ vs. $T_{1}$ and the effect of package $\left(T_{1}, T_{2}\right)$ vs. $T_{2}$. We can also stand for the effect of a programme vs. the effect of the other programme. For example, in the case of exclusive effects, taking the difference yields also a relative effect. 


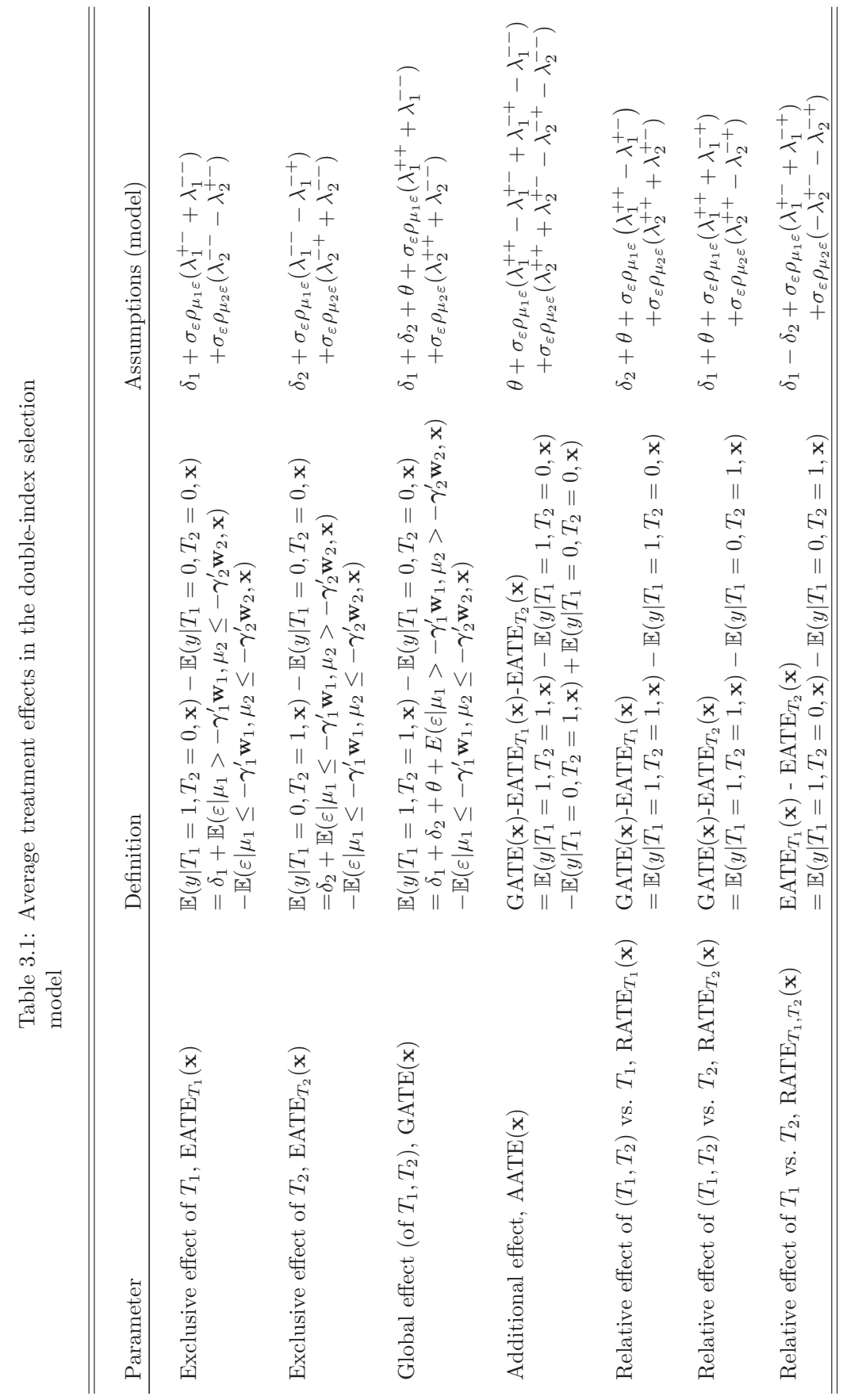


Treatment effects in the generalized Roy model

The Roy model allows to compute the average treatment effects as in the case of the doubleindex endogenous selection model, but also to derive several heterogenous average treatment effects: the effects on the treated and on the untreated. For each, we also compute the associated exclusive, global, additional and relative effects. In addition, some new effects can be identified viz, sequential and substitution effects. Both effects are relevant for policy analysis.

i) Sequential effect. This effect is of particular interest. As part our assessment, deworming and school feeding programs are complementary in nature. Depending on which treatment is firstly implemented, the overall effect will differ. This means that we will not have the same effect if a pupil got school feeding treatment before deworming, and vice versa. Of course the ideal case is to deworm first, hence the rationale of the sequence. Let us denote $T_{1}$ the deworming treatment and $T_{2}$ the canteen treatment. It is expected that the sequence $T_{1} T_{2}$ be more efficient than $T_{2} T_{1}$.

ii) Substitution effect. By substitution effect, we mean replacing one program with another. For example, what would happen if after having started a program, it is stopped and replaced by another. In this, substitution effect is different from the sequential effect. The substitution effect is particularly interesting when the substitution is made with programs targeting the same goal. In the case of the deworming and canteen programs the question arises whether the substitution makes sense. The answer is yes. On one hand, the two programs can have the same goal: improve pupils' performance. On the other hand, if during the implementation of programs, a program appears to be more expensive than anticipated compared to the other, and in case of lack of resources, the organizers may face such choices. In this case, it seems clear that the cheapest program will be substituted for the more expensive one. Experience has shown that the deworming program is cheaper than the canteen because the resources mobilized are cheaper. This fact is supported by cost-effectiveness analysis (see Miguel and Kremer, 2004).

In our data, although both treatments (deworming and canteen) have not been implemented sequentially or not has been substituted, our sampling allows us to identify and estimate these effects. Thus we are able to provide decision makers with powerful policy analysis tools. These effects are summarized in Table (3.2). 


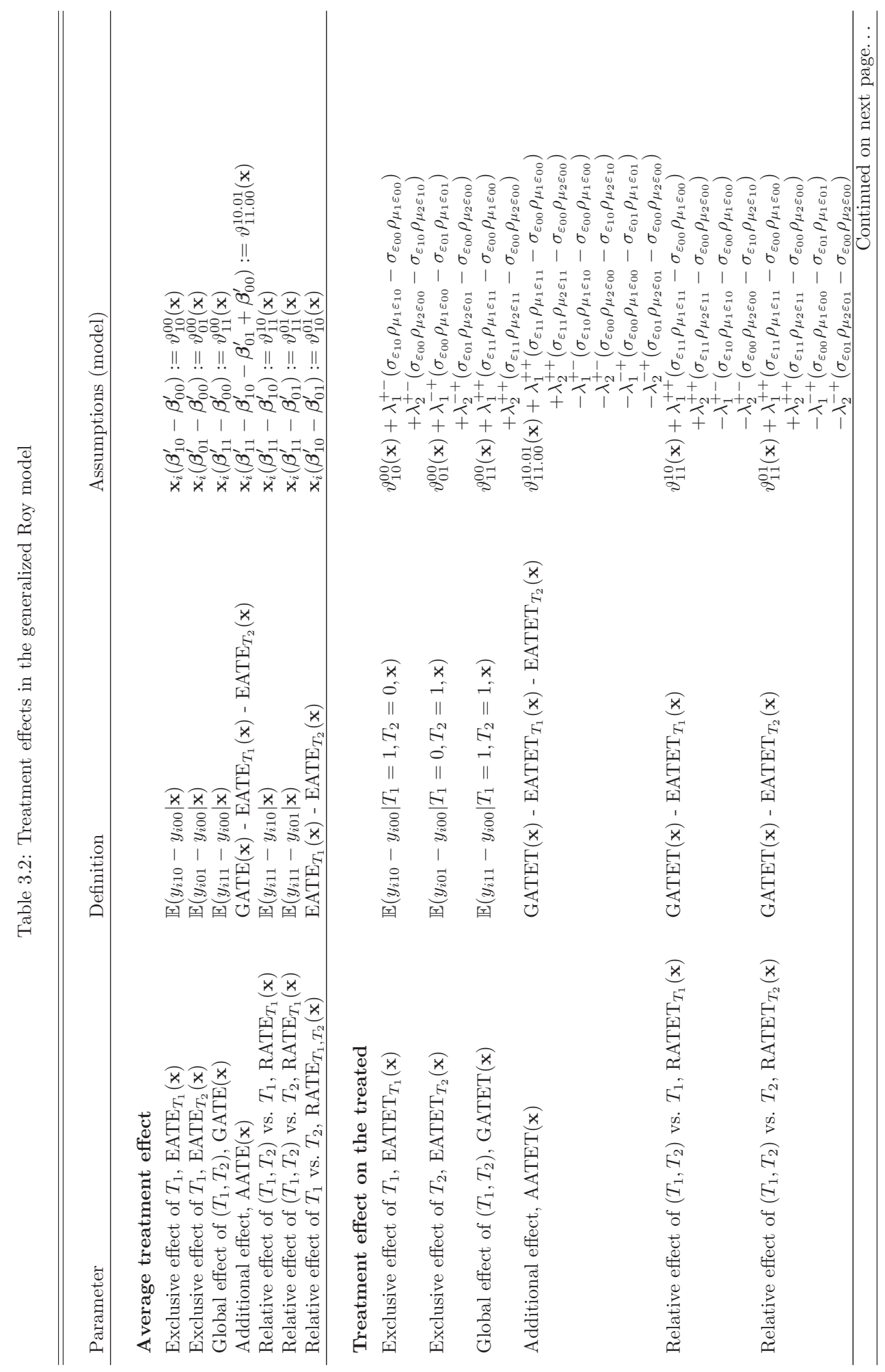



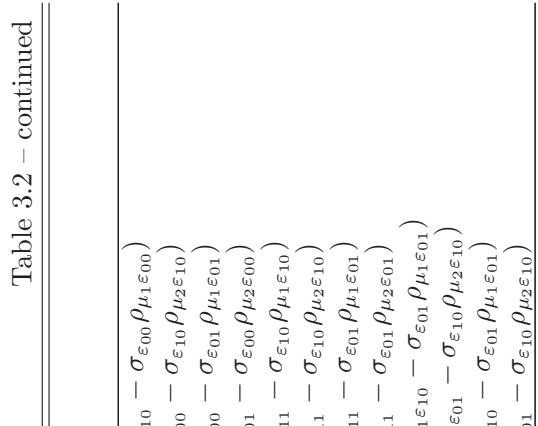

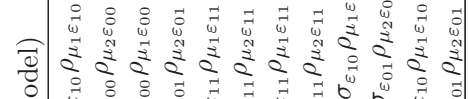
过

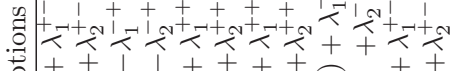

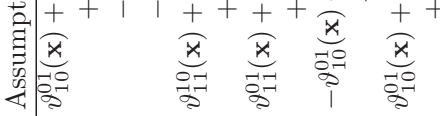

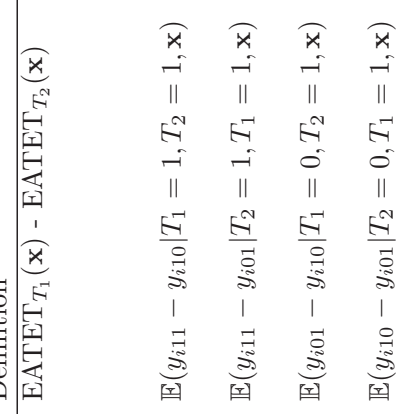

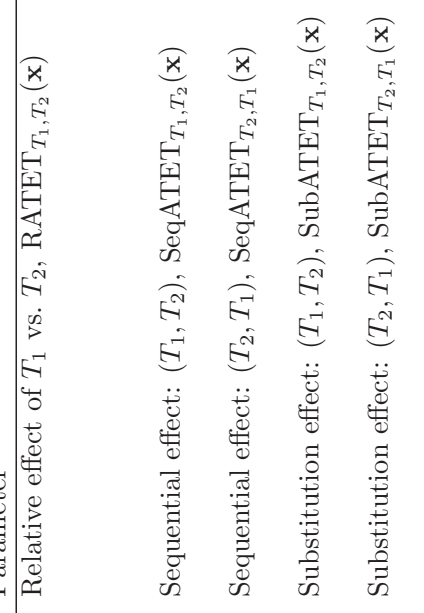
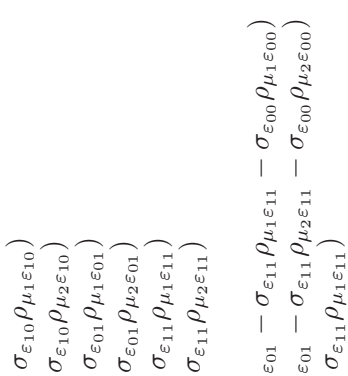

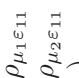

ำ

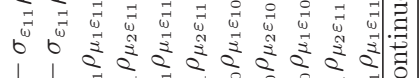

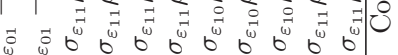

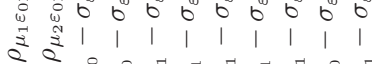

$\begin{array}{lll}1 & 1 & 1\end{array}$

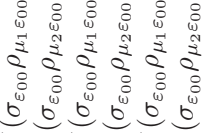

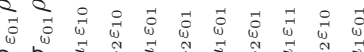

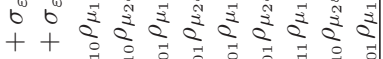

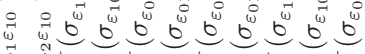

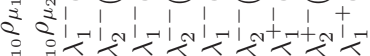

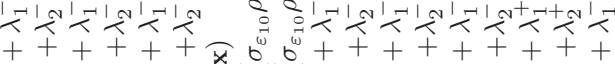

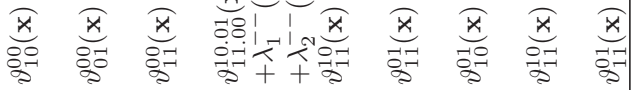
矛

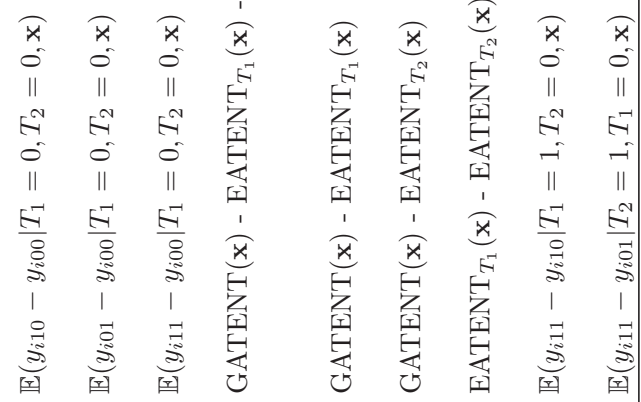

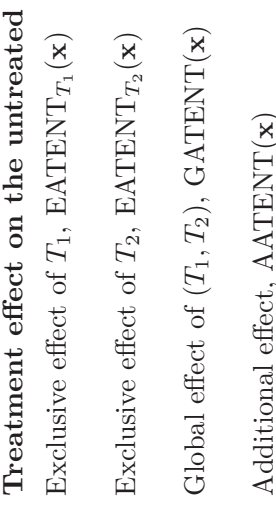

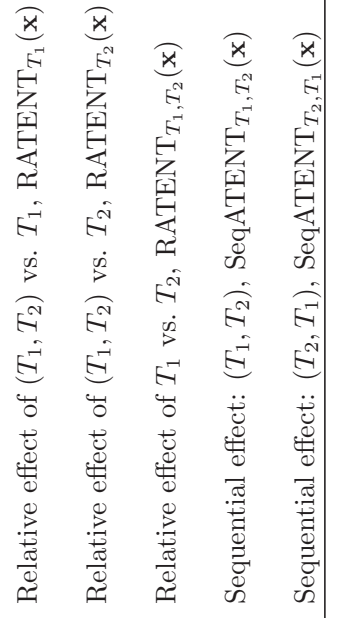




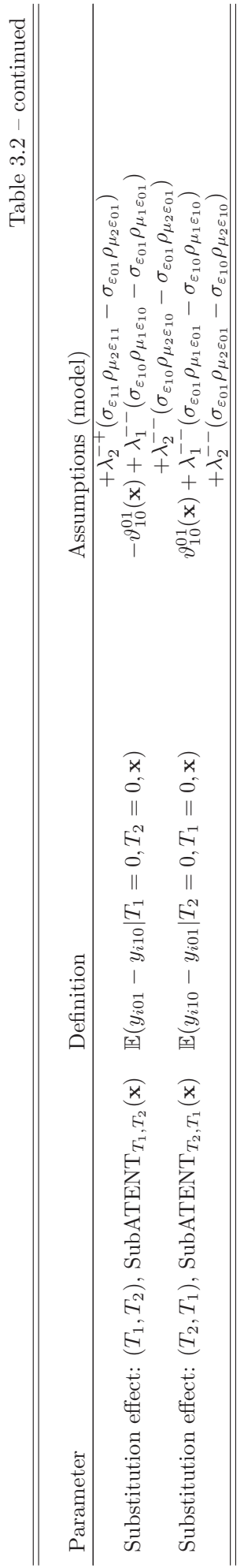




\subsection{Findings}

Remember that from the estimation perspective, this study focuses on two things: the study of the treatment effects from the package of deworming and school meal program alongside the determinants of the academic performance of pupils. Both objectives have motivated our modeling strategy in the previous section. We have estimated the two models presented in the previous section: DISM (Eqs. (1-1), (1-2) and (1-5)) and the generalized Roy (Eqs. (1-1)), (1-4 and 1-24a-1-24d). ${ }^{11}$ Recall that these two specifications are different and they also delivered different treatment effects. The DISM model used the selection processes alongside an outcome equation for the full sample. It can be used to estimate the Average Treatment Effects (ATE), meaning the effect of treatment on the population. The generalized Roy's model is a switching regression framework with four regimes. It also uses the two selection equations but allows for different outcome equations (one for each regime). As a result, we can estimate not only the ATE, but also the Average Treatment Effect on the Treated (ATET) and the treatment effect on the untreated (ATENT). In both cases (DISM and Roy), the double selection given by relations (1-1) and (1-1) is endogenous. The estimation results are provided in Tables B.2, B.3 and B.4 for the score outcomes (aggregate, French and math) and Tables B.6 and B.5 for the rate outcomes (enrollment, promotion and dropout) in the appendix.

\subsubsection{Determinants of school performances: Selections and outcomes}

The bivariate selection equations (1-1 and 1-2 describe the participation mechanisms in the two programs (deworming and school meal). We used the criteria (see Chapter 2 Section 2.3 for criteria) set by World Food Program and the Ministry of National Education of Senegal to motivate the choice of the control variables for the selection equations. Among these criteria, some were recorded by the survey such as be located in an area with food insecurity, or be particularly affected by rising prices. The variables that we use are the existence of a management committee in the school, warehouse, school cooperative, association of pupils' mothers, grant from the Rural Council, distance to home, the total number of pupils in the school, disturbances that delayed the starting of the courses, the gender of pupils and medical box. These factors of selection are the same regardless of the outcome. This implies that we have the same selection equations for each outcome (aggregate score, French score, math score, enrollment rate, promotion rate and dropout rate).

For deworming program, the results show that the total number of pupils, the existence of a management committee of the school, association of pupils' mothers, school cooperative, existence of water point in the school and the gender of pupils have a positive effect on the probability of benefiting from the program. Furthermore, the existence of school infrastructure and various associations in schools promote the supply of food and health programs. The existence of a medicine box in the school reduced the likelihood of benefiting from the deworming program. This suggests that pupils in schools with first aid box are less affected by parasitic worms so that the concerned schools are less likely to get into the deworming program. This may be because schools with medicine box are likely to have already an established medical facility. Such schools would be less need for medical care including deworming.

On the school meals program, the results show that the total number of pupils, the distance between pupils' home and school, the existence association of pupils' mothers, a grant from the rural council, a warehouse, disturbances that have caused delays in starting the academic year act positively on the probability of receiving a meal program at school. On the contrary, the existence of school cooperative reduces the probability of receiving school meal program. Indeed, the school cooperative plays several roles (cleanliness of the school, gardening activities, etc.). In reality, in some schools, the school cooperative is not operational. In other schools, the school

\footnotetext{
${ }^{11}$ All the computations are performed with STATA. Despite the complexity of the procedures, the codes are optimized to run fast.
} 
cooperative plays its role fully and even goes further to organize activities to enable children to enjoy their lunch.

The variable distance to school (distance between school and the pupils' home) is also one of the selection criteria listed by the government for establishing canteens. In our sample, most schools have benefited from the so-called canteens 'price increase'. These were established to respond to the rising prices of food staples. This could explain the fact that we found a positive effect of the distance control on the probability of benefitting from the meal program.

\section{Outcomes}

\section{Scores}

It is usually accepted that pupils in schools that are well equipped with 'appropriate' textbooks are likely to perform better. ${ }^{12}$ Our results show a nonlinear relationship between the number of pupils per manual and test scores. The coefficient of the linear term of the control 'Manual' is positive and significant (for all score outcomes) while its square is negative (for the aggregate and French scores). In other words, scores increase with the number of pupils who share a textbook up to a certain threshold from which the scores drop. This threshold is 4 pupils per textbook for the French score. For the aggregate score, the square term is negative but not significant and the turning point is 7, which corresponds to the maximum of pupils per textbook. Observe that the decreasing part of the curve is out of sample. This result could be explained by the fact that the learning time decreases when the number of pupils by textbook increases. This result corroborates the findings of Michaelowa (2006). According to CONFEMEN (1999), French and mathematics textbooks have a positive impact on learning, with a larger effect for the French textbooks.

Another interesting control variable is class size. Previous studies and experiments that have evaluated the effects of reducing class size on pupils' achievement do not reach a consensus. While some studies show that pupils in reduced size classes have better academic results (Piketty, 2004), others show that the reduction of class size does not necessarily lead pupils to perform better (Davies, 2003). Our results show a negative but not significant effect of class size on scores (see Tables B.2, B.3 in the appendix). Estimates based on the Roy's model (see Table B.4 in the appendix) sometimes show a positive relation, sometimes a negative one depending on the regime. For pupils who benefit from deworming, we find a negative and significant relation between class size and the aggregate and French scores. For those who benefited from the meal program or the package (deworming and meal), the results show a positive and significant relation between class size and test scores. For the untreated group, the effect of class size on French score is negative and significant. Along the lines of Altinok (2006), we could argue that class size does not affect pupils' outcomes in schools with canteens or for those in schools where both program are implemented. However, in schools without meal program or with only the deworming program, we find that class size does impact negatively the score in French. This is consistent with Brossard (2003). It is worthwhile to notice that in the Senegalese context, there is a decline of the French language compared to national languages in particular the 'Wolof' language (ANSD, 2006). ${ }^{13}$

We obtain a nonlinear relationship between the age of teacher and scores. The linear term is negative while the square is positive drawing a $U$ shaped relation. As a result, the age of the teacher has a negative effect on pupils' performance up to a threshold from which the relation becomes positive. This threshold is 32 years for the aggregate score, 34 years for the French

\footnotetext{
${ }^{12} \mathrm{~A}$ well known result in the literature is that of Glewwe et al. (2009). This study evaluates a program of textbook delivered to pupils in Kenya. The authors found that a textbook program has a negative impact on pupils' achievement. The problem was that the textbooks were in English. However, English was not the language commonly used by Kenyan pupils at this level of education.

${ }^{13}$ Wolof is the most widely spoken language in Senegal (by the Wolof ethnic group which is about $45 \%$ of the total population, as well as non-Wolof people). This language, which is also spoken in Gambia and Mauritania, is experiencing a cultural expansion.
} 
score and 31 years for math score. In our sample, the age of the teachers varied between 20 and 53 years with an average age of 31 for the whole sample. Thus the age of teacher impacts positively on scores when it reaches the average age of 31 years. This finding regarding the age of the teacher probably reflects an experience effect. Indeed, if we assume that mastering a classroom is positively related to teachers' experience, we can expect a positive effect of the age of the teacher on pupil's performance (Schwille et al. 1991). It is important to remember that in the literature, there is no consensus on the relation between the age of the teacher and pupils' achievement. Indeed, the age of the teacher can interact with both experience and education. This means that teachers of the same age but with different levels of education and different experience will not necessarily have the same effects on pupil's achievement.

The age of pupils is another potential determinant of their performance. The results obtained from the DISM model (see Tables B.2, B.3 in the appendix) show a positive and significant effect of pupil's age on scores. As documented by Schwille et al. (1991), this relation may be explained by the maturity of older pupils compared to younger pupils. According to UNESCO (1987), the normal age of pupils in primary school is between 6 and 11 years. In our sample, the age of pupils varies between 6 and 15 years. Therefore, there is a gap between the normal age group and the current age of pupils. This may mean that either pupils entered school late, or they have failed several times. As mentioned in the description of variables, if we consider the case of late entry, a positive relation can be observed (Schwille et al. 1991). In the case of repeated repetition, one generally expects a negative relation between age and pupils' performance. High rate of repetition thus indicative a low academic performance of pupils. The estimates provided by the model of Roy (see Table B.4 in the appendix) show a negative relation between the age of pupils and the aggregate and French scores for the group of pupils who benefit from the package. This finding could follow from the fact that this group of students is the youngest on average, meaning the less mature compared to pupils in other groups (see descriptive statistics in Table B.1: the average age is about 9.3 against 9.5, 9.7 and 9.9 respectively for pupils in the deworming, canteen and untreated groups).

Another interesting evidence is the positive relation between education spending and the aggregate and French scores. This result is not surprising because the more parents invest in the education of their kids the higher achievement we could expect. The estimates also show that the 'disturbed courses' control, gender of the teacher, level of study or class of student, absenteeism of teachers, deworming at home and living in a community where parents are not interested in school have a negative effect on pupil scores. Undoubtedly, disturbance and teacher absenteeism decreased the learning time of pupils. According to UNESCO (2005), teacher absenteeism affects much of the time devoted to learning and hence learning outcomes. The result regarding the gender of teacher is consistent with the finding of Jarousse and Mingat (1989). The authors found similar results relying on data from Togo, meaning that pupils with female teachers perform better than those with male teachers. For deworming at home, the relation found could be explained by the nature of the drug used for deworming children. Rural households use either traditional or modern deworming drugs. As mentioned in section 3.2, several cases of side effects in terms of fatigue or trigger diarrhea have been reported. Also, the use of traditional drugs is not without consequences. This could lead to a decline in pupils' performance.

The control variables like Koranic school, early childhood institution, existence of latrine, existence of hand washing device, existence of a college in the village where the school of pupils is located have a positive effect on the scores. These results are expected. For example, the effect of college in the village where the school of pupils is operating can be viewed as indirect effect of more advanced sisters or brothers of pupils in these villages. They could act as mentor to younger ones who are in primary school. Unfortunately, we have no direct information on the fact that a pupil would have a brother or sister in a college. One can also argue that having a college in the village will motivate pupils. The results concerning modern preschool (also known as early childhood institution) and informal school (here Koranic school) are fairly well known 
in the literature. The Koranic school usually found in Muslim countries, is an informal private educational structure that provides religious education based on memorization. The Koranic school is known for developing the capacity of learning and memory because children learn by heart very early.

On the qualification of teachers, the results show that pupils taught by a teacher with the professional qualification CEAP perform better than those supervised by a teacher with another professional qualification (the reference). ${ }^{14} \mathrm{~A}$ surprising result is that pupils taught by teachers without professional qualification perform better than those supervised by teachers with professional degree. In the case of Senegal, CONFEMEN (2007) has reported no evidence or negative correlation between continuing training and pupils' achievement. Especially in rural areas, this can be explained by two factors. Firstly, in practice, it may be that those teachers in the category without professional degree are awaiting graduation because most often they have already passed the written examination. Secondly, they are teachers whose motivation is higher than those with professional degrees. They can be inspected at any time. This control is crucial for their career. The performance of pupils under their care is reflected in the state of their job. As a result, it is likely that this motivation is a push factor which leads them to supervise pupils very well. This may also explain why pupils supervised by these teachers are more effective.

Regarding the academic degree of teachers, the results from the DISM model show that pupils supervised by a teacher with the High School Diploma perform better in mathematics than those supervised by a teacher with the national certificate. As pointed out by Rivers and Sanders (2002), this result suggests that teachers with High School Diploma and more have a higher level of knowledge in mathematics than those with the certificate. When we use the Roy's model, we get a positive relation between the scores and the proportion of teachers with High School Diploma for the group of pupils who got the deworming program. On the contrary, the effect is negative for pupils who received the meal program only or the package. This finding is consistent with the results of CONFEMEN (1999). The results also show that pupils taught by teachers who have received continuing training are less successful in mathematics than those taught by teachers who have not received such training. This result is surprising but could be explained by the length of the continuing training: as pointed out by UNESCO (2000), the impact of the training on pupils' achievement becomes positive only if the training covers several periods, otherwise one can observe a negative effect.

On the geographical area, the results show that pupils living in Diourbel perform better than Fatick (reference) unlike those living in Kolda and Sédhiou that record weak performance. As we have already stated in the data section, Kolda and Sédhiou are isolated southern regions with very weak risk management capacity and who practice mainly livestock. Fatick and Diourbel are located in the center with a good ability to manage risk. These two regions are predominantly involved in agriculture. ${ }^{15}$ In the southern regions, the livestock system is traditional and extensive. The combination of heavy rainfall, dense water system and the availability of crop residues (e.g. rice straw, stalks of millet and corn, peanut vines, etc.) after the rainy season promote the development of a rich and varied pasture capable of maintaining the herd throughout the year (ANSD, 2010). However, the frequency of bushfires at the end of the dry season (by end of May, June and July) leads to food deficits. It is worth noticing that this period also corresponds to the exams period for pupils. This means that at the end of the school year, farmers are concerned by the search of food for livestock. As some pupils are requested by their family to help with the economic activities, which increases their absenteeism and impact their performances negatively.

The coefficient of the treatment dummy $T_{1}$ for the deworming program is negative and sig-

\footnotetext{
${ }^{14}$ It is worth to remember that the most two important professional degrees in Senegal are the CAP (Certificat d'Aptitude Pédagogique, meaning 'Pedagogical Aptitude Certificate') and CEAP (Certificat Elémentaire d'Aptitude Pédagogique, which is 'Basic Pedagogical Aptitude Certificate').

${ }^{15}$ In our sample, the number of head of cattle by household varied between 0 and 500 in the region of Kolda, 0 to 502 in Sédhiou, 0 to 111 in Fatick and 0 to 48 in Diourbel. As for agriculture, we have the following proportions of arable land hold in each region: $97 \%$ in Diourbel, $92 \%$ in Fatick, $96 \%$ in Kolda and 87\% in Sédhiou.
} 
nificant where as the dummy of the meal program is of the opposite sign for all scores except for scores in math for which $T_{2}$ is not significant. Note that these effects are not the treatment effects of the programs as the estimated partial correlation coefficients are also significant. Consequently, the treatment effects will be computed as derived in section 3.4.2. As outlined earlier, an interesting aspect of our specification (DISM) is that it offers the opportunity to investigate whether deworming and meal programs are complementary or substitutes. This is given by the sign of the interaction term $T_{1} T_{2}$. The coefficient is positive and significant for the aggregate and French scores meaning that in the perspective of improving pupils' academic achievement, the two programs are complementary.

Enrollment, promotion and dropout

The estimation results for enrollment, promotion and dropout rates are reported in Tables B.6 and B.5 in the appendix.

Enrollment. The control variables temporary shelters, association of pupils' parents, age of teacher, Koranic school, snack, pupils who eat their fill and literacy of the household head have a positive effect. Particularly we find a nonlinear $U$ shape relation between the age of teacher and the enrollment rate with a threshold at 32 years from which the age of teacher has a positive effect. On the contrary, determinants like class size, health expenditure, distance between pupils' home and their school, disturbances that delays the starting of courses, gender of the teacher, holding a farmland and school expenses have negative effects. The coefficients of these variables are expected sign except for the variable distance from pupils' home to their school for which we do not anticipate a particular sign: the result shows that living less than one kilometer from the school has a negative effect.

Another interesting and intuitive result is that having a literate household head increases the enrollment rate. However, the enrollment rate declines with school expenses. This result is important for education policy in terms of reducing the illiteracy rate in rural areas where the living standard is low. Ideally, if policy makers want to encourage families to send their children to school, they must ensure that the cost of schooling is not too high. Two other results are worth noting: having a teacher male and holding arable land have negative effects. In the first case, in rural areas, the enrollment of girls is not always well received. The promotion of female teachers may be desirable and may help in that respect. In the second case, arable land increases the practice of child labor, which would in turn reduce enrollment. The geographical location or regions also influences the rate of enrollment. Contrary to Kolda and Sédhiou that have a negative effect on the enrollment rate compared to Fatick (reference), Diourbel has a positive effect. The meal program $T_{2}$ has a positive and significant effect.

Relying on the Roy's model, for pupils who participate in the deworming program and those receiving the package, we obtain that the number of classes in temporary shelters have a negative effect. Instead, for the group of pupils in the meal program and those in the untreated group, the effect is positive. For the group receiving the meal program, we have a negative relation between enrollment and the proportion of pupils who have experienced early childhood institution and those who bring a snack at school. Indeed, as the pupils in our sample are at second and fourth year class, if there are no children of school age in the family and even if the pupils had experienced an early childhood institution or bring food to school, it is possible to find a negative relation between these variables and the enrollment rate.

Promotion. The variables temporary shelters, age of teacher, age of pupil, distance to school, association of pupils' parents, gender of the teacher, continuing training, Koranic school, early childhood institution, pupils who eat their fill and ownership of arable land have a positive effect. As in the case of enrollment rate, there is a $\mathrm{U}$ shape nonlinear relation between the age of the teacher and the promotion rate with a turning point at 38 years. However, health expenditure, number of head of livestock owned, disturbance that have caused delay in courses and absenteeism have a negative effect. Note that holding an arable land has a positive effect, while the number of head of livestock affects promotion negatively. As we have already mentioned, this 
result may be related to the economic characteristics of the study areas. On the one hand, Kolda and Sédhiou have low capacity to manage risk and these two regions are those where households have the greatest number of heads of cattle. On the other hand, Diourbel and Fatick are more agriculture oriented. In addition, the practice of livestock based on a system of transhumance in which farmers often move with their families is not beneficial to children who go to school. On the geographical locations, the results show a positive effect of Diourbel and Sédhiou compared to the Fatick region. This effect is negative for Kolda region.

The deworming program has a positive effect while the meal program has a negative and significant effect. The sign of the coefficient of the interaction term $T_{1} T_{2}$ reveals that the two programs are complementary. The estimates derived from the Roy's model support the previous findings except for the control variable eat at fill in the group of meal program.

Dropout. The variable temporary shelters, class size, age of pupil, distance to school, association of pupils' parents, gender of teacher, continuing training, Koranic school, early childhood institution, pupils who eat their fill, ownership of arable land and the existence of a college in the village have a negative effect. Remember that (in the case of the dropout rate), a negative sign on a coefficient is a positive result as we seek to reduce the dropout rate. Instead, the controls health spending, number of head of cattle, disturbances that caused delay in starting the courses, the gender and the literacy of household head have a positive effect on the dropout rate. The coefficient of the variable arable land is negative while the number of head of cattle is positive. This means that children in households that keep livestock are more likely to drop, while the possession of arable land reduces the dropout rate. This result is very interesting in two respects. On the one hand, as we have already widely documented, farmers are mainly located in the regions of Kolda and Sédhiou which are landlocked with low risk management capacity. These farmers often faced problems of feeding livestock at the end of each school year. On the other hand, we can link this result with that obtained from the scores outcome so that the two results complement each other. Indeed, we obtain a negative effect of the variable number of head of cattle on the scores while the effect on the dropout rate is positive. As for regions, the results show that there is a negative relation between the dropout rate and Diourbel and Sédhiou compared to Fatick, unlike the Kolda region where the effect on the dropout rate is positive. Deworming and meal programs have a negative and a positive effect on the dropout rate respectively. The coefficient on the interaction term $T_{1} T_{2}$ shows that both programs are substitutes with the aim of reducing dropouts.

The estimates obtained from the Roy's specification pointed to the same direction as those observed from the DISM model except for temporary shelters, class size and the age of pupils for the deworming regime. With regard to the gender of pupil, the estimation results for the deworming and package regimes show a negative effect of male pupils on dropout rate.

\subsubsection{Effects of meal and deworming on school performances}

As we have described in Section 3.4.2 and summarized in Tables 3.1 and 3.2 the package of deworming and meal programs and the data sampling allow us to estimate a wide range of treatment effects. Depending on the specification, we have computed these effects for the entire population: Average Treatment Effect (ATE) and for subpopulations of treated (ATET) and untreated (ATENT). For each, we distinguish the exclusive, global, additional and relative effects in the case of the DISM model. In addition, we include sequential and substitution effects in the case of the Roy model. The results are presented in Tables 3.3, 3.4 and 3.5 for the score outcomes, and in Tables 3.6 and 3.7 for the enrollment, promotion and dropout rates.

Scores

Exclusive effects. Remember that the exclusive effects are the effects of each program separately. These effects are positive and significant. Thus, having only the deworming program has a positive and significant effect on pupils' achievement (aggregate, French and math). The exclusive effect of meal is also positive and significant. In other words, each program taken separately has 
Table 3.3: Average Treatment Effects for scores: DISM $^{a}$

\begin{tabular}{|c|c|c|c|c|c|c|}
\hline & \multicolumn{3}{|c|}{ FIML $^{b}$} & \multicolumn{3}{|c|}{ Two step } \\
\hline & Aggregate & French & Math & Aggregate & French & Math \\
\hline Excusif: deworming & $3.434^{* * *}$ & $0.055^{*}$ & $6.264^{* * *}$ & $3.700^{* * *}$ & $0.302^{* * *}$ & $6.866^{* * *}$ \\
\hline Excusif: meal & $7.197^{* * *}$ & $4.103^{* * *}$ & $10.158^{* * *}$ & $6.560^{* * *}$ & $3.779^{* * *}$ & $9.329^{* * *}$ \\
\hline Global & $15.097^{* * *}$ & $10.674^{* * *}$ & $19.578^{* * *}$ & $14.099^{* * *}$ & $9.997^{* * *}$ & $18.353^{* * *}$ \\
\hline Additionnal & $4.465^{* * *}$ & $6.515^{* * *}$ & $3.155^{* * *}$ & $3.838^{* * *}$ & $5.915^{* * *}$ & $2.157^{* * *}$ \\
\hline Relative: package vs. deworming & $11.663^{* * *}$ & $10.618^{* * *}$ & $13.313^{* * *}$ & $10.399^{* * *}$ & $9.694^{* * *}$ & $11.486^{* * *}$ \\
\hline Relative: package vs. meal & $7.900^{* * *}$ & $6.571^{* * *}$ & $9.420^{* * *}$ & $7.538^{* * *}$ & $6.218^{* * *}$ & $8.023^{* * *}$ \\
\hline Relative: deworming vs. meal & $-3.763^{* * *}$ & $-4.047^{* * *}$ & $-3.893^{* * *}$ & $-2.860^{* * *}$ & $-3.476^{* * *}$ & $-2.462^{* * *}$ \\
\hline
\end{tabular}

Notes ${ }^{a}$ : Double-Index Selection Model; ${ }^{b}$ : Full Information Maximum Likelihood.

Significance levels (Bootstrap -100 replications-): $\quad *: 10 \% \quad * *: 5 \% \quad * * *: 1 \%$

a positive and significant effect on pupils' achievement. It is important to note that the effect of the meal program is more pronounced than the deworming. The generalized Roy model provides exclusive effects that point to the same direction as those obtained from the DISM specification. Global effects. The global or overall effect is the effect of the package, i.e, the combination of deworming and meal. The global effects are positive and significant for all scores. We observe that the global effects are larger than the exclusive ones. This implies that pupils who get both deworming and meal experienced greater improvement in their academic performance compared to pupils who receive only one of the two programs. This result is very interesting and is consistent with our expectations. As we have mentioned earlier from the estimation results, the two programs are complementary. This means that the global effect is expected to be higher than the sum of exclusive effects. The global effects computed from the generalized Roy model tell the same story as those obtained with the DISM.

Additional effects. This effect is the additional effect of having a program in addition to an other. These effects are positive and significant both from the DISM and the Roy framework. This means that if pupils have already enjoyed the meal (or deworming), then taking deworming (or meal) in addition improves their scores. The sign of this effect can be seen as a corollary of the complementarity of the two programs. However, the additional effects are lower than the global effects. Remember that the additional effect is different from the global and the sequential effects as we have already documented in Section 3.4.2.

Relative effects. Relative effects seek to compare pairwise programs, including the fact of having the package vs. a single program. We observe that the relative effect of the package vs. deworming is positive and significant. The same holds for package vs. meal. It is worth to note that the relative effect of the package vs. deworming is larger than package vs. meal. This is not surprising as the exclusive effect of the meal is higher than that of deworming. We also observe that the relative effect of deworming vs. meal is negative and significant for all scores. This result makes sense because the exclusive effect of meal is larger than the exclusive effect of deworming. The relative effects calculated from the generalized Roy model tell the same story. 


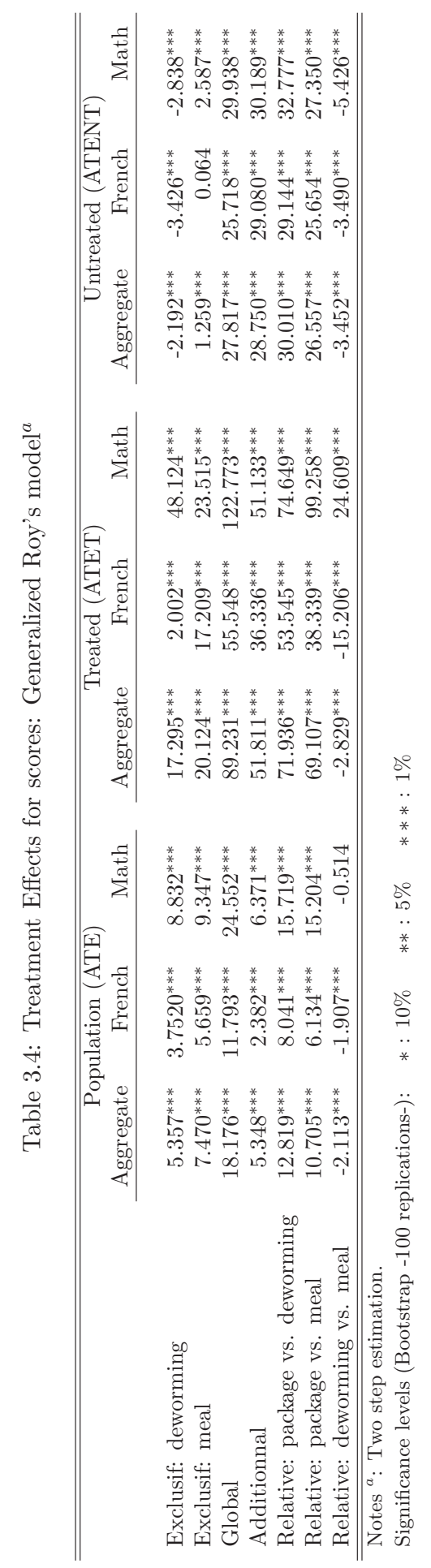


In addition to the average treatment effects (ATE) discussed above, the generalized Roy model allows us to estimate the effects on the treated (ATET) and on the untreated (ATET). As in the case of ATE, here we also have exclusive, global, and additional effects.

Average treatment effects on the treated (ATET). Except for the French score, we observe that the exclusive effects on the treated are larger than their analogue ATE. The global effects of both programs are greater than the exclusive effects and greater than the sum of the exclusive effects. Observe that for the math score, the exclusive effects of deworming are larger than the effects of the meal program. This explains why we have a positive and significant relative effect of deworming vs. meal.

Average treatment effect on the untreated (ATENT). Regarding the effect on the untreated, i.e, the group of pupils who received neither deworming nor meal, the purpose is to inform policy makers on the rationale for extending the programs to a wider population. Such extension could result in significant costs of implementation. The results show that the exclusive effects of deworming are negative while those of the meal program are positive. It is important to note that the combination of the two programs greatly improves pupils' achievement. We also obtain positive additional effects. The relative effects of deworming vs. meal are negative because the exclusive effects of meal are larger than those of deworming. So for untreated pupils, it is recommended to combine the two programs to increase scores.

Table 3.5: Substitution and Sequential Effects for scores: Generalized Roy's model ${ }^{a}$

\begin{tabular}{|c|c|c|c|c|c|c|}
\hline & \multicolumn{3}{|c|}{ Treated (ATET) } & \multicolumn{3}{|c|}{ Untreated (ATENT) } \\
\hline & Aggregate & French & Math & Aggregate & French & Math \\
\hline Sequential: $\left(T_{1}, T_{2}\right)$ & $54.999^{* * *}$ & $33.247^{* * *}$ & $55.144^{* * *}$ & $144.436^{* * *}$ & $130.512^{* * *}$ & $142.742^{* * *}$ \\
\hline Sequential: $\left(T_{2}, T_{1}\right)$ & $68.244^{* * *}$ & $38.043^{* * *}$ & $99.576^{* * *}$ & $-63.891^{* * *}$ & $-67.825^{* * *}$ & $-60.469^{* * *}$ \\
\hline Substitution: $\left(T_{1}, T_{2}\right)$ & $5.104^{* * *}$ & $0.178^{* * *}$ & $6.254^{* * *}$ & $3.452^{* * *}$ & $3.490^{* * *}$ & $5.426^{* * *}$ \\
\hline Substitution: $\left(T_{2}, T_{1}\right)$ & $15.066^{* * *}$ & $1.550^{* * *}$ & $45.748^{* * *}$ & $-3.452^{* * *}$ & $-3.490^{* * *}$ & $-5.426^{* * *}$ \\
\hline
\end{tabular}

Sequential effects. The sequential effect is of particular interest as it accounts for the order in which the programs are implemented. We expect the effect to differ depending on whether pupils got school feeding first and deworming next or vice versa. From a nutritional perspective for example, the ideal order would be to deworm first, hence the rationale of the sequence. Unfortunately, we do not have a nutritional outcome indicator for which it may be expected that the sequence $T_{1} T_{2}$ might be more efficient than $T_{2} T_{1}$. For the group of treated pupils, there are positive sequential effects. However, the sequence $T_{2} T_{1}$ proves to be more effective. Thus, it seems more advantageous to introduce the meal program before deworming. As we have discussed in Section 3.2, this result could be related to the negative perception that some families have towards deworming as consequences of the sides effects of drug used. However, for pupils in the untreated group, it is better to deworming first. For this group, introducing meal program before deworming significantly lowers pupils' achievement.

Substitution effects. For pupils in the treated group, we observe a very interesting result. Indeed, introducing the canteen until a time and replacing it with a deworming program is more beneficial in terms of improving pupils' academic achievement than the reverse. This is important for policy analysis in particular for policy makers and NGOs who fund these sort of programs. Indeed, we know that deworming program is far less expensive than the meal program. We will come back to this aspect in the next section for further details through the cost-effectiveness analysis. What this result says is that, if for one reason or another, we have to substitute a program for another (for example funding limitations), then it is more interesting to replace the meal 
program by the deworming if we seek to improve pupils' academic achievement. This result is consistent with the sequential effect.

For the untreated group, deworming pupils first before replacing the program by meal would positively act on scores. However, unlike pupils in the treated group, it is not beneficial to introduce meal program, and then replace it with deworming. Again, this is consistent with the sequential effect results. Overall, the combination of the two programs is more beneficial in terms of increasing pupils' academic achievement.

Enrollment, promotion and dropout

Enrollment. We expect positive effects of programs on enrollment rate. The estimates give us expected effect for the meal program and unexpected effects for the deworming. The exclusive effect of meal and deworming is respectively positive and negative. Even if the additional effect is positive, it is not high enough to offset the negative impact of deworming. We also obtain that the global effect is negative which means that putting the two programs together decreases the enrollment rate.

The relative effect of the package vs. deworming is positive. This is not surprising because we have an exclusive effect of deworming which is negative and an additional effect which is positive. So the relative effect of the package vs. deworming simply means that when you set as goal to increase enrollment, among deworming alone and the combination of the two programs, it is better to choose a combination of both. The relative effect of the package vs. the meal is negative. This implies that when we set the target of increasing enrollment, the implementation of the meal program alone is better than the package. The relative effect of the meal program compared to deworming is positive. This is normal because the exclusive effect of meal is positive while that of deworming is negative. This means that the meal program would better help to increase enrollment compared to the deworming program.

Table 3.6: Average Treatment Effects for rates

\begin{tabular}{|c|c|c|c|c|c|c|}
\hline & \multicolumn{3}{|c|}{ DISM $^{a}$} & \multicolumn{3}{|c|}{ Roy $^{b}$} \\
\hline & Enrollment & Promotion & Dropout & Enrollment & Promotion & Dropout \\
\hline Exclusif: deworming & $-25.619^{* * *}$ & $-5.198^{* * *}$ & $-1.443^{* * *}$ & $-19.642^{* * *}$ & $-8.290^{* * *}$ & $-1.263^{* * *}$ \\
\hline Exclusif: meal & $11.177^{* * *}$ & $2.727^{* * *}$ & $-4.797^{* * *}$ & $19.644^{* * *}$ & $5.021^{* * *}$ & $-5.430^{* * *}$ \\
\hline Global & $-12.400^{* * *}$ & $7.040^{* * *}$ & $-9.029^{* * *}$ & $-14.415^{* * *}$ & $2.547^{* * *}$ & $-8.100^{* * *}$ \\
\hline Additionnal & $2.041^{* * *}$ & $9.511^{* * *}$ & $-2.788^{* * *}$ & $2.735^{* * *}$ & $5.815^{* * *}$ & $-3.680^{* * *}$ \\
\hline Relative: package vs. deworming & $13.219^{* * *}$ & $12.239^{* * *}$ & $-7.585^{* * *}$ & $17.675^{* * *}$ & $10.837^{* * *}$ & $-9.436^{* * *}$ \\
\hline Relative: package vs. meal & $-23.578^{* * *}$ & $4.313^{* * *}$ & $-4.231^{* * *}$ & $-20.740^{* * *}$ & $1.768^{* * *}$ & $-2.111^{* * *}$ \\
\hline Relative: deworming vs. meal & $36.797^{* * *}$ & $7.925^{* * *}$ & $-3.353^{* * *}$ & $1.550^{* * *}$ & $2.381^{* * *}$ & $-1.660^{* * *}$ \\
\hline
\end{tabular}

Notes ${ }^{a}$ : Double-Index Selection Model, two step estimations; ${ }^{b}$ : Roy model, two step estimations.

Significance levels (Bootstrap -100 replications-): $\quad *: 10 \% \quad * *: 5 \% \quad * * *: 1 \%$

Promotion. Here positive effects are expected. The exclusive effect of deworming is negative while other effects are positive and significant. The global effect shows that combining the two programs is the best option. For the DISM model the global effect is computed as 7.040 which is larger than the effect of the meal (2.727) and deworming (-5.198). For the generalized Roy model, the global effect is computed as 2.547 which is lower than the meal (5.021) and higher than the deworming (-8.290).

The relative effects clearly reveal that the package perform better than the single programs. The relative effect of the meal program vs. deworming shows that if one has to choose between the two alternatives, implementing the meal program better fit the target of achieving a higher rate of promotion. The exclusive effects lead to the same conclusion.

Dropout. Here we expect negative effects. Remember that negative treatment effects here mean 
that the program has reduced the dropout rate. The exclusive effect of deworming is negative as the effect of the meal program. The global effect indicates that the combination of the two managed to reduce significantly the dropout rate compared to the single programs. The global effect is computed as -9.029 in the DISM and -8.100 in the generalized Roy model whereas the figures for the exclusive effects are -1.443 and -4.797 for the deworming and the meal in the DISM and -1.263 and -5.430 in the generalized Roy model respectively.

Table 3.7: Treatment Effects for rates: Generalized Roy's ${ }^{a}$

\begin{tabular}{|c|c|c|c|c|c|c|}
\hline & \multicolumn{3}{|c|}{ Treated (ATET) } & \multicolumn{3}{|c|}{ "Untreated (ATENT) } \\
\hline & Enrollment & Promotion & Dropout & Enrollment & Promotion & Dropout \\
\hline Exclusif: deworming & $-251.724^{* * *}$ & $-59.885^{* * *}$ & $89.095^{* * *}$ & $80.410^{* * *}$ & $-11.976^{* * *}$ & $-0.995^{* * *}$ \\
\hline Exclusif: meal & $-37.906^{* * *}$ & $41.619^{* * *}$ & $-38.952^{* * *}$ & $45.890^{* * *}$ & $-17.996^{* * *}$ & $9.014^{* * *}$ \\
\hline Global & $-381.663^{* * *}$ & $-31.793^{* * *}$ & $14.529^{* * *}$ & $58.663^{* * *}$ & $14.644^{* * *}$ & $-1.869^{* * *}$ \\
\hline Additionnal & $-92.032^{* * *}$ & $-13.527^{* * *}$ & $-35.613^{* * *}$ & $-67.636^{* * *}$ & $44.617^{* * *}$ & $-9.889^{* * *}$ \\
\hline Relative: package vs. deworming & $-129.938^{* * *}$ & $28.091^{* * *}$ & $-74.565^{* * *}$ & $-21.746^{* * *}$ & $26.621^{* * *}$ & $-0.874^{* * *}$ \\
\hline Relative: package vs. meal & $-343.757^{* * *}$ & $-73.413^{* * *}$ & $53.482^{* * *}$ & $12.773^{* * *}$ & $32.641^{* * *}$ & $-10.884^{* * *}$ \\
\hline Relative: deworming vs. meal & $-213.818^{* * *}$ & $-101.504^{* * *}$ & $128.048^{* * *}$ & $34.520^{* * *}$ & $6.020^{* * *}$ & $-10.010^{* * *}$ \\
\hline
\end{tabular}

The relative effect of the package vs. deworming shows that the package performs better in terms of reducing dropouts. The relative effect of the package vs. meal is slightly lower than the exclusive effect of the meal program. The relative effect of meal compared with deworming shows that the former performs better.

\subsection{Policy analysis}

In this section we will first present a treatment effect heterogeneity analysis and then perform a cost-effectiveness analysis. Both analyzes are relevant to the policymaker. Treatment effect heterogeneity analysis is relevant from a policy perspective as it enables to identify subgroups of populations for which treatments are effective. To understand whether all targeted pupils benefit positively from the intervention it is important to explore the effects heterogeneity.

In a context of scarce resources, the effectiveness of an educational policy to promote the quality is determined by the effects of the program on educational outcomes and also the cost to achieve them, hence the need for cost-effectiveness analysis.

\subsubsection{Treatment effect heterogeneity}

In section 3.5, we have summarized the treatment effects by taking the average either over the entire sample (this is the case for the average treatment effect) or over two sub-populations leading to the average treatment effect on the treated (ATET) and the average treatment effect on the untreated (ATENT). All these effects are based on a hypothesis of homogeneity though the ATET and ATENT which can be viewed as heterogenous effect. In other words, the average effects obtained are extrapolated to the population considered. In this section, we study the variation in treatment effects across populations. Treatment effect heterogeneity is an outstanding issue in many impact evaluation studies. As pointed out by Imai and Strauss (2011), the study of heterogeneous treatment effects is relevant from a policy perspective as it enables to identify 
subgroups of populations for which treatments are effective. ${ }^{16}$ Treatment effect is heterogenous if some pupils experience larger treatment effects than others while the treatment is identical for all. This may follow from the characteristics of pupils and likely some unobserved factors.

\section{Scores}

The distribution of the treatments effects for the scores outcomes are plotted in Figures B.1, B.2, B.3, B.4 and B.5 in the Appendix. Two salient pictures can be observed. On one hand, the exclusive ATE effect for meal program has a strong uni-modal distribution which is concentrated around the average. At the same time, the distribution of the exclusive effect of deworming is more heterogeneous, less concentrated and slightly shifted to the right. In other words, the effect of the deworming is more heterogeneous while the effect of the meals is more homogeneous. As a result, the distribution of the exclusive effect of deworming is more dispersed than the effect of meals. This implies that the second order moment of the exclusive deworming effect is greater than that of the meal effect. On the other hand, the distributions of global and additional effects are closely related except for the Average treatment effect on treated (ATET) of math score. The distribution of the sequential and substitution effects shows similar pictures with strong uni-modality.

\section{Enrollment, promotion and dropout}

Figures B.6, B.7, B.8, B.9 and B.10 display the plots of the distributions of treatment effects for the enrollment, promotion and dropout rates. The outstanding findings concern the enrollment rate and the distribution of sequential and substitution effects. Regarding enrollment rate, we observe a bi-modal distribution of the additional ATE with the largest mode having negative values of the treatment effects and a second less pronounced mode which displays positive values of treatment effects. This result indicates a double heterogeneity: one related to the sign of the treatment effects and the other to the multiple modality. Whereas a significant proportion of pupils experiment an additional negative effect, another smaller proportion experiment an additional positive effect. The other distributions on the Figure display uni-modal patterns. Figure B.6 (left) that shows the effects of treatment on the treated, the exclusive and additional effects of deworming have a bi-modal distribution with different signs for each modality. We deduce that the heterogeneity picture is more pronounced than in the previous case. An interesting phenomenon appears in Figure B.9 (left). We observe a mirror-like distribution between the two substitution effects for the promotion rate. While the uni-modality $T_{2} T_{1}$ is displayed around negative treatment effect values, the substitution $T_{1} T_{2}$ reflects the same shape but with a reverse tail and a main mode around positive values of treatment effects.

The main conclusion we draw from the analysis of the distributions is that while some effects are homogeneous (e.g, exclusive ATE for meal and substitution effects for treated and untreated for the scores), other effects are clearly heterogeneous (mainly some effects on enrollment rate, sequential and substitution effects). In other words, pupils react differently to the same intervention.

\subsubsection{Cost-effectiveness analysis}

A major policy issue of impact analysis is whether other alternatives are more effective than the programs under study in terms of cost-effectiveness. Cheung and Perrotta (2011) outlined that there are very few papers that studied the cost per outcome for school meal programs. Relying on an experiment in Cambodia, the authors found that the on-site (school) feeding is the most cost-effective program while distributing take home rations is relatively expensive. They also found that adding the deworming intervention to both on-site feeding and take home ration

\footnotetext{
${ }^{16} \mathrm{It}$ is worthwhile to notice that we are not conducting a statistical inference on causal heterogeneity. Readers interested in this aspect can refer to the studies of Angrist (2004), Horiuchi et al. (2007), Imai and Strauss (2011) and Imai and Ratkovic (2013).
} 
make the full package much more cost-effective thanks to the fact that the complete package attracts many more pupils and the deworming medications are extremely cheap. Miguel and Kremer (2004) found that the cost per additional year of school participation for the deworming is very cost-effective compared to other programs. In this section we conduct a cost-effectiveness analysis for our package. We will compare various alternatives in terms of cost and effectiveness in improving pupils' performance.

A first step in this analysis consists in determining the operation cost of the programs. Tables 3.9 and 3.8 report the average cost per year of running deworming and meal programs. These costs have been calculated using information obtained from the Division of School Canteens (DCS) of the Ministry of Education of Senegal. The World Food Program has planned 16,900 tons of food for a total cost of 7,165,413,861 CFA in 2011. This is intended for 3,400 schools or 560,000 pupils, in elementary schools in few regions, including Fatick, Kolda, Sedhiou, Diourbel and Matam. We used this information to compute cost the per child of the meal program in 2011. To these costs, we added costs related to strengthening capacities and resources of the Division of School Canteens, personnel responsible for the canteens and school management committees.

Table 3.8: Program cost per year: Meal

\begin{tabular}{lcccc}
\hline \hline & Quantity & Currency (CFA) & Number of pupils & Cost per pupil (CFA) \\
\hline Meal & 16,900 & $7,165,413,861$ & 560,000 & \\
Food & 1 & $170,566,943$ & 560,000 & 14000 \\
Other costs ${ }^{a}$ & $7,335,980,804$ & 560,000 & \\
\hline \multicolumn{4}{l}{ Total meal } \\
\hline \multicolumn{2}{l}{ Note: ${ }^{a}$ Strengthening capacities and resources of the DCS, responsible of canteens and } \\
school management committees.
\end{tabular}

For cost related to deworming, we used information obtained through the school canteen division (DCS) and the DCMS of the ministry of education. In fact, for 2011 it was planned for some regions to receive 400,000 tablets of mebendazole for 200,000 pupils due to two tablets per student per year. The estimated cost of a tablet is around $16 \mathrm{CFA}$, therefore the total cost for the drug is 6,400,000 CFA. To these costs, we added costs of transportation, mission of advocacy and supervision that are only estimated costs. To assess the cost-effectiveness we used the cost of a program divided by the percentage of additional outcome due to the program.

Table 3.9: Program cost per year: Deworming

\begin{tabular}{lrrrr}
\hline \hline & Quantity & Currency (CFA) & Number of pupils & Cost per pupil (in CFA) \\
\hline Deworming & & & & \\
Drugs & 400,000 & $6,400,000$ & 200,000 & \\
Advocacy Mission & 1 & $3,734,280$ & & \\
Transport & 1 & 200,000 & & \\
Supervision & 1 & $4,496,000$ & & 74 \\
\hline Total deworming & & $14,830,280$ & 200,000 & \\
\hline \hline
\end{tabular}

\section{Scores}

The results for the cost effectiveness analysis are reported in Tables 3.10 and 3.11. Our findings are consistent with the results in the literature. The deworming program is by far the most cost effective in increasing pupils' achievement compared to the meal program. With the deworming program it takes about $37 \mathrm{CFA}$ per year and per pupil to increase the French score by one point, whereas this figure is about 761,227 for the meal program. AS a result, the meal program is twenty times more expensive. The combination of both programs cost about 237,166 
CFA per pupil and per year to increase the score in French by one point. To increase the score in math by one point, it will take about 2; 557,089 and 107,304 CFA per year per pupil respectively for deworming, meal and the package. It is important to also note that deworming alone is more cost effective in terms of increasing scores than the combination of the two programs.

Table 3.10: Cost effectiveness analysis for scores

\begin{tabular}{|c|c|c|c|c|c|c|}
\hline & \multicolumn{3}{|c|}{$\operatorname{DISM}^{a}$} & \multicolumn{3}{|c|}{ Roy ${ }^{b}$} \\
\hline & Aggregate & French & Math & Aggregate & French & Math \\
\hline Cost deworming only & 74 & 74 & 74 & 74 & 74 & 74 \\
\hline Cost meal only & 13100 & 13100 & 13100 & 13100 & 13100 & 13100 \\
\hline Cost deworming and meal & 13174 & 13174 & 13174 & 13174 & 13174 & 13174 \\
\hline \multicolumn{7}{|l|}{ Percentage of additional score } \\
\hline \multicolumn{7}{|l|}{ ATE } \\
\hline Excusif: deworming & 3.7 & 0.3 & 6.9 & 5.4 & 3.8 & 8.8 \\
\hline Excusif: meal & 6.6 & 3.8 & 9.3 & 7.5 & 5.7 & 9.3 \\
\hline Global & 14.1 & 10 & 18.4 & 18.2 & 11.8 & 24.6 \\
\hline \multicolumn{7}{|l|}{ ATET } \\
\hline Excusif: deworming & & & & 17.3 & 2 & 48.1 \\
\hline Excusif: meal & & & & 20.1 & 17.2 & 23.5 \\
\hline Global & & & & 89.2 & 55.6 & 122.8 \\
\hline Sequential: $(\mathrm{T} 1, \mathrm{~T} 2)$ & & & & 55 & 33.2 & 55.1 \\
\hline Sequential: (T2,T1) & & & & 68.2 & 38 & 99.6 \\
\hline Substitution: (T1,T2) & & & & 5.1 & 0.2 & 6.3 \\
\hline Substitution: (T2,T1) & & & & 15.1 & 1.6 & 45.8 \\
\hline \multicolumn{7}{|c|}{ Cost per percentage of additional score } \\
\hline \multicolumn{7}{|l|}{ ATE } \\
\hline Excusif: deworming & 20 & 246 & 11 & 14 & 20 & 8 \\
\hline Excusif: meal & 1997 & 3467 & 1404 & 1754 & 2315 & 1402 \\
\hline Global & 934 & 1318 & 718 & 725 & 1117 & 537 \\
\hline \multicolumn{7}{|l|}{ ATET } \\
\hline Excusif: deworming & & & & 4 & 37 & 2 \\
\hline Excusif: meal & & & & 651 & 761 & 557 \\
\hline Global package & & & & 148 & 237 & 107 \\
\hline Sequential: (T1,T2) & & & & 240 & 396 & 239 \\
\hline Sequential: (T2,T1) & & & & 193 & 346 & 132 \\
\hline Substitution: (T1,T2) & & & & 2581 & 74012 & 2107 \\
\hline Substitution: (T2,T1) & & & & 874 & 8499 & 288 \\
\hline
\end{tabular}

Notes $^{a}$ : Dummy Index-Selection Model, two step estimations; ${ }^{b}$ : Roy general model, two step estimations.

This makes deworming program being the easiest and least expensive to implement, because it does not require all the material required by the establishment of a canteen. However, at the same time deworming is not the program that has the greatest impact for pupils (in terms of academic results) and for the families given their attitude towards deworming. Also, most families would prefer canteens as they contribute directly to the alleviate food poverty which is a serious problem in rural areas.

The analysis also shows that the introduction of canteen before deworming is more cost effective than the introduction of deworming first. For example, to increase the math score by one point, the meal-deworming sequence cost about $132 \mathrm{CFA}$ and the deworming-meal sequence cost about $240 \mathrm{CFA}$. The substitution of the deworming program to the meal is more cost effective compared to the reverse. Overall, compared to the meal program alone, the combination of both 
programs, whatever the order of implementation or substitution, it should be noted that the deworming alone single is much more cost effective.

\section{Enrollment, promotion and dropout}

The results show that it takes about 1172 CFA to increase the enrollment rate by one percent with the school feeding program. In terms of promotion rate, we find that the package (with $1871 \mathrm{CFA}$ ) is more cost effective than the meal program (4804 CFA). It is not surprising that deworming only is not enough to increase promotions. Indeed, we know that deworming improves the health of children and so it must be combined with another program (here meal) to be more beneficial. 


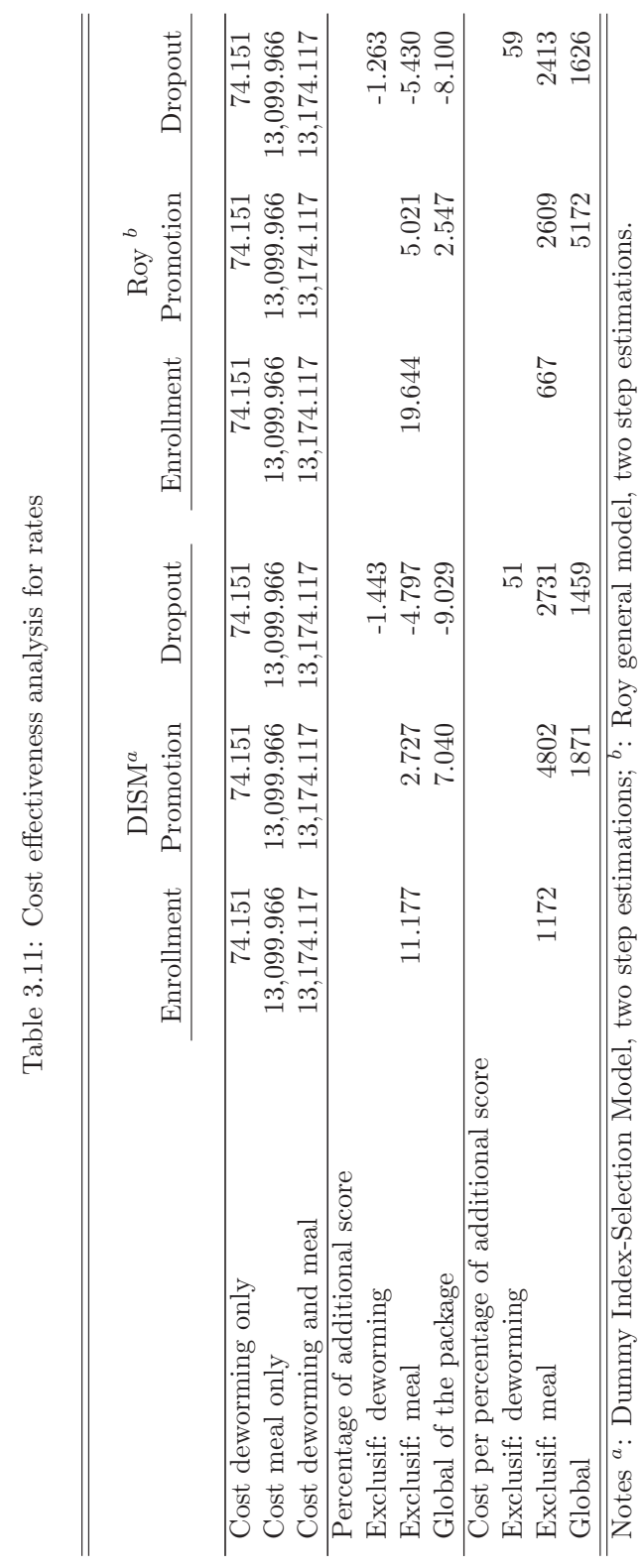


For the dropout rate, we find evidence that deworming is more cost effective that the meal program only and the package. However, the package is more cost effective than the meal program. Indeed, to reduce the dropout rate of $1 \%$, it takes about 51,386 CFA per year and per pupil for the deworming program, 2731 CFA for meal program and 1459 for the package program.

\subsection{Conclusion}

Thanks to a unique observational data set, we are able to assess the impact of deworming and school meals as a true package on pupils' achievement in rural Senegal. In that, our approach is new and can be distinguished from previous contributions in which deworming and school feeding are implemented and studied separately as single programs or a single joint program. From our field experience and in conducting this analysis, several comments come to our mind as regard the role of such interventions.

The main results that emerged from this chapter are the following. i) An interesting aspect of our specification is that it enables to investigate whether deworming and school meals are complementary or substitutable. We observe that the two programs are complementary in the goal of increasing scores and promotion rates. Contrariwise, they are substitutes with the aim of reducing dropouts. ii) We derive a very diverse range of treatment effects. For the score outcomes, we obtain positive and significant additional exclusive and global average treatment effects (ATE). The impact of the meal program on the scores is greater than that the deworming program. The combination of the two programs (package) has a greater impact. This result reinforces the complementary finding. Moreover, the relative effect of the package vs. the deworming alone is greater than that compared to the canteen only. For the average treatment effects on the treated (ATET), the exclusive, global, and additional effects are positive and significant. It should be noted that the effects on the treated are larger than the ATE. For the treatment effects on the untreated (ATENT), the results show that the exclusive effect of deworming is negative while the effect of canteen is positive. The combination of the two programs greatly increases scores. The sequential effects indicate that for the pupils in the treated group, the impact of the package performs better if the school meal is introduced before deworming. For pupils in the untreated group, the reverse sequence is preferable. Substitution effects show that for the treated group, implementing school meals until a time and replace it with a deworming program is more beneficial in terms of enhancing scores compared to the reverse. Regarding enrollment, we obtain an exclusive negative ATE effect for the deworming program, an exclusive positive effect of the meals program, a negative overall effect and an additional positive effect. Moreover, when we set the target of increasing enrollment, the implementation of meals program alone is preferable to deworming or the package. When the objective is to increase the promotion rate or reduce dropout, the package is the best option. iii) The cost-effectiveness analysis indicates that, regarding scores, deworming is far cheaper than the meals program. It also shows that introducing the meals before deworming is more cost-effective than the reverse. As for the promotion rate, the combination of the two programs is more cost-effective than the single meals program. For the dropout rate, deworming is more cost-effective compared to the canteen and the package. However, the package is more cost effective compared to the canteen only.

Finally, results in this chapter suggest several additional areas for future research. Empirically, a multiple outcome framework seems promising. In this study, we have considered six outcomes (aggregate, French and math scores; and enrollment, promotion and dropout rates) separately. It would be interesting to consider the improvement of all these outcome jointly. Evidently, these outcomes are related. For example, in order to record good scores, one needs to be enrolled and not to dropout. Moreover, being successful in terms of academic achievements (via score) may also imply a high probability to be promoted. Another challenge consists in 
extending the double-index selection process to the case of M-selections. This is not only a methodological challenge, but represents a real empirical interest. Indeed, as outlined by Lechner (2001) many evaluation problems (for example the different types of labor market policies in Europe) involve more than two treatments. However, this is not without methodological difficulties. Yet in this study, the likelihood maximization procedure is complex. As for the Roy model, the main difficulty is that the procedure is data consuming. Indeed, all the parameters have to be estimated for each regime. As a result, the problem of parameter inflation is likely. In this sense, the two-stage estimation provides a more manageable alternative. Another extension of this study would also be also to study the impact of the meal and deworming programs on the welfare of households of beneficiary pupils. At the time of writing, none of these issues have been addressed. We speculate that these are promising empirical research avenue. 


\section{CHAPTER 4}

\section{Impact of Meal Program on School Performances: A Randomized Experiment}

\subsection{Introduction}

Experimental studies are increasingly used to measure the impact of policies or development projects. Horiuchi et al. (2007, p. 669) pointed out that "randomized experiments are more likely to yield unbiased estimates of causal effects than typical observational studies. This is because, the randomization of treatment makes the treatment and control groups is equal on average in terms of observed and unobserved characteristics". However, despite their benefits on observational studies, randomized experiments are often faced with difficulties. Likewise, in controlled social science experiments, researchers often do not have perfect control of their target population because participants may choose not to follow instructions on the random assignment treatment or not responding. Therefore, problems of partial adhesion (non-compliance) and attrition (non-response) can coexist. In this regard, some authors, including Horiuchi et al. (2007), Esterling et al. (2011) and Barnard et al. (2003), argued that a good analysis must take problems related to attrition and partial adhesion into account simultaneously. Unfortunately, most of the existing studies do not address one of the two problems, although this leads to invalid causal inference.

In this study, some schools in the treatment group did not receive the school feeding while other schools in the control group have benefited from the program, hence the problem of non-compliance. In addition, during the post-program survey, the academic performance of some pupils has not been collected, hence the presence of attrition or non-response. Given that problems of non-compliance and non response are not produced completely at random (as discussed in descriptive statistics), ignoring them in the estimation of the impact of the program can seriously distort causal inference.

Using experimental data, this study measures the impact of an experimental school feeding program on school performance in the presence of attrition and partial adherence in rural Senegal. It uses a statistical method that can overcome the problems of partial adherence and attrition simultaneously. The study covers four regions of Senegal, two in the center (Diourbel and Fatick) and two in the south of the country (Sédhiou and Kolda). We have two types of outcomes, namely those related to academic performance of pupils (aggregate score, French score and math score) and those related to internal efficiency of schools (enrollment rate, repetition rate, promotion and dropout rates). We use a model that take the two problems in a single framework for estimating the impact of the school feeding program into account (Horiuchi et al., 2007, Barnard et al., 2003). In the presence of partial adherence, a standard intention totreat (ITT) effect which is informative for policy makers, gives a valid inference of the effect of 
assignment on outcome, but usually the goal is to calculate the effect of receiving the treatment rather than the effect of the assignment. That is why we calculate both the ITT effect and the effect of receiving the treatment which is called in the literature by "complier average causal effect (CACE)".

In this study, we also do a cost-effectiveness analysis and cost-benefit analysis. The costbenefit analysis is performed to determine whether the economic gains from the school feeding program exceed the costs involded. The cost-effectiveness analysis is conducted to know if there are other options to achieve the same objectives covered by the feeding at a lower cost. In a poor country, without an evaluation in terms of cost-effectiveness or cost-benefit of different options, we cannot know which policy to prioritize. This is very important because in a context of scarce resources, it is necessary to choose the most cost effective program in improving educational outcomes.

The results show that the school feeding program can increase pupils' performances and enrollment rate, while reducing the dropout rate. The effect of the program is positive for all scores and it is greater for the math score than for the French one. Cost-benefit analysis conducted in this chapter shows that the school feeding program is justified in terms of economic gains while cost-effectiveness analysis shows that the meal program is less effective than the deworming program.

The chapter is organized as follows: Section 4.2 describes the experimental design, the data and variables. In Section 4.3 we present the validation of the experimental design with the descriptive statistics before and after the program. Section 4.4 describes the analytical framework and the model used to measure the program's impact. In Section 4.5, we present a summary of the findings before making a policy analysis in Section 4.6. Section 4.7 concluded the chapter.

\subsection{Experimental design, data and variables}

In this experiment, a system was set up to build the treatment and control groups before the introduction of the program randomly. The intervention consisted of providing hot meals through school canteens located in rural primary schools in Senegal. A monthly contribution of 200 FCFA per pupil is required to purchase other products not included in the food delivered by the WFP, but related to the functioning of the school canteens such as fresh vegetables, fish or meat or even cereals other than maize, because WFP provides only maize during this periode.

Four regions (Fatick, Kolda, Diourbel and Sédhiou) of Senegal were chosen to conduct the experiment. The central regions which are Diourbel and Fatick are mainly composed of farmers, and are closer to the capital (Dakar), while those in the South (Kolda and Sédhiou), with mainly ranchers, are very isolated. These areas are characterized by the presence of very few meal programs, by very marked delays in enrollment, and high prevalence of poverty and vulnerability. Food insecurity in these areas, due to poor harvests, not only reduces household income but also impoverishes the diet. Their children are, therefore mostly at risk of acute malnutrition.

The sample consists of pupils in the second and in fourth year of primary school (CP and CE2 in Senegal), among schools located in Fatick, Diourbel, Kolda and Sédhiou. Several reasons have guided the selection of $\mathrm{CP}$ and $\mathrm{CE} 2$ grades. On the one hand, for reasons of cost of investigation, it was difficult to involve all pupils in each school. However, it should be noted that all pupils benefit from school canteen. On the other hand, at the end of the second year of primary school, if the child cannot read and write, the probability to leave school before completing the cycle is very high. In addition, if after four years of primary education, skills in reading and writing are not vested, it is unlikely that the child can acquire the basic skills that education is supposed to give him. Regarding the duration of treatment exposure, it is about 13 months, so the experiment lasted a little more than one school year.

Different actors have been involved in the implementation of this experiment. The main ones includes; the World Food Program (WFP), the Ministry of Education through its 'Division des 
Cantines Scolaires (DCaS)', the 'Institut National d'Étude et d'Action pour le Développement de l'Éducation (INEADE)', the 'Inspections Départementales de l'Éducation Nationale (IDEN)', which became the 'Inspections de l'Éducation et Formation (IEF)' not very long ago, school directors, teachers and communities. WFP provides food during each quarter and transports them to the beneficiary schools. The DCas provides monitoring and coordination of management of the canteens. At the level of the IEF, the persons in charge of canteens have to monitor their functioning and write quarterly reports which is sent to WFP and to the Division of school canteens. In each school, the operation of the canteen is provided by a Management Committee which includes the school director, other teachers, two representatives of the pupils (one boy and one girl) and a representative of the notables of the village. The management committee has three specialized committees responsible for food management, community participation to the kitchen and meals. Teachers fill tracking sheets of the canteen under the supervision of the director who collects and transmits them to the person in charge of canteens at the IEF. The INEADE was responsible for test designing, recruitment of evaluators and for the marking of tests. Parents of pupils are grouped into associations such as the association of parents or the associations of mothers of pupils. These associations are responsible for ensuring the smooth operation of canteens and the payment financial contribution of FCFA 200 per pupil and per month for purchasing of ingredients which are not provided by the WFP. The parents also supplies firewood for cooking meals and support the construction of kitchens and store rooms for the food. Communities are in charge of providing kitchen utensils for preparing meals. The description of the sampling strategy and the data, will be discussed in the following subsections.

\subsubsection{Sampling strategy}

The sampling strategy has been to first identify the geographic areas (regions) for the project, and to also establish a population of eligible schools based on some criteria which will be listed below. Then, from the population of eligible schools constituted, random sampling was conducted to select a sample of schools for participation in the project. Finally random drawing was conducted at each school for pupils participating to the program. Eligible geographical areas should meet the following criteria: i) be among the poorest regions of Senegal, ii) have a system of standardized assessment in the area and iii) be in a priority area of partners involved in implementation of the project. Primary schools eligible for the experiment came from a set of schools provided by the Department of Education based on the following criteria: i) non availability of meal program and health service package including deworming in schools, ii) having a number of pupils between 50 and 600, iii) have a grade of CP and / or CE2 iv) having a functional management committee and v) be located in a rural area. The selection criteria for pupils are: i) to be in a class of $\mathrm{CP}$ or $\mathrm{CE} 2$ and ii) to be attending the class the day of the random selection of 20 pupils.

Selection of eligible schools for the project

On the basis of eligibility criteria the regions of Kolda, Tambacounda and Matam were selected. Schools potentially or partially eligible in these three regions were isolated according to eligibility criteria by using the data base on schools provided by the Ministry of Education. The number of these eligible schools was 241 in Kolda, 101 in Matam and 143 in Tambacounda, which came up to a total of 485 eligible schools. In terms of regional proportion of potentially eligible schools, it was $49.7 \%$ in Kolda, $20.8 \%$ in Matam and $29.5 \%$ in Tambacounda. This amounts to a number of schools of 79 for Kolda, 32 for Matam and 48 for Tambacounda. As the target population was initially set up for four groups, finally 80 schools were selected for Kolda and 34 for Matam. After a first random selection, it emerged from a monitoring mission that some selected schools did not meet the eligibility requirements of schools because the file provided by the Department of Education contained schools that did not meet the criteria. Indeed, some schools already had a deworming program, whilst others were provided with school meals in recent years. For Matam region in particular, the majority of schools already had canteens and all pupils were dewormed 
and supplemented with iron. Moreover, while some schools were in temporary shelters, others were isolated and did not have water. This is a major drawback for the establishment of school canteens. So schools in Matam region were removed from the sample in favor of those of Sédhiou. Similarly, schools in the region of Tambacounda had received a deworming program initiated by the Ministry of Health and the World Health Organization (WHO). These schools were then replaced by schools in Fatick and Foundiougne. The choice of these new regions was due to the fact that they are areas of intervention of the experiment partners, in particular the WFP.

The sample was then drawn again on the basis of the same selection criteria, but this time using lists provided directly by person in charge of canteens. Particularly those in the IEF instead of the lists that were provided by the Ministry of Education.

Thus, 167 schools located in Diourbel, Fatick, Kolda and Sédhiou were identified as eligible schools. Each region is administratively divided into departments and schools were distributed as shown in the Table 4.1 below. The distribution of the final sample of schools across regions was proportional to the number of eligible schools by region.

Table 4.1: Schools eligible and selected for the experiment, 2009

\begin{tabular}{lccc}
\hline \hline \multirow{2}{*}{ Regions } & Department & Schools eligible & Schools selected \\
\hline \hline \multirow{2}{*}{ Diourbel } & & & \\
& Diourbel & 18 & 11 \\
Fatick & Fatick & 41 & 37 \\
& Foundioune & 41 & 22 \\
Kolda & Kolda & 33 & 29 \\
Sédhiou & Velingara & 20 & 14 \\
\hline \hline Total & Sédhiou & 14 & 7 \\
\hline \hline
\end{tabular}

The sample size for the experiment is 120 schools and these schools were randomized into 4 groups of 30 schools. There was a canteen group (schools that will only be equipped with a canteen), a canteen and deworming group (schools that will receive canteens and deworming), a deworming group (schools that will only receive deworming) and finally a control group (schools that have no canteen and no deworming). Moreover, in the sampling frame used to form the population of eligible schools, some schools were waiting to receive school meals from the WFP. It emerged that these schools were newly equipped with canteens. These schools were then withdrawn from of the sample and were replaced by schools without canteens drawn randomly from a group of replacement schools that had been constituted beforehand. We also observed that four schools randomly classified into the 'canteen group' had the canteens for a long time. These four schools were replaced by four schools randomly chosen among the 19 schools newly equipped with canteens by WFP. The remaining 15 schools were added to the list of replacement schools which contained 47 schools in total. These schools will be used as replacement schools when needed during investigations. Among these 47 schools, 28 had just been newly equipped with a canteen by WFP and 19 had no canteens. Replacement schools without canteens were randomly placed in "replacement" order, and will replace schools in the control group whenever needed. The same procedure was applied to replacement schools with canteens that will replace schools in the treatment group when needed.

\section{Selection of pupils}

Once the schools in the experiment sample have been stabilized, a random selection of pupils within each school was made. In schools where there has been more than one CP or CE2 class, a random drawing was conducted at the first class to have the class in which pupils will be 
drawn randomly. In each given class, 20 pieces of paper were marked among a total number of paper equal to the size of the class. Each pupil was then asked to choose a piece of paper. After the draw, the 20 pupils who happened to take the marked papers were selected for testing. In classes, where the number was less than 20 pupils, pupils who attended the class the day of the draw were selected systematically.

Standardized tests and surveys

After the random selection of pupils, standardized tests were conducted. The selected subjects were mathematics and French and the two disciplines were given at the same time. For tests, the INEADE has conducted supervision, administration, correction and data entry. The INEADE has developed tools in the form of assessment booklets which are submitted to pupils to test their academic performance. The strategy involves the selection of resource persons from the 'Collectifs de Directeurs d'Ecoles (CODEC)' of six administrative departments to run the experiment. Persons selected from the CODEC in charge to conduct the tests were trained before giving tests to pupils in CP and CE2. The completed assessment booklets were then sent to CRES by the IEF. Treatment (correction coding and computerization) of notebooks from standardized tests was conducted by INEADE.

Testing pupils in math and French for the first survey took place before March 2009. After implementation of the program, test administration for the second survey took place throughout the month of June 2010. After the program, a different test was given to pupils passing in higher class and the old test was given to repeaters.

Despite the efforts of coordination, after conducting the first survey, an audit of the entire sample conducted in December 2009 revealed that 20 schools in the control group were already equipped with canteens few years ago. Therefore, these schools were replaced by new schools and pupils who were already tested.

\section{Problems}

During the first survey, in addition to sampling problems, the long distances between communities, difficulties in accessing some schools and a teachers' strike have resulted to the extension of the duration of investigations. This also resulted to delay in giving tests to pupils in some schools. Numerous problems were also encountered during the second survey. Indeed, some schools were already on vacation before the arrival of the persons in charge of the tests. To limit the attrition rate, some children were searched in the fields and tests were given. Also, some classes in temporary shelters were closed early because of the raining season. Consequently, tests to some pupils were conducted under trees. All this justifies another visit after the holidays to test pupils that could not be evaluated at the end of the 2009-2010 school year. Still, some pupils were not found for testing. Another problem is related to the delivery of completed books and questionnaires to persons in charge of canteens and then to CRES. Unlike the baseline survey, this device had not worked well during the second survey, and this caused a delay in the treatment of notebooks and questionnaires.

Furthermore, to implement canteens, the strategy has been to persuade the WFP and the Ministry of Education to direct their annual resources to schools in the treatment group and not to those in the control group. The establishment of school canteens has taken much longer than expected. Canteens have actually started operation in January 2010 except for some which started in December 2009.

About the deworming, drugs had been acquired but the establishment of the medical team was delayed due to a lack of financial support. This was reason for abandoning the deworming in the experiment. That is why we are not able to assess the impact of the deworming program in this chapter.

Thus the four groups formed randomly in advance have been reduced to two groups. The deworming group was added to the control group, whilst the deworming and canteen was added to the canteen group. Finally, we have a sample of 120 schools divided into two groups (treatment and control). 


\subsubsection{Data and variables}

Data

In this research, we used two primary data sets collected by the CRES before and after the implementation of the school feeding program. The information was collected from population of 4,110 students including 2,122 boys and 1,988 girls, enrolled in rural primary schools in Senegal, particularly in CP and CE2 classes. Data collected are based on the characteristics of schools, teachers and classes, the characteristics of pupils and their households as well as on the school census of population located within four miles of the school.

A questionnaire on the school, the teachers and the classes were given by directors selected from the group of the 'Collectifs de Directeurs d'Ecoles (CODEC)'. The questionnaire includes information on school infrastructure, characteristics of teachers and of pupils.

A household survey including the administration of a questionnaire to all households of selected pupils, and the population census within five kilometers around each school was carried out by CRES. For the first survey, the investigation began in March, 2009 and spread until May, 2009. As for the second one, the investigation began on June, 2010 and ended in mid-July, 2010. This survey collected information on pupils' household characteristics, on pupils themselves, on their environment and the community in which they live.

Information sheets were also used to collect data on the operation of school canteens. They were filled by teachers under the supervision of school directors. Unfortunately, these records were not well filled.

In addition to the surveys, data on the academic performance of pupils were collected at the beginning and the end of the 2009-2010 school year through standardized assessments earlier described.

We also used secondary data obtained from the database of the Ministry of Education. These are related to pupil enrollment, school infrastructure, the number of repeaters by level of study and for the entire school, as well as the number of promotion by study level. This secondary data do not contain information on the number of dropouts. So, information on the admission to the college entrance examination from the Directorate of services and examinations were also used to calculate the promotion rate and finally the dropout rate. The list and definition of variables is given in Table A.1 in the appendix.

\section{Measurement of outcome variables}

The outcome variables used are grouped into two categories: variables related to pupils' achievement (aggregate score, French score and math score) and those related to the internal efficiency of schools (repetition rate, promotion rate, dropout and enrollment rates).

Variables related to pupils' performances.

The scores were calculated in terms of items by taking the sum total of correct answers. Missing responses were considered incorrect responses (wrong answer). Each correct answer equal one (1), whilst each incorrect answer is zero (0). The score is the percentage of correct answers. Each score was calculated as follows: for scores in French, it was the total of good responses given by a pupil divided by the total number of items (questions) in French then multiplied by 100. Then this result represents the score of the pupil in French. The same procedure was used to calculate scores for mathematics. The aggregate score is a weighted average of French and math scores based on the number of items in each discipline. In other words, it is the sum total of the items obtained in French and mathematics divided by the total number of questions in mathematics and French, and the result is reported to 100 (multiplied by 100).

Variables related to the internal efficiency of schools

Data collected provide information about the total number of repetitions, dropouts and pupils per school for each grade (CI, CP, CE1, CE2, CM1 and CM2) within each school, as well as enrollment in the first grade (CI) from 2007 to 2010. As there were many missing values for these variables, secondary data from the Ministry of Education were used to impute missing 
data. In addition, the same data have allowed us to have the information on repetition of the school year 2010 which is only reported in 2011. Remember that the experiment was conducted in the 2009-2010 school year. Information on outcomes related to internal efficiency (repetition, dropout, promotion and enrollment) in the data collected was limited to the years 2009-2010. That is why we used secondary data from the ministry of education to calculate outcome variables repetition rate, dropout and promotion rates after program. For the calculation of the repetition rate for the year 2009, we used the ratio of the sum of repeaters of 2009 reported in 2010 to the total number of pupils (Pupils) in the school for 2009 school year.

$$
\text { Repetition }_{\text {rate } 09}=\frac{\sum \text { repeaters }(C I, C P, C E 1, C E 2, C M 1, C M 2)_{10}}{\sum \text { Pupils }(C I, C P, C E 1, C E 2, C M 1, C M 2)_{09}} * 100
$$

For the calculation of the repetition rate for the year 2010, we used the ratio of the sum of repeaters of 2010 reported in 2011 to the total number of pupils at the school in 2010.

$$
\text { Repetition }_{\text {rate }_{10}}=\frac{\sum \text { repeaters }(C I, C P, C E 1, C E 2, C M 1, C M 2)_{11}}{\sum \text { Pupils }(C I, C P, C E 1, C E 2, C M 1, C M 2)_{10}} * 100
$$

To calculate the promotion rate for the baseline year i.e. 2009, we made the connection between the total number promoted in 2009, against the total number of pupils in the school for the same year. For the calculation of the total promoted in 2009 within each school, we used the following equation:

$$
\text { Promoted }_{09}=\text { Pupils }_{10}-\text { newenrollment } 10-\text { totalrepeater }_{10}+\text { examadmitted }_{09}
$$

where Pupils $_{10}$ is the total number of pupils within each school in 2010 , newenrollment 10 refers to pupils who have enrolled for the introductory course -first year of primary school- (CI) in 2010, totalrepeater $s_{10}$ is the total number of repeaters recorded in 2010, and examadmitted 09 is the number of pupils admitted to the secondary school entrance examination in 2009. Note that we do not have any information on transfers between schools, which is why they do not appear in the calculation of the total promoted. Thus, the promotion rate for 2009 is obtained from the following relation:

$$
\text { Promotion }_{\text {rate }_{09}}=\frac{\text { Promoted }_{09}}{\text { Pupils }_{09}} * 100
$$

To calculate the promotion rate in 2010 , that is to say, after the program, we used the same approach.

To calculate the dropout rate by year (2009 and 2010), we used the following relation:

$$
\text { Dropout }_{\text {rate }}=100-\left(\text { Repetition }_{\text {rate }}+\text { Promotion }_{\text {rate }}\right)
$$

For enrollment rate before the program (2009), we took the difference between the new enrolled in the first year of primary school (CI) of 2009 and that of 2008 , which we divided by the new enrolled in 2008 and multiplied by 100 . The following relationship was used:

$$
\text { Enrollment }_{\text {rate }_{09}}=\frac{\text { newenrollment } 09-\text { newenrollment }_{08}}{\text { newenrollment }} * 100 \text {. }
$$

For enrollment rate after the program (2010), we used the same approach.

\section{Choice of control variables}

Control variables were selected based on both their availability in databases and their relevance from the point of view of the literature. Based on the literature on randomized ex- 
periments, control variables explain the performance of pupils (scores), but are not related to treatment. They can be grouped into four categories: the characteristics of schools and teachers, the characteristics of pupils, characteristics of pupils' households, and characteristics of the environment or the community where pupils live.

Characteristics of schools and teachers

Characteristics of schools selected are the number of classes in temporary shelters, the number of pupils per manual, the distance between the school and the home of the pupil and disturbances that have resulted to delays in the start of classes. It is important to remember that in general, schools with the best infrastructure produce the best performance.

Teacher characteristics considered are gender, age, training received by the teacher, academic qualifications and teacher absenteeism.

Pupils characteristics

The variables on the characteristics of students who have been selected are gender, age, class or level of education, Koranic school, institution of childhood and the number of hours of work that the child spends doing housework per day.

Household characteristics

Variables related to household characteristics of those selected are the household size per adult equivalent, literacy of the household head, gender of household head, number of head of livestock that the household owns, possession of cultivable land owned by the household and marital status.

Community characteristics

Characteristics related to the environment of the pupils chosen are the existence of a secondary school ("college") in the village of the pupil, the number of primary schools in the village, and the variable "Koranic school com." which equal to 1 if the pupil live in a community where attending Koranic school prevent children from going to school.

\subsection{Descriptive statistics}

In this section, we are exploring the homogeneity of the treatment and control groups before the implementation of the program, using the pre-program survey; and then present descriptive statistics after implementation of program using the post program survey.

\subsubsection{Descriptive statistics before program}

The aim of the various tests performed in this subsection is to determine if there are any differences in the mean or in the distribution of variables for the treatment group and the control group before the implementation of the program. In other words, we are exploring whether the two groups are homogeneous before introducing the program regarding variables chosen.

In the data, the unit of observation is the pupil while the random assignment to the treatment was conducted at the school level. Therefore, we first conducted, tests at the school level, then at the pupils level.

\section{Homogeneity of the two groups: comparison tests at the school level}

Parametric tests (test of equal mean of Student) and nonparametric (test of equal distributions of Mann-Whitney and Kolmogorov-Smirnov) were performed for each variable. An analysis of multiple variances (MANOVA) and a Hotelling test were performed on all variables taken simultaneously.

A Levene test of equality of variance was conducted before the test of equal mean of Student. Both tests were performed on both dichotomous and continuous variables (Table C.1 and C.2), while the tests of equal distribution of Mann-Whitney and Kolmogorov-Smirnov were performed only on continuous variables (Tables 4.2; 4.3, 4.6 and 4.7). 
The Levene test of equality of variances performed at school level indicates that we can reject the hypothesis of equal variances for the variables household size, primary schools, livestock, number of hours, disturbance and marital status (Table C.1). Therefore, we performed a Student test with unequal variances for these variables, and a Student test with equal variances for the remaining variables.

Regarding the Student test, the last column of Table C.1 shows that, on average, there is no significant difference between schools in the treatment group and those in the control group for most of the variables. We did not observe significant differences between the two groups for all outcomes related to scores and for a number of control variables including: temporary shelters (1.718 temporary shelters for the treatment group against 1.576 for the control group); distance between school and home $(81.4 \%$ of schools in the treatment group against $80.9 \%$ of schools in the control group are located between 0 and $1 \mathrm{~km}$ from the pupils' homes); school manual (1.114 manual for pupils in the treatment group against 1.089 manual for pupils in the control group in average); and literacy of household head (18.0\% for the treatment group and $16.6 \%$ for the control group). The results of the parametric test at the school level indicates that the two groups are homogeneous regarding all outcomes related to internal efficiency of schools except for the enrollment rate for which we observe a significance at $10 \%$. For control variables number of hours ( 0.19 hours per day in the treatment group against 0.13 in the control group); disturbance (84.5 of schools had disturbance in the treatment group against $61.4 \%$ of schools in the control group); and Koranic school in the community (61.6\% of pupils live in a community where attending Koranic school prevents children from going to school in the treatment group against 38.7 pupils in the control group); the treatment group displays, in average, the highest percentage with a significance difference. For the control livestock $(8.9$ head of cattle in the treatment group against $14.8 \%$ in the control group); the control group has the highest average with a significant difference.

The results of the non-parametric tests performed only on continuous variables (Table 4.2 and 4.3) confirm those of the parametric test for some variables. For example, we note that there is no significant difference between the treatment and control groups for all score outcomes and for other variables including temporary shelters, school manual, teacher age, pupil age and primary school.

However, before implementation of the program, with significance levels of $10 \%$ and $5 \%$, there are significant differences between the two groups of schools for a number of variables including enrollment rate, livestock, disturbance and Koranic school and variables. Regarding pupils' scores, on average, there is no significant difference between the two groups. However, on average, the academic results of pupils in the treatment group are lower than those in the comparison group. Likewise, aggregate score, French and math scores are 37.743, 37.602 and 37.871 percent points for the treatment group, whereas these scores are $41.357,41.247$ and 41.467 percent points for the control group respectively. The differences are about four (4) points in favor of pupils in the control group.

In terms of the internal efficiency of schools, there are no differences between the two groups for outcome variables except for the enrollment rate $(-8.021 \%$ for the treatment group and $-26.197 \%$ for the control group). Indeed, schools in the treatment group show the lowest promotion rate, but the highest dropout and repetition rates. In those schools, promotion rate, dropout rate and enrollment rate are $80.617 \%, 14.706 \%$ and $-8.021 \%$ respectively, while those in the control group shows $83.200 \%, 12.564 \%$ and $-26.197 \%$ respectively.

For control variables such as livestock, number of hours, disturbance, Koranic school and Koranic school in the community, there is a significant difference between the two groups.

Also, non-parametric tests show that there are significant differences between the two groups for some variables (Table 4.2 and 4.3). Table 4.2 on the Kolmogorov test reveals a significant difference between schools in the treatment group and those in the control group for variables French score, dropout rate, enrollment rate, and livestock. 
Table 4.2: Kolmogorov-Smirnov test: School level, 2009

\begin{tabular}{|c|c|c|c|c|c|c|c|}
\hline \multirow[b]{2}{*}{ Variable } & \multicolumn{2}{|c|}{ Treatment } & \multicolumn{2}{|c|}{ control } & \multicolumn{2}{|c|}{ Combined } & \multirow{2}{*}{$\begin{array}{r}\text { Corrected } \\
\text { p-value }\end{array}$} \\
\hline & $\mathrm{D}$ & p-value & $\mathrm{D}$ & p-value & $\mathrm{D}$ & p-value & \\
\hline Aggregate score & -0.163 & 0.233 & 0.030 & 0.941 & 0.163 & 0.461 & 0.382 \\
\hline French score & -0.223 & $0.066^{*}$ & 0.043 & 0.903 & 0.223 & 0.132 & $0.093^{*}$ \\
\hline Math score & -0.140 & 0.343 & 0.016 & 0.985 & 0.140 & 0.659 & 0.582 \\
\hline Repetition rate & -0.072 & 0.753 & 0.078 & 0.717 & 0.078 & 0.996 & 0.993 \\
\hline Dropout rate & -0.024 & 0.969 & 0.302 & 0.007 & 0.302 & $0.014^{* *}$ & $0.008^{* * *}$ \\
\hline Enrollment rate & -0.003 & 0.999 & 0.262 & 0.024 & 0.262 & $0.049^{* *}$ & $0.032^{* *}$ \\
\hline Promotion rate & -0.147 & 0.310 & 0.020 & 0.978 & 0.147 & 0.602 & 0.522 \\
\hline Temporary shelters & -0.023 & 0.971 & 0.083 & 0.685 & 0.083 & 0.991 & 0.985 \\
\hline School manual & -0.120 & 0.456 & 0.080 & 0.705 & 0.120 & 0.827 & 0.770 \\
\hline Number of hours & -0.026 & 0.962 & 0.203 & 0.105 & 0.203 & 0.209 & 0.156 \\
\hline Household size $^{a}$ & -0.126 & 0.417 & 0.063 & 0.803 & 0.126 & 0.774 & 0.709 \\
\hline Teacher age & -0.070 & 0.765 & 0.066 & 0.785 & 0.070 & 0.999 & 0.999 \\
\hline Pupil age & -0.180 & 0.171 & 0.093 & 0.622 & 0.180 & 0.340 & 0.269 \\
\hline Primary school & -0.089 & 0.652 & 0.000 & 1.00 & 0.089 & 0.983 & 0.972 \\
\hline Livestock & -0.236 & $0.047^{* *}$ & 0.001 & 1.000 & 0.236 & 0.094 & $0.064^{*}$ \\
\hline
\end{tabular}

Note $^{a}$ : Household size per adult equivalent.

Significance levels: $\quad *: 10 \% \quad * *: 5 \% \quad * * *: 1 \%$

Table 4.3 on the Mann-Whitney test shows that there is a significant difference between the two groups for variables dropout rate, promotion rate, enrollment rate, number of hours and livestock.

Table 4.3: Mann-Whitney test: School level, 2009

\begin{tabular}{|c|c|c|c|c|}
\hline Variable & $\begin{array}{c}\text { Treatment } \\
\text { \# Obs. }\end{array}$ & $\begin{array}{l}\text { Control } \\
\text { \# Obs. }\end{array}$ & $\begin{array}{c}\text { rank. } \\
z\end{array}$ & $\begin{array}{l}\text { test } \\
p>|z|\end{array}$ \\
\hline Aggregate score & 60 & 50 & 1.375 & 0.169 \\
\hline French score & 60 & 50 & 1.615 & 0.106 \\
\hline Math score & 60 & 50 & 1.051 & 0.293 \\
\hline Repetition rate & 60 & 49 & -0.420 & 0.674 \\
\hline Dropout rate & 60 & 49 & -2.365 & $0.018^{* *}$ \\
\hline Promotion rate & 60 & 49 & 1.297 & 0.194 \\
\hline Enrollment rate & 60 & 49 & -2.144 & $0.032^{* *}$ \\
\hline temporary shelters & 60 & 50 & -0.656 & 0.511 \\
\hline School manual & 60 & 50 & 0.525 & 0.599 \\
\hline Number of hours & 60 & 50 & -1.981 & $0.047^{* *}$ \\
\hline Household size $\mathrm{e}^{a}$ & 60 & 50 & 0.600 & 0.548 \\
\hline Teacher age & 60 & 50 & 0.213 & 0.831 \\
\hline Pupil age & 60 & 50 & 0.702 & 0.482 \\
\hline Primary schools & 60 & 49 & 0.557 & 0.577 \\
\hline Livestock & 60 & 50 & 1.945 & $0.051^{*}$ \\
\hline
\end{tabular}

Note $^{a}$ : Household size per adult equivalent.

Significance levels: $\quad *: 10 \% \quad * *: 5 \% \quad * * *: 1 \%$ 
The results from the parametric and non-parametric tests performed at school level indicate that the two groups are homogeneous for most of the variables. The verification of the homogeneity of the two groups vis-à-vis the variables taken simultaneously is needed before a conclusion on whether or not the two groups are homogeneous at the baseline. Therefore, a Hotelling test which is a generalization of the Fisher test and a MANOVA which is a multiple variance analysis were performed.

Table 4.4 on the Hotelling test for equality of means shows that the two groups are identical regarding all variables taken simultaneously.

Table 4.4: Hotelling test: School level, 2009

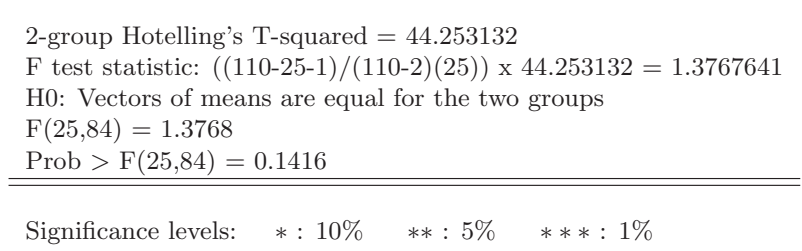

The four tests performed by the MANOVA are all no significant (Table 4.5) and thus confirm the results of the Hotelling test (Table 4.4). This implies that the behavior of the group of variables does not change from one group to another. Therefore, hypothesis on equal mean vectors between the two groups is not rejected overall.

Table 4.5: MANOVA on quantitative variables: School level, 2009

\begin{tabular}{|c|c|c|c|c|c|c|}
\hline $\begin{array}{l}\text { Number of obs }=110 \\
\mathrm{~W}=\text { Wilks' lambda } \mathrm{L}=\text { Lawley-Hotelling trace } \\
\mathrm{P}=\text { Pillai's trace } \mathrm{R}=\text { Roy's largest root } \\
\text { Source }\end{array}$ & Statistic & df & F(df1, & $\mathrm{df2})=$ & $\mathrm{F}$ & Prob $>F$ \\
\hline group & $\begin{array}{ll}\text { W } & 0.7093 \\
\text { P } & 0.2907 \\
\text { L } & 0.4098 \\
\text { R } & 0.4098 \\
\end{array}$ & 1 & $\begin{array}{l}25.0 \\
25.0 \\
25.0 \\
25.0\end{array}$ & $\begin{array}{l}84.0 \\
84.0 \\
84.0 \\
84.0\end{array}$ & $\begin{array}{l}1.38 \\
1.38 \\
1.38 \\
1.38\end{array}$ & $\begin{array}{l}0.1416 \mathrm{e} \\
0.1416 \mathrm{e} \\
0.1416 \mathrm{e} \\
0.1416 \mathrm{e}\end{array}$ \\
\hline Residual & & 108 & & & & \\
\hline Total & & 109 & & & & \\
\hline
\end{tabular}

In conclusion, before implementing the program, the tests at school level show that there were no significant differences between the two groups for the greatest number of variables; whereas tests performed simultaneously using all variables show that there are no significant differences between the two groups. However, it is possible to find significant differences between the treatment group and the control group when tests are carried out on more disaggregated data i.e. at the pupil level because the sample sizes of the two groups become larger.

In what follows, we verify the homogeneity of the two groups by performing tests at the pupils 
level.

\section{Homogeneity of the two groups: comparison tests at the pupil level}

From Table C.2 in the appendix, Levene's test of equality of variances shows that we cannot reject the hypothesis of equal variances for variables enrollment rate, school manual, household size, teacher age, grade, absenteeism, gender of teacher, continuing training, High school diploma and more, national certificate, gender of pupil, literacy of household head, and college. Therefore, a Student test with equal variances was performed for these variables, while a Student test with unequal variance was performed for the remaining variables for which the assumption of equal variances was rejected.

Contrary to the results of the tests performed at the school level, for the majority of variables, Table C.2 in the appendix shows that there is a significant difference between the treatment group and the control group before implementation of the program. This is not difficult to understand because when tests are performed on the more disaggregated data, that is to say, the pupil level, the sample sizes of the two groups become larger.

In terms of pupils' achievement, as mentioned in the case of tests at the school level, pupils' performance in the treatment group were lower than those in the control group. The aggregate score, French score and math score, are 37.158, 37.095 and 37.202 points percentage respectively for the treatment group, while in the control group these scores are 41.872, 41.627 and 42.117 points percentage respectively for aggregate, French and math scores. The differences are around five percent points in favor of pupils in the control group. Significance tests indicate that these differences are significant at the $1 \%$ level as shown in the last column of Table C.2 in the appendix.

For outcome variables related to the internal efficiency of schools, namely the dropout rate ( $17.784 \%$ for the treatment group and $12.204 \%$ for the control group), the promotion rate ( $80.624 \%$ for treatment group and $84.408 \%$ for the control group), the enrollment rate $(-10.085 \%$ for the treatment group against $-35.226 \%$ for the control group), the results show significant differences between the two groups. For the repetition rate $(5.781 \%$ for the treatment group and $5.492 \%$ for the control group), there was no significant difference between the two groups.

For some control variables, schools in the treatment group display the most important means with significant differences. Among others, those variables are temporary shelters, distance to school, number of hours, disturbance, continuing training, high school diploma and more, Koranic school and Koranic school in the community (Table C.2 in the appendix). On average, we noted that in the treatment group, 1.7 classes are in temporary shelters (against 1.6 classes in the control group), $82.5 \%$ of pupils have their house less than one $\mathrm{km}$ from school (against $78.1 \%$ of pupils in the control group), $98 \%$ have an association of parents (against $95 \%$ for the control group), $32 \%$ have an association of mothers (against $18 \%$ for control group) and $61.7 \%$ of teachers received a continuing training against $58.5 \%$ of teachers in the control group. Note that $45.9 \%$ of the teachers in the treatment group have high school diploma and more, while they are $40.1 \%$ in the control group. Pupils in the treatment group work around 0.2 hours per day against 0.14 hours for pupils in the control group.

For variables household size, pupils' age, primary schools, livestock, absenteeism, national certificate, gender of pupil, land and "college", the control group displayed the highest average compared to the treatment group, with significant differences. The household size in control group is 9.883 persons on average against 9.526 persons in the treatment group. About $53 \%$ of pupils in the control group are boys against $50 \%$ of pupils in the treatment group. About $9 \%$ of schools in the control group reported that their teachers are often absent from school against $5 \%$ of schools in the treatment group.

Taking the characteristics of schools and teachers into account, we observed that approximately $58.9 \%$ of teachers have an academic degree equal to national certificate in the control group, as against $54.9 \%$ in the treatment group. Regarding community characteristics, $27 \%$ of schools in 
the control group are in villages with a "college", while $24 \%$ of schools in the treatment group are in a village with "college".

However, for a number of variables, such as school manual, teacher age, gender of teacher, early childhood institution, gender of household head, literacy of head of household and marital status, there is no significant difference between the two groups. Table C.2 in appendix shows that around $18 \%$ of household heads are literate in the treatment and control groups and around $91 \%$ of them are men. About the characteristics of pupils in each group, about $5 \%$ of them have gone through an earlier childhood institution.

Table C.2 shows that, before the implementation of the program, the two groups are homogeneous only for a few variables, while there are significant differences between the two groups for most variables including outcome variables, except the repetition rate. In addition, Kolmogorov tests of equal distribution performed on continuous variables indicate that the two groups are not equal in all variables (Tables 4.6 and 4.7 ).

Table 4.6: Kolmogorov-Smirnov test: pupil level, 2009

\begin{tabular}{|c|c|c|c|c|c|c|c|}
\hline \multirow[b]{2}{*}{ Variable } & \multicolumn{2}{|c|}{ Treatment } & \multicolumn{2}{|c|}{ Control } & \multicolumn{2}{|c|}{ Combined } & \multirow{2}{*}{$\begin{array}{r}\text { Corrected } \\
\text { p-value }\end{array}$} \\
\hline & $\mathrm{D}$ & p-value & $\mathrm{D}$ & $\mathrm{p}$-value & $\mathrm{D}$ & p-value & \\
\hline Aggregate score & -0.092 & 0.000 & 0.000 & 1.000 & 0.092 & 0.000 & $0.000^{* * *}$ \\
\hline French score & -0.091 & 0.000 & 0.000 & 1.000 & 0.091 & 0.000 & $0.000^{* * *}$ \\
\hline Math score & -0.072 & 0.000 & 0.001 & 0.995 & 0.072 & 0.000 & $0.000^{* * *}$ \\
\hline Repetition rate & -0.084 & 0.000 & 0.081 & 0.000 & 0.084 & 0.000 & $0.000^{* * *}$ \\
\hline Dropout rate & 0.001 & 0.995 & 0.304 & 0.000 & 0.304 & 0.000 & $0.000^{* * *}$ \\
\hline Promotion rate & -0.145 & 0.000 & 0.000 & 1.000 & 0.145 & 0.000 & $0.000^{* * *}$ \\
\hline Enrollment rate & -0.004 & 0.960 & 0.306 & 0.000 & 0.306 & 0.000 & $0.000^{* * *}$ \\
\hline Temporary shelters & -0.054 & 0.006 & 0.098 & 0.000 & 0.098 & 0.000 & $0.000^{* * *}$ \\
\hline School manual & -0.130 & 0.000 & 0.064 & 0.001 & 0.130 & 0.000 & $0.000^{* * *}$ \\
\hline Number of hours & 0.000 & 1.000 & 0.029 & 0.222 & 0.029 & 0.440 & 0.423 \\
\hline Household size ${ }^{a}$ & -0.041 & 0.047 & 0.011 & 0.795 & 0.041 & 0.095 & $0.088^{*}$ \\
\hline Teacher age & -0.053 & 0.006 & 0.078 & 0.000 & 0.078 & 0.000 & $0.000^{* * *}$ \\
\hline Pupil age & -0.033 & 0.138 & 0.021 & 0.454 & 0.033 & 0.076 & 0.261 \\
\hline Primary schools & -0.114 & 0.000 & 0.000 & 1.000 & 0.114 & 0.000 & $0.000^{* * *}$ \\
\hline Livestock & -0.096 & 0.000 & 0.000 & 1.000 & 0.096 & 0.000 & $0.000^{* * *}$ \\
\hline
\end{tabular}

The Mann-Whitney test in Table 4.7 shows that the two groups are not homogeneous for continuous variables, except for the following variables: repetition rate, number of hours, teacher age and pupil age.

Table 4.7: Mann-Whitney test: Pupil level, 2009

\begin{tabular}{|c|c|c|c|c|}
\hline & Treatment & Control & $\operatorname{rank}$ & test \\
\hline Variable & \# Obs. & \# Obs. & $z$ & $p>|z|$ \\
\hline Aggregate score & 1886 & 1648 & 6.225 & $0.000^{* * *}$ \\
\hline
\end{tabular}


Table 4.7 - continued

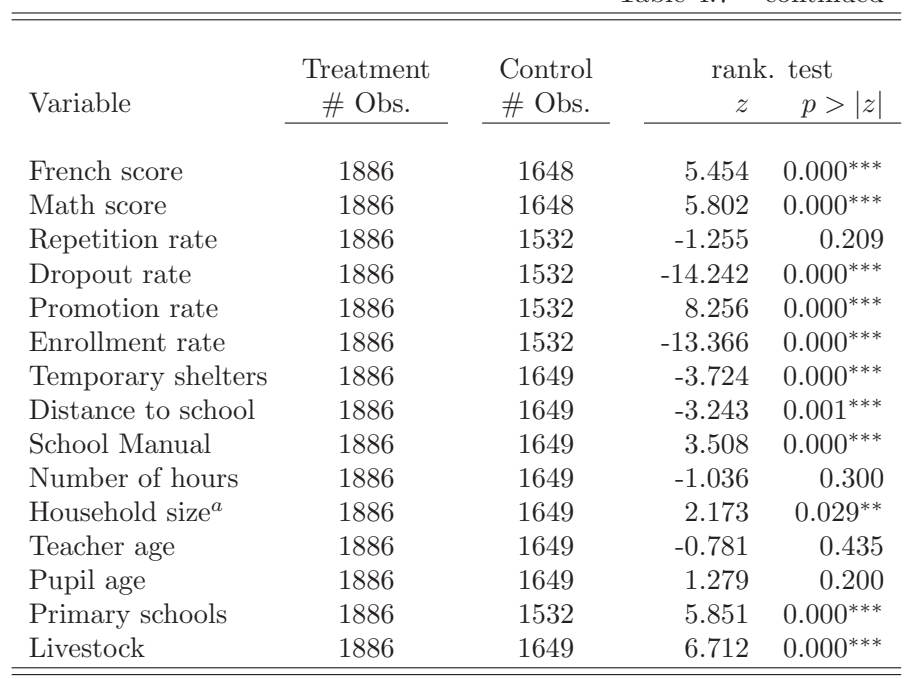

Note $^{a}$ : Household size per adult equivalent.

Significance levels: $\quad *: 10 \% \quad * *: 5 \% \quad * * *: 1 \%$

Considering the results of the parametric and non-parametric tests performed at the pupil level, we can say that the two groups are not, on average, identical to the view of all the variables taken individually. Verification of the homogeneity of the two groups with regard to variables taken simultaneously is needed. Thus, as in the case of the tests performed in the school level, a Hotelling test and a MANOVA were performed.

Table 4.8: Hotelling test: Pupil level, 2009

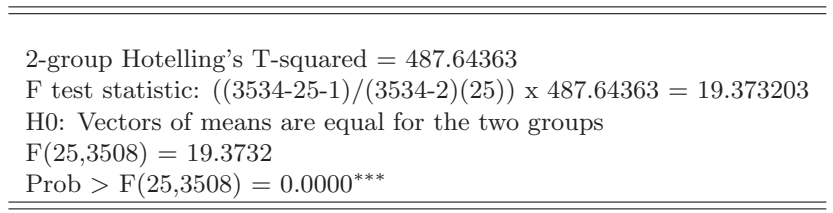

Significance levels: $\quad *: 10 \% \quad * *: 5 \% \quad * * *: 1 \%$

The Hottelling test and the MANOVA performed at the pupil level on all variables indicates that the two groups are not homogeneous at the baseline (Tables 4.8 and 4.9). 
Table 4.9: MANOVA on quantitative variables: Pupil level, 2009

\begin{tabular}{|c|c|c|c|c|c|c|}
\hline $\begin{array}{l}\text { Number of obs }=3418 \\
\mathrm{~W}=\text { Wilks' lambda } \mathrm{L}=\text { Lawley-Hotelling trace } \\
\mathrm{P}=\text { Pillai's trace } \mathrm{R}=\text { Roy's largest root } \\
\text { Source }\end{array}$ & Statistic & df & $\mathrm{F}(\mathrm{df} 1$, & $\mathrm{df} 2)=$ & $\mathrm{F}$ & Prob $>F$ \\
\hline group & $\begin{array}{rr}\text { W } & 0.8787 \\
\text { P } & 0.1213 \\
\text { L } & 0.1381 \\
\text { R } & 0.1381 \\
\end{array}$ & 1 & $\begin{array}{l}25.0 \\
25.0 \\
25.0 \\
25.0\end{array}$ & $\begin{array}{l}3508.0 \\
3508.0 \\
3508.0 \\
3508.0 \\
\end{array}$ & $\begin{array}{l}19.37 \\
19.37 \\
19.37 \\
19.37\end{array}$ & $\begin{array}{l}0.000^{* * *} \mathrm{e} \\
0.000^{* * *} \mathrm{e} \\
0.000^{* * *} \mathrm{e} \\
0.000^{* * *} \mathrm{e}\end{array}$ \\
\hline Residual & & 3532 & & & & \\
\hline Total & & 3533 & & & & \\
\hline
\end{tabular}

In view of the results of testing pupil level, we noted that the two groups are not homogeneous before program implementation unlike the results obtained with tests carried out at school level.

\section{Descriptive statistics on the status of non-response}

In randomized experiments, it is common to encounter problems related to non-response or attrition. Non-response problems occur when we are unable to obtain information on the outcome variable of some observations after program implementation.

As part of this research, despite efforts deployed to prevent attrition problem, from 4110 pupils, 1150 pupils have not been tested in the second survey (after the program). We noted an attrition rate of $27.98 \%$. For the non-response, 518 pupils are part of the control group, while 632 pupils are part of the treatment group. The attrition rate for treatment and control groups are $28.35 \%$ and $26.53 \%$, respectively. In terms of geographic location, on 1150 pupils that have not been tested, $97(8.43 \%)$ are in Diourbel, $266(23.13 \%)$ in Sédhiou, $376(32.7 \%)$ in Fatick and 411 $(35.74 \%)$ in the Kolda region.

The non-response problem is mainly due to the fact that the team responsible for administering the tests went to regions during the rainy season. During this season, some pupils help parents in the fields. In addition, in some schools, classes in temporary shelters were not working because of the rainy season and therefore the pupils were absent. So many pupils were absent on the day of testing.

To reduce the non-response rate, some pupils had been sought in the villages or in the fields with the help of the local administration. Irrespective of this efforts, we noted non-response problem. To understand whether non-response occurred randomly, it is important to compare pupils for which we noted non-response, with those for which we do not have non-response problem.

We distinguish two groups of pupils: the pupils on whom we did not have non-response problem, are now label non-attritors and the pupils on whom we have the non-response problem are now label attritors.

In what follows, we first compare attritors and non-attritors. Then, we make a comparison of the two groups of pupils within each group. Finally, we compare attritors in control group with those in the treatment group (Tables C.3 and C.4).

Comparison between attritors and non-attritors

As suggested by Duflo et al. (2008) it is important to compare attritors and non-attritors to see if non-response has occurred randomly or not. Table C.3 in the appendix allows making 
a comparison between attritors and non-attritors using the baseline survey.

Regarding the academic performances of pupils, we realized that non-attritors have the highest scores, compared to attritors. Similarly, the aggregate score, French score and math score for non-attritors, are 40.516, 40.370 and 40.663 respectively, while those for attritors are 36.082, 35.929 and 36.193. The differences are significant and are about four points in favor of nonattritors. For variables related to internal efficiency of schools, we observe that the repetition rate of attritors that is $6.435 \%$ is significantly higher than repetition rate for non-attritors which is $5.373 \%$. Likewise, the dropout rate of attritors which is $16.865 \%$, is significantly higher than the dropout rate of non-attritors $(14.721 \%)$. The promotion rate of non-attritors which is about $82.882 \%$ is significantly higher than the promotion rate of attritors $(80.739 \%)$. For control variables literacy of household head, one can see that around $19 \%$ of household head for the non-attritors are literate against $16 \%$ for attritors. Also, attritors work in average 0.20 hour per day against 0.16 hour for non-attritors. Regarding school characteristics, one observes that for attritors, on average, we have 1.7 classes in temporary shelters against 1.6 classes for non-attritors (see table C.3). In sum, statistics show that attritors have less literate household heads, the largest number of classes in temporary shelters and mostly live at more than one mile from school compared to non-attritors.

Furthermore, the last column of table C.3 shows that there is no significant differences between the two groups of pupils for variables household size and primary schools. But for other control variables such school manual, pupil age, and Koranic school in the community attritors display the highest value with significant differences.

Table 4.10 on Hottelling test shows that for all outcomes and control variables taken simultaneously, there is a significant difference between the two group of pupils at $1 \%$.

In summary, before the implementation of the program, attritors and non-attritors are not homogeneous.

Table 4.10: Hotelling test: attritors versus non-attritors, 2009

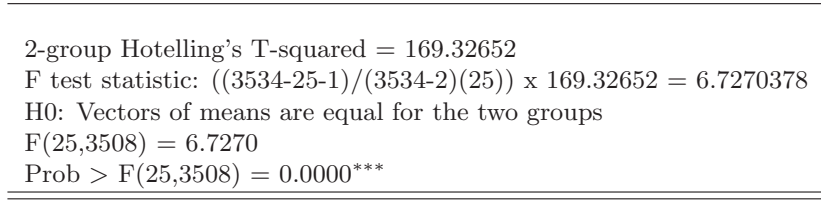

Significance levels: $\quad *: 10 \% \quad * *: 5 \% \quad * * *: 1 \%$

\section{Comparison between attritors and non-attritors within each group}

Table C.3 in the appendix allows us to compare attritors and non-attritors within each group for each variable taken individually using baseline survey.

For the treatment group, Table C.3 indicates that there are significant differences between attritors and non-attritors for all outcome variables. For some of the control variables, household size, literacy of household head, number of hours and Koranic school in the community, significant differences between the two groups of pupils do occur. However, for other control variables, there are significant differences between the two groups of pupils. These variables are among others, temporary shelters (2.017 classes in temporary shelters for attritors against 1.665 for nonattritors), school manual (1.321 pupils per manual for attritors against 1.066 for non-attritors), pupil age (9.86 years for attritors against 9.49 for non-attritors), and land (89\% of households have farmland for attritors against $94 \%$ of households for non-attritors).

For the control group, Table C.3 in the appendix shows that there are significant differences 
between attritors and non-attritors for pupils' achievement. Regarding outcome variables related to the internal efficiency of schools, there are significant differences between the two groups of pupils for promotion and dropout rates. However, we do not find significant difference between the two groups for repetition rate.

Unlike the treatment group, in the control group, there is no significant difference between attritors and non-attritors for most of the control variables. However, for other control variables, there are significant differences between the two groups of pupils. These variables are: temporary shelters, distance to school, number of hours and literacy of household head.

Tables 4.11 and 4.12 related to the hotelling test in the treatment and control groups show that there are significant differences between attritors and non-attritors for all variables (outcomes and controls) taken simultaneously.

Table 4.11: Hotelling test for treatment group: attritors vs. nonattritors, 2009

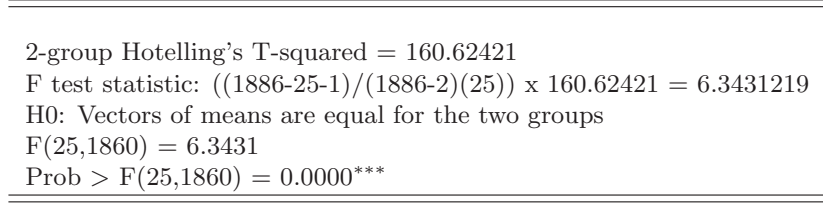

Significance levels: $\quad *: 10 \% \quad * *: 5 \% \quad * * *: 1 \%$

Table 4.11 shows that there is a significant difference between attritors and non-attritors in the treatment group for all variables taken simultaneously.

Table 4.12: Hotelling test for control group: attritors vs. non-attritors, 2009

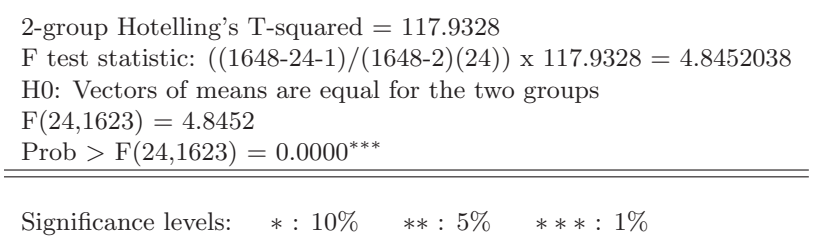

Table 4.12 indicates that there is a significant difference between attritors and those nonattritors in the control group for all variables taken simultaneously.

Comparison of attritors in the treatment group with those in the control group

On pupils' achievement, Table C.4 shows that, on average, attritors in the control group have the highest scores (aggregate, French and math) with significant differences. The aggregate score, French score and math score for attritors in the control group are 39.762, 39.053 and 40.471, while those in the treatment group are $33.172,33.459$ and 32.811 respectively.

Regarding outcome variables related to internal efficiency of schools, one observes that repetition rate and dropout rate in the treatment group $(7.156 \%$ and $18.965 \%$, respectively) are higher than those in the control group $(5.457 \%$ and $14.013 \%$, respectively), with significant differences. 
For variables, household size, livestock and land attritors in the control group have the highest average with significant differences. For variables temporary shelters, school manual, literacy of household head and pupil age, attritors in the treatment group have the highest averages with significant differences.

Table 4.13: Hotelling test on attritors: treatment vs. control, 2009

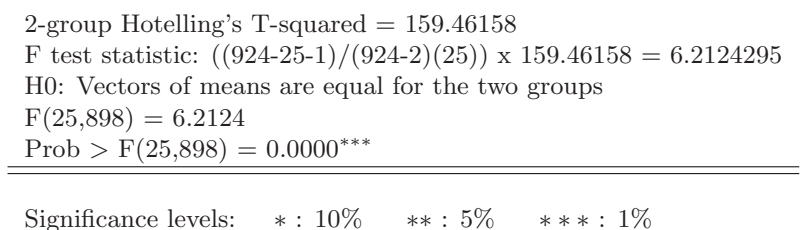

The Hotelling test in Table 4.13 shows that, given all variables, there is a significant difference between attritors in the treatment group and those in the control group.

In conclusion, non-response does not occur randomly. Therefore, it is important to correct the attrition problem when measuring the impact of school canteens on school performances.

We now proceed to compare between the treatment and control group after implementation of the food program.

\subsubsection{Descriptive statistics after program}

Table C.5 in the appendix provides descriptive statistics for treated and control groups after implementation of the meals program, as well as the evolution of each group between the two periods (see last two columns of the table).

Taking academic results into consideration, we observe that on average, the pupils' scores in the treatment group are higher than those in the control group in contrast to what we observed before the implementation of the program. Therefore, the scores (aggregate, French and math) of pupils in the treated group became, 59.851, 56.694 and 62.563. Scores of pupils in the control group became, 54.308, 51.819 and 56.423 for aggregate score, French and math scores, respectively. There is a significant difference in scores between the two groups.

It is important to note that we observe almost a doubling of educational outcomes in the control group. This could be justified by two reasons. First, when the implemented program has a positive effect on academic achievement, the presence of a partial adhesion (non-compliance) can contribute to increase in academic performance of pupils in the control group. Second, this situation can be explained by the fact that the first-pass tests were carried out just after the big holidays that officially lasted for three months, while those in the second passage took place at the end of the school year. The fact that the pupils remained a long time without learning may explain the poor results of the first test compared to second passage.

On the internal efficiency of schools, on average, repetition rate, promotion rate, dropout rate and enrollment rate in the control group are, $1.596 \%, 85.113 \%, 11.323 \%$ and $-22.705 \%$, respectively, while they are $3.830 \%, 80.580 \%, 11.845 \%$ and $-7.439 \%$ for the treatment group. There are significant differences between the two groups.

However, for some variables, we do not find significant differences between the two groups after the experiment. These variables are, among others, dropout rate, temporary shelters, household size, teacher age, pupil age, absenteeism, continuing training, Koranic school, earlier childhood institution, literacy of household head and gender of household head. 
For variables distance to school, number of hours, disturbance, gender of teacher, high school diploma and more, marital status and Koranic school in community, pupils in the treatment group have the highest average with significant differences. For variables school manual, livestock, primary schools, gender of pupil, national certificate, land and college, pupils in the control group have the highest average with significant differences (Table C.5 in the appendix).

In terms of evolution of treated and control groups between the two periods, there are no significant changes in the control group for some variables including temporary shelters, distance to school, household size, livestock, number of hour, primary schools, gender of teacher, continuing training, gender of pupil and college (see last column of Table C.5 in the appendix). In the treated group, the last column of Table C.5 in the appendix shows that there is no significant change between the two periods given a number of variables including distance to school, school manual, livestock, number of hours, land and continuing training.

For example, one observes that the number of classes under temporary shelters in the treatment group decreased from 1.71 to 1.65 between the two periods. However this number remains almost the same for the control group (this number increased from 1.57 to 1.60 with no significant difference).

\section{Partial adherence}

Despite the simplicity offered by randomized experiments compared to observational studies in assessing the impact of social programs, they are not without difficulties. In addition to the attrition problem mentioned above, Table 4.14 shows non-compliance or partial adherence. In other words, all schools assigned to the treatment group did not receive the school feeding program and some schools assigned to the control group received the program. In fact, 62 schools (2160 pupils) are assigned to the treatment group, but only 54 schools (1887 pupils) have actually benefited from the program (87\% of compliance). Out of 58 schools (1950 pupils) assigned to the control group, 46 (1510 pupils) have not benefited from the program (79\% of compliance). So, 8 schools (273 pupils) in the treatment group have not benefited from the program (13\% of non-compliance), while 12 schools (440 pupils) in the control group benefited (21\% of non-compliance). Some schools in the control group were able to receive treatment during the experiment because in addition to WFP which is one of the partners of this experiment, there are other non-governmental organizations (NGO) that implement canteens in rural Senegal. So, some schools in the control group have benefited from the canteens implemented by NGOs. Some schools in the treatment group did not receive treatment for administrative reasons we could not clearly understand. When we called the directors of schools, they reported that they were promised to be receiving food from time to time. They waited in vain without any explanations.

Table 4.14: Treatment planned versus treatment received

\begin{tabular}{lcccc}
\hline \hline & \multicolumn{2}{c}{ Treatment received } & \multirow{2}{*}{ Total } \\
\cline { 2 - 4 } & & 0 & 1 & \\
\hline Planned Treatment & 0 & $46(79 \%)$ & $12(21 \%)$ & 58 \\
& 1 & $8(13 \%)$ & $54(87 \%)$ & 62 \\
Total & & 54 & 66 & 120 \\
\hline \hline
\end{tabular}

Table 4.15 presents comparison between pupils assigned to the treatment group and those who actually received the treatment, and pupils assigned to the treatment group that did not 
received, using the baseline survey.

Table 4.15: Comparison of adhesion status, 2009

\begin{tabular}{|c|c|c|c|c|c|}
\hline \multirow[b]{2}{*}{ Variable } & \multicolumn{2}{|c|}{ Planned treatment, received } & \multicolumn{2}{|c|}{ Planned treatment, not received } & \multirow{2}{*}{$\begin{array}{l}\text { t-test } \\
\text { p-value }\end{array}$} \\
\hline & Mean & Std. Err. & Mean. & Std. Err. & \\
\hline Aggregate score & 36.269 & 17.542 & 43.746 & 24.258 & $0.000^{* * *}$ \\
\hline French score & 36.117 & 19.438 & 44.640 & 24.113 & $0.000^{* * *}$ \\
\hline Math score & 36.412 & 19.768 & 42.918 & 27.046 & $0.000^{* * *}$ \\
\hline Repetition rate & 6.718 & 7.752 & 3.965 & 4.390 & $0.000^{* * *}$ \\
\hline Dropout rate & 17.264 & 11.993 & 18.197 & 11.281 & 0.227 \\
\hline Promotion rate & 76.360 & 12.554 & 77.836 & 11.209 & $0.066^{*}$ \\
\hline Enrollment rate & 17.264 & 11.993 & 18.197 & 11.281 & 0.227 \\
\hline Temporary shelters & 1.921 & 1.383 & 1.294 & 0.957 & $0.000^{* * *}$ \\
\hline Distance to school & 0.822 & 0.382 & 0.897 & 0.304 & $0.002^{* * *}$ \\
\hline School Manual & 1.112 & 1.250 & 1.261 & 0.757 & $0.055^{*}$ \\
\hline Household size ${ }^{a}$ & 10.992 & 6.394 & 13.455 & 9.072 & $0.000^{* * *}$ \\
\hline Teacher age & 32.035 & 5.735 & 28.904 & 4.442 & $0.000^{* * *}$ \\
\hline Pupil age & 9.642 & 1.662 & 9.828 & 1.954 & $0.093^{*}$ \\
\hline Primary school & 1.257 & 0.526 & 1.235 & 0.424 & 0.515 \\
\hline Livestock & 7.074 & 14.499 & 19.566 & 26.855 & $0.000^{* * *}$ \\
\hline
\end{tabular}

Note $^{a}$ :Household size per adult equivalent.

Significance levels: $\quad *: 10 \% \quad * *: 5 \% \quad * * *: 1 \%$

Regarding the academic performance of pupils, the group initially assigned to the program, but that did not receive treatment has the highest scores with significant differences. On internal efficiency, we do not find significant differences between the two groups of pupils for variables dropout rate and enrollment rate. But for the repetition rate, the group of pupils that did not receive treatment displayed the lowest rate, while the group that received the treatment as planned displays the lowest promotion rate. So, the table shows that the partial adhesion did not occurs randomly, which means that it is essential to correct this in order to obtain the effect of the program on those who actually received the treatment.

In summary, four regions in Senegal, namely, Fatick, Kolda, Diourbel and Sédhiou were chosen to host the experiment program. The intervention consisted of providing a hot lunch through the implementation of a school canteen. The sampling strategy consisted first to select geographical locations, then to constitute a population of schools eligible to perform a random selection of 120 schools to participate in the experiment. These schools were randomly divided between a treatment group and a control group. In each school, 40 pupils were selected from the grade of second year (CP) and fourth year (CE2) to participate in the experiment. It is important to note that all pupils in treated school have benefited from school feeding. The variables available are of two types: i) outcome variables (pupils' achievement and internal efficiency of schools) and ii) control variables on schools and teachers characteristics, pupils' characteristics and their households, as well as the environment in which they live. Before the implementation of the program, the results of various test carried out at school level show that there are no differences between the treatment group and the control.

We noted problem of non-compliance and non-response. In the literature, there are ways to correct non-compliance and non-response separately. However, other approaches have been developed to address the problems related to non-response and non-compliance simultaneously (Horiuchi et al., 2007). 


\subsection{Econometrics specification}

As described above, the experiment consists of a set of data concerning two periods. In this section, we present an analytical framework of non-compliance and non-response (Section 4.4.1) before presenting a model taking the two problems into account simultaneously (Section 4.4.2).

\subsubsection{Partial adhesion and attrition: framework}

As mentioned above, in this experiment non-compliance has occurred. Instrumental variables are often used to correct the problem of non-compliance or partial adhesion; however, the method becomes inefficient in the presence of attrition (Horiuchi et al., 2007; Esterling et al., 2011).

As already mentioned in the descriptive statistics, from 4110 pupils, 1150 have not been tested in the investigation of the second survey, while an attrition rate of $27.98 \%$ ( $26.53 \%$ in the control group against $28.35 \%$ in the treatment group) is observed.

The removal of observations with non-response, as is often the case in experimental studies, produces inefficient results and may bias the results (Horiuchi et al., 2007). Even if non-response occurs randomly, removing observations with non-response changes the target population for which causal effects are estimated. This is true even when the missing data is not affected by the assignment mechanism and the treatment, because non-attritors are generally systematically different from attritors. In addition to bias, inefficiency can come from the loss of information due to the exclusion of certain observations in the analysis.

In the literature, several approaches have been developed to face the problems of attrition and partial adhesion simultaneously (Imai, 2006; Horiuchi et al., 2007; Esterling et al., 2011; Barnard et al., 2003).

From the Bayesian modeling framework of Imbens and Rubin (1997) that can process both partial adherence and non-response problems, Horiuchi et al. (2007) developed a model to take account of attrition and non-compliance problems. We use this framework to assess the impact of school feeding program on academic performance in the presence of partial adherence and attrition. Let us first present, in the context of our study, the general statistical framework of random experiments with partial adherence and attrition introduced by Angrist et al. (1996) and generalized by Frangakis and Rubin (2002).

\section{Partial adhesion}

We begin by describing the statistical framework of Angrist et al. (1996) in the context of our study which is on a school feeding program.

Let $Z_{i}$ be the status of initial treatment equal to 1 if it is anticipated that the pupil $i$ receives a school canteen and 0 otherwise. Let $T_{i}(z)$ be a potential treatment variable given the assignment to treatment $Z_{i}=z$. The real or effective treatment that is equal to 1 if the pupil has actually benefited from a school canteen and 0 otherwise can be defined as follows: $T_{i} \equiv Z_{i} T_{i}(1)+\left(1-Z_{i}\right) T_{i}(0)$. If it is anticipated that the pupil $i$ receives access to a school canteen (does not receives access) but it does not benefit (it does benefit) then $Z_{i}=1\left(Z_{i}=0\right)$ and $T_{i}(0)=0\left(T_{i}(0)=1\right)$ then $T_{i}=0\left(T_{i}=1\right)$. In this experiment, $13 \%$ of pupils in the treatment group do not benefit from the program, while $21 \%$ of pupils in the control group does finally benefit from the program.

Given the assignment to treatment, the potential outcome variable is define as follows: $Y_{i}(z) \equiv$ $Y_{i}\left(Z_{i}=z\right)$. The actual outcome variable is defined as: $Y_{i} \equiv Z_{i} Y_{i}(1)+\left(1-Z_{i}\right) Y_{i}(0)$. For example in the group of treatment assignment, $Y(1)$ is observed but $Y(0)$ is not.

We also define two types of individuals, compliers and non-compliers. Compliers are pupils who remain in their initial treatment status $\left(T_{i}(1)=1\right.$ and $\left.T_{i}(0)=0\right)$ while non-compliers are those who do not follow the instructions. That is to say, those who deviate from their initial treatment assignment status. Let $C_{i}$ be a variable of indicator of compliance that equal to 1 if the pupil $i$ is a complier and 0 otherwise. 
One can distinguish three types of non-compliers. The "always-taker" namely those who benefit from the canteen independently of whether they are assigned to the treatment group $\left(T_{i}(1)=\right.$ $\left.T_{i}(0)=1\right)$, the "never-taker" that is to say those who do not benefit from the canteen independently of whether they are assigned to the group of beneficiary $\left(T_{i}(1)=T_{i}(0)=0\right)$ and the "defiers" namely those who benefit from the canteen only when they are assigned to the control group $\left(T_{i}(1)=0\right.$ et $\left.T_{i}(0)=1\right)$.

Table 4.16: Complier versus Noncomplier

\begin{tabular}{|c|c|c|c|}
\hline & & \multicolumn{2}{|c|}{ Planned Treatment } \\
\hline & & $Z_{i}=1$ & $Z_{i}=0$ \\
\hline Treatment received & $\begin{array}{l}T_{i}=1 \\
T_{i}=0\end{array}$ & $\begin{array}{c}\text { C: } Y_{i}(1) \text { observed; } C_{i}=1 \\
\text { NC: } Y_{i}(1) \text { observed; } C_{i}=0\end{array}$ & $\begin{array}{c}\text { NC: } Y_{i}(0) \text { observed; } C_{i}=0 \\
\text { C: } Y_{i}(0) \text { observed; } C_{i}=1\end{array}$ \\
\hline
\end{tabular}

C: Complier; NC: Noncomplier.

In our experiment, unlike Horiuchi et al. (2007), there are always-takers because we have information on the status of adherence of pupils in the control group. Table 4.16 shows that from the observed data, we have the adhesion status of pupils in the treatment and control groups. A high proportion of compliers are paramount for a successful statistical analyzes of randomized experiments. In this study, the proportion of complier is about $83 \%$, which is superior to other proportions found in the context of other randomized experiments. For example, the proportion of compliers is about $70 \%$ in the study conducted by Horiuchi et al. (2007).

\section{Attrition}

In this experiment, despite efforts, attrition has occurred because many pupils were absent on the day of the test for second survey. At first, one considers the approach of random attrition -MAR-. Since attrition may be affected by the assignment to treatment, it is important to introduce a potential response which is an indicator variable, $R_{i}(z)$ for $z \in\{0,1\}$. For example, $R_{i}(1)=1$ and $R_{i}(0)=0$ means that pupils $i$ will be tested if assigned to the treatment group, but will not be tested if assigned to the treatment group. The observed indicator variable is given by $R_{i} \equiv Z_{i} R(1)+\left(1-Z_{i}\right) R(0)$, hence the formalization of the hypothesis of missing at random (MAR):

$$
\begin{aligned}
& P\left(R_{i}(z)=1 \mid Y_{i}(z)=1, T_{i}(z)=t, Z_{i}=z, X_{i}=x\right) \\
= & P\left(R_{i}(z)=1 \mid Y_{i}(z)=0, T_{i}(z)=t, Z_{i}=z, X_{i}=x\right),
\end{aligned}
$$

for all $t, z \in\{0,1\}$, where $X_{i}$ represents a vector of pretreatment variables. This hypothesis implies that after conditioning on planned and actual treatments, on variables as well as on pretreatment covariates, the pattern of attrition is not systematically related to the outcome variable.

Second, the latent ignorability approach is an alternative hypothesis (Barnard et al., 2003; Frangakis and Rubin, 1999). This hypothesis can be formalized as follows:

$$
\begin{aligned}
& P\left(R_{i}(z)=1 \mid Y_{i}(z)=1, C_{i}=c, Z_{i}=z, X_{i}=x\right) \\
= & P\left(R_{i}(z)=1 \mid Y_{i}(z)=0, C_{i}=c, Z_{i}=z, X_{i}=x\right),
\end{aligned}
$$

for all $c, z \in\{0,1\}$. This assumption conditions on covariate $c$ rather than on the observed treatment variable. 
The third hypothesis is the approach of non-ignorability-NI- (Imai, 2006). Contrary to MAR and LI assumptions, NI assumption is appropriate if the missing data mechanism depends directly on the values of the outcome variable itself (probably not observed). Formally we have:

$$
\begin{aligned}
& P\left(R_{i}(1)=1 \mid T_{i}(1)=t, Y_{i}(1)=y, Z_{i}=1, X_{i}=x\right) \\
= & P\left(R_{i}(0)=1 \mid T_{i}(0)=t, Y_{i}(0)=y, Z_{i}=0, X_{i}=x\right)
\end{aligned}
$$

for all $t \in\{0,1\}$. This assumption means that the missing data mechanism does not depend on the assignment to the treatment provided, once we condition on the outcome variable as well as the actual treatment received and observed pretreatment variables. The relevance of each assumption depends on the context of the experience, and sensitivity analysis which plays an important role when considering the robustness in the resulting conclusions (Horiuchi et al., 2007). However, the assumption is made regarding the missing data mechanism. A key point has been to include significant predictors of the outcome variable among the $X_{i}$. Such variables also lead to an effective estimation of causal effects because they help to predict precisely the missing values of the outcome variable. Finally, it is important to note that in our experiment, there are no missing values on the outcome variables before the program. We observe missing values only after the program. ${ }^{1}$

\subsubsection{Partial adhesion and attrition: the model}

The model consists of two parts Bayesian modeling: the first describes the partial adhesion and the second reflects on the outcome variable. For partial adhesion (non-compliance), a probit model with linear predictors is defined as follow:

$$
\mathbb{P}\left(C_{i}=1 \mid \mathbf{x}_{i}\right)=\Phi\left(\mathbf{x}_{i}^{\prime} \boldsymbol{\alpha}\right),
$$

where $\Phi($.$) is the cumulative distribution function of the standard normal, X_{i}$ represents the control variables that may include dummies and $\boldsymbol{\alpha}$ is the coefficient vector to be estimated.

Given the status of adhesion $C_{i}$, the assignment to treatment $Z_{i}$ and observable variables $X_{i}$, the outcome variable is modeled as a linear relation by the following equation:

$$
Y_{i}=\theta C_{i} Z_{i}+\beta C_{i}\left(1-Z_{i}\right)+\mathbf{x}_{i}^{\prime} \gamma+\varepsilon_{i},
$$

where $\varepsilon_{i}$ is an iid error term, $\theta$ and $\beta$ are intercepts related to compliers of the treatment and control groups respectively, and $\gamma$ is the vector of parameters for the $\mathbf{x}_{i}$ which includes an intercept. Combining the two relations (1-4) and (1-5) we can write the likelihood function:

$$
L=\prod_{i=1}^{N}\left[\Phi\left(\mathbf{x}_{i}^{\prime} \boldsymbol{\alpha}\right) \varphi\left(Y_{i} \mid Z_{i}, \mathbf{x}_{i}\right)\right]^{C_{i}}\left[\left\{1-\Phi\left(\mathbf{x}_{i}^{\prime} \boldsymbol{\alpha}\right)\right\} \varphi\left(Y_{i} \mid Z_{i}, \mathbf{x}_{i}\right)\right]^{1-C_{i}}
$$

with $\varphi\left(Y_{i} \mid Z_{i}, \mathbf{x}_{i}\right)=\frac{1}{\sigma_{\varepsilon}} \phi\left(\frac{Y_{i}-\theta C_{i} Z_{i}-\beta C_{i}\left(1-Z_{i}\right)-\mathbf{x}_{i}^{\prime} \gamma}{\sigma_{\varepsilon}}\right)$ where $\phi$ denotes the univariate standard normal density function. This likelihood function cannot be estimated directly because the value of the outcome variable $Y_{i}$ is missing for some pupils due to attrition. We use the imputation based method for missing data in which each iteration of the estimation procedure imputes missing values of $Y_{i}$ using. This process is repeated until convergence holds, Horiuchi et al. (2007). In the estimation procedure, independent prior distributions are assigned to $\theta, \beta$ and $\gamma$ and the joint posterior distribution is sampled using a Markov chain Monte Carlo (MCMC) method.

\footnotetext{
${ }^{1}$ See Barnard et al. (2003) for the problem of missing data before and after the program.
} 


\section{Treatment effects}

Two types of treatment effects can be calculated, the intention to treat effect (ITT) and the complier average causal effect (CACE).

The ITT is given by:

$$
I T T=\frac{1}{N} \sum_{i=1}^{N}\left[Y_{i}(1)-Y_{i}(0)\right]
$$

It represents the effect of being assigned to the treatment group rather than the effect of actually benefiting from the school feeding program. Although the ITT does not represent the effect of treatment actually received, from the perspective of the decision maker who would generalize the program, ITT can be of great interest. However, in the presence of attrition, the estimated effect of ITT is not simpler and requires making additional assumptions about the missing data mechanism. In fact, this simple average difference estimator will be biased (Frangakis and Rubin, 1999).

In addition, we may also be interested in estimating the effect of a program on individuals who actually received the treatment instead of estimating the ITT. Given the assumptions on partial adherence and attrition, it is possible to identify the causal effect on beneficiaries (Angrist et al., 1996).

We focus on the causal effect of treatment on participants. The complier average causal effect $(\mathrm{CACE})$ is defined as follows:

$$
C A C E=\frac{\sum_{i=1}^{N} C_{i}\left[Y_{i}(1)-Y_{i}(0)\right]}{\sum_{i=1}^{N} C_{i}}
$$

In this experiment, CACE represents the causal effect of the program on pupils who benefit from the program. It is important to note that this effect is different from the sample average treatment effect (ATE), which concerns the entire population. As the ITT effect for non-compliers is zero under the assumption that the assignment to treatment does not directly affect the outcome variable, the $C A C E$ will always be larger than the ITT effect.

\subsection{Findings}

In this section, we present the findings of our analysis. We have two types of outcome variables namely those on pupils performance (aggregate score, French score and math) and those on the internal efficiency of schools (enrollment, promotion, repetition and dropout). It is important to remember that we are in the presence of partial adherence and attrition problems. The presence of partial adhesion led us to first compute the intention to treat effect (ITT) before calculating the effect of the program on those who actually received it (CACE). We used a model taking of both the problems of attrition and partial adherence into account. For each outcome variable, we performed the impact of the school feeding by grade and gender of the pupil.

Our inference is based on Monte Carlo samples from three independent Markov chains, each of which has the length of 50,000 and is initiated at different sets of starting values. We found out that all parameters have the values of the Gelman-Rubin (GR) convergence statistic that are less than 1.01 (meaning $1<G R<1.01$ ). This statistic suggests that a satisfactory degree of convergence has been achieved. We discard the first 20,000 draws from each chain and retain the last 30,000 , thus, we based our inference on a combined total of 90,000 posterior draws. ${ }^{2}$

\footnotetext{
${ }^{2}$ Unlike to Chapters 2 and 3 where all computations are performed with STATA, in this Chapter 4, the R software is used for estimations.
} 
In the following subsections, we first present the impact of school feeding on pupils' performances before presenting its effect on the internal efficiency of schools. In order to save space, we summarize the different effects calculated without presenting the integrality of estimates.

\subsubsection{Impact of school feeding on pupils' performances}

For all outcome variables related to pupils' performances (aggregate score, French score and math score) positive effects are expected. Table 4.17 shows the means of the ITT effect and the $\mathrm{CACE}$ on scores. The table clearly shows that the school feeding program has a positive effect on pupils' achievement. So, the results give us expected effects. For the aggregate score, the ITT is 4.318 points of percentage, while the CACE is 7.616 points of percentage with a standard deviations of 0.726 and 1.267 for the ITT effect and the CACE, respectively. The confidence intervals show that these effects are significant.

Table 4.17: Effect of school feeding on pupils' performance

\begin{tabular}{lcccr}
\hline \hline & & \multicolumn{3}{c}{ Confidence interval } \\
& Mean & S.D & $2.5 \%$ & $97.5 \%$ \\
\hline Aggregate score & & & & \\
Intention to treat effect (ITT) & 4.318 & 0.726 & 2.899 & 5.746 \\
Complier average causal effect (CACE) & 7.616 & 1.267 & 5.133 & 10.109 \\
French score & & & & \\
Intention to treat effect (ITT) & 3.769 & 0.817 & 2.180 & 5.377 \\
Complier average causal effect (CACE) & 6.645 & 1.431 & 3.858 & 9.459 \\
Math score & & & & \\
Intention to treat effect (ITT) & 4.740 & 0.804 & 3.171 & 6.311 \\
Complier average causal effect (CACE) & 8.406 & 1.407 & 5.659 & 11.162 \\
\hline \hline
\end{tabular}

For the French score the ITT effect is 3.767 points of percentage and the CACE is 6.645 points of percentage with a standard deviations of 0.817 and 1.431, respectively. For the Math score, the ITT effect is 4.740 points of percentage and the CACE is 8.406 points of percentage with a standard deviations of 0.804 and 1.407 , respectively. Therefore, the effect on math score is higher than that of the French. As expected, one also observes that for all scores, the CACE is greater than the ITT effect. 
Figures 4.1 and 4.2 represent histograms of the posterior distribution of the CACE and the ITT effect for the aggregate score, French and math scores, respectively.

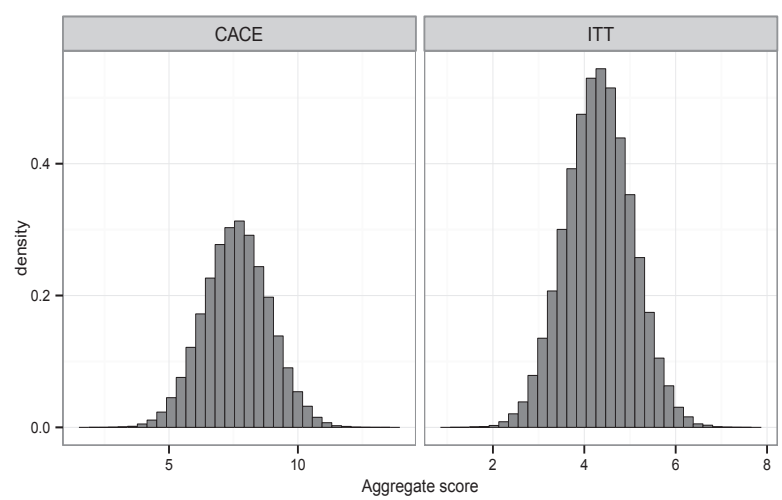

Figure 4.1: Histogram of posterior distribution for aggregate score: CACE and ITT effect.

Figure 4.1 shows the histograms on the distribution of the CACE and the ITT effect for the aggregate score. One can sees that the CACE range between 4.5 and 10.5 points of percentage and the ITT effect varies between 2 and 6.5 points of percentage. 
Figure 4.2 shows the histograms on the distribution of the CACE and ITT effect for French and math scores. Here also, one can sees that the CACE ranges from 4.5 to 10.5 points of percentage and from 4 to 12.5 points of percentage for French and math scores, respectively. The ITT effects which are smaller than the CACE range from 1 to 6 points of percentage and from 2.5 to 7.5 point of percentages for French and math scores, respectively.
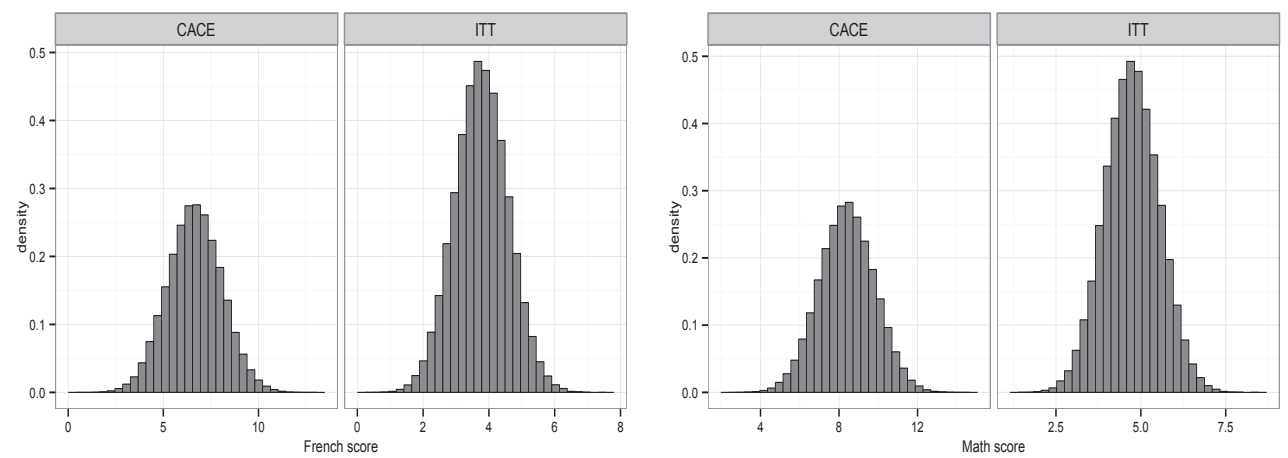

Figure 4.2: Histogram of posterior distribution for French and math scores: CACE and ITT effect.[left]:French score [right]: Math score

For policy makers or NGO or donors, this finding is important because one can see that the effect of the canteen on pupils' performances (scores) is positive everywhere. This means that in average, the impact of the program is positive for all simulated parameters contrary to what we will find in what follows in other outcomes namely the promotion rate.

\section{Impact heterogeneity}

It is interesting to study the effects of the school feeding program on some subgroups. This exercise allows us to understand if the impact of the program is different from one subgroup to another. It is thought for example that the program's impact might differ according to gender or according to the grade (class) of the pupil.

Impact of school feeding on pupils' performance by gender

Table 4.18 gives the ITT effect and the CACE for boys and girls. For aggregate and math scores, the impact of the programme on girls is greater than boys, while in French the effect of the intervention is greater for boys as compared to girls. According to the confidence intervals, we see that all effects are significant. In fact, in the literature, sometimes results are mixed about the relation between the gender of the pupil and performance (Ma, 2007). 


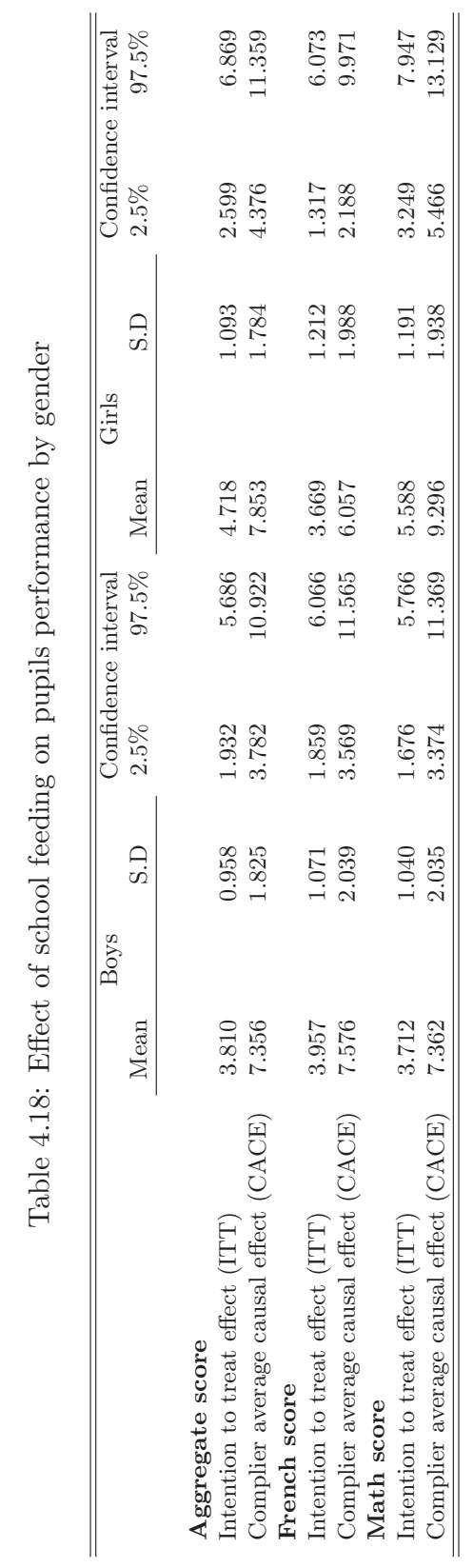


This result on the greater impact of the program on girls' performances that we found could be explained by the fact that the presence of a school canteen can ensure that girls do not come to home to eat lunch, which means that they are exempt from chores they usually perform during hours of the afternoon. Therefore, they are less distracted and can concentrate better on studying.

Impact of school feeding on pupils' performance by grade

We also examined whether the impact of the meal program varies by level of study of the pupil. Table 4.19 shows positive effects everywhere. It also appears that the impact of the program on scores for pupils in grade CE2 is greater than those in grade CP but the difference is small. 


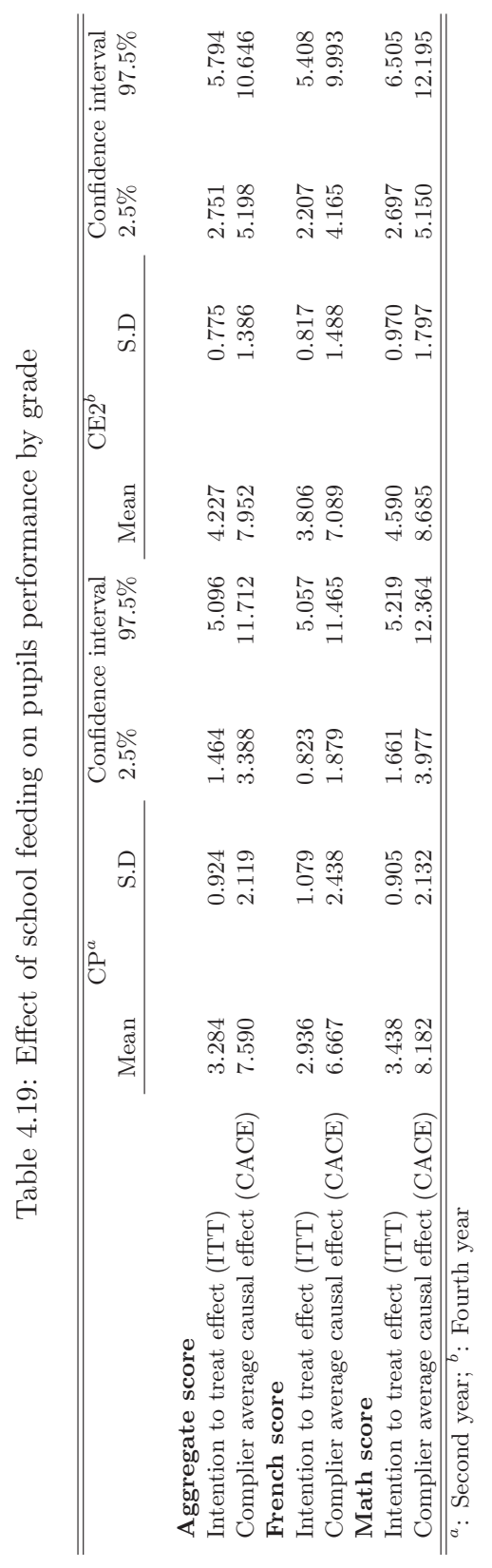




\subsubsection{Impact of canteens on internal efficiency of schools}

The outcome variables related to internal efficiency of schools are enrollment rate, repetition rate, promotion rate and dropout rate. For the enrollment and promotion rates, we expect positive effects, but for the repetition and dropout rates we expect a negative effect. Remember that negative treatment effects on repetition and dropout rates mean that the meal program has reduced the repetition and the dropout rates, so has positive effect on these outcomes. Table 4.20 shows the impact of feeding on the internal efficiency of schools. It also appears that the program lowers dropout rate and increases enrollment rate, which is as expected. However, the program increases the repetition rate and decreases the promotion rate, which is unexpected. This could be explained by the following situation. In order to fight repetition, the Government of Senegal has taken a number of measures that are administrative in nature, namely suppressing repetition during the first year of each step in the primary cycle, and cap at $5 \%$ the rate of repetition at the end of each step. The first years of each step in primary cycle are CI (first year of primary school), CE1 (third year of primary school) and CM1 (Fifth year of primary school). The end of year in each step is CP (second year), CE2 (fourth year) and CM2 (sixth year). Probably, if some schools in the control group apply the measure while some schools in the treatment group did not respect the measure, this can explain the results that we have obtained. However, the effective application of the measure by schools remains to be verified.

Table 4.20: Effect of school feeding on internal efficiency

\begin{tabular}{lrrrr}
\hline \hline & & \multicolumn{3}{c}{ Confidence interval } \\
& Mean & S.D & $2.5 \%$ & $97.5 \%$ \\
\hline Enrollment rate & & & & \\
Intention to treat effect (ITT) & 12.807 & 4.344 & 3.725 & 21.028 \\
Complier average causal effect (CACE) & 7.717 & 2.651 & 2.100 & 12.673 \\
Repetition rate & & & & \\
Intention to treat effect (ITT) & 1.599 & 0.105 & 1.394 & 1.805 \\
Complier average causal effect (CACE) & 2.712 & 0.173 & 2.373 & 3.049 \\
Promotion rate & & & & \\
Intention to treat effect (ITT) & -0.186 & 0.334 & -0.842 & 0.467 \\
Complier average causal effect (CACE) & -0.301 & 0.541 & -1.359 & 0.758 \\
Dropout rate & & & & \\
Intention to treat effect (ITT) & -0.868 & 0.295 & -1.446 & -0.291 \\
Complier average causal effect (CACE) & -1.423 & 0.479 & -2.355 & -0.480 \\
\hline \hline
\end{tabular}

About our results on dropout and enrollment rates, several authors have found the same result in the literature. In using experimental data, Kazianga et al. (2009) in Burkina Faso, found that a school feeding program has a positive effect on enrollment. In Peru, Cueto and Chinen (2007) found that an experimental program of school breakfasts in primary schools lowered the dropout rates of pupils benefiting from the program. 
For the enrollment and dropout rates, figure 4.3 shows the histograms of posterior distribution of the CACE and the ITT effect.
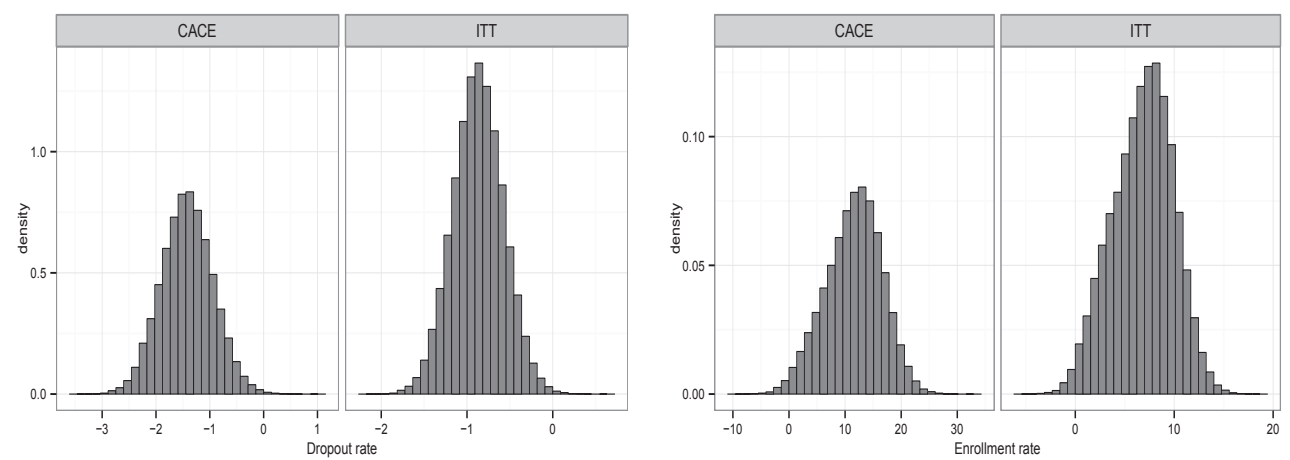

Figure 4.3: Histogram of posterior distribution for dropout and enrollment rates: CACE and ITT effect. [left]: Repetition rate [right]: Promotion rate

For the enrollment rate, one can see on the figure that the ITT effect and the CACE are positive everywhere. The ITT effect ranges from 0 to 16 points of percentage while the CACE is between 0 and 25 points of percentage. For the dropout rate, one can note that the ITT effect and the CACE are negative everywhere. Similarly, the ITT effect is between -2 and 0 while the CACE is between -3 and 0 . These results indicate that the school feeding program lowers the dropout rate and boots enrollment. 
Figure 4.4 shows that the histograms of posterior distribution of the ITT effect and the $\mathrm{CACE}$ for the repetition and promotion rates. For the repetition rate, one observes that the ITT effect and the CACE are everywhere positive. The ITT effect also ranges from 1.2 to 2.0 points of percentages, while the CACE range from 2 and 3.3 points of percentage.
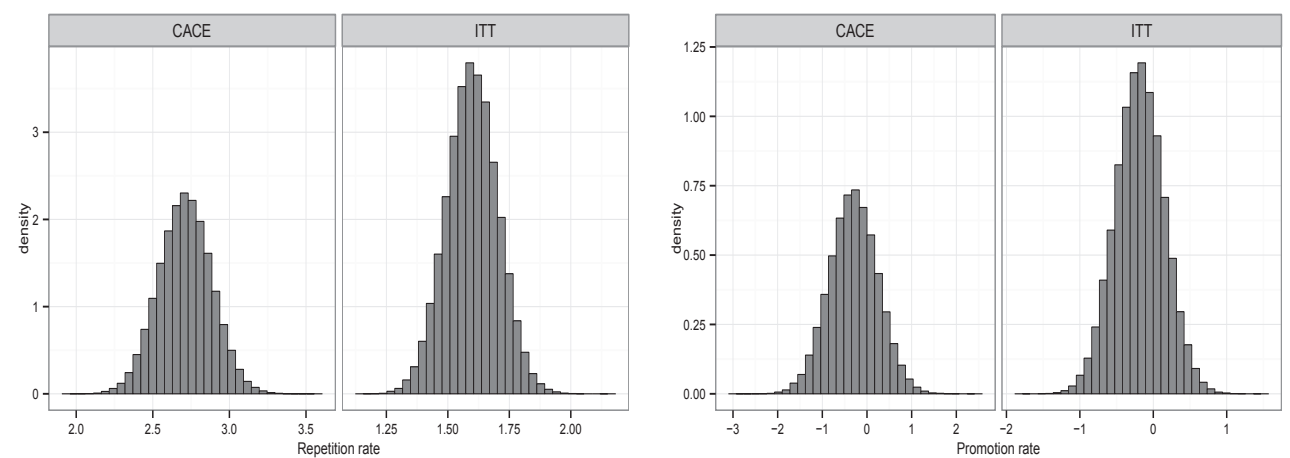

Figure 4.4: Histogram of posterior distribution for repetition and promotion rates: CACE and ITT effect. [left]: Repetition rate [right]: Promotion rate

For the promotion rate, one observes that the ITT effect and the CACE are not everywhere negative. Indeed, the ITT effect is between -1 and 1 points of percentage while the CACE ranges from -2 and 1.9 points of percentage.

\section{Impact heterogeneity}

Here also, we tried to understand whether the impact of school feeding on internal efficiency differs according to gender or according to the grade (class) of the pupil.

Impact of school feeding on internal efficiency by gender

Table 4.21 gives the impact of the meal program on the internal efficiency of schools by gender. It appears that the enrollment rate of boys is greater than that of girls. In contrast, the table shows that girls dropout are lesser than boys in the presence of school canteens. 


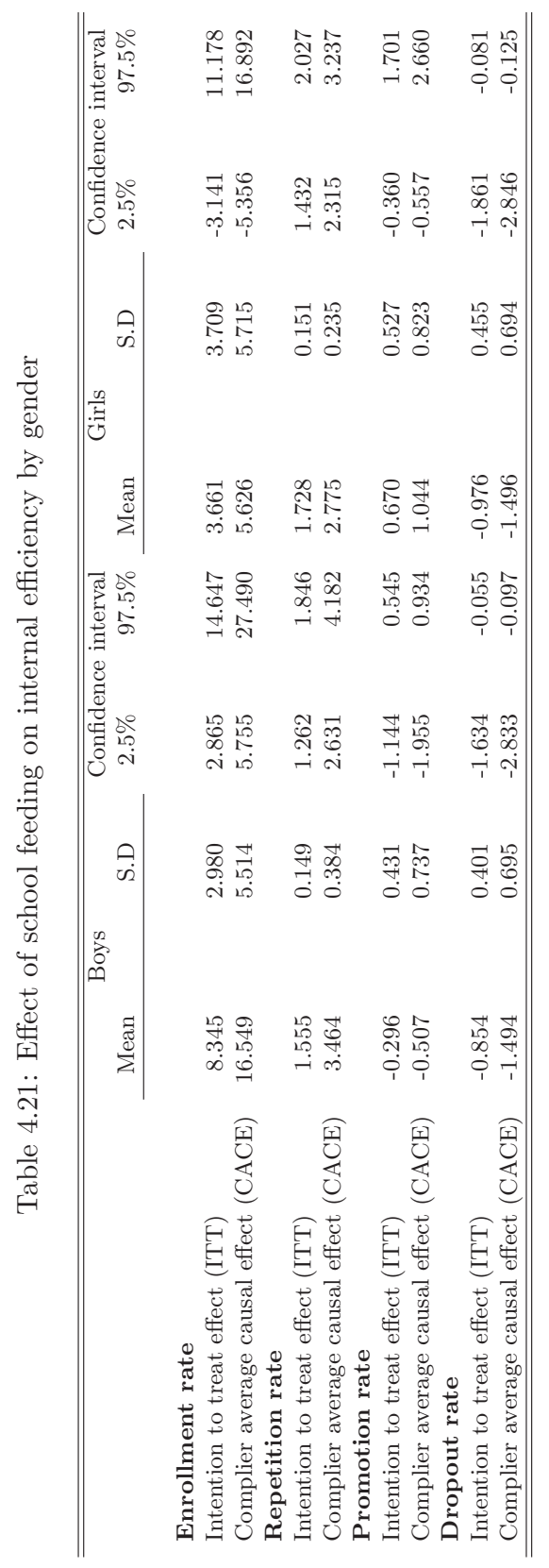


Impact of school feeding on internal efficiency by grade

The impact of the meal program on internal efficiency by grade or class is given in Table 4.22. It appears that the program has a positive impact on the promotion rate of pupils in grade CP. However the result is not significant. 


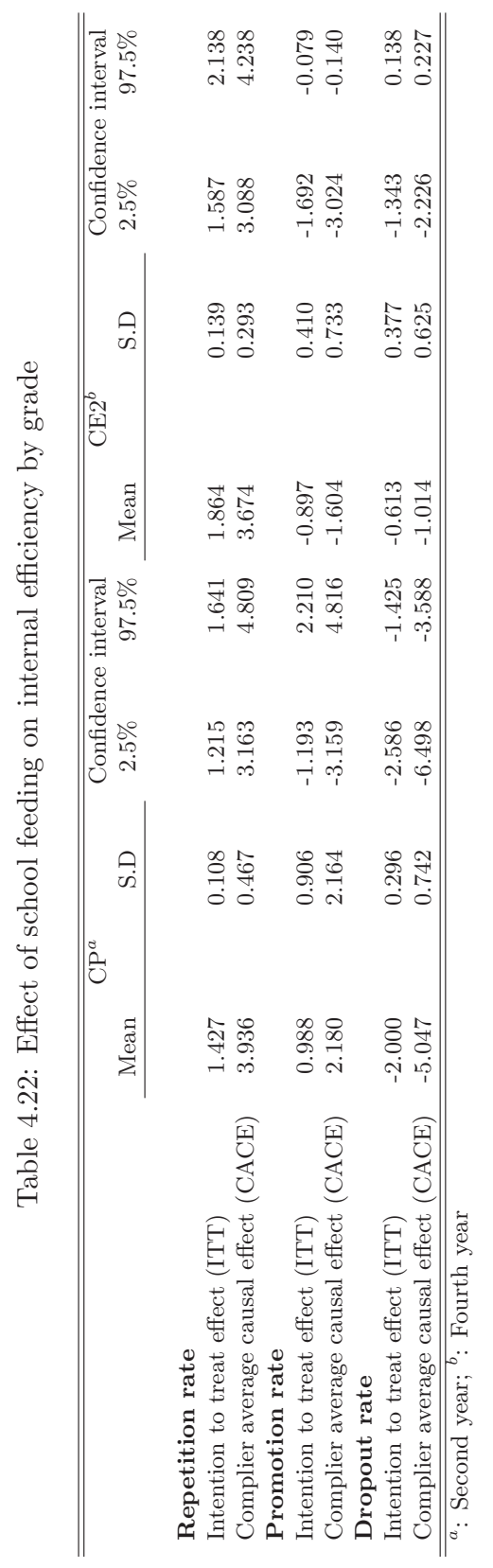


In addition, the table shows that the impact of the program on the reduction of the dropout rate is greater for the youngest pupils which are in class $\mathrm{CP}$ than for those in grade of CE2.

\subsection{Policy analysis}

An important political issue in the analysis of the impact of a program is whether other options are more effective or beneficial than the evaluated program. In a context of scarce resources, the effectiveness of an educational policy to promote quality is determined by the effects of the program on educational outcomes and also the cost to achieve them. The cost-benefit and costeffective analysis allow for a prioritization of levers on which education policy can act to improve on the quality of learning by taking into account resource constraints. The cost-effectiveness analysis is distinct from the cost-benefit one, which assigns a monetary value to the measure of the effect before comparing it with the cost of the intervention. The cost-benefit analysis shows whether a program or policy is relevant, or to choose among several alternatives. It involves comparing the total expected costs of each option to the total expected benefits, to see whether the benefits outweigh the costs and by how much.

\subsubsection{Cost-effectiveness analysis}

The relevance of educational policy options for improving school performance is determined not only on the basis of their positive effects but also their cost. Cost-effectiveness analysis allows one to relate the cost of the policies and their impact on school performance. It provides a framework for reflection on investment options in the presence of resource constraints.

Based on experience in Cambodia, Cheung and Perrotta (2011) noted that there are few studies that address the issue of cost-effectiveness analysis in the assessment of the impact of school feeding programs. The authors found out that school feeding is more profitable than the rations on improving enrollment and attendance. They also found that the combination of school meals and rations with deworming is more profitable due to the fact that the entire package attracts many more pupils, and also drugs against parasites are not expensive. Miguel and Kremer (2004) found that deworming is far more cost effective than other options if the goal is to increase school participation. In this subsection, we conduct a cost-effectiveness analysis of school feeding program compared to another option that is deworming. We will compare the deworming and the canteen in terms of cost and effectiveness in improving academic performance. A similar analysis was previously conducted in Chapter 3, but the purpose of the exercise is to determine whether the conclusion will be the same when the impact of the meal program is from the experimental study.

To conduct the analysis, we need the cost of the meal program and the cost related to each input. For the cost of the canteen, based on information from the ministry of education and the WFP, the annual cost per pupil is approximately 14000 CFA. For the cost of the deworming, based on information from the ministry of education, the annual cost per pupil is approximately 74 CFA.

Knowing the costs of different interventions and the impact of each program on outcomes, we can then combine the cost of each policy and its impact in calculating the cost-effectiveness ratio. By dividing the cost of each option by its effect, we get the cost of producing one unit of a given outcome, for example we will have, for each option, the cost of the improvement of the academic achievement of 1 point. Once this is done, we can then define which program require the lowest cost, for improving educational outcome of 1 point.

As we are not able to assess the impact of the deworming in this chapter, the percentage of additional score for the deworming is taken from the Chapter 3. Dhaliwal et al. (2012) conducted a comparative analysis on cost-effectiveness of various programs implemented in different 
countries but having the same goal. They used results from various analysis conducted in different countries to perform the cost-effectiveness analysis. Their analysis included 11 programs (deworming, free uniform program, iron fortification and deworming, Camera Monitoring of Teachers' Attendance etc.) in six countries (Kenya, India, Nepal, Dominican Republic, Madagascar, and Mexico). The authors pointed out that it is important to have a single year for all programs in a given analysis. In addition, the outcome variable should be the same for all interventions.

Table 4.23 presents the cost and impact of each intervention on the scores as well as the ratio between the cost and percentage of additional scores for each intervention.

\begin{tabular}{lrrr}
\multicolumn{4}{c}{ Table 4.23: Cost effectiveness } \\
\hline \hline & Aggregate score & French score & Math score \\
\hline Cost & 13100 & 13100 & 13100 \\
Canteen & 74 & 74 & 74 \\
Deworming & & & \\
\hline Percentage of additional score & 7.593 & 6.665 & 8.396 \\
Canteen & 17.295 & 2.002 & 48.124 \\
Deworming & & & \\
\hline Cost per percentage of additional score & 1725 & 1965 & 1560 \\
Canteen & 4 & 37 & 2 \\
Deworming & & & \\
\hline \hline
\end{tabular}

As in the previous chapter, this analysis shows that the deworming is by far the most costeffective option in improving pupils' performance. It is important to remember that school feeding can have positive implications on the environment of the school, child health, poverty and well-being of households. However, in a context of scarce resources, sustainability of school feeding programs should depend largely on reducing their cost when the objective is to improve scores.

\subsubsection{Cost-benefit analysis}

In this subsection, we perform a cost-benefit analysis to determine whether the gains from school feeding program exceed the costs engaged. The gains were reduced to the additional incomes generated by increasing the number of years of primary education obtained by the pupils that are beneficiaries of the program. So we did not take into account other benefits that would result from the program like better health, or the welfare resulting from a change in the distribution of income in favor of the poorest households that will generate the increase of their level of primary education. To perform the cost-benefit analysis, we successively estimated the number of additional years of primary education, the additional incomes that are generated by the school feeding program and the cost of the intervention for a pupil.

For the cost of the intervention, as mentioned earlier, based on information from WFP and the Ministry of Education, the annual cost of the school feeding per pupil $(c d)$ was calculated by dividing the annual expenditure of the program by the number of pupils benefiting from the canteen. The annual cost of the canteen per pupil is around 13100 CFA.

With no information allowing direct assessment of the increase in the number of years of education resulting from a long participation in treatment, to calculate the number of additional years of education, (the experiment lasted about 13 months), we simulated the effects on the average years of schooling that we would obtain if we had several years of experimentation. Using the post program survey, a pseudo-cohort was constructed after the program implementation (2010). More precisely, the transition rates by grade were calculated from the first year of primary school (CI) to the sixth and final year of primary school (CM2). Their product gave us the expectation of the number of years of schooling for a child who enters school in 2009. This calculation was 
done for the treatment group and the control group. The difference is 0.018 which is the number of additional years of education gained from the treatment (as).

It is also possible to use Markov transition matrices to evaluate the treatment effect in terms of additional years of education (Behrman et al., 2005).

Moreover, the additional incomes were calculated over the entire period of active life of the future adult with and without participation in the school feeding program. Based on the literature, we assumed that active life begins at age 15 and ends at age 65 . The following approach was used:

i) Using the equation of Mincer (1974) and data from the survey on follow-up of the poverty in Senegal (ESPS) conducted in 2005 among Senegalese households, the performance of an additional year of primary education in Senegal $(t r)$ was estimated. This performance is 6378 CFA. To estimate the performance, we used as dependent variable the consumption per adult equivalent, and as control variables, we used the highest grade completed by the individual, marital status, age, gender, social class, professional experience, mode of principal activity's payment (variable that has as modalities, salary, payment in kind, payment to the task, payment to the profit) and the type of contract.

ii) The ESPS data were also used to estimate the average annual income of the pseudo-cohort at age $15,16, \ldots$, and 65 .

iii) From the estimated annual average income, we computed the present value of the stream of future incomes of a pupil in the control group using the following relation:

$$
Y_{T=0}=\sum_{i=15}^{65} \frac{Y_{i}}{(1+r)^{i}},
$$

with $Y_{i}$ the annual income in millions of CFA francs corresponding to the year of active life $i$, and $r$ the real discount rate which is assumed equal to 3\% and 5\% as in Behrman et al. (2005). iv) Then, the present value of the future incomes of a pupil in the treatment group was calculated from the following relation:

$$
Y_{T=1}=\sum_{i=15}^{65} \frac{Y_{i}(1+t r * a s)}{(1+r)^{i}}
$$

v) The economic gain or benefit of the school feeding program was obtained by calculating the difference between the discounted income of pupils who benefit from the program and those who do not benefit from the program.

vi) Finally, the ratio between economic gain and the cost of the canteen provides a measure of the rate of performance of the intervention. This is 3172 CFA (for a discount of $3 \%$ rate) and 2840 CFA (for a discount of $5 \%$ ), hence come to the conclusion that the school feeding program is a worthwhile investment because its economic benefits exceed the costs involved. However, this result underestimates the benefits of the program because it does not account for the improved health and social well-being due to program participation. We can thus say that the program is justified in terms of economic gains.

\subsection{Conclusion}

In this chapter, using experimental data, we have assessed the impact of a school feeding program on school performances in the presence of attrition and partial adherence. The study used a statistical method that can overcome the problems of partial adherence and attrition simultaneously. It covers four regions of Senegal namely Diourbel, Fatick, Sédhiou and Kolda. We used outcome variables related to the academic performance of pupils (aggregate score, French score and Math score) and to internal efficiency of schools (enrollment rate, repetition rate, promotion and dropout rates). We also conducted a cost-effectiveness and cost-benefit analysis. 
Baseline data were used to check the random assignment to the treatment. To this end, several tests (Kolmogorov-Smirnov test, Mann-Whitney and Student tests) were performed. The results show that on average, the two groups are homogeneous when the tests are carried out at the school level. However, when tests are performed at student level, the results show that differences exist between the two groups.

The results that emerge from this study show that the school feeding program can significantly increase pupils' performances and enrollment rate, while reducing the dropout rate. The posterior distribution of the ITT effect and the CACE shows that the impact of the program is not homogeneous across outcomes. In fact the impact of the program is positive for all scores (aggregate, French and math) and it is more important in math score compared to French score. The impact is also greater for girls compared to boys in the math score, while in French score the impact is greater for boys than girls. For policy analysis, results suggest that despite the justification of the meal program in terms of economic gains, the cost-effectiveness analysis show that the deworming program is more effective.

In future research, it would be important to take two types of heterogeneity in measuring the impact of the school feeding program into account. In the case study, schools do not prepare meals every day of the week and the number of days per week they prepare meal varies from one school to another (this number of days of preparation is between 2 and 5 days per week). In addition, canteen meals are prepared and served at different places and by different people. Pupils who share the meal cannot consume exactly the same amount of food per each meal served; hence the need to take the heterogeneity of treatment into account (which is caused by differences in treatment) in estimating the causal effect of the program. Moreover, given the same treatment, the magnitude of treatment effects may vary from one unit treated to another. Such heterogeneity is known as heterogeneity of treatment effect. Thus, the magnitude of treatment effects may depend on the beneficiaries.

Another extension of this study would also be to study the long term impact of the school feeding program by taking the impact of the program on health, nutrition and social welfare into account. 
CHAPTER 5

General Conclusion

Program evaluation is an important tool to inform policy makers about the effectiveness of programs and the efficient allocation of resources. Several studies have examined the impact of school feeding and deworming programs on school performances in developing countries. However, conflicting evidence still exists regarding the effects of these programs on school performance. There is also little evidence on the cost effectiveness of these programs.

In Senegal, although nutritional and health experiences have been developed in recent years, no empirical study have been conducted to assess the impacts of meal and deworming programs on school performances, and also address issues related to the complementarity or substitutability of the two programs. This thesis aims at filling this gap. We use experimental and observational data and rely on both program evaluation and structural approaches to fill the gap in knowledge. The thesis is organized in 5 chapters.

Chapter 1 presents the motivation and objectives of the thesis, which also gives the history of meal and deworming programs in Senegal. It also summarizes the debates between program evaluation approach and structural approaches recently raised by some authors before highlighting the contributions and the organization of the thesis.

Chapter 2 uses a unique and recent data set from school feeding and deworming programs in rural Senegal to assess their effects on pupils' achievement measured by test scores, while elaborating on the determinants of test scores. We also used observational data on approximately 5000 pupils. The sample contains data on school and teacher characteristics, pupils' achievements (aggregate score, score in French and math score), pupils' and household characteristics, community characteristics, shocks variables, geographical location and treatment indicators. In this chapter, we used both program evaluation (Propensity Score Matching) and structural approaches. For the structural approaches, we used the dummy endogenous selection model originally developed by Heckman (1979) and the Roy (1951) model. We used Full Information Maximum Likelihood (FIML) and the Control Function (CF) approach as procedures of estimation. We computed the average effect of each intervention on all sample (ATE), on the treated (ATET) and on the non-treated (ATENT) depending on the model used. In the dummy endogenous selection model (Heckman selection framework), we identified only the ATE. In the PSM, we identified the ATET; and in the Roy model in addition to the ATE and ATET, we also calculated the ATENT. Regarding the ATENT, i.e, the average effect on the group of pupils who do not receive the meal program, the purpose is to inform policy makers on the rationale for extending the programs to this subpopulation of pupils. In addition, in Chapter 2 we distinguished between deworming at home and deworming in school.

Chapter 3 develops a model for two treatments to assess the impact of school feeding and 
deworming programs as a package on school performances, while elaborating on the determinants of these performances. Using the same observational data as in Chapter 2, this chapter addresses issues related to the impact of each program taken in isolation, but also the impact of the combination of the two interventions and several kind of effects (global effects, exclusive effects, relative effects, additional effects, sequential and substitution effects). In the chapter, we also tested the complementarity versus substitutability of the two programs. In addition to the outcome variables used in Chapter 2, Chapter 3 also assesses the impact of the two interventions on internal efficiency of schools measured by enrollment, promotion and dropout rates. The chapter proposes a structural framework for double-index selection where treatment variables are endogenous. Firstly, we use a double endogenous selection model; and secondly use a generalized Roy (1951) framework. For each specification, we propose two estimation procedures, the Full Information Maximum Likelihood (FIML) and the Control Function (two-step estimation) à la Heckman $(1978,1979)$.

Chapter 4 uses experimental data to assess the effects of meal programs on school performances in the presence of attrition and partial adherence. We used a statistical method developed by Horiuchi et al. (2007). This method allows us to overcome the problems of partial adherence and attrition simultaneously. In the presence of partial adherence, an intention to-treat (ITT) effect which is informative for policy makers gives a valid inference of the impact of assignment on outcome, but it is also important to know the effect of receiving the treatment rather than the effect of the assignment. That is why we calculate not only the ITT effect, but also the effect of actually receiving the treatment, namely the Complier Average Causal Effect (CACE). The analysis in this chapter cover four regions of Senegal, two in the center (Diourbel and Fatick) and two in the south of the country (Sédhiou and Kolda). We used two types of outcomes, namely those related to academic performance of pupils (aggregate score, French score and Math score) and those related to internal efficiency of schools (enrollment rate, repetition rate, promotion and dropout rates). It is important to remember that in this chapter, it was not possible to address issues related to the impact of the deworming program on school performances because as mentioned in Section 4.2, the deworming component was removed to the experiment.

Chapter 5 summarizes the key findings from all chapters (Section 5.1), gives some policy implications (Section 5.2), discusses the limitations of the thesis (Section 5.3) and presents the need for some future research (5.4).

\subsection{Summary of findings}

Chapter 2 presents several sets of estimates on the determinants of pupils' performances. The results show that most of the control variables are of the expected sign and significant. Some others do not conform to intuition. However, they become highly interesting when put in the specific rural Africa context.

For controls related to school characteristics, as expected, temporary shelters have negative effects on pupils' performance (aggregate, French and math scores).

For control number of pupils per manual, results show that the number of pupils per manual increases the scores until a point from which it has a negative effect on performance. This variable ranges between 0.2 to 7 pupils per manual. Our reading is that even if school manual has a capacity to improve pupils' competence, as the number of pupils per manual become higher, this impact scores negatively because it decreases the learning time as the number of pupils per manual increases.

For the control class size, the results show a U-shaped relation with aggregate and math scores. The performance decreases with increasing class size which is consistent with some findings in the literature on performance. However, starting from a certain threshold, the performance 
increases which is also consistent with other findings.

The teacher's age also shows a similar U-shaped picture. The linear term is negative while the square term is positive for scores. This means that the teacher's age affects the scores negatively until a certain threshold at which additional age positively affects the performances. Control variable gender of teacher $($ male $=1)$ has a negative effect on scores as expected. However, controls water point, professional qualification and academic qualification (High School Diploma and more) have positive effect on pupils' performance. Regarding professional qualification, the results show that pupils supervised by a teacher with the vocational qualification CAP and CEAP perform better than those taught by a teacher with another vocational degree which is the reference. The surprising result is that pupils taught by a teacher with no professional diploma perform better than those supervised by a teacher with another vocational degree. This result is consistent with a finding of CONFEMEN (2007) in Senegal. For the academic qualification of teachers, the results show that pupils supervised by a teacher with the "High School Diploma and more" perform better in mathematics as compared to those supervised by a teacher with the national certificate.

Regarding controls related to pupils' characteristics, variable pupil's age shows an inverted U-shaped relation, meaning that, older pupils have a negative effect on performance. Controls related to gender of pupils, Koranic school, early childhood institution, pupils eat at fill impact performances positively. However, the pupil's study level (class, grade; CE2=1) impact scores negatively.

Regarding household characteristics, the results show that education expenditure and household size have a positive effect on performances, as expected.

For community characteristics, to live in a community where some parents are not interested in school has a negative effect on scores, while to live in a village having a college shows a positive one.

Regarding shocks variables, controls number of pregnancies registered in school, absenteeism of teacher, disturbed courses and health expenditure impact scores negatively. Regarding regions, results show that pupils living in Diourbel perform better than those in Fatick.

About the impact of the meal and deworming programs on pupils' performances, the following are the main results emerging from the Chapter 2. The meal program can increase pupils' scores significantly. Also, the effect of the meal program is more important for pupils who are in a school with an association of mothers. Deworming in school has a positive effect on pupils' performance, while deworming at home has a negative impact on pupils' scores. Our reading is that, it is essential to assist rural households to deworm children at home. This will avoid the use of traditional medicines for deworming children. In addition, the deworming program significantly increases the scores of pupils who are in a school with an association of pupils' mothers or a school project.

In terms of policy analysis, the results show that the impact of programs varies depending on pupils' gender, the existence of an association around the school or the educational level of the pupil. Also, the Local Average Treatment Effect (LATE), reveals that in increasing scores, the most cost-effective way is to combine the canteen and the association of parents compared to other options including management committee, school project, school cooperative and reduction of teacher absence.

In Chapter 3, results on the determinants of scores are consistent with those in Chapter 2. In addition, results show that control deworming at home impacts scores negatively, while existence of latrine and existence of hand washing devices in school impact scores positively. About the geographical area, pupils living in Diourbel perform better than those in Fatick. However, pupils in Kolda and Sédhiou perform less than those in Fatick.

In addition, temporary shelters, association of pupils' parents, age of teacher, Koranic school, snack, pupils who eat their fill, and literacy of the household head have a positive effect on 
enrollment rate. ${ }^{1}$ Another interesting and intuitive result is that having a literate household head increases the enrollment rate. In contrast, the enrollment rate declines with school expenses. This result is important for education policy in terms of reducing the illiteracy rate in rural areas where the living standard is very low. Unquestionably, if policy makers want to encourage families to send their children to school, it would be interesting to ensure that the cost of schooling is not too high. Two other results are worth noting: having a male teacher and holding arable land have negative effects on enrollment.

Control variables temporary shelters, age of teacher, age of pupil, distance to school, association of pupils' parents, gender of the teacher, continuing training, Koranic school, early childhood institution, pupils who eat their fill and ownership of arable land have a positive effect on the promotion rate.

For the outcome of dropout rate, results show that the variables temporary shelters, class size, age of pupil, distance to school, association of pupils' parents, gender of teacher, vocational training, Koranic school, early childhood institution, pupils who eat their fill, ownership of arable land and the existence of a college in the village have negative effects on the outcome. In contrast, controls health expenditure, number of head of cattle, disturbances that caused delay in starting the courses, and the gender of household head have positive effects on the dropout rate. Remember that in the case of the dropout rate, negative indicator on a coefficient is a positive result as the objective is to reduce the dropout rate.

As a whole, our findings about the determinants of school performances conducted in Chapter 2 and Chapter 3 suggest that although temporary shelters allow to reduce dropout rate and to increase enrollment and promotion rates, they have negative impact on pupils' achievements (scores). In addition, even if school manual can improve the competence of pupils, when the number of pupils per manual become high (more than 4 pupils per manual) this effect seems to become negative.

In terms of the impacts of the interventions, the main results from Chapter 3 are the following. For the score outcomes, the study shows a positive and significant additional, exclusive and global average treatment effects (ATE). The impact of the meals program on the scores is greater than that from the deworming program. The combination of the two programs (package) has greater impacts than that for each program taken in isolation. This result indicates that, if the aim is to increase the scores, the two programs should be complementary. Moreover, the relative effect of the package versus the deworming alone is greater than that the effect of the canteen only. For the average treatment effects on the treated (ATET), the exclusive, global, and additional effects are all positive and significant. It should be noted that the effects on the treated are larger than the ATE. For the treatment effects on the untreated (ATENT), the results show that the exclusive effect of deworming is negative while the one on canteen is positive. The combination of the two programs greatly increases the scores. The sequential effects indicate that for the pupils in the treated group, the impact of the package performs better if the school meal is introduced before deworming. For pupils in the untreated group, the reverse sequence is preferable.

For the internal efficiency of schools, results on the impact on enrollment rate show a negative exclusive ATE effect for the deworming program, a positive exclusive ATE for the meal program, a negative overall effect and a positive additional effect. Moreover, when we set the target for increasing enrollment, the implementation of the meal program alone is preferable to deworming or the package. When the objective is to increase the promotion rate or reduce dropout, the package is the best option.

Also, findings show that the meal and deworming programs are complementary in the goal of increasing scores and promotion rates. On the contrary, the two programs are substitutes when the aim is to reduce dropouts.

\footnotetext{
${ }^{1}$ Estimations related to internal efficiency of school in chapter 2 are not presented for issue of space. Findings in Chapter 3 are consistent with those in Chapter 2 in terms of outcomes related to internal efficiency of schools.
} 
The cost-effectiveness analysis conducted indicates that, regarding scores, deworming is far cheaper than the meals program. It also shows that introducing the meals before deworming is more cost-effective than the reverse. For the promotion rate, the combination of the two programs is more cost-effective than the school feeding program only. For the dropout rate, deworming is more cost-effective than the canteen and the package. However, the package is more cost-effective than the single school feeding.

In Chapter 4, using baseline data, we checked the random assignment to the treatment by performing several tests (Kolmogorov-Smirnov test, Mann-Whitney and Student tests). The test results show that on average, the two groups are relatively homogeneous when the tests are carried out at school level. By contrast, when tests are performed at pupil level, we observe some differences between the two groups. Our inference is based on Monte Carlo samples from three independent Markov chains. Each of these samples has the length of 50,000 and is initiated at different sets of starting values. We found out that all parameters have values of the Gelman-Rubin convergence statistic that are less than 1.01, meaning between 1 and 1.01. So, a satisfactory degree of convergence has been achieved and we based our inference on a combined total of 90,000 posterior draws.

The main results emerging from the chapter are as follows.

The school feeding program can significantly increase pupils' performances and enrollment rate, while reducing the dropout rate. However, the program increases the repetition rate and decreases the promotion rate, which is unexpected. This may follow from the measures taken by the Government of Senegal consisting in suppressing repetition during the first year of each step in the primary cycle and capping at $5 \%$ the rate of repetition at the end of each step. This also indicates that the effect of the meal program is positive for all scores (aggregate, French and math) and is greater in the math score than in the French score. One also noted that the CACE is always greater than the ITT effect. In addition, the effect of the program is greater for girls than for boys in the math score, while we observe the contrary for the French score. In terms of promotion rate, the results show that the program do not increases except for the promotion rate of girls and the pupils who are in the grade $\mathrm{CP}$.

The cost-benefit analysis conducted in this chapter shows that the meal program is justified in terms of economic gains and the result of the cost-effectiveness analysis, which is consistent with the findings presented in Chapter 3.

Furthermore, the findings in Chapter 2 are consistent with those in Chapter 3 in terms of the determinants of performances and treatment effects. In both chapters, the results show that the meal and deworming programs have positive effects on performances. In addition, we do not notice contradictory findings on the impact of the meal program on school performances in Chapters 2, 3 and 4. One can take results in those chapters as complementary for the following reasons. First, the experimental data used in Chapter 4 do not allow us to assess the impact of the deworming program, because this program was removed from the experiment. Second, in Chapter 4, we do not address several issues presented in Chapters 2 and 3 because of the data and the method used.

From our analysis, it appears that using a structural approach allows to conduct a richer analysis on the effects calculation and policy analysis.

Overall, from this thesis, it appears that the meal and deworming programs have positive impact on school performances. Therefore, these two interventions seem to be means of improving pupils' achievements and enrollment rates, while reducing the dropout rates.

\section{$5.2 \quad$ Policy implications}

The results of this thesis have some policy implications. The findings in terms of determinants of performances suggest that despite the fact that temporary shelters allow to reduce dropout rates, while having positive effects on enrollment and promotion rates, they contribute towards 
reducing pupils' achievement (scores). So, putting up temporary shelters owing to the lack of classrooms appears to be a necessary evil. However, to ensure the quality of education to improve pupils' achievements, it is also important not to use temporary shelters for a long time. Unfortunately, our research cannot give a clear idea about the optimal duration of temporary shelters.

For control number of pupil per manual, results show that even if school manual has a capacity to improve pupils' competence, when the number of pupils per manual becomes higher than 4 pupils per manual, this impacts scores negatively. Therefore, although the ideal is to ensure a manual per pupil especially for key courses (namely French and math), our results suggest that reducing the number of pupil per manual below four (4) pupils contribute towards improving student achievement. Also, implementing a policy gearing towards reducing teachers' absenteeism and disturbances (strikes, deluge etc.), resulting to delays in the start of the courses will improve educational quality.

Furthermore, it is important to promote more experienced teachers (from 33 years old) and female teachers. Encouraging the construction of colleges in villages will also contribute to improving pupils' performances. In addition, encouraging parents to put their children in childhood institution or a Koranic school will contribute towards improving academic achievement, enrollment and help reducing dropout rate.

Results also suggest, that improving the literacy rate for household heads in rural areas, reducing the cost of schooling and encouraging the creation of associations of pupil's parents can contribute to increase the enrollment rate.

Finally, encouraging teachers to have professional qualification CAP and CEAP, promoting teachers with High school diploma and more as an academic qualification, and increasing the duration of continuing training for teachers are some ways of improving pupils' achievement.

In terms of impact of the interventions, implementing meal and deworming programs in primary schools seems to be an effective means of improving school performances. For better success of these programs, community involvement through associations of pupils' mothers and parents are more appropriate.

In addition, the combination of meal and deworming is more beneficial in terms of increasing pupils's achievements. The sequence of implementation does matter. The two programs are complementary in the goal of increasing scores and promotion rates. They are substitutes with reducing dropouts. Also, our results suggest that it is essential to assist rural households in deworming children at home, because this will avoid the use of traditional medicines.

Cost-effectiveness analysis conducted in this thesis indicates that, regarding scores, deworming is far cheaper than the meals program. It also shows that introducing the meals before deworming is more cost-effective than the reverse. For the promotion rate, the combination of the two programs is more cost-effective than the school feeding program only. For the dropout rate, deworming is more cost-effective than the canteen or the package. However, the package is more cost effective than school feeding alone. Another interesting economic analysis performed in this thesis is a cost-benefit analysis. The results show that the meal program is justified in terms of economic gains because costs engaged are less than the benefits realized.

Furthermore, several school meal programs were initiated in developing countries. However, their impact on educational outcomes is often contradictory. Over the last decade, a heated debate has taken place over the quality of these programs to attract and retain pupils at school, as well as increasing their performances. Therefore, doubts were expressed regarding the objectives of these programs to improve school and academic performances of pupils. These critiques point out that in the context of acute poverty; school feeding programs contribute more towards reducing food poverty. Indeed, some parents send their children to schools where these programs are implemented so that they can receive food rations. Another issue regarding the role of school meals is their ability to reduce child labor. Child labor is widely practiced in rural communities. This allows some families to generate additional income for the household. As food 
costs constitute a significant share of household's spending in rural areas, it is likely that some parents send their children to school in the hope of thereby reducing their food expenditures.

However this thesis does not address the issues of poverty reduction and child labor.

Regarding deworwing programs, it is worthwhile noticing that the perception of families about this intervention is mixed or even negative. Several families have serious reservations due to side effects of deworming drugs or to simple prejudices. It is therefore quite possible that the result of this program is contrary to what is expected. Major effort related to information sharing and education must be conducted to convince families and children about the merits of such interventions. These considerations should lead analysts to be cautious about the conclusions of impact assessments.

In this thesis, one of the novelties of the interventions we evaluated has been to combine them as a package. Intuitively, deworming and meals are complementary as both programs could mutually reinforce each other. Moreover, the order or the sequence in which the programs are administered makes sense and can play on the results. This thesis provided answers to these questions.

\subsection{Limitations of the thesis}

This thesis has several limitations. Firstly, due to the lack of data on anthropometric measures, it does not address the issue of the impact of meal and deworming programs on the nutritional status of pupils. Also, it does not address issues about the impact of interventions on child labor.

In addition, the thesis does not develop a structural model that overcomes attrition and partial adhesion simultaneously in an experimental setting.

Another limitation is related to the deworming program in Chapter 2. As mentioned earlier in the introduction of Chapter 2, the deworming program is not the sum of the deworming in school and at home. Also, we do not take the combination of deworming in school and at home into account. In addition, we distinguish between deworming at school and at home, but the number of observations is so small that we have not been able to produce estimates using all estimation methods.

Furthermore, the experimental data used in Chapter 4 does not allow us to assess the impact of the deworming program in an experimental setting. Also, in Chapter 4, it would be important to consider the two types of heterogeneity in measuring the impact of the school feeding program. Another limitation is that the thesis does not address the issue related to the effects of meal and deworming programs on household consumption and welfare. Finally, the multilevel aspect of the data (pupils, households, school and communities) was not taken into account for all chapters.

\subsection{Future research}

The work done in this thesis has led to reflections that suggest several additional areas for future research. A first development is the multiple outcome framework. In this thesis, we have considered the outcomes variables (aggregate, French and math scores; enrollment, promotion, repetition and dropout rates) separately. It would be interesting to consider the improvement of these outcomes jointly. Evidently, these outcomes are related. For example, in order to record good scores, one needs to be enrolled and not to be a dropout. Moreover, being successful in terms of academic achievements (via scores) may also imply a high probability to be promoted or a low probability of repeating. Another area of research would be to use models with continuous treatments (see Hirano and Imbens, 2004 and Florence et al., 2004 for analysis with continuous treatment). 
In Chapter 3, a big challenge consists in extending the model developed for double treatment to the case of M-treatment. This is not only a methodological challenge, but represents a real empirical interest. As outlined by Lechner (2001), many evaluation problems (for example the different types of labor market policies in Europe) involve more than two treatments. However, this is not without methodological difficulties. With regard to the first model discussed in the Chapter 3, we see the kernel $K\left(y_{i}, \mathbf{x}_{i},\right)$, which depends on the four regimes. This makes the maximization procedure complex. As for the Roy model, the main difficulty is that the procedure includes data consuming. All the parameters have to be estimated for each regime. As a result, the problem related to parameter inflation is likely. For both models, our experience is that the algorithms are very sensitive to the choice of initial values. In this regard, the two-stage estimation provides a more manageable alternative. At the time of writing, none of these issues have been addressed. We speculated that these could be a promising empirical research avenue. Another future research would be to develop structural model that could simultaneously overcome the attrition and partial adherence problems, and to test the method using our experimental data (Lalonde, 1986).

Concerning Chapter 4, future research would be to take two types of heterogeneity in measuring the impact of the school feeding program (Horiuchi et al., 2007) into account. Schools do not prepare every day of the week, and as such, the number of days allocates to preparation of meals per week varies from one school to another (this number of days of preparation is between 2 and 5 days per week). In addition, canteen meals are prepared and served at different places and by different people. Pupils who share the meal cannot consume exactly the same amount of food pert meal served; hence the need to take heterogeneity of treatment (which is caused by differences in treatment) in estimating the causal effect of the program into consideration. Moreover, given the same treatment, the magnitude of treatment effects may vary from one pupil treated to another. Such heterogeneity is known as heterogeneity of treatment effect. Therefore, the magnitude of treatment effects may depend on the beneficiaries.

Horiuchi et al. (2007) have modeled the treatment effect as an unknown function of an observed variable, regarded as $D_{i}$ heterogeneity variable. The main difference between the two sources of heterogeneity is the nature of the heterogeneity variable $D_{i}$. Treatment effect heterogeneity $D_{i}$ can be regarded as an observed characteristic of pupils (for example gender). On the other hand, a treatment heterogeneity $D_{i}$ measures the observed level of treatment or dose of treatment. While the first case $D_{i}$ was observed before assignment to treatment variable, in the second case, $D_{i}$ describes the treatment itself and can be influenced by treatment allocation.

As future research, we also intend to use panel data in the case of multiple treatments. In fact, the Ministry of Education runs an annual campaign to collect information from every school. Some of the data include: the characteristics of schools, characteristics of teachers, characteristics of students, school infrastructures (whether or not there is a school project, school feeding, deworming program, iron supplementation etc.), dropout, repetition etc. The Ministry, with support from WFP has set up a tool called 'Balise' (Base de données Alimentation Scolaire au Senegal). This project allows having semestrial data on school feeding in all schools in Senegal. So from this information, we can construct panel data between ten year periods (2003-2013). In addition to school feeding programs and deworming (intestinal parasite), the impact of iron supplementation and vitamin A on educational outcomes will be measured.

Another future research line would be to study the long term impact of the school feeding and deworming programs by taking into account the impact of the program on health, nutrition and social welfare.

Overall, this thesis provides empirical evidence on the impact of meal and deworming programs on school performances using both experimental and non-experimental studies. To our knowledge, this is the first study to reveal that the combination of meal and deworming programs as package would be more effective in terms of increasing school performances, while giving the effect of each intervention and several other effects to inform policy makers. 
Adelman, S. W., D. O. Gilligan, and K. Lehrer (2008): "How Effective are Food for Education Programs? A Critical Assessment of the Evidence from Developing Countries," Food Policy Review 9, International Food Policy Research Institute IFPRI.

Ahmed, A. (2004): "Impact of feeding children in school: Evidence from Bangladesh," International Food Policy Research Institute, Washington, D.C. Mimeo.

Ahmed, A., And C. Del Ninno (2002): "The Food For Education Program in Bangladesh: An Evaluation of its Impact on Educational Attainment and Food Security," International Food Policy Research Institute, (Discussion Paper 138).

Akakpo, K. (2004): "Mesure d'Impact du Programme d'Alimentation Scolaire du PAM dans les Ecoles Publiques Rurales au Sénégal," UNESCO, BREDA.

Altinok, N. (2006): Les sources de la qualité de l'éducation. In C. Bourreau-Dubois \& B. Jeandidier (Eds.), Économie Sociale et Droit. Économie Sociale et solidaire, Famille et éducation, Protection sociale, 2, Actes des XXVIèmes Journées de l'AES, l'harmattan edn.

Angrist, J. (2004): "Treatment Effects Heterogeneity in Theory and Practice," The Economic Journal, (114), C52-C83.

Angrist, J., G. W. Imbens, and D. Rubin (1996): "Identification of Causal Effects Using Instrumental Variables," Journal of the American Statistical Association, (91), 444-472.

Angrist, J. D., And V. Lavy (2001): "Does Teacher Training Effect Pupil Learning? Evidence from Matched Comparisons in Jerusalem Public Schools," Journal of Labour Economics, 19(2), 343-369.

Anh, T. S., J. Knodel, and J. Friedman (1998): "Family Size and Children's Education in Vietnam," Demography, (35(1)), 57-70.

ANSD (2006): Résultats définitifs du troisième recensement général de la population et de l’habitat du Sénégal 2002. Rapport national de présentation, décembre 2006.

(2010): Situation Economique et Sociale régionale, Kolda 2010. ANSD/SRSD Kolda.

Attanasio, O., C. Meghir, and A. Santiago (2009): "Education Choices in Mexico: Using a Structural Model and a Randomized Experiment to Evalute Progresa," IFS/EDEPO Working Paper. 
Banerjee, A., S. Cole, E. Duflo, and L. Linden (2007): "Remedying Education: Evidence from Two Randomized Experiments in India," Quarterly Journal of Economics, (122), 12351264 .

Banerjee, A., And E. Duflo (2009): "The Experimental Approach to Development Economics," NBER Working Paper, (14467).

Barnard, J., C. E. Frangakis, J. L. Hill, and D. B. Rubin (2003): "Principal Stratification Approach to Broken Randomized Experiments: A Case Study of School Choice Vouchers in New York (with Discussion)," Journal of the American Statistical Association, 98, 299-311.

Barnard, J., C. E. Frangaskis, and J. L. Hill (2003): "Principal Stratification Approach to Broken Randomized Experiments: ACase Study of School Choice Vouchers in New York (with Discussion)," American Statistical Association, (98 (462)), 299-311.

Behrman, J., P. Sengupta, and P. Todd (2005): "Progressing through Progresa: An Impact Assessment of a School Subsidy Experiment in Mexico," Economic Development and Cultural Change, 54(1), 238-275.

Blake, J. (1989): "Family Size and Achievement," CA: University of California Press, Berkeley.

Blundell, R., and M. C. Dias (2008): "Alternative Approaches to Evaluation in Empirical Microeconomics," The Institute for Fiscal Studies working paper, (26).

Bobonis, G., E. Miguel, and C. P. Sharma (2006): "Iron Deficiency Anemia and Shool Participation," Journal of Human Ressources, (41(4)), 692-721.

Bressoux, P. (1996): "The effect of teachers' training on pupils' achievement: The case of elementary school in France," School Effectiveness and school Improvement, 7(3), 252-279.

Brodaty, T., B. Crépon, and D. Fougère (2001): "Using matching estimators to evaluate alternative youth employment programs : Evidence from France, 1986-1988," In M. Lechner, and F. Pfeiffer (eds), Econometric Evaluation of Labour Market Policies. Heidelberg: Physica/Springer., pp. 85-124.

Brossard, M. (2003): Rétention, redoublement et qualité dans les écoles publiques primaires béninoises. Quel diagnostic? Quelles pistes de politiques éducatives? Dakar: Pôle de Dakar.

Buchmann, C. (2000): "Family structure, parental perceptions and child labor in Kenya: what factors determine who is enrolled in school?," Social Forces, (78(4)), 1349-1378.

Caliendo, M., and S. Kopeinig (2008): "Some Pratical Guidance For the Implementation of the Propensity Score Matching," Journal of Economic Surveys, (22), 31-72.

Chernichovsky, D. (1985): "Socioeconomic and demographic aspects of school enrollment and attendance in rural Botswana," Economic Development and Cultural Change, (33(2)), 319-332.

Cheung, M., and M. Perrotta (2011): "The Impact of a Food For Education Program on Schooling in Cambodia," Institute for International Economic Studies, (766).

Clotfelter, C., H. Ladd, and J. Vigdor (2006): "Teacher-Student Matching and the Assessment of Teacher Effectiveness," NBER Working Papers, 11936.

Cohen, E., and R. A. Rossmiller (1987): "Research on effective schools: implications for less developed countries," Comparative Education Review, 31 (3), 377-399. 
Coleman, J. S., E. Q. Campbell, C. J. Hobson, J. McPartland, A. M. Mood, F. D. WeinfELd, AND R. L. YORK (1966): "Equality of educational opportunity," Washington D.C.: U.S. Department of education and welfare.

CONFEMEN (1999): "Les facteurs de l'efficacité dans l'enseignement primaire : les résultats du programme PASEC sur neuf pays d'Afrique et de l'Océan indien," Dakar: Secrétariat Technique Permanent de la CONFEMEN.

(2007): "Évaluation PASEC Sénégal," Dakar : Secrétariat Technique Permanent de la CONFEMEN.

Cueto, S., And M. Chinen (2007): "Educational Impact of a School Breakfast Programme in Rural Peru," International Journal of Educational Development.

Curtis, L., And S. Phipps (2000): "La pauvreté et le bien-être de l'enfance au Canada et aux Etats-Unis : La façon dont nous mesurons la pauvreté a-t-elle de l'importance?," Développement des ressources humaines Canada, Document de travail no SP-556-01-03F.

Davies, P. (2003): "The School Class Size Debate: Does Size Matter?," In Education Forum, Briefing Paper, 6.

DCAS (2011): "Guide de mise en place et de gestion des cantines scolaires," Rapport ministère de l'éducation, Division des Cantines Scolaires (DCaS).

DCMS (2003): "Etude opérationnelle du contrôle de l'anémie chez les écoliers Sénégalais," Rapport Division Contrôle Médical Scolaire (DCMS), Ministère de l'Education Sénégal.

Deaton, A. S. (2009): "Instruments of Development: Randomization in the Tropics, and the Search for the Elusive Keys to Economic Development," National Bureau of Economic Research Working Paper 14690.

(2010): "Instruments, Randomization, and Learning about Development," Journal of Economic Literature, (48), 424-455.

Dhaliwal, J. J., E. Duflo, R. Glennerster, and C. Tulloch (2012): "Comparative Cost-Effectiveness Analysis to Inform Policy in Developing Countries: A General Framework with Applications for Education," Abdul Latif Jameel Poverty Action Lab (J-PAL), MIT.

Diagne, A. (2012): Le Sénégal face aux défi de l'éducation, Enjeux et perspectives pour le XXIeme siécle. Karthala et cres edn.

Diambomba, M. (1997): "La qualité de l'éducation en République Centrafricaine: effets des facteurs individuel et scolaire sur le rendement des élèves dans l'enseignement primaire centrafricain," Québec : GERPS/CONFEMEN.

Duflo, E. (2004): "Scaling Up and Evaluation," In Annual World Bank Conference on Development Economics, Accelerating Development, ed. François Bourguignon and Boris Pleskovic Washington, D.C.: World Bank; Oxford and New York: Oxford University Press, pp. 341-369.

Duflo, E., P. Dupas, M. Kremer, and S. Sinei (2006): "Education and HIV/AIDS Prevention: Evidence from a randomized evaluation in Western Kenya," World Bank Policy Research Working Paper, (402).

Duflo, E., R. Glennerster, and M. Kremer (2008): "Using Randomization in Development Economics Research: A toolkit," Handbook of Development Economics. In: Schultz, T.P., Strauss, J. (Eds.), (Volume 4), Chapitre 61. 
El-Hioui, M., A. Sounlem, A. O. T. Ahami, Y. Aboussaleh, S. Rusinek, and K. Dik (2008): "Caractéristiques sociodémographiques et anthropométriques en relation avec la performance scolaire dans une école rurale de la ville de Kenitra (Maroc)," Antropo, 17, 25-33.

Esterling, K. M., M. A. Neblo, and D. M. J. Lazer (2011): "Estimating Treatment Effects in the Presence of Noncompliance and Nonresponse: The Generalized Endogenous Treatment Model," Political Analysis Advance Access.

Felouzis, G. (1997): L'efficacité des enseignants. Paris : PUF, coll. Pédagogie d'aujourd'hui.

Florens, J. P., J. J. Heckman, C. Meghir, and E. J. Vytlacil (2004): "Identification of Treatment Effects Using Control Functions in Models with Continuous, Endogenous Treatment and Heterogeneous Effects," Econometrica, (76), 1191-1206.

Frangakis, C. E., and D. B. Rubin (1999): "Addressing Complications of Intention-to-Treat Analysis in the Combined Presence of All-or-None Treatment- Noncompliance and Subsequent Missing Outcomes," Biometrika, (86(2)), 365-379.

(2002): "Principal Stratification in Causal Inference," Biometrics, (58(1)).

Freedman, D. A. (2006): "Statistical Models for Causation: What Inferential Leverage Do They Provide?," Evaluation Review, (30(6)), 691-713.

Frolich, M. (2004): "Programme Evaluation with Multiple Treatments," Journal of Economic Surveys, 18, 181-224.

Fu, M. L., L. Cheng, S. H. Tu, and W. H. Pan (2007): "Association between unhealthful eating patterns and unfavorable overall school performance in children," Journal of the American association, pp. 1935-1943.

Fuller, B., and P. Clarke (1994): "Raising School Effects While Ignoring Culture? Local Conditions and the Influence of Classroom Tools, Rules, and Pedagogy," Review of Educational Research, 64(1), 119-157.

Fuller, B., And X. Liang (1999): "Which girls stay in school? The influence of family economy, social demands, and ethnicity in South Africa," In C. H. Bledsoe, J. B. Casterline, J. A. Johnson-Kuhn et G. Haaga (Eds.), National Academy Press, pp. 181-215.

Glass, G. V., And M. L. Smith (1979): "Meta analysis of research on class size and Achievement," Educational Evaluation and Policy Analysis, 1(1), 2-16.

Glewwe, P., M. Kremer, and S. Moulin (2009): "Many children Left Behind? Textbooks and Test Scores in Kenya," American Economics Journal, (1), 112-135.

Glewwe, P., And A. E. Miguel (2008): "The impact of child health and nutrition on education in less developed countries," Handbook of Development Economics, Volume 4.

Grantham-McGregor, S. M., S. P. Walker, and S. Chang (2000): "Nutritional deficiencies and later behavioural development," Proceedings of the Nutrition Society, 59, 47-54.

Greene, W. H. (2011): "Econometric Analysis," Pearson, (7th Edition).

Grigorenko, E. L., A. Klin, D. L. Pauls, R. Senft, C. Hooper, and F. Volkmar (2002): "A descriptive study of hyperlexia in a clinically referred sample of children with developmental delays," Journal of Autism and Developmental Disorders, 32, 3-12. 
Heckman, J. J. (1976): "The Common Structure of Statistical Models of Truncation, Sample Selection and Limited Dependent Variables and a Simple Estimator for Such Models," Annals of Economic and Social Measurement, 5, 475-492.

(1978): "Dummy Endogenous Variables in a Simultaneous Equation System," Econometrica, 46, 931-959.

— (1979): "Sample Selection Bias as a Specification Error," Econometrica, 47, 153-162.

(1992): "Randomization and social policy evaluation," In: Manski, C., Garfinkel, I. (Eds.), Evaluating Welfare and Training Programs. Harvard Univ. Press, Cambridge, MA.

(2010): "Building Bridges Between Structural and Program Approaches to Evaluating Policy," Journal of Economic Literature, (48), 356-398.

Heckman, J. J., and C. Taber (2008): "Roy Model," The New Palgrave Dictionary of Economics, ed. by S. N. Durlauf, and L. E. Blume. Basingstoke: Palgrave Macmillan.

Heckman, J. J., and S. Urzua (2009): "Comparing IV with Structural Models: What Simple IV Can and Cannot Identify," National Bureau of Economic Research Working Paper, 14706.

Heckman, J. J., and E. J. Vytlacil (1998): "Instrumental Variables Methods for the Correlated Random Coefficient Model," Journal of Human Resources, 33, 974-987.

(2007a): "Econometric Evaluation of Social Programs, Part I: Causal Models, Structural Models and Econometric Policy Evaluation," in Handbook of Econometrics, ed. by J. J. Heckman, and E. E. Leamer, vol. 6B, chap. 70, pp. 4779-4874. Elsevier, North-Holland.

(2007b): "Econometric Evaluation of Social Programs, Part II: Using the marginal treatment effect to organize alternative economic estimators to evaluate social programs and to forecast their effects in new environments," in Handbook of Econometrics, ed. by J. J. Heckman, and E. E. A. E. Leamer, vol. 6B, chap. 70, pp. 4875-5144. Elsevier, North-Holland.

Hirano, K., and G. W. Imbens (2004): "The Propensity Score with Continuous Treatments," Draft chapter for Missing Data and Bayesian Methods in Practice: Contributions by Donald Rubin $\breve{S}$ Statistical Family, Wiley, forthcoming.

Horiuchi, Y., K. Imai, and N. Taniguchi (2007): "Designing and Analysing Randomized Experiments: Application to a Japanese Election Survey Experiment," American Journal of Political Science, (51), 669-687.

HoxBy, C. (2000): "The Effect of Class Size on Student Achievement: Evidence from Population Variation," Quarterly Journal of Economics, 115(4), 1239-1285.

ImaI, K. (2006): "Statistical Analysis of Randomized Experiments with Nonignorable Missing Binary Outcomes," Technical report. Princeton University.

ImAi, K., AND M. RatKovic (2013): "Estimating treatment effect heterogeneity in randomized program evaluation," The Annals of Applied Statistics, (4), 443-470.

ImAi, K., AND A. Strauss (2011): "Estimation of heterogeneous treatment effects from randomized experiments, with application to the optimal planning of the get-out-the-vote campaign," Political Analysis, (19), 1-19.

Imbens, G. W. (2010): "Better LATE Than Nothing: Some Comments on Deaton (2009) and Heckman and Urzua (2009)," Journal of Economic Literature, (48), 399-423. 
Imbens, G. W., And J. D. Angrist (1994): "Identification and Estimation of Local Average Treatment Effects," Econometrica, 62(2).

Imbens, G. W., And D. B. Rubin (1997): "Bayesian Inference for Causal Effects in Randomized Experiments with Noncompliance," Annals of Statistics, (25(1)), 305-327.

Imbens, G. W., and J. M. Wooldridge (2009): "Recent Developments in the Econometrics of Program Evaluation," Journal of Economic Literature, (47), 5-86.

Jacoby, E., Cueto, and E. Pollitt (1996): "Benefits of a School Breakfast Programme Among Andean Children in Huaraz, Peru," Food and Nutrition Bulletin, (17), 54-64.

Jarousse, J., And A. Mingat (1989): "Les effets des conditions d'enseignement sur les acquisitions des élèves à l'école primaire : le cas du Togo," Dijon : IREDU.

Kane, T. J., J. E. Rockoff, and D. O. Staiger (2008): "What Does Certification Tell Us About Teacher Effectiveness? Evidence from New York City," Economics of Education Review, 27(6), 615-631.

Kazianga, H., D. De Walque, and H. Alderman (2009): "Educational and Health Impacts of Two School Feeding Schemes: Evidence from a Randomized Trial in Rural Burkina Faso," The World Bank Policy Research Working Paper, (4976).

Kotz, S., N. Balakrishnan, and N. L. Johnson (2000): "Continuous Multivariate Distributions," John Wiley \& Sons, INC, 1, $2^{\text {nd }}$.

Krueger, A. B. (1999): "Experimental Estimates of Education Production Functions," Quarterly Journal of Economics, 114, 497-532.

Kvalsvig, J. D., R. M. Cooppan, and K. J. Connolly (1991): "The Effects of Parasite Infections on Cognitive Processes in Children," Annals of Tropical Medecine and Parasitology, (85(5)), 551-568.

Lalonde, R. J. (1986): "Evaluating the Econometric Evaluations of Training Programs with Experimental Data," American Economic Review, (76(4)).

LaWson-Body, N. (1993): "Influence de l'origine sociale sur le rendement scolaire au Togo," Thèse de doctorat, Université de Laval.

LECHNER, M. (2001): "Identification and estimation of causal effect of multiples treatments under the conditional independence assumption," In M. Lechner, and F. Pfeiffer (eds), Econometric Evaluation of Labour Market Policies. Heidelberg: Physica/Springer., pp. 43-58.

LIoyd, C., AND A. Blanc (1996): "Children's schooling in Sub-Saharan Africa: the role of fathers, mothers and others," Population and Development Review, (22(2)), 265-298.

Lockheed, M. E., B. Fuller, and A. P. Nyirongo (1989): "Family Effects on Students; Achievement in Thailand and Malawi," Sociology of Education, 62(4), 239-256.

Lockheed, M. E., S. C. Vail, and B. Fuller (1986): "How textbooks affect achievement in developing countries: evidence from Thailand," Educational Evaluation and Policy Analysis, 8(4), 379-392.

Lugaz, C. (2006): "Les programmes d'alimentation scolaire: définition, mise en œuvre, impact," UNESCO, IIPE, Cahiers de recherche de l'IIPE.

MA, X. (2007): "Gender differences in learning outcomes," Document de référence pour le rapport mondial de suivi sur l'EPT. 
Maddala, G. S. (1983): "Quantitative and Limited Dependent Variable Models in Econometrics," Cambridge: Cambridge University Press.

Marschak, J. (1953): "Economic Measurements for Policy and Prediction," In Studies in Econometric Method, ed. William C. Hood and Tjalling C. Koopmans, 1-26. New York: Wiley.

McEwan, P. (2013): "The impact of Chile's school feeding program on education outcomes," Economics of Education Review, (32), 122-139.

Michaelowa, K. (2006): "Des intrants rentables : une méta-analyse des évaluations du SACMEQ et du PASEC. In Éduquer plus et mieux. Écoles et programmes d'alphabétisation de développement de la petite enfance : comment assurer l'efficacité des apprentissages?," ADEA/IIPE/UNESCO, pp. 76-80.

Miguel, E., And M. Kremer (2004): "Worms: Identifying Impacts on Education and Health in the Presence of Treatment Externalities," Econometrica, (72(1)), 159-217.

Mincer, J. A. (1974): "Schooling, Experience and Earnings," National Bureau of Economic Research.

Muthén, B. (1990): "Moments of the Censored and Truncated Bivariate Normal Distribution," British Journal of Mathematical and Statistical Psychology, 43, 131-143.

Nokes, C., C. V. D. Bosch, and D. A. P. Bundy (1998): "The Effects of Iron Deficiency and Anemia on Mental and Motor Performance, Educational Achievement, and Behavior in Children: A Report of the International Nutritional Anemia Consultative Group," USAID:Washington, DC.

Nokes, C., S. M. Grantham-Mcgregor, A. W. Sawyer, E. S. Cooper, B. A. RobinSON, AND D. A. P. Bundy (1992): "Moderate-to-Heavy Infection of Trichuris Trichiura Affect Cognitive Function in Jamaican School Children," Parasitology, (104), 539-547.

Olken, B. (2007): "Monitoring Corruption: Evidence from a Field Experiment in Indonesia," Journal of Political Economy, (115), 200-249.

OMS (2000): "Le Rapport de Santé dans le Monde 2000," Organisation Mondiale de la Santé Génève.

PAM (2003): "La vulnérabilité structurelle à l'insécurité alimentaire en milieu rural," Gouvernement du Sénégal, Programme Alimentaire Mondial, VAM Unit, Rapport Sénégal.

(2006): "Education et développement de l'enfant," Programme Alimentaire Mondial: Sénégal. mimeo, (10451.0-Activité 2).

Piketty, T. (2004): "L'impact de la taille des classes et de la ségrégation sociale sur la réussite scolaire dans les écoles françaises : une estimation à partir du panel primaire 1997.," Document de travail EHESS, Paris.

Pollitt, E. (1995): "Does Breakfast Make a Difference in School?," Journal of the American Dietetic Association, (95(10)), 1134-1139.

Pollitt, E., P. Hathirat, N. Kotchabhakadi, L. Missel, and A. Valyasevi (1989): "Iron deficiency and education achievement in Thailand," American Journal of Clinical Nutrition, (50(3)), 687-697.

Powell, C. A., S. P. Walker, S. M. Chang, and S. M. Grantham-McGregor (1998): "Nutrition and Education: A Randomized Trial of the Effects of Breakfast in Rural Primary School Children," American Journal of Clinical Nutrition, (68), 873-879. 
Rivers, J. C., And W. L. SAnders (2002): "Teacher quality and equity in educational opportunity: Findings and policy implications," In L. T. Izumi, W. M. Evers (Eds.). Stanford, CA: Hoover Press, pp. 13-23.

Roy, A. (1951): "Some Thoughts on the Distribution of Earnings," Oxford Economic Papers, $3,135-146$.

Ryan, B. A., And G. R. Adams (1998): "Relations familiales et succès scolaire des enfants : données de l'Enquête longitudinale nationale sur les enfants et les jeunes," Document de travail, Ottawa : Développement des ressources humaines Canada, (W-98-13F).

Schwille, J., T. Eisemon, F. Ukobizoba, R. Houang, D. Kwon, and R. Prouty (1991): "Is grade repetition always wasteful? New data and unanswered questions," In BRIDGES Research Report, Cambridge, MA: Harvard Institute for International Development.

SEShadRI, S., AND T. Gopaldas (1989): "Impact of iron supplementation on cognitive functions in preschool and school-aged children: the Indian experience," American Journal of Clinical Nutrition, (50(3)), 675-686.

Simeon, D., and S. Grantham-McGregor (1989): "Effects of Missing Breakfast on the Cognitive Functions of School Children with Differing Nutritional Status," American Journal of Clinical Nutrition, (49), 646-653.

Soewondo, S., M. Husaini, and E. Pollitt (1989): "Effects of iron deficiency on attention and learning processes of preschool children: Bandung, Indonesia," American Journal of Clinical Nutrition, (50(3)), 667-674.

Tan, J. P., J. Lane, and G. Lassibille (1999): "Student outcomes in Philippine Elementary Schools: An Evaluation of Four Experiments," World Bank Economic Review, (13(3)).

TARAs, H. (2005): "Nutrition and Student Performance at School," Journal of School Health, 75(6), 199-213.

Todd, P., And K. I. Wolpin (2006): "Assessing the Impact of a School Subsidy Program in Mexico: Using a Social Experiment to Validate a Dynamic Behavioral Model of Child Schooling and Fertility," American Economic Review, (96(5)), 1384-1417.

UNESCO (1987): "Les politiques de l'éducation et de la formation en Afrique subsaharienne. Problématique, orientation, perspectives," UNESCO, Paris.

(2000): "Forum mondial sur l'éducation. Éducation pour tous: tenir nos engagements collectifs," UNESCO, Dakar.

(2005): "Lignes directrices pour des prestations de qualité dans l'enseignement supérieur transfrontalier," UNESCO, Paris.

Vermeersch, C., And M. Kremer (2004): "School Meals, Educational Achievement and School Competition: Evidence from a Randomized Evaluation," Oxford University, Oxford Mimeo.

Whaley, S. E., M. Sigman, C. Neumann, N. Bwibo, D. Guthrie, R. Weiss, S. AlBER, And S. P. Meurphy (2003): "The Impact of Dietary Intervention on the Cognitive Development of Kenyan School Children," American Society for Nutritional Sciences, (133).

Wooldridge, J. M. (2003): "Further Results on Instrumental Variables Estimation of Average Treatment Effects in the Correlated Random Coefficient Model," Economics Letters, 79, 185191. 


\section{APPENDIX $A$}

\section{Appendix Chapter 2}

\section{Appendix A}

\section{Appendix A.1 List and definition of variables}

Table A.1: List and definition of variables

\begin{tabular}{|c|c|c|}
\hline Variable name & Definition & Nature \\
\hline \multicolumn{3}{|l|}{ Outcome variables } \\
\hline Aggregate score & Aggregate test scores in French and Mathematics & continuous \\
\hline French score & Test scores in French & continuous \\
\hline Math score & Test scores in Mathematics & continuous \\
\hline Enrollment rate & Enrollment rate of the school & continuous \\
\hline Repetition rate & Repetition rate of the school & continuous \\
\hline Promotion rate & Promotion rate of the school & continuous \\
\hline Dropout rate & Dropout rate of the school & continuous \\
\hline \multicolumn{3}{|l|}{ Treatment indicators } \\
\hline Meal & Meal program indicator & binary $($ yes $=1)$ \\
\hline Deworming & Deworming in school/ at home indicator & binary $($ yes $=1)$ \\
\hline Deworming in school & Deworming in school program indicator & binary $($ yes $=1)$ \\
\hline Deworming at home & Deworming at home program indicator & binary $($ yes $=1)$ \\
\hline \multicolumn{3}{|c|}{ Schools and teachers characteristics } \\
\hline Temporary shelters & Number of classes in temporary shelters & continuous \\
\hline School manual ${ }^{a}$ & Number of pupils per manual in the school & continuous \\
\hline Total pupils & Total number of pupils per school & continuous \\
\hline Class size & Number of pupils by class & continuous \\
\hline Teacher's age & Age of the teacher (in year) & continuous \\
\hline Number of pregnancies & $\begin{array}{l}\text { Number of pregnancies in the school during } \\
\text { the school year }\end{array}$ & continuous \\
\hline Number of marriages & $\begin{array}{l}\text { Number of marriages in the school during } \\
\text { the school year }\end{array}$ & continuous \\
\hline Distance to school & Distance between school to pupils's home $(\mathrm{km})$ & binary $(0-1 \mathrm{~km}=1)$ \\
\hline Gender of teacher & Gender of the teacher & binary $($ male $=1)$ \\
\hline No professional qualif. & Teachers without professional qualification & binary $($ yes $=1)$ \\
\hline
\end{tabular}


Table A.1 - continued

\begin{tabular}{|c|c|c|}
\hline Variable name & Definition & Nature \\
\hline Professional qualif. CAP & $\begin{array}{l}\text { Teachers with a "Certificat d'Aptitude Pedagogique" } \\
\text { as professional qualification }\end{array}$ & binary (yes=1) \\
\hline Professional qualif. CEAP & Teachers with a 'Certificat Elémentaire d'Aptitude & \\
\hline & Pédagogique" as professional qualification & binary $($ yes $=1)$ \\
\hline Other professional qualif. & Teachers with other professional qualification & binary $($ yes $=1)$ \\
\hline High school diploma \& more & $\begin{array}{l}\text { Teachers having as academic qualification: } \\
\text { baccalaureate or undergraduate or bachelor }\end{array}$ & binary $($ yes $=1)$ \\
\hline National certificate & $\begin{array}{l}\text { Teachers having as academic qualification: } \\
\text { national certificate 'brevet' }\end{array}$ & binary (yes=1) \\
\hline Continuing training & Teachers have received continuing training & binary (yes=1) \\
\hline Absenteeism & $\begin{array}{l}\text { Indicates whether the teachers of the schools } \\
\text { are often absent or not }\end{array}$ & binary (yes=1) \\
\hline Playground & Existence of a playground in the school & binary (yes $=1$ ) \\
\hline Medicine box & Existence of a medicine box in the school & binary $($ yes $=1$ ) \\
\hline Toilets & Existence of separate toilets in the school & binary (yes=1) \\
\hline Management committee & Existence of a management committee in the school & binary (yes=1) \\
\hline School project & Existence of a school project in the school & binary $($ yes $=1)$ \\
\hline Cooperative school & Existence of a cooperative school in the school & binary (yes=1) \\
\hline Association of parents & $\begin{array}{l}\text { Existence of a Association of pupil's parents } \\
\text { in the school }\end{array}$ & binary $($ yes=1) \\
\hline Association of mothers & $\begin{array}{l}\text { Existence of a Association of pupil's mothers } \\
\text { in the school }\end{array}$ & binary $($ yes $=1)$ \\
\hline Rural council grant & School receives a grant from the rural council & binary (yes $=1$ ) \\
\hline Water point & Existence of a water point in the school & binary (yes=1) \\
\hline Disturbed courses & Disturbances that caused delay of the start courses & binary (yes=1) \\
\hline Storage & Existence of a storage -warehouse- in the school & binary (yes=1) \\
\hline Meals near school & Opportunity of the pupils to have meal near school & binary $($ yes $=1$ ) \\
\hline Hands washing & Existence of a hands washing device in the school & binary (yes=1) \\
\hline Pupils characteristics & & \\
\hline Pupil's age & Age of the pupil (in year) & continuous \\
\hline Number of hours ${ }^{b}$ & $\begin{array}{l}\text { The number of hours of work that the } \\
\text { child spends doing housework per day }\end{array}$ & continuous \\
\hline Gender of pupil & Gender of the pupil & binary $($ boy $=1)$ \\
\hline Grade & Education level of the pupil & binary $(\mathrm{CE} 2=1)$ \\
\hline Koranic school & Pupils who attended a islamic school & binary $($ yes $=1)$ \\
\hline Early childhood inst. & Pupils who attended an early child institution & binary (yes=1) \\
\hline Sick last 3 months & Pupils who have been sick in the last 3 months & binary $($ yes $=1)$ \\
\hline Pupils eat fill & Pupils who eat to their fill at home & binary (yes=1) \\
\hline Snack & Child who brings a snack to school & binary $($ yes $=1)$ \\
\hline Households characteristics & & \\
\hline Food expenditure & $\begin{array}{l}\text { Monthly Food expenditure of household } \\
\text { per adult equivalent (in } \log , \mathrm{cfa}^{a} \text { ) }\end{array}$ & continuous \\
\hline Health expenditure & Annual health expenditure of household $\left(\log , \mathrm{cfa}^{c}\right)$ & continuous \\
\hline Education expenditure & Annual education expenditure of household $\left(\mathrm{log}, \mathrm{cfa}^{c}\right)$ & continuous \\
\hline Household size & Household size per adult equivalent & continuous \\
\hline Livestock & Number of head of livestock that the household owns & continuous \\
\hline Gender of household head & Gender of the household head & binary $($ male $=1)$ \\
\hline Lit. of household head & Head of household is literate in French & binary $($ yes $=1)$ \\
\hline Marital status & Married people & binary (yes=1) \\
\hline Land & $\begin{array}{l}\text { Possession of cultivable land owned } \\
\text { by the household }\end{array}$ & binary $($ yes=1) \\
\hline Communities characteristics & & \\
\hline Primary schools & Number of primary schools in the community & continuous \\
\hline
\end{tabular}


Table A.1 - continued

\begin{tabular}{|c|c|c|}
\hline Variable name & Definition & Nature \\
\hline College & Existence of a college in the school's village & binary $($ yes $=1)$ \\
\hline Parents school & $\begin{array}{l}\text { Child living in a community where parents } \\
\text { are not interested in school }\end{array}$ & binary (yes $=1$ ) \\
\hline Koranic school com. & $\begin{array}{l}\text { Child living in a community where attending Koranic } \\
\text { school prevent children from going to school }\end{array}$ & binary $($ yes $=1)$ \\
\hline \multicolumn{3}{|c|}{ Geographical localization } \\
\hline Diourbel & Region Diourbel & binary $($ yes $=1)$ \\
\hline Fatick & Region Fatick & binary $($ yes $=1)$ \\
\hline Kolda & Region Kolda & binary $($ yes $=1$ ) \\
\hline Sédhiou & Region Sédhiou & binary $($ yes $=1)$ \\
\hline
\end{tabular}

Note $^{a}$ : This variable is created using total pupil and number of school manual; ${ }^{b}$ : This variable is created by doing the report between the total sum of hours spend in each housework by sevent $(7) ;^{c}$ : Currency of the French colonies in Africa.

\section{Appendix A.2 Map of Senegal: geographical areas benefiting form the pro- grams}

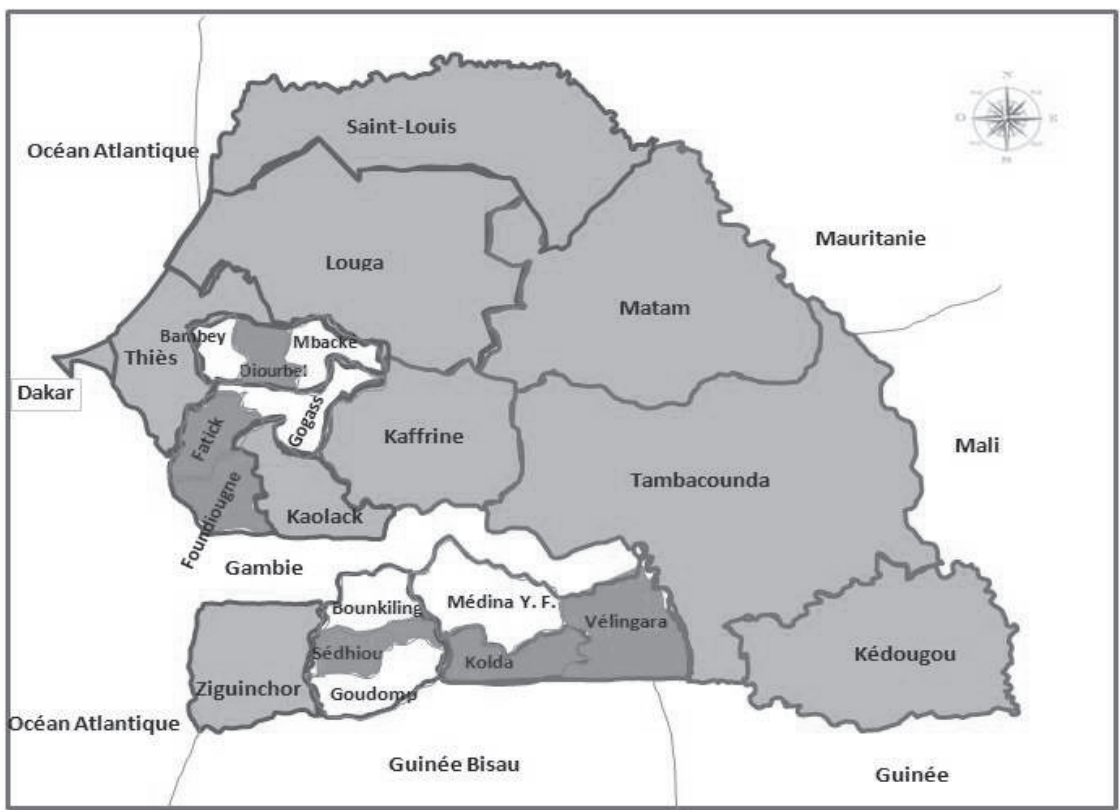

The blue color indicates the geographical areas benefiting form the programs.

Figure A.1: Geographical areas covered by the meal and deworming programs 


\section{Appendix A.3 Summary statistics}

Table A.2: Summary statistics: Meal

\begin{tabular}{|c|c|c|c|c|c|}
\hline \multirow[b]{2}{*}{ Variable } & \multicolumn{2}{|c|}{ Treatment group } & \multicolumn{2}{|c|}{ Control group } & \multirow{2}{*}{$\begin{array}{l}\text { t-test } \\
p>|t|\end{array}$} \\
\hline & Mean & Std. Dev. & Mean & Std. Dev. & \\
\hline Aggregate score & 42.445 & 18.883 & 37.587 & 19.458 & $0.000^{* * *}$ \\
\hline French score & 41.877 & 21.162 & 38.228 & 21.171 & $0.000^{* * *}$ \\
\hline Math score & 42.890 & 21.110 & 36.949 & 21.339 & $0.000^{* * *}$ \\
\hline Temporary shelters & 1.686 & 1.318 & 1.503 & 1.168 & $0.001^{* * *}$ \\
\hline School manual & 1.523 & 3.544 & 1.077 & 1.092 & $0.000^{* * *}$ \\
\hline Total pupils & 187.877 & 127.701 & 187.440 & 118.995 & 0.939 \\
\hline Class size & 27.946 & 12.181 & 35.834 & 14.395 & $0.000^{* * *}$ \\
\hline Number of pregnancies & 0.257 & 0.516 & 0.089 & 0.331 & $0.000^{* * *}$ \\
\hline Number of marriages & 0.172 & 0.378 & 0.321 & 0.698 & $0.000^{* * *}$ \\
\hline Teacher's age & 32.368 & 5.464 & 31.223 & 5.496 & $0.000^{* * *}$ \\
\hline Pupil's age & 9.688 & 1.693 & 9.902 & 1.780 & $0.010^{* *}$ \\
\hline Number of hours & 0.379 & 0.888 & 0.328 & 0.573 & 0.104 \\
\hline Food expenditure ${ }^{a}$ & 11.495 & 0.526 & 11.462 & 0.520 & 0.186 \\
\hline Education expenditure ${ }^{a}$ & 7.636 & 1.176 & 7.512 & 1.366 & $0.046^{* *}$ \\
\hline Health expenditure $^{a}$ & 7.778 & 1.670 & 7.440 & 2.128 & $0.000^{* * *}$ \\
\hline Household size ${ }^{b}$ & 10.930 & 6.260 & 11.451 & 6.924 & 0.101 \\
\hline Livestock & 9.900 & 20.740 & 12.534 & 21.116 & $0.008^{* * *}$ \\
\hline Distance to school & 0.817 & 0.386 & 0.780 & 0.414 & $0.052^{*}$ \\
\hline Playground & 0.916 & 0.276 & 0.747 & 0.434 & $0.000^{* * *}$ \\
\hline Management committee & 0.629 & 0.483 & 0.530 & 0.499 & $0.000^{* * *}$ \\
\hline School project & 0.084 & 0.278 & 0.149 & 0.356 & $0.000^{* * *}$ \\
\hline Cooperative school & 0.699 & 0.458 & 0.682 & 0.465 & 0.434 \\
\hline Association of parents & 0.983 & 0.126 & 0.961 & 0.192 & $0.007^{* * *}$ \\
\hline Association of mothers & 0.438 & 0.496 & 0.153 & 0.360 & $0.000^{* * *}$ \\
\hline Rural council grant & 0.924 & 0.263 & 0.618 & 0.485 & $0.000^{* * *}$ \\
\hline Water point & 0.438 & 0.496 & 0.395 & 0.489 & $0.062^{*}$ \\
\hline Disturbed courses & 0.902 & 0.297 & 0.713 & 0.452 & $0.000^{* * *}$ \\
\hline Meals near school & 0.319 & 0.466 & 0.285 & 0.451 & 0.107 \\
\hline Storage & 0.440 & 0.496 & 0.183 & 0.387 & $0.000^{* * *}$ \\
\hline Medicine box & 0.060 & 0.238 & 0.133 & 0.339 & $0.000^{* * *}$ \\
\hline Gender of teacher & 0.794 & 0.404 & 0.840 & 0.366 & $0.009^{* * *}$ \\
\hline No professional qualif. ${ }^{c}$ & 0.199 & 0.399 & 0.316 & 0.465 & $0.000^{* * *}$ \\
\hline Professional qualif. CAP & 0.145 & 0.352 & 0.162 & 0.369 & 0.302 \\
\hline Professional qualif. CEAP & 0.349 & 0.477 & 0.188 & 0.391 & $0.000^{* * *}$ \\
\hline Other professional qualif. & 0.306 & 0.461 & 0.332 & 0.471 & 0.239 \\
\hline High school diploma \& more & 0.433 & 0.496 & 0.448 & 0.497 & 0.541 \\
\hline National certificate & 0.566 & 0.496 & 0.551 & 0.497 & 0.541 \\
\hline Continuing training & 0.450 & 0.497 & 0.654 & 0.475 & $0.000^{* * *}$ \\
\hline Absenteeism & 0.037 & 0.190 & 0.080 & 0.271 & $0.000^{* * *}$ \\
\hline Gender of pupil & 0.479 & 0.499 & 0.515 & 0.499 & 0.127 \\
\hline Grade & 0.505 & 0.500 & 0.499 & 0.500 & 0.789 \\
\hline Koranic school & 0.285 & 0.452 & 0.296 & 0.456 & 0.618 \\
\hline Early childhood inst. & 0.037 & 0.190 & 0.067 & 0.250 & $0.007^{* * *}$ \\
\hline Snack & 0.050 & 0.219 & 0.099 & 0.299 & $0.000^{* * *}$ \\
\hline Sick last 3 months & 0.277 & 0.448 & 0.244 & 0.430 & 0.112 \\
\hline Pupils eat fill & 0.879 & 0.326 & 0.883 & 0.321 & 0.793 \\
\hline Gender of household head & 0.923 & 0.266 & 0.895 & 0.306 & $0.043^{* *}$ \\
\hline Lit. of household head & 0.158 & 0.365 & 0.157 & 0.364 & 0.950 \\
\hline Marital status & 0.959 & 0.197 & 0.953 & 0.211 & 0.544 \\
\hline
\end{tabular}


Table A.2 - continued

\begin{tabular}{|c|c|c|c|c|c|}
\hline \multirow[b]{2}{*}{ Variable } & \multicolumn{2}{|c|}{ Treatment group } & \multicolumn{2}{|c|}{ Control group } & \multirow{2}{*}{$\begin{array}{l}\text { t-test } \\
p>|t|\end{array}$} \\
\hline & Mean & Std. Dev. & Mean & Std. Dev. & \\
\hline Land & 0.946 & 0.225 & 0.947 & 0.224 & 0.934 \\
\hline Parents school & 0.491 & 0.500 & 0.489 & 0.500 & 0.937 \\
\hline College & 0.133 & 0.340 & 0.239 & 0.426 & $0.000^{* * *}$ \\
\hline Diourbel & 0 & 0 & 0.079 & 0.270 & $0.000^{* * *}$ \\
\hline Fatick & 0.672 & 0.469 & 0.425 & 0.494 & $0.000^{* * *}$ \\
\hline Kolda & 0.185 & 0.389 & 0.454 & 0.498 & $0.000^{* * *}$ \\
\hline Sédhiou & 0.141 & 0.349 & 0.039 & 0.195 & $0.000^{* * *}$ \\
\hline
\end{tabular}

\# Observations $\quad 613 \quad 1756$

Note $^{a}$ : In log; ${ }^{b}:$ Household size per adult equivalent; ${ }^{c}:$ qualification.

Significance levels: $\quad *: 10 \% \quad * *: 5 \% \quad * * *: 1 \%$

Table A.3: Summary statistics: Deworming

\begin{tabular}{|c|c|c|c|c|c|}
\hline \multirow[b]{2}{*}{ Variable } & \multicolumn{2}{|c|}{ Treatment group } & \multicolumn{2}{|c|}{ Control group } & \multirow{2}{*}{$\begin{array}{l}\text { t-test } \\
p>|t|\end{array}$} \\
\hline & Mean & Std. Dev. & Mean & Std. Dev. & \\
\hline Aggregate score & 37.495 & 18.480 & 37.587 & 19.458 & 0.913 \\
\hline French score & 37.680 & 20.777 & 38.228 & 21.171 & 0.554 \\
\hline Math score & 37.161 & 19.910 & 36.949 & 21.339 & 0.818 \\
\hline Temporary shelters & 1.633 & 1.138 & 1.503 & 1.168 & $0.011^{* *}$ \\
\hline School manual & 0.913 & 0.894 & 1.077 & 1.092 & $0.000^{* * *}$ \\
\hline Total pupils & 223.104 & 149.087 & 187.440 & 118.995 & $0.000^{* * *}$ \\
\hline Class size & 35.752 & 12.887 & 35.834 & 14.395 & 0.894 \\
\hline Number of pregnancies & 0.147 & 0.402 & 0.089 & 0.331 & $0.000^{* * *}$ \\
\hline Number of marriages & 0.305 & 0.731 & 0.321 & 0.698 & 0.593 \\
\hline Teacher's age & 32.619 & 5.799 & 31.223 & 5.496 & $0.000^{* * *}$ \\
\hline Pupil's age & 9.659 & 1.785 & 9.902 & 1.780 & $0.002^{* * *}$ \\
\hline Number of hours & 0.268 & 0.504 & 0.328 & 0.573 & $0.014^{* *}$ \\
\hline Food expenditure ${ }^{a}$ & 11.458 & 0.542 & 11.462 & 0.520 & 0.866 \\
\hline Education expenditure $^{a}$ & 7.774 & 1.370 & 7.512 & 1.366 & $0.000^{* * *}$ \\
\hline Health expenditure $^{a}$ & 7.251 & 2.269 & 7.440 & 2.128 & $0.049^{* *}$ \\
\hline Household size ${ }^{b}$ & 11.429 & 6.413 & 11.451 & 6.924 & 0.941 \\
\hline Livestock & 8.306 & 13.432 & 12.534 & 21.116 & $0.000^{* * *}$ \\
\hline Distance to school & 0.856 & 0.350 & 0.780 & 0.414 & $0.000^{* * *}$ \\
\hline Playground & 0.800 & 0.276 & 0.747 & 0.434 & $0.005^{* * *}$ \\
\hline Management committee & 0.673 & 0.469 & 0.530 & 0.499 & $0.000^{* * *}$ \\
\hline School project & 0.079 & 0.270 & 0.149 & 0.356 & $0.000^{* * *}$ \\
\hline Cooperative school & 0.729 & 0.444 & 0.682 & 0.465 & $0.022^{* *}$ \\
\hline Association of parents & 0.983 & 0.127 & 0.961 & 0.192 & $0.004^{* * *}$ \\
\hline Association of mothers & 0.248 & 0.432 & 0.153 & 0.360 & $0.000^{* * *}$ \\
\hline Rural council grant & 0.656 & 0.475 & 0.618 & 0.485 & $0.072 *$ \\
\hline Water point & 0.526 & 0.499 & 0.395 & 0.489 & $0.000^{* * *}$ \\
\hline Disturbed courses & 0.659 & 0.474 & 0.713 & 0.452 & $0.007^{* * *}$ \\
\hline Meals near school & 0.265 & 0.441 & 0.285 & 0.451 & 0.313 \\
\hline Storage & 0.264 & 0.441 & 0.183 & 0.387 & $0.000^{* * *}$ \\
\hline Medicine box & 0.119 & 0.324 & 0.133 & 0.339 & 0.335 \\
\hline Gender of teacher & 0.823 & 0.381 & 0.840 & 0.366 & 0.297 \\
\hline
\end{tabular}


Table A.3 - continued

\begin{tabular}{|c|c|c|c|c|c|}
\hline \multirow[b]{2}{*}{ Variable } & \multicolumn{2}{|c|}{ Treatment group } & \multicolumn{2}{|c|}{ Control group } & \multirow{2}{*}{$\begin{array}{l}\text { t-test } \\
p>|t|\end{array}$} \\
\hline & Mean & Std. Dev. & Mean & Std. Dev. & \\
\hline No professional qualif. ${ }^{c}$ & 0.216 & 0.411 & 0.316 & 0.465 & $0.000^{* * *}$ \\
\hline Professional qualif. CAP & 0.264 & 0.441 & 0.162 & 0.369 & $0.000^{* * *}$ \\
\hline Professional qualif. CEAP & 0.194 & 0.395 & 0.188 & 0.391 & 0.739 \\
\hline Other professional qualif. & 0.325 & 0.468 & 0.332 & 0.471 & 0.736 \\
\hline High school diploma \& more & 0.519 & 0.499 & 0.448 & 0.497 & $0.001^{* * *}$ \\
\hline National certificate & 0.480 & 0.499 & 0.551 & 0.497 & $0.001^{* * *}$ \\
\hline Continuing training & 0.547 & 0.498 & 0.654 & 0.475 & $0.000^{* * *}$ \\
\hline Absenteeism & 0.114 & 0.319 & 0.080 & 0.271 & $0.006^{* * *}$ \\
\hline Gender of pupil & 0.493 & 0.500 & 0.515 & 0.499 & 0.328 \\
\hline Garde & 0.473 & 0.499 & 0.499 & 0.500 & 0.236 \\
\hline Koranic school & 0.276 & 0.447 & 0.296 & 0.456 & 0.322 \\
\hline Early childhood inst. & 0.119 & 0.324 & 0.067 & 0.250 & $0.000^{* * *}$ \\
\hline Snack & 0.124 & 0.330 & 0.099 & 0.299 & $0.068^{*}$ \\
\hline Sick last 3 months & 0.402 & 0.490 & 0.244 & 0.430 & $0.000^{* * *}$ \\
\hline Pupils eat fill & 0.922 & 0.268 & 0.883 & 0.321 & $0.004^{* * *}$ \\
\hline Gender of household head & 0.880 & 0.324 & 0.895 & 0.306 & 0.300 \\
\hline Lit. of household head & 0.216 & 0.411 & 0.157 & 0.364 & $0.000^{* * *}$ \\
\hline Marital status & 0.956 & 0.204 & 0.953 & 0.211 & 0.751 \\
\hline Land & 0.943 & 0.230 & 0.947 & 0.224 & 0.753 \\
\hline Parents school & 0.536 & 0.499 & 0.489 & 0.500 & $0.032^{* *}$ \\
\hline College & 0.202 & 0.402 & 0.239 & 0.426 & $0.047^{* *}$ \\
\hline Diourbel & 0.020 & 0.141 & 0.079 & 0.270 & $0.000^{* * *}$ \\
\hline Fatick & 0.627 & 0.483 & 0.425 & 0.494 & $0.000^{* * *}$ \\
\hline Kolda & 0.310 & 0.463 & 0.454 & 0.498 & $0.000^{* * *}$ \\
\hline Sédhiou & 0.041 & 0.198 & 0.039 & 0.195 & 0.892 \\
\hline
\end{tabular}

$\begin{array}{lll}\text { \# Observations } & 731 & 1756\end{array}$

Note $^{a}:$ In log; ${ }^{b}$ : Household size per adult equivalent; ${ }^{c}:$ qualification.

Significance levels: $\quad *: 10 \% \quad * *: 5 \% \quad * * *: 1 \%$

\section{Appendix A.4 Further estimation results}

Table A.4: Propensity score estimation: Probit regression

\begin{tabular}{|c|c|c|c|c|}
\hline \multirow[b]{2}{*}{ Variable } & \multicolumn{2}{|c|}{ Meal } & \multicolumn{2}{|c|}{ Deworming } \\
\hline & Coef. & Std. Err. & Coef. & Std. Err. \\
\hline Total pupils & $0.0008^{* * *}$ & 0.0001 & $0.0006^{* * *}$ & 0.0001 \\
\hline Distance to school & $0.253^{* * *}$ & 0.055 & - & - \\
\hline Management committee & - & - & $0.206^{* * *}$ & 0.053 \\
\hline Association of mothers & $0.470^{* * *}$ & 0.051 & $0.229^{* * *}$ & 0.060 \\
\hline Cooperative school & - & - & $0.188^{* * *}$ & 0.052 \\
\hline Rural council grant & $0.607^{* * *}$ & 0.053 & $0.055^{* * *}$ & 0.020 \\
\hline Water point & $0.111^{* *}$ & 0.052 & $0.140^{* *}$ & 0.059 \\
\hline Disturbed courses & $0.254^{* * *}$ & 0.051 & - & - \\
\hline Storage & $0.470^{* * *}$ & 0.054 & - & - \\
\hline Gender of pupil & $-0.073^{*}$ & 0.043 & $-0.080^{*}$ & 0.047 \\
\hline Lit. of household head & - & - & $0.158^{* * *}$ & 0.060 \\
\hline Intercept & $-1.673^{* * *}$ & 0.085 & $-1.121^{* * *}$ & 0.059 \\
\hline
\end{tabular}




\begin{tabular}{|c|c|c|}
\hline & Meal & Deworming \\
\hline Variable & Coef. Std. Err. & Coef. Std. Err. \\
\hline \multicolumn{3}{|l|}{ LR test } \\
\hline$\chi_{2}$ (d.o.f) & $156.94(17)$ & $248.51(13)$ \\
\hline$P>\chi_{2}$ & 0.000 & 0.000 \\
\hline Pseudo $\mathrm{R}^{2}$ & 0.0714 & 0.0596 \\
\hline Log likelihood & -1019.997 & -1959.846 \\
\hline \# Observations & 3398 & 3625 \\
\hline
\end{tabular}

Significance levels: $\quad *: 10 \% \quad * *: 5 \% \quad * * *: 1 \%$

Table A.5: Determinants of scores: Meal, $\mathrm{HSF}^{a}$ regression $\left(2-\right.$ step $\left.^{b}\right)$

Variable

Performance Eq.

Temporary shelters

School manual

School manual squared

Class size

Class size squared

Number of pregnancies

Number of marriages

Teacher's age

Teacher's age square

Pupil's age

Pupil's age square

Number of hours

Food expenditure ${ }^{c}$

Education expenditure ${ }^{c}$

Health expenditure ${ }^{c}$

Household size ${ }^{d}$

Livestock

Distance to school

Playground

Water point

Disturbed courses

Meals near school

Gender of teacher

No professional qualif. ${ }^{e}$

Professional qualif. CAP

Professional qualif. CEAP

High school diploma \& more

Continuing training

Absenteeism

Gender of pupil

Garde

Koranic school

Early childhood inst.
Aggregate score

Coef. Std. Err.

$-0.749^{* *}$

$6.698^{* * *}$

$-0.613^{* * *}$

$-0.237^{* *}$

0.001

$-3.586^{* * *}$

$-0.494$

$-1.390^{* *}$

$0.023^{* *}$

$7.113^{* * *}$

$-0.284^{* * *}$

$-0.030$

$-0.749$

$0.937^{* * *}$

$-0.270$

$0.211^{* * *}$

0.007

1.366

0.400

$2.424^{* * *}$

$-6.363^{* * *}$

$-0.120$

$-4.799^{* * *}$

$6.271^{* * *}$

$2.755^{*}$

$4.483^{* * *}$

0.216

$-0.198$

$-1.174$

$1.422^{* *}$

$-12.658^{* * *}$

$2.706^{* * *}$

$4.902^{* * *}$

0.345

1.107

0.195

0.114

0.001

1.035

0.736

0.657

0.009

2.109

0.097

0.534

0.767

0.296

0.186

0.065

0.019

0.939

1.060

0.884

1.170

0.912

1.144

1.043

1.413

1.098

0.889

0.790

1.600

0.722

1.032

0.812

1.563
French score

Coef. Std. Err.

\begin{tabular}{l} 
French score \\
Coef. $\quad$ Std. Err. \\
\hline
\end{tabular}

$-0.433$

$6.779^{* * *}$

$-0.939^{* * *}$

$-0.076$

$-0.0002$

$-3.757^{* * * *}$

$-1.240$

$-1.123$

0.016

$8.439^{* * *}$

$-0.348^{* * *}$

$-0.114$

$-1.088$

$1.149^{* * * *}$

$-0.189$

$0.215^{* * *}$

$-0.004$

1.425

0.085

$1.584^{*}$

$-5.076^{* * *}$

$-1.270$

$-3.920^{* * *}$

$5.056^{* * *}$

$3.088^{* *}$

$2.025 *$

$-1.237$

$-0.370$

$-0.406$

0.703

$-19.557^{* * *}$

$2.630^{\text {*** }}$

$5.811^{* * *}$

\subsection{9}

1.185

0.209

0.122

0.001

1.108

0.788

0.703

0.010

2.257

0.104

0.572

0.822

0.317

0.199

0.070

0.020

0.997

1.134

0.940

1.244

0.976

1.225

1.117

1.512

1.175

0.952

0.845

1.714

0.766

1.105

0.869

1.673
Math score Coef. Std. Err.

$-1.151^{* * *}$
$6.608^{* * *}$

0.386

1.240

$-0.395^{* * *} \quad 0.128$

$0.004^{* *} \quad 0.001$

$-3.303^{* * *} \quad 1.158$

$0.173 \quad 0.825$

$-1.533^{* *} \quad 0.735$

$0.027^{* *} \quad 0.011$

$5.914^{* *} \quad 2.361$

$-0.228^{* *} \quad 0.109$

$0.050 \quad 0.596$

$\begin{array}{ll}-0.537 & 0.859\end{array}$

$0.747^{* *} \quad 0.332$

$-0.363^{*} \quad 0.208$

$0.206^{* * *} \quad 0.073$

$0.020 \quad 0.021$

$1.316 \quad 1.062$

$0.651 \quad 1.187$

$3.270^{* * *} \quad 1.000$

$-7.615^{* * *} \quad 1.321$

$1.096 \quad 1.022$

$-5.729^{* * *} \quad 1.280$

$7.477^{* * *} \quad 1.167$

$2.643^{*} \quad 1.582$

$6.807^{* * *} \quad 1.229$

$1.646^{*} \quad 0.994$

$\begin{array}{ll}-0.159 & 0.884\end{array}$

$\begin{array}{ll}-1.939 & 1.789\end{array}$

$1.926^{* *} \quad 0.818$

$-5.707^{* * *} \quad 1.155$

$2.839^{* * *} \quad 0.909$

$3.915^{* *} \quad 1.751$ 
Table A.5 - continued

\begin{tabular}{|c|c|c|c|c|c|c|}
\hline \multirow[b]{2}{*}{ Variable } & \multicolumn{2}{|c|}{ Aggregate score } & \multicolumn{2}{|c|}{ French score } & \multicolumn{2}{|c|}{ Math score } \\
\hline & Coef. & Std. Err. & Coef. & Std. Err. & Coef. & Std. Err. \\
\hline Snack & 1.472 & 1.315 & 1.477 & 1.408 & 1.686 & 1.471 \\
\hline Sick last 3 months & 0.069 & 0.825 & 0.723 & 0.883 & -0.495 & 0.922 \\
\hline Pupils eat fill & $2.899^{* *}$ & 1.165 & $2.615^{* *}$ & 1.248 & 3.125 & 1.303 \\
\hline Gender of household head & $-1.156^{*}$ & 1.366 & -2.291 & 1.462 & 0.050 & 1.528 \\
\hline Lit. of household head & -1.660 & 1.007 & -1.170 & 1.078 & $-2.397^{* *}$ & 1.127 \\
\hline Marital status & 1.153 & 1.873 & 2.366 & 2.006 & -0.0179 & 2.094 \\
\hline Land & -2.319 & 1.670 & -1.013 & 1.787 & $-3.377^{*}$ & 1.869 \\
\hline Parents school & $-3.075^{* * *}$ & 0.823 & $-3.940^{* * *}$ & 0.881 & $-2.066^{* *}$ & 0.921 \\
\hline College & $7.274^{* * *}$ & 1.110 & $6.668^{* * *}$ & 1.187 & $7.928^{* * *}$ & 1.244 \\
\hline Diourbel & $6.731^{* * *}$ & 1.805 & $4.937^{* *}$ & 1.930 & $8.595^{* * *}$ & 2.023 \\
\hline Kolda & -0.725 & 1.298 & -0.099 & 1.389 & -1.209 & 1.454 \\
\hline Sédhiou & -0.887 & 1.909 & $-3.681^{*}$ & 2.045 & 1.773 & 2.134 \\
\hline Meal & 3.055 & 2.137 & $4.873^{* *}$ & 2.274 & 0.996 & 2.410 \\
\hline Intercept & 26.737 & 17.820 & 21.579 & 19.074 & 30.573 & 19.948 \\
\hline \multicolumn{7}{|l|}{ Selection Eq. } \\
\hline Total pupils & $0.0006^{*}$ & 0.0003 & $0.0006^{*}$ & 0.0003 & $0.0006^{*}$ & 0.0003 \\
\hline Distance to school & $0.1276^{*}$ & 0.075 & $0.127^{*}$ & 0.075 & $0.127^{*}$ & 0.075 \\
\hline Management committee & 0.089 & 0.070 & 0.089 & 0.0702 & 0.089 & 0.070 \\
\hline Association of mothers & $0.707^{* * *}$ & 0.072 & $0.707^{* * *}$ & 0.072 & $0.707^{* * *}$ & 0.072 \\
\hline Cooperative school & -0.012 & 0.068 & -0.012 & 0.068 & -0.012 & 0.068 \\
\hline Rural council grant & 0.800 & 0.087 & 0.800 & 0.087 & 0.800 & 0.087 \\
\hline Water point & -0.035 & 0.076 & -0.035 & 0.076 & -0.035 & 0.076 \\
\hline Disturbed courses & $0.986^{* * *}$ & 0.096 & $0.986^{* * *}$ & 0.096 & $0.986^{* * *}$ & 0.096 \\
\hline Storage & $0.841^{* * *}$ & 0.074 & $0.841^{* * *}$ & 0.074 & $0.841^{* * *}$ & 0.074 \\
\hline Gender of pupil & -0.088 & 0.062 & -0.088 & 0.062 & -0.088 & 0.062 \\
\hline Intercept & $-2.701^{* * *}$ & 0.161 & $-2.701^{* * *}$ & 0.161 & $-2.701^{* * *}$ & 0.161 \\
\hline hasard $\lambda$ & $3.967^{* * *}$ & 1.365 & 1.653 & 1.455 & $6.401^{* * *}$ & 1.535 \\
\hline$\rho$ & 0.229 & - & 0.077 & - & 0.327 & - \\
\hline$\sigma$ & 17.271 & - & 18.313 & - & 19.562 & - \\
\hline$\lambda$ & 3.967 & 1.365 & 1.653 & 1.455 & 6.401 & 1.535 \\
\hline \# Observations & \multicolumn{2}{|c|}{2369} & \multicolumn{2}{|c|}{2369} & \multicolumn{2}{|c|}{2369} \\
\hline Wald $\chi_{2}(50)$ & \multicolumn{2}{|c|}{718.99} & \multicolumn{2}{|c|}{888.99} & \multicolumn{2}{|c|}{618.01} \\
\hline$P>\chi_{2}$ & \multicolumn{2}{|c|}{0.000} & \multicolumn{2}{|c|}{0.000} & \multicolumn{2}{|c|}{0.000} \\
\hline
\end{tabular}

Notes $^{a}$ : Heckman selection framework; ${ }^{b}$ : Two step; ${ }^{c}:$ In log; ${ }^{d}$ : Household size per adult equivalent; ${ }^{e}:$ qualification. Significance levels (bootstrap): $\quad *: 10 \% \quad * *: 5 \% \quad * * *: 1 \%$

Table A.6: Determinants of scores: Deworming, $\mathrm{HSF}^{a}$ regression $\left(2-\right.$ step $\left.^{b}\right)$

\begin{tabular}{|c|c|c|c|c|c|c|}
\hline \multirow[b]{2}{*}{ Variable } & \multicolumn{2}{|c|}{ Aggregate score } & \multicolumn{2}{|c|}{ French score } & \multicolumn{2}{|c|}{ Math score } \\
\hline & Coef. & Std. Err. & Coef. & Std. Err. & Coef. & Std. Err. \\
\hline Performance Eq. & & & & & & \\
\hline Temporary shelters & 0.452 & 0.390 & 0.228 & 0.412 & 0.663 & 0.440 \\
\hline School manual & 1.335 & 1.097 & $2.479^{* *}$ & 1.157 & 0.323 & 1.239 \\
\hline School manual squared & 0.091 & 0.190 & -0.304 & 0.201 & $0.472^{* *}$ & 0.215 \\
\hline Class size & -0.161 & 0.119 & 0.140 & 0.125 & $-0.469^{* * *}$ & 0.134 \\
\hline Class size squared & 0.0007 & 0.001 & $-0.003^{* *}$ & 0.001 & $0.004^{* * *}$ & 0.001 \\
\hline Number of pregnancies & $-6.593^{* * *}$ & 1.116 & $-8.530^{* * *}$ & 1.176 & $-4.600^{* * *}$ & 1.253 \\
\hline
\end{tabular}


Table A.6 - continued

\begin{tabular}{|c|c|c|c|c|c|c|}
\hline \multirow[b]{2}{*}{ Variable } & \multicolumn{2}{|c|}{ Aggregate score } & \multicolumn{2}{|c|}{ French score } & \multicolumn{2}{|c|}{ Math score } \\
\hline & Coef. & Std. Err. & Coef. & Std. Err. & Coef. & Std. Err. \\
\hline Number of marriages & -0.056 & 0.677 & 0.192 & 0.714 & -0.285 & 0.764 \\
\hline Teacher's age & $-2.580^{* * *}$ & 0.629 & $-3.014^{* * *}$ & 0.663 & $-2.122^{* * *}$ & 0.709 \\
\hline Teacher's age square & $0.036^{* * *}$ & 0.009 & $0.040^{* * *}$ & 0.009 & $0.032^{* * *}$ & 0.010 \\
\hline Pupil's age & $6.947^{* * *}$ & 1.953 & $7.452^{* * *}$ & 2.058 & $6.325^{* * *}$ & 2.196 \\
\hline Pupil's age square & $-0.276^{* * *}$ & 0.092 & $-0.301^{* * *}$ & 0.097 & $-0.245^{* *}$ & 0.103 \\
\hline Number of hours & -0.131 & 0.627 & -0.143 & 0.661 & -0.083 & 0.706 \\
\hline Food expenditure $^{c}$ & 0.712 & 0.738 & -0.015 & 0.778 & $1.359^{*}$ & 0.831 \\
\hline Education expenditure ${ }^{c}$ & 0.235 & 0.275 & 0.262 & 0.290 & 0.214 & 0.309 \\
\hline Health expenditure ${ }^{c}$ & 0.078 & 0.167 & 0.123 & 0.176 & 0.029 & 0.187 \\
\hline Household size ${ }^{d}$ & $0.266^{* * *}$ & 0.063 & $0.243^{* * *}$ & 0.067 & $0.288^{* * *}$ & 0.071 \\
\hline Livestock & -0.0007 & 0.020 & -0.020 & 0.021 & 0.020 & 0.023 \\
\hline Distance to school & 1.083 & 0.909 & 1.450 & 0.959 & 0.640 & 1.024 \\
\hline Playground & $1.741^{*}$ & 0.972 & $1.833^{*}$ & 1.024 & $1.903^{*}$ & 1.094 \\
\hline Water point & -0.775 & 1.013 & $-2.553^{* *}$ & 1.075 & 0.959 & 1.182 \\
\hline Disturbed courses & $-4.042^{* * *}$ & 0.998 & $-2.396^{* *}$ & 1.052 & $-5.881^{* * *}$ & 1.121 \\
\hline Meals near school & -0.553 & 0.907 & $-1.681^{*}$ & 0.957 & 0.568 & 1.025 \\
\hline Gender of teacher & $-3.710^{* * *}$ & 1.145 & $-2.301^{*}$ & 1.207 & $-5.178^{* * *}$ & 1.287 \\
\hline No professional qualif. ${ }^{e}$ & 0.224 & 1.040 & -1.233 & 1.097 & 1.712 & 1.171 \\
\hline Professional qualif. CAP & -1.063 & 1.262 & -1.546 & 1.331 & -0.456 & 1.420 \\
\hline Professional qualif. CEAP & $4.493^{* * *}$ & 1.164 & $3.134^{* *}$ & 1.229 & $5.465^{* * *}$ & 1.317 \\
\hline High school diploma \& more & $1.745^{* *}$ & 0.882 & 0.950 & 0.930 & $2.323^{* *}$ & 0.993 \\
\hline Continuing training & -0.456 & 0.795 & -0.367 & 0.839 & -0.460 & 0.896 \\
\hline Absenteeism & $-4.931^{* * *}$ & 1.335 & $-3.156^{* *}$ & 1.408 & $-6.734^{* * *}$ & 1.507 \\
\hline Gender of pupil & 0.769 & 0.700 & 0.289 & 0.744 & 1.329 & 0.825 \\
\hline Grade & $-14.213^{* * *}$ & 0.984 & $-21.154^{* * *}$ & 1.037 & $-7.323^{* * *}$ & 1.107 \\
\hline Koranic school & $3.562^{* * *}$ & 0.798 & $4.144^{* * *}$ & 0.841 & $3.206^{* * *}$ & 0.898 \\
\hline Early childhood inst. & 2.105 & 1.319 & $2.900^{* *}$ & 1.390 & 1.336 & 1.478 \\
\hline Snack & -0.305 & 1.153 & 0.009 & 1.215 & -0.583 & 1.294 \\
\hline Sick last 3 months & -0.181 & 0.771 & 0.216 & 0.813 & -0.717 & 0.866 \\
\hline Pupils eat fill & 0.429 & 1.192 & 1.004 & 1.257 & -0.177 & 1.344 \\
\hline Gender of household head & -0.667 & 1.266 & -1.322 & 1.334 & -0.173 & 1.422 \\
\hline Lit. of household head & -0.129 & 1.027 & 0.125 & 1.091 & -0.273 & 1.205 \\
\hline Marital status & 2.816 & 1.795 & 2.399 & 1.891 & 3.209 & 2.016 \\
\hline Land & -2.365 & 1.594 & -2.270 & 1.679 & -2.513 & 1.790 \\
\hline Parents school & $-3.040^{* * *}$ & 0.801 & $-2.808^{* * *}$ & 0.844 & $-3.413^{* * *}$ & 0.903 \\
\hline College & $1.721^{*}$ & 1.026 & 0.821 & 1.082 & $2.747^{* *}$ & 1.157 \\
\hline Diourbel & 1.274 & 1.686 & 0.914 & 1.779 & 1.730 & 1.904 \\
\hline Kolda & $-9.912^{* * *}$ & 1.236 & $-10.037^{* * *}$ & 1.304 & $-9.766^{* * *}$ & 1.397 \\
\hline Sédhiou & $-8.668^{* * *}$ & 2.268 & $-7.127^{* * *}$ & 2.391 & $-10.053^{* * *}$ & 2.553 \\
\hline Deworming & -3.969 & 6.257 & 4.887 & 6.633 & $-13.797^{*}$ & 7.259 \\
\hline Intercept & $45.704^{* * *}$ & 16.753 & $57.735^{* * *}$ & 17.664 & $35.401^{*}$ & 18.866 \\
\hline Selection Eq. & & & & & & \\
\hline Total pupils & 0.0004 & 0.0002 & 0.0004 & 0.0002 & 0.0004 & 0.0002 \\
\hline Management committee & $0.269^{* * *}$ & 0.058 & $0.269^{* * *}$ & 0.058 & $0.269^{* * *}$ & 0.058 \\
\hline Association of mothers & $0.209^{* * *}$ & 0.071 & $0.209^{* * *}$ & 0.071 & $0.209^{* * *}$ & 0.071 \\
\hline Cooperative school & 0.095 & 0.060 & 0.095 & 0.060 & 0.095 & 0.060 \\
\hline Water point & $0.167^{* * *}$ & 0.065 & $0.167^{* * *}$ & 0.065 & $0.167^{* * *}$ & 0.065 \\
\hline Medicine box & $-0.154^{*}$ & 0.083 & $-0.154^{* * *}$ & 0.083 & $-0.154^{* * *}$ & 0.083 \\
\hline Gender of pupil & -0.064 & 0.053 & -0.064 & 0.053 & -0.064 & 0.053 \\
\hline Lit. of household head & $0.166^{* *}$ & 0.069 & $0.166^{* *}$ & 0.069 & $0.166^{* *}$ & 0.069 \\
\hline Intercept & $-0.947^{* * *}$ & 0.071 & $-0.947^{* * *}$ & 0.071 & $-0.947^{* * *}$ & 0.071 \\
\hline hasard $\lambda$ & 2.045 & 3.779 & -3.856 & 4.005 & $8.487^{*}$ & 4.381 \\
\hline$\rho$ & 0.120 & - & -0.214 & - & 0.424 & - \\
\hline
\end{tabular}


Table A.6 - continued

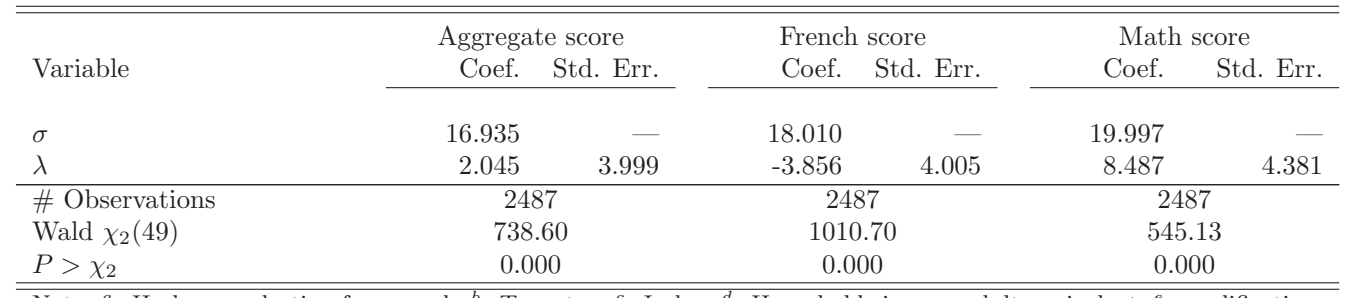

Notes $^{a}$ : Heckman selection framework; ${ }^{b}$ : Two step; ${ }^{c}$ : In log; ${ }^{d}$ : Household size per adult equivalent; ${ }^{e}$ : qualification. Significance levels (bootstrap): $\quad *: 10 \% \quad * *: 5 \% \quad * * *: 1 \%$

Table A.7: Determinants of scores: Meal, Roy regression $\left(\mathrm{ML}^{a}\right)$

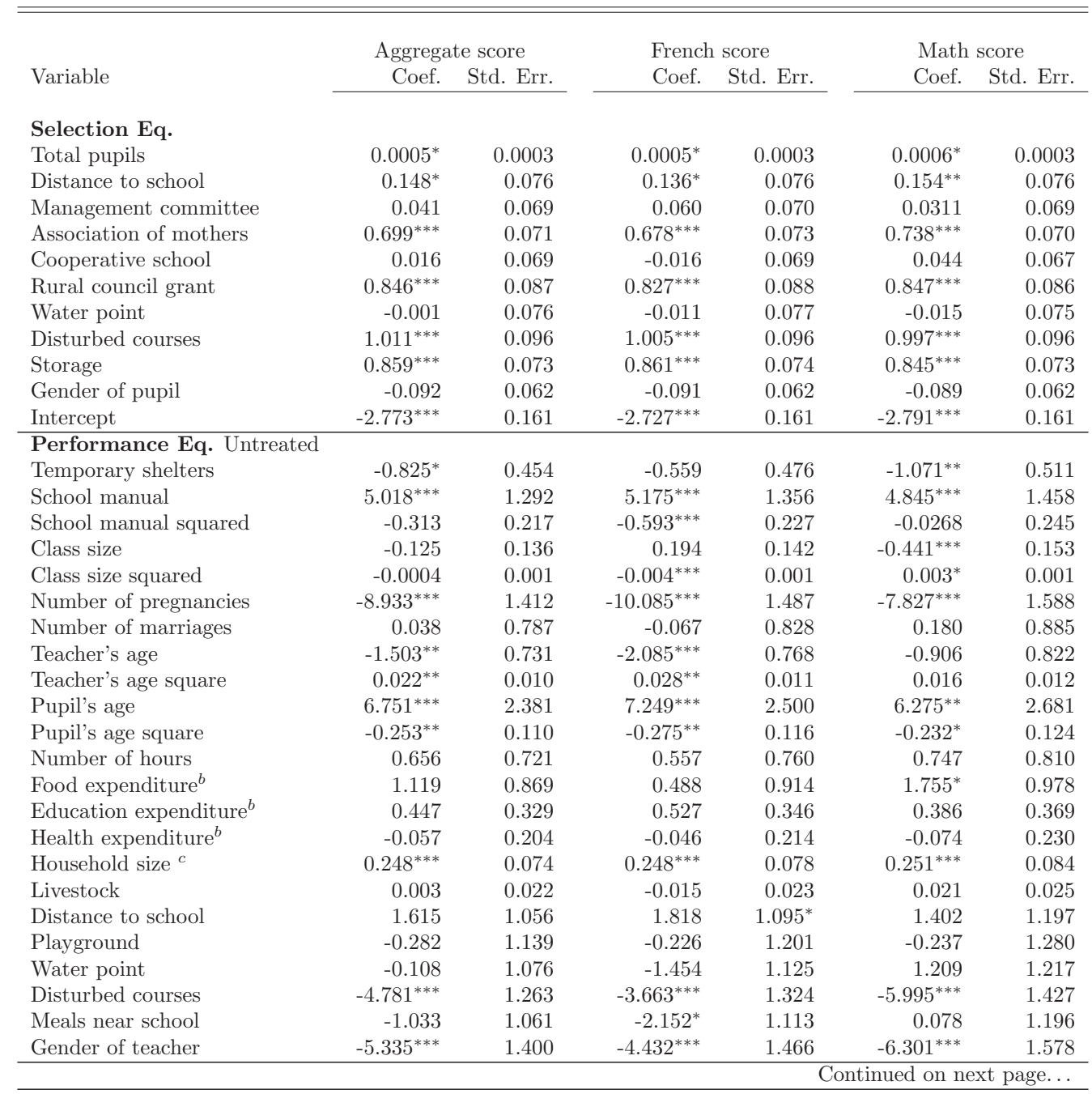


Table A.7 - continued

\begin{tabular}{|c|c|c|c|c|c|c|}
\hline \multirow[b]{2}{*}{ Variable } & \multicolumn{2}{|c|}{ Aggregate score } & \multicolumn{2}{|c|}{ French score } & \multicolumn{2}{|c|}{ Math score } \\
\hline & Coef. & Std. Err. & Coef. & Std. Err. & Coef. & Std. Err. \\
\hline No professional qualif. ${ }^{d}$ & $3.906^{* * *}$ & 1.279 & 1.999 & 1.344 & $5.839^{* * *}$ & 1.440 \\
\hline Professional qualif. CAP & 0.713 & 1.581 & 0.311 & 1.659 & 1.100 & 1.783 \\
\hline Professional qualif. CEAP & $6.431^{* * *}$ & 1.400 & $5.279^{* * *}$ & 1.466 & $7.597^{* * *}$ & 1.577 \\
\hline High school diploma \& more & $2.276^{* *}$ & 1.059 & 1.044 & 1.111 & $3.531^{* * *}$ & 1.194 \\
\hline Continuing training & 0.538 & 0.940 & -0.410 & 0.985 & 1.520 & 1.060 \\
\hline Absenteeism & -1.710 & 1.670 & -0.207 & 1.813 & $-3.443^{*}$ & 1.859 \\
\hline Gender of pupil & $1.568^{*}$ & 0.833 & 1.231 & 0.864 & $1.928^{* *}$ & 0.944 \\
\hline Grade & $-14.670^{* * *}$ & 1.180 & $-21.884^{* * *}$ & 1.241 & $-7.496^{* * *}$ & 1.327 \\
\hline Koranic school & $3.969^{* * *}$ & 0.940 & $4.207^{* * *}$ & 0.988 & $3.762^{* * *}$ & 1.058 \\
\hline Early childhood inst. & $4.946^{* * *}$ & 1.703 & $5.521^{* * *}$ & 1.787 & $4.389^{* *}$ & 1.919 \\
\hline Snack & 2.216 & 1.419 & 2.121 & 1.497 & 2.271 & 1.594 \\
\hline Sick last 3 months & -0.823 & 0.952 & -0.488 & 1.001 & -1.160 & 1.071 \\
\hline Pupils eat fill & -0.005 & 1.355 & 0.006 & 1.426 & 0.014 & 1.523 \\
\hline Gender of household head & -1.213 & 1.537 & -2.224 & 1.614 & -0.249 & 1.731 \\
\hline Lit. of household head & -1.156 & 1.168 & 0.404 & 1.228 & $-2.665^{* *}$ & 1.314 \\
\hline Marital & 1.489 & 2.098 & 1.377 & 2.210 & 1.644 & 2.359 \\
\hline Land & 0.543 & 1.918 & 1.144 & 2.017 & -0.093 & 2.158 \\
\hline Parents school & $-3.825^{* * *}$ & 0.979 & $-4.634^{* * *}$ & 1.026 & $-3.105^{* * *}$ & 1.104 \\
\hline College & $3.320^{* * *}$ & 1.240 & $2.699^{* *}$ & 1.299 & $3.959^{* * *}$ & 1.397 \\
\hline Diourbel & $3.442^{*}$ & 1.909 & 2.196 & 2.003 & $4.424^{* *}$ & 2.144 \\
\hline Kolda & $-7.967^{* * *}$ & 1.499 & $-7.833^{* * *}$ & 1.574 & $-8.158^{* * *}$ & 1.689 \\
\hline Sédhiou & -1.768 & 2.777 & -1.526 & 2.923 & -1.987 & 3.126 \\
\hline Intercept & 18.555 & 19.833 & $34.993^{*}$ & 20.826 & 1.463 & 22.328 \\
\hline \multicolumn{7}{|l|}{ Performance Eq. Treated } \\
\hline Temporary shelters & -1.055 & 1.019 & 0.749 & 1.134 & $-2.686^{* *}$ & 1.160 \\
\hline School manual & 2.483 & 11.960 & -9.088 & 13.346 & 5.472 & 13.560 \\
\hline School manual squared & 5.274 & 5.111 & 7.731 & 5.699 & 6.540 & 5.802 \\
\hline Class size & $-0.951^{* * *}$ & 0.283 & $-1.201^{* * *}$ & 0.314 & $-0.708^{* *}$ & 0.321 \\
\hline Class size squared & $0.017^{* * *}$ & 0.004 & $0.020^{* * *}$ & 0.004 & $0.014^{* * *}$ & 0.004 \\
\hline Number of pregnancies & $-20.212^{* * *}$ & 3.352 & $-13.855^{* * *}$ & 3.732 & $-27.037^{* * *}$ & 3.814 \\
\hline Number of marriages & -0.707 & 3.067 & -0.445 & 3.415 & -2.231 & 3.503 \\
\hline Teacher's age & -1.054 & 1.597 & 1.803 & 1.771 & -2.508 & 1.821 \\
\hline Teacher's age square & 0.022 & 0.023 & -0.021 & 0.026 & $0.045^{*}$ & 0.0271 \\
\hline Pupil's age & 4.883 & 3.739 & $7.664^{*}$ & 4.160 & 2.026 & 4.254 \\
\hline Pupil's age square & -0.152 & 0.172 & -0.292 & 0.192 & -0.0136 & 0.196 \\
\hline Number of hours & 0.540 & 0.677 & 0.680 & 0.753 & 0.397 & 0.770 \\
\hline Total expenditure ${ }^{b}$ & -1.146 & 1.410 & -1.495 & 1.568 & -1.510 & 1.604 \\
\hline Education expenditure $^{b}$ & 0.423 & 0.579 & 0.696 & 0.644 & 0.151 & 0.659 \\
\hline Health expenditure ${ }^{b}$ & -0.115 & 0.397 & 0.312 & 0.442 & -0.590 & 0.452 \\
\hline Household size ${ }^{c}$ & 0.004 & 0.120 & 0.018 & 0.134 & -0.038 & 0.137 \\
\hline Livestock & -0.039 & 0.034 & -0.035 & 0.038 & -0.039 & 0.039 \\
\hline Distance to school & 0.126 & 1.836 & 1.366 & 2.042 & -1.422 & 2.091 \\
\hline Playground & 7.048 & 6.225 & 3.688 & 6.958 & $15.423^{* *}$ & 7.070 \\
\hline Water point & $10.068^{* * *}$ & 1.927 & $8.927^{* * *}$ & 2.147 & $10.647^{* * *}$ & 2.190 \\
\hline Disturbed courses & -2.427 & 3.879 & 6.796 & 4.288 & $-12.795^{* * *}$ & 4.456 \\
\hline Meals near school & -3.308 & 2.584 & -1.516 & 2.874 & $-6.047^{* *}$ & 2.948 \\
\hline Gender of teacher & $-4.079^{*}$ & 2.458 & -3.867 & 2.733 & -3.103 & 2.797 \\
\hline No professional qualif. ${ }^{d}$ & $13.694^{* * *}$ & 2.416 & $19.318^{* * *}$ & 2.687 & $8.147^{* * *}$ & 2.748 \\
\hline Professional qualif. CAP & $6.599^{*}$ & 3.347 & $8.402^{* *}$ & 3.714 & 6.062 & 3.812 \\
\hline Professional qualif. CEAP & -2.338 & 2.004 & $-6.714^{* * *}$ & 2.232 & 2.176 & 2.282 \\
\hline High school diploma \& more & $-9.828^{* * *}$ & 1.968 & $-11.317^{* * *}$ & 2.182 & $-8.084^{* * *}$ & 2.246 \\
\hline Continuing training & -1.149 & 1.582 & 2.596 & 1.758 & $-6.058^{* * *}$ & 1.810 \\
\hline Absenteeism & -3.806 & 8.841 & -5.349 & 9.849 & 3.304 & 10.043 \\
\hline
\end{tabular}


Table A.7 - continued

Variable

Gender of pupil

Garde

Koranic school

Early childhood inst.

Snack

Sick last 3 months

Pupils eat fill

Gender of household head

Lit. of household head

Marital status

Land

Parents school

College

Diourbel

Kolda

Sédhiou

Intercept

$\rho 0$

$\rho 1$

Log likelihood

\# Observations

Wald $\chi_{2}(10)$

$P>\chi_{2}$

\begin{tabular}{|c|c|c|c|c|c|}
\hline \multicolumn{2}{|c|}{ Aggregate score } & \multicolumn{2}{|c|}{ French score } & \multicolumn{2}{|c|}{ Math score } \\
\hline Coef. & Std. Err. & Coef. & Std. Err. & Coef. & Std. Err. \\
\hline-0.268 & 1.188 & -2.003 & 1.326 & 0.566 & 1.351 \\
\hline$-13.499^{* * *}$ & 1.979 & $-21.352^{* * *}$ & 2.202 & $-6.090^{* * *}$ & 2.252 \\
\hline-2.054 & 1.429 & -1.991 & 1.589 & -1.905 & 1.626 \\
\hline 0.242 & 3.261 & 0.165 & 3.626 & -0.003 & 3.710 \\
\hline-1.195 & 2.787 & -0.500 & 3.102 & -0.342 & 3.172 \\
\hline 0.086 & 1.352 & 1.583 & 1.504 & -1.161 & 1.538 \\
\hline $5.596^{* * *}$ & 1.944 & $6.002^{* * *}$ & 2.162 & $4.629^{* *}$ & 2.212 \\
\hline 0.813 & 2.538 & -0.610 & 2.821 & 3.082 & 2.888 \\
\hline 1.250 & 1.760 & 0.138 & 1.957 & 1.278 & 2.004 \\
\hline 0.511 & 3.363 & 4.874 & 3.740 & -4.163 & 3.827 \\
\hline$-5.607^{*}$ & 2.922 & -2.767 & 3.253 & $-7.029^{* *}$ & 3.323 \\
\hline $5.833^{* *}$ & 2.382 & 0.955 & 2.652 & $11.709^{* * *}$ & 2.706 \\
\hline $13.869^{* * *}$ & 3.544 & $10.073^{* *}$ & 3.947 & $16.694^{* * *}$ & 4.027 \\
\hline$-5.84 \mathrm{e}-13$ & & $-9.39 \mathrm{e}-15$ & 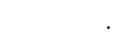 & $4.89 \mathrm{e}-14$ & \\
\hline $23.015^{* * *}$ & 3.628 & $20.230^{* * *}$ & 4.039 & $24.933^{* * *}$ & 4.122 \\
\hline $7.017^{* *}$ & 3.395 & 2.207 & 3.776 & $10.196^{* * *}$ & 3.863 \\
\hline 33.819 & 36.477 & -27.247 & 40.462 & $80.928^{*}$ & 41.571 \\
\hline $0.428^{* * *}$ & 0.076 & $0.253^{* * *}$ & 0.105 & $0.489^{* * *}$ & 0.066 \\
\hline 0.086 & 0.151 & 0.187 & 0.145 & -0.068 & 0.154 \\
\hline-1098 & .795 & -1114 & .915 & -1127 & .584 \\
\hline 23 & & 23 & & 23 & \\
\hline 483 & & 468 & & 494 & \\
\hline 0.0 & & 0.0 & & 0.0 & \\
\hline
\end{tabular}

Notes ${ }^{a}$ :Maximum Likelihood; ${ }^{b}$ In $\log ;{ }^{c}$ : Household size per adult equivalent; ${ }^{d}$ : qualification.

Significance levels (bootstrap-100 replications-): $\quad *: 10 \% \quad * *: 5 \% \quad * * *: 1 \%$

Table A.8: Determinants of scores: Deworming, Roy regression $\left(\mathrm{ML}^{a}\right)$

\begin{tabular}{|c|c|c|c|c|c|c|}
\hline \multirow[b]{2}{*}{ Variable } & \multicolumn{2}{|c|}{ Aggregate score } & \multicolumn{2}{|c|}{ French score } & \multicolumn{2}{|c|}{ Math score } \\
\hline & Coef. & Std. Err. & Coef. & Std. Err. & Coef. & Std. Err. \\
\hline \multicolumn{7}{|l|}{ Selection Eq. } \\
\hline Total pupils & $0.0005^{* * *}$ & 0.0002 & $0.0005^{* *}$ & 0.0002 & $0.0006^{* * *}$ & 0.0002 \\
\hline Management committee & 0.063 & 0.050 & 0.085 & 0.055 & 0.077 & 0.049 \\
\hline Association of mothers & $0.200^{* * *}$ & 0.058 & $0.190^{* * *}$ & 0.060 & $0.201^{* * *}$ & 0.059 \\
\hline Cooperative school & $0.139^{* * *}$ & 0.051 & $0.124^{* * *}$ & 0.053 & $0.137^{* * *}$ & 0.051 \\
\hline Water point & $0.161^{* * *}$ & 0.062 & $0.172^{* * *}$ & 0.063 & $0.155^{* *}$ & 0.062 \\
\hline Medicine box & $-0.381^{* * *}$ & 0.074 & $-0.303^{* * *}$ & 0.080 & $-0.401^{* * *}$ & 0.070 \\
\hline Gender of pupil & -0.035 & 0.053 & -0.045 & 0.053 & -0.031 & 0.053 \\
\hline Lit. of household head & $0.164^{* *}$ & 0.068 & $0.167^{* *}$ & 0.068 & $0.158^{* *}$ & 0.068 \\
\hline Intercept & $-0.873^{* * *}$ & 0.064 & $-0.871^{* * *}$ & 0.066 & $-0.889^{* * *}$ & 0.064 \\
\hline \multicolumn{7}{|c|}{ Performance Eq. Untreated } \\
\hline Temporary shelters & -0.526 & 0.450 & -0.380 & 0.472 & -0.693 & 0.508 \\
\hline School manual & $4.382^{* * *}$ & 1.290 & $4.801^{* * *}$ & 1.350 & $3.936^{* * *}$ & 1.456 \\
\hline School manual squared & -0.250 & 0.217 & $-0.560^{* *}$ & 0.227 & 0.068 & 0.245 \\
\hline Class size & -0.073 & 0.135 & $0.234^{*}$ & 0.141 & $-0.378^{* *}$ & 0.152 \\
\hline Class size squared & -0.0008 & 0.001 & $-0.004^{* * *}$ & 0.001 & $0.003^{*}$ & 0.001 \\
\hline Number pregnancies & $-8.949^{* * *}$ & 1.418 & $-10.086^{* * *}$ & 1.488 & $-7.826^{* * *}$ & 1.599 \\
\hline
\end{tabular}


Table A.8 - continued

\begin{tabular}{|c|c|c|c|c|c|c|}
\hline \multirow[b]{2}{*}{ Variable } & \multicolumn{2}{|c|}{ Aggregate score } & \multicolumn{2}{|c|}{ French score } & \multicolumn{2}{|c|}{ Math score } \\
\hline & Coef. & Std. Err. & Coef. & Std. Err. & Coef. & Std. Err. \\
\hline Number of marriages & -0.193 & 0.795 & -0.223 & 0.832 & -0.136 & 0.896 \\
\hline Teacher's age & $-1.550^{* *}$ & 0.734 & $-2.134^{* * *}$ & 0.769 & -0.959 & 0.827 \\
\hline Teacher's age square & $0.023^{* *}$ & 0.011 & $0.029^{* * *}$ & 0.011 & 0.017 & 0.012 \\
\hline Pupil's age & $6.512^{* * *}$ & 2.387 & $7.111^{* * *}$ & 2.501 & $5.923^{* *}$ & 2.690 \\
\hline Pupil's age square & $-0.240^{* *}$ & 0.111 & $-0.268^{* *}$ & 0.116 & $-0.212^{*}$ & 0.125 \\
\hline Number of hours & 0.583 & 0.726 & 0.523 & 0.761 & 0.656 & 0.818 \\
\hline Food expenditure ${ }^{b}$ & 1.105 & 0.874 & 0.469 & 0.915 & $1.716^{*}$ & 0.985 \\
\hline Education expenditure $^{b}$ & 0.506 & 0.330 & 0.559 & 0.346 & 0.456 & 0.372 \\
\hline Health expenditure $^{b}$ & -0.078 & 0.204 & -0.056 & 0.214 & -0.101 & 0.230 \\
\hline Household size ${ }^{c}$ & $0.260^{* * *}$ & 0.075 & $0.255^{* * *}$ & 0.078 & $0.265^{* * *}$ & 0.084 \\
\hline Livestock & 0.008 & 0.022 & -0.011 & 0.023 & 0.028 & 0.025 \\
\hline Distance to school & 1.477 & 1.039 & 1.735 & 1.089 & 1.197 & 1.172 \\
\hline Playground & 0.139 & 1.138 & 0.022 & 1.194 & 0.262 & 1.282 \\
\hline Water point & -0.099 & 1.091 & -1.344 & 1.158 & 1.160 & 1.230 \\
\hline Disturbed courses & $-6.153^{* * *}$ & 1.227 & $-4.521^{* * *}$ & 1.288 & $-7.792^{* * *}$ & 1.383 \\
\hline Meals near school & -1.116 & 1.066 & $-2.219^{* *}$ & 1.116 & 0.017 & 1.203 \\
\hline Gender of teacher & -5.883 & 1.401 & $-4.723^{* * *}$ & 1.470 & $-7.055^{* * *}$ & 1.578 \\
\hline No professional qualif. ${ }^{d}$ & $3.897^{* * *}$ & 1.287 & 1.947 & 1.347 & $5.853^{* * *}$ & 1.452 \\
\hline Professional qualif. CAP & 0.339 & 1.579 & 0.198 & 1.659 & 0.459 & 1.782 \\
\hline Professional qualif. CEAP & $6.467^{* * *}$ & 1.403 & $5.349^{* * *}$ & 1.469 & $7.592^{* * *}$ & 1.584 \\
\hline High school diploma \& more & $2.366^{* *}$ & 1.061 & 1.123 & 1.111 & $3.642^{* * *}$ & 1.197 \\
\hline Continuing training & 0.480 & 0.942 & -0.393 & 0.986 & 1.367 & 1.062 \\
\hline Absenteeism & $-4.140^{* *}$ & 1.627 & -1.642 & 1.704 & $-6.593^{* * *}$ & 1.834 \\
\hline Gender of pupil & $1.715^{* *}$ & 0.839 & 1.280 & 0.870 & $2.147^{* *}$ & 0.950 \\
\hline Grade & $-14.930^{* * *}$ & 1.186 & $-22.015^{* * *}$ & 1.242 & $-7.848^{* * *}$ & 1.338 \\
\hline Koranic school & $4.158^{* * *}$ & 0.942 & $4.334^{* * *}$ & 0.987 & $3.976^{* * *}$ & 1.062 \\
\hline Early childhood inst. & $4.659^{* * *}$ & 1.701 & $5.411^{* * *}$ & 1.785 & $3.933^{* *}$ & 1.917 \\
\hline Snack & 1.765 & 1.425 & 1.877 & 1.495 & 1.673 & 1.607 \\
\hline Sick last 3 months & -0.925 & 0.957 & -0.543 & 1.003 & -1.308 & 1.078 \\
\hline Pupils eat fill & 0.111 & 1.365 & 0.090 & 1.429 & 0.138 & 1.539 \\
\hline Gender of household head & -1.393 & 1.538 & -2.328 & 1.613 & -0.464 & 1.733 \\
\hline Lit. of household head & -0.335 & 1.213 & 1.006 & 1.276 & -1.707 & 1.371 \\
\hline Marital status & 1.970 & 2.105 & 1.661 & 2.208 & 2.263 & 2.372 \\
\hline Land & 0.599 & 1.924 & 1.186 & 2.018 & -0.028 & 2.167 \\
\hline Parents school & $-4.507^{* * *}$ & 0.969 & $-4.986^{* * *}$ & 1.015 & $-4.029^{* * *}$ & 1.093 \\
\hline College & $3.601^{* * *}$ & 1.241 & $2.852^{* *}$ & 1.299 & $4.360^{* * *}$ & 1.400 \\
\hline Diourbel & 2.104 & 1.908 & 1.471 & 1.999 & 2.813 & 2.149 \\
\hline Kolda & $-8.038^{* * *}$ & 1.507 & $-7.896^{* * *}$ & 1.577 & $-8.222^{* * *}$ & 1.699 \\
\hline Sédhiou & -2.964 & 2.781 & -2.280 & 2.916 & -3.488 & 3.134 \\
\hline Intercept & 21.546 & 19.914 & $37.381^{*}$ & 20.877 & 5.766 & 22.448 \\
\hline Performance Eq. Treated & & & & & & \\
\hline Temporary shelters & $3.279^{* * *}$ & 0.772 & $2.501^{* * *}$ & 0.859 & $4.208^{* * *}$ & 0.858 \\
\hline School manual & $-4.664^{* *}$ & 2.167 & $-4.489^{*}$ & 2.447 & $-4.819^{* *}$ & 2.435 \\
\hline School manual squared & $0.655^{*}$ & 0.399 & 0.511 & 0.445 & 0.697 & 0.456 \\
\hline Class size & -0.337 & 0.229 & -0.029 & 0.256 & $-0.560^{* *}$ & 0.259 \\
\hline Class size squared & 0.004 & 0.002 & 0.0001 & 0.003 & $0.007^{* *}$ & 0.003 \\
\hline Number of pregnancies & -2.873 & 1.774 & $-4.489^{* *}$ & 1.974 & -1.541 & 1.968 \\
\hline Number of marriages & 1.217 & 1.284 & 1.217 & 1.421 & 1.662 & 1.429 \\
\hline Teacher's age & $-2.890^{* *}$ & 1.144 & $-2.635^{* *}$ & 1.295 & $-2.933^{* *}$ & 1.256 \\
\hline Teacher's age square & $0.037^{* *}$ & 0.016 & 0.030 & 0.018 & $0.040^{* *}$ & 0.018 \\
\hline Pupil's age & $10.136^{* * *}$ & 3.249 & $11.109^{* * *}$ & 3.561 & $9.334^{* *}$ & 3.648 \\
\hline Pupil's age square & $-0.462^{* * *}$ & 0.157 & $-0.512^{* * *}$ & 0.172 & $-0.424^{* *}$ & 0.177 \\
\hline Number of hours & -1.535 & 1.145 & -1.281 & 1.267 & $-2.080^{*}$ & 1.260 \\
\hline
\end{tabular}


Table A.8 - continued

\begin{tabular}{|c|c|c|c|c|c|c|}
\hline \multirow[b]{2}{*}{ Variable } & \multicolumn{2}{|c|}{ Aggregate score } & \multicolumn{2}{|c|}{ French score } & \multicolumn{2}{|c|}{ Math score } \\
\hline & Coef. & Std. Err. & Coef. & Std. Err. & Coef. & Std. Err. \\
\hline Food expenditure ${ }^{b}$ & 0.015 & 1.260 & -0.973 & 1.403 & 0.352 & 1.393 \\
\hline Education expenditure ${ }^{b}$ & -0.158 & 0.456 & -0.127 & 0.507 & -0.265 & 0.492 \\
\hline Health expenditure ${ }^{b}$ & $0.509^{*}$ & 0.260 & $0.638^{* *}$ & 0.288 & 0.318 & 0.288 \\
\hline Household size ${ }^{c}$ & 0.140 & 0.111 & 0.133 & 0.122 & 0.124 & 0.124 \\
\hline Livestock & 0.018 & 0.044 & -0.002 & 0.050 & 0.043 & 0.048 \\
\hline Distance to school & -0.484 & 1.742 & -0.944 & 1.906 & -0.523 & 1.931 \\
\hline Playground & $5.616^{* * *}$ & 1.791 & $5.818^{* * *}$ & 1.982 & $5.988^{* * *}$ & 2.009 \\
\hline Water point & 1.292 & 2.057 & 0.850 & 2.290 & 1.565 & 2.271 \\
\hline Disturbed courses & $-4.707^{* * *}$ & 1.835 & $-3.655^{*}$ & 2.017 & $-6.336^{* * *}$ & 2.038 \\
\hline Meals near school & -0.808 & 1.707 & -1.551 & 1.873 & 0.296 & 1.883 \\
\hline Gender of teacher & 0.596 & 1.874 & 0.545 & 2.063 & 1.133 & 2.108 \\
\hline No professional qualif. ${ }^{d}$ & $-4.451^{* *}$ & 1.762 & $-5.444^{* * *}$ & 1.959 & $-3.745^{*}$ & 1.956 \\
\hline Professional qualif. CAP & -1.593 & 2.021 & -2.382 & 2.243 & -0.275 & 2.233 \\
\hline Professional qualif. CEAP & -2.247 & 2.112 & $-3.985^{*}$ & 2.371 & -2.523 & 2.354 \\
\hline High school diploma \& more & -1.812 & 1.624 & -0.491 & 1.806 & $-4.206^{* *}$ & 1.790 \\
\hline Continuing training & $-3.748^{* *}$ & 1.450 & -1.674 & 1.623 & $-5.348^{* * *}$ & 1.600 \\
\hline Absenteeism & -0.234 & 2.418 & 1.140 & 2.668 & -1.133 & 2.675 \\
\hline Gender of pupil & -2.376 & 1.460 & $-3.495^{* *}$ & 1.550 & -0.877 & 1.656 \\
\hline Grade & $-10.886^{* * *}$ & 1.726 & $-18.956^{* * *}$ & 1.846 & -2.913 & 1.978 \\
\hline Koranic school & 2.183 & 1.405 & $3.767^{* *}$ & 1.565 & 1.913 & 1.552 \\
\hline Early childhood inst. & -0.595 & 1.955 & -0.021 & 2.170 & -1.804 & 2.158 \\
\hline Snack & -1.994 & 1.819 & -0.842 & 2.011 & -3.093 & 2.025 \\
\hline Sick last 3 months & -0.622 & 1.204 & -0.145 & 1.336 & -1.653 & 1.339 \\
\hline Pupils eat fill & -3.188 & 2.199 & -0.608 & 2.471 & $-6.040^{* *}$ & 2.434 \\
\hline Gender of household head & -0.151 & 2.068 & -0.757 & 2.284 & -0.411 & 2.332 \\
\hline Lit. of household head & $4.449^{* *}$ & 1.897 & $4.498^{* *}$ & 2.044 & $5.146^{* *}$ & 2.146 \\
\hline Marital status & 3.100 & 3.090 & 3.551 & 3.449 & 2.388 & 3.455 \\
\hline Land & $-6.599^{* *}$ & 2.615 & $-9.738^{* * *}$ & 2.889 & $-4.846^{*}$ & 2.916 \\
\hline Parents school & -0.189 & 1.468 & 0.667 & 1.634 & -2.122 & 1.610 \\
\hline College & -1.010 & 1.896 & -2.987 & 2.100 & 1.648 & 2.113 \\
\hline Diourbel & $-9.725^{* *}$ & 4.208 & -6.473 & 4.653 & $-13.753^{* * *}$ & 4.751 \\
\hline Kolda & $-13.875^{* * *}$ & 2.220 & $-13.365^{* * *}$ & 2.458 & $-14.992^{* * *}$ & 2.489 \\
\hline Sédhiou & $-20.292^{* * *}$ & 3.912 & $-14.785^{* * *}$ & 4.250 & $-25.096^{* * *}$ & 4.368 \\
\hline Intercept & 31.901 & 28.981 & 36.269 & 32.504 & 30.293 & 32.372 \\
\hline$\rho 0$ & $0.406^{* * *}$ & 0.097 & $0.289^{*}$ & 0.150 & $0.444^{* * *}$ & 0.088 \\
\hline$\rho 1$ & $0.910^{* * *}$ & 0.025 & $0.878^{* * *}$ & 0.040 & $0.925^{* * *}$ & 0.024 \\
\hline Log likelihood & -11933 & .474 & -1208 & .921 & -1222 & .351 \\
\hline \# Observations & 248 & & 24 & & 24 & \\
\hline Wald $\chi_{2}(8)$ & 96. & & 82. & & 106 & \\
\hline$P>\chi_{2}$ & 0.00 & & 0.0 & & 0.0 & \\
\hline
\end{tabular}

Notes $^{a}$ : Maximum Likelihood; ${ }^{b}$ In log; ${ }^{c}$ : Household size per adult equivalent; ${ }^{d}$ : qualification.

Significance levels (bootstrap -100 replications-): $\quad *: 10 \% \quad * *: 5 \% \quad * * *: 1 \%$

Table A.9: Determinants of scores: Meal, Roy regression (2-step $\left.{ }^{a}\right)$

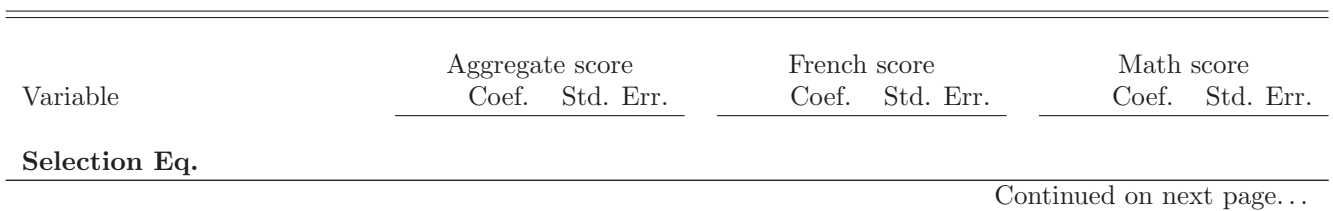


Table A.9 - continued

\begin{tabular}{|c|c|c|c|c|c|c|}
\hline \multirow[b]{2}{*}{ Variable } & \multicolumn{2}{|c|}{ Aggregate score } & \multicolumn{2}{|c|}{ French score } & \multicolumn{2}{|c|}{ Math score } \\
\hline & Coef. & Std. Err. & Coef. & Std. Err. & Coef. & Std. Err. \\
\hline Total pupils & $0.0008^{* * *}$ & 0.0001 & $0.0008^{* * *}$ & 0.0001 & $0.0008^{* * *}$ & 0.0001 \\
\hline Distance to school & $0.259^{* * *}$ & 0.056 & $0.259^{* * *}$ & 0.056 & $0.259^{* * *}$ & 0.056 \\
\hline Management committee & -0.068 & 0.049 & -0.068 & 0.049 & -0.068 & 0.049 \\
\hline Association of mothers & $0.507^{* * *}$ & 0.052 & $0.507^{* * *}$ & 0.052 & $0.507^{* * *}$ & 0.052 \\
\hline Cooperative school & $-0.171^{* * *}$ & 0.047 & $-0.171^{* * *}$ & 0.047 & $-0.171^{* * *}$ & 0.047 \\
\hline Rural council grant & $0.607^{* * *}$ & 0.053 & $0.607^{* * *}$ & 0.053 & $0.607^{* * *}$ & 0.053 \\
\hline Water point & $0.124^{* *}$ & 0.053 & $0.124^{* *}$ & 0.053 & $0.124^{* *}$ & 0.053 \\
\hline Disturbed courses & $0.270^{* * *}$ & 0.052 & $0.270^{* * *}$ & 0.052 & $0.270^{* * *}$ & 0.052 \\
\hline Storage & $0.513^{* * *}$ & 0.055 & $0.513^{* * *}$ & 0.055 & $0.513^{* * *}$ & 0.055 \\
\hline Gender of pupil & $-0.077^{*}$ & 0.043 & $-0.077^{*}$ & 0.043 & $-0.077^{*}$ & 0.043 \\
\hline Intercept & $-1.579^{* * *}$ & 0.088 & $-1.579^{* * *}$ & 0.088 & $-1.579^{* * *}$ & 0.088 \\
\hline \multicolumn{7}{|l|}{ Performance Eq. Untreated } \\
\hline Temporary shelters & -0.764 & 0.480 & -0.475 & 0.490 & $-1.056^{*}$ & 0.535 \\
\hline School manual & $4.960^{* * *}$ & $1.365^{* * *}$ & 5.049 & 1.248 & $4.867^{* * *}$ & 1.701 \\
\hline School manual squared & -0.293 & 0.207 & $-0.576^{* * *}$ & 0.182 & -0.010 & 0.275 \\
\hline Class size & -0.110 & 0.121 & $0.217^{*}$ & 0.116 & $-0.439^{* * *}$ & 0.159 \\
\hline Class size squared & -0.0006 & 0.001 & $-0.004^{* * *}$ & 0.001 & $0.003^{*}$ & 0.002 \\
\hline Number of pregnancies & $-9.030^{* * *}$ & 1.811 & $-10.075^{* * *}$ & 1.664 & $-7.995^{* * *}$ & 2.145 \\
\hline Number of marriages & 0.054 & 1.073 & -0.117 & 1.089 & 0.226 & 1.163 \\
\hline Teacher's age & $-1.484^{* *}$ & 0.715 & $-2.119^{* * *}$ & 0.768 & -0.849 & 0.797 \\
\hline Teacher's age square & $0.022^{* *}$ & 0.010 & $0.029^{* *}$ & 0.011 & 0.016 & 0.011 \\
\hline Pupil's age & $6.554^{* * *}$ & 2.226 & $7.105^{* * *}$ & 2.225 & $6.002^{* *}$ & 2.643 \\
\hline Pupil's age square & $-0.244^{* *}$ & 0.101 & $-0.268^{* * *}$ & 0.103 & $-0.220^{*}$ & 0.118 \\
\hline Number of hours & 0.623 & 0.796 & 0.547 & 0.797 & 0.702 & 0.917 \\
\hline Food expenditure ${ }^{b}$ & 1.169 & 1.010 & 0.489 & 1.034 & $1.842^{*}$ & 1.119 \\
\hline Education expenditure $^{b}$ & 0.436 & 0.402 & 0.530 & 0.460 & 0.340 & 0.388 \\
\hline Health expenditure $^{b}$ & -0.073 & 0.217 & -0.051 & 0.227 & -0.094 & 0.244 \\
\hline Household size ${ }^{c}$ & $0.248^{* * *}$ & 0.068 & $0.251^{* * *}$ & 0.070 & $0.245^{* * *}$ & 0.080 \\
\hline Livestock & 0.003 & 0.025 & -0.013 & 0.023 & 0.020 & 0.030 \\
\hline Distance to school & $2.190^{* *}$ & 1.109 & $2.022^{*}$ & 1.040 & $2.357^{*}$ & 1.313 \\
\hline Playground & -0.478 & 1.153 & -0.119 & 1.229 & -0.830 & 1.314 \\
\hline Water point & 0.692 & 1.134 & -1.365 & 1.174 & $2.749^{* *}$ & 1.341 \\
\hline Disturbed courses & $-6.121^{* * *}$ & 1.157 & $-4.432^{* * *}$ & 1.157 & $-7.815^{* * *}$ & 1.378 \\
\hline Meals near school & -1.022 & 0.991 & $-2.148^{* *}$ & 1.061 & 0.098 & 1.122 \\
\hline Gender of teacher & $-5.397^{* * *}$ & 1.385 & $-4.445^{* * *}$ & 1.421 & $-6.377^{* * *}$ & 1.564 \\
\hline No professional qualif. ${ }^{d}$ & $3.950^{* * *}$ & 1.465 & 1.969 & 1.601 & $5.930^{* * *}$ & 1.500 \\
\hline Professional qualif. CAP & 0.208 & 1.654 & -0.012 & 1.611 & 0.404 & 1.996 \\
\hline Professional qualif. CEAP & $6.143^{* * *}$ & 1.565 & $5.196^{* * *}$ & 1.686 & $7.091^{* * *}$ & 1.727 \\
\hline High school diploma \& more & $2.388^{* *}$ & 1.043 & 1.127 & 1.112 & $3.664^{* * *}$ & 1.258 \\
\hline Continuing training & 0.537 & 0.967 & -0.405 & 1.017 & 1.486 & 1.097 \\
\hline Absenteeism & -2.067 & 1.477 & -0.971 & 1.701 & $-3.184^{* *}$ & 1.604 \\
\hline Gender of pupil & $1.541^{*}$ & 0.790 & 1.291 & 0.870 & $1.790^{* *}$ & 0.852 \\
\hline Grade & $-14.689^{* * *}$ & 1.272 & $-21.925^{* * *}$ & 1.225 & $-7.452^{* * *}$ & 1.547 \\
\hline Koranic school & $3.995^{* * *}$ & 0.976 & $4.262^{* * *}$ & 1.029 & $3.726^{* * *}$ & 1.043 \\
\hline Early childhood inst. & $4.765^{* *}$ & 1.871 & $5.432^{* * *}$ & 1.951 & $4.098^{* *}$ & 2.037 \\
\hline Snack & 1.981 & 1.730 & 1.927 & 1.794 & 2.048 & 1.972 \\
\hline Sick last 3 months & -0.867 & 1.005 & -0.509 & 1.017 & -1.225 & 1.112 \\
\hline Pupils eat fill & 0.028 & 1.442 & 0.069 & 1.532 & -0.011 & 1.520 \\
\hline Gender of household head & -1.311 & 1.516 & -2.300 & 1.652 & $-0.328^{* *}$ & 1.683 \\
\hline Lit. of household head & -1.171 & 1.158 & 0.434 & 1.232 & -2.771 & 1.355 \\
\hline Marital status & 1.640 & 1.818 & 1.550 & 2.112 & 1.745 & 2.235 \\
\hline Land & 0.466 & 1.884 & 1.135 & 2.077 & -0.220 & 2.077 \\
\hline Parents school & $-3.983^{* * *}$ & 1.008 & $-4.771^{* * *}$ & 1.090 & $-3.213^{* * *}$ & 1.097 \\
\hline
\end{tabular}


Table A.9 - continued

\begin{tabular}{|c|c|c|c|c|c|c|}
\hline \multirow[b]{2}{*}{ Variable } & \multicolumn{2}{|c|}{ Aggregate score } & \multicolumn{2}{|c|}{ French score } & \multicolumn{2}{|c|}{ Math score } \\
\hline & Coef. & Std. Err. & Coef. & Std. Err. & Coef. & Std. Err. \\
\hline College & $3.443^{* * *}$ & 1.197 & $2.787^{* *}$ & 1.309 & $4.110^{* * *}$ & 1.340 \\
\hline Diourbel & 2.856 & 2.066 & 1.867 & 2.127 & 3.830 & 2.354 \\
\hline Kolda & -7.741 & 1.494 & -7.807 & 1.525 & $-7.668^{* * *}$ & 1.767 \\
\hline Sédhiou & $-1.489^{* * *}$ & 3.074 & -1.757 & 2.735 & -1.211 & 3.755 \\
\hline lambda0 & $7.322^{* * *}$ & 2.279 & 2.655 & 2.413 & $11.936^{* * *}$ & 2.589 \\
\hline Intercept & 19.949 & 20.432 & $35.802^{*}$ & 19.731 & 4.213 & 24.637 \\
\hline \# Observations & \multicolumn{2}{|c|}{1756} & \multicolumn{2}{|c|}{1756} & \multicolumn{2}{|c|}{1756} \\
\hline Adj R-squared & \multicolumn{2}{|c|}{0.2367} & \multicolumn{2}{|c|}{0.2885} & \multicolumn{2}{|c|}{0.1988} \\
\hline \multicolumn{7}{|l|}{ Performance Eq. Treated } \\
\hline Temporary shelters & -1.094 & 1.261 & 0.671 & 1.285 & $-2.664^{*}$ & 1.517 \\
\hline School manual & 5.570 & 15.996 & -3.133 & 15.367 & 4.800 & 19.815 \\
\hline School manual squared & 4.114 & 6.384 & 5.507 & 6.328 & 6.752 & 7.866 \\
\hline Class size & $-0.918^{* * *}$ & 0.295 & $-1.132^{* * *}$ & 0.325 & $-0.716^{*}$ & 0.390 \\
\hline Class size squared & $0.016^{* * *}$ & 0.004 & $0.019^{* * *}$ & 0.004 & $0.014^{* *}$ & 0.005 \\
\hline Number of pregnancies & $-20.273^{* * *}$ & 3.309 & $-13.959^{* * *}$ & 3.684 & $-27.009^{* * *}$ & 3.710 \\
\hline Number of marriages & -0.203 & 3.168 & 0.472 & 3.576 & -2.199 & 3.741 \\
\hline Teacher's age & -0.936 & 2.227 & 2.065 & 2.233 & -2.497 & 2.532 \\
\hline Teacher's age square & 0.020 & 0.034 & -0.025 & 0.034 & 0.045 & 0.038 \\
\hline Pupil's age & 4.895 & 3.217 & $7.681^{* *}$ & 3.724 & 2.009 & 3.986 \\
\hline Pupil's age square & -0.153 & 0.141 & $-0.294^{*}$ & 0.170 & -0.012 & 0.173 \\
\hline Number of hours & 0.542 & 0.652 & 0.683 & 0.784 & 0.403 & 0.724 \\
\hline Food expenditure $^{b}$ & -1.181 & 1.452 & -1.559 & 1.627 & -1.515 & 1.675 \\
\hline Education expenditure $^{b}$ & 0.413 & 0.616 & 0.672 & 0.897 & 0.155 & 0.618 \\
\hline Health expenditure $^{b}$ & -0.094 & 0.452 & 0.352 & 0.465 & -0.598 & 0.573 \\
\hline Household size ${ }^{c}$ & 0.004 & 0.124 & 0.018 & 0.152 & -0.038 & 0.139 \\
\hline Livestock & -0.040 & 0.034 & -0.037 & 0.037 & -0.038 & 0.041 \\
\hline Distance to school & 0.869 & 2.083 & 2.920 & 2.293 & -1.654 & 2.472 \\
\hline Playground & 5.012 & 6.837 & -0.169 & 7.645 & $15.656^{*}$ & 8.172 \\
\hline Water point & $10.532^{* * *}$ & 1.911 & $9.904^{* * *}$ & 2.190 & $10.458^{* * *}$ & 2.326 \\
\hline Disturbed courses & -2.587 & 3.428 & $6.291^{*}$ & 3.800 & $-12.346^{* * *}$ & 4.068 \\
\hline Meals near school & -2.913 & 2.571 & -0.778 & 2.882 & $-6.046^{* *}$ & 2.868 \\
\hline Gender of teacher & $-4.078^{*}$ & 2.372 & -3.868 & 2.627 & -3.120 & 2.928 \\
\hline No professional qualif. ${ }^{d}$ & $13.836^{* * *}$ & 2.299 & $19.651^{* * *}$ & 2.587 & $8.094^{* *}$ & 3.293 \\
\hline Professional qualif. CAP & $7.396^{* *}$ & 3.701 & $9.999^{* * *}$ & 3.820 & 5.917 & 4.795 \\
\hline Professional qualif. CEAP & -2.227 & 1.797 & $-6.528^{* * *}$ & 2.112 & 2.186 & 2.421 \\
\hline High school diploma \& more & $-10.023^{* * *}$ & 1.624 & $-11.728^{* * *}$ & 2.017 & $-8.082^{* * *}$ & 1.834 \\
\hline Continuing training & -0.735 & 1.666 & $3.403^{*}$ & 2.004 & $-6.106^{* * *}$ & 1.900 \\
\hline Absenteeism & -6.215 & 10.253 & -10.161 & 10.563 & 3.956 & 12.433 \\
\hline Gender of pupil & -0.408 & 1.123 & $-2.278^{*}$ & 1.389 & 0.595 & 1.236 \\
\hline Grade & $-13.611^{* * *}$ & 1.793 & $-21.590^{* * *}$ & 2.092 & $-6.060^{* * *}$ & 2.257 \\
\hline Koranic school & -2.060 & 1.467 & -2.005 & 1.533 & -1.892 & 1.695 \\
\hline Early childhood inst. & 0.330 & 4.368 & 0.342 & 4.526 & -0.054 & 4.844 \\
\hline Snack & -1.267 & 2.736 & -0.655 & 2.517 & -0.344 & 3.388 \\
\hline Sick last 3 months & 0.090 & 1.560 & 1.591 & 1.647 & -1.163 & 1.804 \\
\hline Pupils eat fill & $5.653^{* * *}$ & 1.734 & $6.114^{* * *}$ & 1.939 & $4.632^{* *}$ & 1.987 \\
\hline Gender of household head & 0.902 & 2.374 & -0.435 & 2.884 & 3.030 & 2.813 \\
\hline Lit. of household head & 1.375 & 1.822 & 0.382 & 1.871 & 1.277 & 2.068 \\
\hline Marital status & 0.456 & 3.079 & 4.756 & 3.586 & -4.126 & 4.080 \\
\hline Land & $-5.645^{*}$ & 3.016 & -2.843 & 2.947 & -7.017 & 3.633 \\
\hline Parents school & $6.174^{* *}$ & 2.889 & 1.614 & 3.117 & $11.598^{* * *}$ & 3.402 \\
\hline College & $14.494^{* * *}$ & 3.328 & $11.325^{* * *}$ & 3.445 & $16.480^{* * *}$ & 4.278 \\
\hline Diourbel & - & - & - & - & - & - \\
\hline Kolda & $23.804^{* * *}$ & 4.279 & $21.846^{* * *}$ & 4.900 & $24.660^{* * *}$ & 4.713 \\
\hline
\end{tabular}


Table A.9 - continued

\begin{tabular}{|c|c|c|c|c|c|c|}
\hline \multirow[b]{2}{*}{ Variable } & \multicolumn{2}{|c|}{ Aggregate score } & \multicolumn{2}{|c|}{ French score } & \multicolumn{2}{|c|}{ Math score } \\
\hline & Coef. & Std. Err. & Coef. & Std. Err. & Coef. & Std. Err. \\
\hline Sédhiou & $7.671^{* *}$ & 3.460 & 3.489 & 4.309 & $10.073^{* *}$ & 3.905 \\
\hline lambda1 & 4.613 & 5.119 & $9.846^{*}$ & 5.779 & -2.009 & 5.675 \\
\hline Intercept & 28.076 & 43.501 & -39.195 & 44.620 & 81.794 & 52.081 \\
\hline \# Observations & \multicolumn{2}{|c|}{613} & \multicolumn{2}{|c|}{613} & \multicolumn{2}{|c|}{613} \\
\hline Adj R-squared & \multicolumn{2}{|c|}{0.3983} & \multicolumn{2}{|c|}{0.4099} & \multicolumn{2}{|c|}{0.3759} \\
\hline
\end{tabular}

Notes ${ }^{a}$ :Two step ${ }^{b}$ In $\log ;{ }^{c}$ : Household size per adult equivalent; ${ }^{d}$ : qualification.

Significance levels (bootstrap -100 replications-): $\quad *: 10 \% \quad * *: 5 \% \quad * * *: 1 \%$

Table A.10: Determinants of scores: Deworming, Roy regression $\left(2-\right.$ step $\left.^{a}\right)$

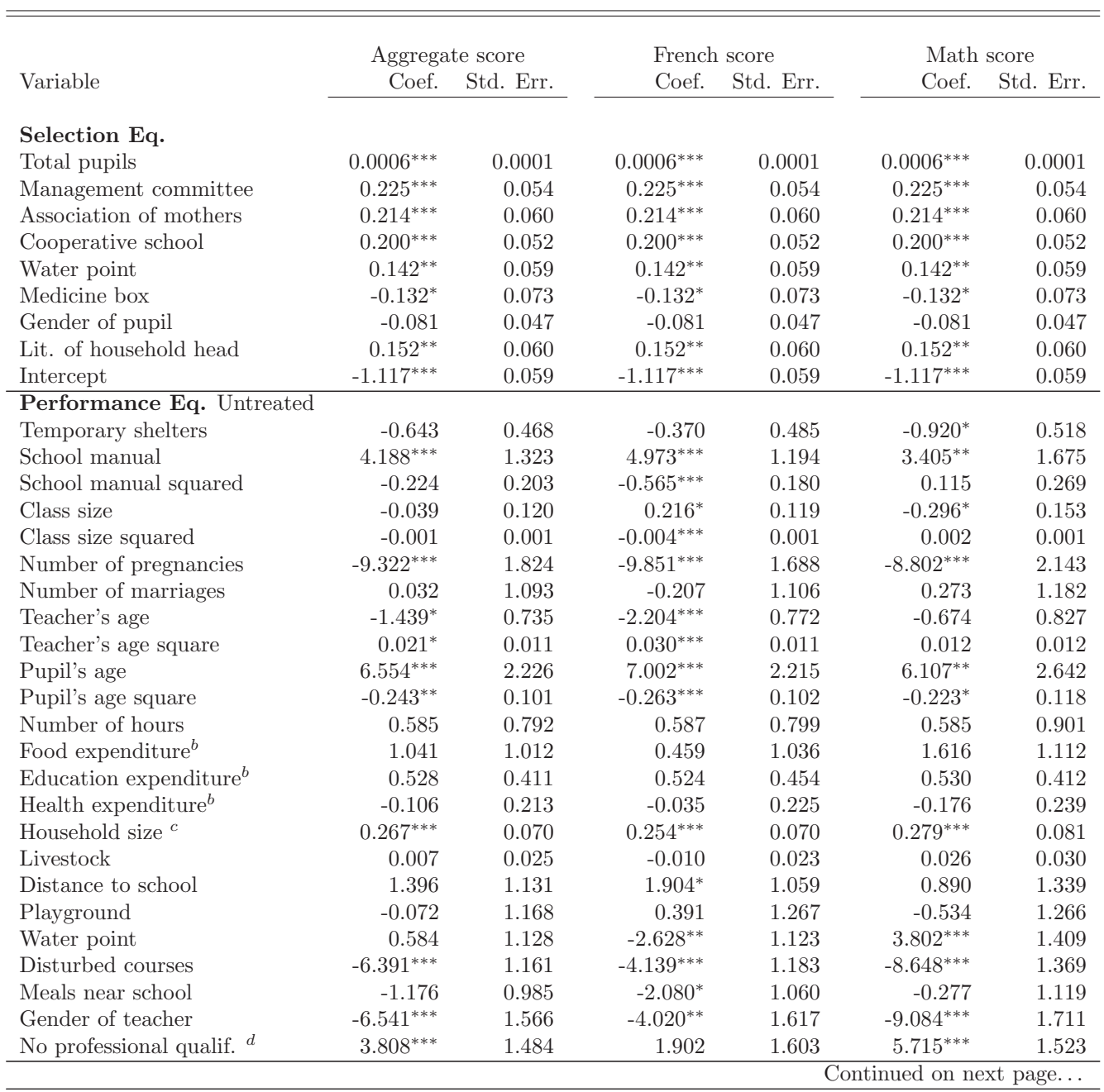


Table A.10 - continued

\begin{tabular}{|c|c|c|c|c|c|c|}
\hline \multirow[b]{2}{*}{ Variable } & \multicolumn{2}{|c|}{ Aggregate score } & \multicolumn{2}{|c|}{ French score } & \multicolumn{2}{|c|}{ Math score } \\
\hline & Coef. & Std. Err. & Coef. & Std. Err. & Coef. & Std. Err. \\
\hline Professional qualif. CAP & 1.014 & 1.791 & -0.725 & 1.796 & 2.728 & 2.094 \\
\hline Professional qualif. CEAP & $6.774^{* * *}$ & 1.617 & $5.044^{* * *}$ & 1.730 & $8.503^{* * *}$ & 1.772 \\
\hline High school diploma \& more & $2.421^{* *}$ & 1.055 & 1.076 & 1.104 & $3.7820^{* * *}$ & 1.284 \\
\hline Continuing training & 0.753 & 0.966 & -0.578 & 1.031 & $2.090^{*}$ & 1.078 \\
\hline Absenteeism & $-3.589^{* *}$ & 1.395 & -2.050 & 1.669 & $-5.137^{* * *}$ & 1.473 \\
\hline Gender of pupil & $1.383^{*}$ & 0.827 & $1.640^{*}$ & 0.911 & 1.126 & 0.872 \\
\hline Grade & $-14.900^{* * *}$ & 1.262 & $-21.945^{* * *}$ & 1.227 & $-7.852^{* * *}$ & 1.509 \\
\hline Koranic school & $4.239^{* * *}$ & 0.967 & $4.247^{* * *}$ & 1.026 & $4.228^{* * *}$ & 1.036 \\
\hline Early childhood inst. & $4.903^{* * *}$ & 1.847 & $5.220^{* * *}$ & 1.940 & $4.587^{* *}$ & 2.004 \\
\hline Snack & 1.896 & 1.699 & 1.734 & 1.799 & 2.072 & 1.903 \\
\hline Sick last 3 months & -0.884 & 1.012 & -0.504 & 1.019 & -1.264 & 1.128 \\
\hline Pupils eat fill & 0.231 & 1.461 & 0.119 & 1.535 & 0.342 & 1.565 \\
\hline Gender of household head & -1.387 & 1.512 & -2.321 & 1.646 & -0.458 & 1.677 \\
\hline Lit. of household head & 0.124 & 1.281 & -0.146 & 1.287 & 0.397 & 1.529 \\
\hline Marital status & 1.914 & 1.802 & 1.719 & 2.096 & 2.123 & 2.247 \\
\hline Land & 0.485 & 1.902 & 1.142 & 2.060 & -0.188 & 2.099 \\
\hline Parents school & $-4.805^{* * *}$ & 1.015 & $-4.746^{* * *}$ & 1.123 & $-4.877^{* * *}$ & 1.078 \\
\hline College & $3.730^{* * *}$ & 1.199 & $2.708^{* *}$ & 1.307 & $4.763^{* * *}$ & 1.336 \\
\hline Diourbel & 2.260 & 2.030 & 1.971 & 2.135 & 2.539 & 2.312 \\
\hline Kolda & $-8.064^{* * *}$ & 1.501 & $-7.958^{* * *}$ & 1.508 & $-8.163^{* * *}$ & 1.783 \\
\hline Sédhiou & -1.915 & 3.095 & -2.706 & 2.827 & -1.109 & 3.702 \\
\hline Lambda0 & $13.151^{* *}$ & 5.857 & -6.592 & 6.067 & $32.851^{* * *}$ & 6.567 \\
\hline Intercept & 22.951 & 20.950 & $33.533^{*}$ & 19.960 & 12.480 & 25.147 \\
\hline \# Observations & \multicolumn{2}{|c|}{1756} & \multicolumn{2}{|c|}{1756} & \multicolumn{2}{|c|}{1756} \\
\hline Adj R-squared & \multicolumn{2}{|c|}{0.2342} & \multicolumn{2}{|c|}{0.2884} & \multicolumn{2}{|c|}{0.1995} \\
\hline \multicolumn{7}{|l|}{ Performance Eq. Treated } \\
\hline Temporary shelters & $2.831^{* * *}$ & 0.896 & 1.610 & 1.009 & $4.058^{* * *}$ & 0.942 \\
\hline School manual & -3.551 & 2.622 & -2.703 & 2.788 & -4.306 & 3.319 \\
\hline School manual squared & 0.521 & 0.554 & 0.278 & 0.514 & 0.758 & 0.824 \\
\hline Class size & -0.417 & 0.274 & -0.037 & 0.260 & $-0.792^{* *}$ & 0.358 \\
\hline Class size squared & 0.005 & 0.003 & -0.00005 & 0.003 & $0.010^{* *}$ & 0.004 \\
\hline Number of pregnancies & -2.888 & 1.875 & $-5.212^{* * *}$ & 1.731 & -0.439 & 2.261 \\
\hline Number of marriages & 2.091 & 1.536 & 2.121 & 1.660 & 2.039 & 1.613 \\
\hline Teacher's age & $-4.290^{* * *}$ & 1.696 & $-3.892^{* *}$ & 1.748 & $-4.683^{* *}$ & 1.926 \\
\hline Teacher's age square & $0.058^{* * *}$ & 0.024 & $0.049^{*}$ & 0.025 & $0.066^{* *}$ & 0.027 \\
\hline Pupil's age & $10.784^{* * *}$ & 3.250 & $11.405^{* * *}$ & 3.661 & $9.765^{* * *}$ & 3.654 \\
\hline Pupil's age square & $-0.485^{* * *}$ & 0.154 & $-0.525^{* * *}$ & 0.171 & $-0.429^{* *}$ & 0.176 \\
\hline Number of hours & $-2.386^{* *}$ & 1.108 & -1.933 & 1.281 & $-2.764^{* *}$ & 1.160 \\
\hline Food expenditure ${ }^{b}$ & 0.172 & 1.293 & -0.821 & 1.561 & 0.726 & 1.338 \\
\hline Education expenditure $^{b}$ & -0.385 & 0.414 & -0.238 & 0.477 & -0.541 & 0.473 \\
\hline Health expenditure $^{b}$ & 0.445 & 0.299 & $0.555^{*}$ & 0.330 & 0.353 & 0.319 \\
\hline Household size ${ }^{c}$ & 0.143 & 0.106 & 0.093 & 0.120 & 0.181 & 0.114 \\
\hline Livestock & 0.010 & 0.048 & -0.023 & 0.053 & 0.051 & 0.055 \\
\hline Distance to school & -1.099 & 2.176 & -0.773 & 2.216 & -1.552 & 2.469 \\
\hline Playground & $6.174^{* * *}$ & 2.036 & $5.795^{* *}$ & 2.327 & $7.313^{* * *}$ & 2.206 \\
\hline Water point & -1.961 & 2.057 & -2.925 & 2.166 & -1.093 & 2.255 \\
\hline Disturbed courses & $-4.382^{*}$ & 2.283 & -2.627 & 2.351 & $-6.368^{* *}$ & 2.541 \\
\hline Meals near school & -0.819 & 1.807 & -2.296 & 1.980 & 0.634 & 2.011 \\
\hline Gender of teacher & 0.328 & 1.979 & 0.266 & 2.195 & 0.723 & 2.003 \\
\hline No professional qualif. ${ }^{d}$ & $-3.837^{* *}$ & 1.696 & $-4.734^{* *}$ & 1.920 & -3.094 & 1.901 \\
\hline Professional qualif. CAP & -2.060 & 1.679 & $-3.030^{*}$ & 1.845 & -0.845 & 1.958 \\
\hline Professional qualif. CEAP & 0.859 & 2.338 & -0.848 & 2.570 & 1.092 & 2.744 \\
\hline High school diploma \& more & -1.161 & 1.816 & 0.355 & 1.782 & -3.431 & 2.131 \\
\hline
\end{tabular}


Table A.10 - continued

\begin{tabular}{|c|c|c|c|c|c|c|}
\hline \multirow[b]{2}{*}{ Variable } & \multicolumn{2}{|c|}{ Aggregate score } & \multicolumn{2}{|c|}{ French score } & \multicolumn{2}{|c|}{ Math score } \\
\hline & Coef. & Std. Err. & Coef. & Std. Err. & Coef. & Std. Err. \\
\hline Continuing training & $-3.335^{*}$ & 1.776 & -1.061 & 1.801 & $-5.414^{* * *}$ & 2.069 \\
\hline Absenteeism & -2.414 & 3.027 & -0.972 & 3.302 & -3.822 & 3.283 \\
\hline Gender of pupil & -1.619 & 1.109 & $-2.731^{* *}$ & 1.217 & -0.178 & 1.242 \\
\hline Grade & $-13.284^{* * *}$ & 1.778 & $-20.143^{* * *}$ & 1.989 & $-6.536^{* * *}$ & 1.961 \\
\hline Koranic school & $3.052^{*}$ & 1.739 & $4.369^{* *}$ & 1.794 & 2.576 & 1.934 \\
\hline Early childhood inst. & 0.547 & 2.205 & 1.074 & 2.339 & -0.137 & 2.470 \\
\hline Snack & -2.987 & 1.892 & -2.018 & 2.057 & $-3.971^{*}$ & 2.070 \\
\hline Sick last 3 months & 0.393 & 1.329 & 0.635 & 1.437 & -0.306 & 1.486 \\
\hline Pupils eat fill & -1.378 & 1.866 & 1.357 & 2.195 & $-4.204^{* *}$ & 1.961 \\
\hline Gender of household head & -1.214 & 2.307 & -1.007 & 2.574 & -2.025 & 2.510 \\
\hline Lit. of household head & 2.492 & 1.654 & 2.114 & 1.762 & $3.323^{*}$ & 1.815 \\
\hline Marital status & $5.161^{*}$ & 3.035 & 4.698 & 3.599 & $5.672^{*}$ & 3.117 \\
\hline Land & $-8.462^{* *}$ & 3.795 & $-10.113^{* * *}$ & 3.723 & $-6.994^{*}$ & 4.133 \\
\hline Parents school & -0.420 & 1.807 & 1.086 & 1.935 & -2.316 & 2.022 \\
\hline College & -0.595 & 1.951 & -2.542 & 1.988 & 1.687 & 2.300 \\
\hline Diourbel & $-8.254^{*}$ & 4.742 & -5.898 & 4.115 & -10.411 & 6.410 \\
\hline Kolda & $-13.948^{* * *}$ & 2.485 & $-14.005^{* * *}$ & 2.657 & $-13.959^{* * *}$ & 2.630 \\
\hline Sédhiou & $-17.810^{* * *}$ & 3.855 & $-12.991^{* * *}$ & 3.826 & $-22.427^{* * *}$ & 4.384 \\
\hline lambda1 & 8.056 & 5.178 & 6.824 & 5.579 & $10.476^{*}$ & 5.736 \\
\hline Intercept & $69.269^{* *}$ & 34.146 & $71.107^{* *}$ & 35.996 & $73.724^{*}$ & 39.091 \\
\hline \# Observations & 73 & & & & $7:$ & \\
\hline Adj R-squared & 0.24 & & 0.2 & & 0.1 & \\
\hline
\end{tabular}

Notes ${ }^{a}$ :Two-step ${ }^{b}$ In log; ${ }^{c}$ : Household size per adult equivalent; ${ }^{d}$ : qualification.

Significance levels (bootstrap -100 replications-): $\quad *: 10 \% \quad * *: 5 \% \quad * * *: 1 \%$ 
Table A.11: Matching test: Meal

\begin{tabular}{|c|c|c|c|c|c|c|c|}
\hline \multirow[b]{2}{*}{ Variable } & \multirow[b]{2}{*}{ Sample } & \multicolumn{2}{|c|}{ Mean } & \multirow[b]{2}{*}{$\%$ bias } & \multirow{2}{*}{$\begin{array}{l}\% \text { bias } \\
\text { reduction }\end{array}$} & \multicolumn{2}{|c|}{ t-test } \\
\hline & & Treated & Control & & & $\mathrm{t}$ & $P>|t|$ \\
\hline \multirow[t]{2}{*}{ Total pupils } & unmatched & 232.81 & 192.25 & 25.4 & & 7.90 & $0.000^{* * *}$ \\
\hline & matched & 199.5 & 207.77 & -5.2 & 79.6 & -1.36 & 0.173 \\
\hline \multirow[t]{2}{*}{ Distance to school } & unmatched & 0.843 & 0.776 & 17.3 & & 4.96 & 0.000 \\
\hline & matched & 0.830 & 0.824 & 1.6 & 90.6 & 0.41 & 0.683 \\
\hline \multirow[t]{2}{*}{ Association of mothers } & unmatched & 0.372 & 0.179 & 44.0 & & 13.84 & $0.000^{* * *}$ \\
\hline & matched & 0.313 & 0.338 & -5.7 & 87.2 & -1.27 & 0.203 \\
\hline \multirow[t]{2}{*}{ Rural council grant } & unmatched & 0.801 & 0.589 & 47.3 & & 13.81 & $0.000^{* * *}$ \\
\hline & matched & 0.823 & 0.802 & 4.7 & 90.1 & 1.30 & 0.193 \\
\hline \multirow[t]{2}{*}{ Water point } & unmatched & 0.540 & 0.433 & 21.6 & & 6.51 & $0.000^{* * *}$ \\
\hline & matched & 0.477 & 0.518 & -8.2 & 61.9 & -1.97 & 0.049 \\
\hline \multirow[t]{2}{*}{ Disturbed courses } & unmatched & 0.718 & 0.653 & 14.1 & & 4.21 & $0.000^{* * *}$ \\
\hline & matched & 0.727 & 0.690 & 7.9 & 43.7 & 1.95 & 0.051 \\
\hline \multirow[t]{2}{*}{ Storage } & unmatched & 0.330 & 0.143 & 44.9 & & 14.27 & $0.000^{* * *}$ \\
\hline & matched & 0.288 & 0.271 & 4.2 & 90.7 & 0.93 & 0.353 \\
\hline \multirow{2}{*}{ Gender of pupil } & unmatched & 0.498 & 0.517 & -3.7 & & -1.13 & 0.260 \\
\hline & matched & 0.497 & 0.492 & 0.9 & 75.7 & 0.22 & 0.827 \\
\hline
\end{tabular}

Significance levels: $\quad *: 10 \% \quad * *: 5 \% \quad * * *: 1 \%$

Table A.13: Matching test: Deworming

\begin{tabular}{|c|c|c|c|c|c|c|c|}
\hline \multirow[b]{2}{*}{ Variable } & \multirow[b]{2}{*}{ Sample } & \multicolumn{2}{|c|}{ Mean } & \multirow[b]{2}{*}{$\%$ bias } & \multirow{2}{*}{$\begin{array}{l}\text { bias } \\
\text { reduction }\end{array}$} & \multicolumn{2}{|c|}{ t-test } \\
\hline & & Treated & Control & & & $\mathrm{t}$ & $P>|t|$ \\
\hline \multirow{2}{*}{ Total pupils } & unmatched & 241.65 & 192.25 & 31.5 & & 8.58 & $0.000^{* * *}$ \\
\hline & matched & 242.47 & 236.17 & 4.0 & 87.3 & 0.80 & 0.425 \\
\hline \multirow[t]{2}{*}{ Management committee } & unmatched & 0.695 & 0.531 & 34.2 & & 8.80 & $0.000^{* * *}$ \\
\hline & matched & 0.687 & 0.678 & 1.7 & 94.9 & 0.37 & 0.709 \\
\hline \multirow[t]{2}{*}{ Association of mothers } & unmatched & 0.244 & 0.179 & 15.9 & & 4.29 & $0.000^{* * *}$ \\
\hline & matched & 0.235 & 0.224 & 2.8 & 82.7 & 0.55 & 0.579 \\
\hline \multirow[t]{2}{*}{ Cooperative school } & unmatched & 0.728 & 0.601 & 27.1 & & 6.95 & $0.000^{* * *}$ \\
\hline & matched & 0.733 & 0.728 & 1.0 & 96.5 & 0.21 & 0.834 \\
\hline \multirow[t]{2}{*}{ Water point } & unmatched & 0.557 & 0.433 & 25.1 & & 6.60 & $0.000^{* * *}$ \\
\hline & matched & 0.554 & 0.550 & 0.7 & 97.2 & 0.15 & 0.882 \\
\hline \multirow{2}{*}{ Gender of pupil } & unmatched & 0.493 & 0.517 & -4.8 & & -1.27 & 0.205 \\
\hline & matched & 0.494 & 0.496 & -0.5 & 90.6 & -0.09 & 0.925 \\
\hline \multirow[t]{2}{*}{ Lit. of household head } & unmatched & 0.236 & 0.166 & 17.4 & & 4.56 & $0.000^{* * *}$ \\
\hline & matched & 0.234 & 0.230 & 0.9 & 95.0 & 0.17 & 0.866 \\
\hline
\end{tabular}

Significance levels: $\quad *: 10 \% \quad * *: 5 \% \quad * * *: 1 \%$

Table A.15: Deworming in school: $\mathrm{HSF}^{a}$ regression $\left(\mathrm{FIML}^{b}\right)$ 
Table A.15 - continued

Variable

\begin{tabular}{|c|c|c|c|c|c|}
\hline \multicolumn{2}{|c|}{ Aggregate score } & \multicolumn{2}{|c|}{ French score } & \multicolumn{2}{|c|}{ Math score } \\
\hline Coef. & Std. Err. & Coef. & Std. Err. & Coef. & Std. Err. \\
\hline 0.025 & 1.025 & -1.768 & 1.084 & 1.848 & 1.155 \\
\hline-0.541 & 1.223 & -0.476 & 1.294 & -0.432 & 1.381 \\
\hline $3.958^{* * *}$ & 1.164 & $2.561^{* *}$ & 1.227 & $4.861^{* * *}$ & 1.313 \\
\hline $1.672^{*}$ & 0.878 & 0.925 & 0.925 & $2.200^{* *}$ & 0.989 \\
\hline-0.575 & 0.810 & -0.587 & 0.852 & -0.482 & 0.913 \\
\hline$-4.958^{* * *}$ & 1.277 & $-2.402^{*}$ & 1.343 & $-7.428^{* * *}$ & 1.438 \\
\hline 0.531 & 0.728 & -0.125 & 0.760 & 1.293 & 0.814 \\
\hline$-14.586^{* * *}$ & 0.986 & $-21.465^{* * *}$ & 1.040 & $-7.622^{* * *}$ & 1.107 \\
\hline $3.366^{* * *}$ & 0.800 & $3.813^{* * *}$ & 0.843 & $3.185^{* * *}$ & 0.901 \\
\hline 1.633 & 1.286 & $2.513^{*}$ & 1.364 & 0.847 & 1.455 \\
\hline-0.311 & 1.139 & 0.059 & 1.203 & -0.544 & 1.284 \\
\hline-0.418 & 0.755 & -0.268 & 0.798 & -0.767 & 0.851 \\
\hline-0.019 & 1.189 & 0.473 & 1.252 & -0.507 & 1.342 \\
\hline-0.496 & 1.245 & -1.134 & 1.317 & -0.161 & 1.404 \\
\hline-0.240 & 0.927 & 0.548 & 0.980 & -0.988 & 1.046 \\
\hline 2.741 & 1.762 & 2.410 & 1.865 & 3.082 & 1.988 \\
\hline-2.194 & 1.577 & -2.159 & 1.665 & -2.383 & 1.779 \\
\hline$-3.313^{* * *}$ & 0.795 & $-3.255^{* * *}$ & 0.838 & $-3.476^{* * *}$ & 0.897 \\
\hline $2.025^{*}$ & 1.037 & 0.642 & 1.086 & $3.331^{* * *}$ & 1.170 \\
\hline 1.109 & 1.712 & 0.228 & 1.800 & 2.108 & 1.923 \\
\hline$-8.390^{* * *}$ & 1.251 & $-8.779^{* * *}$ & 1.316 & $-8.063^{* * *}$ & 1.408 \\
\hline$-9.785^{* * *}$ & 2.273 & $-8.342^{* * *}$ & 2.403 & $-10.888^{* * *}$ & 2.557 \\
\hline$-20.104^{* * *}$ & 2.321 & $-21.916^{* * *}$ & 2.689 & $-18.968^{* * *}$ & 2.671 \\
\hline $46.775^{* * *}$ & 16.755 & $55.615^{* * *}$ & 17.634 & $38.458^{* *}$ & 18.891 \\
\hline-0.00006 & 0.0002 & -0.0002 & 0.0003 & -0.00001 & 0.0003 \\
\hline $0.181^{* *}$ & 0.078 & $0.228^{* * *}$ & 0.078 & $0.199^{* *}$ & 0.079 \\
\hline $0.454^{* * *}$ & 0.0759 & $0.417^{* * *}$ & 0.0781 & $0.490^{* * *}$ & 0.077 \\
\hline $0.429^{* * *}$ & 0.081 & $0.377^{* * *}$ & 0.081 & $0.423^{* * *}$ & 0.081 \\
\hline $0.505^{* * *}$ & 0.083 & $0.506^{* * *}$ & 0.083 & $0.496^{* * *}$ & 0.083 \\
\hline-4.461 & 98.194 & -5.440 & 2142.0 & -5.973 & 16813.54 \\
\hline-0.050 & 0.068 & -0.056 & 0.069 & -0.051 & 0.069 \\
\hline$-1.865^{* * *}$ & 0.103 & $-1.817^{* * *}$ & 0.1027 & $-1.883^{* * *}$ & 0.102 \\
\hline $0.727^{* * *}$ & 0.089 & $0.648^{* * *}$ & 0.095 & 0.674 & 0.088 \\
\hline $2.886^{* * *}$ & 0.019 & $2.928^{* * *}$ & 0.019 & $2.997^{* * *}$ & 0.019 \\
\hline 0.621 & 0.054 & 0.570 & 0.064 & 0.588 & 0.058 \\
\hline 17.931 & 0.350 & 18.697 & 0.369 & 20.039 & 0.387 \\
\hline 11.139 & 1.141 & 10.665 & 1.355 & 11.783 & 1.330 \\
\hline
\end{tabular}

No professional qualif. ${ }^{e}$

Professional qualif. CAP

Professional qualif. CEAP

High school diploma \& more

Continuing training

Absenteeism

Gender of pupil

Grade

Koranic school

Early childhood inst.

Snack

Sick last 3 months

Pupils eat fill

Gender of household head

Lit. of household head

Marital status

Land

Parents school

College

Diourbel

Kolda

Sédhiou

Deworming in school

Selection Eq.

Total pupils

Management committee

Association of mothers

Cooperative school

Water point

Medicine box

Gender of pupil

Intercept

atanh $\rho$

$\ln \sigma$

$\rho$

1.141

10.665

LR test $\left(H_{0}: \rho=0\right)$

\begin{tabular}{lccc} 
LR test $\left(H_{0}: \rho=0\right)$ & 38.08 & 24.07 & 35.23 \\
$\chi_{2}(1)$ & 0.000 & 0.000 & 0.5725 \\
$P>\chi_{2}$ & -11306.085 & -11442.162 & -11601.186 \\
\hline Log likelihood & 2487 & 2487 & 2487 \\
$\#$ Observations & 795.27 & 1048.52 & 587.95 \\
Wald $\chi_{2}(46)$ & 0.000 & 0.000 & 0.000 \\
$P>\chi_{2}$ & -
\end{tabular}

Notes $^{a}$ : Heckman selection framework; ${ }^{b}$ : Full Information Maximum Likelihood; ${ }^{c}$ : In log; ${ }^{d}$ : Household size per adult equivalent; ${ }^{e}$ : qualification.

Significance levels: $\quad *: 10 \% \quad * *: 5 \% \quad * * *: 1 \%$ 
Table A.16: Deworming in school: $\mathrm{HSF}^{a}$ regression $\left(2-\right.$ step $\left.^{b}\right)$

\begin{tabular}{|c|c|c|c|c|c|c|}
\hline \multirow[b]{2}{*}{ Variable } & \multicolumn{2}{|c|}{ Aggregate score } & \multicolumn{2}{|c|}{ French score } & \multicolumn{2}{|c|}{ Math score } \\
\hline & Coef. & Std. Err. & Coef. & Std. Err. & Coef. & Std. Err. \\
\hline \multicolumn{7}{|l|}{ Performance Eq. } \\
\hline Temporary shelters & 0.603 & 0.395 & 0.452 & 0.417 & $0.761^{*}$ & 0.445 \\
\hline School manual & 0.608 & 1.106 & 1.388 & 1.165 & -0.059 & 1.245 \\
\hline School manual squared & 0.158 & 0.193 & -0.170 & 0.202 & $0.476^{* *}$ & 0.217 \\
\hline Class size & -0.188 & 0.119 & 0.131 & 0.125 & $-0.515^{* * *}$ & 0.134 \\
\hline Class size squared & 0.001 & 0.001 & $-0.003^{* *}$ & 0.001 & $0.005^{* * *}$ & 0.001 \\
\hline Number of pregnancies & $-6.298^{* * *}$ & 1.095 & $-8.309^{* * *}$ & 1.166 & $-4.229^{* * *}$ & 1.226 \\
\hline Number of marriages & -0.539 & 0.692 & -0.270 & 0.724 & -0.783 & 0.781 \\
\hline Teacher's age & $-2.308^{* * *}$ & 0.629 & $-2.552^{* * *}$ & 0.662 & $-2.035^{* * *}$ & 0.708 \\
\hline Teacher's age square & $0.032^{* * *}$ & 0.009 & $0.033^{* * *}$ & 0.009 & $0.031^{* * *}$ & 0.010 \\
\hline Pupil's age & $7.003^{* * *}$ & 1.947 & $7.358^{* * *}$ & 2.055 & $6.508^{* * *}$ & 2.188 \\
\hline Pupil's age square & $-0.279^{* * *}$ & 0.091 & $-0.299^{* * *}$ & 0.096 & $-0.253^{* *}$ & 0.103 \\
\hline Number of hours & -0.114 & 0.628 & -0.073 & 0.661 & -0.114 & 0.707 \\
\hline Food expenditure ${ }^{c}$ & 0.675 & 0.739 & -0.005 & 0.778 & 1.284 & 0.831 \\
\hline Education expenditure ${ }^{c}$ & 0.284 & 0.278 & 0.348 & 0.292 & 0.230 & 0.314 \\
\hline Health expenditure ${ }^{c}$ & 0.080 & 0.165 & 0.125 & 0.175 & 0.031 & 0.186 \\
\hline Household size $^{d}$ & $0.273^{* * *}$ & 0.063 & $0.257^{* * *}$ & 0.066 & $0.289^{* * *}$ & 0.071 \\
\hline Livestock & -0.003 & 0.020 & -0.025 & 0.021 & 0.018 & 0.023 \\
\hline Distance to school & 0.731 & 0.913 & 1.227 & 0.962 & 0.192 & 1.027 \\
\hline Playground & 1.002 & 0.976 & 1.236 & 1.030 & 1.088 & 1.098 \\
\hline Water point & 0.947 & 1.034 & -0.411 & 1.066 & $2.216^{*}$ & 1.177 \\
\hline Disturbed courses & $-4.786^{* * *}$ & 1.005 & $-2.973^{* * *}$ & 1.062 & $-6.749^{* * *}$ & 1.129 \\
\hline Meals near school & 0.081 & 0.926 & -1.119 & 0.970 & 1.263 & 1.045 \\
\hline Gender of teacher & $-4.141^{* * *}$ & 1.121 & $-2.822^{* *}$ & 1.188 & $-5.477^{* * *}$ & 1.257 \\
\hline No professional qualif. ${ }^{e}$ & 0.118 & 1.031 & -1.588 & 1.088 & 1.849 & 1.158 \\
\hline Professional qualif. CAP & -0.779 & 1.226 & -0.736 & 1.305 & -0.690 & 1.373 \\
\hline Professional qualif. CEAP & $3.935^{* * *}$ & 1.178 & $2.602^{* *}$ & 1.236 & $4.870^{* * *}$ & 1.328 \\
\hline High school diploma \& more & $1.904^{* *}$ & 0.880 & 1.059 & 0.928 & $2.521^{* *}$ & 0.990 \\
\hline Continuing training & -0.429 & 0.811 & -0.624 & 0.853 & -0.196 & 0.913 \\
\hline Absenteeism & $-4.592^{* * *}$ & 1.287 & $-2.321^{*}$ & 1.351 & $-6.939^{* * *}$ & 1.451 \\
\hline Gender of pupil & 0.565 & 0.730 & -0.036 & 0.746 & 1.265 & 0.834 \\
\hline Grade & $-14.157^{* * *}$ & 0.983 & $-21.018^{* * *}$ & 1.036 & $-7.339^{* * *}$ & 1.106 \\
\hline Koranic school & $3.483^{* * *}$ & 0.803 & $3.879^{* * *}$ & 0.846 & $3.287^{* * *}$ & 0.903 \\
\hline Early childhood inst. & 2.003 & 1.299 & $2.864^{* *}$ & 1.379 & 1.172 & 1.455 \\
\hline Snack & -0.120 & 1.145 & 0.144 & 1.211 & -0.364 & 1.286 \\
\hline Sick last 3 months & -0.409 & 0.760 & -0.192 & 0.801 & -0.778 & 0.854 \\
\hline Pupils eat fill & 0.220 & 1.192 & 0.616 & 1.254 & -0.213 & 1.343 \\
\hline Gender of household head & -0.691 & 1.254 & -1.305 & 1.328 & -0.236 & 1.407 \\
\hline Lit. of household head & -0.295 & 0.932 & 0.510 & 0.988 & -1.051 & 1.046 \\
\hline Marital status & 2.737 & 1.775 & 2.416 & 1.881 & 3.037 & 1.992 \\
\hline Land & $-2.645^{*}$ & 1.586 & -2.378 & 1.677 & $-2.929^{*}$ & 1.781 \\
\hline Parents school & $-3.180^{* * *}$ & 0.800 & $-3.145^{* * *}$ & 0.841 & $-3.358^{* * *}$ & 0.901 \\
\hline College & 1.424 & 1.034 & 0.390 & 1.086 & $2.556^{* *}$ & 1.164 \\
\hline Diourbel & 1.057 & 1.713 & 0.268 & 1.794 & 1.881 & 1.933 \\
\hline Kolda & $-8.399^{* * *}$ & 1.287 & $-9.026^{* * *}$ & 1.355 & $-7.789^{* * *}$ & 1.448 \\
\hline Sédhiou & $-8.524^{* * *}$ & 2.277 & $-7.342^{* * *}$ & 2.401 & $-9.672^{* * *}$ & 2.562 \\
\hline Deworming in school & $-19.898^{* * *}$ & 4.653 & $-17.270^{* * *}$ & 4.808 & $-22.862^{* * *}$ & 5.288 \\
\hline Intercept & $43.842^{* * *}$ & 16.777 & $55.165^{* * *}$ & 17.669 & $33.853^{*}$ & 18.881 \\
\hline Selection Eq. & & & & & & \\
\hline Total pupils & -0.0005 & 0.0003 & -0.0005 & 0.0003 & -0.0005 & 0.0003 \\
\hline Management committee & $0.393^{* * *}$ & 0.080 & $0.393^{* * *}$ & 0.080 & $0.393^{* * *}$ & 0.080 \\
\hline
\end{tabular}


Table A.16 - continued

\begin{tabular}{|c|c|c|c|c|c|c|}
\hline \multirow[b]{2}{*}{ Variable } & \multicolumn{2}{|c|}{ Aggregate score } & \multicolumn{2}{|c|}{ French score } & \multicolumn{2}{|c|}{ Math score } \\
\hline & Coef. & Std. Err. & Coef. & Std. Err. & Coef. & Std. Err. \\
\hline Association of mothers & $0.491^{* * *}$ & 0.083 & $0.491^{* * *}$ & 0.083 & $0.491^{* * *}$ & 0.083 \\
\hline Cooperative school & $0.235^{* * *}$ & 0.083 & $0.235^{* * *}$ & 0.083 & $0.235^{* * *}$ & 0.083 \\
\hline Water point & $0.517^{* * *}$ & 0.085 & $0.517^{* * *}$ & 0.085 & $0.517^{* * *}$ & 0.085 \\
\hline Medicine box & -5.628 & & -5.628 & & -5.628 & . \\
\hline Gender of pupil & -0.079 & 0.071 & -0.079 & 0.071 & -0.079 & 0.071 \\
\hline Intercept & $-1.770^{* * *}$ & 0.100 & $-1.770^{* * *}$ & 0.100 & $-1.770^{* * *}$ & 0.100 \\
\hline hasard $\lambda$ & $11.090^{* * *}$ & 2.605 & $8.029^{* * *}$ & 2.702 & $14.113^{* * *}$ & 2.955 \\
\hline$\rho$ & 0.619 & - & 0.438 & - & 0.689 & - \\
\hline$\sigma$ & 17.915 & - & 18.295 & - & 20.477 & - \\
\hline$\lambda$ & 11.090 & 3.999 & 8.029 & 2.702 & 14.113 & 2.955 \\
\hline \# Observations & \multicolumn{2}{|c|}{2487} & \multicolumn{2}{|c|}{2487} & \multicolumn{2}{|c|}{2487} \\
\hline Wald $\chi_{2}(48)$ & \multicolumn{2}{|c|}{778.56} & \multicolumn{2}{|c|}{1049.67} & \multicolumn{2}{|c|}{583.62} \\
\hline$P>\chi_{2}$ & \multicolumn{2}{|c|}{0.000} & \multicolumn{2}{|c|}{0.000} & \multicolumn{2}{|c|}{0.000} \\
\hline
\end{tabular}

Notes $^{a}$ : Heckman selection framework; ${ }^{b}$ : Two step; ${ }^{c}$ : In log; ${ }^{d}$ : Household size per adult equivalent; ${ }^{e}$ : qualification.

Significance levels: $\quad *: 10 \% \quad * *: 5 \% \quad * * *: 1 \%$

Table A.17: Deworming at home: $\mathrm{HSF}^{a}$ regression $\left(\mathrm{FIML}^{b}\right)$

\begin{tabular}{|c|c|c|c|c|c|c|}
\hline \multirow[b]{2}{*}{ Variable } & \multicolumn{2}{|c|}{ Aggregate score } & \multicolumn{2}{|c|}{ French score } & \multicolumn{2}{|c|}{ Math score } \\
\hline & Coef. & Std. Err. & Coef. & Std. Err. & Coef. & Std. Err. \\
\hline \multicolumn{7}{|l|}{ Performance Eq. } \\
\hline Temporary shelters & 0.516 & 0.387 & 0.197 & 0.408 & $0.783^{*}$ & 0.436 \\
\hline School manual & 1.264 & 1.082 & $2.269^{* *}$ & 1.142 & 0.519 & 1.214 \\
\hline School manual squared & 0.086 & 0.189 & -0.291 & 0.199 & $0.433^{* *}$ & 0.212 \\
\hline Class size & -0.178 & 0.117 & 0.117 & 0.124 & $-0.483^{* * *}$ & 0.132 \\
\hline Class size squared & 0.001 & 0.001 & $-0.002^{*}$ & 0.001 & $0.005^{* * *}$ & 0.001 \\
\hline Number of pregnancies & $-6.418^{* * *}$ & 1.099 & $-8.487^{* * *}$ & 1.161 & $-4.402^{* * *}$ & 1.239 \\
\hline Number of marriages & -0.013 & 0.673 & 0.082 & 0.711 & -0.073 & 0.758 \\
\hline Teacher's age & $-2.576^{* * *}$ & 0.619 & $-2.881^{* * *}$ & 0.654 & $-2.283^{* * *}$ & 0.697 \\
\hline Teacher's age square & $0.036^{* * *}$ & 0.009 & $0.037^{* * *}$ & 0.009 & $0.034^{* * *}$ & 0.010 \\
\hline Pupil's age & $7.198^{* * *}$ & 1.945 & $7.511^{* * *}$ & 2.051 & $6.690^{* * *}$ & 2.195 \\
\hline Pupil's age square & $-0.289^{* * *}$ & 0.091 & $-0.307^{* * *}$ & 0.096 & $-0.262^{* * *}$ & 0.103 \\
\hline Number of hours & -0.288 & 0.633 & -0.257 & 0.667 & -0.273 & 0.713 \\
\hline Food expenditure ${ }^{c}$ & 1.099 & 0.798 & 0.381 & 0.840 & $1.771^{* *}$ & 0.891 \\
\hline Education expenditure $^{c}$ & 0.060 & 0.299 & 0.088 & 0.314 & 0.022 & 0.334 \\
\hline Health expenditure $^{c}$ & 0.232 & 0.183 & 0.283 & 0.193 & 0.192 & 0.205 \\
\hline Household size ${ }^{d}$ & $0.260^{* * *}$ & 0.063 & $0.238^{* * *}$ & 0.066 & $0.280^{* * *}$ & 0.071 \\
\hline Livestock & -0.001 & 0.020 & -0.020 & 0.021 & 0.019 & 0.023 \\
\hline Distance to school & 1.071 & 0.901 & 1.275 & 0.951 & 0.818 & 1.014 \\
\hline Playground & $1.756^{*}$ & 0.944 & 1.529 & 0.997 & $2.253^{* *}$ & 1.064 \\
\hline Water point & $-1.655^{*}$ & 0.941 & $-2.683^{* * *}$ & 0.990 & -0.757 & 1.054 \\
\hline Disturbed courses & $-4.135^{* * *}$ & 0.981 & $-2.550^{* *}$ & 1.035 & $-5.821^{* * *}$ & 1.108 \\
\hline Meals near school & -0.512 & 0.899 & -1.545 & 0.950 & 0.495 & 1.013 \\
\hline Gender of teacher & $-3.514^{* * *}$ & 1.115 & $-2.500^{* *}$ & 1.176 & $-4.632^{* * *}$ & 1.259 \\
\hline No professional qualif. ${ }^{e}$ & 0.099 & 1.028 & -1.329 & 1.083 & 1.681 & 1.159 \\
\hline Professional qualif. CAP & -1.095 & 1.231 & -1.342 & 1.299 & -0.840 & 1.388 \\
\hline Professional qualif. CEAP & $4.310^{* * *}$ & 1.166 & $2.964^{* *}$ & 1.229 & $5.311^{* * *}$ & 1.313 \\
\hline
\end{tabular}


Table A.17 - continued

\begin{tabular}{|c|c|c|c|c|c|c|}
\hline \multirow[b]{2}{*}{ Variable } & \multicolumn{2}{|c|}{ Aggregate score } & \multicolumn{2}{|c|}{ French score } & \multicolumn{2}{|c|}{ Math score } \\
\hline & Coef. & Std. Err. & Coef. & Std. Err. & Coef. & Std. Err. \\
\hline High school diploma \& more & $1.471^{*}$ & 0.881 & 0.908 & 0.925 & $1.922^{*}$ & 0.997 \\
\hline Continuing training & -0.432 & 0.789 & -0.297 & 0.834 & -0.487 & 0.889 \\
\hline Absenteeism & $-4.980^{* * *}$ & 1.259 & $-2.722^{* *}$ & 1.329 & $-7.376^{* * *}$ & 1.421 \\
\hline Gender of pupil & 0.938 & 0.753 & 0.279 & 0.791 & $1.708^{* *}$ & 0.839 \\
\hline Grade & $-13.985^{* * *}$ & 0.985 & $-20.816^{* * *}$ & 1.043 & $-7.195^{* * *}$ & 1.108 \\
\hline Koranic school & $3.639^{* * *}$ & 0.792 & $4.230^{* * *}$ & 0.837 & $3.276^{* * *}$ & 0.893 \\
\hline Early childhood inst. & 2.021 & 1.304 & $2.814^{* *}$ & 1.376 & 1.274 & 1.470 \\
\hline Snack & -0.467 & 1.140 & -0.104 & 1.203 & -0.791 & 1.286 \\
\hline Sick last 3 months & $-3.781^{* * *}$ & 0.996 & $-3.725^{* * *}$ & 1.081 & $-4.364^{* * *}$ & 1.135 \\
\hline Pupils eat fill & 0.595 & 1.191 & 0.954 & 1.258 & 0.131 & 1.340 \\
\hline Gender of household head & -0.891 & 1.255 & -1.444 & 1.326 & $-0.466^{* *}$ & 1.416 \\
\hline Lit. of household head & -1.459 & 1.042 & -0.697 & 1.099 & -2.291 & 1.164 \\
\hline Marital status & 2.902 & 1.784 & 2.504 & 1.886 & 3.271 & 2.008 \\
\hline Land & $-2.817^{*}$ & 1.575 & $-2.740^{*}$ & 1.659 & -2.779 & 1.778 \\
\hline Parents school & $-2.820^{* * *}$ & 0.7951 & $-2.773^{* * *}$ & 0.840 & $-3.071^{* * *}$ & 0.894 \\
\hline College & 1.587 & 1.011 & 0.881 & 1.067 & $2.550^{* *}$ & 1.139 \\
\hline Diourbel & 1.311 & 1.676 & 1.119 & 1.767 & 1.626 & 1.887 \\
\hline Kolda & $-9.838^{* * *}$ & 1.224 & $-9.812^{* * *}$ & 1.291 & $-9.840^{* * *}$ & 1.381 \\
\hline Sédhiou & $-9.195^{* * *}$ & 2.231 & $-6.891^{* * *}$ & 2.351 & $-11.118^{* * *}$ & 2.517 \\
\hline Deworming at home & $19.156^{* * *}$ & 2.983 & $20.067^{* * *}$ & 3.460 & $20.339^{* * *}$ & 3.563 \\
\hline Intercept & $37.668^{* *}$ & 17.066 & $51.619^{* * *}$ & 17.985 & 25.231 & 19.201 \\
\hline \multicolumn{7}{|l|}{ Selection Eq. } \\
\hline Food expenditure & -0.066 & 0.055 & -0.072 & 0.055 & -0.064 & 0.055 \\
\hline Education expenditure & 0.018 & 0.020 & 0.022 & 0.020 & 0.017 & 0.021 \\
\hline Health expenditure & $-0.025^{* *}$ & 0.013 & $-0.025^{* *}$ & 0.013 & $-0.026^{* *}$ & 0.013 \\
\hline Sick last 3 months & $0.604^{* * *}$ & 0.060 & $0.608^{* * *}$ & 0.060 & $0.608^{* * *}$ & 0.060 \\
\hline Water point & $0.105^{*}$ & 0.059 & $0.103^{*}$ & 0.059 & $0.105^{*}$ & 0.059 \\
\hline Gender of pupil & -0.045 & 0.057 & -0.050 & 0.057 & -0.033 & 0.057 \\
\hline Lit. of household head & $0.205^{* * *}$ & 0.074 & $0.203^{* * *}$ & 0.074 & $0.206^{* * *}$ & 0.074 \\
\hline Intercept & -0.308 & 0.627 & -0.263 & 0.632 & -0.330 & 0.628 \\
\hline $\operatorname{atanh} \rho$ & $-0.712^{* * *}$ & 0.115 & $-0.691^{* * *}$ & 0.125 & -0.667 & 0.120 \\
\hline $\ln \sigma$ & $2.920^{* * *}$ & 0.029 & $2.969^{* * *}$ & 0.031 & $3.029^{* * *}$ & 0.030 \\
\hline$\rho$ & -0.612 & 0.072 & -0.598 & 0.080 & -0.583 & 0.079 \\
\hline$\sigma$ & 18.553 & 0.550 & 19.482 & 0.613 & 20.678 & 0.623 \\
\hline$\lambda$ & -11.361 & 1.641 & -11.664 & 1.909 & -12.060 & 1.969 \\
\hline \multicolumn{7}{|l|}{ LR test $\left(H_{0}: \rho=0\right)$} \\
\hline$\chi_{2}(1)$ & \multicolumn{2}{|c|}{7.09} & \multicolumn{2}{|c|}{5.38} & \multicolumn{2}{|c|}{6.24} \\
\hline$P>\chi_{2}$ & \multicolumn{2}{|c|}{0.007} & \multicolumn{2}{|c|}{0.020} & \multicolumn{2}{|c|}{0.012} \\
\hline Log likelihood & \multicolumn{2}{|c|}{-11725.921} & \multicolumn{2}{|c|}{-11859.491} & \multicolumn{2}{|c|}{-12020.072} \\
\hline \# Observations & \multicolumn{2}{|c|}{2487} & \multicolumn{2}{|c|}{2487} & \multicolumn{2}{|c|}{2487} \\
\hline Wald $\chi_{2}(46)$ & \multicolumn{2}{|c|}{780.21} & \multicolumn{2}{|c|}{1053.13} & \multicolumn{2}{|c|}{572.34} \\
\hline$P>\chi_{2}$ & \multicolumn{2}{|c|}{0.000} & \multicolumn{2}{|c|}{0.000} & \multicolumn{2}{|c|}{0.000} \\
\hline
\end{tabular}

Notes $^{a}$ : Heckman selection framework; ${ }^{b}$ :Full Information Maximum Likelihood; ${ }^{c}$ : In log; ${ }^{d}$ : Household size per adult equivalent; ${ }^{e}$ : qualification.

Significance levels: $\quad *: 10 \% \quad * *: 5 \% \quad * * *: 1 \%$ 
Table A.18: Deworming at home: $\mathrm{HSF}^{a}$ regression (2-step)

\begin{tabular}{|c|c|c|c|c|c|c|}
\hline \multirow[b]{2}{*}{ Variable } & \multicolumn{2}{|c|}{ Aggregate score } & \multicolumn{2}{|c|}{ French score } & \multicolumn{2}{|c|}{ Math score } \\
\hline & Coef. & Std. Err. & Coef. & Std. Err. & Coef. & Std. Err. \\
\hline \multicolumn{7}{|l|}{ Performance Eq. } \\
\hline Temporary shelters & 0.433 & 0.387 & 0.148 & 0.408 & 0.697 & 0.436 \\
\hline School manual & 1.566 & 1.080 & $2.521^{* *}$ & 1.138 & 0.788 & 1.216 \\
\hline School manual squared & 0.056 & 0.189 & -0.319 & 0.199 & $0.412^{*}$ & 0.213 \\
\hline Class size & -0.170 & 0.118 & 0.136 & 0.125 & $-0.488^{* * *}$ & 0.133 \\
\hline Class size squared & 0.0008 & 0.001 & $-0.003^{* *}$ & 0.001 & $0.004^{* * *}$ & 0.001 \\
\hline Number of pregnancies & $-6.629^{* * *}$ & 1.110 & $-8.656^{* * *}$ & 1.174 & $-4.585^{* * *}$ & 1.248 \\
\hline Number of marriages & -0.0217 & 0.675 & 0.112 & 0.712 & -0.138 & 0.759 \\
\hline Teacher's age & $-2.658^{* * *}$ & 0.623 & $-2.961^{* * *}$ & 0.657 & $-2.340^{* * *}$ & 0.701 \\
\hline Teacher's age square & $0.037^{* * *}$ & 0.009 & $0.039^{* * *}$ & 0.009 & $0.035^{* * *}$ & 0.010 \\
\hline Pupil's age & $6.989^{* * *}$ & 1.952 & $7.536^{* * *}$ & 2.059 & $6.323^{* * *}$ & 2.196 \\
\hline Pupil's age square & $-0.277^{* * *}$ & 0.092 & $-0.305^{* * *}$ & 0.097 & $-0.244^{* *}$ & 0.103 \\
\hline Number of hours & -0.147 & 0.629 & -0.124 & 0.663 & -0.128 & 0.708 \\
\hline Food expenditure $^{b}$ & 1.007 & 0.819 & 0.208 & 0.851 & $1.750^{*}$ & 0.929 \\
\hline Education expenditure $^{b}$ & 0.114 & 0.303 & 0.161 & 0.315 & 0.061 & 0.345 \\
\hline Health expenditure $^{b}$ & 0.189 & 0.201 & 0.216 & 0.210 & 0.168 & 0.229 \\
\hline Household size ${ }^{c}$ & $0.261^{* * *}$ & 0.063 & $0.238^{* * *}$ & 0.067 & $0.283^{* * *}$ & 0.071 \\
\hline Livestock & 0.0008 & 0.020 & -0.017 & 0.021 & 0.021 & 0.023 \\
\hline Distance to school & 1.095 & 0.905 & 1.265 & 0.954 & 0.842 & 1.018 \\
\hline Playground & $1.777^{*}$ & 0.951 & 1.516 & 1.003 & $2.324^{* *}$ & 1.070 \\
\hline Water point & -1.448 & 0.970 & $-2.425^{* *}$ & 1.012 & -0.609 & 1.100 \\
\hline Disturbed courses & $-4.016^{* * *}$ & 0.989 & $-2.539^{* *}$ & 1.044 & $-5.648^{* * *}$ & 1.113 \\
\hline Meals near school & -0.558 & 0.905 & $-1.598^{*}$ & 0.954 & 0.459 & 1.019 \\
\hline Gender of teacher & $-3.707^{* * *}$ & 1.127 & $-2.565^{* *}$ & 1.190 & $-4.866^{* * *}$ & 1.267 \\
\hline No professional qualif. ${ }^{d}$ & 0.358 & 1.031 & -1.166 & 1.088 & $1.959^{*}$ & 1.161 \\
\hline Professional qualif. CAP & -1.263 & 1.238 & -1.436 & 1.306 & -0.998 & 1.393 \\
\hline Professional qualif. CEAP & $4.581^{* * *}$ & 1.168 & $3.153^{* * *}$ & 1.231 & $5.611^{* * *}$ & 1.314 \\
\hline High school diploma \& more & $1.791^{* *}$ & 0.883 & 1.029 & 0.931 & $2.330^{* *}$ & 0.993 \\
\hline Continuing training & -0.369 & 0.795 & -0.222 & 0.838 & -0.436 & 0.894 \\
\hline Absenteeism & $-5.165^{* * *}$ & 1.270 & $-2.892^{* *}$ & 1.340 & $-7.558^{* * *}$ & 1.429 \\
\hline Gender of pupil & 0.914 & 0.728 & 0.232 & 0.752 & $1.701^{* *}$ & 0.830 \\
\hline Grade & $-14.239^{* * *}$ & 0.983 & $-21.143^{* * *}$ & 1.038 & $-7.373^{* * *}$ & 1.107 \\
\hline Koranic school & $3.671^{* * *}$ & 0.800 & $4.223^{* * *}$ & 0.844 & $3.335^{* * *}$ & 0.900 \\
\hline Early childhood inst. & 2.106 & 1.314 & $2.852^{* *}$ & 1.388 & 1.361 & 1.477 \\
\hline Snack & -0.272 & 1.148 & 0.076 & 1.214 & -0.606 & 1.291 \\
\hline Sick last 3 months & -2.906 & 2.632 & -2.230 & 2.793 & -3.972 & 2.951 \\
\hline Pupils eat fill & 0.420 & 1.195 & 0.807 & 1.260 & -0.034 & 1.345 \\
\hline Gender of household head & -0.669 & 1.264 & -1.332 & 1.334 & -0.177 & 1.422 \\
\hline Lit. of household head & -1.187 & 1.251 & -0.218 & 1.307 & -2.178 & 1.415 \\
\hline Marital status & 2.706 & 1.794 & 2.320 & 1.893 & 3.059 & 2.019 \\
\hline Land & -2.347 & 1.587 & -2.362 & 1.676 & -2.365 & 1.784 \\
\hline Parents school & $-3.002^{* * *}$ & 0.797 & $-2.942^{* * *}$ & 0.841 & $-3.214^{* * *}$ & 0.897 \\
\hline College & $1.808^{*}$ & 1.017 & 1.026 & 1.073 & $2.758^{* *}$ & 1.144 \\
\hline Diourbel & 1.429 & 1.675 & 1.223 & 1.765 & 1.792 & 1.885 \\
\hline Kolda & $-9.887^{* * *}$ & 1.232 & $-9.844^{* * *}$ & 1.299 & $-9.900^{* * *}$ & 1.386 \\
\hline Sédhiou & $-8.540^{* * *}$ & 2.240 & $-6.525^{* * *}$ & 2.368 & $-10.402^{* * *}$ & 2.518 \\
\hline Deworming at home & 14.368 & 13.612 & 11.905 & 14.484 & 18.158 & 15.239 \\
\hline Intercept & $40.490^{* *}$ & 17.595 & $54.649^{* * *}$ & 18.491 & 27.556 & 19.844 \\
\hline Selection Eq. & & & & & & \\
\hline Food expenditure & -0.069 & 0.056 & -0.069 & 0.056 & -0.069 & 0.056 \\
\hline Education expenditure & 0.026 & 0.022 & 0.026 & 0.022 & 0.026 & 0.022 \\
\hline
\end{tabular}


Table A.18 - continued

\begin{tabular}{|c|c|c|c|c|c|c|}
\hline \multirow[b]{2}{*}{ Variable } & \multicolumn{2}{|c|}{ Aggregate score } & \multicolumn{2}{|c|}{ French score } & \multicolumn{2}{|c|}{ Math score } \\
\hline & Coef. & Std. Err. & Coef. & Std. Err. & Coef. & Std. Err. \\
\hline Health expenditure & $-0.027^{* *}$ & 0.013 & $-0.027^{* *}$ & 0.013 & $-0.027^{* *}$ & 0.013 \\
\hline Sick last 3 months & $0.623^{* * *}$ & 0.061 & $0.623^{* * *}$ & 0.061 & $0.623^{* * *}$ & 0.061 \\
\hline Water point & 0.092 & 0.060 & 0.092 & 0.060 & 0.092 & 0.060 \\
\hline Gender of pupil & -0.023 & 0.059 & -0.023 & 0.059 & -0.023 & 0.059 \\
\hline Lit. of household head & $0.200^{* * *}$ & 0.075 & $0.200^{* * *}$ & 0.075 & $0.200^{* * *}$ & 0.075 \\
\hline Intercept & -0.337 & 0.647 & -0.337 & 0.647 & -0.337 & 0.647 \\
\hline hasard $\lambda$ & -8.603 & 7.738 & -6.997 & 8.235 & -10.784 & 8.662 \\
\hline$\rho$ & -0.481 & - & -0.379 & - & -0.529 & 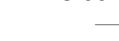 \\
\hline$\sigma$ & 17.853 & - & 18.417 & - & 20.349 & - \\
\hline$\lambda$ & -8.603 & 7.738 & -6.997 & 8.235 & -10.784 & 8.662 \\
\hline \# Observations & 24 & & 24 & & & \\
\hline Wald $\chi_{2}(53)$ & 841 & & 111 & & & \\
\hline$P>\chi_{2}$ & 0.0 & & 0.0 & & & \\
\hline
\end{tabular}

Notes $^{a}$ : Heckman selection framework; ${ }^{b}$ : In $\log ;{ }^{c}$ : Household size per adult equivalent; ${ }^{d}$ : qualification. Significance levels: $\quad *: 10 \% \quad * *: 5 \% \quad * * *: 1 \%$ 
APPENDIX B

Appendix Chapter 3

\section{Appendix B}

\section{Appendix B.1 Computation of outcome variables related to internal efficiency}

For the internal efficiency of schools, we used the enrollment, promotion and dropout rates that we calculated for year 2009 .

For enrollment rate, we took the difference between the new enrolled in the first year of primary school (CI) of 2009 'newenrollment 09 ' and those of 2008 'newenrollment $t_{08}$ ' that we divided by the new enrolled in 2008 'newenrollment $t_{08}$ ' and multiplied by 100 . We used the following relationship:

$$
\text { Enrollment }_{\text {rate }}=\frac{\text { newenrollment } 09-\text { newenrollment } 08}{\text { newenrollment } 08} * 100 \text {. }
$$

To calculate the promotion rate, we made the connection between the total promoted in 2009 'Promoted ${ }_{09}$ ' and the total number of pupils in the school for the same year and multiplied by 100. To compute the total promoted 'Promoted 09 ' we used the following equation:

$$
\text { Promoted }_{09}=\text { Pupils }_{10}-\text { newenrollment } 10-\text { totalrepeater }_{10}+\text { examadmitted }_{09}
$$

where 'Pupils $s_{10}$ ' is the total number of pupils within each school in 2010 , 'newenrollment 10 ' refers to pupils who have enrolled for the introductory course -first year of primary school- (CI) in 2010, 'totalrepeater $s_{10}$ ' is the total number of repeaters recorded in 2010 , and 'examadmitted 09 ' is the number of pupils admitted to the secondary school entrance examination in 2009. It is important to note that we do not have any information on transfers between schools, that is why they do not appear in the calculation of the total promoted. So, the promotion rate for 2009 is obtained from the following relation:

$$
\text { Promotion }_{\text {rate }}=\frac{\text { Promoted }_{09}}{\text { Pupils }_{09}} * 100
$$

To compute the dropout rate, we used the following relation:

$$
\text { Dropout }_{\text {rate }}=100-\left(\text { Repetiotion }_{\text {rate }_{09}}+\text { Promotion }_{\text {rate }_{09}}\right) ;
$$

To compute the repetition rate we made the ratio between the sum of repeaters of 2009 reported in 2010 and the total number of pupils '(Pupils)' in the school for 2009. 
Repetition $_{\text {rate }_{09}}=\frac{\sum \text { repeaters }(C I, C P, C E 1, C E 2, C M 1, C M 2)_{10}}{\sum \text { Pupils }(C I, C P, C E 1, C E 2, C M 1, C M 2)_{09}} * 100$

\section{Appendix B.2 General framework for mean of trivariate truncated normal distributions}

Let $x, y$ and $z$ denote three random variables following a trivariate normal distribution with mean $\boldsymbol{\mu}$ and covariance matrix $\boldsymbol{\Sigma}$, i.e.,

$$
(x, y, z) \sim N\left[\left(\begin{array}{l}
\mu_{x} \\
\mu_{y} \\
\mu_{z}
\end{array}\right),\left(\begin{array}{ccc}
\sigma_{x}^{2} & & \\
\rho_{x y} \sigma_{x} \sigma_{y} & \sigma_{y}^{2} & \\
\rho_{x z} \sigma_{x} \sigma_{z} & \rho_{y z} \sigma_{y} \sigma_{z} & \sigma_{z}^{2}
\end{array}\right)\right] .
$$

In this appendix we establish various neat relations regarding the mean of trivariate truncated normal distribution. These relations are used in the estimation strategies of this chapter 3 and also proved useful in deriving treatment effects. The formulas we get also have the advantage of being easy to write in terms of code for most statistical and econometric software. Before proceeding, let's briefly recall the well-known formulae for the mean of univariate and bivariate truncation.

\section{Univariate truncation}

The conditional expectation $\mathbb{E}(x \mid x>a)$ involving the univariate truncated normal distribution is given by

$$
\mathbb{E}(x \mid x>a)=\mu_{x}+\sigma_{x} \lambda_{x_{a}}\left[\left(\frac{a-\mu_{x}}{\sigma_{x}}\right)\right]
$$

where $\lambda_{x_{a}}$ denotes the inverse Mill's ratio and equals $\frac{\phi_{1}\left(\frac{a-\mu_{x}}{\sigma_{x}}\right)}{1-\Phi_{1}\left(\frac{a-\mu_{x}}{\sigma_{x}}\right)}$ with $\phi_{1}$ and $\Phi_{1}$ denoting respectively the probability distribution function (pdf) and the cumulative distribution function (cdf) of the univariate standard normal distribution. Similarly, the conditional expectation involving the univariate truncated (from above) normal distribution is given by

$$
\mathbb{E}(x \mid x \leq b)=\mu_{x}-\sigma_{x} \lambda_{x_{b}}\left[\left(\frac{b-\mu_{x}}{\sigma_{x}}\right)\right]
$$

where $\lambda_{x_{b}}$ equals $\frac{\phi_{1}\left(\frac{b-\mu_{x}}{\sigma_{x}}\right)}{1-\Phi_{1}\left(\frac{b-\mu_{x}}{\sigma_{x}}\right)}$.

\section{Bivariate truncation}

The conditional expectation $E(x \mid y>a)$ involving the bivariate truncated (from below) normal distribution is written as

$$
\begin{aligned}
\mathbb{E}(x \mid y>a) & =\mu_{x}+\frac{\operatorname{cov}(x, y)}{\sigma_{y}} \lambda_{y_{a}} \\
& =\mu_{x}+\frac{\rho_{x y} \sigma_{x} \sigma_{y}}{\sigma_{y}} \lambda_{y_{a}} \\
& =\mu_{x}+\rho_{x y} \sigma_{x} \lambda_{y_{a}},
\end{aligned}
$$


where $\operatorname{cov}(x, y)=\rho_{x y} \sigma_{x} \sigma_{y}$ and $\lambda_{y_{a}}=\frac{\phi_{1}\left(\frac{a-\mu_{y}}{\sigma_{y}}\right)}{1-\Phi_{1}\left(\frac{a-\mu_{y}}{\sigma_{y}}\right)}$.

Similarly, we can show that

$$
\mathbb{E}(x \mid y \leq b)=\mu_{x}-\frac{\operatorname{cov}(x, y)}{\sigma_{y}} \lambda_{y_{b}}
$$

where $\lambda_{y_{b}}=\frac{\phi_{1}\left(\frac{b-\mu_{y}}{\sigma_{y}}\right)}{1-\Phi_{1}\left(\frac{b-\mu_{y}}{\sigma_{y}}\right)}$.

In what follow, we establish the analogue formulae for the case of trivariate truncation

\section{Trivariate truncation}

Now, we are interested in the expressions of $\mathbb{E}(x \mid y>a, z>b), \mathbb{E}(x \mid y \leq a, z \leq b), \mathbb{E}(x \mid y>a, z \leq$ b) and $\mathbb{E}(x \mid y \leq a, z>b)$

These conditional expectations involve the trivariate truncated normal distribution. More specifically, we make use of the conditional distribution of $x \mid y, z$. The mean of that distribution is given by

$$
\mathbb{E}(x \mid y, z)=\mu_{x}+\frac{\sigma_{x} \rho_{x y \cdot z}}{\sigma_{y}}\left(y-\mu_{y}\right)+\frac{\sigma_{x} \rho_{x z \cdot y}}{\sigma_{z}}\left(z-\mu_{z}\right),
$$

where $\rho_{x y . z}$ and $\rho_{x z . y}$ are derived from and related to the partial correlations, and are given by

$$
\rho_{x y . z}=\frac{\rho_{x y}-\rho_{x z} \rho_{y z}}{1-\rho_{y z}^{2}} ; \rho_{x z . y}=\frac{\rho_{x z}-\rho_{x y} \rho_{y z}}{1-\rho_{y z}^{2}} .
$$

Expression of $\mathbb{E}(x \mid y>a, z>b)$

We can derive $\mathbb{E}(x \mid y>a, z>b)$ by using equation (B). Hence,

$$
\begin{aligned}
\mathbb{E}(x \mid y>a, z>b) & =\mu_{x}+\frac{\sigma_{x} \rho_{x y \cdot z}}{\sigma_{y}} \mathbb{E}\left(y-\mu_{y} \mid y>a, z>b\right)+\frac{\sigma_{x} \rho_{x z \cdot y}}{\sigma_{z}} \mathbb{E}\left(z-\mu_{z} \mid y>a, z>b\right) \\
& =\mu_{x}+\frac{\sigma_{x} \rho_{x y \cdot z}}{\sigma_{y}}\left[\mathbb{E}(y \mid y>a, z>b)-\mu_{y}\right]+\frac{\sigma_{x} \rho_{x z \cdot y}}{\sigma_{z}}\left[\mathbb{E}(z \mid y>a, z>b)-\mu_{z}\right] .
\end{aligned}
$$

Thus, we need to derive the expressions of $\mathbb{E}(y \mid y>a, z>b)$ and $\mathbb{E}(z \mid y>a, z>b)$.

i) Computation of $\mathbb{E}(y \mid y>a, z>b)$

Since

$$
f_{1}(y \mid y>a, z>b)=\underbrace{\frac{f_{2}(y, z)}{P[y>a, z>b]}}_{\equiv \Gamma}, 1
$$

the conditional expectation can be written as

$$
\begin{aligned}
\mathbb{E}(y \mid y>a, z>b) & =\mathbb{E}\left(y z^{0} \mid y>a, z>b\right) \\
& =\Gamma^{-1} \int_{a}^{\infty} \int_{b}^{\infty} y f(y, z) d y d z .
\end{aligned}
$$

\footnotetext{
${ }^{1}$ The subscripts 1 and 2 denote respectively the univariate and the bivariate pdf of cdf of the normal or standard normal distribution.
} 
By making the variable changes $Y=\frac{y-\mu_{y}}{\sigma_{y}}$ and $Z=\frac{z-\mu_{z}}{\sigma_{z}}$ so that $d y=\sigma_{y} d Y$ and $d z=$ $\sigma_{z} d Z$, the expectation can be written as

$$
\begin{aligned}
\mathbb{E}(y \mid y>a, z>b) & =\underbrace{\Phi_{2}^{-1}\left[\frac{\mu_{y}-a}{\sigma_{y}}, \frac{\mu_{z}-b}{\sigma_{z}}, \rho_{y z}\right]}_{\equiv \Phi_{2}^{-1}} \int_{\frac{a-\mu_{y}}{\sigma_{y}}}^{\infty} \int_{\frac{b-\mu_{z}}{\sigma_{z}}}^{\infty}\left(\sigma_{y} Y+\mu_{y}\right) \frac{\phi_{2}(Y, Z)}{\sigma_{y} \sigma_{z}} \sigma_{y} \sigma_{z} d Y d Z \\
& =\sigma_{y} \Phi_{2}^{-1} \int_{\frac{a-\mu_{y}}{\sigma_{y}}}^{\infty} \int_{\frac{b-\mu_{z}}{\sigma_{z}}}^{\infty} Y \phi_{2}(Y, Z) d Y d Z+\mu_{y} \Phi_{2}^{-1} \underbrace{\int_{\frac{a-\mu_{y}}{\sigma_{y}} \int_{\frac{b-\mu_{z}}{\sigma_{z}}}^{\infty} \phi_{2}(Y, Z) d Y d Z}^{\infty}}_{\equiv \Phi_{2}} \\
& =\sigma_{y} \underbrace{\Phi_{2}^{-1} \int_{\frac{a-\mu_{y}}{\sigma_{y}} \int_{\frac{b-\mu_{z}}{\sigma_{z}}}^{\infty} Y \phi_{2}(Y, Z) d Y d Z}^{\infty}}_{\mathbb{E}(Y-10)}+\mu_{y} . \underbrace{\infty}_{\left.\frac{a-\mu_{y}}{\sigma_{y}}, Z>\frac{b-\mu_{z}}{\sigma_{z}}\right)}
\end{aligned}
$$

Since $Y$ and $Z$ have a bivariate standard normal distribution, we can use the result of Rosenbaum (1961) or, more generally, moment generating function of Muthén (1990) to show that

$$
\begin{aligned}
\mathbb{E}\left(Y \mid Y>\frac{a-\mu_{y}}{\sigma_{y}}, Z>\frac{b-\mu_{z}}{\sigma_{z}}\right)= & \Phi_{2}^{-1}\left[\phi_{1}\left(\frac{a-\mu_{y}}{\sigma_{y}}\right) \Phi_{1}\left(\frac{\rho_{y z} \frac{a-\mu_{y}}{\sigma_{y}}-\frac{b-\mu_{z}}{\sigma_{z}}}{\sqrt{1-\rho_{y z}^{2}}}\right)\right. \\
& \left.+\rho_{y z} \phi_{1}\left(\frac{b-\mu_{z}}{\sigma_{z}}\right) \Phi_{1}\left(\frac{\rho_{y z} \frac{b-\mu_{z}}{\sigma_{z}}-\frac{a-\mu_{y}}{\sigma_{y}}}{\sqrt{1-\rho_{y z}^{2}}}\right)\right] .
\end{aligned}
$$

Hence,

$$
\begin{aligned}
\mathbb{E}(y \mid y>a, z>b)= & \sigma_{y} \Phi_{2}^{-1}\left[\phi_{1}\left(\frac{a-\mu_{y}}{\sigma_{y}}\right) \Phi_{1}\left(\frac{\rho_{y z} \frac{a-\mu_{y}}{\sigma_{y}}-\frac{b-\mu_{z}}{\sigma_{z}}}{\sqrt{1-\rho_{y z}^{2}}}\right)\right. \\
& \left.+\rho_{y z} \phi_{1}\left(\frac{b-\mu_{z}}{\sigma_{z}}\right) \Phi_{1}\left(\frac{\rho_{y z} \frac{b-\mu_{z}}{\sigma_{z}}-\frac{a-\mu_{y}}{\sigma_{y}}}{\sqrt{1-\rho_{y z}^{2}}}\right)\right]+\mu_{y} .
\end{aligned}
$$

ii) Computation of $\mathbb{E}(z \mid y>a, z>b)$

Similarly, we can derive $\mathbb{E}(z \mid y>a, z>b)$ as

$$
\begin{aligned}
\mathbb{E}(z \mid y>a, z>b)= & \sigma_{z} \Phi_{2}^{-1}\left[\phi_{1}\left(\frac{b-\mu_{z}}{\sigma_{z}}\right) \Phi_{1}\left(\frac{\rho_{y z} \frac{b-\mu_{z}}{\sigma_{z}}-\frac{a-\mu_{y}}{\sigma_{y}}}{\sqrt{1-\rho_{y z}^{2}}}\right)\right. \\
& \left.+\rho_{y z} \phi_{1}\left(\frac{a-\mu_{y}}{\sigma_{y}}\right) \Phi_{1}\left(\frac{\rho_{y z} \frac{a-\mu_{y}}{\sigma_{y}}-\frac{b-\mu_{z}}{\sigma_{z}}}{\sqrt{1-\rho_{y z}^{2}}}\right)\right]+\mu_{z} .
\end{aligned}
$$

For notational convenience, let us define $\Lambda_{1}$ and $\Lambda_{2}$ as

$$
\begin{aligned}
& \Lambda_{1} \equiv \phi_{1}\left(\frac{a-\mu_{y}}{\sigma_{y}}\right) \Phi_{1}\left(\frac{\rho_{y z} \frac{a-\mu_{y}}{\sigma_{y}}-\frac{b-\mu_{z}}{\sigma_{z}}}{\sqrt{1-\rho_{y z}^{2}}}\right) \\
& \Lambda_{2} \equiv \phi_{1}\left(\frac{b-\mu_{z}}{\sigma_{z}}\right) \Phi_{1}\left(\frac{\rho_{y z} \frac{b-\mu_{z}}{\sigma_{z}}-\frac{a-\mu_{y}}{\sigma_{y}}}{\sqrt{1-\rho_{y z}^{2}}}\right) .
\end{aligned}
$$


Hence, $\mathbb{E}(x \mid y>a, z>b)$ can be written as

$$
\begin{aligned}
\mathbb{E}(x \mid y>a, z>b)= & \mu_{x}+\frac{\sigma_{x} \rho_{x y . z}}{\sigma_{y}}\left[\sigma_{y} \Phi_{2}^{-1}\left(\Lambda_{1}+\rho_{y z} \Lambda_{2}\right)+\mu_{y}-\mu_{y}\right] \\
& +\frac{\sigma_{x} \rho_{x z . y}}{\sigma_{z}}\left[\sigma_{z} \Phi_{2}^{-1}\left(\Lambda_{2}+\rho_{y z} \Lambda_{1}\right)+\mu_{z}-\mu_{z}\right] .
\end{aligned}
$$

Replacing $\rho_{x y . z}$ and $\rho_{x z . y}$ by their expressions, and simplifying the previous expression yield

$$
\begin{aligned}
\mathbb{E}(x \mid y>a, z>b)= & \mu_{x}+\frac{\sigma_{x}\left(\rho_{x y}-\rho_{x z} \rho_{y z}\right)}{1-\rho_{y z}^{2}} \Phi_{2}^{-1}\left(\Lambda_{1}+\rho_{y z} \Lambda_{2}\right) \\
& +\frac{\sigma_{x}\left(\rho_{x z}-\rho_{x y} \rho_{y z}\right)}{1-\rho_{y z}^{2}} \Phi_{2}^{-1}\left(\Lambda_{2}+\rho_{y z} \Lambda_{1}\right) .
\end{aligned}
$$

Factorizing yields

$$
\begin{aligned}
\mathbb{E}(x \mid y>a, z>b)= & \mu_{x}+\frac{\sigma_{x} \Phi_{2}^{-1}}{1-\rho_{y z}^{2}} \Lambda_{1}\left[\rho_{x y}-\rho_{x z} \rho_{y z}+\rho_{x z} \rho_{y z}-\rho_{x y} \rho_{y z}^{2}\right] \\
& +\frac{\sigma_{x} \Phi_{2}^{-1}}{1-\rho_{y z}^{2}} \Lambda_{2}\left[\rho_{x z}-\rho_{x y} \rho_{y z}+\rho_{x y} \rho_{y z}-\rho_{x z} \rho_{y z}^{2}\right]
\end{aligned}
$$

which can also be written as

$$
\begin{aligned}
\mathbb{E}(x \mid y>a, z>b) & =\mu_{x}+\frac{\sigma_{x} \Phi_{2}^{-1}}{1-\rho_{y z}^{2}} \Lambda_{1} \rho_{x y}\left(1-\rho_{y z}^{2}\right)+\frac{\sigma_{x} \Phi_{2}^{-1}}{1-\rho_{y z}^{2}} \Lambda_{2} \rho_{x z}\left(1-\rho_{y z}^{2}\right) \\
& =\mu_{x}+\sigma_{x} \Phi_{2}^{-1} \Lambda_{1} \rho_{x y}+\sigma_{x} \Phi_{2}^{-1} \Lambda_{2} \rho_{x z} .
\end{aligned}
$$

The final expression of $\mathbb{E}(x \mid y>a, z>b)$ is given by

$$
\begin{aligned}
\mathbb{E}(x \mid y>a, z>b)= & \mu_{x}+\frac{\sigma_{x} \rho_{x y} \phi_{1}\left(\frac{a-\mu_{y}}{\sigma_{y}}\right)}{\Phi_{2}\left[\frac{\mu_{y}-a}{\sigma_{y}}, \frac{\mu_{z}-b}{\sigma_{z}}, \rho_{y z}\right]} \Phi_{1}\left(\frac{\rho_{y z} \frac{a-\mu_{y}}{\sigma_{y}}-\frac{b-\mu_{z}}{\sigma_{z}}}{\sqrt{1-\rho_{y z}^{2}}}\right) \\
& +\frac{\sigma_{x} \rho_{x z} \phi_{1}\left(\frac{b-\mu_{z}}{\sigma_{z}}\right)}{\Phi_{2}\left[\frac{\mu_{y}-a}{\sigma_{y}}, \frac{\mu_{z}-b}{\sigma_{z}}, \rho_{y z}\right]} \Phi_{1}\left(\frac{\rho_{y z} \frac{b-\mu_{z}}{\sigma_{z}}-\frac{a-\mu_{y}}{\sigma_{y}}}{\sqrt{1-\rho_{y z}^{2}}}\right) .
\end{aligned}
$$

Expression of $\mathbb{E}(x \mid y \leq a, z \leq b)$

In this case, $\mathbb{E}(y \mid y>a, z>b)$ and $\mathbb{E}(z \mid y>a, z>b)$ are replaced by $\mathbb{E}(y \mid y \leq a, z \leq b)$ and $\mathbb{E}(z \mid y \leq a, z \leq b)$ in equation (B-7). Hence, we need to derive $\mathbb{E}(y \mid y \leq a, z \leq b)$ and $\mathbb{E}(z \mid y \leq a, z \leq b)$. Equation (B-8) is now written as

$$
f_{1}(y \mid y \leq a, z \leq b)=\frac{f_{2}(y, z)}{\mathbb{P}[y \leq a, z \leq b]} .
$$

Using similar derivations as previously and results from Muthén (1990), $\mathbb{E}(y \mid y \leq a, z \leq b)$ is given by

$$
\begin{aligned}
\mathbb{E}(y \mid y \leq a, z \leq b)= & \sigma_{y} \Phi_{2}^{-1}\left[\frac{a-\mu_{y}}{\sigma_{y}}, \frac{b-\mu_{z}}{\sigma_{z}}, \rho_{y z}\right]\left[-\phi_{1}\left(\frac{a-\mu_{y}}{\sigma_{y}}\right) \Phi_{1}\left(\frac{\frac{b-\mu_{z}}{\sigma_{z}}-\rho_{y z} \frac{a-\mu_{y}}{\sigma_{y}}}{\sqrt{1-\rho_{y z}^{2}}}\right)\right. \\
& \left.-\rho_{y z} \phi_{1}\left(\frac{b-\mu_{z}}{\sigma_{z}}\right) \Phi_{1}\left(\frac{\frac{a-\mu_{y}}{\sigma_{y}}-\rho_{y z} \frac{b-\mu_{z}}{\sigma_{z}}}{\sqrt{1-\rho_{y z}^{2}}}\right)\right]+\mu_{y} .
\end{aligned}
$$


Similarly, $\mathbb{E}(z \mid y \leq a, z \leq b)$ is derived as

$$
\begin{aligned}
\mathbb{E}(z \mid y \leq a, z \leq b)= & \sigma_{z} \Phi_{2}^{-1}\left[\frac{a-\mu_{y}}{\sigma_{y}}, \frac{b-\mu_{z}}{\sigma_{z}}, \rho_{y z}\right]\left[-\phi_{1}\left(\frac{b-\mu_{z}}{\sigma_{z}}\right) \Phi_{1}\left(\frac{\frac{a-\mu_{y}}{\sigma_{y}}-\rho_{y z} \frac{b-\mu_{z}}{\sigma_{z}}}{\sqrt{1-\rho_{y z}^{2}}}\right)\right. \\
& \left.-\rho_{y z} \phi_{1}\left(\frac{a-\mu_{y}}{\sigma_{y}}\right) \Phi_{1}\left(\frac{\frac{b-\mu_{z}}{\sigma_{z}}-\rho_{y z} \frac{a-\mu_{y}}{\sigma_{y}}}{\sqrt{1-\rho_{y z}^{2}}}\right)\right]+\mu_{z} .
\end{aligned}
$$

Using similar calculations as previously, $\mathbb{E}(x \mid y \leq a, z \leq b)$ is derived as

$$
\begin{aligned}
\mathbb{E}(x \mid y \leq a, z \leq b)= & \mu_{x}-\frac{\sigma_{x} \rho_{x y} \phi_{1}\left(\frac{a-\mu_{y}}{\sigma_{y}}\right)}{\Phi_{2}\left[\frac{a-\mu_{y}}{\sigma_{y}}, \frac{b-\mu_{z}}{\sigma_{z}}, \rho_{y z}\right]} \Phi_{1}\left(\frac{\frac{b-\mu_{z}}{\sigma_{z}}-\rho_{y z} \frac{a-\mu_{y}}{\sigma_{y}}}{\sqrt{1-\rho_{y z}^{2}}}\right) \\
& -\frac{\sigma_{x} \rho_{x z} \phi_{1}\left(\frac{b-\mu_{z}}{\sigma_{z}}\right)}{\Phi_{2}\left[\frac{a-\mu_{y}}{\sigma_{y}}, \frac{b-\mu_{z}}{\sigma_{z}}, \rho_{y z}\right]} \Phi_{1}\left(\frac{\frac{a-\mu_{y}}{\sigma_{y}}-\rho_{y z} \frac{b-\mu_{z}}{\sigma_{z}}}{\sqrt{1-\rho_{y z}^{2}}}\right) .
\end{aligned}
$$

Expression of $\mathbb{E}(x \mid y>a, z \leq b)$

The calculation of $\mathbb{E}(x \mid y>a, z \leq b)$ involves $\mathbb{E}(y \mid y>a, z \leq b)$ and $\mathbb{E}(z \mid y>a, z \leq b)$ in equation (B-7). The latter two expectations are given by

$$
\begin{aligned}
\mathbb{E}(y \mid y>a, z \leq b)= & \sigma_{y} \Phi_{2}^{-1}\left[\frac{\mu_{y}-a}{\sigma_{y}}, \frac{b-\mu_{z}}{\sigma_{z}},-\rho_{y z}\right]\left[\phi_{1}\left(\frac{a-\mu_{y}}{\sigma_{y}}\right) \Phi_{1}\left(\frac{\frac{b-\mu_{z}}{\sigma_{z}}-\rho_{y z} \frac{a-\mu_{y}}{\sigma_{y}}}{\sqrt{1-\rho_{y z}^{2}}}\right)\right. \\
& \left.-\rho_{y z} \phi_{1}\left(\frac{b-\mu_{z}}{\sigma_{z}}\right) \Phi_{1}\left(\frac{\rho_{y z} \frac{b-\mu_{z}}{\sigma_{z}}-\frac{a-\mu_{y}}{\sigma_{y}}}{\sqrt{1-\rho_{y z}^{2}}}\right)\right]+\mu_{y}
\end{aligned}
$$

and

$$
\begin{aligned}
\mathbb{E}(z \mid y>a, z \leq b)= & \sigma_{z} \Phi_{2}^{-1}\left[\frac{\mu_{y}-a}{\sigma_{y}}, \frac{b-\mu_{z}}{\sigma_{z}},-\rho_{y z}\right]\left[-\phi_{1}\left(\frac{b-\mu_{z}}{\sigma_{z}}\right) \Phi_{1}\left(\frac{\rho_{y z} \frac{b-\mu_{z}}{\sigma_{z}}-\frac{a-\mu_{y}}{\sigma_{y}}}{\sqrt{1-\rho_{y z}^{2}}}\right)\right. \\
& \left.+\rho_{y z} \phi_{1}\left(\frac{a-\mu_{y}}{\sigma_{y}}\right) \Phi_{1}\left(\frac{\frac{b-\mu_{z}}{\sigma_{z}}-\rho_{y z} \frac{a-\mu_{y}}{\sigma_{y}}}{\sqrt{1-\rho_{y z}^{2}}}\right)\right]+\mu_{z} .
\end{aligned}
$$

Hence, $\mathbb{E}(x \mid y>a, z \leq b)$ is given by

$$
\begin{aligned}
\mathbb{E}(x \mid y>a, z \leq b)= & \mu_{x}+\frac{\sigma_{x} \rho_{x y} \phi_{1}\left(\frac{a-\mu_{y}}{\sigma_{y}}\right)}{\Phi_{2}\left[\frac{\mu_{y}-a}{\sigma_{y}}, \frac{b-\mu_{z}}{\sigma_{z}},-\rho_{y z}\right]} \Phi_{1}\left(\frac{\frac{b-\mu_{z}}{\sigma_{z}}-\rho_{y z} \frac{a-\mu_{y}}{\sigma_{y}}}{\sqrt{1-\rho_{y z}^{2}}}\right) \\
& -\frac{\sigma_{x} \rho_{x z} \phi_{1}\left(\frac{b-\mu_{z}}{\sigma_{z}}\right)}{\Phi_{2}\left[\frac{\mu_{y}-a}{\sigma_{y}}, \frac{b-\mu_{z}}{\sigma_{z}},-\rho_{y z}\right]} \Phi_{1}\left(\frac{\rho_{y z} \frac{b-\mu_{z}}{\sigma_{z}}-\frac{a-\mu_{y}}{\sigma_{y}}}{\sqrt{1-\rho_{y z}^{2}}}\right) .
\end{aligned}
$$

Expression of $\mathbb{E}(x \mid y \leq a, z>b)$

Finally, the calculation of $\mathbb{E}(x \mid y \leq a, z>b)$ involves $\mathbb{E}(y \mid y \leq a, z>b)$ and $\mathbb{E}(z \mid y \leq a, z>b)$ 
in equation (B-7), the expressions of which are given by

$$
\begin{aligned}
\mathbb{E}(y \mid y \leq a, z>b)= & \sigma_{y} \Phi_{2}^{-1}\left[\frac{a-\mu_{y}}{\sigma_{y}}, \frac{\mu_{z}-b}{\sigma_{z}},-\rho_{y z}\right]\left[-\phi_{1}\left(\frac{a-\mu_{y}}{\sigma_{y}}\right) \Phi_{1}\left(\frac{\rho_{y z} \frac{a-\mu_{y}}{\sigma_{y}}-\frac{b-\mu_{z}}{\sigma_{z}}}{\sqrt{1-\rho_{y z}^{2}}}\right)\right. \\
& \left.+\rho_{y z} \phi_{1}\left(\frac{b-\mu_{z}}{\sigma_{z}}\right) \Phi_{1}\left(\frac{\frac{a-\mu_{y}}{\sigma_{y}}-\rho_{y z} \frac{b-\mu_{z}}{\sigma_{z}}}{\sqrt{1-\rho_{y z}^{2}}}\right)\right]+\mu_{y}
\end{aligned}
$$

and

$$
\begin{aligned}
\mathbb{E}(z \mid y \leq a, z>b)= & \sigma_{z} \Phi_{2}^{-1}\left[\frac{a-\mu_{y}}{\sigma_{y}}, \frac{\mu_{z}-b}{\sigma_{z}},-\rho_{y z}\right]\left[\phi_{1}\left(\frac{b-\mu_{z}}{\sigma_{z}}\right) \Phi_{1}\left(\frac{\frac{a-\mu_{y}}{\sigma_{y}}-\rho_{y z} \frac{b-\mu_{z}}{\sigma_{z}}}{\sqrt{1-\rho_{y z}^{2}}}\right)\right. \\
& \left.-\rho_{y z} \phi_{1}\left(\frac{a-\mu_{y}}{\sigma_{y}}\right) \Phi_{1}\left(\frac{\rho_{y z} \frac{a-\mu_{y}}{\sigma_{y}}-\frac{b-\mu_{z}}{\sigma_{z}}}{\sqrt{1-\rho_{y z}^{2}}}\right)\right]+\mu_{z} .
\end{aligned}
$$

Hence, $\mathbb{E}(x \mid y \leq a, z>b)$ is given by

$$
\begin{aligned}
\mathbb{E}(x \mid y \leq a, z>b)= & \mu_{x}-\frac{\sigma_{x} \rho_{x y} \phi_{1}\left(\frac{a-\mu_{y}}{\sigma_{y}}\right)}{\Phi_{2}\left[\frac{a-\mu_{y}}{\sigma_{y}}, \frac{\mu_{z}-b}{\sigma_{z}},-\rho_{y z}\right]} \Phi_{1}\left(\frac{\rho_{y z} \frac{a-\mu_{y}}{\sigma_{y}}-\frac{b-\mu_{z}}{\sigma_{z}}}{\sqrt{1-\rho_{y z}^{2}}}\right) \\
& +\frac{\sigma_{x} \rho_{x z} \phi_{1}\left(\frac{b-\mu_{z}}{\sigma_{z}}\right)}{\Phi_{2}\left[\frac{a-\mu_{y}}{\sigma_{y}}, \frac{\mu_{z}-b}{\sigma_{z}},-\rho_{y z}\right]} \Phi_{1}\left(\frac{\frac{a-\mu_{y}}{\sigma_{y}}-\rho_{y z} \frac{b-\mu_{z}}{\sigma_{z}}}{\sqrt{1-\rho_{y z}^{2}}}\right) .
\end{aligned}
$$

\section{Appendix B.3 Descriptive statistics and further estimations results}

\section{Descriptive statistics}




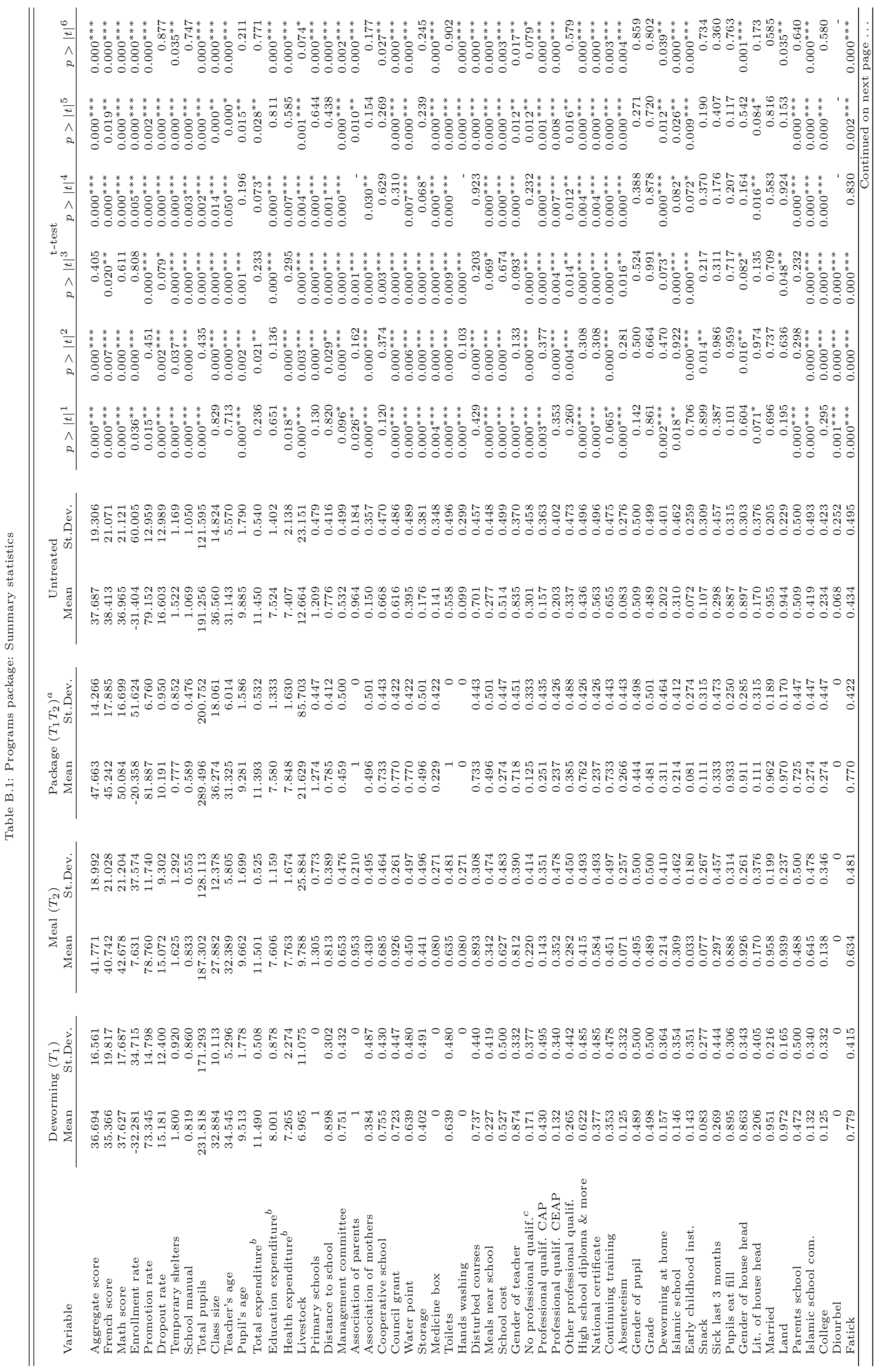




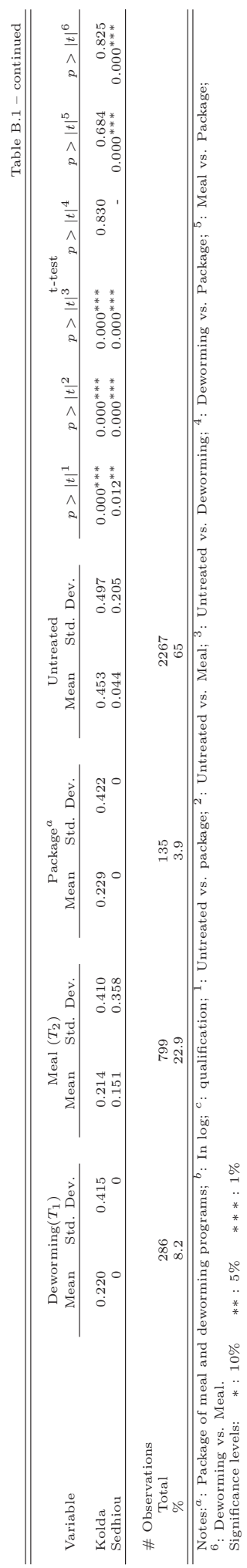




\section{Further estimation results}

Table B.2: Determinants of scores: DISM $^{a}$ model, FIML $^{b}$

\begin{tabular}{|c|c|c|c|c|c|c|}
\hline \multirow[b]{2}{*}{ Variable } & \multicolumn{2}{|c|}{ Aggregate score } & \multicolumn{2}{|c|}{ French score } & \multicolumn{2}{|c|}{ Math score } \\
\hline & Coef. & Std. Err. & Coef. & Std. Err. & Coef. & Std. Err. \\
\hline \multicolumn{7}{|l|}{ Selection Eq. Deworming } \\
\hline Total pupils & $0.0005^{*}$ & 0.0002 & $0.0004^{* *}$ & 0.0002 & $0.0006^{* * *}$ & 0.0002 \\
\hline Management committee & 0.014 & 0.059 & 0.010 & 0.060 & -0.026 & 0.060 \\
\hline Medicine box & $-0.414^{* * *}$ & 0.090 & $-0.360^{* * *}$ & 0.091 & $-0.400^{* * *}$ & 0.092 \\
\hline Association of mothers & $0.386^{* * *}$ & 0.059 & $0.363^{* * *}$ & 0.060 & $0.406^{* * *}$ & 0.060 \\
\hline Cooperative school & $0.331^{* * *}$ & 0.068 & $0.296^{* * *}$ & 0.069 & $0.317^{* * *}$ & 0.069 \\
\hline Water point & $0.441^{* * *}$ & 0.066 & $0.460^{* * *}$ & 0.067 & $0.439^{* * *}$ & 0.067 \\
\hline Gender of pupil & -0.043 & 0.055 & -0.047 & 0.056 & -0.048 & 0.056 \\
\hline Intercept & $-1.823^{* * *}$ & 0.079 & $-1.792^{* * *}$ & 0.080 & $-1.812^{* * *}$ & 0.079 \\
\hline \multicolumn{7}{|l|}{ Selection Eq. Meal } \\
\hline Total pupils & $0.0004^{*}$ & 0.0002 & $0.0004^{* *}$ & 0.0002 & $0.0004^{* *}$ & 0.0002 \\
\hline Distance to school & 0.022 & 0.061 & 0.028 & 0.062 & 0.021 & 0.061 \\
\hline Management committee & -0.041 & 0.055 & -0.047 & 0.055 & -0.044 & 0.054 \\
\hline Association of mothers & $0.631^{* * *}$ & 0.057 & $0.646^{* * *}$ & 0.058 & $0.631^{* * *}$ & 0.057 \\
\hline Cooperative school & 0.080 & 0.055 & 0.077 & 0.056 & 0.085 & 0.055 \\
\hline Rural council grant & $0.717^{* * *}$ & 0.068 & $0.691^{* * *}$ & 0.068 & $0.738^{* * *}$ & 0.068 \\
\hline Water point & -0.009 & 0.062 & 0.003 & 0.062 & -0.020 & 0.062 \\
\hline Disturbed courses & $0.911^{* * *}$ & 0.072 & $0.917^{* * *}$ & 0.072 & $0.909^{* * *}$ & 0.072 \\
\hline Storage & $0.831^{* * *}$ & 0.058 & $0.834^{* * *}$ & 0.058 & $0.817^{* * *}$ & 0.058 \\
\hline Gender of pupil & -0.060 & 0.050 & -0.060 & 0.050 & -0.061 & 0.049 \\
\hline Intercept & $-2.435^{* * *}$ & 0.119 & $-2.430^{* * *}$ & 0.119 & $-2.436^{* * *}$ & 0.119 \\
\hline \multicolumn{7}{|l|}{ Performance Eq. } \\
\hline Temporary shelters & 0.342 & 0.316 & 0.367 & 0.338 & 0.189 & 0.355 \\
\hline School manual & $3.150^{* * *}$ & 0.915 & $3.576^{* * *}$ & 0.979 & $2.944^{* * *}$ & 1.021 \\
\hline School manual squared & -0.096 & 0.159 & $-0.450^{* * *}$ & 0.170 & 0.240 & 0.177 \\
\hline Class size & -0.013 & 0.024 & -0.025 & 0.026 & -0.007 & 0.027 \\
\hline Teacher's age & $-3.102^{* * *}$ & 0.528 & $-1.974^{* * *}$ & 0.562 & $-3.976^{* * *}$ & 0.588 \\
\hline Teacher's age square & $0.048^{* * *}$ & 0.007 & $0.028^{* * *}$ & 0.008 & $0.064^{* * *}$ & 0.008 \\
\hline Pupil's age & $0.833^{* * *}$ & 0.226 & $0.831^{* * *}$ & 0.244 & $0.811^{* * *}$ & 0.253 \\
\hline Education expenditure ${ }^{c}$ & $0.507^{* *}$ & 0.244 & $0.729^{* * *}$ & 0.263 & 0.320 & 0.273 \\
\hline Health expenditure ${ }^{c}$ & -0.052 & 0.150 & 0.029 & 0.162 & -0.165 & 0.168 \\
\hline Livestock & -0.007 & 0.010 & -0.011 & 0.011 & -0.0004 & 0.011 \\
\hline Disturbed courses & $-4.963^{* * *}$ & 0.918 & $-4.177^{* * *}$ & 0.988 & $-6.080^{* * *}$ & 1.020 \\
\hline Meals near school & 0.124 & 0.727 & -0.554 & 0.783 & 0.727 & 0.814 \\
\hline Toilets & $2.787^{* *}$ & 0.762 & $1.626^{* *}$ & 0.820 & $3.791^{* * *}$ & 0.850 \\
\hline Hands washing & $3.078^{* *}$ & 1.219 & $4.058^{* * *}$ & 1.303 & $2.401^{*}$ & 1.358 \\
\hline Gender of teacher & $-4.555^{* * *}$ & 0.913 & $-4.118^{* * *}$ & 0.986 & $-5.147^{* * *}$ & 1.023 \\
\hline No professional qualif. ${ }^{d}$ & $3.274^{* * *}$ & 0.870 & $2.400^{* *}$ & 0.934 & $4.399^{* * *}$ & 0.971 \\
\hline Professional qualif. CAP & -0.167 & 1.060 & 0.782 & 1.150 & -0.819 & 1.186 \\
\hline Professional diploma CEAP & $3.081^{* * *}$ & 0.919 & 1.265 & 0.990 & $4.594^{* * *}$ & 1.027 \\
\hline High school diploma \& more & 0.661 & 0.721 & -0.653 & 0.773 & $1.638^{* *}$ & 0.803 \\
\hline Continuing training & -0.701 & 0.672 & 0.107 & 0.722 & $-1.643^{* *}$ & 0.750 \\
\hline Absenteeism & $-4.025^{* * *}$ & 1.135 & -1.667 & 1.226 & $-6.371^{* * *}$ & 1.269 \\
\hline Gender of pupil & 0.670 & 0.627 & -0.291 & 0.662 & $1.543^{* *}$ & 0.699 \\
\hline Grade & $-11.209^{* * *}$ & 0.815 & $-17.697^{* * *}$ & 0.876 & $-4.605^{* * *}$ & 0.912 \\
\hline Deworming at home & $-1.595^{* *}$ & 0.749 & $-1.650^{* *}$ & 0.807 & -1.324 & 0.838 \\
\hline Islamic school & $2.669^{* * *}$ & 0.676 & $2.561^{* * *}$ & 0.727 & $2.927^{* * *}$ & 0.756 \\
\hline Early childhood inst. & 1.920 & 1.197 & $2.712^{* *}$ & 1.295 & 1.207 & 1.344 \\
\hline Snack & -1.104 & 1.015 & -1.094 & 1.094 & -0.750 & 1.137 \\
\hline
\end{tabular}


Table B.2 - continued

\begin{tabular}{|c|c|c|c|c|c|c|}
\hline \multirow[b]{2}{*}{ Variable } & \multicolumn{2}{|c|}{ Aggregate score } & \multicolumn{2}{|c|}{ French score } & \multicolumn{2}{|c|}{ Math score } \\
\hline & Coef. & Std. Err. & Coef. & Std. Err. & Coef. & Std. Err. \\
\hline Sick last 3 months & 0.005 & 0.655 & 0.251 & 0.705 & -0.293 & 0.732 \\
\hline Pupils eat fill & $1.956^{* *}$ & 0.990 & $1.944^{*}$ & 1.063 & $1.922^{*}$ & 1.107 \\
\hline Gender of household head & 0.130 & 1.103 & -0.486 & 1.190 & 0.581 & 1.235 \\
\hline Lit. of household head & -0.116 & 0.799 & -0.080 & 0.861 & -0.353 & 0.895 \\
\hline Married & 1.654 & 1.568 & 2.753 & 1.691 & 0.588 & 1.754 \\
\hline Land & -1.949 & 1.358 & -1.299 & 1.465 & -2.417 & 1.519 \\
\hline Parents school & $-2.047^{* *}$ & 0.654 & $-2.802^{* * *}$ & 0.697 & $-1.221^{*}$ & 0.728 \\
\hline College & $6.235^{* * *}$ & 0.905 & $4.496^{* * *}$ & 0.971 & $8.050^{* * *}$ & 1.016 \\
\hline Diourbel & $4.484^{* * *}$ & 1.639 & 2.531 & 1.751 & $6.590^{* * *}$ & 1.828 \\
\hline Kolda & $-3.818^{* * *}$ & 0.952 & $-4.474^{* * *}$ & 1.025 & $-2.870^{* * *}$ & 1.063 \\
\hline Sedhiou & $-4.194^{*}$ & 1.530 & $-5.255^{* * *}$ & 1.633 & -2.783 & 1.713 \\
\hline Deworming $\left(T_{1}\right)$ & $-17.155^{* * *}$ & 2.516 & $-18.719^{* * *}$ & 3.008 & $-15.190^{* * *}$ & 2.816 \\
\hline Meal $\left(T_{2}\right)$ & $3.488^{*}$ & 1.948 & $4.147^{* *}$ & 2.076 & 2.966 & 2.048 \\
\hline$T_{1} T_{2}$ & $3.600^{*}$ & 2.097 & $5.868^{* *}$ & 2.268 & 2.216 & 2.342 \\
\hline Intercept & $82.369^{* * *}$ & 9.427 & $69.068^{* * *}$ & 10.037 & $92.011^{* * *}$ & 10.520 \\
\hline$\rho 12$ & $-0.071^{*}$ & 0.039 & $-0.066^{*}$ & 0.039 & $-0.067^{*}$ & 0.039 \\
\hline$\rho 13$ & $0.567^{* * *}$ & 0.058 & $0.494^{* * *}$ & 0.070 & $0.528^{* * *}$ & 0.060 \\
\hline$\rho 23$ & 0.098 & 0.068 & -0.010 & 0.068 & $0.181^{* * *}$ & 0.063 \\
\hline$s_{e} 3$ & $18.383^{* * *}$ & 0.335 & $19.414^{* * *}$ & 0.358 & $20.500^{* * *}$ & 0.360 \\
\hline Log likelihood & \multicolumn{2}{|c|}{-17710.647} & \multicolumn{2}{|c|}{-17970.804} & \multicolumn{2}{|c|}{-18098.279} \\
\hline \# Observations & \multicolumn{2}{|c|}{3487} & \multicolumn{2}{|c|}{3487} & \multicolumn{2}{|c|}{3487} \\
\hline Wald $\chi_{2}(7)$ & \multicolumn{2}{|c|}{210.25} & \multicolumn{2}{|c|}{191.39} & \multicolumn{2}{|c|}{209.36} \\
\hline$P>\chi_{2}$ & \multicolumn{2}{|c|}{0.000} & \multicolumn{2}{|c|}{0.000} & \multicolumn{2}{|c|}{0.000} \\
\hline
\end{tabular}

Notes $^{a}$ : Double-Index Selection Model; ${ }^{b}$ : Full Information Maximum Likelihood; ${ }^{c}$ : In log; ${ }^{d}$ : qualification. Significance levels (bootstrap): $\quad *: 10 \% \quad * *: 5 \% \quad * * *: 1 \%$

Table B.3: Determinants of scores: DISM ${ }^{a}$ model, 2-step ${ }^{b}$

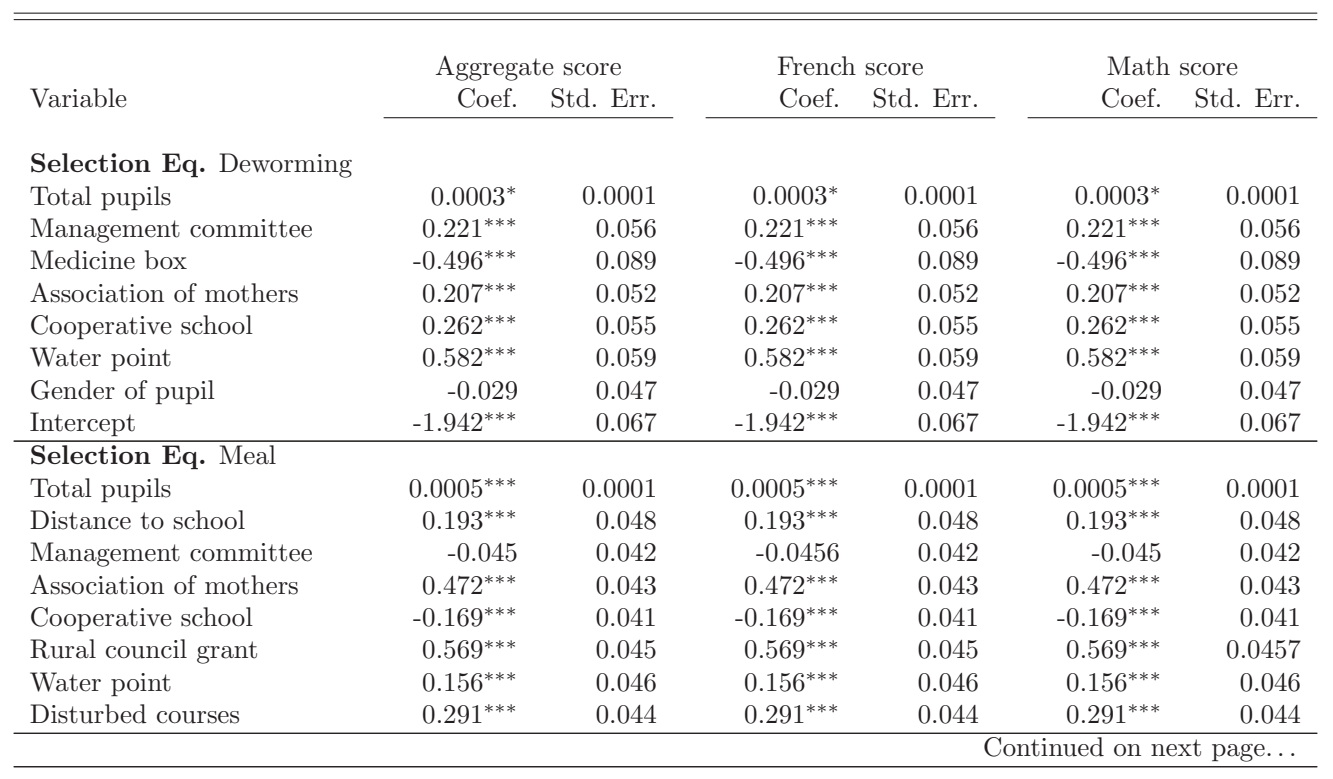


Table B.3 - continued

\begin{tabular}{|c|c|c|c|c|c|c|}
\hline \multirow[b]{2}{*}{ Variable } & \multicolumn{2}{|c|}{ Aggregate score } & \multicolumn{2}{|c|}{ French score } & \multicolumn{2}{|c|}{ Math score } \\
\hline & Coef. & Std. Err. & Coef. & Std. Err. & Coef. & Std. Err. \\
\hline Storage & $0.390^{* * *}$ & 0.044 & $0.390^{* * *}$ & 0.044 & $0.390^{* * *}$ & 0.044 \\
\hline Gender of pupil & -0.041 & 0.037 & -0.041 & 0.037 & -0.041 & 0.037 \\
\hline Intercept & $-1.482^{* * *}$ & 0.075 & $-1.482^{* * *}$ & 0.075 & $-1.482^{* * *}$ & 0.075 \\
\hline \multicolumn{7}{|l|}{ Performance Eq. } \\
\hline Temporary shelters & 0.374 & 0.306 & 0.369 & 0.343 & 0.297 & 0.358 \\
\hline School manual & $3.273^{* * *}$ & 0.966 & $3.611^{* * *}$ & 1.011 & $2.939^{* *}$ & 1.188 \\
\hline School manual squared & -0.096 & 0.156 & $-0.450^{* * *}$ & 0.148 & 0.257 & 0.223 \\
\hline Class size & -0.015 & 0.024 & -0.027 & 0.024 & -0.025 & 0.031 \\
\hline Teacher's age & $-2.979^{* * *}$ & 0.493 & $-1.912^{* * *}$ & 0.534 & $-3.935^{* * *}$ & 0.548 \\
\hline Teacher's age square & $0.046^{* * *}$ & 0.007 & $0.027^{* * *}$ & 0.008 & $0.063^{* * *}$ & 0.008 \\
\hline Pupil's age & $0.867^{* * *}$ & 0.240 & $0.836^{* * *}$ & 0.260 & $0.870^{* * *}$ & 0.269 \\
\hline Education expenditure $^{c}$ & $0.544^{* *}$ & 0.240 & $0.762^{* *}$ & 0.298 & 0.348 & 0.257 \\
\hline Health expenditure $^{c}$ & -0.082 & 0.158 & 0.008 & 0.158 & -0.182 & 0.186 \\
\hline Livestock & -0.001 & 0.015 & -0.007 & 0.015 & 0.004 & 0.017 \\
\hline Disturbed courses & $-5.787^{* * *}$ & 0.664 & $-4.392^{* * *}$ & 0.732 & $-7.295^{* * *}$ & 0.792 \\
\hline Meals near school & -0.049 & 0.791 & -0.653 & 0.850 & 0.564 & 0.853 \\
\hline Toilets & $2.984^{* * *}$ & 0.725 & $1.733^{* *}$ & 0.773 & $4.147^{* * *}$ & 0.831 \\
\hline Hands washing & $3.459^{* * *}$ & 1.172 & $4.337^{* * *}$ & 1.314 & $2.663^{* *}$ & 1.244 \\
\hline Gender of teacher & $-4.751^{* * *}$ & 0.936 & $-4.263^{* * *}$ & 1.028 & $-5.389^{* * *}$ & 1.021 \\
\hline No professional qualif. ${ }^{d}$ & $3.652^{* * *}$ & 0.837 & $2.565^{* * *}$ & 0.935 & $4.839^{* * *}$ & 0.911 \\
\hline Professional qualif. CAP & -0.148 & 1.207 & 0.672 & 1.301 & -0.664 & 1.254 \\
\hline Professional qualif. CEAP & $3.134^{* * *}$ & 1.023 & 1.269 & 1.145 & $4.762^{* * *}$ & 1.093 \\
\hline High school diploma \& more & 0.616 & 0.693 & -0.618 & 0.743 & $1.673^{* *}$ & 0.850 \\
\hline Continuing training & -0.829 & 0.722 & -0.015 & 0.754 & $-1.683^{* *}$ & 0.836 \\
\hline Absenteeism & $-4.13^{* * *}$ & 1.114 & -1.989 & 1.239 & $-6.209^{* * *}$ & 1.132 \\
\hline Gender of pupil & 0.809 & 0.578 & -0.154 & 0.592 & $1.672^{* *}$ & 0.688 \\
\hline Grade & $-11.063^{* * *}$ & 0.806 & $-17.586^{* * *}$ & 0.913 & $-4.501^{* * *}$ & 0.874 \\
\hline Deworming at home & $-1.564^{*}$ & 0.809 & $-1.626^{*}$ & 0.839 & -1.348 & 0.918 \\
\hline Islamic school & $2.799^{* * *}$ & 0.707 & $2.652^{* * *}$ & 0.702 & $3.103^{* * *}$ & 0.841 \\
\hline Early childhood inst. & $2.172^{* *}$ & 1.038 & $2.898^{* *}$ & 1.125 & 1.432 & 1.206 \\
\hline Snack & -1.017 & 0.966 & -1.029 & 0.994 & -0.722 & 1.131 \\
\hline Sick last 3 months & 0.053 & 0.672 & 0.287 & 0.746 & -0.246 & 0.732 \\
\hline Pupils eat fill & $2.010^{*}$ & 1.052 & $1.947^{*}$ & 1.057 & 1.964 & 1.224 \\
\hline Gender of household head & 0.042 & 0.941 & -0.547 & 1.145 & 0.558 & 1.036 \\
\hline Lit. of household head & -0.143 & 0.823 & -0.060 & 0.875 & -0.383 & 0.936 \\
\hline Married & 1.612 & 1.452 & 2.697 & 1.695 & 0.570 & 1.589 \\
\hline Land & -1.995 & 1.491 & -1.330 & 1.600 & -2.450 & 1.626 \\
\hline Parents school & $-1.927^{* * *}$ & 0.621 & $-2.770^{* * *}$ & 0.724 & -1.086 & 0.669 \\
\hline College & $6.251^{* * *}$ & 1.039 & $4.575^{* * *}$ & 1.038 & $8.048^{* * *}$ & 1.188 \\
\hline Diourbel & $4.561^{* * *}$ & 1.359 & $2.652^{*}$ & 1.571 & $6.481^{* * *}$ & 1.538 \\
\hline Kolda & $-3.836^{* * *}$ & 0.959 & $-4.400^{* * *}$ & 0.975 & $-3.142^{* * *}$ & 1.152 \\
\hline Sedhiou & $-4.149^{* * *}$ & 1.471 & $-5.324^{* * *}$ & 1.575 & $-2.825^{*}$ & 1.726 \\
\hline Deworming $\left(T_{1}\right)$ & $-18.845^{* * *}$ & 5.258 & $-19.479^{* * *}$ & 5.427 & $-19.717^{* * *}$ & 5.851 \\
\hline Meal $\left(T_{2}\right)$ & $4.266^{*}$ & 2.520 & $5.064^{*}$ & 2.635 & 3.397 & 2.903 \\
\hline$T_{1} T_{2}$ & $4.066^{*}$ & 2.087 & $6.098^{* * *}$ & 2.326 & 2.442 & 2.338 \\
\hline$h_{1}\left(T_{1}, T_{2}\right)$ & $11.161^{* * *}$ & 2.713 & $9.772^{* * *}$ & 2.855 & $13.181^{* * *}$ & 3.054 \\
\hline$h_{2}\left(T_{1}, T_{2}\right)$ & 1.418 & 1.622 & -0.701 & 1.718 & $3.577^{*}$ & 1.861 \\
\hline Intercept & $80.332^{* * *}$ & 8.341 & $67.714^{* * *}$ & 9.065 & $91.690^{* * *}$ & 9.416 \\
\hline \# Observations & \multicolumn{2}{|c|}{3487} & \multicolumn{2}{|c|}{3487} & \multicolumn{2}{|c|}{3487} \\
\hline Wald $\chi_{2}(43)$ & \multicolumn{2}{|c|}{1395.70} & \multicolumn{2}{|c|}{1866.94} & \multicolumn{2}{|c|}{1243.89} \\
\hline$P>\chi_{2}$ & \multicolumn{2}{|c|}{0.000} & \multicolumn{2}{|c|}{0.000} & \multicolumn{2}{|c|}{0.000} \\
\hline Adj R-squared & \multicolumn{2}{|c|}{0.166} & \multicolumn{2}{|c|}{0.203} & \multicolumn{2}{|c|}{0.145} \\
\hline
\end{tabular}


Table B.3 - continued

\begin{tabular}{|c|c|c|c|}
\hline & Aggregate score & French score & Math score \\
\hline Variable & Coef. Std. Err. & Coef. Std. Err. & Coef. Std. Err. \\
\hline
\end{tabular}

Notes ${ }^{a}$ : Double-Index Selection Model $;{ }^{b}$ : Two step; ${ }^{c}$ : In log; ${ }^{d}$ : qualification.

Significance levels (bootstrap -100 replications-): $\quad *: 10 \% \quad * *: 5 \% \quad * * *: 1 \%$

Table B.4: Determinants of scores: Generalized Roy's model, 2step $^{a}$

\begin{tabular}{|c|c|c|c|c|c|c|}
\hline \multirow[b]{2}{*}{ Variable } & \multicolumn{2}{|c|}{ Aggregate score } & \multicolumn{2}{|c|}{ French score } & \multicolumn{2}{|c|}{ Math score } \\
\hline & Coef. & Std. Err. & Coef. & Std. Err. & Coef. & Std. Err. \\
\hline \multicolumn{7}{|l|}{ Selection Eq. Deworming } \\
\hline Total pupils & $0.0003^{*}$ & 0.0001 & $0.0003^{*}$ & 0.0001 & $0.0003^{*}$ & 0.0001 \\
\hline Management committee & $0.221^{* * *}$ & 0.056 & $0.221^{* * *}$ & 0.056 & $0.221^{* * *}$ & 0.056 \\
\hline Medicine box & $-0.496^{* * *}$ & 0.089 & $-0.496^{* * *}$ & 0.089 & $-0.496^{* * *}$ & 0.089 \\
\hline Association of mothers & $0.207^{* * *}$ & 0.052 & $0.207^{* * *}$ & 0.052 & $0.207^{* * *}$ & 0.052 \\
\hline Cooperative school & $0.262^{* * *}$ & 0.055 & $0.262^{* * *}$ & 0.055 & $0.262^{* * *}$ & 0.055 \\
\hline Water point & $0.582^{* * *}$ & 0.059 & $0.582^{* * *}$ & 0.059 & $0.582^{* * *}$ & 0.059 \\
\hline Gender of pupil & -0.029 & 0.047 & -0.029 & 0.047 & -0.029 & 0.047 \\
\hline Intercept & $-1.942^{* * *}$ & 0.067 & $-1.942^{* * *}$ & 0.067 & $-1.942^{* * *}$ & 0.067 \\
\hline \multicolumn{7}{|l|}{ Selection Eq. Meal } \\
\hline Total pupils & $0.0005^{* * *}$ & 0.0001 & $0.0005^{* * *}$ & 0.0001 & $0.0005^{* * *}$ & 0.0001 \\
\hline Distance to school & $0.193^{* * *}$ & 0.048 & $0.193^{* * *}$ & 0.048 & $0.193^{* * *}$ & 0.048 \\
\hline Management committee & -0.045 & 0.042 & -0.0456 & 0.042 & -0.045 & 0.042 \\
\hline Association of mothers & $0.472^{* * *}$ & 0.043 & $0.472^{* * *}$ & 0.043 & $0.472^{* * *}$ & 0.043 \\
\hline Cooperative school & $-0.169^{* * *}$ & 0.041 & $-0.169^{* * *}$ & 0.041 & $-0.169^{* * *}$ & 0.041 \\
\hline Rural council grant & $0.569^{* * *}$ & 0.045 & $0.569^{* * *}$ & 0.045 & $0.569^{* * *}$ & 0.0457 \\
\hline Water point & $0.156^{* * *}$ & 0.046 & $0.156^{* * *}$ & 0.046 & $0.156^{* * *}$ & 0.046 \\
\hline Disturbed courses & $0.291^{* * *}$ & 0.044 & $0.291^{* * *}$ & 0.044 & $0.291^{* * *}$ & 0.044 \\
\hline Storage & $0.390^{* * *}$ & 0.044 & $0.390^{* * *}$ & 0.044 & $0.390^{* * *}$ & 0.044 \\
\hline Gender of pupil & -0.041 & 0.037 & -0.041 & 0.037 & -0.041 & 0.037 \\
\hline Intercept & $-1.482^{* * *}$ & 0.075 & $-1.482^{* * *}$ & 0.075 & $-1.482^{* * *}$ & 0.075 \\
\hline \multicolumn{7}{|l|}{ Performance Eq. Deworming } \\
\hline Temporary shelters & $-3.374^{* *}$ & 1.479 & $-3.232^{*}$ & 1.917 & $-4.523^{* * *}$ & 1.741 \\
\hline Class size & $-0.321^{* *}$ & 0.154 & $-0.495^{* * *}$ & 0.167 & -0.195 & 0.161 \\
\hline Teacher's age & $-8.972^{* * *}$ & 2.039 & $-10.737^{* * *}$ & 2.416 & $-7.080^{* * *}$ & 2.427 \\
\hline Teacher's age square & $0.125^{* * *}$ & 0.029 & $0.141^{* * *}$ & 0.035 & $0.106^{* * *}$ & 0.033 \\
\hline Pupil's age & $1.594^{* *}$ & 0.602 & $2.361^{* * *}$ & 0.705 & 0.733 & 0.637 \\
\hline High school diploma \& more & $8.193^{* * *}$ & 2.615 & $10.269^{* * *}$ & 2.958 & $7.295^{* *}$ & 2.839 \\
\hline Absenteeism & 4.796 & 5.956 & 3.182 & 6.778 & $11.155^{*}$ & 5.952 \\
\hline Gender of pupil & -0.917 & 1.439 & -0.884 & 1.706 & 0.101 & 1.824 \\
\hline Grade & $-16.738^{* * *}$ & 2.343 & $-25.852^{* * *}$ & 2.818 & $-7.910^{* * *}$ & 2.528 \\
\hline Deworming at home & -3.087 & 2.211 & -0.425 & 2.626 & -2.631 & 3.083 \\
\hline Islamic school & -0.064 & 2.038 & 1.593 & 2.701 & 0.418 & 2.508 \\
\hline Early childhood inst. & -2.572 & 2.821 & -2.322 & 2.786 & -4.188 & 3.342 \\
\hline Snack & -3.450 & 3.166 & -1.729 & 3.580 & -2.574 & 3.731 \\
\hline Pupils eat fill & $6.722^{* * *}$ & 2.498 & $9.624^{* * *}$ & 2.834 & 3.494 & 3.008 \\
\hline Gender of household head & 2.225 & 2.829 & 0.573 & 3.116 & 2.107 & 3.688 \\
\hline Lit. of household head & 1.075 & 1.999 & 1.938 & 2.011 & 1.396 & 2.369 \\
\hline Married & 2.795 & 4.993 & 0.393 & 5.960 & 2.584 & 6.012 \\
\hline$\lambda_{1}^{+-}$ & $24.389^{*}$ & 13.036 & 18.653 & 15.745 & $38.823^{* * *}$ & 13.657 \\
\hline
\end{tabular}


Table B.4 - continued

\begin{tabular}{|c|c|c|c|c|c|c|}
\hline \multirow[b]{2}{*}{ Variable } & \multicolumn{2}{|c|}{ Aggregate score } & \multicolumn{2}{|c|}{ French score } & \multicolumn{2}{|c|}{ Math score } \\
\hline & Coef. & Std. Err. & Coef. & Std. Err. & Coef. & Std. Err. \\
\hline$\lambda_{2}^{+-}$ & 4.648 & 6.433 & 7.436 & 7.489 & 5.561 & 6.661 \\
\hline Intercept & $146.152^{* * *}$ & 49.514 & $195.332^{* * *}$ & 60.408 & $86.224^{* *}$ & 54.921 \\
\hline \# Observations & \multicolumn{2}{|c|}{320} & \multicolumn{2}{|c|}{320} & \multicolumn{2}{|c|}{320} \\
\hline Wald $\chi_{2}(19)$ & \multicolumn{2}{|c|}{244.51} & \multicolumn{2}{|c|}{298.29} & \multicolumn{2}{|c|}{135.96} \\
\hline$P>\chi_{2}$ & \multicolumn{2}{|c|}{0.000} & \multicolumn{2}{|c|}{0.000} & \multicolumn{2}{|c|}{0.000} \\
\hline Adj R-squared & \multicolumn{2}{|c|}{0.356} & \multicolumn{2}{|c|}{0.401} & \multicolumn{2}{|c|}{0.227} \\
\hline \multicolumn{7}{|l|}{ Performance Eq. Meal } \\
\hline Temporary shelters & -0.576 & 0.579 & -0.333 & 0.573 & -0.953 & 0.661 \\
\hline Class size & $0.377^{* * *}$ & 0.043 & $0.368^{* * *}$ & 0.051 & $0.405^{* * *}$ & 0.051 \\
\hline Teacher's age & 1.068 & 1.347 & 1.315 & 1.467 & 1.180 & 1.362 \\
\hline Teacher's age square & -0.005 & 0.020 & -0.010 & 0.022 & -0.005 & 0.021 \\
\hline Pupil's age & -0.256 & 0.431 & -0.453 & 0.501 & -0.152 & 0.455 \\
\hline High school diploma \& more & $-2.768^{* *}$ & 1.289 & $-3.568^{* * *}$ & 1.335 & -1.976 & 1.434 \\
\hline Absenteeism & $5.027^{*}$ & 3.058 & -0.043 & 3.140 & $10.013^{* * *}$ & 3.261 \\
\hline Gender of pupil & 1.396 & 1.238 & -0.710 & 1.324 & $2.866^{* *}$ & 1.391 \\
\hline Grade & $-4.917^{* * *}$ & 1.454 & $-9.533^{* * *}$ & 1.660 & -0.122 & 1.614 \\
\hline Deworming at home & $-3.531^{* *}$ & 1.717 & $-4.292^{* *}$ & 1.813 & -2.743 & 1.886 \\
\hline Islamic school & $-6.009^{* * *}$ & 1.181 & $-7.180^{* * *}$ & 1.321 & $-4.715^{* * *}$ & 1.413 \\
\hline Early childhood inst. & 4.712 & 3.445 & 5.665 & 4.023 & 3.260 & 3.616 \\
\hline Snack & $-4.253^{*}$ & 2.277 & -2.717 & 2.635 & $-4.379^{*}$ & 2.618 \\
\hline Pupils eat fill & $7.617^{* * *}$ & 1.568 & $7.492^{* * *}$ & 1.667 & $7.586^{* * *}$ & 1.854 \\
\hline Gender of household head & 1.271 & 2.524 & -1.371 & 3.162 & $4.352^{*}$ & 2.550 \\
\hline Lit. of household head & 0.031 & 1.772 & -1.396 & 1.901 & 0.884 & 1.981 \\
\hline Married & -2.955 & 2.871 & 2.301 & 3.371 & $-8.175^{* *}$ & 3.684 \\
\hline$\lambda_{1}^{-+}$ & $15.248^{* * *}$ & 5.588 & $16.141^{* * *}$ & 5.917 & $13.537^{* *}$ & 6.754 \\
\hline$\lambda_{2}^{-+}$ & $5.570^{* *}$ & 2.265 & $5.468^{* *}$ & 2.449 & $5.903^{* *}$ & 2.700 \\
\hline Intercept & -1.559 & 21.626 & -1.500 & 24.074 & -7.209 & 21.917 \\
\hline \# Observations & \multicolumn{2}{|c|}{915} & 91 & & 91 & \\
\hline Wald $\chi_{2}(19)$ & 326 & & 277 & & 291 & \\
\hline$P>\chi_{2}$ & 0.0 & & 0.0 & & 0.0 & \\
\hline Adj R-squared & 0.1 & & 0.1 & & 0.1 & \\
\hline Performance Eq. Package & & & & & & \\
\hline Temporary shelters & $5.109^{* * *}$ & 1.588 & $4.772^{* * *}$ & 1.809 & $5.447^{* * *}$ & 1.925 \\
\hline Class size & $1.094^{* * *}$ & 0.167 & $0.918^{* * *}$ & 0.184 & $1.270^{* * *}$ & 0.189 \\
\hline Teacher's age & $-45.806^{* * *}$ & 7.100 & $-41.943^{* * *}$ & 8.370 & $-49.670^{* * *}$ & 8.188 \\
\hline Teacher's age square & $0.662^{* * *}$ & 0.099 & $0.605^{* * *}$ & 0.120 & $0.718^{* * *}$ & 0.114 \\
\hline Pupil's age & $-2.376^{*}$ & 1.239 & $-2.580^{*}$ & 1.390 & -2.171 & 1.334 \\
\hline High school diploma \& more & $-18.169^{* * *}$ & 4.026 & $-21.428^{* * *}$ & 4.280 & $-14.911^{* * *}$ & 4.606 \\
\hline Absenteeism & $-28.398^{* * *}$ & 9.427 & $-18.943^{*}$ & 10.260 & $-37.853^{* * *}$ & 11.122 \\
\hline Gender of pupil & -1.522 & 2.410 & -2.182 & 2.674 & -0.862 & 2.848 \\
\hline Grade & -4.743 & 5.163 & -8.657 & 5.915 & -0.830 & 5.300 \\
\hline Deworming at home & $-5.012^{*}$ & 2.752 & -4.619 & 3.328 & -5.405 & 3.366 \\
\hline Islamic school & 1.816 & 3.682 & 1.069 & 3.938 & 2.563 & 4.386 \\
\hline Early childhood inst. & $-14.486^{* *}$ & 6.811 & -10.298 & 6.825 & $-18.675^{* *}$ & 9.133 \\
\hline Snack & 1.302 & 5.644 & 1.527 & 5.840 & 1.077 & 7.072 \\
\hline Pupils eat fill & -0.029 & 4.594 & 1.950 & 4.358 & -2.009 & 6.155 \\
\hline Gender of household head & -2.709 & 4.383 & -2.363 & 4.686 & -3.054 & 4.773 \\
\hline Lit. of household head & -3.546 & 3.994 & -5.005 & 3.866 & -2.087 & 4.795 \\
\hline Married & -4.312 & 6.624 & -4.114 & 7.045 & -4.510 & 6.797 \\
\hline$\lambda_{1}^{++}$ & $80.384^{* * *}$ & 18.355 & $68.143^{* * *}$ & 20.381 & $92.624^{* * *}$ & 22.789 \\
\hline$\lambda_{2}^{++}$ & $-47.396^{*}$ & 27.553 & -49.361 & 31.404 & -45.432 & 32.294 \\
\hline Intercept & $731.730^{* * *}$ & 105.850 & $695.734^{* * *}$ & 127.702 & $767.727^{* * *}$ & 123.242 \\
\hline \# Observations & 16 & & 16 & & 16 & \\
\hline
\end{tabular}


Table B.4 - continued

\begin{tabular}{|c|c|c|c|c|c|c|}
\hline \multirow[b]{2}{*}{ Variable } & \multicolumn{2}{|c|}{ Aggregate score } & \multicolumn{2}{|c|}{ French score } & \multicolumn{2}{|c|}{ Math score } \\
\hline & Coef. & d. Err. & Coef. & Err. & Coef. & Std. Err. \\
\hline Wald $\chi_{2}(19)$ & \multicolumn{2}{|c|}{299.54} & \multicolumn{2}{|c|}{410.47} & \multicolumn{2}{|c|}{219.96} \\
\hline$P>\chi_{2}$ & \multicolumn{2}{|c|}{0.000} & \multicolumn{2}{|c|}{0.000} & \multicolumn{2}{|c|}{0.000} \\
\hline Adj R-squared & \multicolumn{2}{|c|}{0.476} & \multicolumn{2}{|c|}{0.507} & \multicolumn{2}{|c|}{0.373} \\
\hline \multicolumn{7}{|l|}{ Performance Eq. Untreated } \\
\hline Temporary shelters & $-0.601^{*}$ & 0.313 & $-0.571^{*}$ & 0.319 & $-0.636^{*}$ & 0.356 \\
\hline Class size & -0.036 & 0.026 & $-0.080^{* * *}$ & 0.026 & 0.007 & 0.031 \\
\hline Teacher's age & -0.471 & 0.526 & -0.555 & 0.511 & -0.384 & 0.620 \\
\hline Teacher's age square & 0.005 & 0.007 & 0.005 & 0.007 & 0.005 & 0.009 \\
\hline Pupil's age & $0.656^{* *}$ & 0.298 & $0.522^{*}$ & 0.311 & $0.789^{* *}$ & 0.322 \\
\hline High school diploma \& more & 0.874 & 0.694 & 0.128 & 0.695 & $1.627^{*}$ & 0.819 \\
\hline Absenteeism & $-3.556^{* * *}$ & 1.261 & -0.128 & 1.353 & $-7.001^{* * *}$ & 1.353 \\
\hline Gender of pupil & 1.130 & 0.728 & 0.254 & 0.705 & $2.003^{* *}$ & 0.855 \\
\hline Grade & $-13.060^{* * *}$ & 0.959 & $-18.900^{* * *}$ & 1.012 & $-7.215^{* * *}$ & 1.113 \\
\hline Deworming at home & $-1.965^{*}$ & 1.022 & -1.535 & 1.063 & $-2.389^{* *}$ & 1.098 \\
\hline Islamic school & $2.252^{* * *}$ & 0.780 & $1.864^{* *}$ & 0.810 & $2.640^{* *}$ & 0.909 \\
\hline Early childhood inst. & $5.931^{* * *}$ & 1.531 & $6.724^{* * *}$ & 1.568 & $5.154^{* * *}$ & 1.729 \\
\hline Snack & $-2.311^{*}$ & 1.258 & -1.879 & 1.411 & $-2.728^{* *}$ & 1.335 \\
\hline Pupils eat fill & $4.699^{* * *}$ & 1.309 & $3.382^{* *}$ & 1.339 & $6.016^{* * *}$ & 1.418 \\
\hline Gender of household head & $-3.243^{* *}$ & 1.373 & $-3.234^{* *}$ & 1.384 & $-3.261^{* *}$ & 1.646 \\
\hline Lit. of household head & $2.260^{* *}$ & 1.030 & $3.086^{* * *}$ & 1.031 & 1.440 & 1.168 \\
\hline Married & 0.879 & 1.813 & 1.202 & 1.769 & 0.570 & 2.187 \\
\hline$\lambda_{1}^{--}$ & $14.650^{* * *}$ & 3.452 & $15.842^{* * *}$ & 3.285 & $13.511^{* * *}$ & 4.162 \\
\hline$\lambda_{2}^{--}$ & $-5.559^{* * *}$ & 1.936 & $-4.644^{* *}$ & 2.101 & $-6.439^{* * *}$ & 2.111 \\
\hline Intercept & $46.542^{* * *}$ & 9.254 & $55.706^{* * *}$ & 9.063 & $37.308^{* * *}$ & 10.935 \\
\hline \# Observations & 25 & & 25 & & 25 & \\
\hline Wald $\chi_{2}(19)$ & 518 & & 872 & & 232 & \\
\hline$P>\chi_{2}$ & 0.0 & & 0.0 & & 0.0 & \\
\hline Adj R-squared & 0.1 & & 0.1 & & 0.0 & \\
\hline
\end{tabular}

Notes $^{a}:$ Two-step; ${ }^{b}:$ In log.

Significance levels (bootstrap -100 replications-): $\quad *: 10 \% \quad * *: 5 \% \quad * * *: 1 \%$

Table B.5: Determinants of rates: Generalized Roy's model, (2step $^{a}$ )

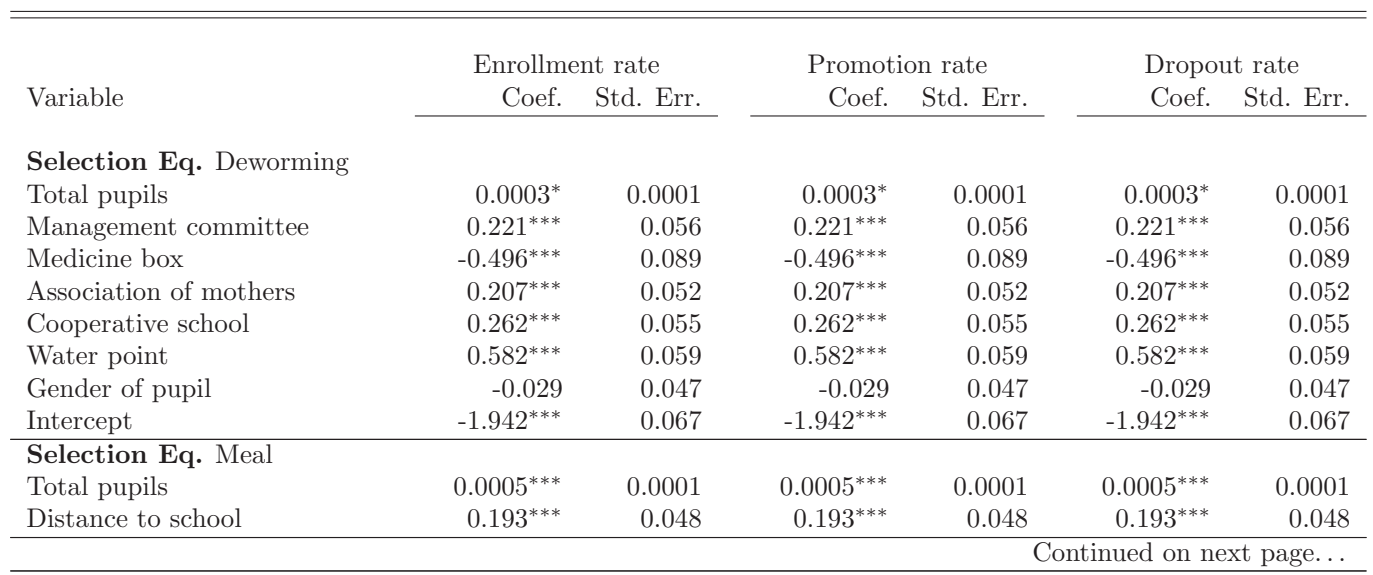


Table B.5 - continued

\begin{tabular}{|c|c|c|c|c|c|c|}
\hline \multirow[b]{2}{*}{ Variable } & \multicolumn{2}{|c|}{ Enrollment rate } & \multicolumn{2}{|c|}{ Promotion rate } & \multicolumn{2}{|c|}{ Dropout rate } \\
\hline & Coef. & Std. Err. & Coef. & d. Err. & Coef. & td. Err. \\
\hline Management committee & -0.045 & 0.042 & -0.0456 & 0.042 & -0.045 & 0.042 \\
\hline Association of mothers & $0.472^{* * *}$ & 0.043 & $0.472^{* * *}$ & 0.043 & $0.472^{* * *}$ & 0.043 \\
\hline Cooperative school & $-0.169^{* * *}$ & 0.041 & $-0.169^{* * *}$ & 0.041 & $-0.169^{* * *}$ & 0.041 \\
\hline Rural council grant & $0.569^{* * *}$ & 0.045 & $0.569^{* * *}$ & 0.045 & $0.569^{* * *}$ & 0.0457 \\
\hline Water point & $0.156^{* * *}$ & 0.046 & $0.156^{* * *}$ & 0.046 & $0.156^{* * *}$ & 0.046 \\
\hline Disturbed courses & $0.291^{* * *}$ & 0.044 & $0.291^{* * *}$ & 0.044 & $0.291^{* * *}$ & 0.044 \\
\hline Storage & $0.390^{* * *}$ & 0.044 & $0.390^{* * *}$ & 0.044 & $0.390^{* * *}$ & 0.044 \\
\hline Gender of pupil & -0.041 & 0.037 & -0.041 & 0.037 & -0.041 & 0.037 \\
\hline Intercept & $-1.482^{* * *}$ & 0.075 & $-1.482^{* * *}$ & 0.075 & $-1.482^{* * *}$ & 0.075 \\
\hline \multicolumn{7}{|c|}{ Performance Eq. Deworming } \\
\hline Temporary shelters & $-17.718^{* * *}$ & 1.372 & $-4.563^{* * *}$ & 0.832 & $3.877^{* * *}$ & 0.560 \\
\hline Class size & $-1.833^{* * *}$ & 0.180 & -0.191 & 0.120 & $0.375^{* * *}$ & 0.078 \\
\hline Pupil's age & -0.802 & 0.562 & -0.471 & 0.361 & $0.507^{* *}$ & 0.239 \\
\hline Education expenditure $^{b}$ & $6.397^{* * *}$ & 1.258 & $3.490^{* * *}$ & 0.941 & $-2.114^{* * *}$ & 0.619 \\
\hline Health expenditure $^{b}$ & -0.322 & 0.328 & -0.201 & 0.237 & 0.178 & 0.159 \\
\hline Livestock & $-0.129^{*}$ & 0.077 & -0.038 & 0.069 & 0.044 & 0.049 \\
\hline Distance to school & $14.758^{* * *}$ & 2.762 & $5.703^{* * *}$ & 2.191 & -1.429 & 1.410 \\
\hline Gender of teacher & $21.138^{* * *}$ & 3.323 & $11.811^{* * *}$ & 1.792 & $-11.421^{* * *}$ & 1.419 \\
\hline Continuing training & - & - & $-3.469^{* *}$ & 1.620 & - & - \\
\hline Gender of pupil & 2.710 & 2.052 & 1.385 & 1.296 & $-1.721^{* *}$ & 0.858 \\
\hline Islamic school & $6.191^{* * *}$ & 2.337 & $4.217^{* *}$ & 1.651 & $-2.940^{* *}$ & 1.139 \\
\hline Early childhood inst. & 1.763 & 2.816 & -2.447 & 1.688 & $1.849^{*}$ & 1.122 \\
\hline Snack & $5.863^{*}$ & 3.286 & $3.731^{*}$ & 2.002 & $-2.670^{* *}$ & 1.273 \\
\hline Sick last 3 months & - & - & -0.805 & 1.075 & 0.277 & 0.725 \\
\hline Pupils eat fill & $28.923^{* * *}$ & 3.800 & $15.421^{* * *}$ & 2.141 & $-10.674^{* * *}$ & 1.595 \\
\hline Gender of household head & 2.794 & 3.136 & 2.558 & 1.988 & -1.799 & 1.376 \\
\hline Lit. of household head & 3.206 & 2.482 & 1.500 & 1.773 & -0.470 & 1.275 \\
\hline Married & -4.358 & 5.155 & -1.001 & 3.477 & 0.673 & 2.356 \\
\hline Land & -4.573 & 3.032 & $-4.560^{* *}$ & 1.837 & $2.547^{*}$ & 1.464 \\
\hline$\lambda_{1}^{+-}$ & $-65.351^{* * *}$ & 8.487 & -3.835 & 6.480 & $23.827^{* * *}$ & 4.441 \\
\hline$\lambda_{2}^{+-}$ & $-38.792^{* * *}$ & 4.391 & $8.434^{* * *}$ & 2.998 & -3.129 & 2.201 \\
\hline Intercept & $95.461^{* * *}$ & 27.436 & $40.598^{*}$ & 21.126 & -9.200 & 14.720 \\
\hline \# Observations & \multicolumn{2}{|c|}{288} & \multicolumn{2}{|c|}{286} & \multicolumn{2}{|c|}{286} \\
\hline Wald $\chi_{2}($ d.o.f $)$ & \multicolumn{2}{|c|}{$2276.27(19)$} & \multicolumn{2}{|c|}{$1213.66(21)$} & \multicolumn{2}{|c|}{$2028.79(20)$} \\
\hline$P>\chi_{2}$ & \multicolumn{2}{|c|}{0.000} & \multicolumn{2}{|c|}{0.000} & \multicolumn{2}{|c|}{0.000} \\
\hline Adj R-squared & \multicolumn{2}{|c|}{0.790} & \multicolumn{2}{|c|}{0.528} & \multicolumn{2}{|c|}{0.696} \\
\hline \multicolumn{7}{|l|}{ Performance Eq. Meal } \\
\hline Temporary shelters & $5.558^{* * *}$ & 0.577 & $2.835^{* * *}$ & 0.321 & $-0.994^{* * *}$ & 0.259 \\
\hline Class size & $-0.773^{* * *}$ & 0.100 & $0.143^{* * *}$ & 0.026 & $-0.242^{* * *}$ & 0.027 \\
\hline Pupil's age & 0.193 & 0.762 & -0.173 & 0.220 & -0.236 & 0.164 \\
\hline Education expenditure $^{b}$ & $-2.202^{*}$ & 1.152 & -0.033 & 0.370 & -0.056 & 0.305 \\
\hline Health expenditure $^{b}$ & -0.351 & 0.565 & $-0.733^{* * *}$ & 0.219 & $0.498^{* * *}$ & 0.178 \\
\hline Livestock & -0.101 & 0.087 & -0.012 & 0.016 & -0.024 & 0.023 \\
\hline Distance to school & -4.514 & 3.076 & $2.597^{* *}$ & 1.077 & -0.873 & 1.049 \\
\hline Gender of teacher & $-34.665^{* * *}$ & 4.519 & $6.140^{* * *}$ & 1.028 & -1.204 & 0.782 \\
\hline Continuing training & - & - & -1.240 & 0.940 & - & - \\
\hline Gender of pupil & 1.057 & 1.926 & -0.492 & 0.700 & 0.556 & 0.582 \\
\hline Islamic school & 3.015 & 2.301 & 1.302 & 0.827 & 0.765 & 0.726 \\
\hline Early childhood inst. & $-14.563^{* * *}$ & 4.928 & 2.457 & 2.100 & $-4.149^{* * *}$ & 1.562 \\
\hline Snack & $-14.347^{* * *}$ & 3.786 & $-2.507^{*}$ & 1.318 & 1.587 & 1.175 \\
\hline Sick last 3 months & - & - & -0.930 & 0.848 & 0.948 & 0.673 \\
\hline Pupils eat fill & 2.785 & 3.002 & $-2.704^{* *}$ & 1.207 & 0.653 & 0.885 \\
\hline Gender of household head & $13.535^{*}$ & 6.919 & 0.829 & 1.775 & 1.815 & 1.179 \\
\hline
\end{tabular}


Table B.5 - continued

\begin{tabular}{|c|c|c|c|c|c|c|}
\hline \multirow[b]{2}{*}{ Variable } & \multicolumn{2}{|c|}{ Enrollment rate } & \multicolumn{2}{|c|}{ Promotion rate } & \multicolumn{2}{|c|}{ Dropout rate } \\
\hline & Coef. & Std. Err. & Coef. & td. Err. & Coef. & td. Err. \\
\hline Lit. of household head & $14.765^{* * *}$ & 3.149 & $2.875^{* * *}$ & 0.831 & -0.282 & 0.736 \\
\hline Married & -0.469 & 6.573 & -1.701 & 1.784 & -1.243 & 1.878 \\
\hline Land & -3.955 & 5.090 & 1.093 & 1.395 & 1.562 & 1.186 \\
\hline$\lambda_{1}^{-+}$ & $42.324^{* * *}$ & 7.314 & $-11.011^{* * *}$ & 2.738 & $7.086^{* * *}$ & 2.395 \\
\hline$\lambda_{2}^{-}+$ & $-25.219^{* * *}$ & 4.571 & $15.937^{* * *}$ & 1.547 & $-9.221^{* * *}$ & 1.220 \\
\hline Intercept & $70.729^{* * *}$ & 16.113 & $60.811^{* * *}$ & 5.263 & $27.725^{* * *}$ & 4.447 \\
\hline \# Observations & \multicolumn{2}{|c|}{827} & \multicolumn{2}{|c|}{822} & \multicolumn{2}{|c|}{822} \\
\hline Wald $\chi_{2}($ d.o.f $)$ & \multicolumn{2}{|c|}{$379.93(19)$} & \multicolumn{2}{|c|}{$273.15(21)$} & \multicolumn{2}{|c|}{$212.57(20)$} \\
\hline$P>\chi_{2}$ & \multicolumn{2}{|c|}{0.000} & \multicolumn{2}{|c|}{0.000} & \multicolumn{2}{|c|}{0.000} \\
\hline Adj R-squared & \multicolumn{2}{|c|}{0.271} & \multicolumn{2}{|c|}{0.222} & \multicolumn{2}{|c|}{0.172} \\
\hline \multicolumn{7}{|l|}{ Performance Eq. Package } \\
\hline Temporary shelters & $-13.834^{* * *}$ & 0.584 & $5.512^{* * *}$ & 0.182 & $-0.868^{* * *}$ & 0.014 \\
\hline Class size & -0.013 & 0.024 & 0.004 & 0.007 & -0.0004 & 0.0006 \\
\hline Pupil's age & -0.047 & 0.040 & $0.032^{* *}$ & 0.015 & -0.001 & 0.001 \\
\hline Education expenditure $^{b}$ & 0.001 & 0.068 & 0.003 & 0.023 & -0.0004 & 0.001 \\
\hline Health expenditure $^{b}$ & 0.021 & 0.063 & -0.011 & 0.022 & 0.0009 & 0.001 \\
\hline Livestock & $0.0006^{* *}$ & 0.0003 & -0.0002 & 0.0001 & 0.00002 & 0.00001 \\
\hline Distance to school & $9.046^{* * *}$ & 0.340 & $-2.847^{* * *}$ & 0.106 & $0.237^{* * *}$ & 0.008 \\
\hline Gender of teacher & $0.554^{*}$ & 0.335 & $-0.232^{*}$ & 0.118 & 0.015 & 0.008 \\
\hline Continuing training & - & - & -0.123 & 0.085 & - & - \\
\hline Gender of pupil & $1.784^{* * *}$ & 0.094 & $0.700^{* * *}$ & 0.031 & $-0.079^{* * *}$ & 0.002 \\
\hline Islamic school & $-0.502^{* * *}$ & 0.149 & $0.158^{* * *}$ & 0.048 & $-0.012^{* * *}$ & 0.004 \\
\hline Early childhood inst. & -0.387 & 0.255 & 0.106 & 0.074 & $-0.010^{*}$ & 0.006 \\
\hline Snack & -0.226 & 0.200 & 0.068 & 0.052 & -0.006 & 0.004 \\
\hline Sick last 3 months & - & - & 0.017 & 0.025 & -0.0005 & 0.002 \\
\hline Pupils eat fill & 0.340 & 0.250 & -0.107 & 0.094 & 0.006 & 0.007 \\
\hline Gender of household head & -0.209 & 0.142 & 0.061 & 0.048 & -0.005 & 0.004 \\
\hline Lit. of household head & -0.053 & 0.140 & 0.020 & 0.046 & -0.002 & 0.003 \\
\hline Married & 0.117 & 0.186 & -0.054 & 0.062 & 0.004 & 0.005 \\
\hline Land & -0.187 & 0.292 & 0.042 & 0.083 & -0.006 & 0.007 \\
\hline$\lambda_{1}^{++}$ & $-167.168^{* * *}$ & 0.797 & -1.094 & 0.264 & $0.989^{* * *}$ & 0.021 \\
\hline$\lambda_{2}^{++}$ & $72.112^{* * *}$ & 2.696 & $-23.553^{* * *}$ & 0.827 & $1.983^{* * *}$ & 0.069 \\
\hline Intercept & $191.992^{* * *}$ & 1.855 & $101.787^{* * *}$ & 0.540 & $7.386^{* * *}$ & 0.047 \\
\hline \# Observations & \multicolumn{2}{|l|}{135} & 1 & & 13 & \\
\hline Wald $\chi_{2}($ d.o.f $)$ & $3.66 \mathrm{e}+0$ & $(19)$ & 3270 & & $1.09 \mathrm{e}+$ & $(20)$ \\
\hline$P>\chi_{2}$ & 0.00 & & 0.0 & & 0.0 & \\
\hline Adj R-squared & 0.9 & & 0. & & 0. & \\
\hline Performance Eq. Untreated & & & & & & \\
\hline Temporary shelters & $2.571^{* * *}$ & 0.843 & $0.599^{* * *}$ & 0.194 & $-0.828^{* * *}$ & 0.171 \\
\hline Class size & $-0.449^{* * *}$ & 0.070 & $-0.027^{*}$ & 0.015 & 0.014 & 0.015 \\
\hline Pupil's age & -0.899 & 0.594 & 0.133 & 0.140 & $-0.233^{*}$ & 0.134 \\
\hline Education expenditure $^{b}$ & $4.867^{* * *}$ & 0.917 & 0.067 & 0.173 & -0.131 & 0.177 \\
\hline Health expenditure $^{b}$ & $-3.649^{* * *}$ & 0.585 & $-0.367^{* * *}$ & 0.105 & $0.346^{* * *}$ & 0.102 \\
\hline Livestock & $-0.413^{* * *}$ & 0.044 & $-0.074^{* * *}$ & 0.010 & $0.089^{* * *}$ & 0.010 \\
\hline Distance to school & $-12.540^{* * *}$ & 3.487 & $4.037^{* * *}$ & 0.629 & $-4.219^{* * *}$ & 0.608 \\
\hline Gender of teacher & $-20.712^{* * *}$ & 2.761 & $1.542^{* *}$ & 0.684 & 0.722 & 0.665 \\
\hline Continuing training & - & - & $2.225^{* * *}$ & 0.549 & - & - \\
\hline Gender of pupil & 0.149 & 2.072 & 0.733 & 0.539 & -0.741 & 0.528 \\
\hline Islamic school & 0.865 & 2.351 & $1.985^{* * *}$ & 0.474 & $-1.364^{* * *}$ & 0.447 \\
\hline Early childhood inst. & 4.222 & 3.705 & $2.277^{* * *}$ & 0.708 & $-2.908^{* * *}$ & 0.624 \\
\hline Snack & 1.371 & 3.371 & -0.387 & 0.829 & -0.380 & 0.899 \\
\hline Sick last 3 months & - & - & 0.332 & 0.517 & -0.222 & 0.496 \\
\hline Pupils eat fill & $31.105^{* * *}$ & 2.854 & $4.286^{* * *}$ & 0.966 & $-6.112^{* * *}$ & 1.044 \\
\hline
\end{tabular}


Table B.5 - continued

\begin{tabular}{|c|c|c|c|c|c|c|}
\hline \multirow[b]{2}{*}{ Variable } & \multicolumn{2}{|c|}{ Enrollment rate } & \multicolumn{2}{|c|}{ Promotion rate } & \multicolumn{2}{|c|}{ Dropout rate } \\
\hline & Coef. & Std. Err. & Coef. & d. Err. & Coef. & l. Err. \\
\hline Gender of household head & $-20.150^{* * *}$ & 4.069 & 1.075 & 0.866 & 0.434 & 0.696 \\
\hline Lit. of household head & $17.791^{* * *}$ & 3.112 & -0.466 & 0.659 & -0.081 & 0.652 \\
\hline Married & -4.055 & 7.092 & -1.591 & 1.104 & $1.900^{*}$ & 1.039 \\
\hline Land & $-7.900^{* *}$ & 3.738 & $3.034^{* * *}$ & 1.068 & $-3.034^{* * *}$ & 1.076 \\
\hline$\lambda_{1}^{--}$ & $107.293^{* * *}$ & 10.615 & $20.796^{* * *}$ & 2.161 & $-22.859^{* * *}$ & 2.143 \\
\hline$\lambda_{2}^{-}-$ & $23.885^{* * *}$ & 6.695 & $-20.390^{* * *}$ & 1.233 & $19.688^{* * *}$ & 1.195 \\
\hline Intercept & -19.412 & 13.954 & $73.448^{* * *}$ & 2.788 & $22.035^{* * *}$ & 2.870 \\
\hline \# Observations & \multicolumn{2}{|c|}{2312} & \multicolumn{2}{|c|}{2299} & \multicolumn{2}{|c|}{2299} \\
\hline Wald $\chi_{2}($ d.o.f $)$ & \multicolumn{2}{|c|}{$1727.77(19)$} & \multicolumn{2}{|c|}{$812.10(21)$} & \multicolumn{2}{|c|}{$1033.09(20)$} \\
\hline$P>\chi_{2}$ & \multicolumn{2}{|c|}{0.000} & \multicolumn{2}{|c|}{0.000} & \multicolumn{2}{|c|}{0.000} \\
\hline Adj R-squared & \multicolumn{2}{|c|}{0.283} & \multicolumn{2}{|c|}{0.154} & \multicolumn{2}{|c|}{0.184} \\
\hline
\end{tabular}

Notes $^{a}:$ Two-step; ${ }^{b}:$ In log.

Significance levels (bootstrap -100 replications-): $\quad *: 10 \% \quad * *: 5 \% \quad * * *: 1 \%$

Table B.6: Determinants of rates: $\operatorname{DISM}^{a}$ model, $\left(2-\right.$ step $\left.^{b}\right)$

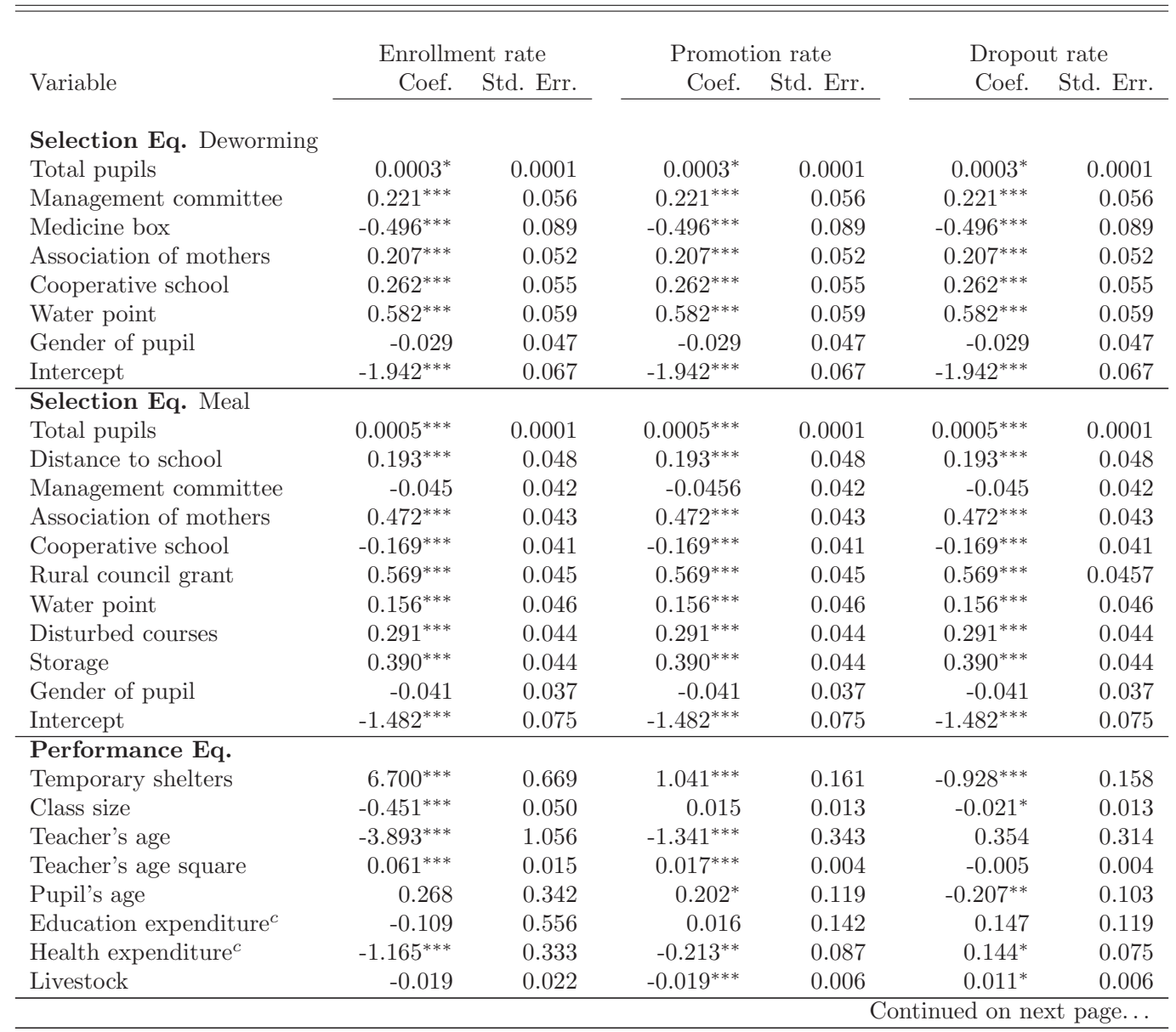


Table B.6 - continued

\begin{tabular}{|c|c|c|c|c|c|c|}
\hline \multirow[b]{2}{*}{ Variable } & \multicolumn{2}{|c|}{ Enrollment rate } & \multicolumn{2}{|c|}{ Promotion rate } & \multicolumn{2}{|c|}{ Dropout rate } \\
\hline & Coef. & Std. Err. & Coef. & Err. & Coef. & d. Err. \\
\hline Primary schools & 0.241 & 0.982 & - & - & - & - \\
\hline Distance to school & $-7.556^{* * *}$ & 2.038 & $3.995^{* * *}$ & 0.477 & $-2.959^{* * *}$ & 0.470 \\
\hline Association of parents & $10.440^{* * *}$ & 2.377 & $6.021^{* * *}$ & 0.787 & $-3.703^{* * *}$ & 0.468 \\
\hline Disturbed courses & $-4.018^{* *}$ & 1.630 & $-4.573^{* * *}$ & 0.485 & $5.258^{* * *}$ & 0.411 \\
\hline Meals near school & 1.121 & 1.325 & -0.598 & 0.385 & -0.529 & 0.406 \\
\hline Gender of teacher & $-6.145^{* * *}$ & 2.114 & $3.749^{* * *}$ & 0.495 & $-1.203^{* * *}$ & 0.453 \\
\hline Continuing training & - & - & $1.315^{* * *}$ & 0.459 & $-1.090^{* *}$ & 0.423 \\
\hline Absenteeism & -0.113 & 3.067 & $-1.761^{* * *}$ & 0.578 & -0.155 & 0.606 \\
\hline Gender of pupil & 1.159 & 1.144 & 0.416 & 0.403 & -0.535 & 0.356 \\
\hline Islamic school & $2.991^{* *}$ & 1.251 & $1.405^{* * *}$ & 0.394 & $-0.983^{* *}$ & 0.394 \\
\hline Early childhood inst. & -3.321 & 2.120 & $1.913^{* * *}$ & 0.696 & $-2.313^{* * *}$ & 0.609 \\
\hline Snack & $4.155^{* *}$ & 1.843 & 0.277 & 0.683 & -0.143 & 0.715 \\
\hline Sick last 3 months & - & - & -0.525 & 0.378 & 0.416 & 0.374 \\
\hline Pupils eat fill & $5.852^{* * *}$ & 2.042 & $2.307^{* * *}$ & 0.799 & $-3.261^{* * *}$ & 0.788 \\
\hline Gender of household head & -3.988 & 2.741 & 0.790 & 0.657 & $0.884^{*}$ & 0.499 \\
\hline Lit. of household head & $10.834^{* * *}$ & 1.614 & -0.524 & 0.497 & $1.009^{* *}$ & 0.453 \\
\hline Married & -0.213 & 3.435 & -0.627 & 0.870 & -0.243 & 0.823 \\
\hline Land & $-4.760^{*}$ & 2.424 & $1.643^{* *}$ & 0.744 & $-1.459^{*}$ & 0.797 \\
\hline School cost & $-3.771^{* *}$ & 1.462 & & & & \\
\hline Islamic school com. & 1.030 & 1.432 & - & - & $1.167^{* * *}$ & 0.431 \\
\hline College & - & - & -0.260 & 0.623 & $-1.213^{* *}$ & 0.586 \\
\hline Diourbel & $13.551^{* * *}$ & 3.995 & $5.728^{* * *}$ & 0.890 & $-4.680^{* * *}$ & 0.858 \\
\hline Kolda & $-76.376^{* * *}$ & 1.858 & $-5.258^{* * *}$ & 0.509 & $5.558^{* * *}$ & 0.534 \\
\hline Sedhiou & $-18.183^{* * *}$ & 2.721 & $9.914^{* * *}$ & 0.740 & $-6.079^{* * *}$ & 0.815 \\
\hline Deworming $\left(T_{1}\right)$ & -8.007 & 10.196 & $7.548^{* *}$ & 2.917 & $-9.645^{* * *}$ & 2.887 \\
\hline Meal $\left(T_{2}\right)$ & $58.966^{* * *}$ & 5.924 & $-18.048^{* * *}$ & 1.646 & $13.065^{* * *}$ & 1.608 \\
\hline$T_{1} T_{2}$ & 1.623 & 3.601 & $9.494^{* * *}$ & 1.339 & $-2.799^{* * *}$ & 1.001 \\
\hline$h_{1}\left(T_{1}, T_{2}\right)$ & $-9.007^{*}$ & 5.140 & $-6.171^{* * *}$ & 1.486 & $3.943^{* * *}$ & 1.438 \\
\hline$h_{2}\left(T_{1}, T_{2}\right)$ & $-28.215^{* * *}$ & 3.804 & $12.224^{* * *}$ & 1.059 & $-10.518^{* * *}$ & 1.009 \\
\hline Intercept & $65.993^{* * *}$ & 20.599 & $93.063^{* * *}$ & 5.516 & $14.921^{* * *}$ & 5.268 \\
\hline \# Observations & 34 & & 34 & & 34 & \\
\hline Wald $\chi_{2}($ d.o.f $)$ & 19968. & $6(34)$ & 2534.0 & & 3453.2 & \\
\hline$P>\chi_{2}$ & 0.0 & & 0.0 & & 0.0 & \\
\hline Adj R-squared & 0.6 & & 0.2 & & 0.2 & \\
\hline
\end{tabular}

Notes ${ }^{a}$ : Double-Index Selection Model; ${ }^{b}$ : Two step; ${ }^{c}$ : In log.

Significance levels (bootstrap -100 replications-): $\quad *: 10 \% \quad * *: 5 \% \quad * * *: 1 \%$ 


\section{Appendix B.4 Figures on the treatment effect heterogeneity}
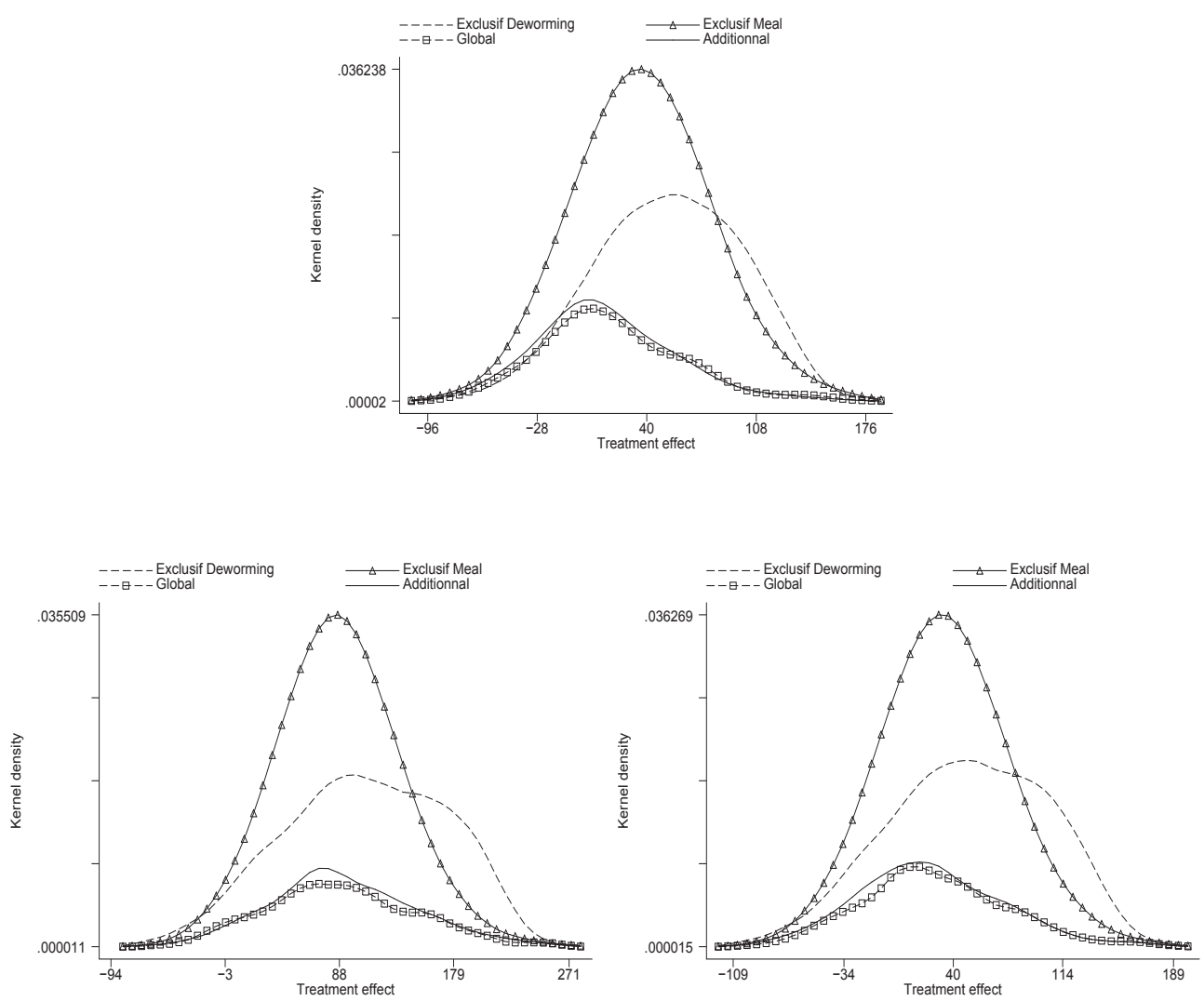

Figure B.1: Distribution of treatment effects for aggregate score. [Top]: Average Treatment Effect (ATE) [Bottom-left]: Average Treatment Effect on the Treated (ATET). [Bottom-right]: Average Treatment Effect on the Nontreated (ATENT) 

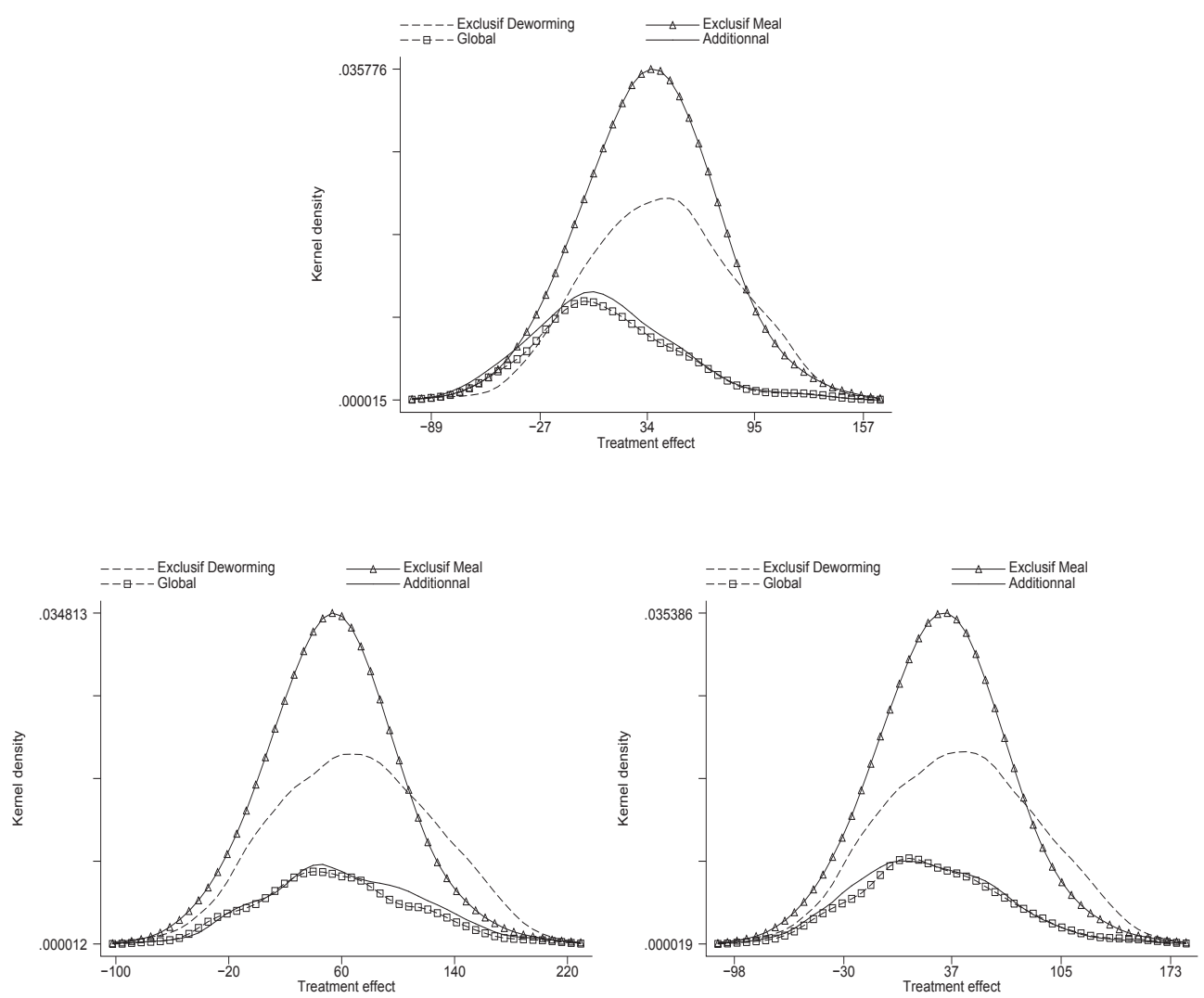

Figure B.2: Distribution of treatment effects for French score. [Top]: Average Treatment Effect (ATE) [Bottom-left]: Average Treatment Effect on the Treated (ATET). [Bottom-right]: Average Treatment Effect on the Nontreated (ATENT) 

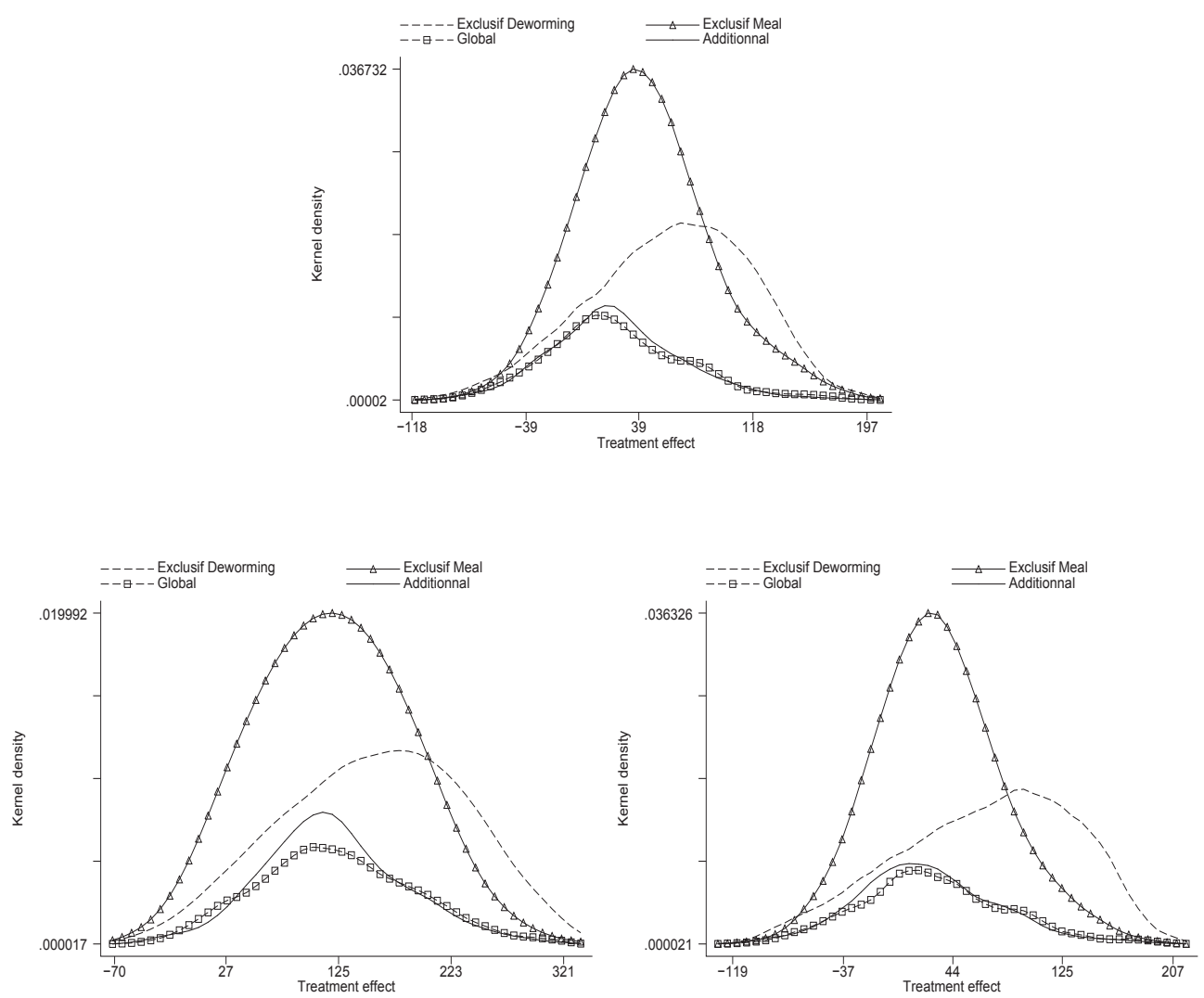

Figure B.3: Distribution of treatment effects for math score. [Top]: Average Treatment Effect (ATE) [Bottom-left]: Average Treatment Effect on the Treated (ATET). [Bottom-right]: Average Treatment Effect on the Nontreated (ATENT) 

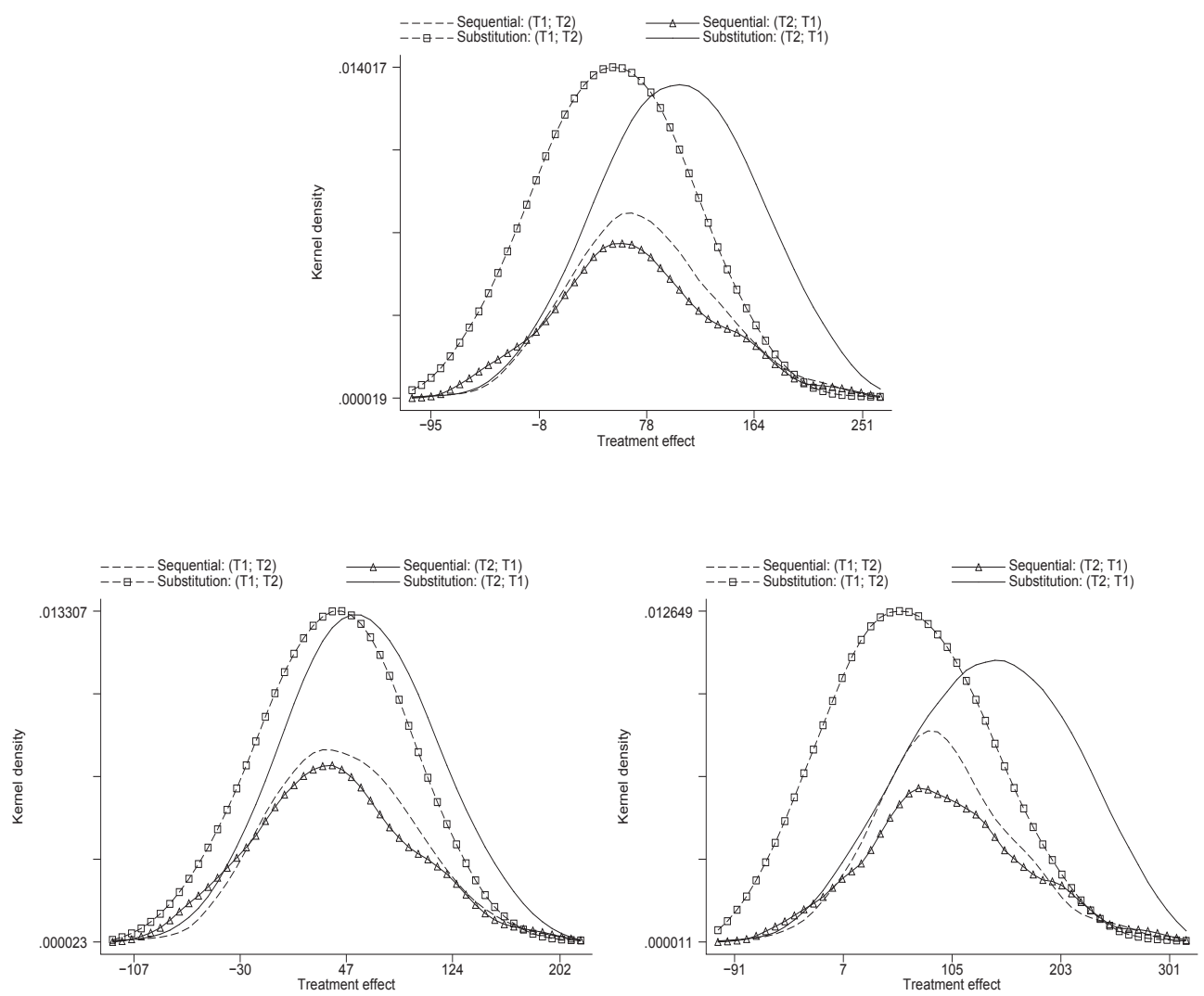

Figure B.4: Distribution of sequential and substitution effects on the treated. [Top]: Aggregate score [Bottom-left]: French score. [Bottom-right]: Math score 

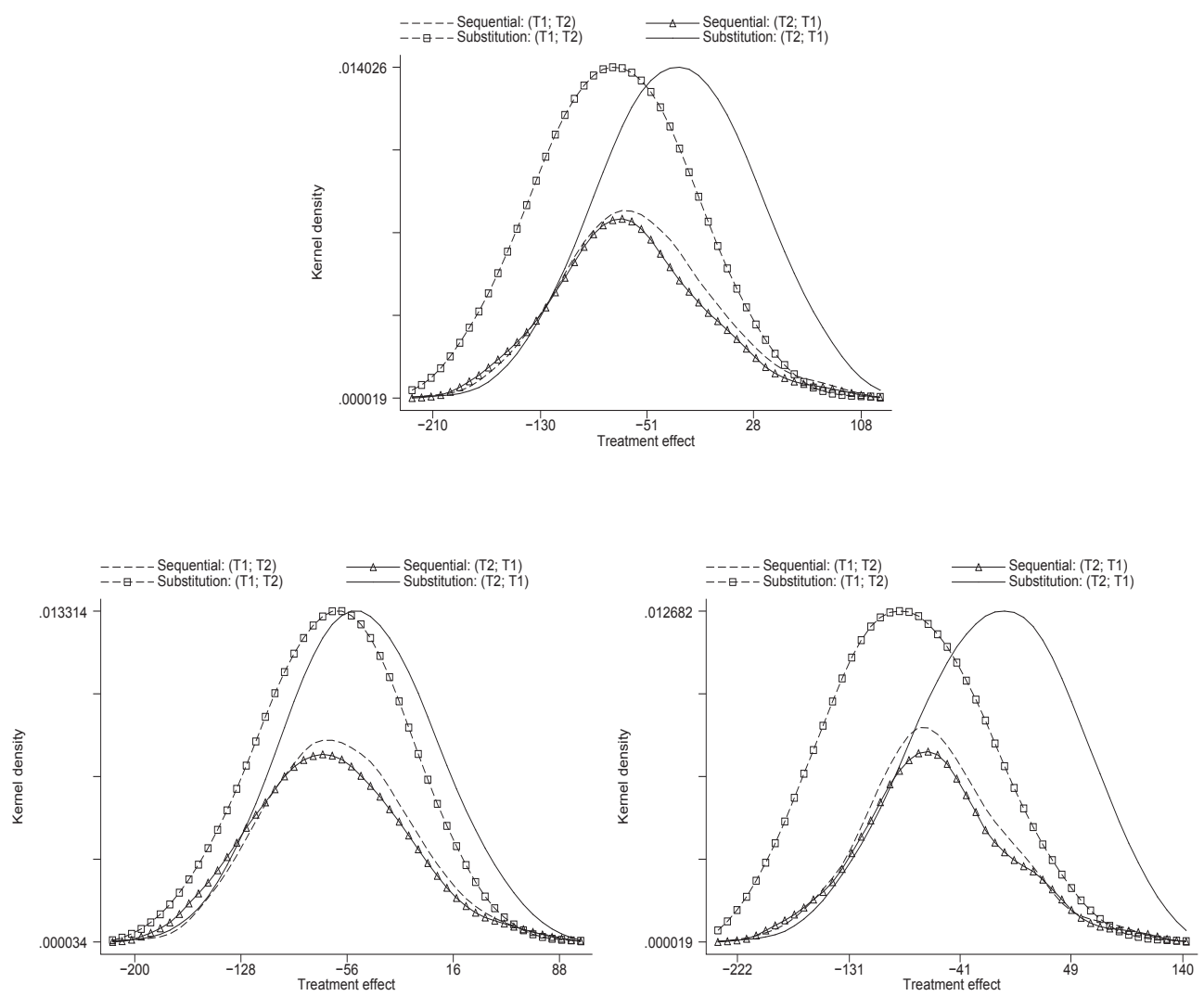

Figure B.5: Distribution of sequential and substitution effects on the nontreated. [Top]: Aggregate score [Bottom-left]: French score. [Bottom-right]: Math score 

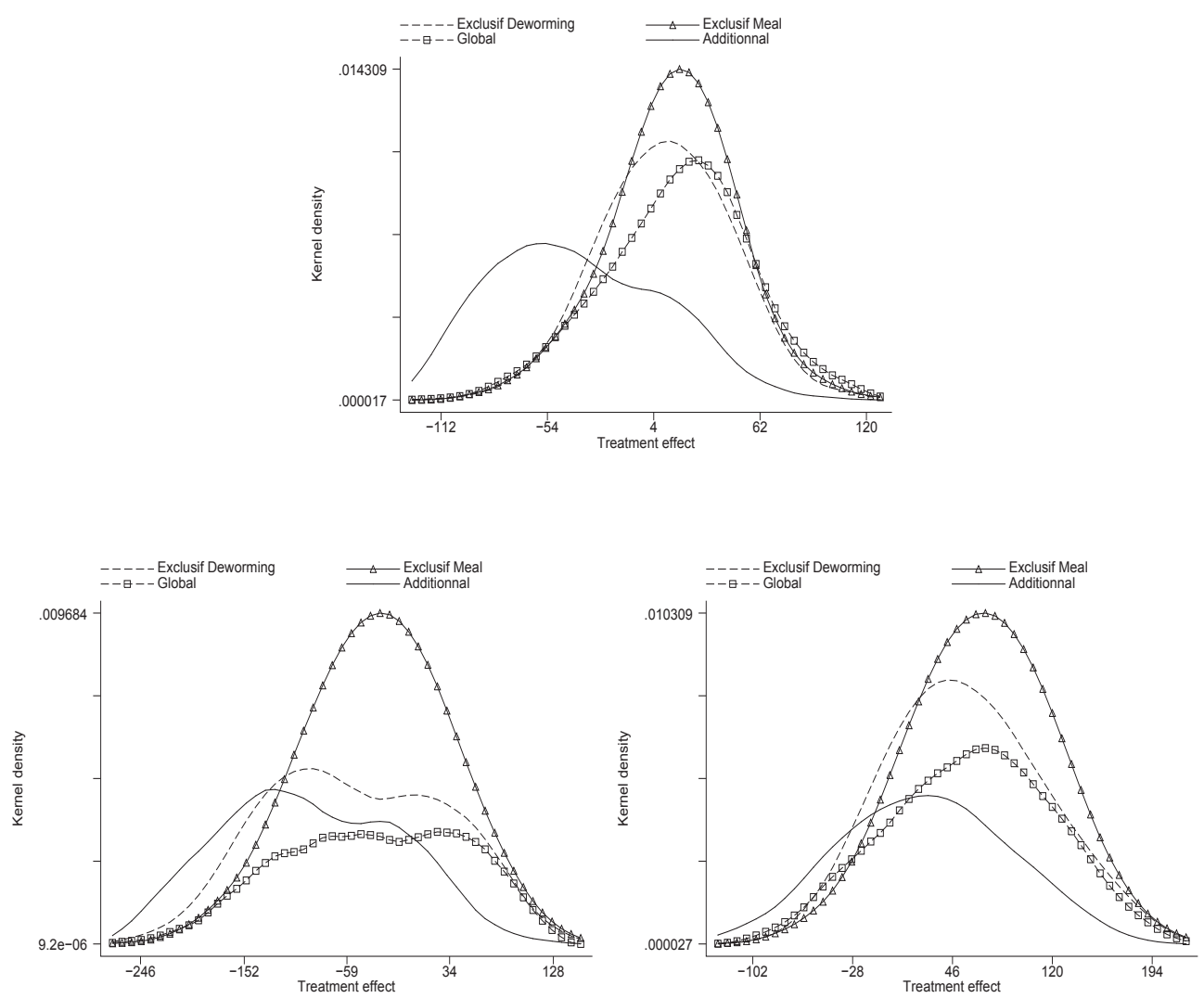

Figure B.6: Distribution of treatment effects for enrollment rate. [Top]: Average Treatment Effect (ATE) [Bottom-left]: Average Treatment Effect on the Treated (ATET). [Bottom-right]: Average Treatment Effect on the Nontreated (ATENT) 

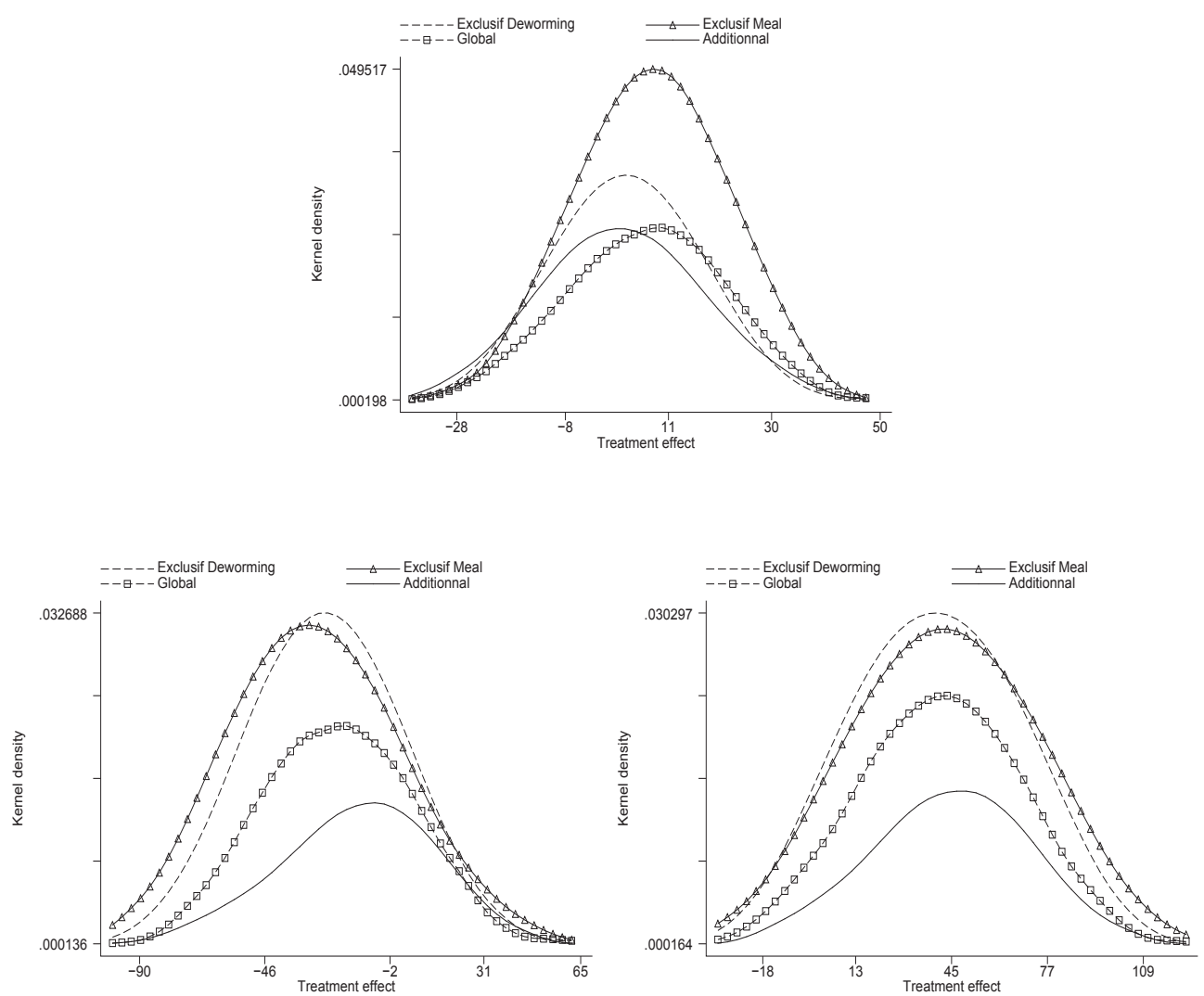

Figure B.7: Distribution of treatment effects for promotion rate. [Top]: Average Treatment Effect (ATE) [Bottom-left]: Average Treatment Effect on the Treated (ATET). [Bottom-right]: Average Treatment Effect on the Nontreated (ATENT) 

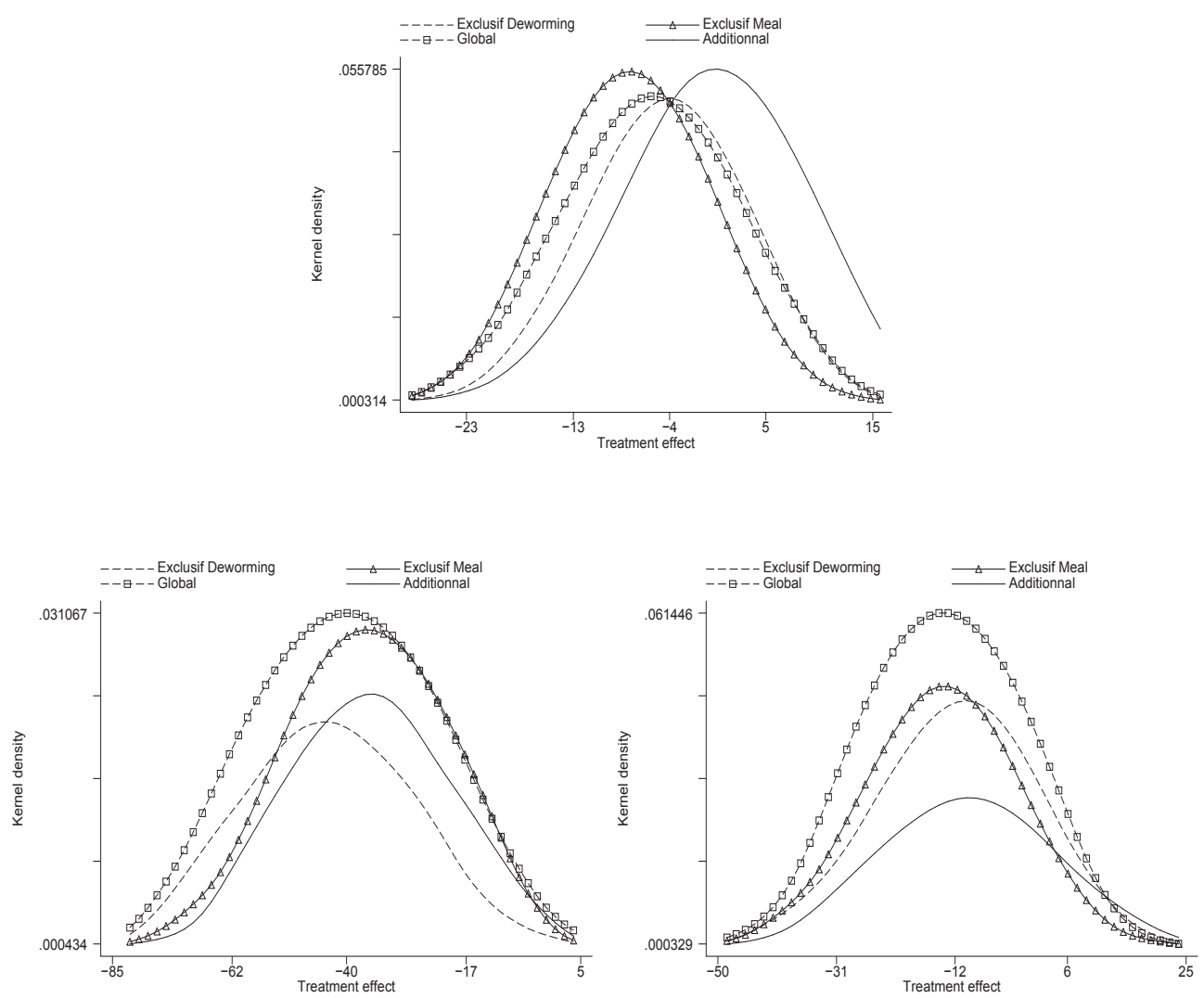

Figure B.8: Distribution of treatment effects for dropout rate. [Top]: Average Treatment Effect (ATE) [Bottom-left]: Average Treatment Effect on the Treated (ATET). [Bottom-right]: Average Treatment Effect on the Nontreated (ATENT) 

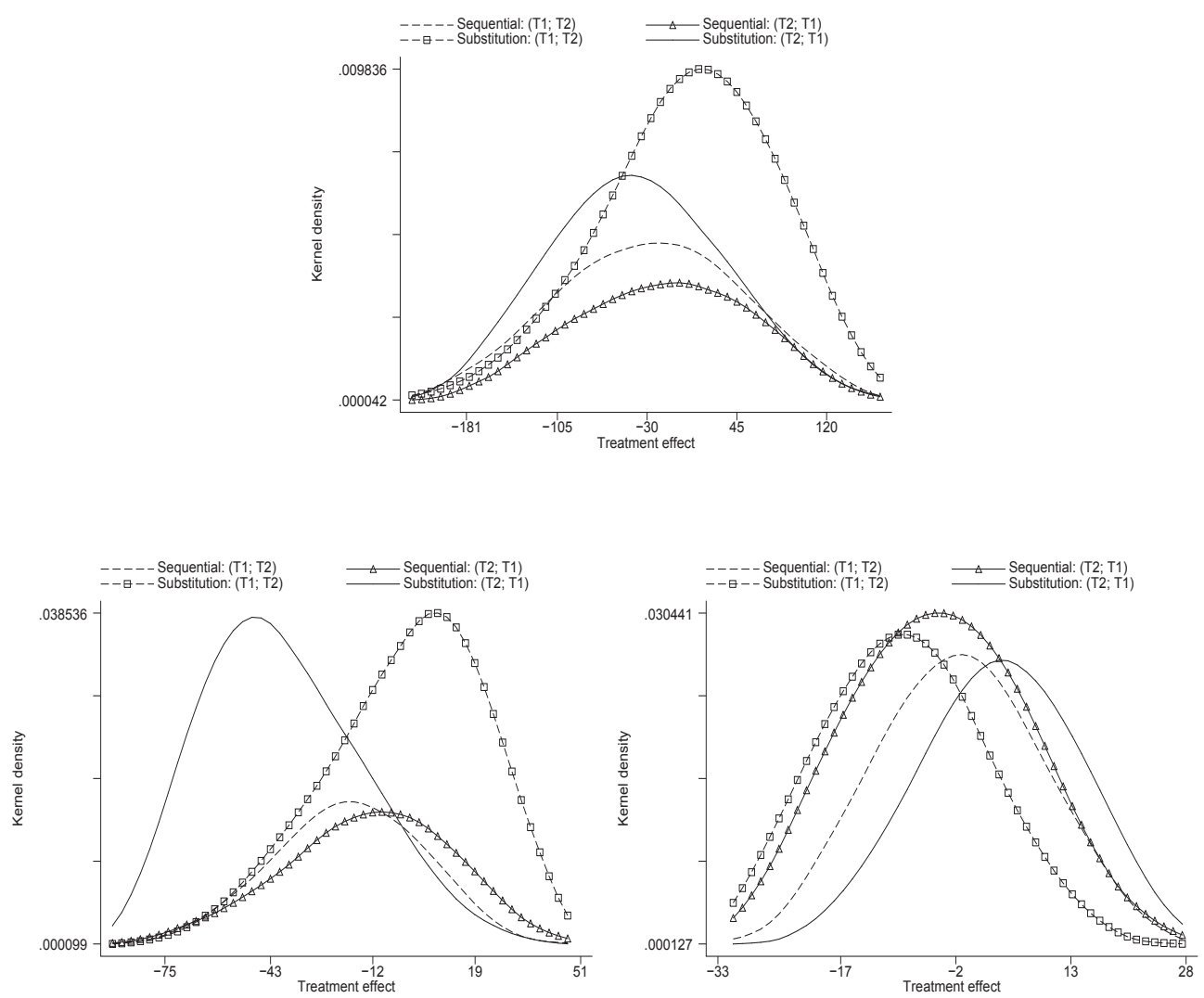

Figure B.9: Distribution of sequential and substitution effects on the treated. [Top]: Enrollment rate [Bottom-left]: Promotion rate. [Bottom-right]: Dropout rate 

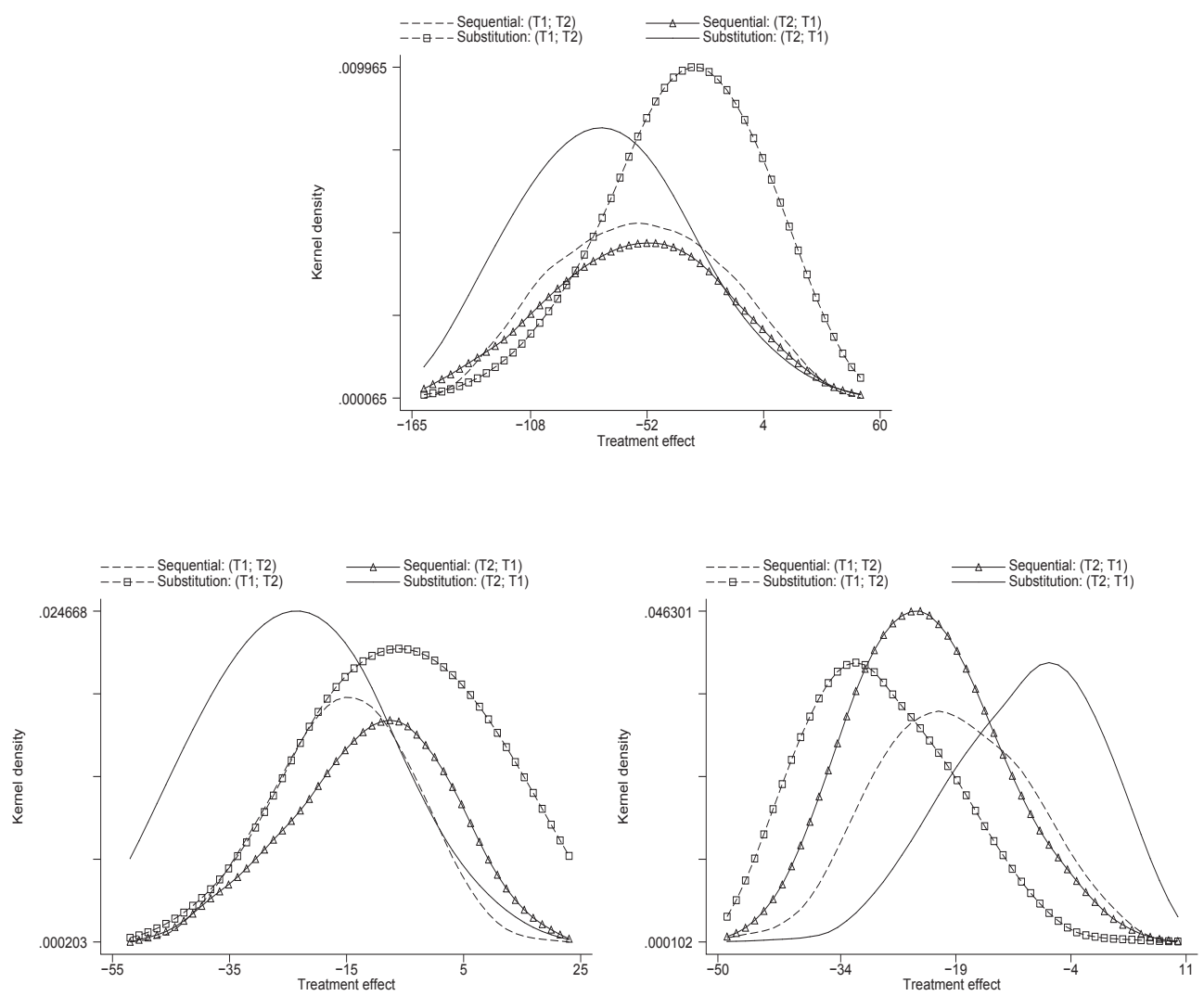

Figure B.10: Distribution of sequential and substitution effects on the nontreated. [Top]: Enrollment rate [Bottom-left]: Promotion rate. [Bottom-right]: Dropout rate 


\section{APPENDIX C}

Appendix Chapter 4

Appendix $\mathrm{C}$

Appendix C.1 Descriptive statistics before program 


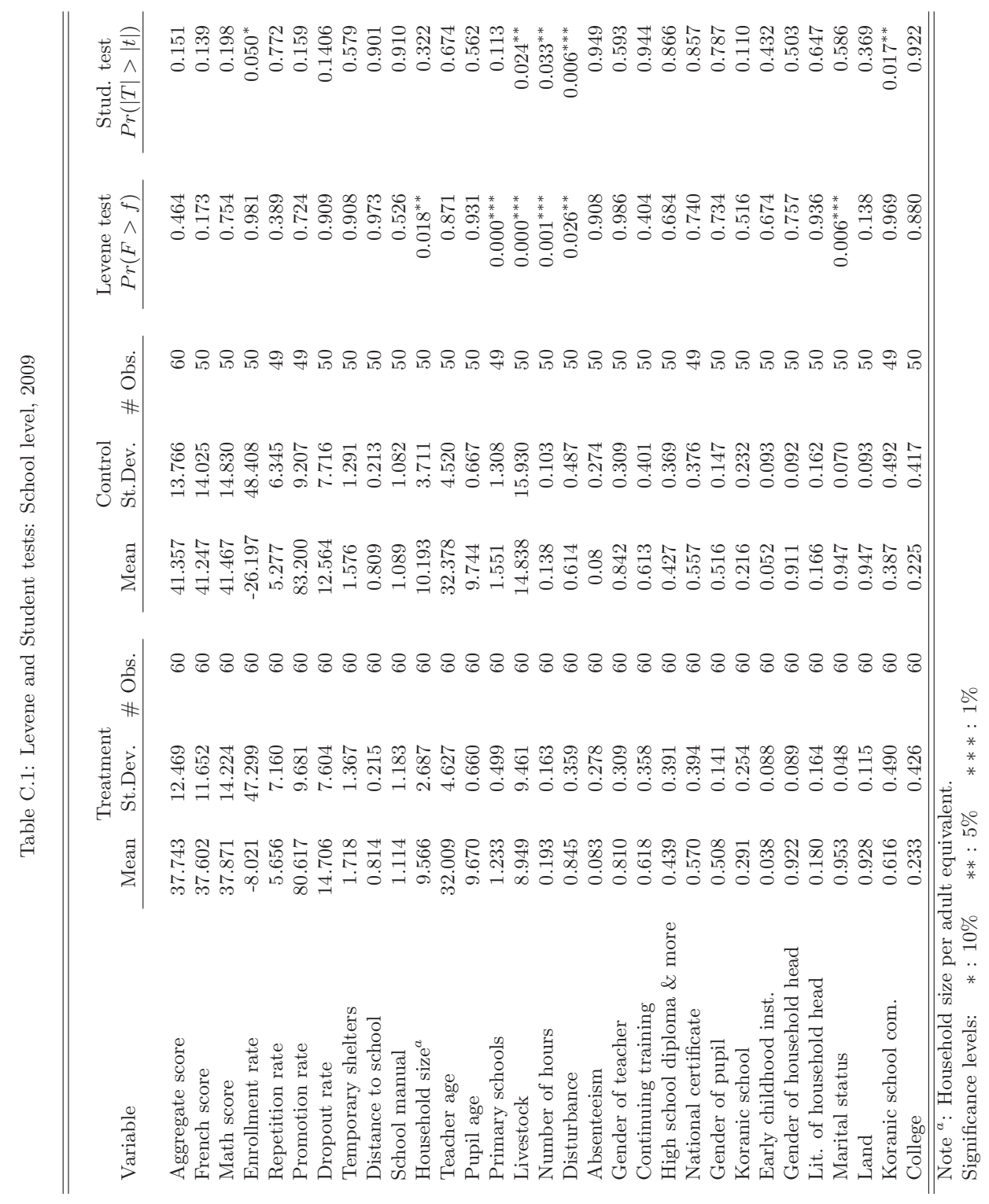




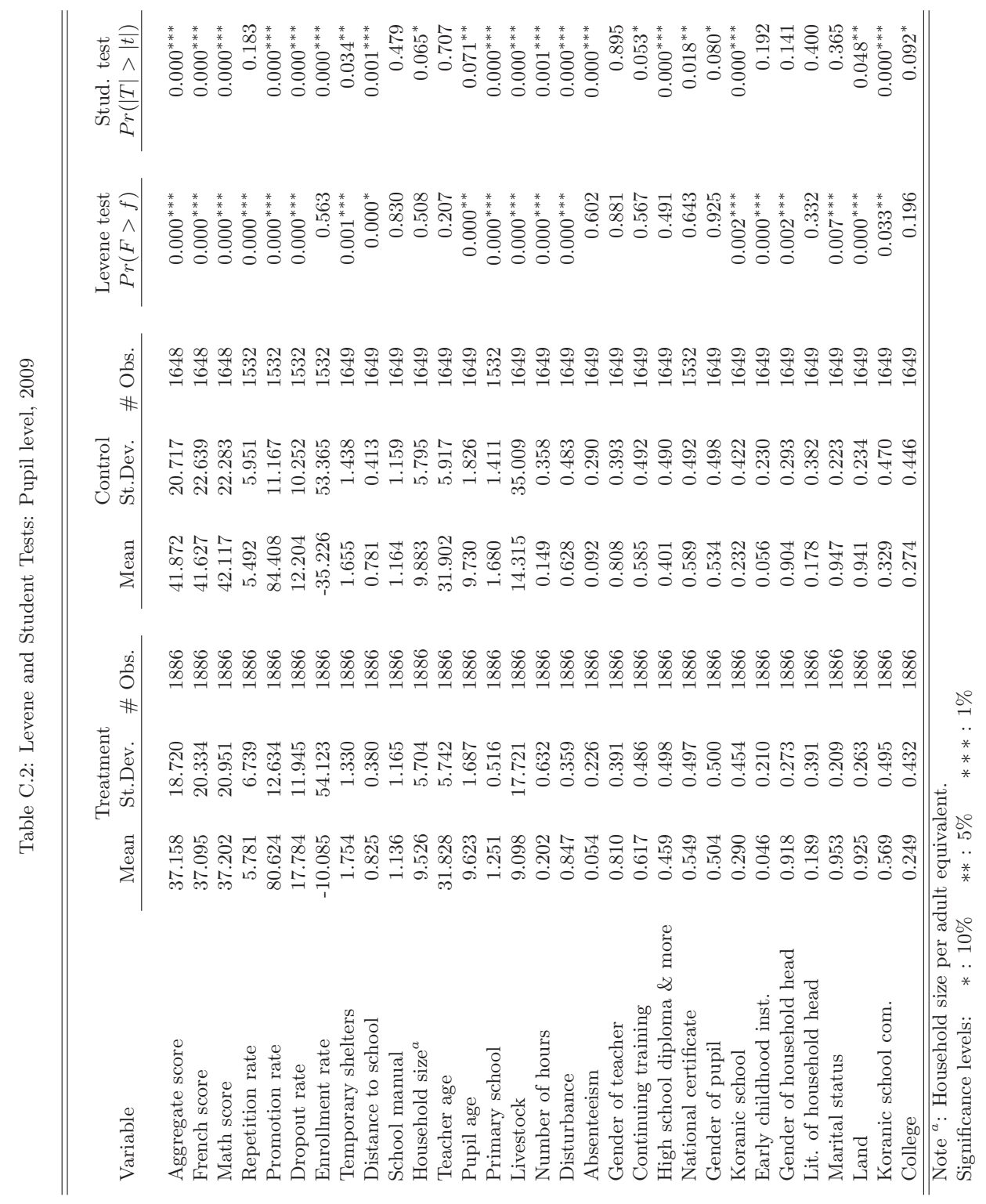




\section{Appendix C.2 Comparison of attritors and non attritors}

Table C.3: Descriptive statistics on the attrition status, 2009

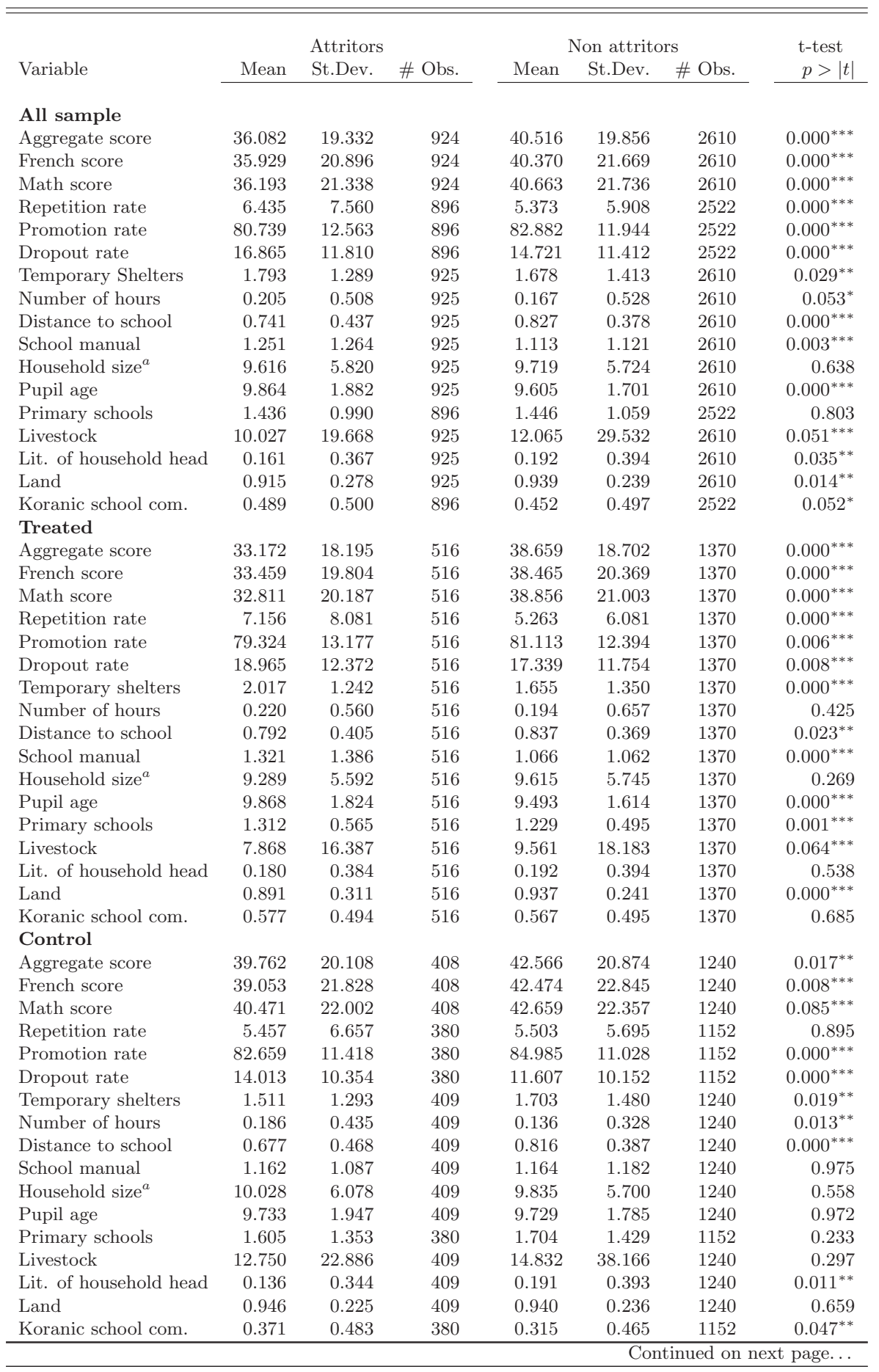


Table C.3 - continued

\begin{tabular}{|c|c|c|c|c|c|c|}
\hline \multirow[b]{2}{*}{ Variable } & \multicolumn{3}{|c|}{ Attritors } & \multicolumn{2}{|c|}{ Non attritors } & t-test \\
\hline & Mean & St.Dev. & \# Obs. & St.Dev. & \# Obs. & $p>|t|$ \\
\hline
\end{tabular}

Note $^{a}$ : Household size per adult equivalent.

Significance levels: $\quad *: 10 \% \quad * *: 5 \% \quad * * *: 1 \%$

Table C.4: Descriptive statistics of attritors by group, 2009

\begin{tabular}{|c|c|c|c|c|c|c|c|}
\hline \multirow[b]{2}{*}{ Variable } & \multicolumn{3}{|c|}{ Attritors of control } & \multicolumn{3}{|c|}{ Attritors of treatment } & \multirow{2}{*}{$\begin{array}{l}\text { t-test } \\
p>|t|\end{array}$} \\
\hline & Mean & St.Dev. & \# Obs. & Mean & St.Dev. & \# Obs. & \\
\hline Aggregate score & 39.762 & 20.108 & 408 & 33.172 & 18.195 & 516 & $0.000^{* * *}$ \\
\hline French score & 39.053 & 21.828 & 408 & 33.459 & 19.804 & 516 & $0.000^{* * *}$ \\
\hline Math score & 40.471 & 22.002 & 408 & 32.811 & 20.187 & 516 & $0.000^{* * *}$ \\
\hline Repetition rate & 5.457 & 6.676 & 380 & 7.156 & 8.081 & 516 & $0.000^{* * *}$ \\
\hline Promotion & 82.659 & 11.418 & 380 & 79.324 & 13.177 & 516 & $0.000^{* * *}$ \\
\hline Dropout rate & 14.013 & 10.354 & 380 & 18.965 & 12.372 & 516 & $0.000^{* * *}$ \\
\hline Temporary shelters & 1.511 & 1.293 & 409 & 2.017 & 1.242 & 516 & $0.000^{* * *}$ \\
\hline Distance to school & 0.677 & 0.468 & 409 & 0.792 & 0.405 & 516 & $0.000^{* * *}$ \\
\hline Number of hours & 0.186 & 0.435 & 409 & 0.220 & 0.560 & 516 & 0.312 \\
\hline School manual & 1.162 & 1.087 & 409 & 1.321 & 1.386 & 516 & $0.057^{* *}$ \\
\hline Household size $^{a}$ & 10.028 & 6.078 & 409 & 9.289 & 5.592 & 516 & $0.055^{* *}$ \\
\hline Pupil age & 9.733 & 1.947 & 409 & 9.968 & 1.824 & 516 & $0.058^{* *}$ \\
\hline Primary schools & 1.605 & 1.353 & 380 & 1.312 & 0.565 & 516 & $0.000^{* * *}$ \\
\hline Livestock & 12.750 & 22.886 & 409 & 7.868 & 16.387 & 516 & $0.000^{* * *}$ \\
\hline Lit. of household head & 0.136 & 0.344 & 409 & 0.180 & 0.384 & 516 & $0.075^{* *}$ \\
\hline Land & 0.946 & 0.225 & 409 & 0.891 & 0.311 & 516 & $0.002^{* * *}$ \\
\hline Koranic school com. & 0.371 & 0.483 & 380 & 0.577 & 0.494 & 516 & $0.000^{* * *}$ \\
\hline
\end{tabular}

Note $^{a}$ : Household size per adult equivalent.

Significance levels: $\quad *: 10 \% \quad * *: 5 \% \quad * * *: 1 \%$

\section{Appendix C.3 Descriptive statistics after program}




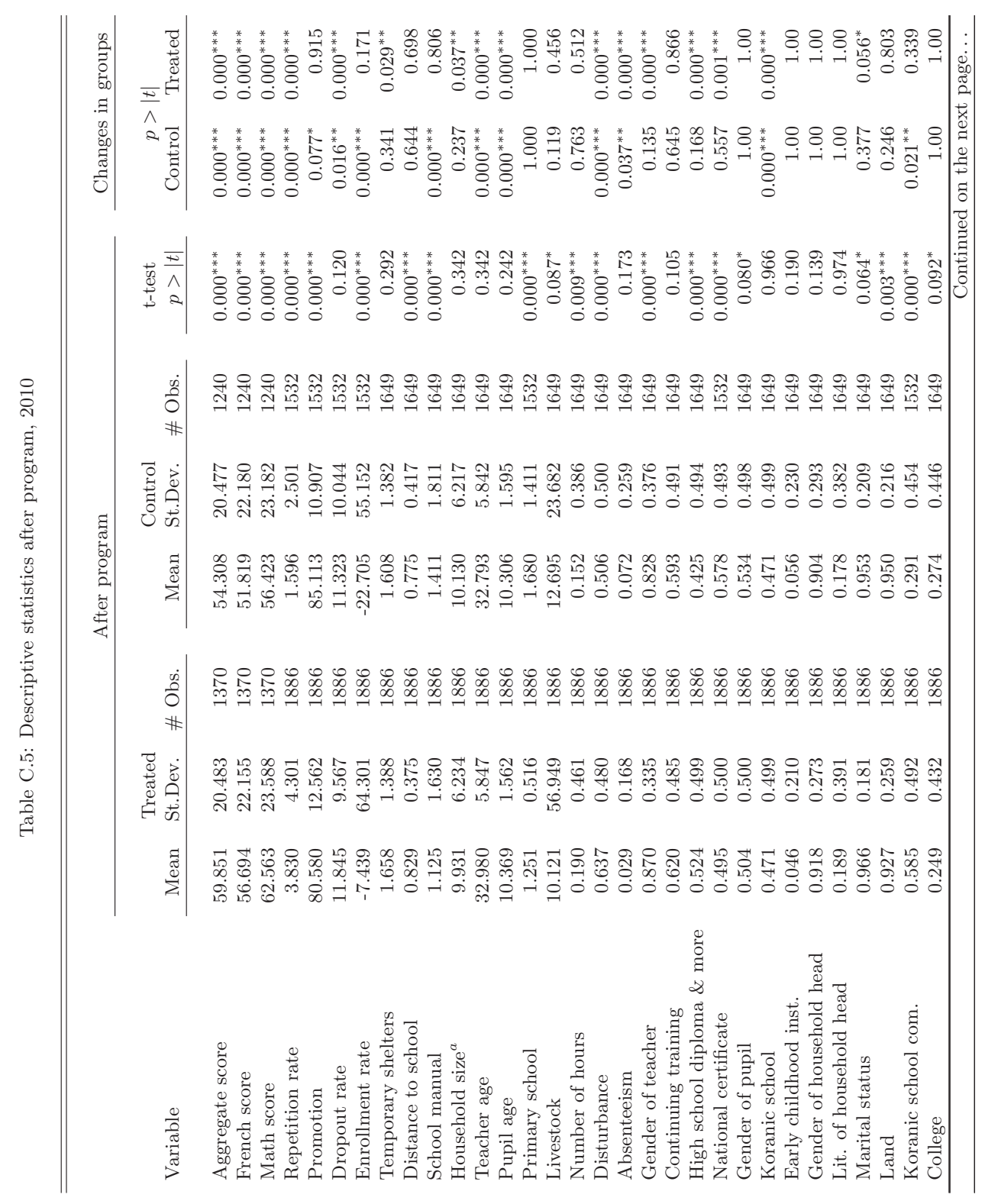




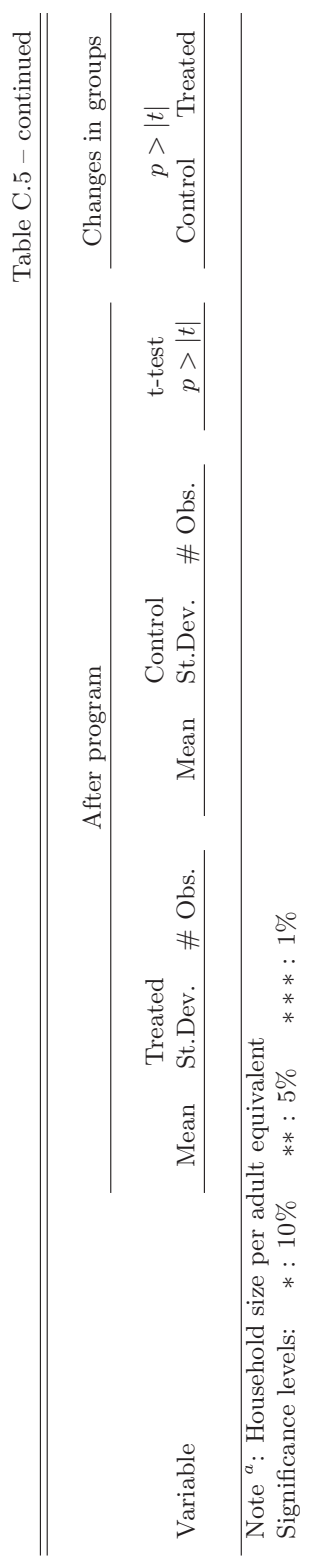


Programmabeoordeling is een belangrijk instrument om beleidsmakers te informeren over de doeltreffendheid van programma's en de doeltreffende toewijzing van hulpmiddelen. In verschillende studies werd de impact onderzocht van schoolmaaltijd- en ontwormingsprogramma's op de onderwijsresultaten in ontwikkelingslanden. Er bestaan echter nog altijd tegenstrijdige bewijzen over de doeltreffendheid van die programma's met betrekking tot de schoolresultaten. Er zijn ook weinig bewijzen van de kostenefficiëntie van die programma's. Hoewel er in de laatste jaren in Senegal voedings-en gezondheidservaring ontwikkeld is, is er geen empirisch onderzoek voor de beoordeling van de impact van schoolmaaltijd- en ontwormingsprogramma's op de schoolresultaten dat ook thema's behandelt met betrekking tot de complementariteit of vervangbaarheid van de twee programma's.

In deze scriptie wordt ernaar gestreefd dit hiaat op te vullen. We maken gebruik van experimentele en observationele gegevens en vertrouwen op zowel programmabeoordeling als op een structurele aanpak. De scriptie is opgedeeld in 5 hoofdstukken. Na een introductie in hoofdstuk 1 wordt er in hoofdstuk 2 gebruik gemaakt van observationele gegevens om de impact van maaltijden en ontworming als aparte programma's te beoordelen. We bestuderen de impact van deze programma's op de schoolprestaties gemeten door proefresultaten, terwijl er verder wordt gegaan op de factoren die de proefresultaten bepalen. Dit verklaart dat de maaltijd- en ontwormingsprogramma's de resultaten van leerlingen kunnen verhogen. De impact van elk programma is belangrijker voor leerlingen die op school zitten in samenhang met hun moeders. Ontwormen op school heeft een positief effect op de prestaties van de leerlingen, terwijl de ontworming thuis een negatieve impact heeft op hun resultaten. Daarnaast verhoogt het ontwormingsprogramma de resultaten aanzienlijk van leerlingen die op school zitten in associateie met hun moeders of schoolproject. Onder de determinanten van de proefresultaten hebben de onderwijsuitgaven, de grootte van het huishouden, de Koranschool, de instelling voor de vroege kinderjaren en het bestaan van een school in het dorp een positief effect op de prestaties van de leerlingen, terwijl controles zoals verstoorde cursussen, tijdelijke onderkomens, het geslacht en lerarenafwezigheid een negatieve invloed op hun cijfers. Op het gebied van de beleidsanalyse toont het Plaatselijke Gemiddelde Behandelingseffect (Local Average Treatment Effect, LATE) dat wanneer het doel is om de resultaten te verhogen het de meest kostenefficiënte manier is om de kantine en de oudervereniging te combineren in vergelijking met andere opties waaronder het beheercomité, het schoolproject, de schoolcoöperatie en de vermindering van de lerarenafwezigheid.

In hoofdstuk 3 worden de waarnemingsgegevens gebruikt om de voedings- en ontwormingsprogramma's van de school te beoordelen als een pakket voor de schoolprestaties (totaalscore, score voor Frans, score voor wiskunde, inschrijving, percentage zittenblijvers en vroegtijdige schoolverlaters), terwijl er verder wordt ingegaan op de determinanten van die prestaties. In dit hoofdstuk wordt een model ontwikkeld voor twee behandelingen, wat het mogelijk maakt verschillende be- 
handelingseffecten in te schatten, terwijl ook onderwerpen in verband met de complementariteit of vervangbaarheid van de programma's worden behandeld. Er is gebleken dat zowel maaltijdals ontwormingsprogramma's de behaalde cijfers verhogen, maar de combinatie van de twee programma's is nuttiger voor de verhoging van de prestaties van de leerlingen. De volgorde van de uitvoering speelt geen rol. De twee programma's zijn complementair om de cijfers en slaagpercentages te verhogen. Zij zijn echter substituten om het aantal vroegtijdige schoolverlaters te verminderen. Uit de kosten-batenanalyse blijkt dat ontworming veruit goedkoper is dan schoolmaaltijden. De uitvoering van maaltijdprogramma's vóór ontworming is echter kostenefficiënter dan omgekeerd.

Op het vlak van de bepalende factoren van de cijfers komen de in dit hoofdstuk vastgestelde resultaten overeen met die in hoofdstuk 2. Bovendien heeft ontworming thuis een negatieve invloed op de cijfers, terwijl het bestaan van latrines en van een apparaat om de handen te wassen op school een positief effect heeft op de cijfers.

Een interessant en intuitief resultaat is dat het hebben van een geletterd gezinshoofd het inschrijvingspercentage verhoogt. Het inschrijvingspercentage daalt daarentegen samen met de schoolkosten. Dit resultaat is belangrijk voor het onderwijsbeleid met het oog op de vermindering van de analfabetiseringsgraad in landelijke gebieden waar de levensstandaard zeer laag is. Als beleidsmakers gezinnen inderdaad willen aanmoedigen om hun kinderen naar school te sturen, zou het interessant zijn om ervoor te zorgen dat de onderwijskosten niet te hoog zijn. Er zijn nog twee vermeldenswaardige resultaten: het hebben van een mannelijke leraar en bezitten van bouwland hebben een negatieve invloed op de inschrijvingscijfers.

Samengevat suggereren de bevindingen uit het oogpunt van de bepalende factoren van de schoolresultaten in hoofdstuk 2 en 3 dat hoewel opvangcentra het percentage van vroegtijdige schoolverlaters verminderen en een positieve invloed hebben op de inschrijvings- en slaagpercentages, ze ook bijdragen tot de vermindering van de prestaties (cijfers) van de leerlingen. Als schoolboeken bovendien de competentie van de leerlingen kunnen verbeteren, lijkt het effect op de scores negatief te zijn, wanneer het aantal leerlingen per handboek hoger wordt (meer dan 4 leerlingen per handboek). Het doorvoeren van een beleid om de lerarenafwezigheid en storingen te verminderen zal de kwaliteit van het onderwijs verbeteren. Het lijkt ook belangrijk om meer ervaren leerkrachten (vanaf 33 jaar oud) en vrouwelijke leraren te bevorderen. Ten slotte kunnen de prestaties van de leerlingen onder andere verbeterd worden door de leraren aan te moedigen om de beroepskwalificaties CAP en CEAP te halen, leraren met een diploma van de middelbare school en meer als academische kwalificatie te ondersteunen en de duur van de voortgezette lerarenopleiding te verhogen.

In hoofdstuk 4 wordt gebruik gemaakt van experimentele gegevens om de impact van het maaltijdprogramma op de schoolresultaten te beoordelen in de aanwezigheid van afbouwing en gedeeltelijke naleving. De schattingsresultaten tonen aan dat het schoolmaaltijdprogramma de prestaties van de leerlingen en het inschrijvingspercentage aanzienlijk verhoogt en het percentage van vroegtijdige schoolverlaters vermindert. Het programma verhoogt daarentegen het percentage zittenblijvers en vermindert het slaagpercentage, wat onverwacht is. Dit kan voortvloeien uit de door de Senegalese regering genomen maatregelen die eruit bestaan het zittenblijven te elimineren tijdens het eerste jaar van elke stap in de lagere schoolcyclus en wordt beperkt tot $5 \%$ van het maximaal percentage zittenblijvers op het einde van elke stap.

Er is ook vastgesteld dat het maaltijdprogramma een groter effect heeft voor meisjes ten opzichte van jongens in het geval van de wiskundescore, terwijl jongens beter presteren dan de meisjes voor Frans.

Uit de kosten-batenanalyse in dit hoofdstuk blijkt dat het maaltijdprogramma gerechtvaardigd is uit het oogpunt van de economische voordelen, terwijl het resultaat van de kosten-batenanalyse overeenkomt met de bevindingen in hoofdstuk 3 .

In hoofdstuk 5 wordt een overzicht gegeven van de belangrijkste bevindingen van alle hoofdstukken plus enkele beleidsimplicaties. Er wordt ook ingegaan op de beperkingen van de scriptie 
en de noodzaak van toekomstige onderzoeksprojecten wordt gerechtvaardigd.

Over het algemeen worden er in deze scriptie empirische bewijzen verstrekt van de impact van maaltijd- en ontwormingsprogramma's op de schoolresultaten door middel van zowel experimentele als niet-experimentele onderzoeken. Voor zover we weten, is dit het eerste onderzoek dat aantoont dat de combinatie van maaltijd- en ontwormingsprogramma als pakket doeltreffender zou zijn om de schoolresultaten te verhogen, terwijl het effect van elke tussenkomst en diverse andere effecten gegeven worden om de beleidsmakers te informeren. 
Fatoumata Lamarana Diallo Sow was born and grew up in Labé, Guinea on July 31, 1980. She completed her high school education in Mathematical Science at Labé in 1999. In 2004, Mrs. Sow holds a master degree in Economics from Gamal Abdel Nasser University in Conakry, Guinea after one year spent at the 'Centre Préparatoire Commun (CPC)' of the Polytechnic Institute at the same university where she successfully completed the year. In 2006, she received her Post-graduate diploma in International Economics from the University Cheikh Anta Diop (UCAD) in Dakar thanks to a scholarship from the 'Programme de Troisième Cycle Interuniversitaire (PTCI)'. To obtain her post-graduate diploma, she wrote on the topic 'Analyzing multidimensional poverty in Guinea using the fuzzy set approach' for which she got a research grant from the African Economic Research Consortium (AERC) based in Nairobi. Since 2008, she has been following the 'Nouveau Programme de Troisième Cycle Inter-universitaire (NPTCI)' in Africa as a $\mathrm{PhD}$ fellow. She also completed specialization courses in Economics of Human Resources in the PhD program. In 2010, and 2011 Mrs. Sow has been a visiting PhD fellow at the United Nations University-Maastricht Economic and Social Research Institute on Innovation and Technology (UNU-MERIT) in Maastricht, Netherlands. Parallel to her PhD research, Mrs. Sow is a teaching assistant at the faculty of Economics at UCAD. She is also a research assistant at the 'Consortium pour la Recherche Economique et Sociale (CRES)', where she participates actively in the research activities of the institute since 2007. In addition, since 2012, Mrs. Sow is a member of the Knowledge Platform Development Policies created by the Ministry of Foreign Affairs in the Netherlands.

During the research for her $\mathrm{PhD}$, Mrs. Sow attended several conferences and seminars including the African Econometric Society (AES) Conference on 'Econometric Modelling in Africa', at the Kenya School of Monetary Studies, July, 2011, Nairobi, Kenya; the Center for the Study of African Economies (CSAE) Conference 2010 'Economic Development in Africa' University of Oxford, March, 2010 Oxford, United Kingdom and the Africa Research Workshop, October, 2011 at UNU-MERIT. In addition, she is a member of the African Economic Research Consortium (AERC) network and the Partnership for Economic Policy (PEP) network, and has received fellowships and grants from NPTCI, AERC, UNU-MERIT and the 'Ministère de l'Enseignement Supérieur et de la Recherche' of Senegal. 


\section{UNITED NATIONS UNIVERSITY}

\section{UNU-MERIT}

\section{4}

\section{Fatoumata Lamarana Diallo}

Evaluation of Meal and Deworming Programs for Primary Schools in Rural Senegal

\section{3}

\section{Anant Kamath}

Information Sharing through Informal Interaction in Low-Tech Clusters

\section{Flavia Pereira de Carvalho}

What we talk about when we talk about Brazilian Mulitantionals: an investigation on Brazilian FDI, economic structure, innovation and the relationship between them

\section{Jun Hou}

Complementarity in Innovation and Development: A Cross-country Comparison

\section{Rufin Baghana}

Impacts of Government Incentives to R\&D, Innovation and Productivity:

A Microeconometric Analysis of the Québec Case

\section{Lilia I. Stubrin}

High-Tech Activities in Emerging Countries: A Network perspective on the Argentinean biotech activity

\section{2}

\section{Abdul Waheed}

Innovation Determinants

and Innovation as a Determinant:

Evidence from Developing Countries

\section{Bilal Mirza}

Energy Poverty and Rural Energy Markets in Pakistan

\section{Benjamin Engelstätter}

Enterprise Software and Video Games: An Empirical Analysis

\section{Fulvia Farinelli}

Natural Resources, Innovation and Export Growth: The Wine Industry in Chili and Argentina

\section{Rodolfo Lauterbach}

Innovation in Manufacturing: From Product Variety and Labor Productivity Growth to Economic

Development in Chile

\section{Kirsten Wiebe}

Quantitative Assessment of Sustainable Development and Growth in Sub-Saharan Africa.

\section{Julio Miguel Rosa}

Organizational Strategies, Firms' Performance and Spatial Spillovers. The Canadian Case in Research and Development.

\section{Johannes Wilhelmus Marie Boels}

Joseph Schumpeter, honderd jaar economische ontwikkeling. Een historisch-theoretische beschouwing.

\section{1}

\section{Daniel Vertesy}

Interrupted Innovation: Emerging economies in the structure of the global aerospace industry.

\section{Tina Saebi}

Successfully managing alliance portfolios: an alliance capability view.

\section{Nora Engel}

Tuberculosis in India - A case of innovation and control.

\section{Evans Mupela}

Connectivity and growth in Sub-Saharan Africa: The role of communication satellites

\section{Nantawan Kwanjai}

Cross cultural intelligence amid intricate cultural webs - A tale of the UnDutchables in the land of 1002 smiles 


\section{Lina Sonne}

Innovation in Finance to Finance Innovation:

Supporting pro-poor entrepreneur-based

innovation

\section{0}

\section{Fernando Santiago}

Human Resources Management Practices and Learning for Innovation in Developing Countries:

Pharmaceutical Firms in Mexico

\section{Zakaria Babutsidze}

Essays on Economies with Heterogenous Interacting Consumers

\section{Bertha Vallejo}

Learning and Innovation Under Changing Market Conditions: The Auto Parts Industry in Mexico

\section{Donatus Ayitey}

Technical Change, Competitiveness and Poverty Reduction: A Study of the Ghanaian Apparel Industry

\section{Sergey Fillipov}

Multinational Subsidiary Evolution: Corporate Change in New EU Member States

\section{Asel Doranova}

Technology Transfer and Learning under the Kyoto regime; Exploring the Technological Impact of CDM projects in developing countries

\section{9}

\section{Alexis Habiyaremye}

From Primary Commodity Dependence to Diversification and Growth". "Absorptive Capacity and Technological Catch Up in Botswana and Mauritius".

\section{Yoseph Getachew}

The Role of Public Capital in Economic Development

\section{Sandra Leitner}

Embodied Technological Change and Patterns of Investment in Austrian Manufacturing

\section{Semih Akçomak}

The Impact of Social Capital on Economic and Social Outcomes

\section{Abraham Garcia}

The Role of Demand in Technical Change

\section{Saurabh Arora}

Coherence in socio-technical systems: a network perspective on the innovation process

\section{8}

\section{Rutger Daems}

Medicines for the developing world

\section{Johannes Hanel}

Assessing Induced Technology - Sombart's

Understanding of Technical Change in the History of Economics

\section{Rifka Weehuizen}

Mental Capital: the economic significance of mental health

\section{Danielle Cloodt}

The relationship between R\&D partnership formation, social embeddedness and innovative performance

50. Sabine Fuss

Sustainable Energy Development under Uncertainty

\section{7}

\section{Tobias Kronenberg}

Reconciling Environmental Conservation with Economic Prosperity: The Feasibility of Double Dividends in the Short and Long Run

\section{Viktoria Kravtsova}

Assessing the Impact of Foreign Direct Investment in Transition Economies

\section{Suhail Sultan}

The Competitive Advantage of Small and Medium Sized Enterprises: The Case of Jordan's Natural Stone Industry

\section{6}

46. Bulat Sanditov

Essays on Social Learning and Imitation 


\section{Mamata Parhi}

Dynamics of New Technology Diffusion: A Study of the Indian Automotive Industry

\section{Andreas Reinstaller}

Social structures and the innovation process: Their role in the demand of firms and consumers

\section{Rose Kiggundu}

Innovation systems and development : the journey of a Beleaguered Nile Perch Fishery in Uganda

\section{Thomas Pogue}

The Evolution of Research Collaboration in South African Gold Mining: 1886-1933

\section{Geoffrey Gachino}

Foreign Direct Investment, Spillovers and Innovation: The Case of Kenyan Manufacturing Industry

\section{0. Önder Nomaler}

Technological Change, International Trade and Growth - An Evolutionary, Multi-Agents-Based Modeling Approach

\section{5}

\section{Samia Satti Osman Mohamed-Nour} Change and Skill Development in the Arab Gulf Countries

\section{Elad Harison}

Intellectual Property Rights: Economics and Policy Analysis

\section{Daniel Dalohoun}

The relationship between R\&D partnership formation, social embeddedness and innovative performance: a multi-level approach of social embeddedness

\section{Müge Ozman}

Networks, Organizations and Knowledge

\section{Bas Straathof}

Product variety and economic growth - The counteracting effects of scale and idiosyncrasy

\section{Wilfred Schoenmakers}

Knowledge Flows between Multinational

Companies: A Patent Data Analysis

\section{Myriam Cloodt}

Mergers and Acquisitions (M\&As) in High-Tech Industries: Measuring the Post-M\&A Innovative Performance of Companies

\section{4}

\section{Paola Criscuolo}

R\&D Internationalisation and Knowledge Transfer. Impact on MNEs and their Home Countries

\section{Maarten Verkerk}

Trust and Power on the Shop Floor

\section{Gottfried Leibbrandt}

Adoption, harmonization and succession of network technologies across countries

\section{Mark Sanders}

Skill Biased Technical change - Its Origins, the Interaction with the Labour Market and Policy Implications

\section{3}

\section{Nadine Roijakkers}

Inter-firm cooperation in high-tech industries: a study of R\&D partnerships in pharmaceutical biotechnology

\section{Viki Sonntag}

Speed, Scale and Sustainability

\section{Masaru Yarime}

From End-of-Pipe Technology to Clean Technology

\section{Stéphane Malo}

The combinatorial Chemistry Revolution Sustaining a Superior Performance Position through Technological Learning

\section{2}

\section{Annelies Hogenbirk}

Determinants of Inward Foreign Direct Investment: the Case of the Netherlands 
2001

\section{John Adeoti}

Technology Investment in Pollution Control in SubSaharan Africa: The Case of the Nigerian

Manufacturing Industry

\section{Edward Huizenga}

Innovation Management: How Frontrunners Stay Ahead. An Empirical Study on Key Success Factors in the ICT sector

\section{0}

\section{Machiel van Dijk}

Technological Change and the Dynamics of Industries. Theoretical Issues and Empirical evidence from Dutch Manufacturing

\section{9}

\section{Jan Cobbenhagen}

Managing Innovation at the Company Level: A Study on Non-Sector-Specific Success Factors

\section{Marjolein Caniëls}

Regional Growth Differentials: The Impact of Locally Bounded Knowledge Spillovers

\section{8}

\section{Aldo Geuna}

Resource allocation and knowledge production: Studies in the economics of university research

\section{6}

\section{Reinoud Joosten}

Dynamics, Equilibria, and Values

\section{Hugo Kruiniger}

Investment, R\&D, and the Financing Decisions of the Firm

\section{5}

\section{Hans van Meijl}

Endogenous Technological Change: The Case of Information Technology. Theoretical Considerations and Empirical Results

\section{René Kemp}

Environmental Policy and Technical Change. A Comparison of the Technological Impact of Policy Instruments

\section{Rohini Acharya}

The Impact of New Technologies on Economic Growth and Trade. A Case Study of Biotechnology

\section{Geert Duysters}

The Evolution of Complex Industrial Systems. The Dynamics of Major IT Sectors

\section{Marjan Groen}

Technology, Work and Organisation, A Study of the Nursing Process in Intensive Care Units

\section{4}

\section{Huub Meijers}

On the Diffusion of Technologies in a Vintage Framework; Theoretical Considerations and Empirical Results

\section{Theon van Dijk}

The Limits of Patent Protection. Essays on the Economics of Intellectual Property Rights

\section{Hans Voordijk}

Naar Integrale Logistiek in Bedrijfsketens, Ontwikkelingen in de Bouw

\section{3}

\section{Paul Diederen}

Technological Progress in Enterprises and Diffusion of Innovations. Theoretical Reflections and Empirical Evidence.

\section{Ben Dankbaar}

Economic Crisis and Institutional Change. The crisis of Fordism from the perspective of the automobile industry

\section{Hanno Roberts}

Accountability and Responsibility: The Influence of Organisation Design on Management Accounting 
1992

\section{Bart Verspagen}

Uneven Growth Between Interdependent

Economies. An Evolutionary View on Technology

Gaps, Trade and Growth

\section{Sjoerd Romme}

A Self-organization Perspective on Strategy

Formation

\section{9}

\section{John Spangenberg}

Economies of Scale, and Atmosphere in Research Organisations

\section{8}

\section{John Hagedoorn}

Evolutionary and heterodox innovation analysis : a study of industrial and technological development in process control and information technology 